\title{
Handbook of Solar Energy Data
} for South-Facing Surfaces in the United States

Volume I: An Insolation, Array Shadowing, and Reflector Augmentation Model

Jeff H. Smith

January 15,1980

Prepared for

U.S. Department of Energy

Through an agreement with

National Aeronautics and Space Administration

by

Jet Propulsion Laboratory

California Institute of Technology

Pasadena, California

(.JPL Publication 79-103, VOLUME I) 


\section{DISCLAIMER}

This report was prepared as an account of work sponsored by an agency of the United States Government. Neither the United States Government nor any agency Thereof, nor any of their employees, makes any warranty, express or implied, or assumes any legal liability or responsibility for the accuracy, completeness, or usefulness of any information, apparatus, product, or process disclosed, or represents that its use would not infringe privately owned rights. Reference herein to any specific commercial product, process, or service by trade name, trademark, manufacturer, or otherwise does not necessarily constitute or imply its endorsement, recommendation, or favoring by the United States Government or any agency thereof. The views and opinions of authors expressed herein do not necessarily state or reflect those of the United States Government or any agency thereof. 


\section{DISCLAIMER}

Portions of this document may be illegible in electronic image products. Images are produced from the best available original document. 


\section{Handbook of Solar Energy Data for South-Facing Surfaces in the United States}

Volume I: An Insolation, Array Shadowing, and Reflector Augmentation Model

Jeff $H$. Smith

January 15,1980

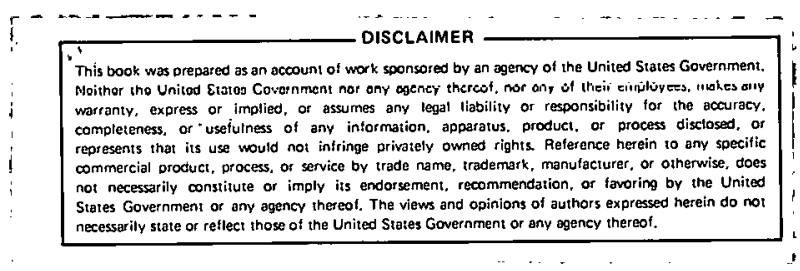

Prepared for

U.S. Department of Energy

Through an agreement with

National Aeronautics and Space Administration

by

Jet Propulsion Laboratory

California Institute of Technology

Pasadena, California

(JPL Publication 79-103, VOLUME I) 
Prepared by the Jet Propulsion Laboratory, California Institute of Technology, for the Department of Energy through an agreement with the National Aeronautics and Space Administration.

The JPL Luw-Cost Solar Array Project is sponsored by the Department of Energy (DOE) and forms part of the Solar Photovoltaic Conversion Program to initiate a majur effort toward the development of low-cost șlar arrays.

This report was prepared as an account of work sponsored by the United States Government. Neither the United States nor the United States Department of Energy, nor any of their employees, nor any of their contractors, subcontractors, or their employees, makes any warranty, express or implied, or assumes any legal liability or responsibility for the accuracy, completeness or usefulness of any information, apparatus, product or process disclosed, or represents that its use would not infringe privately owned rights. 
This handbook provides estimates of average available solar insolation to fixed, flat-plate, south-facing collector surfaces at various array tilt angles at numerous sites in the United States. This first volume contains average daily, total insolation estimates, by month, and annual totals for 235 locations. The second and third volumes contain the daily profiles by hour used to compute the daily totals for the 235 locations (at selected array tilt angles).

A model that estimates the direct, diffuse, and reflected components of total insolation on an hourly, daily, and monthly basis is presented. A shadow loss model and a reflector augmentation model providing estimates of the losses and galns assoclaced with various fixed array geometries are also described. These models can be used with the insolation model provided or with other recorded data.

A FORTRAN computer program with user's guide is presented. The program can be used to generate additional handbook values or to examine the effects of array shadowing and fixed reflector augmentation effects on a daily, monthly, or annual basis. Array shadowing depends on location, array size, array tilt, array separation, and time. The program can be used to examine trade-offs between array spacing and insolation losses due to shadowing. The reflector augmentation program can be used to examine trade-offs among array size and tilt, separation, and reflector tilt to determine the combination of design values that optimize the economic objectives or technical criteria of the system. 


\section{THIS PAGE}

\section{WAS INTENTIONALLY LEFT BLANK}




\section{ACKNOWLEDGMENTS}

Thanks is extended to the Low-Cost Solar Array Project at JPL and especially to Donald Rapp, Paul Sutton, and Peter Tsou, whose numerous suggestions and encouragement helped make this handbook a reality. The author would also like to acknowledge the assistance of Glenn Zucman, who verified the insolation model with an APL program. 
THIS PAGE

\section{WAS INTENTIONALLY LEFT BLANK}




\section{CONTENTS}

GLOSSARY -

I. INTRODUCTION

II. THE INSOLATION MODEL -

A. NOMENCLATURE AND ASSUMPTIONS --

B. PREVIOUS WORK - $2-2$

C. CALCULATION PROCEDURE -

D. SPECTRAL CONSIDERATIONS - 2-15

III. ARRAY SHADOWING -

A. THE PROBLEM - $3-1$

B. THE MODEL -

IV. REFLECTOR AUGMENTATION -

A. THE PROBLEM -

B. THE MODEL -

V. DISCUSSION -

A. INSOLATION MODEL SENSITIVITY

B. INSOLATION MODEL VERIFICATION -

C. SHADOW AND REFLECTOR MODELS -

VI. SOLINS COMPUTER PROGRAM USER'S GUIDE -- -

A. SUBPROGRAM INS -

B. SUBPROGRAMS SHADOW AND REFL -

C. COMPUTER CONFIGURATION REQUIRED --

MEMORY AND TIMING --ー-

D. ACCURACY OF RESULTS - 
E. CONTROL CARD REQUIREMENTS - 6-2

F. USER INSTRUCTIONS - 6-3

REFERENCES -

\section{APPENDICES}

A. LISTING OF SOLINS AND RELATED COMPUTER PROGRAMS --

B. SOLAR CLIMATE PARAMETER DATA FOR 235 LOCALITIES ------ B-1

C. AVERAGE DAILY SOLAR INSOLATION DATA BY MONTH AND ANNUAL TOTALS FOR 235 LOCALITIES -

D. AVERAGE HOURLY AND TOTAL DAILY INSOLATION DATA FOR 235 LOCALITIES -

\section{Figures}

2-1a. Cross Section of the Earth Showing Definitions of $\tau, \theta_{t}, \phi$, and $(\phi-\tau)$

2-1b. Angles Used in Solar Insolation Calculation ----- 2-5

2-2. Solar Spectral Irradiance, Standard Curve, Solar Constant $\left(1.377 \mathrm{~kW} / \mathrm{m}^{2}\right)$

3-1. Shadow Problam Gametry -

4-1. Reflector Augmentation Geometry - 4-2

6-1. SOLINS Deck Setup - 6-2 
2-1. Solar Declination ( $\delta$ ), the Ratio of Solar Radiation Intensity at Normal Incidence Outside Earth's Atmosphere to the Solar Constant $(\gamma)$, and Atmospheric Absorption Coefficient (B) $2-6$

2-2a. The Value of the $\mathrm{m}$ Factor $\left(\mathrm{kWh} / \mathrm{m}^{2}\right)$

2-2b. The Value of the $m$ Factor (BTU/ft ${ }^{2}$ ) ---

2-3. Reflectivity Values for 15 Characteristic Surfaces (Integrated Over Solar Spectrum and Angle of Incidence)

2-4. Average Reflectivity Values for 12 Representative Winter Landscapes 2-14 
THIS PAGE

\section{WAS INTENTIONALLY LEFT BLANK}




\section{GLOSSARY}

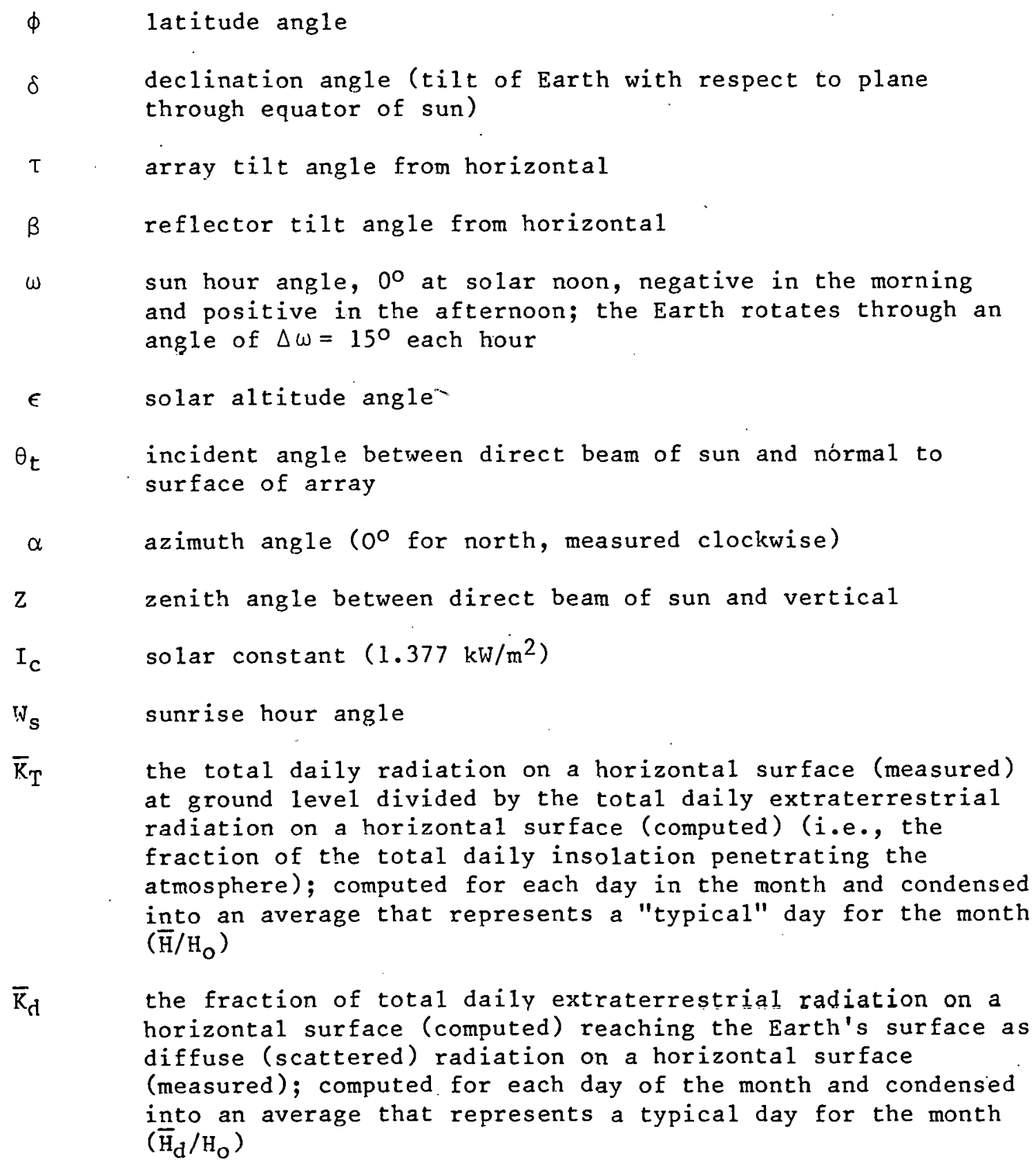

$\overline{\mathrm{K}}_{\mathrm{d}}$ the fraction of total daily extraterrestrial radiation on a horizontal surface (computed) reaching the Earth's surface as diffuse (scattered) radiation on a horizontal surface (measured); computed for each day of the month and condensed into an average that represents a typical day for the month $\left(\overline{\mathrm{H}}_{\mathrm{d}} / \mathrm{H}_{\mathrm{O}}\right)$

$\overline{\mathrm{H}}$ the average daily total radiation reaching a horizontal surface on the ground (measured) during a given month

$\mathrm{H}_{\mathrm{O}}$ daily total extraterrestrial radiation on a horizontal surface for a given month

monthly adjustment to the solar constant to account for Earth-sun distance variation 


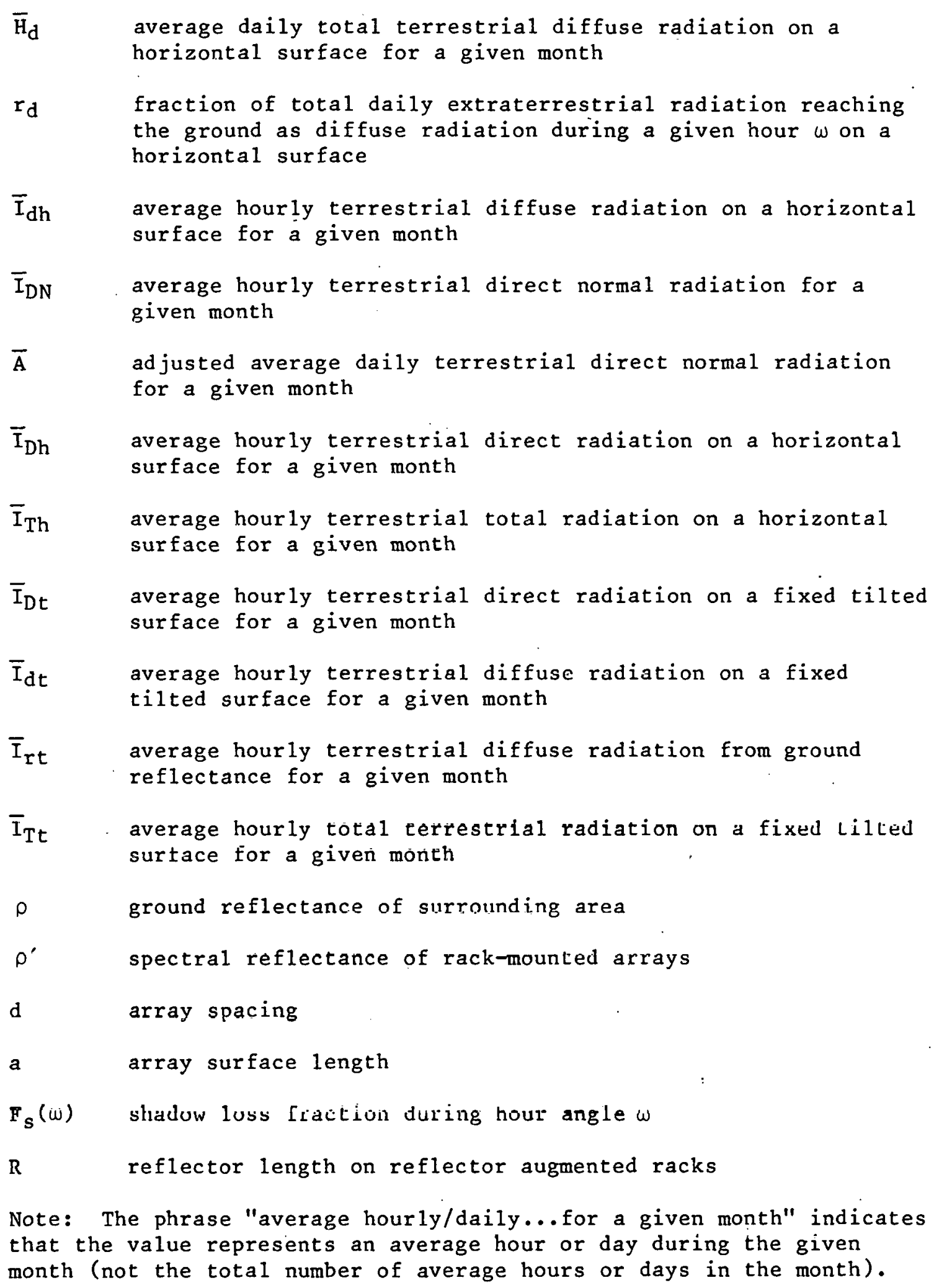




\section{SECTION I}

\section{INTRODUCTION}

This document is designed for engineers, designers, and homeowners. For the homeowner this handbook provides a quick reference for obtaining estimates of available solar insolation for numerous locations and array tilt angles. For the engineers and designers it provides a model and computer program that considers the effects of array shadowing and reflector augmentation as design variables.

The first objective of this publication is to provide a handbook reference document for estimating available solar insolation on southfacing fixed surfaces. The user interested in such data is referred directly to the Appendices. Hourly and daily insolation estimates are provided for 235 U.S. sites at a variety of array tilt angles. In the event the tilt angle is to be adjusted monthly or fixed at some intermediate angle other than the handbook angles, a computer program is provided to aid the user with more detailed requirements. The computer program is based on a parametric solar insolation model that takes into account the site specific solar climate of a particular location. (This document does not consider tracking surfaces although the computer program in Appendix A could be modified to do so.) The computer program enables the engineer/designer to conduct extensive sensitivity analyses on the orientation and location of solar collectors.

This publication is directed toward the engineer/designer who is interested in average insolation estimates that represent the typical solar climate of a particular site. In this sense, the model presented here is an "aggregate" model and does not attempt to model the atmospheric physics in a detailed fashion. In particular, hourly trends within a day are not modeled; that is, a symetric daily profile is presented. The latest available input data required by this model are provided in Appendix $B$ of this document.

The second objective of this publication is to address insolation problems specific to residential/commercial applications. Specifically, it is concerned with the placement of solar collectors on residential housing units and commercial properties (shopping centers, parking lots, etc.) where the amount of space available for collectors is limited by shadowing problems, energy output requirements, and costs. The array spacing problem is also relevant to central station solar power plants although spacing may not be as critical due to the larger land areas involved. The document addresses the relationships among available insolation, spacing, and shadowing for south-facing surfaces -- the costs associated with various spacing arrangements are not addressed. The reflector augmentation problem is related to the shadowing model. Given a tandem rack array design (see page 4-2), a reflective surface built into the support structure of the arrays yields a higher level of insolation at the collector surface. The insolation available to such a surface depends not only upon the 
amount of reflector augmentation, but also on array spacing and sun elevation angles at which shadowing occurs.

A multipurpose package of programs has been written based on the models presented in this report. Details of this package may be found in Section VI and Appendix A. For the user interested in south-facing fixed-surface data, Appendices $C$ and $D$ contain data for 235 sites. Shadowing and reflector augmentation data are not presented due to the many possible combinations of input parameters. In order to obtain insolation estimates that consider shadowing and reflector augmentation, Sections VI and Appendix A should be consulted for directions on how to run the program.

It should be noted that the modeling of the spectral response of photovoltaic cells by time of day and type of material has not been considered here due to the complexities associated with modeling light spectrum changes. This is discussed more in Section II.

Sections II, III, IV, and V present the models used to generate the handbook data and computer program package as well as a discussion of the assumptions and accuracy of the techniques. A careful examination of these sections is advised prior to altering the computer program. 


\section{SECTION II}

THE INSOLATION MODEL

There are basically two ways that solar radiation data can be used for design purposes in the photovoltaics area. One way is to use past hourly recorded data for a particular site or similar locality and estimate through computer simulation what the performance of the system and its components would have been under these historical conditions. Future performance can then be predicted based on this historical analysis. The second method is to use data that correspond to specific types of days (clear, average, "typical," etc.) to calculate the performance of the system and its components.

The first method can account for the transient nature of the process. However, the use of this technique is 1 imited by the cost and complexity associated with the use of detailed models and computer programs and the almost complete absence of good hourly data for solar radiation on tilted surfaces. The primary source of solar radiation data in the United States is the National Weather Service and its network of stations. Data collection began in 1902 with the first measurements of the direct component of the solar radiation at normal incidence being made in Asheville, N.C.

Until 1977 there were 86 stations covering the United States and the West Indies. However, due to errors in data collection and instrument calibration, the accuracy of these data was not guaranteed. Reference 1 gives a complete history of the network. In 1977 a new network with approximately 30-35 stations was established with the aid of the U.S. Department of Energy. This network collects only horizontal surface (direct, diffuse, and total) data by hour.

With the increasing emphasis on the development of solar energy use, much of the past solar radiation data has been rehabilitated. Major. work has been done in an effort to smooth the data record using model's to fill in gaps due to missing data or improperly calibrated instruments. The acronym used to describe this new data base is SOLMET (Reference 2), which consists of solar and meteorological data for 27 sites throughout the United States.

The second method -- using solar radiation data for specific types of days and orientations -- has the advantage that computing insolation estimates for surfaces of arbitrary orientation is a simple computational procedure. However, the input data required are aggregated solar insolation measurements. The approach adopted here was to use the latest available SOLMET derived input data to calibrate an average or typical day insolation model and thus combine the advantages of both methods. 


\section{A. NOMENCLATURE AND ASSUMPTIONS}

The model presented here yields insolation levels for "typical days" -- a term with specific meaning. The method of procedure may be outlined as follows. The amount of solar radiation intensity outside the atmosphere is relatively constant, so the "typical" amount selected for a given month is the quantity around the midpoint ( 15 th or 16th). The proportion of this radiation that actually reaches the Earth's surface varies from day to day (hour to hour, in fact) depending upon the weather characteristics of the location. The amount of solar radiation reaching the Earth's surface during a given month, divided by the total radiation outside the atmosphere during the given month, is used as an estimate of the average or "typical" fraction of radiation reaching the ground. The direct, diffuse, and reflected components of the total radiation are derived from thio fraction and are referred to as typical estimates since they represent the available insolation one could expect on any day in the particular month. Thus, all estimates generated by the model are estimates of the long-run average insolation.

A number of assumptions are associated with the use of this model. First, it is assumed that most of the diffuse radiation comes from an origin near the sun. In other words, the scattering of the solar radiation is mostly forward scattering. Second, it is assumed that the diffuse component is uniformly distributed over the sky. In general, for clear days there is 1 ittle variation in diffuse solar radiation across the United States.* However, while this may not be true for particular partly cloudy days, it is probably sufficient for the "typical" estimate. It is further assumed that the effects on the average culal insolation of tilting the surface (Reference 3 ) are accounted for by the atmospheric attenuation adjustment contained in the model discussed later.

\section{B. PREVIOUS WORK}

Liu and Jordan (References 4-6) conducted extensive analyses during the early 1960s on the available solar radiation data and developed an approach that can be used to estimate the available daily solar radiation for each month of the year for numerous locations in the United States and Canada. Using this approach, it is possible to take the daily total radiation on a horizontal surface, divide the daily total into its direct and diffuse (scattered radiation) components, convert each component into hourly values, and then compute the hourly value of either component on a surface of any orientation desired. In addition, with the availability of the rehabilitated SOLMET data, the calfbration of this model has been extended and improved.

*This can be seen in the relationship between $\overline{\mathrm{K}}_{\mathrm{T}}$ and $\overline{\mathrm{K}}_{\mathrm{d}}$ (page 2-7) where the percentage of the total daily extraterrestrial radiation that reaches the ground as diffuse ranges from $12 \%$ to $19 \%$. 
This Section presents a version of the Liu and Jordan model modified by Kusuda and Ishii at the National Bureau of Standards (Reference 7) to account for early morning and late afternoon errors. These errors are caused by increased atmospheric absorption of the direct radiation beam at extreme sun-hour angles. (During early morning and late afternoon, the direct radiation must pass through a longer length of atmosphere.) The model was adapted to allow for varying array orientations and the algorithm for calculating the total radiation on a tilted surface follows. Furthermore, the model has been updated to include the most recent insolation input data and the revised solar constant $\left(1.377 \mathrm{~kW} / \mathrm{m}^{2}\right)$. The model yields hourly solar radiation values for a given site location (latitude), month (declination), tilt angle, array orientation, and reflectivity. A number of examples are presented in Appendix A of this report.

\section{CALCULATION PROCEDURE}

The calculation procedure is a modification of a method developed by Kusuda and Ishii, which is based on the historical model of Liu and Jordan. For brevity, the approaches are presented in combined form. Details may be found in Reference 7 .

The relevant relationships derived by Liu and Jordan (References 4-6) are:

(1) The monthly average daily diffuse radiation on a horizontal surface as a function of the monthly average daily total radiation on a horizontal surface;

(2) The ratio of the hourly diffuse radiation to the daily diffuse radiation (both on a horizontal surface) as a function of the number of daylight hours;

(3) The ratio of the hourly total radiation to the daily total radiation (both on a horizontal surface) as a function of the number of daylight hours.

The relationships were based on available solar radiation data from Blue Hill, Mass.; Nice, France; Helsingfors, Finland; and Kew Observatory, London, England. More recent data have led to minor adjustments in the diffuse component relationships (Reference 8 ). However, comparisons of these adjustments are inconclusive due to the small differences involved and the limited data. The approach used here retains the original Liu and Jordan results for the diffuse component.

Liu and Jordan (References 6,9) also compiled for each of 12 months, at 80 different locations in the United States and Canada, the "average" daily total radiation on a horizontal surface $(\overline{\mathrm{H}})$ and the ratio $\overline{\mathrm{K}}_{\mathrm{T}}=$ $\overline{\mathrm{H}} / \mathrm{H}_{\mathrm{O}}$, where $\mathrm{H}_{\mathrm{O}}$ is the daily total extraterrestrial radiation on a horizontal surface calculated for the 15 th or 16 th of each month. It should be noted that the choice of the midpoint of the month is somewhat arbitrary since the monthly variation in daily extraterrestrial 
radiation is on $1 y+1.5 \%$. Using the rehabilitated SOLMET data base, the National Climatic Center has updated the data used for computing the $\overline{\mathrm{K}}_{\mathrm{T}}$ fractions (Reference 10 ). Using regression models, the data base has been extended to cover 248 locations. These values were used to generate the 235 selected handbook sites in Appendices C and D. Since the $\overline{\mathrm{K}}_{\mathrm{T}}$ values are site specific and reflect the cloudiness and weather conditions of the site, they more accurately represent the expected insolation levels than do the so-called "clear day" estimates of the earlier literature in this area. The $\overline{\mathrm{K}}_{\mathrm{T}}$ ratio represents the fraction of the total average daily extraterrestrial radiation that is transmitted through the atmosphere and thus provides a useful index for the solar climate of a particular location.

The step-by-step technique for using the above relationships to compute the incident solar radiation on a horizontal or tilted surface follows. Figures $2-1 a$ and $2-1 b$ illustrate the relevant angles involved in the procedure.

\section{Step 1}

The average daily total extraterrestrial radiation $\left(\mathrm{H}_{\mathrm{O}}\right)$ on a horizontal surface for a given site (latitude) and month (declination angle) must be calculated first. This calculation will give the total available radiation on a horizontal surface before the radiation beam passes through the atmosphere and is broken into its direct and diffuse components.

The expression is:

$$
H_{0}=\frac{24}{\pi} \times \gamma \times I_{c} \times\left(\cos \phi \times \cos \delta \times \sin W_{S}+W_{S} \times \sin \phi \times \sin \delta\right)
$$

where

$$
\begin{aligned}
& \mathrm{H}_{\mathrm{O}}=\text { daily total extraterrestrial radiacion on a liurizuntal } \\
& \text { surface }\left(\mathrm{kWh} / \mathrm{m}^{2} / \mathrm{day}\right) \\
& \gamma=\text { ratio of solar radiation intensity at normal incidence } \\
& I_{c}=\text { solar constant, } 1.377 \mathrm{~kW} / \mathrm{m}^{2} \\
& \phi=1 \text { atitude, radians* } \\
& \delta=\text { solar declination àngle, radiaus; uonthly voluco are yiveu } \\
& \text { in Table 2-1; the declination is the angular position of } \\
& \text { the sun at solar noon with respect to the plane of the } \\
& \text { equator (north positive) } \\
& \mathrm{W}_{\mathrm{S}}=\text { sunrise hour angle (radians) }=\cos ^{-1}(-\tan \phi \tan \delta)
\end{aligned}
$$

*Degrees are converted to radians by multiplying by $\pi / 180$. All the trigonometric functions used here require radian arguments. 


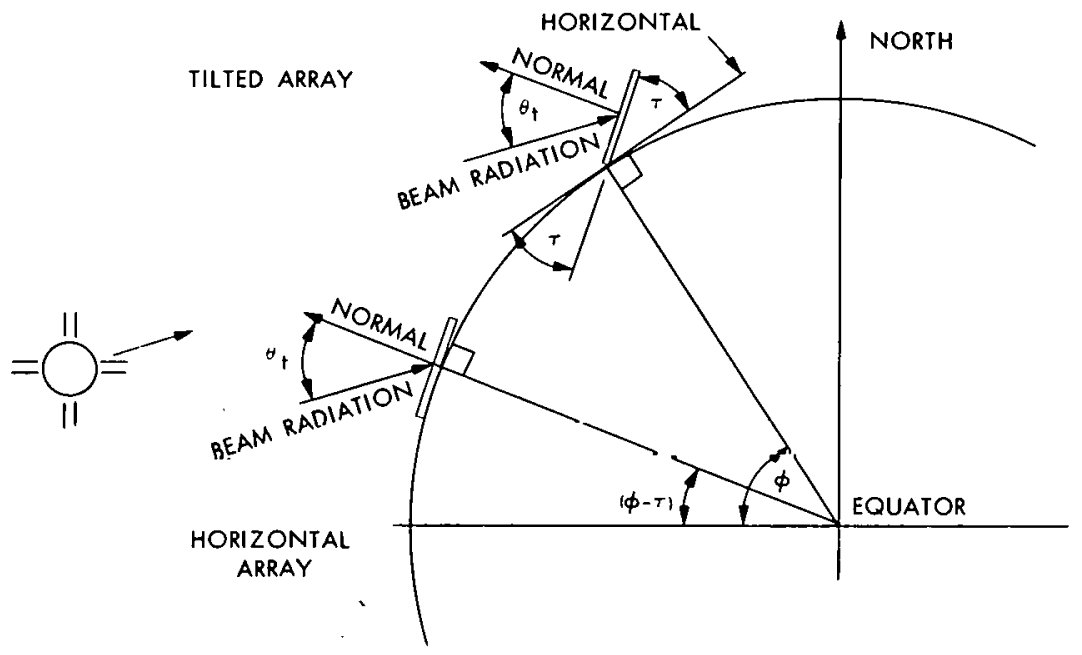

Figure 2-1a. Cross Section of the Earth Showing Definitions of $\tau, \theta_{t}, \phi$, and $(\phi-\tau)$ (Modified from Reference 11)

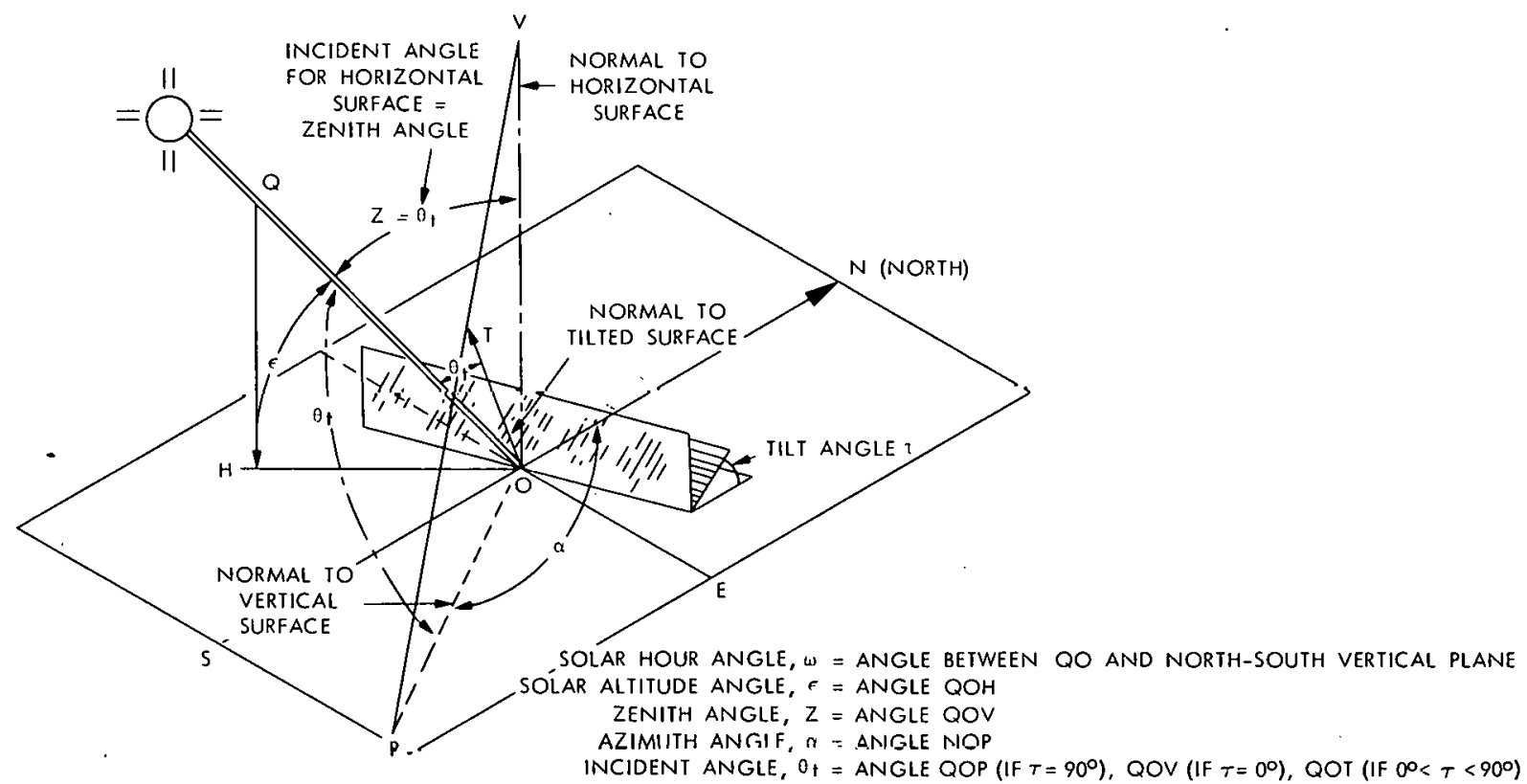

Figurc 2-1b. Angles Used in Sular Insolation Calculation (Modified from Reference 7) 
Table 2-1. Solar Declination ( $\delta$ ), the Ratio of Solar Radiation Intensity at Normal Incidence Outside Earth's Atmosphere to the Solar Constant $(\gamma)$, and Atmospheric Absorption Coefficient (B) (Reference 7)

\begin{tabular}{lccc}
\hline Month & $\begin{array}{c}\text { Declination } \\
\text { (Degrees } \begin{array}{c}\text { Minutes) } \\
\delta\end{array}\end{array}$ & $\begin{array}{c}\text { Ratio } \\
\gamma\end{array}$ & $\begin{array}{c}\text { Atmospheric } \\
\text { Absorption } \\
\text { Coefficient } \\
\text { B }\end{array}$ \\
\hline 1 January & -19.51 & 1.0300 & 0.142 \\
2 February & -10.28 & 1.0207 & 0.144 \\
3 March & 0.20 & 1.0057 & 0.156 \\
4 Apri1 & 11.56 & 0.9875 & 0.180 \\
5 May & 20.14 & 0.9727 & 0.196 \\
6 June & 23.27 & 0.9670 & 0.205 \\
7 July & 20.26 & 0.9692 & 0.207 \\
8 August & 12.03 & 0.9785 & 0.201 \\
9 September & 0.37 & 1.0267 & 0.177 \\
10 October & -10.47 & 1.0133 & 0.160 \\
11 November & -19.58 & 1.0327 & 0.149 \\
12 December & -23.27 & & 149 \\
\hline
\end{tabular}


It should be noted that all times are expressed as angles of the sun's position relative to a vertical plane oriented north to south.

Step 2

The next step is to obtain the monthly average daily total radiation on a horizontal surface, $\overrightarrow{\mathrm{H}}$, from Reference 10 or other available sources and calculate the ratio:

$$
\overline{\mathrm{K}}_{\mathrm{T}}=\overline{\mathrm{H}} / \mathrm{H}_{\mathrm{O}}
$$

The value $\overline{\mathrm{K}}_{\mathrm{T}}$ represents the average fraction of daily total extraterrestrial radiation that penetrated the atmosphere. If the value of $\overline{\mathrm{K}}_{\mathrm{T}}$ is available, this calculation may be omittcd. Appendix B contains a table of values for 235 locations in the United States (Reference 10) derived from the rehabilitated SOLMET data base.

\section{Step 3}

Liu and Jordan determined the relationship between the fractions of extraterrestrial radiation that were daily diffuse $\left(\bar{K}_{d}\right)$ and daily total radiation $\left(\overline{\mathrm{K}}_{\mathrm{T}}\right)$ (on a monthly average basis) for any given month. This empirical relationship is given by:

$\begin{array}{lc}\overline{\mathrm{K}}_{\mathrm{T}} & \overline{\mathrm{K}}_{\mathrm{d}} \\ 0.3 & 0.179 \\ 0.4 & 0.183 \\ 0.5 & 0.188 \\ 0.6 & 0.174 \\ 0.7 & 0.149 \\ 0.75 & 0.125\end{array}$

For example, if $60 \%$ of the average daily extraterrestrial radiation is reaching the Earth at a particular location, $17.4 \%$ of the average daily extraterrestrial radiation which reaches the ground is diffuse (scattered by the atmosphere).

The $\overline{\mathrm{K}}_{\mathrm{T}}, \overline{\mathrm{K}}_{\mathrm{d}}$ relationship is used to determine the daily diffuse radiation on a horizontal surface, $\overline{\mathrm{H}}_{\mathrm{d}}$, by:

$$
\overline{\mathrm{H}}_{\mathrm{d}}=\overline{\mathrm{K}}_{\mathrm{d}} \times \overline{\mathrm{H}}_{\mathrm{o}}
$$

\section{Step 4}

The next step is to take the daily value above and convert it to an hourly value. This is done by calculating the value of a factor, $r_{d}$, from the following equation that gives the fraction, $r_{d}$, of average daily extraterrestrial radiation that reaches the ground as diffuse radiation during a given hour: 


$$
r_{d}=\frac{\pi}{24} \times \frac{\cos \omega-\cos W_{s}}{\sin W_{s}-W_{s} \times \cos W_{s}}
$$

where $\omega=$ the hour angle corresponding to a given hour of the day (solar time), radians.

Note that the use of this relationship "smooths" the average daily diffuse profile into a symmetric function as mentioned earlier. Also note that solar time and standard time are different. Solar time is a local phenomenon, determined so that solar noon occurs when the sun is most nearly overhead. Thus, twó observers displaced east and west of each other would measure different solar times at the same Pacific Standard Time (PST). The following table illustrates the relationship hetween solar time and hour angle:

$$
\text { Solar Time Hour Angle } \omega \text { (Degrees) }
$$

Solar Noon: $\begin{array}{rlr}12 & \text { Noon } & 0 \\ 1 & \text { P.M. } & 15^{\circ} \\ 2 & \text { P.M. } & 30^{\circ} \\ & \cdot & \text {. } \\ & \text {. } & \text {. }\end{array}$

Using $r_{d}$, the average hourly terrestrial diffuse radiation on a horizontal surface $\left(\bar{I}_{d h}\right)$ is the average daily diffuse radialiun multiplied by the fraction available during hour $\omega *$ :

$$
\overline{\mathrm{I}}_{\mathrm{dh}}=\mathrm{r}_{\mathrm{d}} \times \overline{\mathrm{H}}_{\mathrm{d}}
$$

\section{Step 5}

This step involves the calculation of the direct normal component of the hourly radiation adjusted to more accurately accounl fur llie larger amount of atmosphere the beam must pass through near the sunrise and sunset hours. 'The details of this procedure may bc found in Reference 7.

\footnotetext{
*A small d subscript indicates a diffuse component and a large D denotes a direct component. The second letter of the subscript denotes whether the surface is horizontal ( $h)$, tilted $(t)$, or normal to the direct beam $(N)$. A large $T$ denotes total radiation amounts.
} 
The value of the factor $m$, which represents a correction for the increased atmospheric absorption of the direct normal component, can be determined from Table 2-2.* So, the hourly direct normal radiation is calculated using:

$$
\overline{\mathrm{I}}_{\mathrm{DN}}=\overline{\mathrm{A}} \times \exp \left(-\mathrm{B} / \cos \theta_{\mathrm{t}}\right)(\text { with } \tau=0)
$$

where

$$
\begin{aligned}
& \begin{aligned}
\overline{\mathrm{I}}_{\mathrm{DN}}= & \text { average hourly terrestrial direct normal } \\
& \text { radiation }\left(\mathrm{kWh} / \mathrm{m}^{2}\right)
\end{aligned} \\
& \bar{A}=\left(\bar{K}_{T}-\bar{K}_{d}\right) \times f \times H_{O}\left(k W h / m^{2}\right) ; \text { the } \\
& \text { correction factor } m=f \times H_{O} \text { where } f \text { is a } \\
& \text { function of latitude and time of year } \\
& B=\text { an atmospheric absorption coefficient } \\
& \text { (see Table 2-1) that describes the varying } \\
& \text { beam attenuation by the atmosphere during } \\
& \text { the year } \\
& \theta_{t}=\text { the angle of incidence between the sun's } \\
& \text { direct beam and a normal to the array surface. } \\
& \text { It is this variable that takes into account } \\
& \text { the azimuthal orientation, } \alpha \text {, and tilt angle, } \tau \text {, } \\
& \text { of the surface. }
\end{aligned}
$$

The equation for $\theta_{t}$ is derived from Reference 12:

$$
\cos \theta_{t}=\left\{\begin{array}{c}
(\sin \phi \times \cos \tau+\cos \phi \times \sin \tau \times \cos \alpha) \times \sin \delta \\
+(\cos \phi \times \cos \tau-\sin \phi \times \sin \tau \times \cos \alpha) \times \cos \delta \\
\cos \omega-\sin \tau \times \sin \alpha \times \cos \delta \times \sin \omega \\
\text { if } \cos \theta_{t}>0 \\
0 \quad \text { if } \cos \theta_{t} \leq 0
\end{array}\right.
$$

*The computer program presented in Section VI of this document generates thesc $\mathrm{m}$ factors interually. 
Table 2-2a. The Value of the $m$ Factor $\left(\mathrm{kWh} / \mathrm{m}^{2}\right)$

LAT.

MONTH

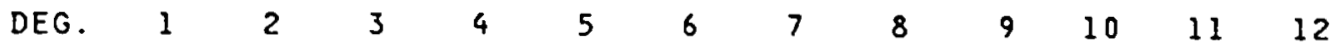

$\begin{array}{llllllllllllllllllll}0 . & 1.77 & 1.74 & 1.74 & 1.77 & 1.81 & 1.83 & 1.83 & 1.81 & 1.77 & 1.77 & 1.78 & 1.78\end{array}$

1. $\quad \begin{array}{lllllllllllll}1.77 & 1.74 & 1.74 & 1.77 & 1.80 & 1.83 & 1.83 & 1.81 & 1.77 & 1.77 & 1.78 & 1.78\end{array}$

2. $\quad \begin{array}{llllllllllllll}1.77 & 1.74 & 1.74 & 1.77 & 1.80 & 1.82 & 1.82 & 1.81 & 1.77 & 1.77 & 1.78 & 1.79\end{array}$

3. $\quad \begin{array}{lllllllllllllll}1.77 & 1.74 & 1.74 & 1.77 & 1.80 & 1.82 & 1.82 & 1.80 & 1.77 & 1.77 & 1.79 & 1.79\end{array}$

4. $\quad \begin{array}{lllllllllllllllll}1.78 & 1.74 & 1.74 & 1.77 & 1.80 & 1.82 & 1.82 & 1.80 & 1.77 & 1.77 & 1.79 & 1.79\end{array}$

5. $\quad 1.781 .751 .741 .771 .791 .81 \quad 1.82 \quad 1.801 .771 .771 .791 .80$

6. $\quad \begin{array}{lllllllllllllll}1.78 & 1.75 & 1.74 & 1.77 & 1.79 & 1.81 & 1.81 & 1.80 & 1.77 & 1.78 & 1.80 & 1.80\end{array}$

7. $\quad 1.791 .751 .741 .771 .791 .81 \quad 1.811 .801 .771 .781 .801 .80$

8. $\quad \begin{array}{lllllllllllllll}1.79 & 1.75 & 1.74 & 1.76 & 1.79 & 1.81 & 1.81 & 1.80 & 1.77 & 1.78 & 1.80 & 1.81\end{array}$

9. $\quad 1.791 .751 .791 .761 .791 .801 .811 .801 .771 .781 .811 .81$

10. $\quad \begin{array}{lllllllllllllllll}1.80 & 1.76 & 1.74 & 1.76 & 1.78 & 1.80 & 1.81 & 1.80 & 1.77 & 1.79 & 1.81 & 1.82\end{array}$

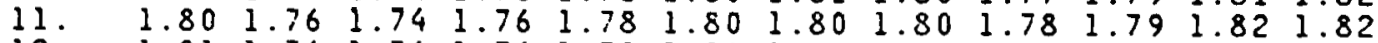

12. $\quad \begin{array}{llllllllllllllll}1.81 & 1.76 & 1.74 & 1.76 & 1.78 & 1.80 & 1.80 & 1.80 & 1.78 & 1.79 & 1.82 & 1.83\end{array}$

13. $\quad \begin{array}{lllllllllllll}1.81 & 1.76 & 1.75 & 1.76 & 1.78 & 1.80 & 1.80 & 1.80 & 1.78 & 1.80 & 1.83 & 1.83\end{array}$

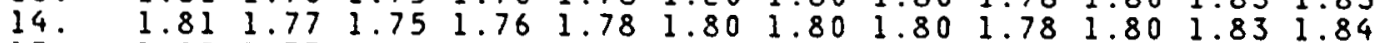

15. $\quad \begin{array}{lllllllllllll}1.82 & 1.77 & 1.75 & 1.76 & 1.78 & 1.79 & 1.80 & 1.80 & 1.78 & 1.80 & 1.84 & 1.84\end{array}$

16. $\quad 1.821 .771 .751 .77 \quad 1.781 .791 .801 .801 .781 .811 .841 .85$

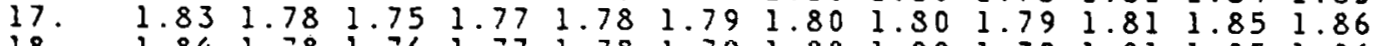

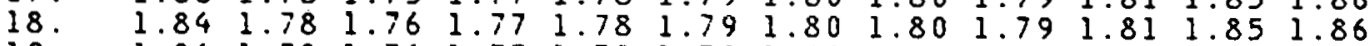

19. $\quad 1.84 .1 .781 .761 .771 .78 \quad 1.791 .801 .801 .791 .821 .861 .87$

20. $\quad \begin{array}{lllllllllllllllll}1.85 & 1.79 & 1.76 & 1.77 & 1.78 & 1.79 & 1.80 & 1.80 & 1.79 & 1.82 & 1.87 & 1.88\end{array}$

21. $1.861 .791 .76 \quad 1.77 \quad 1.78 \quad 1.79 \quad 1.801 .811 .801 .831 .871 .89$

22. $\quad \begin{array}{lllllllllllll}1.86 & 1.80 & 1.77 & 1.77 & 1.78 & 1.79 & 1.80 & 1.81 & 1.80 & 1.83 & 1.88 & 1.90\end{array}$

23. $\quad 1.871 .801 .771 .771 .781 .791 .801 .811 .801 .841 .891 .91$

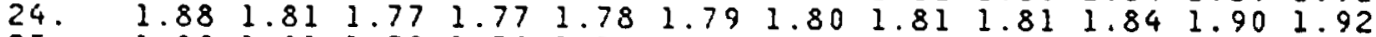

25. $\quad \begin{array}{llllllllllll}1.89 & 1.81 & 1.78 & 1.78 & 1.78 & 1.79 & 1.80 & 1.81 & 1.81 & 1.85 & 1.91 & 1.93\end{array}$

26. $\quad 1.901 .82 \quad 1.78 \quad 1.78 \quad 1.78 \quad 1.791 .801 .82 \quad 1.81 \quad 1.861 .921 .94$

27. $\quad 1.91 \quad 1.821 .781 .78 \quad 1.78 \quad 1.791 .801 .82 \quad 1.82 \quad 1.861 .931 .95$

28. $1.921 .831 .791 .781 .781 .791 .801 .82 \quad 1.82 \quad 1.871 .941 .96$

29. $1.931 .841 .791 .791 .791 .79 \quad 1.81 \quad 1.82 \quad 1.831 .881 .951 .98$

30. $\quad 1.94 \quad 1.841 .791 .791 .791 .801 .811 .831 .831 .891 .961 .94$

$31 . \quad 1.951 .851 .801 .791 .791 .801 .811 .831 .841 .891 .972 .01$

33. $\quad 1.961 .86 \quad 1.801 .791 .791 .801 .811 .83 \quad 1.841 .901 .992 .02$

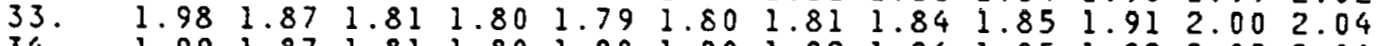

34. $\quad 1.991 .871 .811 .801 .801 .801 .821 .841 .841 .851 .922 .022 .06$

35. $2.01 \quad 1.881 .82 \quad 1.80 \quad 1.801 .801 .82 \quad 1.841 .86 \quad 1.93 \quad 2.032 .08$

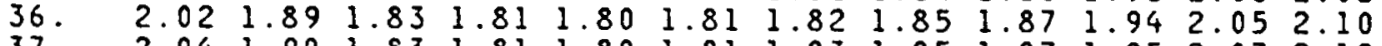

37. $2.041 .901 .831 .81 \quad 1.80 \quad 1.81 \quad 1.831 .851 .871 .952 .072 .12$

$38.22 .061 .911 .841 .821 .811 .81 \quad 1.831 .861 .881 .972 .092 .15$

$39.2 \quad 2.081 .921 .841 .821 .811 .821 .831 .861 .891 .982 .112 .18$

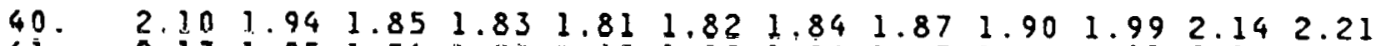

41. $\quad 2.131 .951 .861 .831 .821 .821 .841 .871 .912 .012 .162 .24$

42. $2.151 .961 .871 .84 \quad 1.821 .831 .841 .881 .922 .02 \quad 2.192 .28$

43. $\quad 2.181 .981 .881 .84 \quad 1.83 \quad 1.83 \quad 1.851 .891 .932 .042 .22 \quad 2.32$

44. $\quad 2.21 \quad 1.991 .891 .851 .831 .83 \quad 1.851 .891 .942 .062 .262 .36$

45. $2.252 .011 .891 .851 .831 .84 \quad 1.861 .901 .952 .082 .292 .41$

46. $\quad 2.292 .03 \quad 1.90 \quad 1.86 \quad 1.84 \quad 1.84 \quad 1.86 \quad 1.911 .96 \quad 2.102 .342 .47$

47. $\quad 2.332 .051 .921 .871 .841 .851 .871 .92 \quad 1.972 .122 .382 .53$

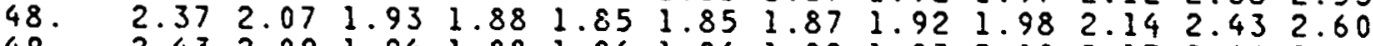

49. 2.432 .091 .941 .881 .861 .861 .881 .932 .002 .172 .492 .68

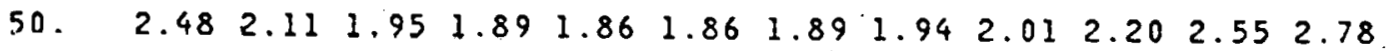


Table 2-2b. The Value of the m Factor (BTU/ft ${ }^{2}$ )

LAT.

MONTH

$\begin{array}{lllllllllllll}\text { DEG. } & 1 & 2 & 3 & 4 & 5 & 6 & 7 & 8 & 9 & 10 & 11 & 12\end{array}$

0. $\quad 561.552 .551 .563 .573,581.581 .574 .561 .561 .565 .565$.

1. $561,552,551,562,572,580,580,574,561.561 .566,566$.

2. $562.553 .551,562.571,579.579 .573 .561 .562 .566 .567$.

3. 563.553 .551 .561 .571 .578 .578 .573 .561 .562 .567 .568$.

4. $\quad 564.554,551.561,570,577,577,572 a 561.563 .568,569$.

5. $\quad 565.554,552.561,569,576,576,572.562 .563 .569 .570$.

6. 566.555 .552 .560 .568 .575 .575 .572 .562 .564 .570 .571$.

7. $\quad 567.555 .552,560,568,574.575 .571 .562,565.571 .573$.

8. $568.556,552,560,567,573,574,571.562,565.572 .574$.

9. $569.531 .533 .564 .567,573.373 .571 .363 .360 .574 .575$.

10. 570.558 .553 .560 .566 .572 .573 .571 .563 .567 .575 .577$.

11. 572.558.553.560.566.571.573.571.563.568.576.578.

12. 573.559 .554 .560 .565 .571 .572 .571 .564 .569 .578 .580$.

13. 574.560.554,560.565,570,572,571.564,570.579.582.

14. $576.561 .555 .560,565,570,572.571 .565 .571 .581 .584$.

15. 577.562 .555 .560 . 565.570.571. 571.566.572. 583. 585.

$16.579 .563 .556 .560 .565,569.571 \cdot 571.566 .573 .584 .587$.

17. 581.564 .557 .560 .564 .569 .571 .572 .567 .574 .586 .590$.

18. 583.565.557, 561, 564,569.571.572.568, 576.588: 592.

19. 585.566 .558 .561 .564 .569 .571 .572 .569 .577 .590 .594$.

20. 587.568 .559 .561 .564 .568 .571 .573 .569 .579 .592 .597$.

21. 589.569 .560 .562 .564 .568 .571 .573 .570 .580 .595 .599$.

22. 591.570 .560 .562 .565 .568 .571 .574 .571 .582 .597 .602$.

23. 594.572.561.563.565.568.571.574.572.583.600.605.

24. 596.574.562.563.565.568.571.575.574.585.602.608.

25. 599.575.563.564.565.569.572.575.575.587.605.612.

26. 602.577 .564 .565 .565 .569 .572 .576 .576 .589 .608 .615$.

27. 605.579.566.565.566.569.572.577.577.591.612.619.

28. 608.581 .567 .566 .566 .569 .573 .578 .579 .594 .615 .623$.

29. 611. 583.568.567.567.570.573.579.580.596.619.627.

30. 615.585.569.568.567.570.574.580.582.598.622.631.

31. $\quad 619.587 .571 .568 .568 .570 .574 .581 .583 .601 .627 .636$.

32. 623.590.572.569.568.571.575.582.585.604.631.641.

33. 627.592.574.570.569,572.576.583.587.607.635.647.

34. $632.595 .576 .572 .570^{\circ} .572 .577 .584 .589 .610 .640 .653$.

35. 637.598 .577 .573 .571 .573 .577 .585 .591 .613 .646 .659$.

36. 642.601.579.574.571.574.578.587.593.617.651.666.

37. 648.604.581.575.572.574.579.588.595.620.657.674.

38.654 .607 .583 .577 .573 .575 .580 .590 .597 .624 .664 .682$.

39.660 .611 .586 .578 .574 .576 .581 .591 .600 .628 .671 .691$.

40. 668.615 .588 .580 .575 .577 .582 .593 .602 .633 .679 .700$.

41. $675.619 .590 .581 .57 \% .578 .584 .595 .605 .637 .687 .711$.

42. 684.623 .593 .583 .578 .579 .585 .597 .608 .642 .696 .722$.

43. 693.628.595.585.579.580.586.599.611.648.706.735.

44. $\quad 703.633 .598 .587 .581 .582 .588 .601 .614 .653 .716 .749$.

45. $\quad 714.638 .601 .589 .582 .583 .589 .603 .618 .659 .728 .765$.

46. $\quad 726.643 .604 .591 .584 .585 .591 .605 .621 .666 .741 .783$.

47.739 .649 .608 .593 .585 .586 .593 .608 .625 .673 .755 .802$.

48.6754 .656 .611 .595 .587 .588 .595 .611 .629 .680 .771 .825$.

49. 770.663 .615 .598 .589 .589 .597 .613 .634 .689 .789 .851$.

$50 . \quad 788.671 .619 .600 .591 .591 .599 .616 .638 .698 .809 .881$. 
The azimuth angle, $\alpha$, is measured clockwise by a north-facing observer

where $\alpha=\left\{\begin{aligned} 0^{\circ} & \text { for north orientation, } \\ 90^{\circ} & \text { for east orientation, } \\ 180^{\circ} & \text { for south orientation, etc. }\end{aligned}\right.$

and $\tau=$ the array surface tilt angle from the horizontal.

Note that for a south-facing surface at any tilt angle

$$
\cos \theta_{t}=\sin (\phi-\tau) \times \sin \delta+\cos (\phi-\tau) \times \cos \delta \times \cos \omega
$$

Step 6

The average hourly terrestrial direct radiation on a horizontal surface is given by:

$$
\bar{I}_{D h}=\bar{I}_{D N} \times \cos \theta_{t}(w i t h \tau=0)
$$

\section{Step 7}

The average hourly terrestrial total radiation on a horizontal surface is given by the sum of the hourly direct and diffuse components:

$$
\overline{\mathrm{I}}_{\mathrm{Th}}=\overline{\mathrm{I}}_{\mathrm{Dl} \mathrm{l}}+\overline{\mathrm{I}}_{\mathrm{dl} \mathrm{t}}
$$

Step 8

The average hourly terrestrial direct radiation on a tilted surface is given by:

$$
\overline{\mathrm{I}}_{D t}=\overline{\mathrm{I}}_{\mathrm{DN}} \times \cos \theta_{t} \text { (with } \tau=\text { given array tilt angle) }
$$

Step 9

The average hourly terrestrial diffuse radiation on a tilted surface is given by:

$$
\bar{I}_{d t}=\frac{1+\cos \tau}{2} \times \bar{I}_{d h}
$$

Step 10

Since the array is tilted up at some angle, $\tau$, it receives reflected radiation from the surrounding area that it faces. This reflerter radiation will vary depending on whether there is high reflectivity (e.g, snow) or low reflectivity (e.g., forest). Tables $2-3$ and $2-4$ show average reflectivities for various surroundings. Hunn and Calafell (Reference 13) have developed a method for ascertaining these average reflectivities. They conclude: 
Table 2-3. Reflectivity Values for 15 Characteristic Surfaces (Integrated Over Solar Spectrum and Angle of Incidence) (Reference 13)

\author{
Surface
}

Average

Reflectivity

1. Snow (freshly fallen or with ice film)

0.75

2. Water surfaces (relatively large incidence

0.07 angies)

3. Soils (clay, loam, etc.)

0.14

4. Earth roads

0.04

5. Coniferous forest (winter)

0.07

6. Forests in autumn, ripe field crops, plants

0.26

7. Weathered blacktop

0.10

8. Weathered concrete

0.22

9. Dead leaves

0.30

10. Dry grass

0.20

11. Green grass

0.26

12. Bituminous and gravel roof

0.13

13. Crushed rock surface

0.20

14. Building surfaces, dark (red brick, dark paints, etc.)

15. Building surfaces, light (light brick, 1ight paints, etc.)

0.60 
Table 2-4. Average Reflectivity Values for 12 Representative Winter Landscapes (Reference 13)

Surface Description

Average

Reflectivity

Rural Areas

Fields with Snow Cover

1. Field with wooded area in background

$0.66,0.73$

2. Open field (soil and dry grass) near road

$0.61,0.70$

3. Trees dispersed in field

0.62

Wooded Areas

4. Conifer forest (with heavy snow cover)

0.61

5. Deciduous forest (with heavy snow cover)

0.72

Water

6. Open water

0.16

7. Ice/snow-covered water

0.68

8. Partially open waterway (trees and houses in background)

$0.43,0.66$

Urban Areas

9. Ürban areas (commercial, institutional)

$0.16,0.38$

10. Residertial areas (dwellings and roadway)

$0.21,0.35,0.45$

11. Educational inotitution

$0.36,0.142$

12. Recreational area (park)

0.49 
(1) An average ground reflectivity of 0.6 to 0.7 , similar to that used by Liu and Jordan (Reference 14), is accurate for most rural landscapes in winter and where snow cover is predominant. The exception to this is for locations adjacent to open, or partially open, bodies of water, where a considerably lower value of reflectivity is obtained.

(2) For urban areas, no characteristic ground reflectivity for winter may be specified due to the wide variation in landscape details possible. However, the range for ground reflectivity for urban areas in winter is 0.16 to 0.49 , considerably lower than that used by Liu and Jordan.

(3) For al1 landscapes considered, ground reflectivities are quite sensitive to the fraction of field of view in snow cover.

Thus, the ground reflectivity is an input parameter denoted here by $\rho$. The average hourly terrestrial reflected radiation from the ground is given by:

$$
\overline{\mathrm{I}}_{\mathrm{rt}}=\frac{1-\cos \mathrm{T}}{2} \times \rho \times \overline{\mathrm{I}}_{\mathrm{Th}}
$$

where $\rho$ is the average ground reflectance.

Step 11

The final step is the calculation of the average hourly terrestrial total radiation on a tilted surface. This total is the sum of the direct, diffuse, and reflected components calculated in steps 8-10.

$$
\overline{\mathrm{I}}_{\mathrm{Tt}}=\overline{\mathrm{I}}_{\mathrm{Dt}}+\overline{\mathrm{I}}_{\mathrm{dt}}+\overline{\mathrm{I}}_{\mathrm{rt}}
$$

Since the model divides daily values into hourly intensities (kilowatts per square meter), the intensity over one hour can be integrated to get kilowatt-hours per square meter (if it is assumed intensity is relatively constant over the hour). So at this point, the units of $\overline{\mathrm{I}}_{\mathrm{Tt}}$ are kilowatts during liuur $\omega$ per square meter.

\section{SPECTRAL CONSIDERATIONS}

As mentioned earlier, the spectral response of solar cells is limited in comparison to the spectrum of light. The standard curve for the spectral distribution of light was in part established by the JPL Mariner Mars probe (References 15 and 16) and is illustrated in Figure 2-2. The area under this standard curve represents the solar constant $I_{c}$, defined earlier. Since the establishment of this constant, an adjustment has been made due to Frolich (Reference 17) updating the constant to $1.377 \mathrm{~kW} / \mathrm{m}^{2}$. This new value is used throughout this document. Superimposed on the same curve is the range of a typical solar cell relative response curve. As shown, the region that the solar cell responds to is smaller than the overail spectrum. 
In fact, the extraterrestrial radiation available to a solar cell can be obtained by multiplying the cell spectral response curve by the standard curve of Figure 2-2 and calculating the area under the resulting curve.

In order to model the transmission of light through the atmosphere, a Rayleigh type equation (Reference 11) could be used to generate transmission coefficients for each wavelength. However, the difficulty in using a transmission equation is determining values of the atmospheric constants in the equations. Furthermore, data that represent profiles over time of these constants are not generally available.

Thus the use of a non-spectrum oriented model will over-estimate the actual radiation to which a solar cell will respond. However, since this model estimates available insolation; it is implicitly assumed that, in the case of photovoltaics, conversion efficiencies must account for this difference when calculating power output from available insolation.

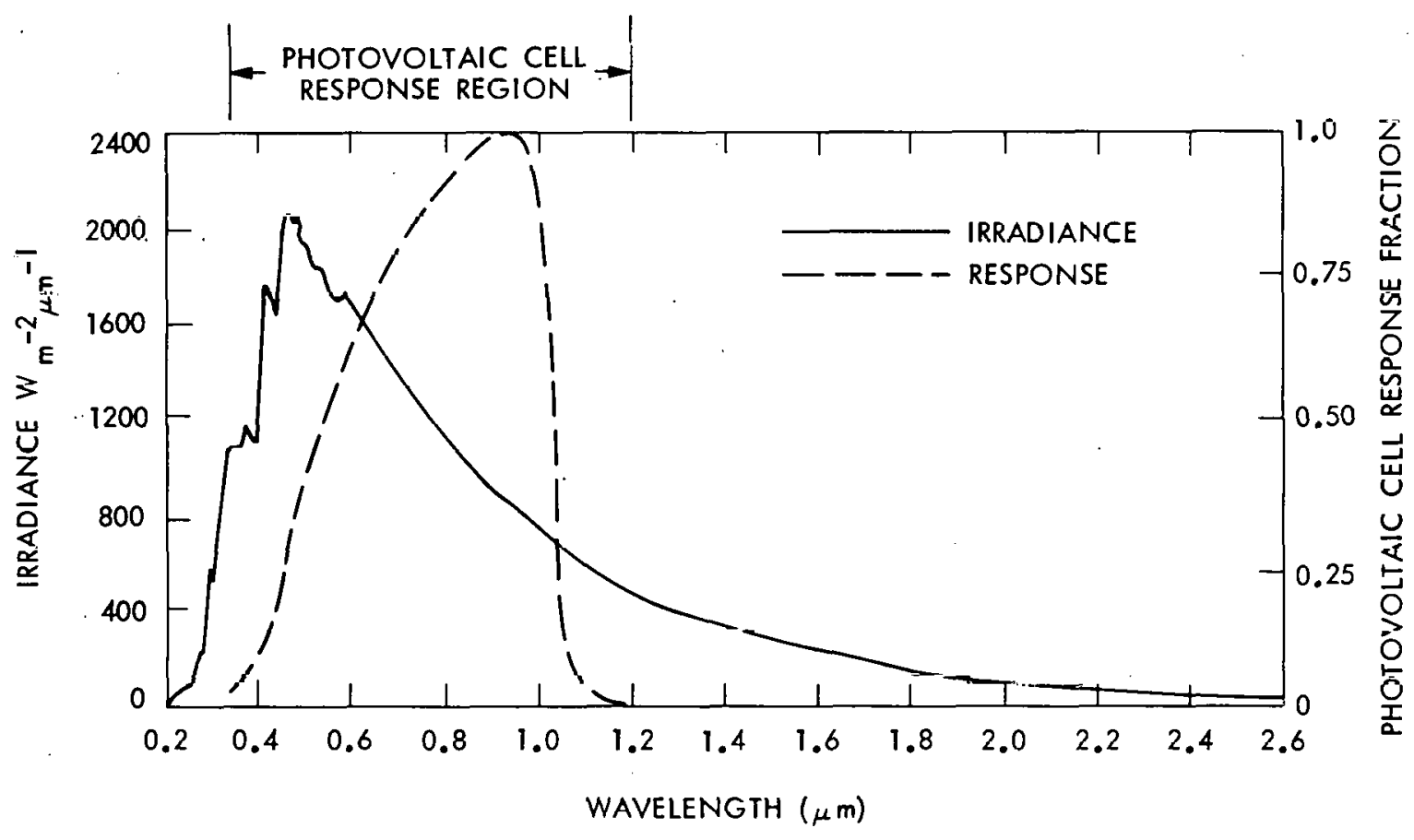

Figure 2-2. Solar Spectral Irradiance, Standard Curve, Solar Constant $\left(1.377 \mathrm{~kW} / \mathrm{m}^{2}\right)$ 


\section{SECTION III}

\section{ARRAY SHADOWING}

\section{A. THE PROBLEM}

The problem of array shadowing arises when arrays are placed too close together and the lower part of an array is caught in the shadow of the array in front of it. The model presented here is based on a model that assumes fixed flat-plate photovoltaic arrays facing south at some tilt angle (see Figure 3-1) and sufficiently close to cause shadowing at some point during the year (References 18 and 19 ).* It is further assumed that the array length in the east-west direction is large relative to the array height and that cells within the arrays are connected in horizontal series strings so that shadowing does not cause an open circuit condition within modules.

The solar insolation loss caused by shadowing is the product of the available insolation to an unshadowed array times the fraction of the array in the shadow of the adjacent array to the south. So, available insolation energy loss is the time integral of the insolation intensity 1oss.

B. THE MODEL

To calculate the available insolation intensity (or energy) lost due to shadowing, the following parameters are required (see Figure 3-1): the length of the array surface, the array tilt angle, the sun elevation angle, and the array spacing.

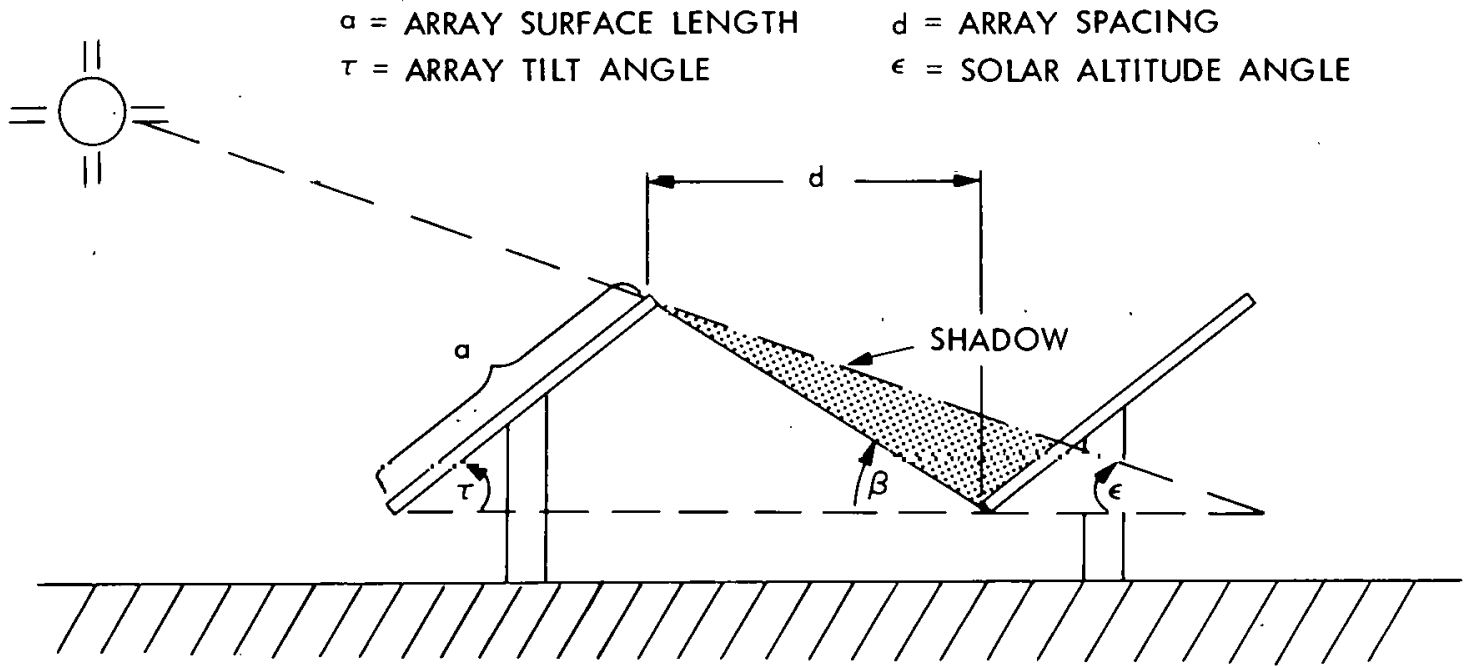

Figure 3-1. Shadow Problem Gepmetry

*The front line of arrays will not receive any shadowing and must be considered separately. 
For a given distance, $d$, between arrays, it can be shown that the fraction of available energy lost during hour $\omega$ is given by:

$$
F_{S}(\omega)= \begin{cases}1 & \text { if } \epsilon \leq 0 \\ 1-(\cos \tau+d / a) \frac{\sin \epsilon}{\sin (\epsilon+\tau)} & \text { if } 0<\epsilon<\beta \text { (Equation 3-1) } \\ 0 & \text { if } \epsilon \geq \beta\end{cases}
$$

where $\quad F_{S}(\omega)=$ shadow loss fraction during hour angle $\omega$

$\tau=$ array tilt angle (radians)

$d=$ array spacing (oame unite as a)

$a$ = array surface length (same units as $d$ )

sin $\epsilon=$ sine of sun elevation angle (a function of $\omega$ )

$=\cos \phi \cos \delta \cos \omega+\sin \phi \sin \delta$ where $\phi, \omega$, and $\delta$ are as defined in Section II (pages 2-4 and 2-8).

Since the above values are hourly, in order to calculate the energy losses over time, hourly power values (assuming the power during the hour is constant) can be summed using:

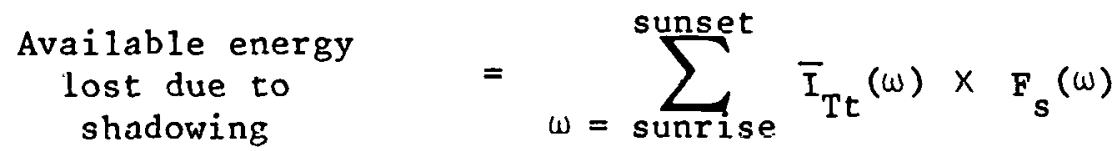

since the power values from Section II are discrete.* This sum represents a daily insolation estimate. To compute energy losses on a monthly or annual basis, the daily value must be multiplied by the number of days in the particular month and the sum taken over months in the year.

The program discussed in Section VI can be used to trade-off the energy losses due to. shadowing with the total amount of space required for the system. The effects of shadowing become more pronounced in the higher latitudes so different spacings are required, depending on the economic parameters of the design.

It should be noted that this model is not restricted to the insolation model of Section II and recorded data could be used.

*Similarly, if the available energy is to be calculated, $\left(1-F_{\mathbf{S}}(\omega)\right)$ must be substituted for $F_{s}(\omega)$ in the above expression. 


\section{REFLECTOR AUGMENTATION}

\section{A. THE PROBLEM}

The question of reflector augmentation is concerned with a specific array design known as the tandem rack design (Reference 20). The geometry of this design is illustrated in Figure 4-1 and the derivation of the model for solar noon may be found in References 18 and 19 . Again it is assumed that the arrays are south facing, but in this case, the north side of the array support structure is covered with a highly reflective surface (such as Mylar) facing the adjacent array. The insolation available to the array surface is determined by calculating an augmentation factor. This augmentation factor represents the additional insolation from the reflective surface.

This model is especially useful in conducting trade-offs among array spacing, tilt angles, and reflector angles.

\section{B. THE MODEL}

Examining Figure $4-1$, it can be seen that when the sun elevation angle, $\epsilon$, is less than the reflector angle, $\beta$, there is no reflector augmentation, and in fact there are losses due to shadowing. In this case, the expression for hourly insolation is given by:

$$
\overline{\mathrm{I}}_{\mathrm{Tt}}(\omega) \times\left(1-\mathrm{F}_{\mathrm{S}}(\omega)\right)
$$

where $\overline{\mathrm{I}}_{\mathrm{Tt}}(\omega)$ is the total insolation available to the surface during hour $\omega$, and $F_{\mathbf{S}}(\omega)$ is the shadow loss fraction, during hour $\omega$ (see Section III).

However, when the sun elevation angle is greater than the reflector tilt, reflection occurs. If the augmentation of array illumination by reflected sunlight is given by $\Delta I / I$, where $\Delta I$ is the additional insolation and $I$ is the available insolation without reflectors, the multiplier that yields the total insolation at the collector surface is given by $1+\Delta I / I$.

For a specular reflectance, $\rho^{\prime}$, the expression for the augmentation multiplier is given by:

$$
1+\Delta I / I=1+\rho^{\prime} \times \sin \left(\epsilon-\beta^{\prime}\right) \times \frac{R-L}{a} \quad(\text { for } B \leq \epsilon \leq 2 \beta)
$$

where: $B$ is the reflector tilt angle $\left(=\tan ^{-1}[a \times(\sin \tau) / d]\right)$; $a$ is the array length in the meridian plane; $R$ is the reflector length in the meridian $p l a n e ;$ and $L$ is the portion of the reflector length for which reflected insolation is not intercepted by the adjacent array for solar elevation angles greater than $2 \beta$. 

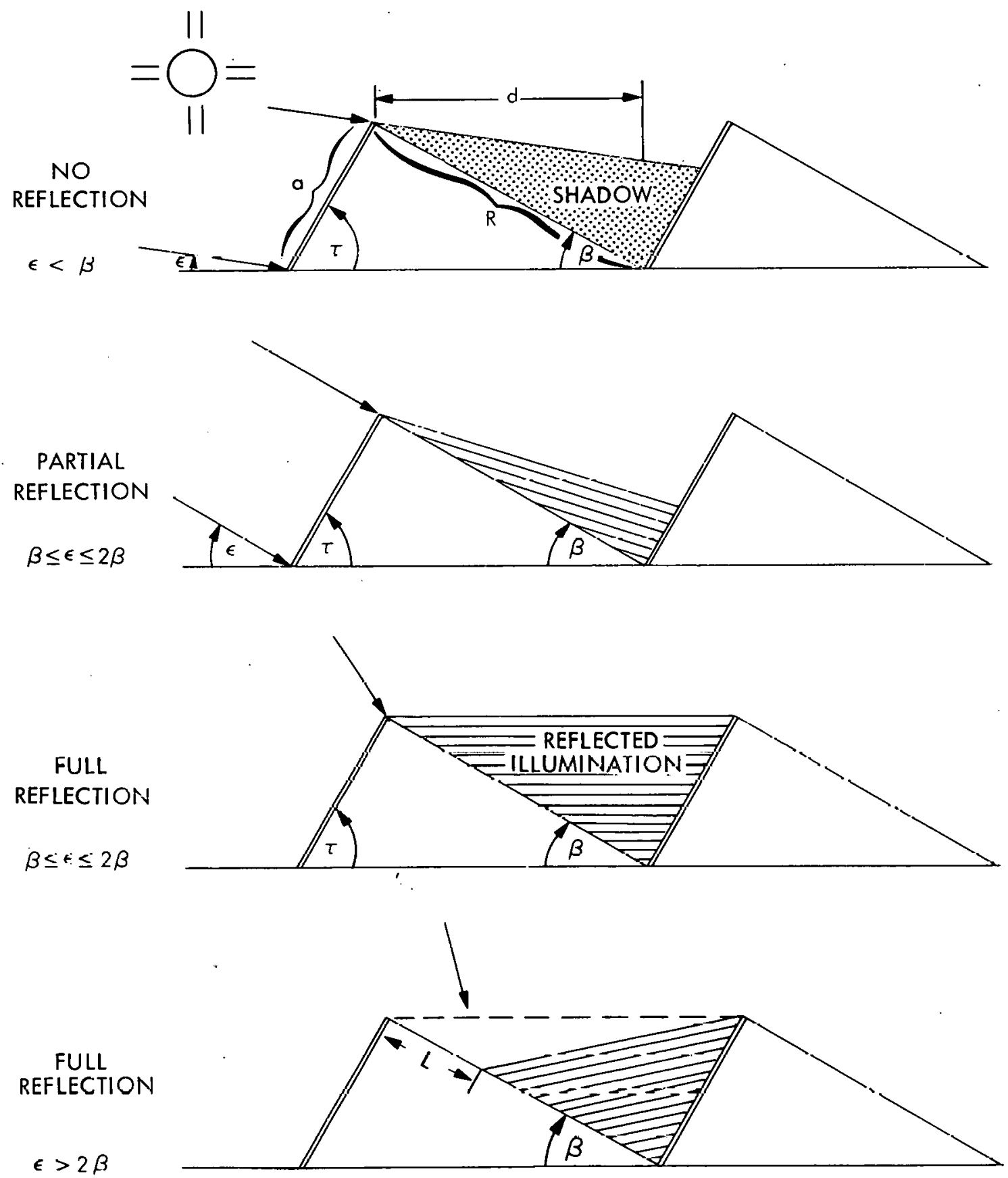

Figure 4-1. Reflector Augmentation Geometry 
Since $L=0$ for $\epsilon \leq 2 \beta$, a simpler form can be used for sun elevation angles less than twice the reflector inclination angle:

$$
1+\Delta I / I=1+\rho^{\prime} \times \sin (\epsilon-\beta) \times \frac{\sin \tau}{\sin \beta}
$$

( for $\beta \leq \epsilon \leq 2 \beta$ )

(Equation 4-1)

And for sun elevations greater than twice the reflector inclination, the multiplier is corrected for reflector losses by:

$I+\Delta I / I=$

$$
\begin{array}{r}
1+\rho^{\prime} \times \sin \tau \times \sin (\epsilon-\beta) \times\left[\csc \beta-\frac{\cot \beta+\cot \tau}{\frac{\sin \beta}{\tan (\epsilon-2 R)}+\cos \beta}\right] \\
\quad(\text { for } \epsilon>2 \beta) \\
\quad \text { (Equation 4-2) }
\end{array}
$$

So, for a given hourly insolation estimate from section. II or recorded data, multiplication by $(1+\Delta I / I)$ yields the total available plus reflected insolation on the array surface. That is,

$$
\bar{I}(\omega)= \begin{cases}\overline{\mathrm{I}}_{\mathrm{Tt}}(\omega) \times(1-(\text { Equation } 3-1 *)) & \text { if } \epsilon<\beta \\ \bar{I}_{\mathrm{Tt}}(\omega) \times(\text { Equation } 4-1) & \text { if } \beta \leq \epsilon \leq 2 \beta \\ \overline{\mathrm{I}}_{\mathrm{Tt}}(\omega) \times(\text { Equation } 4-2) & \text { if } \epsilon>2 \beta\end{cases}
$$

Using the energy equation in Section III, this model can be used to determine the optimal spacing and reflector tilt to maximize energy output by multiplying the insolation level by either the shadow factor or the augmentation factor during hour $\omega$, depending on whether $\epsilon$ is less than or greater than the reflector tilt, $\beta$. The computer program discussed in Section VI can be used to determine the optimal reflector angle and array separation distance for a particular site.

Again, recorded data may be used with this model if desired.

*Equation 3-1 is on page 3-2. 
SECTION V

DISCUSSION

A number of models have been presented that enable the solar array system engineer/designer to examine insolation parameters related to the system design. These models are concerned solely with the available insolation that can be expected for a particular location and array spacing, with or without reflecting surfaces. The estimates are average values intended for use in engineering applications. The advantages of the approach in this document are that sensitivity analyses can easily be performed and, given the appropriate cost function for array spacing, an optimal array spacing can be calculated.

\section{A. INSOLATION MODEL SENSITIVITY}

Certain limitations should be considered in using this model. The accuracy of the insolation model depends primarily on the $\overline{\mathrm{K}}_{\mathrm{T}}$ values. If an hourly $\overline{\mathrm{K}}_{\mathrm{T}}$ profile for a typical day were available, it would capture the shape of the profile more accurately. When $\bar{K}_{T}$ values greater than 0.75 occur, the tabulated correlations between $\bar{K}_{T}$ and $\mathrm{K}_{\mathrm{d}}$ in Section II can introduce extrapolation error. Sunny locations can yield $\overline{\mathrm{K}}_{\mathrm{T}}$ values greater than 0.8 , which requires extrapolation into the range where no correlated $\bar{K}_{d}$ values exist. In the model and program presented here, extrapolation is limited to the upper bound of $\overline{\mathrm{K}}_{\mathrm{T}}=0.80$.

In applications where load matching within the day is important, the insolation model presented here may be deficient. (Again, this depends on the randomness of the solar climate for the location.) Climates with highly variable cloud patterns will cause additional error; however, since the $\overline{\mathrm{K}}_{\mathrm{T}}$ values are derived from monthly average values, daily and monthly totals should be reliable.

A sensitivity analysis was conducted in order to determine the effect of changes in the solar climate parameter $\overline{\mathrm{K}}_{\mathrm{T}}$ on the total insolation $\bar{I}_{T t}$. It was found that for a horizontal surface, there is essentially a one-to-one correspondence between percentage changes in $\overline{\mathrm{K}}_{\mathrm{T}}$ and the resulting changes in $\overline{\mathrm{I}}_{\mathrm{Tt}}$. However, as the array is tilted upward, the $\mathrm{K}_{\mathrm{T}}$ value is more sensitive in winter months and less sensitive in the summer months; that is, the $\overline{\mathrm{K}}_{\mathrm{T}}$ parameter sensitivity depends on the relationship between the orientation of the array surface and the sun elevation angle. In the higher north latitudes, the magnitudes of the sensitivities increase due to the more dramatic effects of changes in the declination and sun elevation angles.

\section{B. INSOLATION MODEL VERIFICATION}

A weakness of the current model is the difficulty in evaluating its accuracy. With errors in data collection, comparisons are difficult to evaluate. The lack of detailed hourly insolation data for various till 
angles at numerous sites is a further problem. Some work has been done in Reference 12 (pages 263-265); however, since clear-day profiles were used, this error analysis has limited applicability.

Comparing the insolation model to three months of data collected at JPL (for a south-facing surface inclined at 15 degrees) yielded daily total estimates within $3 \%$ of the data values. These results are limited by the short record, but, as mentioned earlier, the model takes the daily amount of radiation and distributes it in a symmetric fashion about solar noon, thus introducing variation at the hourly level. The sum of the hourly absolute deviations of the model estimates from the data was much larger ( $6 \%$ to $8 \%$ ); therefore, this model is to be used cautiously for applications where the profile within the day is important since it does not capture hourly trends. However, uncertainty ahnut the trends in hourly data may exceed the error in using the model, depending on the micro-climate of the location. Improvement could be arhieved using hourly $\bar{K}_{T}$ values if the dita were available.

The primary source of error is the data (total radiation on a horizontal surface) used to estimate the $\overline{\mathrm{K}}_{\mathrm{T}}$ parameters and which limit the accuracy of the model. Since the recorded data may have measurement inaccuracies, these errors translate into model inaccuracy. Because of this, small differences cannot be considered very significant (Reference 21). Similarly, the extrapolation of this model to a site without recorded data must be done cautiously, since the model does not consider solar energy availability variations on a micro-cllimate scale and local topography might create local differences in solar insolation patterns.

Two components are influenced by the sunrise hour and array orientation. As can be seen from the definition of $\cos \theta_{t}$ in section II, the direct normal component is defined to be zero when llie sun is behind the array. Thus, this component is unaffected. However, the diffuse and reflected components depend on the sunrise hour angle vid the $r_{d}$ term. Since the diffuoc and raflecter components are small during the sunrise/sunset hours, the error introduced by using the horizontal surface sunrise angle is not believed to be significant. However, the effect of spectrum changes on morning and afternoon insolation values may complicate this problem. The actual sunrise time that the surface "sees" may be calculated by setting the expression for $\cos 0_{t}$ equal to zero and solving ileratively for $\omega=W_{S}$. The author knows of no detailed study of the errors introduced by non-south-facing tilted surfaces. Thus, the user of the SOLINS subroutine provided with this document should be aware of the above problems with non-south-facing tilted arrays.

Finally, it should be noted that the model produces estimates of total available insolation and that photovoltaic cells respond only to certain wavelengths of the spectrum -- a spectrum that changes during the day. Thus, actual available insolation will be less for solar cells since they respond only to a certain region of the spectrum (see Section II-D above). This problem is in a sense a conversion efficiency problem specific to the technology of photovoltaic conversion and was not addressed in this document. 


\section{SHADOW AND REFLECTOR MODELS}

The shadow loss and reflector augmentation models provide estimates of the insolation energy losses and gains associated with these problems. Obviously, there are a number of shortcomings. The accuracy of these models depends directly on the accuracy of the insolation data input. The assumption that there is a one-to-one relationship between shadowing and insolation losses may be an optimistic estimate in the case where partial shadowing severely reduces module output. Another drawback of this model is that it is limited to rectangular-shaped collectors -- non-uniform shadowing is not considered. The reflector augmentation model shares this problem. In addition, there may be double counting of reflected insolation in areas of low ground reflectance. The specular and ground reflectance must be chosen carefully; e.g., if there are no reflective objects (mountains, trees, etc.) facing the arrays, it may be sufficient to set the ground reflectance to zero and adjust the specular reflectance. This is an area requiring further examination.

The remainder of this document presents a SOLINS computer program user's guide; a listing of SOLINS and related computer programs; and insolation profile data for 235 sites in the United States for various south-facing tilt angles. The computer program also computes shadow losses and reflector augmentation estimates on a monthly and annual basis. The values computed were verified by sampling $3 \%$ of the sites and reproducing. all of the calculations using an APL computer program first and then by hand calculation. 


\section{SECTION VI}

\section{SOLINS COMPUTER PROGRAM USER'S GUIDE}

The model presented here and the resulting computer program (called SOLINS) 1 isted in Appendix A were written to support work being performed for the Low-Cost Solar Array Project at the Jet Propulsion Laboratory. In addition to calculating hourly and daily insolation estimates by month, the program calculates monthly and annual total available energy with array shadowing and/or reflector augmentation (that is, a reflecting surface facing the array surface). A copy of the program is available from the Computer Software Management and Information Center (COSMIC) operated for NASA by the Information Services Division of the University of Georgia Computer Center (Reference 22 ).

Eight subprograms are contained in the SOLINS package. In addition, the user must provide a main program that will call SOLINS. Appendix A illustrates a sample main program and each of the eight subprograms. The example main program runs each of three options available with SOLINS and the resulting outputs are shown in Appendix A. Also included in Appendix $A$ is the program that generated the $m$ factor tables in Section II.

\section{A. SUBPROGRAM INS}

The program is based upon a modified version of the National Bureau of Standards model (see Section II) and calculates the direct, diffuse, and reflected components of total insolation on a tilted surface with a given azimuthal orientation (south facing, east facing, etc.). The subroutine that produces these estimates (called INS) generates a monthly, average day profile by hour of the insolation energy. The model uses a parameter that captures the monthly solar climate, so the estimates include clouding factors and other weather patterns -- they are not clear day estimates. The user of the program specifies the latitude of the site, the array tilt, the azimuth, the month, the solar climate parameter, the ground reflectance, and the printout specification.

\section{B. SUBPROGRAMS SHADOW AND REFL}

In order to generate the shadowing and reflector estimates, the user must also specify the array spacing, the array surface length, and the spectral reflectance. Given the user's design data, the information required to run the program is the site latitude and solar climate parameter. This will be discussed in further detail later in this Section. 


\section{COMPUTER CONFIGURATION REQUIRED -- MEMORY AND TIMING}

The program was written in FORTRAN IV using the IBM $370 / 3032$ at the California Institute of Technology. The program was designed to run in a batch mode and requires a maximum of 128 kilobytes of storage. The execution time is between 3 and $20 \mathrm{CPU}$ seconds, depending on the specific user requirements.

\section{ACCURACY OF RESULTS}

Due to the uncertainties associated with insolation data (see Section V, page 5-2), all calculations are performed in single precision. Thus, output tables have accuracy no greater than three significant figures. The program output was verified by hand calculation of a sampling of sites.

\section{E. CONTROL CARD REQUIREMENTS}

The deck setup shown in Figure $6-1$ is required to run the program. 'First are the control cards, which consist of a job card with accounting information specific to the site installation and compiler instructions (cards for an IBM installation are given in Figure 6-1) which are instructions to the operating system. Next, the main program and the SOLINS package are tollowed by a control card (installation specific) and then any data that are to be read in by the main program. At the end of the deck is a card that indicates the end of the job. This card is also installation specific. The program given here has no.data included with it.

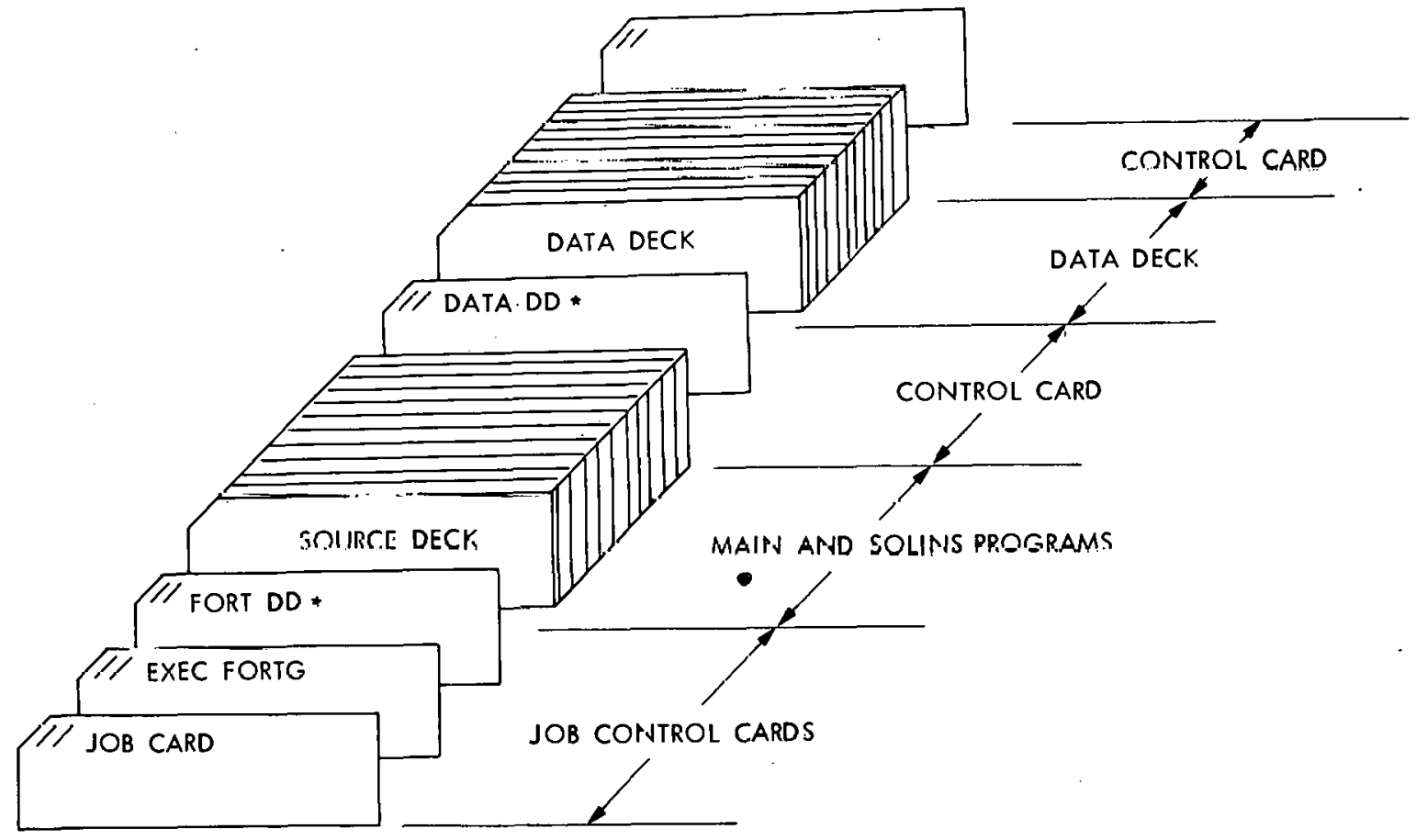

Figure 6-1. SOLINS Deck Setup 


\section{F. USER INSTRUCTIONS}

The user has three options available with the solar insolation program package (SOLINS):

Option 1 -- Calculate the insolation tables only. That is, for one or all months, calculate the average hourly and daily insolation for a tilted surface at a given azimuthal orientation.

Option 2 -- Calculate the insolation at five different array spacings (one tilt angle only) and on a monthly or annual basis. The unobstructed insolation is also calculated and a fraction of total monthly and annual energy lost due to shadowing is computed.

Option 3 -- Calculate the reflector augmentation at a particular array spacing (one tilt angle only) and the fraction energy gain, by month or annually.

Each of these options has a distinct set of requirements and a general set of input rules that apply to all three.

The general input data requirements are that the variables ZKT (the 12 solar climate values), XlAT (the latitude), TOWN (the location), and RHO(the ground reflectance) must be specified when SOLINS is called. XLAT is represented in the form degrees and minutes (e.g., 32.51 is equivalent to $32^{\circ} 51^{\prime}$ ).

In addition to the general input data requirements, the parameters of the subroutine call must be specified. The CALL statement is of the following form:

\section{CALL SOLINS (ISWTCH, MONTH, IPRINT)}

where

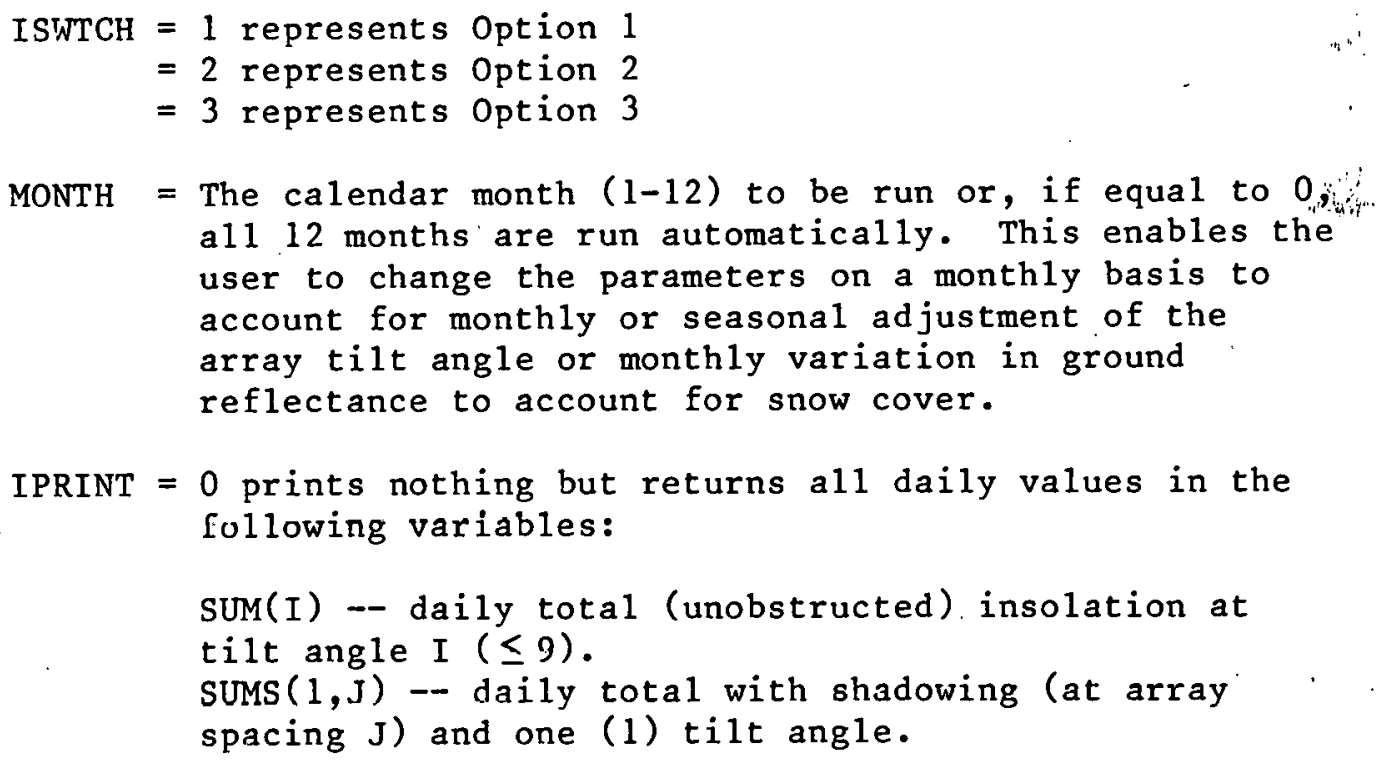


SUMRE(1) -- daily total with reflector augmentation at one (1) tilt angle.

$=1$ prints the hourly and daily tables only;

$=2$ prints only the shadow subroutine results;

$=3$ prints the hourly and daily tables associated with the INS subroutine (which is called by SHADOW) as well as the output from SHADOW;

$=4$ prints the results of subroutine REFL only;

$=5$ prints the hourly and daily tables associated with the INS subroutine (which is called by REFL) as well as the output results from REFL.

The labulated output is in kilowatt-hours per square meter. If other units are desired, the user must redefine the variable RST in the INS subroutine from $1.377 \mathrm{~kW} / \mathrm{m}^{2}$ to the equivalent. value in the now units (e.g., $\left.437 \mathrm{BTU} /\left(\mathrm{hr}-\mathrm{ft}^{2}\right)\right)$. Therefore, depending on the needs of the user, a variety of outputs are possible.

To aid the user, an optional subroutine DATCK (ISWTCH) has been included. This subprogram should be called prior to calling SOLINS and it will perform a cursory examination of the input data by checking ranges of values. It should be noted that DATCK does not have to be run for SOLINS to work; in fact, it may be removed from the package if desired.

The only program restriction is that the calling program to soliNs must contain the following labeled common statement:

COMMON/SOL/ZKT(12), XLAT, RHO, TOWN(5), TILT(9), NO, NA, X(5), IUNIT, SREFL, BETA, ALNGTH, $\operatorname{SIJM}(9), \operatorname{SUM}(9,5), \operatorname{SUMRE}(9)$, $\operatorname{ZIT}(2 / 1,9), \operatorname{SHD}(24,1,5), \operatorname{RF} . F(24,1)$

where these variable names are reserved fur use by solins. In uddition, the output unit must be specified using the integer variable IUNIT. This concludes the general requirements of the SOLINS package.

Option 1

The Option 1 ( TSWTCH $=1$ ) of the SOLINS subprogram computes the hourly and daily insolation estimates by calling the INS subprogram. The requirements for using this option are:

1. Up to but not more than mine tilt angles may be run al ous time. The tilt angles (in decimal degrees) are stored in TILT(I) and a zero tilt angle may be used.

2. The number of tilt angles must be specified with the integer variable NA $(\leq 9)$. 
3. The dzimuthal orientation must be specified with the integer variable NO $(1 \leq$ NO $\leq 9)$, where

$$
\begin{array}{ll}
1=\text { South } & 6=\text { Northeast } \\
2=\text { Southwest } & 7=\text { East } \\
3=\text { West } & 8=\text { Southeast } \\
4=\text { Northwest } & 9=\text { Horizontal } \\
5=\text { North } &
\end{array}
$$

If the user requires an azimuthal orientation angle somewhere between the above choices, the variable WAZ would have to be redefined with that angle $\left(0^{\circ}=\right.$ north, azimuth measured clockwise).

4. The ground reflectance must be specified using the real variahle RHO (a valuc of 0.2 is typically used).

5. The general input requirements must be satisfied.

\section{Option 2}

The Option 2 (ISWTCH = 2) of the SOLINS subprogram computes the monthly total insolation estimates including shadowing by calling the subprogram, SHADOW, which in turn calls the INS subprogram. If the MONTH parameter is set equal to zero, the annual totals are also computed. The requirements for using this option are:

1. Al1 of the Option 1 restrictions must be satisfied except the number of tilt angles, NA, must equal one.

2. The real variable ALNGTH, the array surface length (same units as $X(\bullet))$, must be specified (see Figure 3-1).

3. The real vector $X(\bullet)$ of five array spacing values must be specified (same units as ALNGTH).

4. The azimuthal orientation must be south facing (NO $=1$ ).

5. The tilt angle should not equal zero (reduindant to purpose of Option 2).

Option 3

The Option 3 (ISWTCH = 3) of the SOLINS subprogram computes the total insolation estimates including shadowing and reflector augmentation by calling the subprogram REFL, which in turn calls the INS subprogram. If MONTH $=0$, the annual totals are also computed. The requirements for using this option are:

1. All the Option 2 restrictions must be satisfied -- except the array spacing value must be stored in $X(1)$ (array spacing is not allowed to vary within a single call to REFL). 
2. The real variable SREFL must contain the spectral reflectance value of the reflective surface. If no reflective surface is present, SREFL $=0.0$.

A sample main program with the corresponding output may be found in Appendix A. While this program package does not allow for every possible user need, a listing of the programs is presented in Appendix A to enable the user to adapt the program to individual requirements. 


\section{REFERENCES}

1. Jessup, E., "A Brief History of the Solar Radiation Program," Report and Recommendations of the Solar Energy Data Workshop, November 29-30, 1973, Report No. NSF-RA-N-74-062, National Oceanic and Atmospheric Administration, Asheville, N.C., September 1974.

2. SOLMET, Hourly Solar Radiation-Surface Meteorological Observations, Vol. 1, Users Manua1, TD-9724, December 1977, and Vol. 2, Final Report, February 1979, National Climatic Center, National Oceanic and Atmospheric Administration, Asheville, N.C.

3. Rapp, D., and Oxley, D., "On the Relation Between Global Insolation on Horizontal and Tilted Surfaoco," Energy Cumverston, Vo1. 18, pp. 39-43, 1978.

4. Liu, B. Y., and Jordan, R. C., "The Interrelationship and Characteristic Distribution of Direct, Diffuse, and Total Solar Radiation," Solar Energy, Vol. 4, No. 3, pp. 1-19, 1960.

5. Liu, B. Y., and Jordan, R. C., "Daily Insolation on Surfaces Tilted Toward the Equator," ASHRAE Journal, Vol. 3, No. 10, pp. 53-59, October 1961 .

6. Liu, B. Y., and Jordan, R. C., "A Rational Procedure for Predicting the Long-Term Average Performance of Flat-Plate Solar Energy Collectors," Solar Energy, Vol. 7, No. 2, pp. 53-74, 1963.

7. Kusuda, T., and Ishii, K., Hourly Solar Radiation Data for Vertical and Horizontal Surfaces on Average Days in the United States and Canada, National Bureau of Standards Building Science Series 96, Center for Building Technology, Institute for Applied Technology, Washington, D.C., April 1977.

8. Klein, S. A., Beckman, W. A., and Duffie, J. A., "Monthly Average Solar Radiation on Inclined Surfaces for 261 North American Cities," Solar Energy Lab Report No. 14-2, University of Wisconsin, Madison, Wis., August 1978.

9. Liu, B. Y., and Jordan, R. C., "Availability of Solar Energy for Flat-Plate Solar Heat Collectors," Low Temperature Engineering Applications of Solar Energy, Chapter 1, pp. 1-18, 1967, published as Chapter 5, Applications of Solar Energy for Heating and Cooling of Buildings, American society of Heating, Refrigerating and Air-Conditioning Engineers, Inc. (ASHRAE), New York, N.Y., 1976.

10. Cinquemani, V., Owenby, J. R., and Baldwin, R. G., "Input Data for Solar Systems," Environmental Data and Information Service, National Climatic Center, Asheville, N.C., Interagency Agreement No. E(49-26)-1041, Revised August 1979. 
11. Duffie, J. A., and Beckman, W. A., Solar Energy Thermal Processes, pp. 10-12, J. Wiley and Sons, New York, N.Y., 1974.

12. California Solar Data Manual, pp. 248, 263-265, Energy and Environment Division, Lawrence Berkeley Laboratory, University of California, Berkeley, Calif., January 1977.

13. Hunn, B. D., and Calafell, D. 0., "Determination of Average Ground Reflectivity for Solar Collectors," Technical Note, Solar Energy, Vol. 19, pp. 87-89, December 1975.

14. Liu, B. Y., and Jordan, R. C., "The Long-Term Average Performance of Flat-Plate Solar Energy Collectors," Solar Energy, Vo1. 7, pp. $53-74,1963$.

15. Thekaekara, M. P., "Solar Energy Outside the Earth's Atmusphere," Solar Energy, Vol. 14, pp. 109-127, 1973.

16. Drummond, A. J., and Thekaekara, M. P., The Extraterrestrial Solar Spectrum, Institute of Environmenta1 Science, Mt. Prospect, I11., 1973.

17. Frolich, C., "Contemporary Measures of the Solar Constant," The Solar Output and Its Variation, Edited by 0. R. White, Colorado Associated University Press, Boulder, Colo., pp. 93-109, 1977.

18. Gemme11, W. M., et al, Shopping Center Applications of Photovoltaic Solar Power Systems: System Design Report for Spectrolab, Inc., Facilities Systems Engineering Corp., Los Angeles, Calif., under ERDA Contract No. E(11-1)-2748, February 1976.

19. Photovoltaic Systems Concept Study, No. ALO-2748-12, Vol. III, Chapter 6, pp. 29-35, Epectrolah, Tne.., Sylmar, Calif., April 1977.

20. Terrestrial Central Station Array Life-Cycle Analysis Support St.udy, DOE/JPL/954848-78/1, p. 10, Bechtel National, Inc., San Francisco, Calif., August 1978.

21. Boes, E. C., et al, Availability of Direct, Total, and Diffuse Solar Radiation to Fixed and I'racking Cullectors in the U.S.A., Energy Report No. SAND 77-0885, Sandia Laboratories, Albuquerque, N. Mex., August 1977.

22. Computer Software Management and Information Center (COSMIC), Information Services Division of the University of Georgia Computer Center, Athens, Ga., Program No. NPO-14787, December 1978 . 
APPENDIX A

LISTING OF SOLINS AND RELATED COMPUTER PROGRAMS

An example main program is given followed by the SOLINS, DATCK, SHADOW, REFL, and INS subroutines. Subroutine INS is followed by its FUNCTION subprograms, ZD, SHDLOS, and AUG. The sample main program runs each of the SOLINS three options.

The first option call in the main program generates the monthly tables of insolation values (kilowatt-hours/square meter) as shown on pages A-23 through A-26.

The second option call in the main program generates the monthly ineolation estimates and annual cotals with and without array shadowing for five array spacings. In addition, the fraction of energy lost due to shadowing is printed (page A-27). The monthly losses are computed by multiplying the daily amounts (with and without shadowing) by the number of days in the given month and computing a percentage loss.

The third option call of the main program makes several passes through the reflector augmentation subroutine while varying the array spacing (pages A-28 through A-30). It can be seen that a maximum occurs at a spacing of 7 meters; however, a more detailed search (finer increments) would be required to determine the global optimum. Again, the daily amounts (before multiplication by the number of days in the month) are printed.

Finally, the program used to generate Tables $2-2 a$ and $2-2 b$ on pages $2-10$ and $2-11$ is given on page $A-31$. 


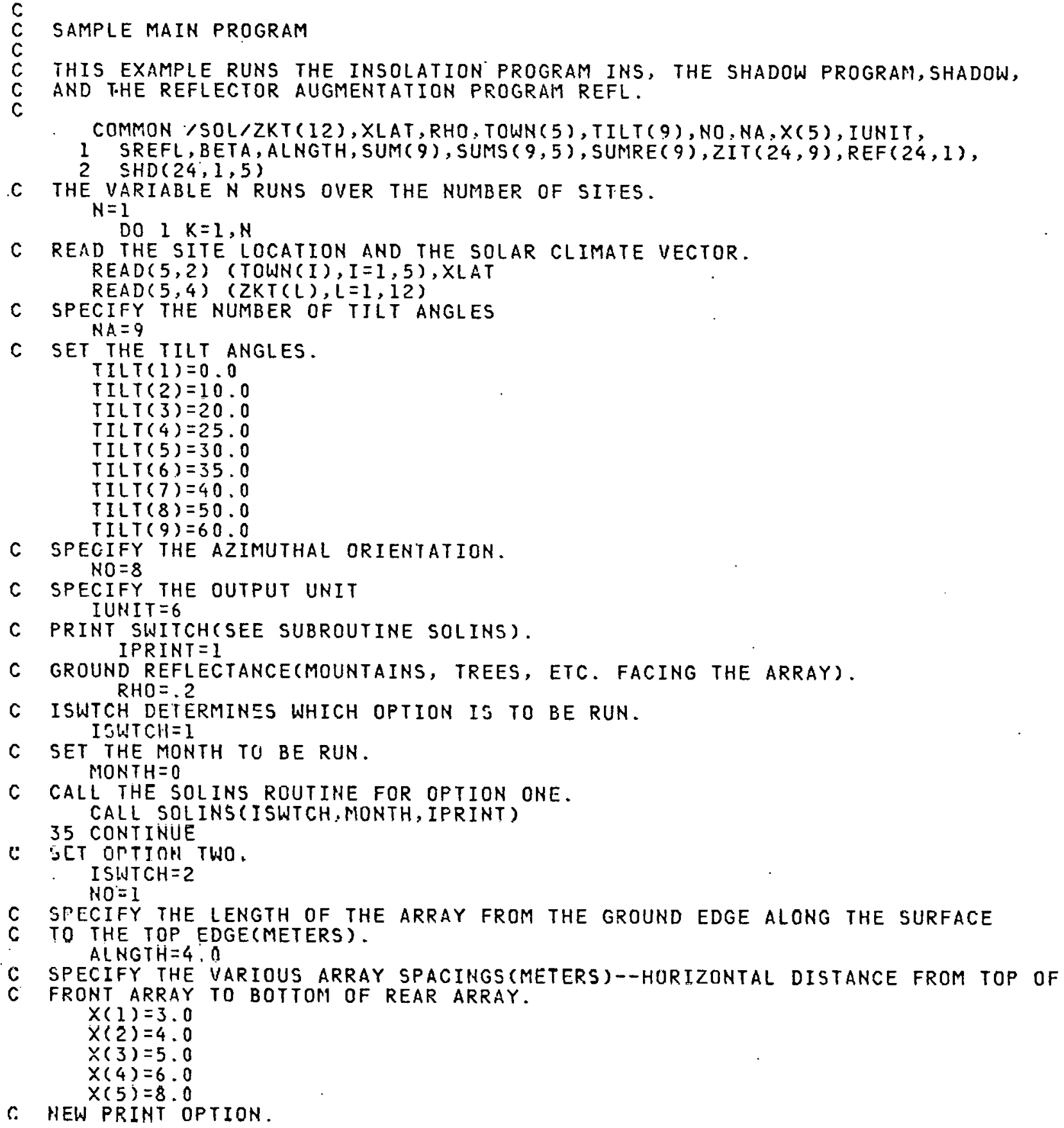

c. HEW PRINT OPTION. 
IPRINT $=2$

C RUN ALL THE MONTHS.

MOHTH $=0$

C SET A NEW TILT ANGLE.

C RESET THE NUMBER OF TILT ANGLES TO ONE. $N A=1$

C CHECK THE DATA FOR OPTION TWO.

CALL DATCK (ISWTCH)

C CALL SOLINS TO RUN OPTION TWO.

CAII SOI TNST. ISIUTCH, MONTH, ITRINT)

C TURN SWITCH TO OPTION THREE--THE REFLECTOR AUGMENTATION PROBLEM.

ISWTCH $=3$

C SPECIFY THE SPECTRAL REFLECTANCE(IF REFLECTORS ARE PRESENT). SREFL $=0.75$

C RUN THE ENTIRE YEAR.

MONTH $=0$

C RESET THE PRINT OPTION.

C RESET THE ARRAY SPACING. $X(1)=3$.

C CHECK THE DATA FOR OPTION THREE.

CALL DATCK (ISWTCH)

C CALL SOLINS FIVE TIMES USING OPTION THREE. DO $5 \mathrm{~J}=1,5$

CALL SOLINS (ISWTCH, MONTH, IPRINT)

C INCREMENT THE ARRAY SPACING. $X(1)=X(1)+4.0$

5 CONTINUE

1 CONTINUE

2 FORMAT $(5 A 4, F 10.0)$

4 FORMAT (12F5.3)

3 STOP

END 


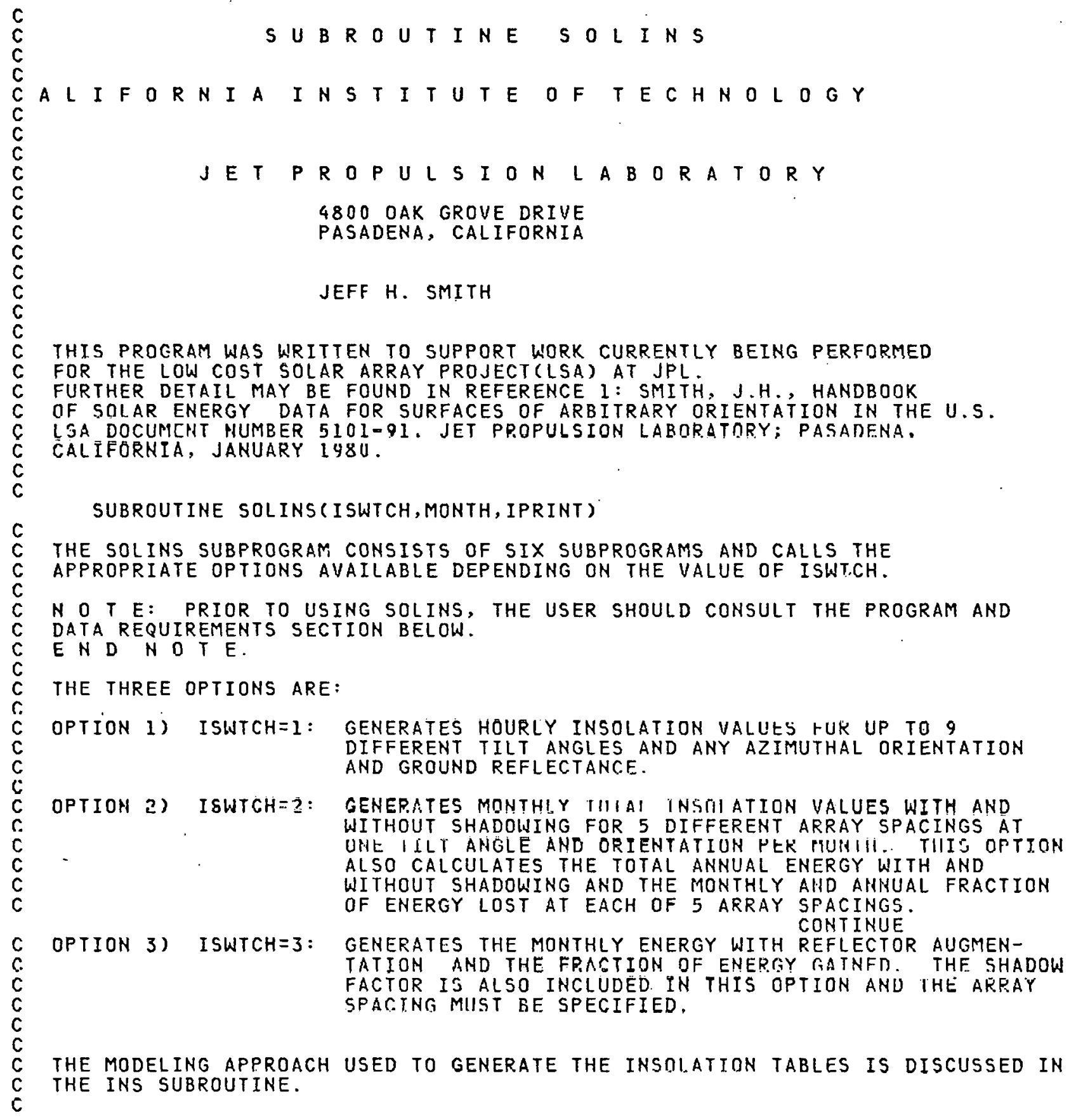


C THE FOLLOWING VALUES OF IPRINT DETERMINE THE INTERMEDIATE AND FINAL OUTPUT C OBTAINED.

C

C IPRINT $=0$ NO PRINTOUT

C IPRIHT =1 HOURLY/DAILY (H/D) TABLE ONLY

C IPRINT $=2$

C IPRINT $=3$

C IPRINT $=4$

C IPRINT $=5$

SHADOW TABLE ONLY

" "AND H/D TABLES

REFLECTOR TABLES ONLY

COMMON /SOL/ZKT (12), XLAT, RHO, TOWN(5), TILT(9), NO, NA,X(5), IUNIT,

I SREFL, BETA, ALNGTH, SUM(9), SUMS $(9,5), \operatorname{SUMRE}(9), Z I T(24,9), R E F(24,1)$,

$2 \operatorname{SHD}(24,1,5)$

C

THE FOLLOHIHC GO TO STATEMEIT CALLS THE APBKUYKLAIE UPIIUN.

GO TO $(1,2,3)$, ISWTCH

C

C

THE FOLLOWING VARIABLES ARE DEFINED FOR COMPATIBILITY PURPOSES ONLY-

PROGRAMMING REQUIREMIENT ONLY

1 DO $10 \mathrm{~K}=1,5$

$10 X(K)=1.0$

$B E T A=1.0$

CALL INS (ISUTCH, MONTH, IPRINT)

RETURN

2 CALL SHADOW(ISWTCH, MONTH, IPRINT)

RETURN

3 CALL REFL ( ISWTCH, MONTH, IPRINT)

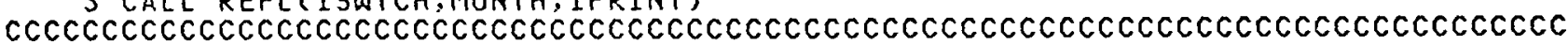

$C$
$C$
$c$
$C$
$C$
$C$
$C$
$C$
$C$
$C$
$C$

R E Q U I R E M E N T S

ASSOCIATED WITH EACH OPTION IS A SET OF RULES WHICH MUST BE ADHERED TO IN ORDER FOR THE PROGRAM TO EXECUTE SUCCESSFULLY. THESE REQUIREMENTS MUST BE SATISFIED PRIOR TO CALLING SOLINS.

IN ADDITION TO THE OPTION REQUIREMENTS THERE ARE A NUMBER OF INPUT DATA AND PROGRAM REQUIREMENTS WHICH ARE ENUMERATED BELOW.

\section{INPUT DATA REQUIREMENTS}

CONTINUE

SINCE THE SOLINS PROGRAM IS USED AS A SUBROUTINE, THF ONIY DATA REQUIREMENTS ARE THAT THE VARIABLES ZKT, XLAT, AND TOWN BE SPECIFIED WHEN SOLINS(OR DATCK IF USED) IS CALLED. ZKT SHOULD CONTAIN 12 SOLAR CLIMATE VALUES FOR JANUARY(ZKT(1)) THROUGH DECEMBER (ZKT(12)). TYPICAL VALUES MAY BE FOUND IN THE REF. DOC. TOWN CONTAINS THE SITE NAME (UP TO 20 CHARACTERS) WITH FORMAT (5A4). XLAT IS SPECIFIED IN DEGREES AND MINUTES SEPARATED BY A DECIMAL POINT (DEG.MIN)

\section{CONTINUE}


THE PROGRAM DATCK DOES NOT HAVE TO BE RUN PRIOR TO CALLING SOLINS. THE MAIN PROGRAM MUST CONTAIN THE FOLLOWING COMMON STATEMENT:

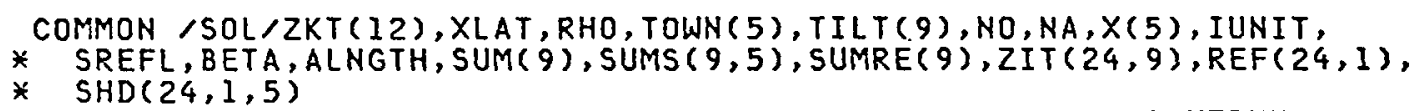

THE UNITS OF THE TABLES MAY BE CONVERTED BY REPLACING THE VARIABLE' RST

(THE SOLAR CONSTANT) WITH ITS EQUIVALENT VALUE IN THE DESIRED UNITS.

RST APPEARS IN THE BEGINNING OF THE INS SUBROUTINE.

N O T E: THF VARIABLES USED IN THE COMMON STATEMENT ARE RESERVED FOR SOLINS AND ITS SUBROUTINES.

THE ARRAY SUM(I) WILL CONTAIN THE DAILY TOTAL INSOLATION FOR ARRAY

TILT ANGLE I FOR MONTH(NO SHADOWING OR REFLECTOR AUGMENTATION).

THE ARRAY SUMS(I,J) WILL CONTAIN THE DAILY TOTAL INSOLATION (WITH SHADOWING) FOR ARRAY SPACING J AND ONE ARRAY TILT ANGLE FOR MONTH.

THE VARIABLE SUMRE(I) WILL CONTAIN THE DAILY TOTAL INSOLATION WITH REFLECTOR AUGMENTATION AND SHADOWING FOR ONE ARRAY TILT ANGLE AND EN D N M T E .

\section{OPTION 1 REQUIREMENTS}

CONTINUE

UP TO BUT NOT MORE THAN 9 TILT ANGLES MAY BE RUN AT ONE TIMECSTORED IN THE ARRAY NAMED TILT). A ZERO TILT ANGLE MAY BE USED.

THE NUMBER OF ANGLES MUST BE SPECIFIED WITH THE INTEGER VARIABLE NA.

THE AZIMUTHAL ORIENTATION MUST BE SPECIFIED WITH THE INTEGER VARIABLE NO: WHERE NO

$$
\begin{aligned}
& =\text { URIENIAIIUN HUMBER AND } \\
& 1=\text { SOUTH } \\
& 2=\text { SOUTHWEST } \\
& 3=\text { WEST } \\
& 4=\text { NORTHWEST } \\
& 5=\text { NORTH } \\
& 6=\text { NORTHEAST } \\
& 7=\text { EAST } \\
& 8=\text { SOUTHEAST } \\
& 9=\text { HORIZ. }
\end{aligned}
$$

THE OUTPUT UNIT MUST BE SPECIFIED USING THE INTEGER VARIABLE IUNIT.

THE GROUND REFLECTANCE MUST BE SPECIFIED USING THE REAL VARIABLE RHO.

THE REAL VECTOR ZKT MUST BE SPECIFIED.

THE TOWN VECTOR SHOULD BE SPECIFIED.

THE LATITUDE XLAT MUST BE SPECIFIED.

THE INTEGER VARIABLE MONTH MUST BE SPECIFIED:

MONTH =DETERMINES WHETHER ALL 12 MDNTHIY TABLES ARE COMPUTED $(=-1)$, OR ONLY ONE MONTHS TABLES ARE COMPUTEDCE.G. MONTH $=3$ FOR MARCH). 


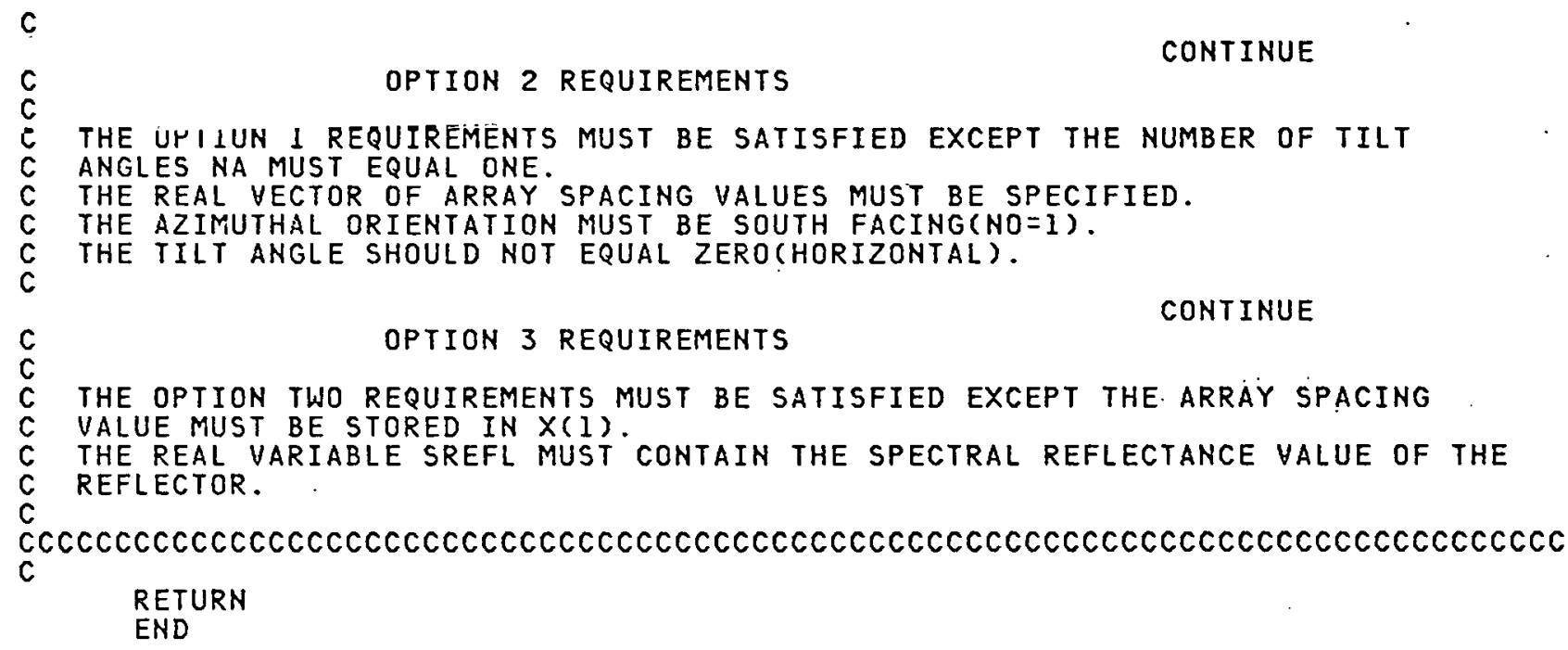




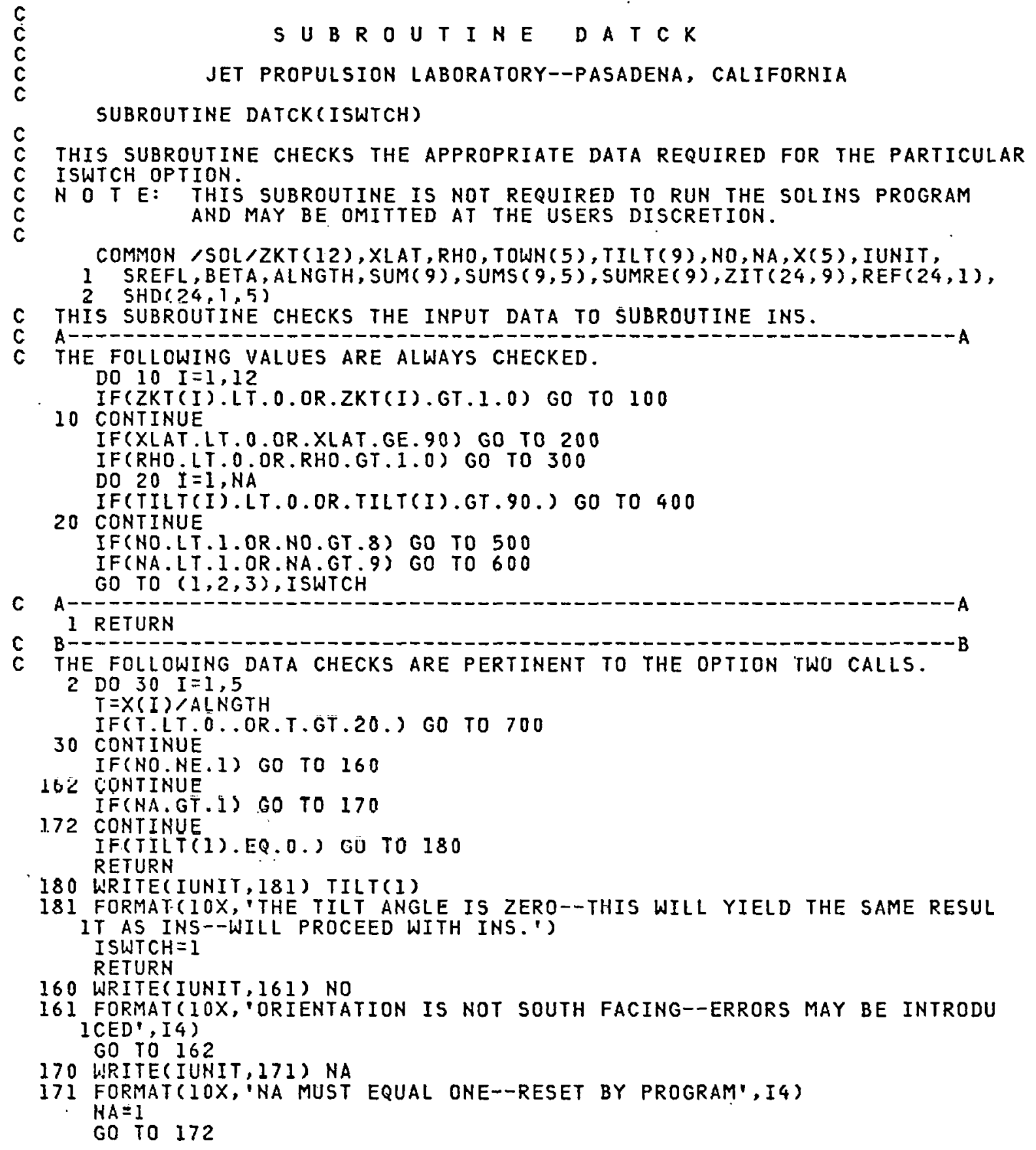




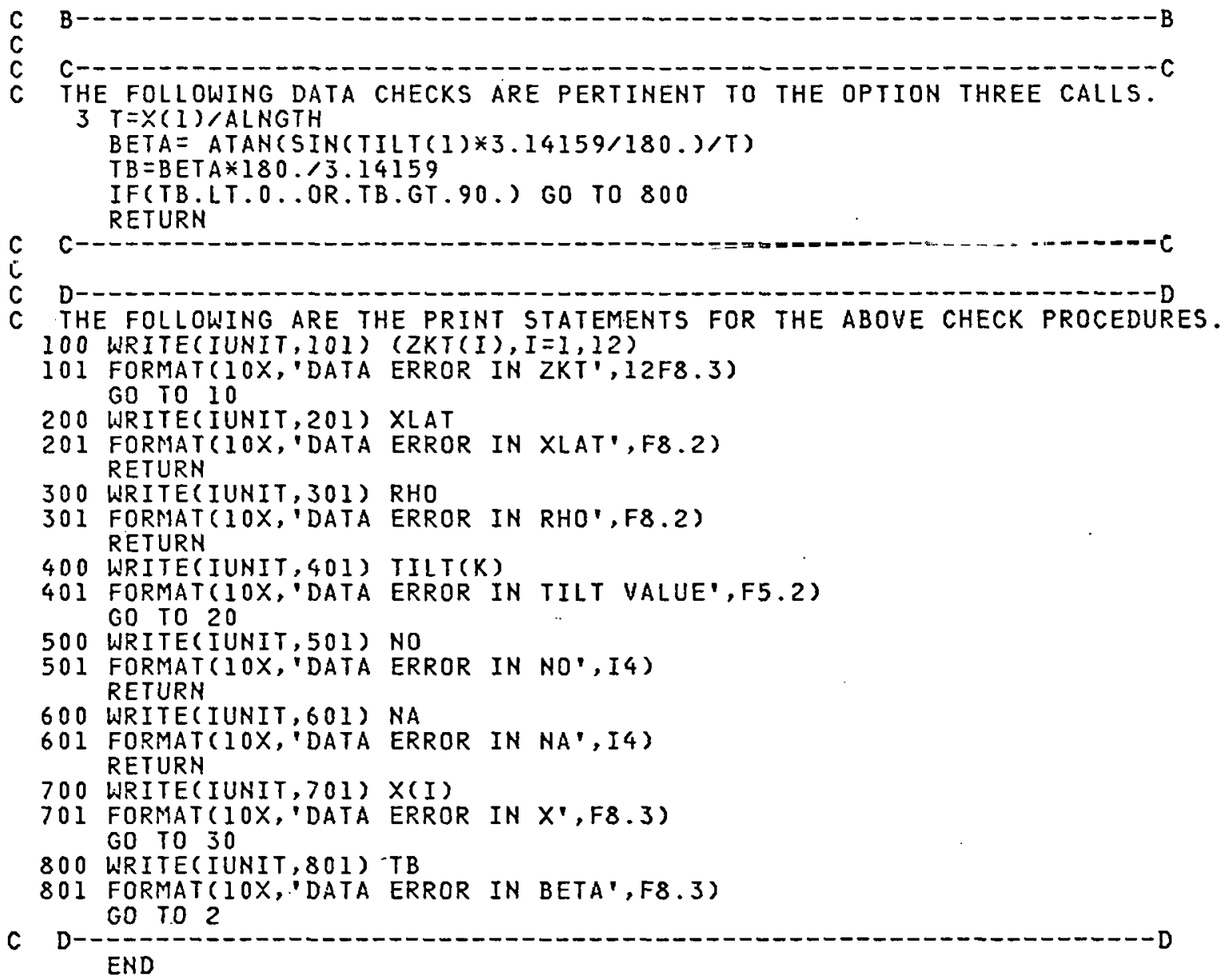




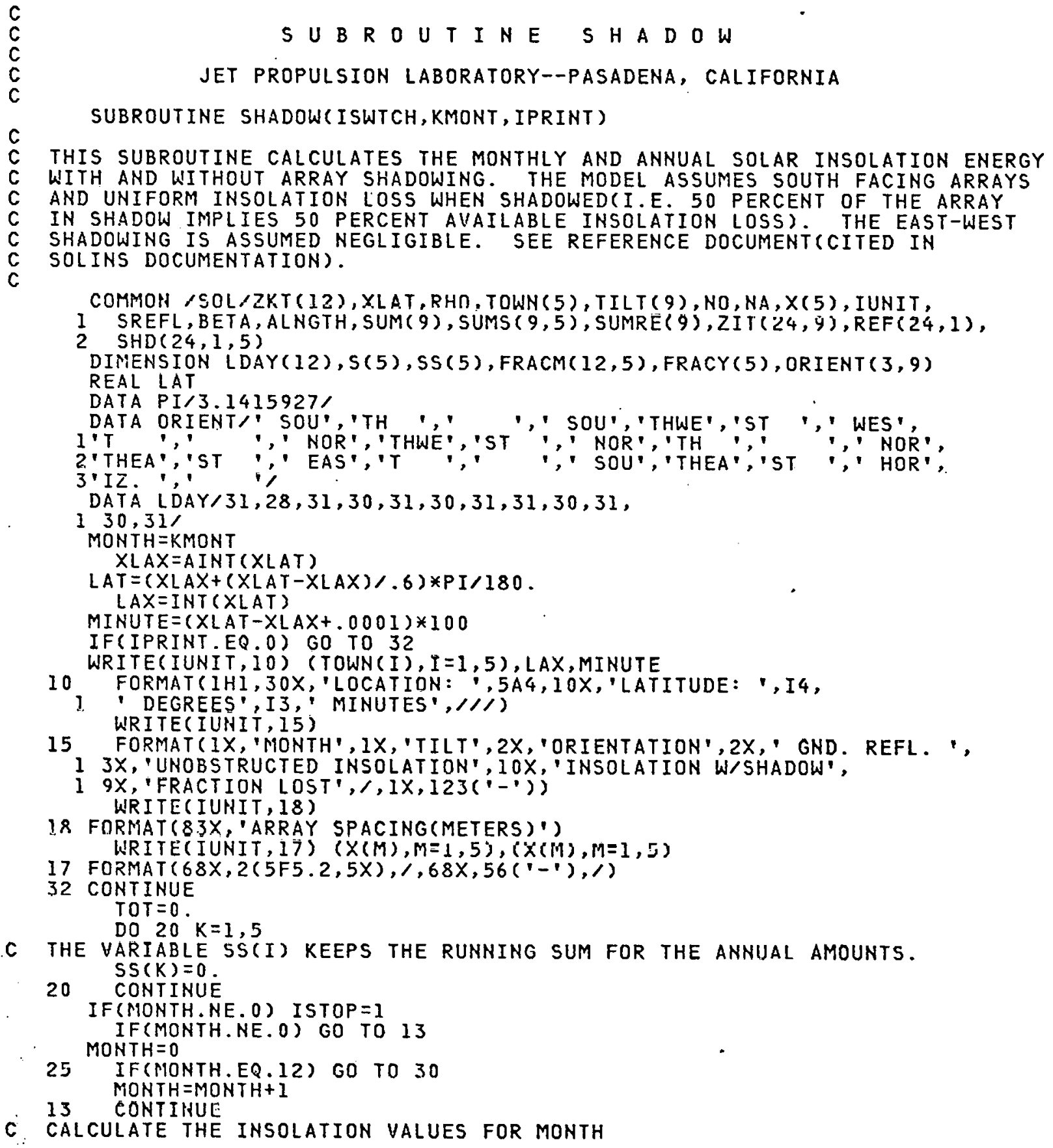


$c$

C CALL INS (ISWTCH, MONTH, IPRINT)

C CALCULATE THE MONTHLY INSOLATION $C=\operatorname{SUM}(1) * \operatorname{LAYY}(M D N T H)$

DO $3 \quad I=1,5$

- $3 \quad S(I)=0$.

C CALCULATE THE ANNUAL INSOLATION THUS FAR.(IF RERUIRED) DO 1 I $1,1,5$ $S(I)=S(I)+S U M S(I, I) * L$ DAY $(M O N T H)$ $S S(I)=S(I)+S S(I)$

C CALCULATE THE MONTHLY FRACTION OF ENERGY LOST DUE TO SHADOWING.

1 FRACM (MONTH, I ) $=A B S(1 .-S(I) / C)$ TOT $=$ TOT +C

IF (IPRINT.EQ.0) GO TO 33

C OUTPUT THE MONTHLY RESULTS.

WRITE (IUNIT , 6) MONTH, TIL $(1$ ( 1$),(\operatorname{ORIENT}(J, N O), J=1,3), \operatorname{RHO}, \operatorname{SUM}(1)$,

1 ( $\operatorname{SUMS}(1, K), K=1,5),($ FRACM $(M O N T H, I), I=1,5)$

6 FORMAT ( IX, I $3,2 X, F 5.2,4 X, 3 A 4,3 X, F 4.2,15 X, F 4.2,15 X$,

$12(5 F 5.2,5 X), / 3$

33 CONTINUE

IF (ISTOP.EQ.1) GO TO 29

2 GO TO 25

30 CONTINUE

IF (ISTOP.EQ.1) GO TO 29

DO 5 L $=1,5$

C CALCULATE THE ANNUAL FRACTION OF ENERGY LOST.

5 FRACY $(L)=I-S S(L) / T O T$

IF(IPRINT.EQ.0) GO TO 29

C OUTPUT THE ANNUAL RESULTS. WRITE(IUNIT,7) TOT, (SS(L), L=1,5), (FRACY $(L), L=1,5)$

7 FORMAT(IX,123(1-1),1/,30X, ANNUAL AMOUNTS: ', F10.2,10X,5F10.2,1, 124X,'ANNUAL FRACTION LOST:',20X,5F10.2)

29 RETURN

END 


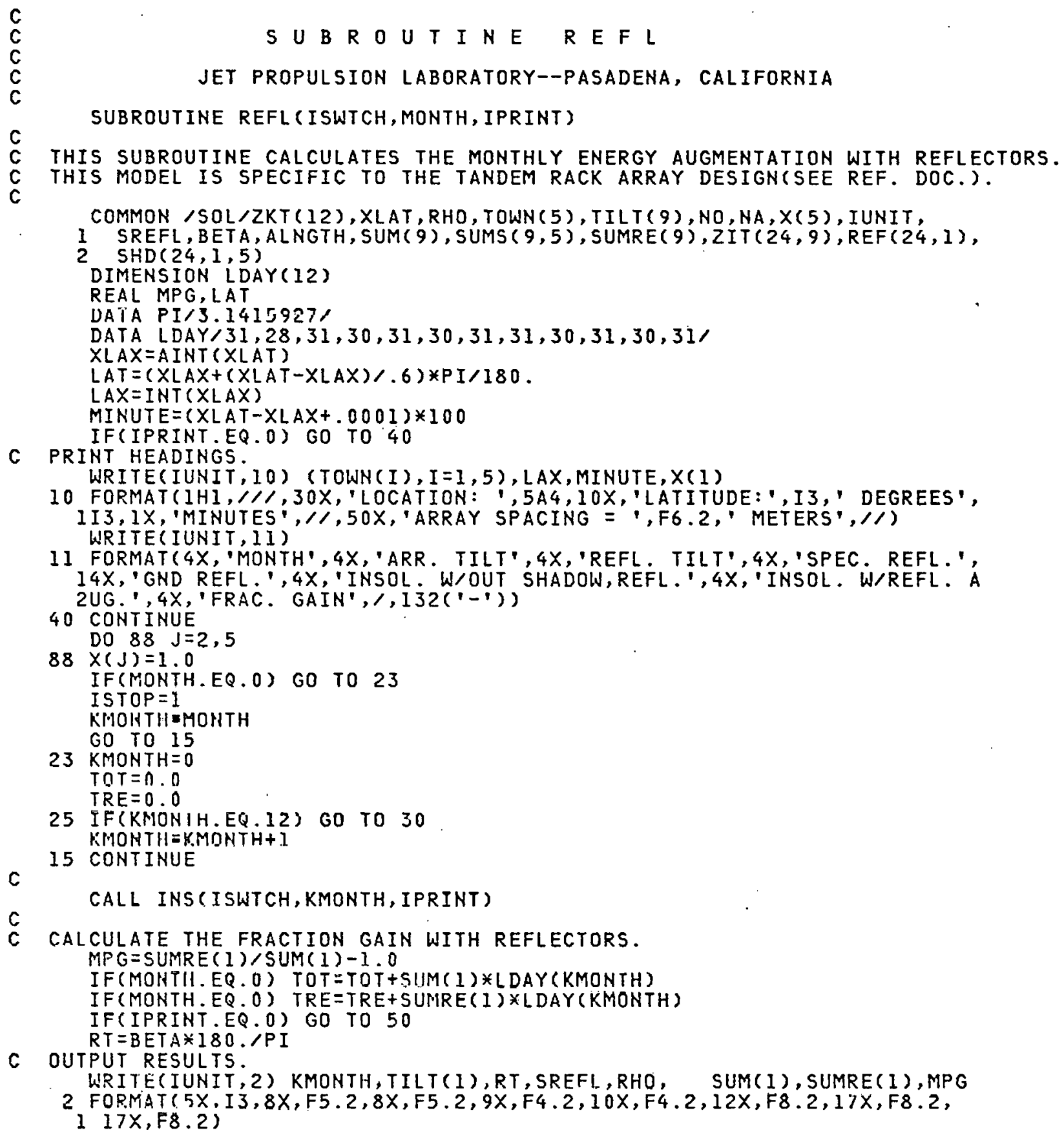


50 CONTINUE

IF(ISTOP.EQ.1) GO TO 13

GO TO 25

30 CONTINUE

IF (IPRINT.EQ.0) GO TO 13

IF(MONTH.NE.O) GO TO 13

ANF $=$ TRE $/ T O T-1.0$

WRITE(IUNIT,62) TOT, TRE, ANF

62 FORMAT $(1 X, 131(-1), / 1,56 X$, ANNUAL AMOUNTS: ', F10.2,15X,FI0.2,15X, $1 F \perp 0.2)$

13 RETURN

END 


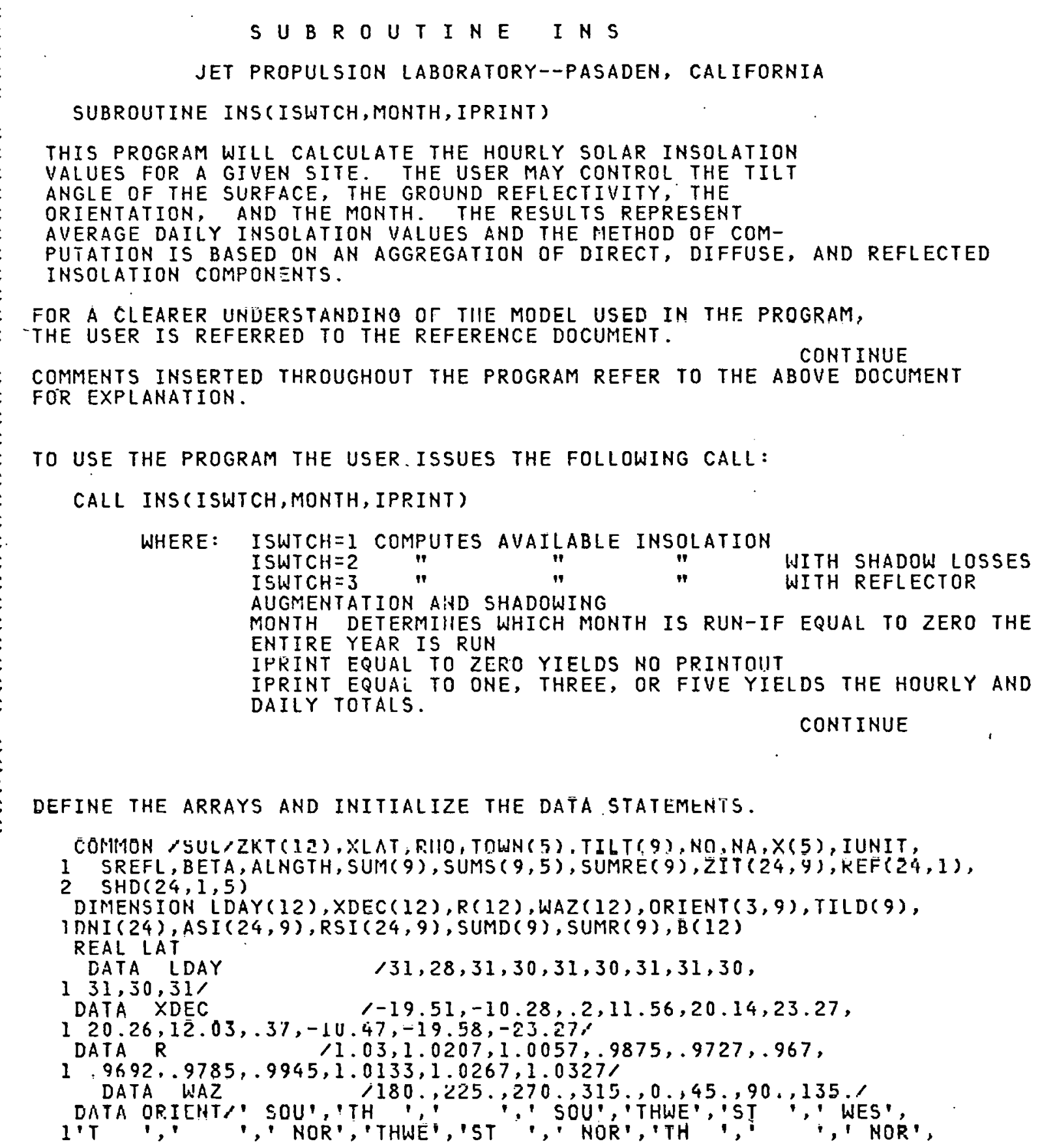




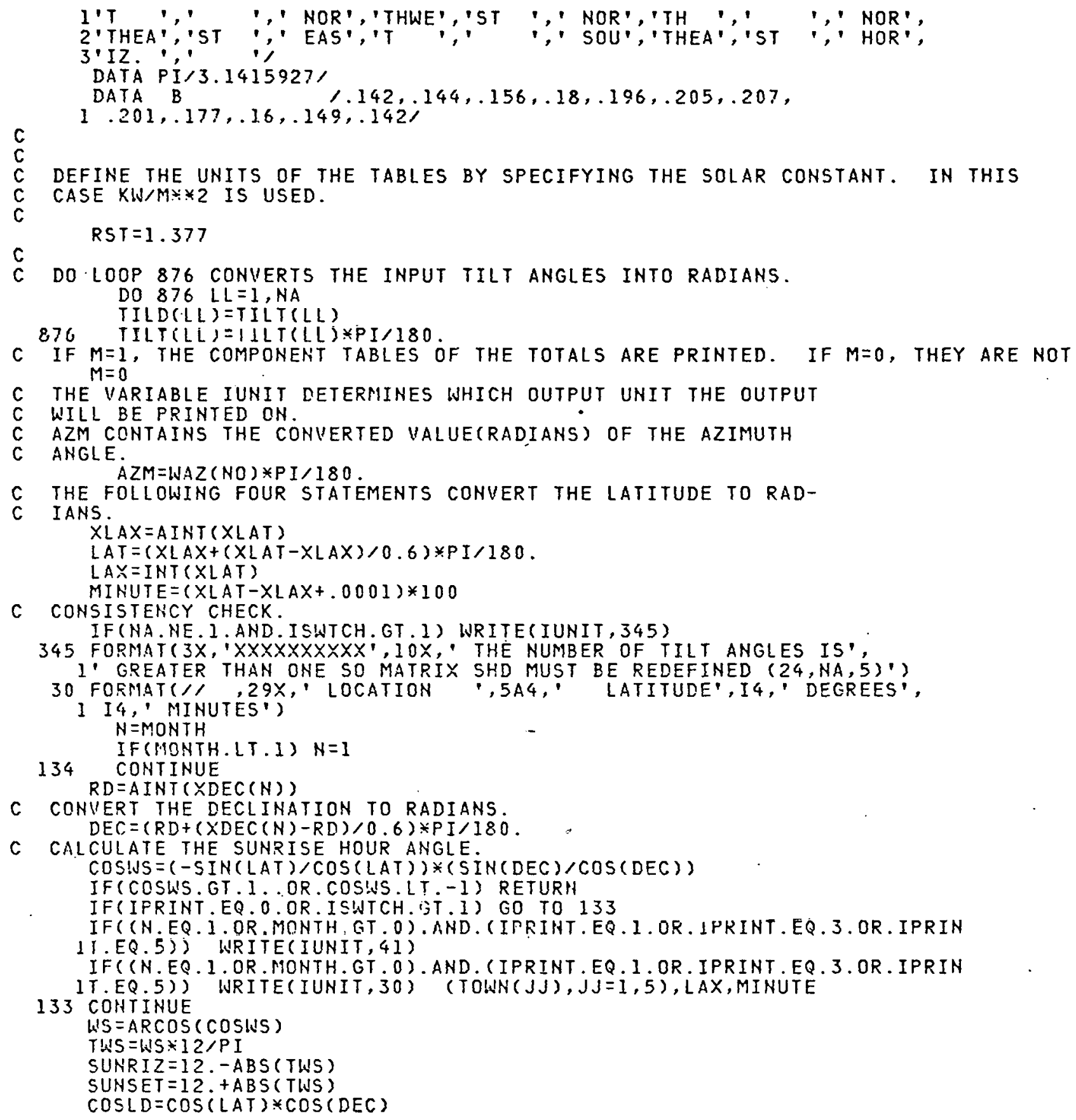




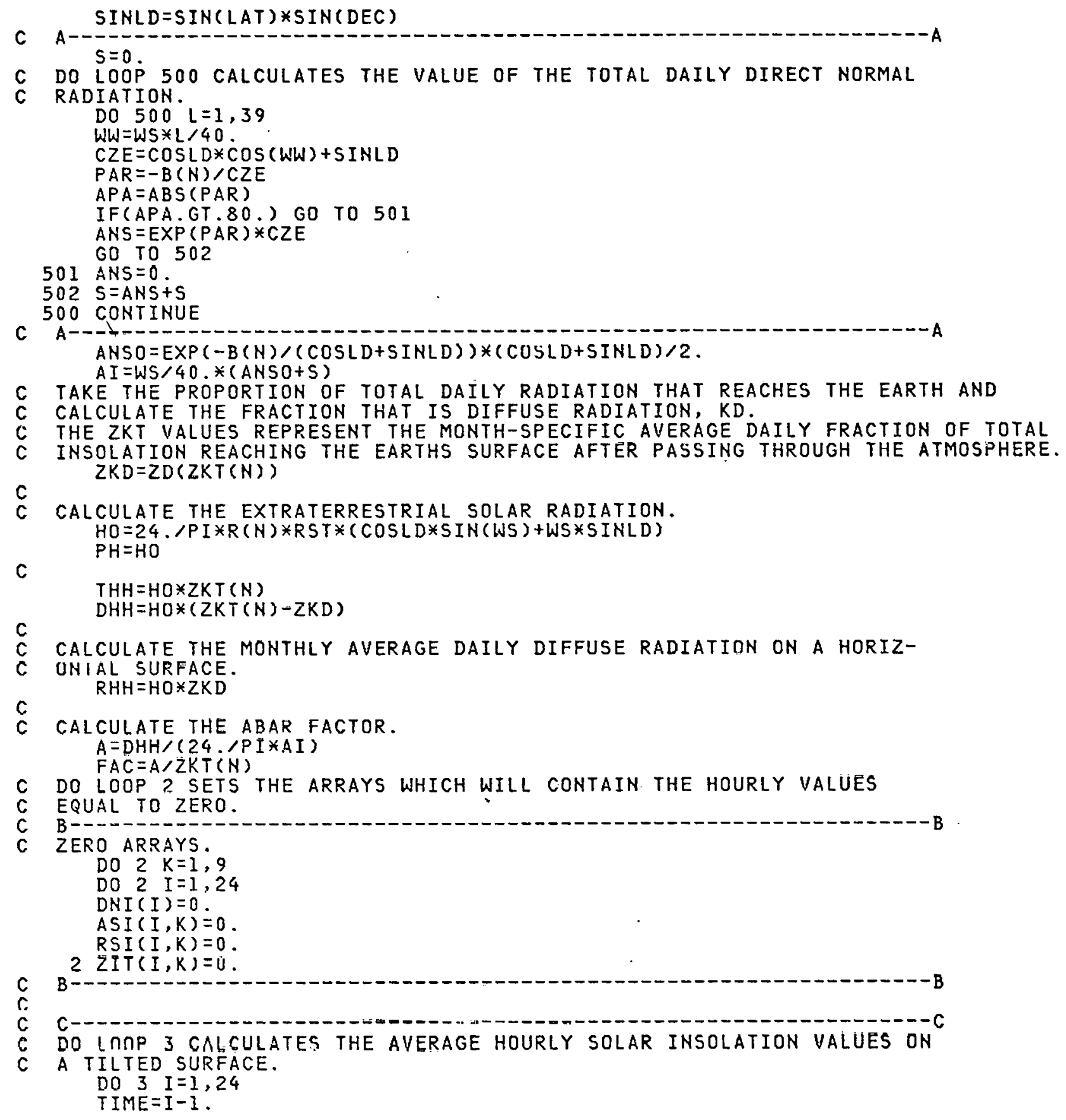




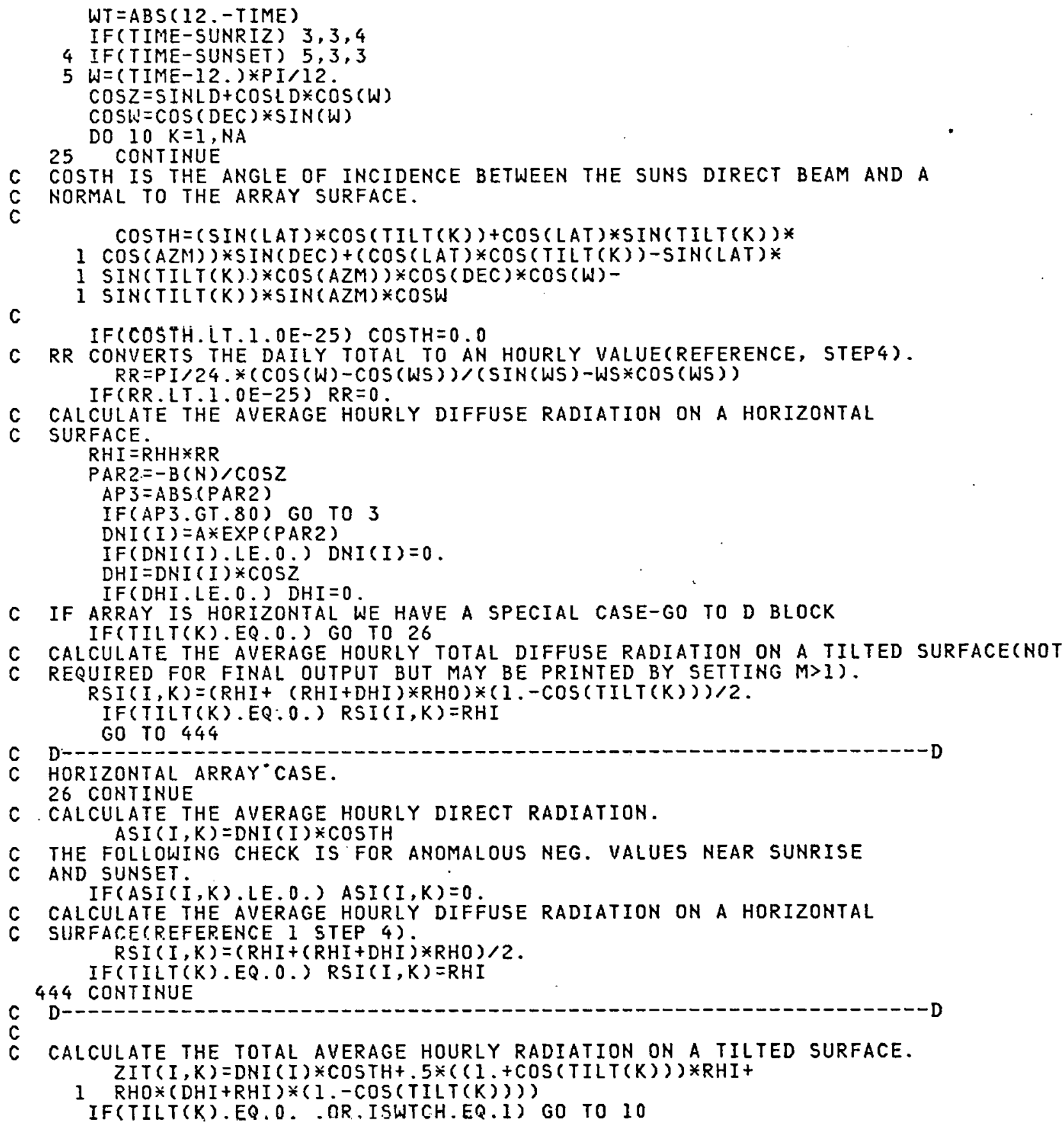




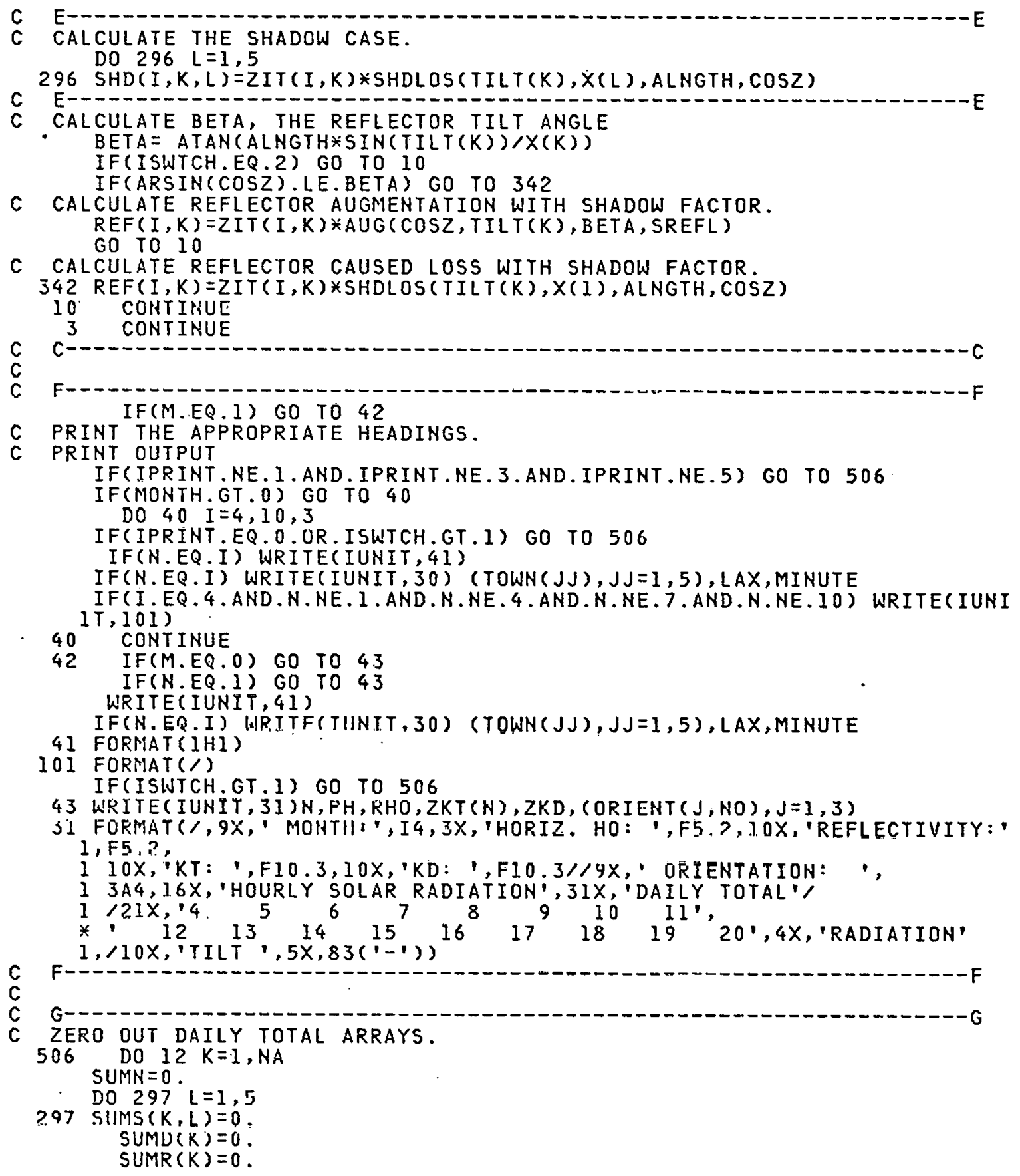




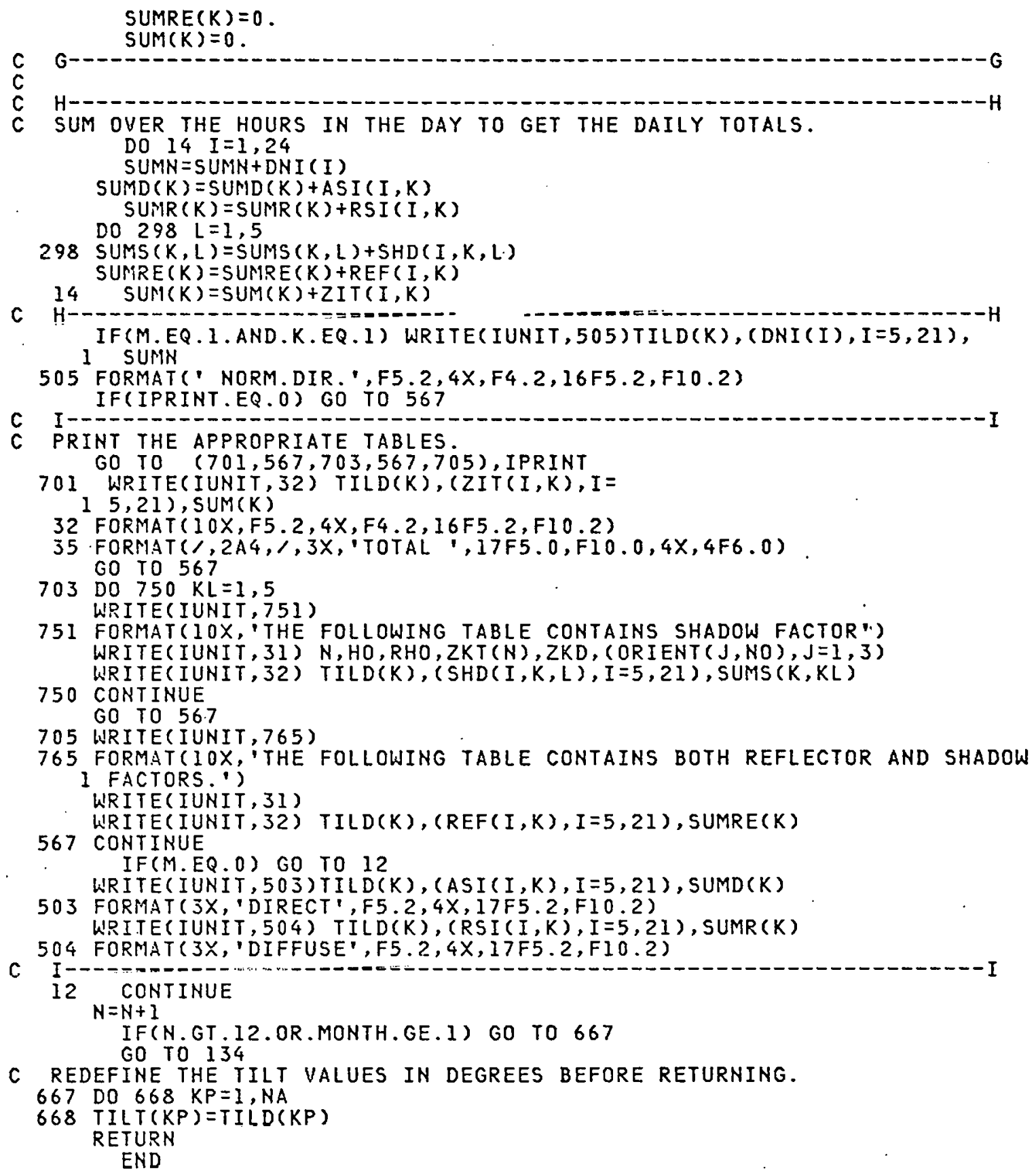




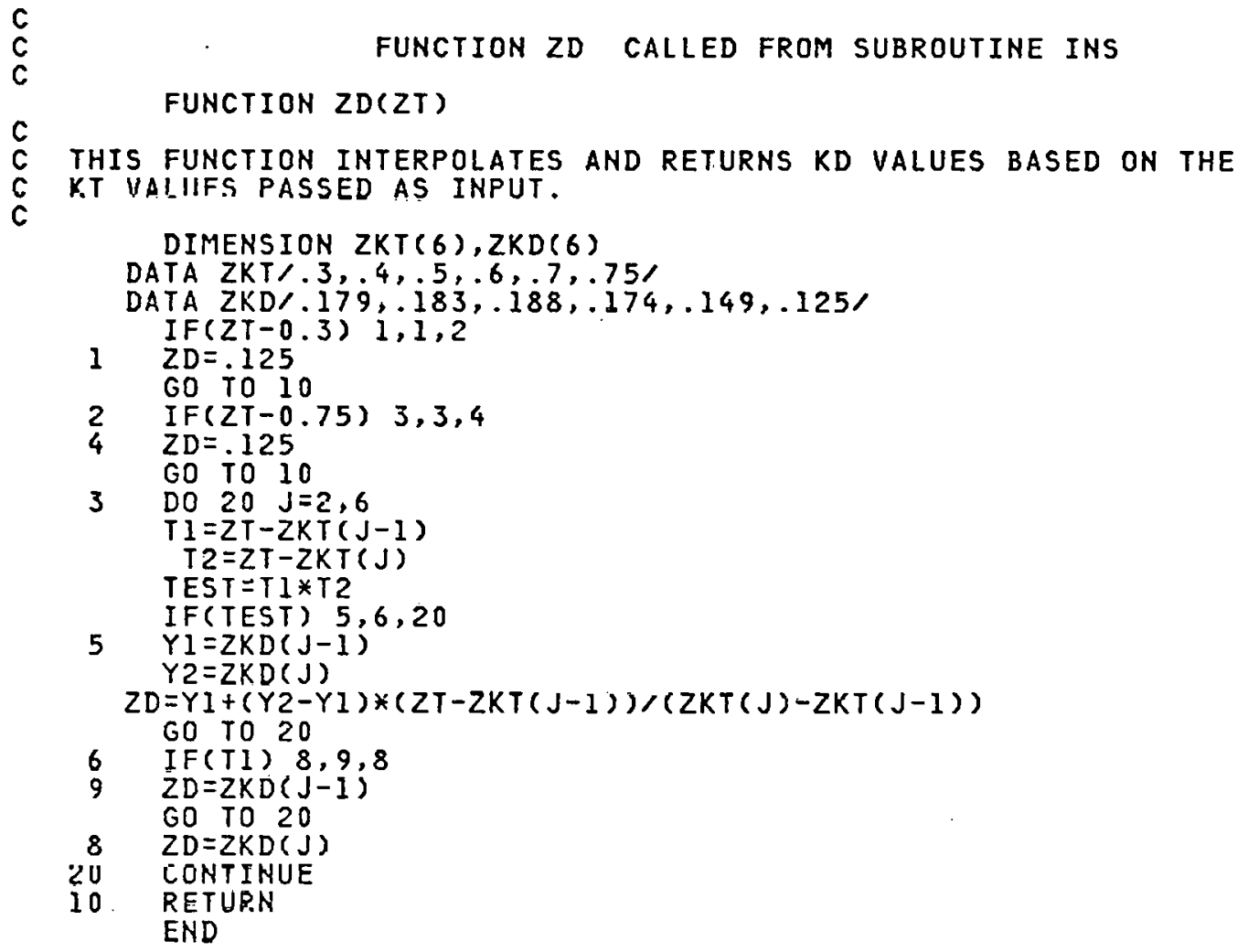




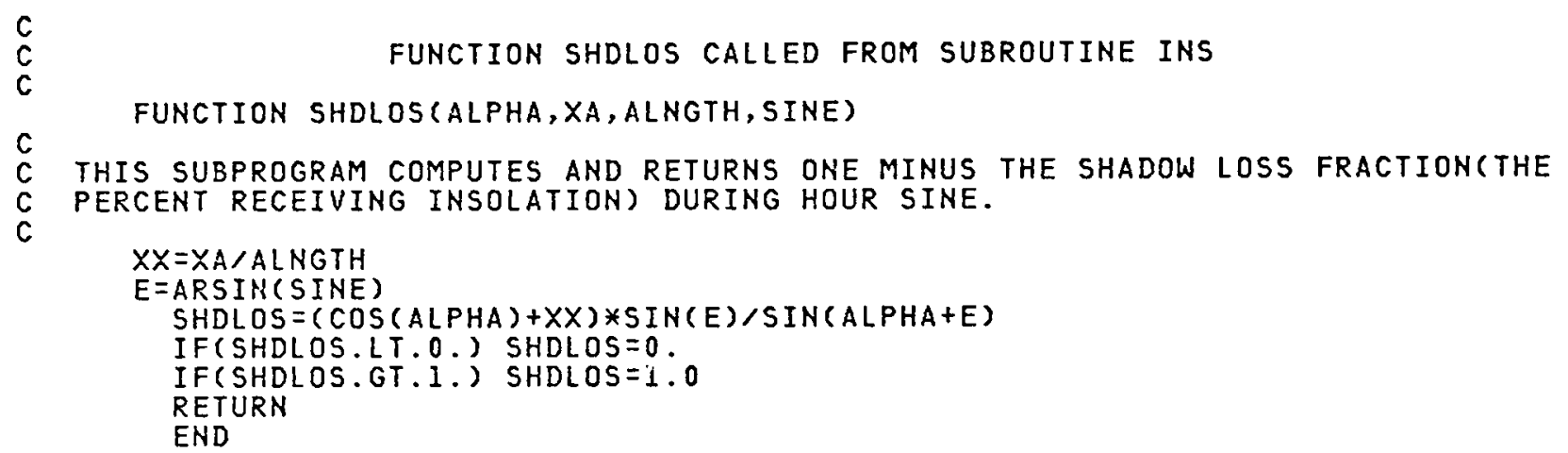


$c$
$c$

FUNCTION AUG CALLED FROM SUBROUTINE INS

FUNCTION AUG (G,T,B, SREFL)

C

C THIS SUBPROGRAM COMPUTES AND.RETURNS ONE PLUS THE INCREASE IN HOURLY ENERGY C DUE TO REFLECTOR AUGMENTATION.

$E=A R S I N(G)$

IF (E.LT.2.*B) GO TO 1

$A U G=S R E F L * S I N(T) * S I N(E-B) *(1 . / S I N(B)-(\operatorname{COS}(B) / S I N(B)$

$1+\operatorname{COS}(T) / \operatorname{SIN}(T)) /(\operatorname{SIN}(B) * \operatorname{COS}(E-2 . * B) / S \operatorname{IN}(E-2 . * B)$

$1+\cos (B))+1.0$

RETURN

$1 A \cup G=S R E F L * S I N(E-B) * S I N(T) / S I N(B)+1.0$

RETURN

END 


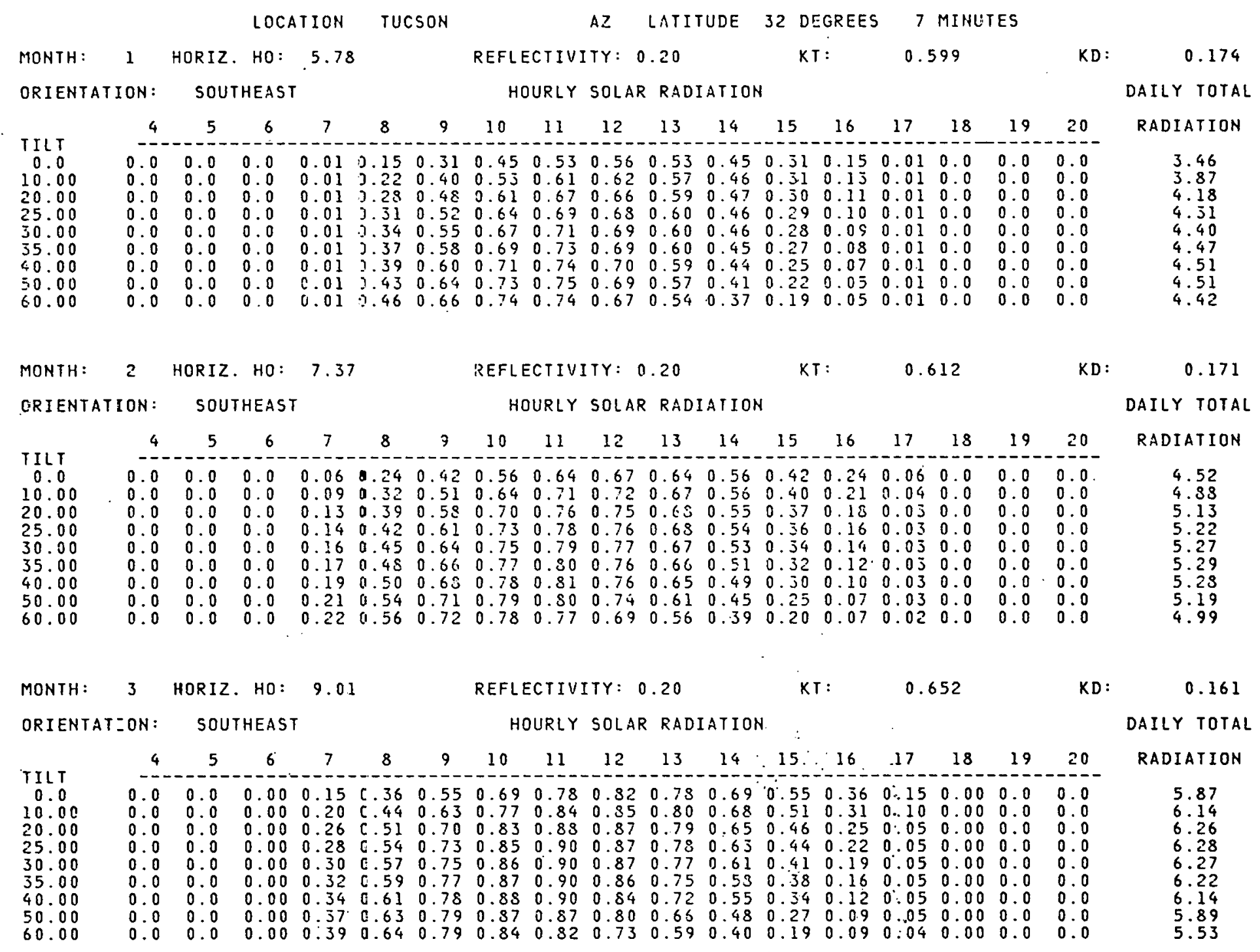




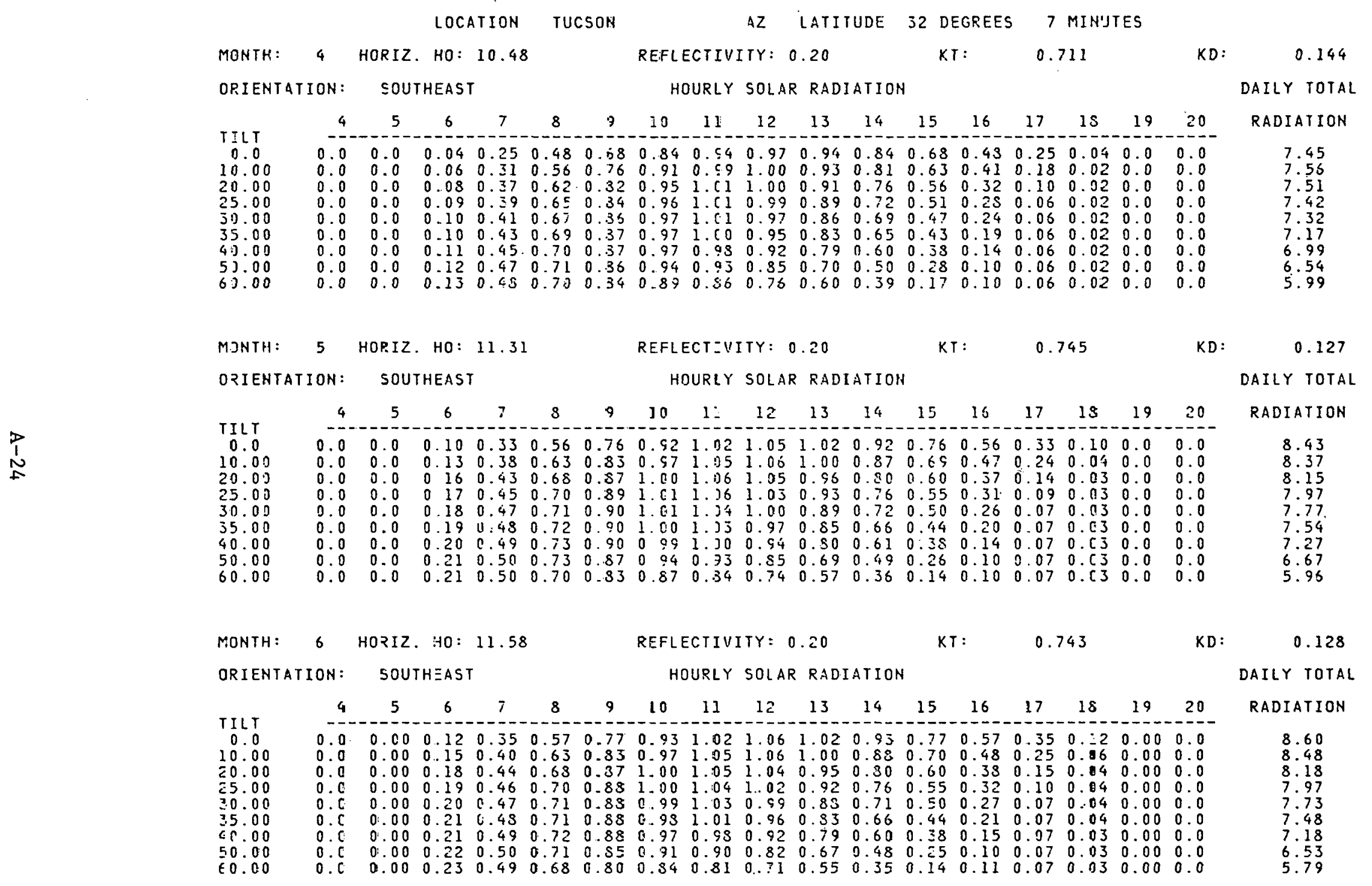




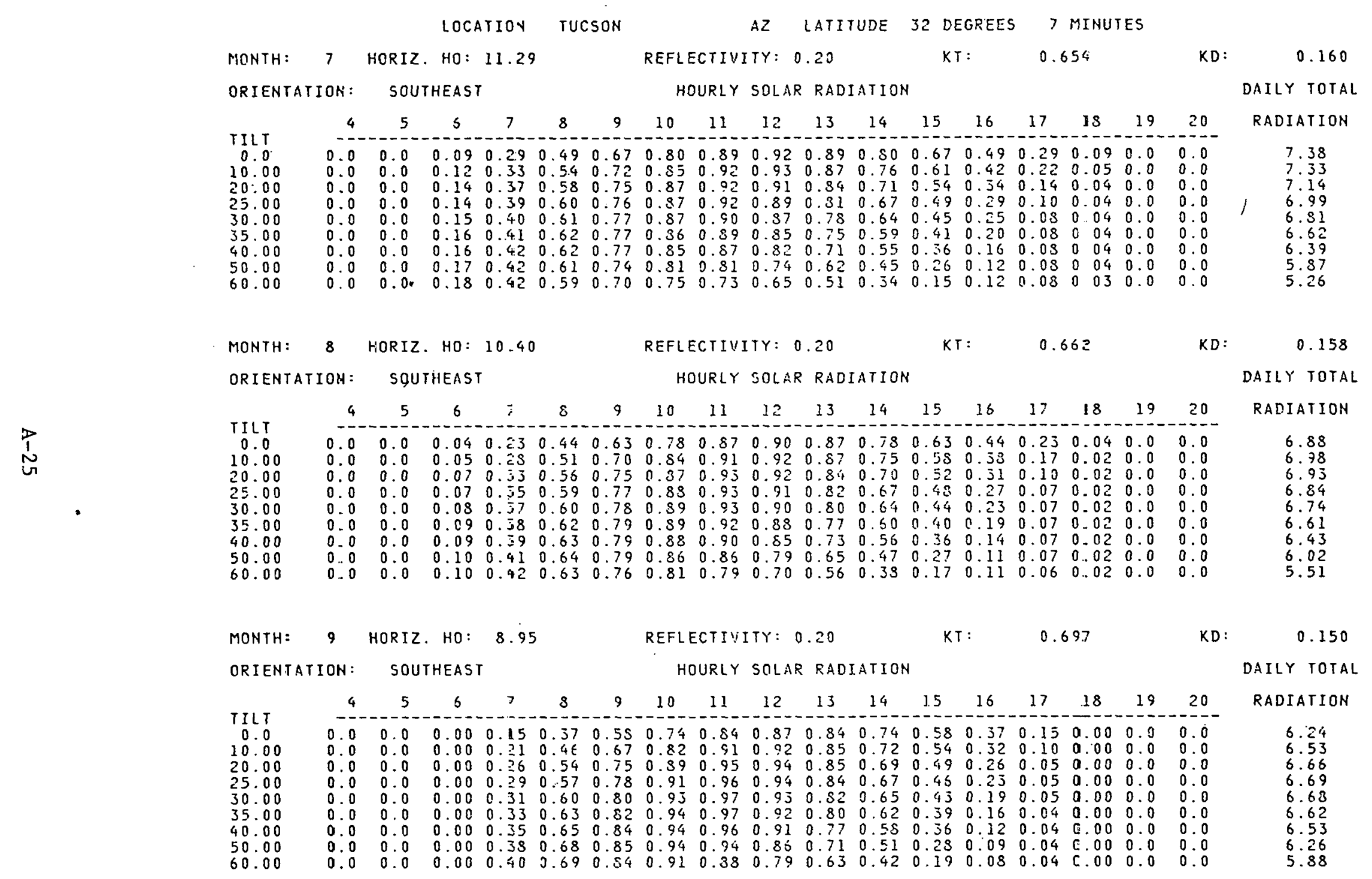




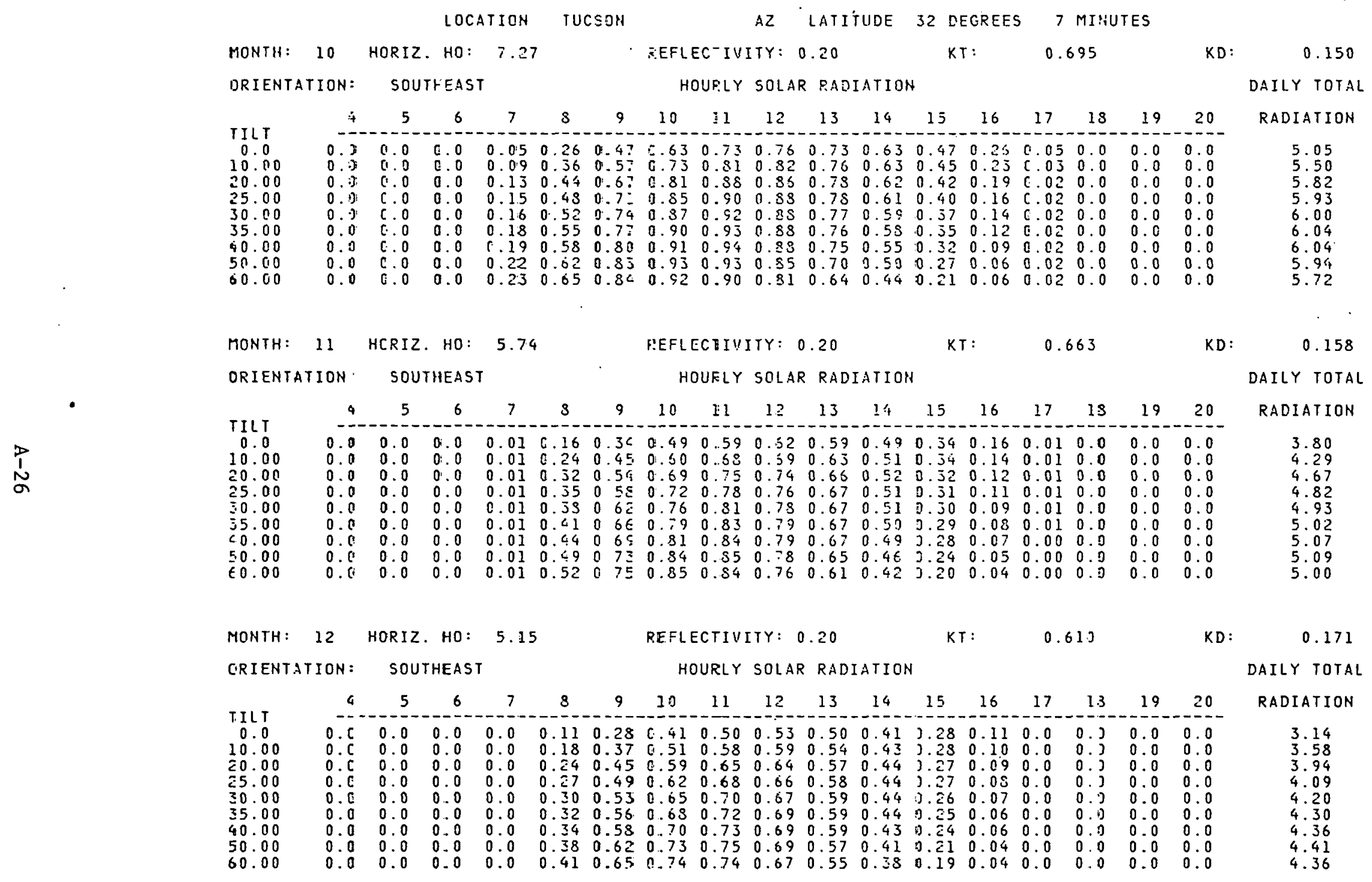




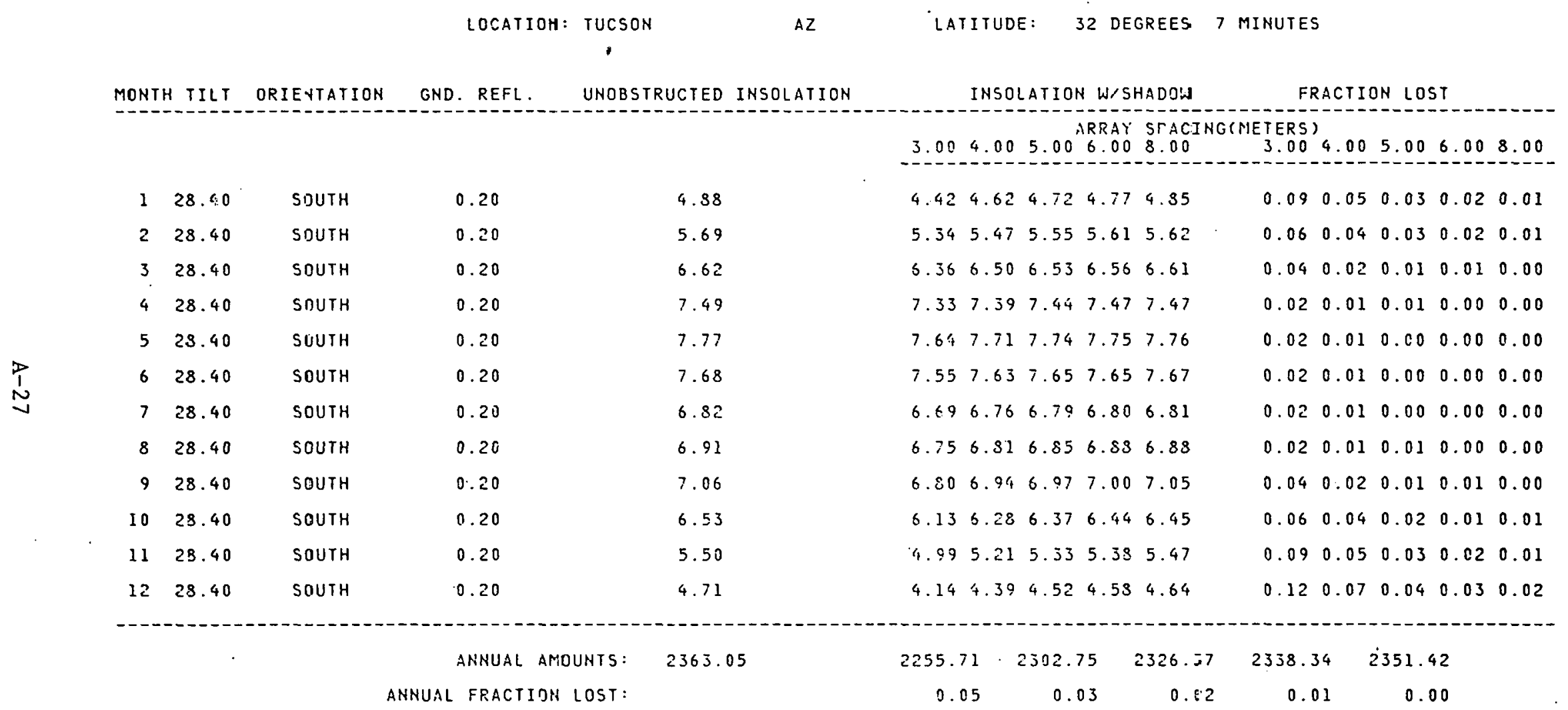


AFRAY SPACING $=3.00$ METERS

MONTH ARR. TILT REFL. TILT SPEC. REFL. GHE REFL. INSOL. W/OUT SHADOW,REFL. INSOL. W/REFL. AUG. FRAC. GAIN

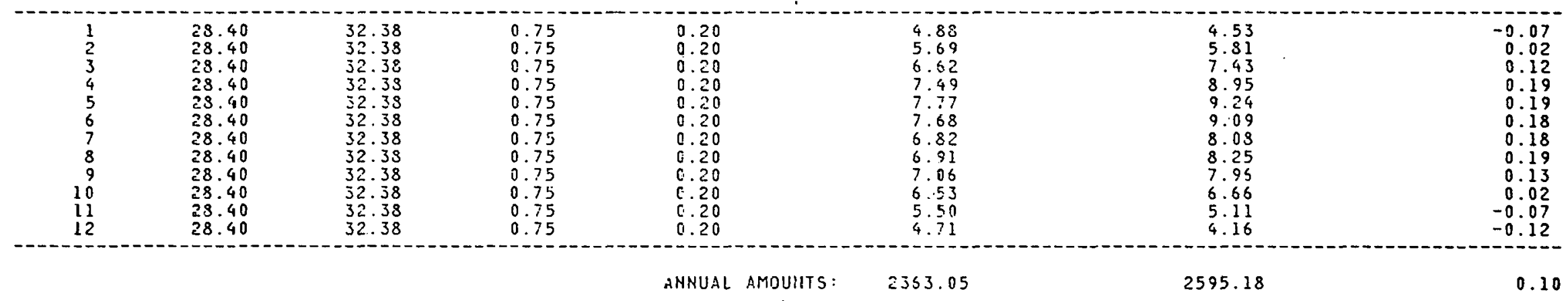

$\underset{\substack{P \\ N \\ N}}{\infty}$
LOCATION: TUCSON
AZ
LATITUDE: 32 JEGREES 7 MINUTES

ARREY SPACING $=7.00$ METERS

MONTH ARR. TILT REFL. TILT SPEC. REFL. GND REFL. INSOL. W/OUT SHADOW,REFL. INSOL. W/REFL. AUG. FRAC. GAIN

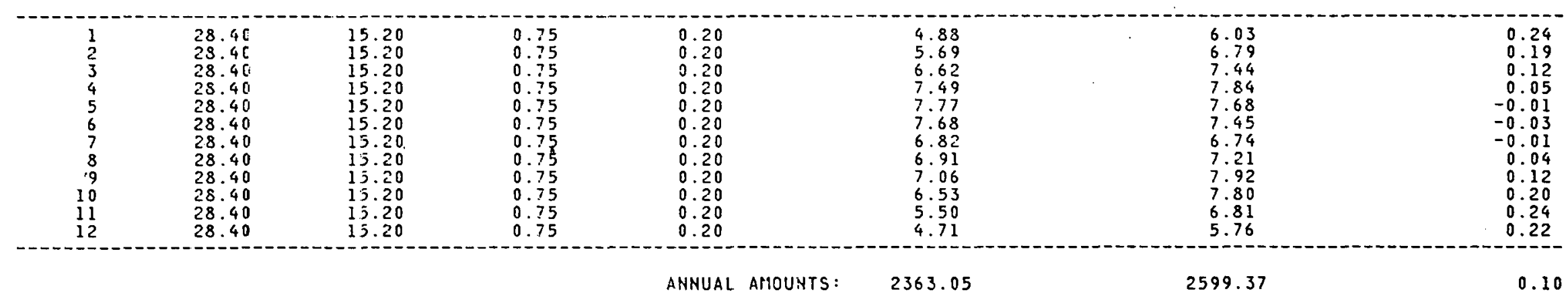


ARRAY SPACING $=11.00$ NIETERS

MONTH ARP. IILT REFL. TILT SPEC. REFL. GND REFL. INSOL. W/OUT SHADOW,REF.. INSOL. W/REFL. AUG. FRAC. GAIN

\begin{tabular}{|c|c|c|c|c|c|c|c|}
\hline $\begin{array}{r}1 \\
2 \\
3 \\
4 \\
5 \\
6 \\
7 \\
8 \\
9 \\
10 \\
11 \\
12\end{array}$ & $\begin{array}{l}28.40 \\
28.40 \\
28.40 \\
28.40 \\
28.40 \\
28.40 \\
28.40 \\
28.40 \\
28.40 \\
28.40 \\
28.40 \\
28.40\end{array}$ & $\begin{array}{l}9.31 \\
9.81 \\
9.81 \\
9.81 \\
9.81 \\
9.81 \\
9.81 \\
9.81 \\
9.81 \\
9.81 \\
9.81 \\
9.81\end{array}$ & $\begin{array}{l}0.75 \\
0.75 \\
0.75 \\
0.75 \\
0.75 \\
0.75 \\
0.75 \\
0.75 \\
0.75 \\
0.75 \\
0.75 \\
0.75\end{array}$ & $\begin{array}{l}0.20 \\
0.20 \\
0.20 \\
0.20 \\
0.20 \\
0.20 \\
0.20 \\
0.20 \\
0.20 \\
0.20 \\
0.20 \\
0.20\end{array}$ & $\begin{array}{r}4.88 \\
5.69 \\
6.62 \\
7.49 \\
7.77 \\
7.68 \\
6.82 \\
6.91 \\
7.05 \\
6.53 \\
5.50 \\
4.71\end{array}$ & $\begin{array}{l}5.82 \\
6.35 \\
6.79 \\
6.99 \\
6.79 \\
6.56 \\
5.97 \\
6.43 \\
7.21 \\
7.29 \\
6.57 \\
5.78\end{array}$ & $\begin{array}{r}0.19 \\
0.12 \\
0.03 \\
-0.07 \\
-0.13 \\
-0.15 \\
-0.13 \\
-0.07 \\
0.02 \\
0.12 \\
0.119 \\
0.23\end{array}$ \\
\hline & & & & ANNUAL & 2363.05 & 388.89 & 0.01 \\
\hline
\end{tabular}

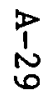

LOCATIOR: TUCSON AZ LATITUDE: 32 DEGREES 7 MINUTES

ARRAY SPACIHG $=15.00$ NIETERS

MONTH ARR. TILT REFL. TILT SPEC. REFL. GND REFL. INSOL. W/OUT SHADOW,REFL. INSOL. W/REFL. AUG. FRAC. GAIN

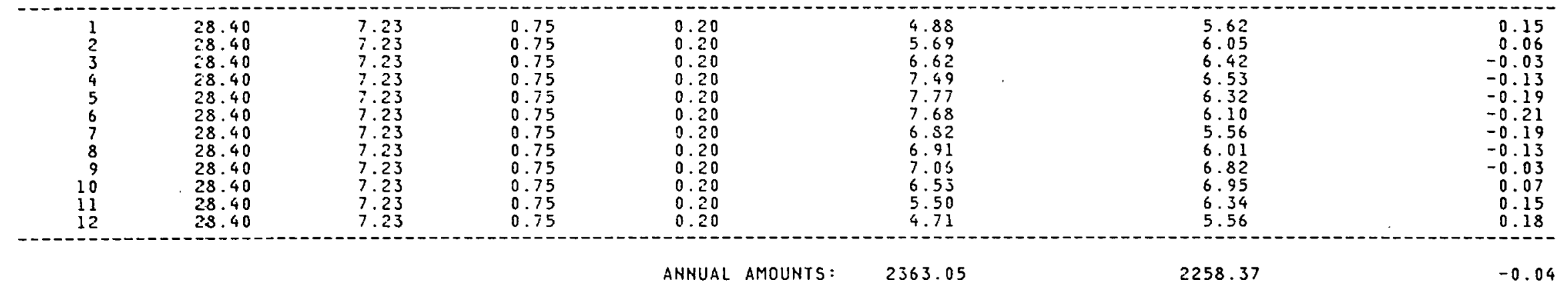


ARFAY SPACING $=19.00$ MEIERS

FRAC. GAIN

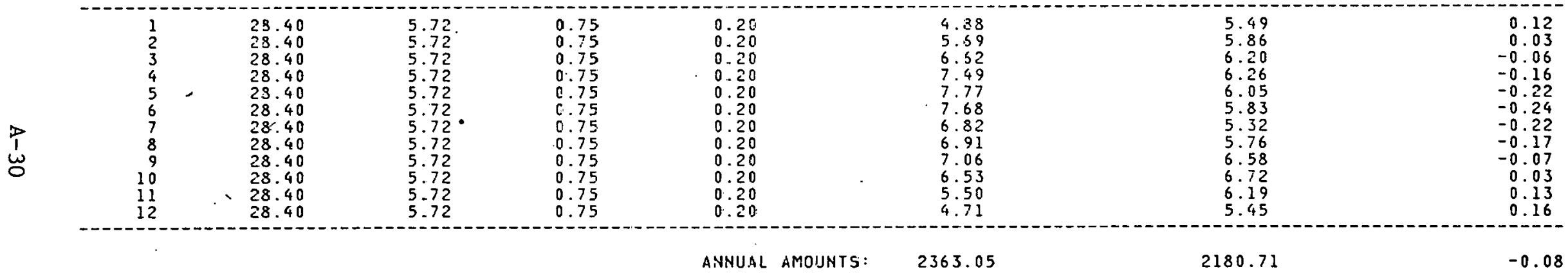


C

THIS PROGRAM GENERATES THE M FACTOR TABLE.

DIMENSION DDEC(12), B(12), DLAT(51), FAC(12), R(12)

DATA DDEC $1-19.51,-10.28,2.2$

$111.56,20.14,23.27,20.26,12.03, .37,-10.47,-19.58,-23.27$,

DATA B $\quad .142, .144, .156, .18, .196, .205, .207, .201$,

$1.177, .16, .149, .142 /$

DATA R /1.03,1.0207,1.0057,.9875,.9727,.967,

$1.9692,99785, .9945,1.0133,1.0267,1.0327$

$P I=3.1416$

C SET THE DUTPUT UNIT

C SET THE SOLAR CONSTANT(AND THUS THE UNITS). $S C=1.377$

WRITE (IUNIT, 60)

60 FORMAT,, $20 X$, THE VALUE OF THE M FACTOR ') WRITE (TUNTT, 70 )

70 FORMAT $(/, 5 X$, 'LAT.', 27X, 'MONTH')

WRITE (IUNIT, 80 )

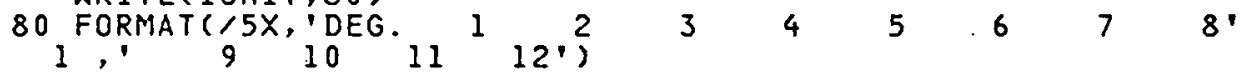

WR ITE (IUNIT, 81 )

81 FORMAT $(/)$

DO $10 I=I, 5 I$

$\operatorname{DLAT}(I)=I-1$.

$X L A T=I N T(D L A T(I))$

RLAT $=(X L A T+(D L A T(I)-X L A T) / .6) * P I / 180$.

DO $20 \mathrm{~J}=1,12$

XDEC $=\operatorname{INT}($ DDEC (J))

$R D E C=(X D E C+(D D E C(J)-X D E C) / .6) * P I / 180$

$\operatorname{COSWS}=(-\operatorname{SIN}(R L A T) / \operatorname{COS}(R L A T)) *(\operatorname{SIN}(R D E C) / \operatorname{COS}(R D E C))$

WS $=A R \operatorname{COS}($ COSWS $)$

DWS $=$ WS $* 180 . / P I$

$\operatorname{COSLD}=\operatorname{COS}(R L A T) * \operatorname{COS}(R D E C)$

$S I N L D=S I N(R L A T) * S I N(R D E C)$

$S=0$.

DO $30 \quad N=1,39$

$W=W S * N / 40$.

$\operatorname{COS} Z=\operatorname{COS} L D * \cos (W)+S I N L D$

$P A R=-B(J) / C O S Z$

$A P A=A B S(P A R)$

IF (APA.GT.80) GO TO 40

ANS $=E X P(P A R) * \operatorname{COSZ}$

GO TO 50

40 ANS $=0$.

$50 \quad S=A N S+S$

30 CONTINUE

ANSO-EXP $(-B(J) /(C O S L D+3 I N L D)) *(C O S L U+S I N L D) / 2$

AINT $=$ WS $/ 40 . *($ ANSO+S $)$

$T H O=R(J) * S C *(C O S L D * S I N(W S)+W S * S I N L D)$

FAC $(J)=T H O / A I N T$

20 CONTINUE

WR ITE(IUNIT, 90) DLAT(I), (FAC(MM), MM=1,12)

90 FORMAT $(5 X, F 3.0,2 X, 12 F 5,2)$

DO $1 K=10,50,10$

IF (I.EQ.K) WRITE(IUNIT, 81)

1 CONTINUL

10 CONTINUE

STOP

END 
The following pages provide solar climate parameter data for 235 localities in the United States (see Reference 10). Latitudes are in degree-minute form.

$$
\begin{gathered}
\overline{\mathrm{K}}_{\mathrm{T}}=\frac{\text { average daily total radiation for month } \mathrm{M}}{\text { average daily total extraterrestrial radiation for month } \mathrm{M}} \\
\mathrm{M}=1,2, \ldots . ., 12
\end{gathered}
$$

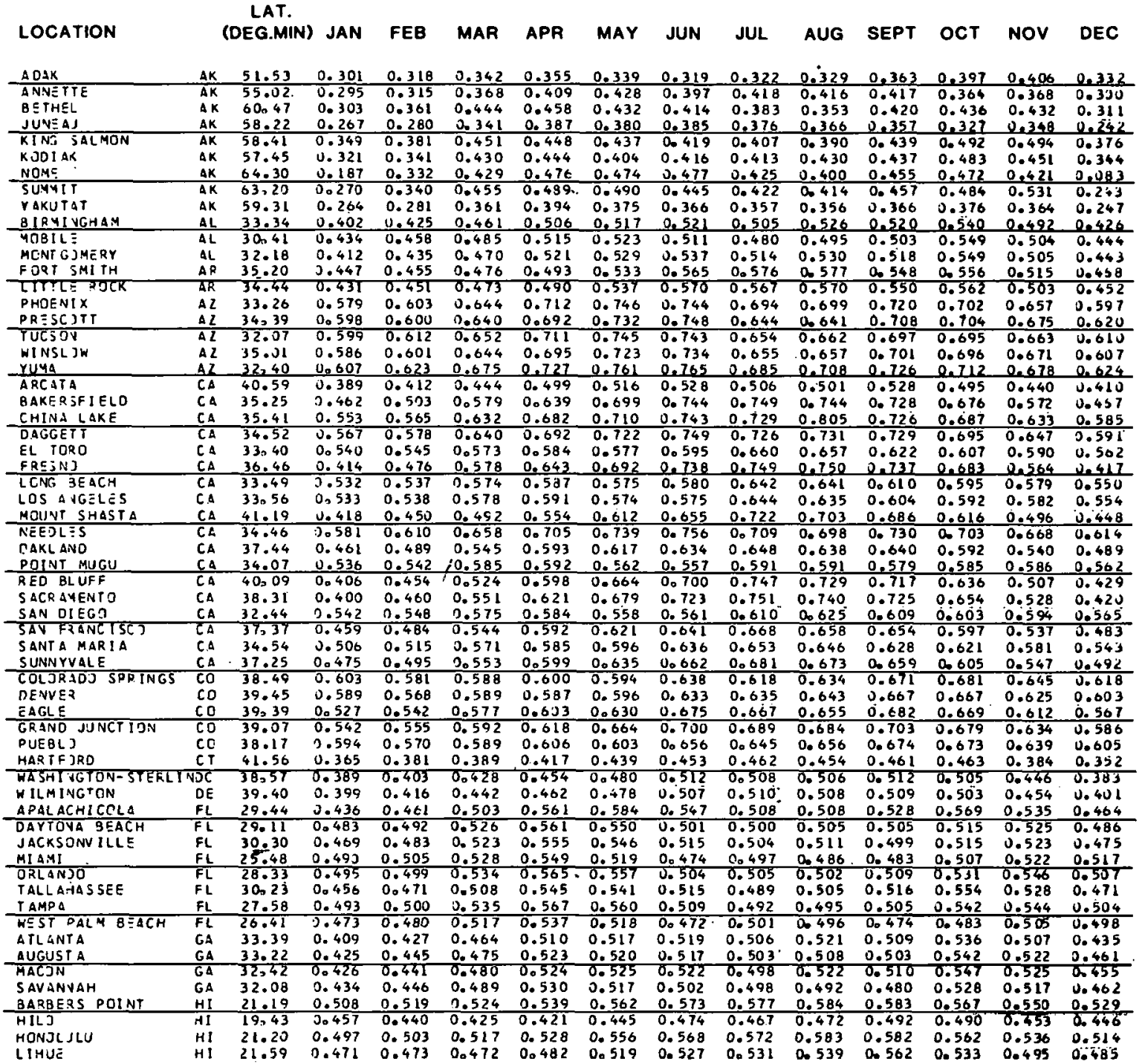




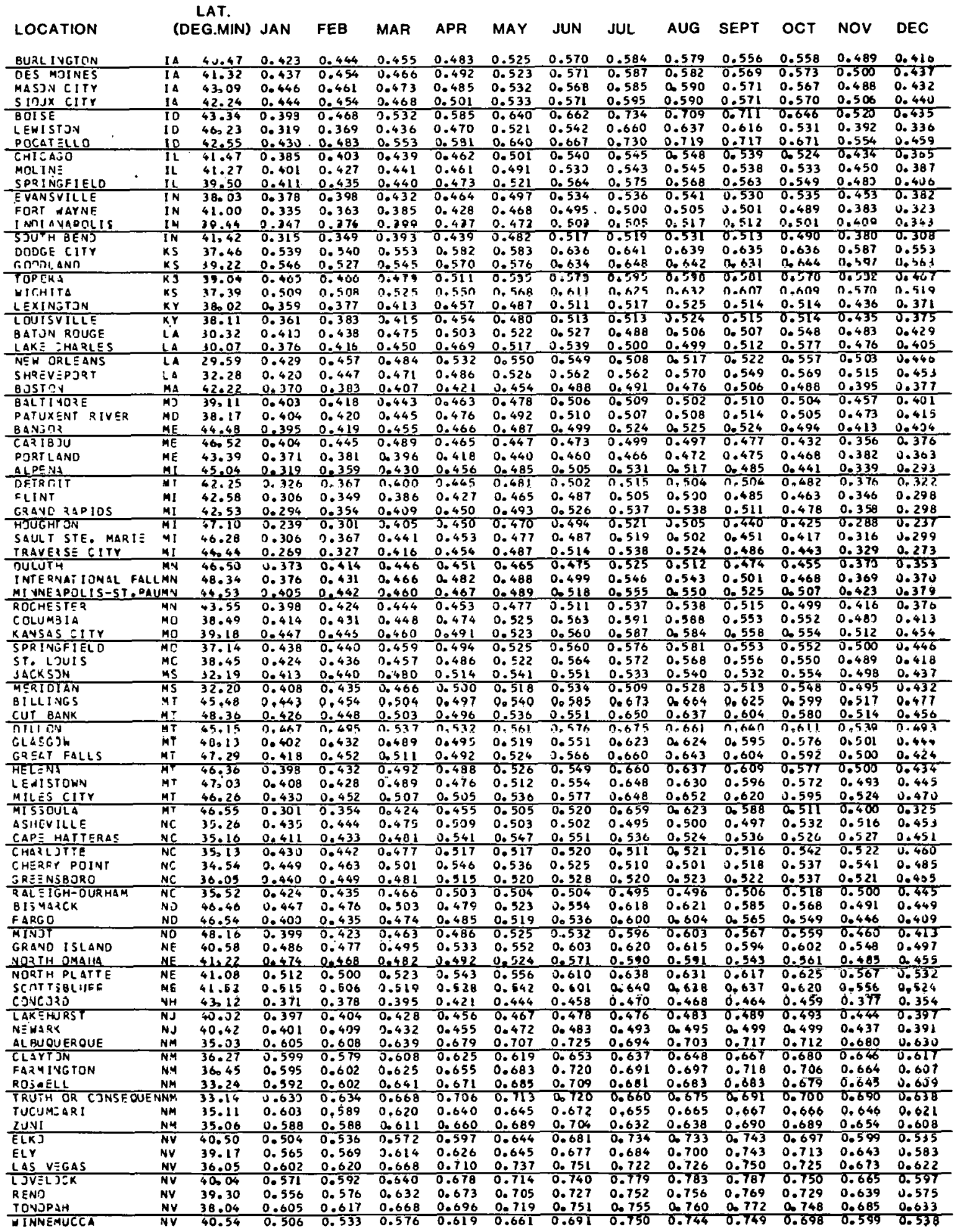




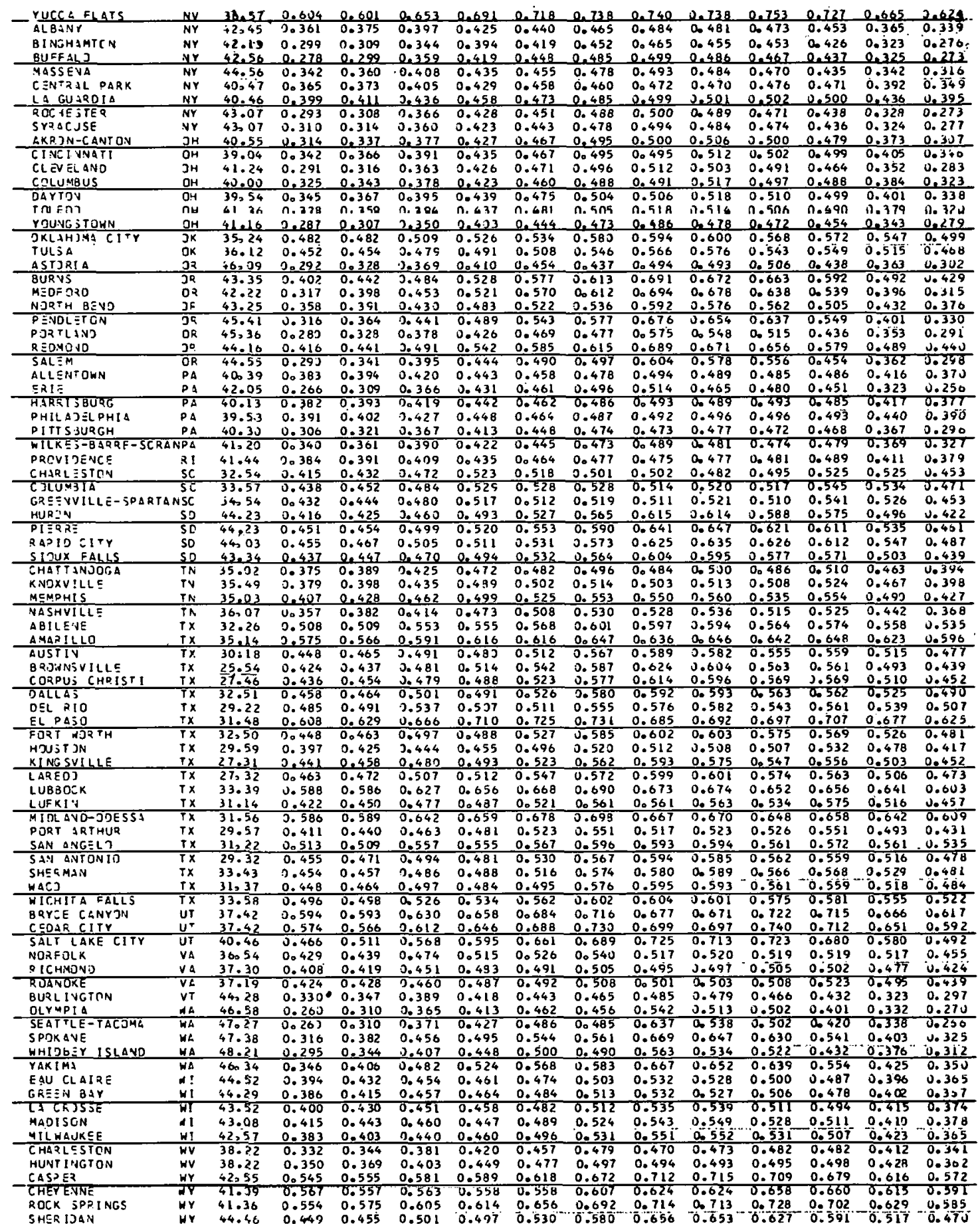




\section{APPENDIX C \\ AVERAGE DAILY SOLAR INSOLATION DATA BY MONTH AND ANNUAL TOTALS FOR 235 LOCALITIES}

The following tables contain average daily insolation estimates and annual totals for south-facing surfaces at tilt angles from 0 to 90 degrees for 235 localities in the United States. The intermediate angles are provided to facilitate interpolation. The average ground reflectivity assumed is 0.20 . Each array tilt angle has 12 estimates associated with it -- one for each month -- plus the total annual amount. The average daily amount for the entire year is computed by dividing the annual total by 365 . The values within the tables are in units of kilowatt hours per square meter. Localities are listed in alphabetical order by state and then by city within the state.

The tables were printed using an IBM 3800 laser printer. 
AVER.AGE DAIL TOTAL TERRESTRZAL INSOLAFION ESTIMATES (KWH/SQ. M)

$\begin{aligned} \text { SITE: } & \text { ADAK } \\ \text { LATITUDE: } & \text { 5: DEGREES } 53 \text { MINLITES }\end{aligned}$

ALERAGE IAILY AMOUNTS BY MONTH

\begin{tabular}{|c|c|c|c|c|c|c|c|c|c|c|c|c|c|c|}
\hline $\begin{array}{l}\text { ARRAY } \\
\text { TILT }\end{array}$ & $\begin{array}{l}---1 \\
J\end{array}$ & $F$ & $M$ & $A$ & $M$ & $\sqrt{3}$ & J & $A$ & 5 & 0 & $N$ & D & $\begin{array}{l}\text { ANNUAL } \\
\text { AMOUNT }\end{array}$ & $\begin{array}{c}\text { AVERAGE } \\
\text { DAY }\end{array}$ \\
\hline 0.0 & 0.73 & 1.36 & $2 . \geq 5$ & 3.25 & 3.72 & 3.72 & 3.53 & 2.99 & 2.39 & 1.66 & 0.97 & 0.59 & 827.7 & 2.3 \\
\hline 10.0 & 0.90 & 1.56 & 2.47 & 3.40 & 3.79 & 3.76 & 3.60 & 3.12 & 2.63 & 1.98 & 1.28 & 0.79 & 892.2 & 2.4 \\
\hline 15.0 & 0.98 & 1.66 & 2.35 & 3.46 & 3.80 & 3.76 & 3.60 & 3.17 & 2.72 & 2.12 & 1.43 & 0.89 & 917.8 & 2.5 \\
\hline 20.0 & 1.05 & 1.74 & 2.63 & 3.49 & 3.80 & 3.73 & 3.60 & 3.19 & 2.80 & 2.25 & 1.57 & 0.98 & 938.9 & 2.6 \\
\hline 25.0 & 1.12 & 1.81 & 2.69 & 3.51 & 3.77 & 3.69 & 3.57 & 3.20 & 2.87 & 2.37 & 1.70 & 1.07 & 955.4 & 2.6 \\
\hline 30.0 & 1.18 & 1.87 & 2.73 & 3.51 & 3.73 & 3.64 & 3.53 & 3.20 & 2.92 & 2.47 & 1.82 & 1.15 & 966.9 & 2.6 \\
\hline 35.0 & 1.24 & 1.92 & 2.76 & 3.49 & 3.67 & 3.57 & 3.48 & 3.18 & 2.96 & 2.56 & 1.92 & 1.22 & 973.4 & 2.7 \\
\hline 40.0 & 1.29 & 1.97 & 2.78 & 3.45 & 3.60 & 3.48 & 3.40 & 3.14 & 2.98 & 2.63 & 2.02 & 1.28 & 974.8 & 2.7 \\
\hline 45.0 & 1.33 & 2.00 & 2.78 & 3.40 & 3.50 & 3.38 & 3.31 & 3.09 & 2.98 & 2.69 & 2.10 & 1.34 & 971.1 & 2.7 \\
\hline 50.0 & 1.36 & 2.02 & 2.77 & 3.33 & 3.39 & 3.27 & 3.21 & 3.03 & 2.97 & 2.73 & 2.17 & 1.38 & 962.3 & 2.6 \\
\hline 60.0 & 1.40 & 2.02 & 2.70 & 3.14 & 3.14 & 3.01 & 2.97 & 2.86 & 2.90 & 2.76 & 2.26 & 1.45 & 931.4 & 2.6 \\
\hline 70.0 & 1.41 & 1.78 & 2.5 .7 & 2.90 & 2.84 & 2.70 & 2.69 & 2.63 & 2.76 & 2.73 & 2.29 & 1.48 & 881.8 & 2.4 \\
\hline 80.0 & 1.38 & 1.89 & 2.39 & 2.60 & 2.49 & 2.35 & 2.36 & 2.37 & 2.57 & 2.63 & 2.27 & 1.48 & 814.5 & 2.2 \\
\hline 90.0 & 1.32 & 1.77 & 2.16 & 2.26 & 2.12 & 2.01 & 2.01 & 2.06 & 2.32 & 2.46 & 2.18 & 1.43 & 732.8 & 2.0 \\
\hline
\end{tabular}


AVERAGE DAILY TOTAL TERRESTRIAL INSOLATION ESTIMATES (KWH;SQ. M)

SITE:
LANNETTE
LATUDE:

AVERAGE DAILY AMOUNTS BY MONTH

\begin{tabular}{|c|c|c|c|c|c|c|c|c|c|c|c|c|c|c|}
\hline ARRAY & J & $F$ & $M$ & A & $M$ & $J$ & J & A & $s$ & 0 & N & D & $\begin{array}{l}\text { ANNUAL } \\
\text { AMOUNT }\end{array}$ & $\begin{array}{l}\text { AVERAGE } \\
\text { DAY }\end{array}$ \\
\hline 0.0 & 0.56 & 1.18 & 2.26 & 3.62 & 4.64 & 4.62 & 4.54 & 3.66 & 2.56 & 1.33 & 0.69 & 0.39 & 916.0 & 2.5 \\
\hline 10.0 & $0^{\prime} .80$ & 1.38 & 2.51 & 3.85 & 4.78 & 4.70 & 4.67 & 3.90 & 2.88 & 1.60 & 0.94 & 0.60 & 994.7 & 2.7 \\
\hline 15.0 & 0.91 & 1.47 & 2.62 & $\ddot{3.93}$ & 4.82 & 4.71 & 4.71 & 3.98 & 3.01 & 1.72 & 1.06 & 0.71 & 1026.3 & 2.8 \\
\hline 20.0 & 1.01 & $1 . .56$ & 2.72 & 4.00 & 4.83 & 4.70 & 4.71 & 4.05 & 3.13 & 1.84 & 1.18 & 0.80 & 1052.4 & 2.9 \\
\hline 25.0 & 1.11 & 1.63 & 2.80 & 4.04 & 4.82 & 4.66 & 4.70 & 4.09 & 3.24 & 1.94 & 1.28 & 0.90 & 1073.3 & 2.9 \\
\hline 30.0 & 1.20 & 1.70 & 2.86 & 4.05 & 4.79 & 4.61 & 4.67 & 4.11 & 3.32 & 2.04 & 1.38 & 0.98 & 1088.6 & 3.0 \\
\hline 35.0 & 1.29 & 1.75 & 2.91 & 4.05 & 4.73 & 4.53 & 4.61 & 4.11 & 3.38 & 2.12 & 1.77 & 1.07 & 1097.9 & 3.0 \\
\hline 40.0 & 1.36 & 1.80 & 2.95 & 4.03 & 4.64 & 4.43 & 4.53 & 4.08 & 3.43 & 2.18 & 1.55 & 1.14 & 1101.2 & 3.0 \\
\hline 45.0 & 1.43 & 1.84 & 2.96 & 3.98 & 4.54 & 4.31 & 4.42 & 4.04 & 3.45 & 2.24 & 1.53 & 1.20 & 1098.3 & 3.0 \\
\hline 50.0 & 1.49 & 1.86 & 2.96 & 3.91 & 4.40 & 4.17 & 4.29 & 3.97 & 3.46 & 2.28 & 1.69 & 1.26 & 1089.4 & 3.0 \\
\hline 60.0 & 1.57 & 1.88 & 2.91 & 3.72 & 4.09 & 3.84 & 3.98 & 3.77 & 3.41 & 2.32 & 1.77 & 1.35 & 1055.2 & 2.9 \\
\hline 20.0 & 1.61 & 1.86 & 2.80 & 3.46 & 3.71 & 3.46 & 3.61 & 3.50 & 3.28 & 2.31 & 1.81 & $1: 40$ & 999.7 & 2.7 \\
\hline 80.0 & 1.61 & 1.79 & 2.62 & 3.12 & 3.26 & 3.01 & 3.18 & 3.17 & 3.08 & 2.23 & 1.80 & 1.41 & 922.9 & 2.5 \\
\hline 90.0 & 1.56 & 1.69 & 2.39 & 2.73 & 2.76 & 2.54 & 2.69 & 2.77 & 2.81 & 2.11 & 1.75 & 1.39 & 827.7 & 2.3 \\
\hline
\end{tabular}


AVERAGE DEILY TOTAL TERRESTREAL INSOLATION ESTIMATES (KWH/SQ. M)

SITE: BETHEL $\quad$ AK.

AVERAGE DAI:Y AMOUNTS BY MONTH

\begin{tabular}{|c|c|c|c|c|c|c|c|c|c|c|c|c|c|c|}
\hline ARRAY & $J$ & $F$ & $M$ & A & $M$ & 3 & $J$ & A & $\mathrm{S}$ & 0 & $N$ & $D$ & $\begin{array}{l}\text { ANNUAL } \\
\text { AMOUNT }\end{array}$ & $\begin{array}{c}\text { AVERAGE } \\
\text { DAY }\end{array}$ \\
\hline 0.0 & $0.3 i$ & 1.00 & 2.33 & 3.79 & 4.58 & 4.79 & 4.07 & 2.90 & 2.21 & 1.17 & 0.43 & 0.15 & $845^{\circ} .0$ & 2.3 \\
\hline 10.0 & 0.45 & 1.27 & 2.72 & 4.11 & 4.76 & 4.91 & 4.22 & 3.10 & 2.56 & 1.54 & 0.72 & 0.28 & 933.6 & 2.6 \\
\hline 15.0 & 0.52 & 1.40 & 2.89 & 4.23 & 4.82 & 4.94 & 4.26 & 3.18 & 2.71 & 1.72 & 0.36 & 0.34 & 970.9 & 2.7 \\
\hline 20.0 & 0.57 & 1.52 & 3.05 & 4.34 & 4.85 & 4.94 & 4.28 & 3.24 & 2.85 & 1.88 & 0.39 & 0.39 & 1003.0 & 2.7 \\
\hline 25.0 & 0.65 & 1.62 & 3.19 & $4 .+2$ & 4.86 & 4.92 & 4.28 & 3.28 & 2.98 & 2.04 & 1.12 & 0.45 & 1029.6 & 2.8 \\
\hline 30.0 & 0.71 & 1.72 & 3.31 & 4.47 & 4.85 & 4.88 & 4.26 & 3.31 & 3.08 & 2.18 & 1.24 & 0.50 & 1051.0 & 2.9 \\
\hline 35.0 & 0.77 & 1.81 & 3.41 & 4.50 & 4.81 & 4.82 & 4.23 & 3.32 & 3.17 & 2.30 & 1.35 & 0.55 & 1067.0 & 2.9 \\
\hline 40.0 & 0.82 & 1.89 & 3.49 & 4.51 & 4.75 & 4.74 & 4.17 & 3.31 & 3.24 & $2.4 \hat{2}$ & 1.45 & 0.59 & 1077.0 & 3.0 \\
\hline 45.0 & 0.86 & 1.96 & 3.54 & 4.49 & 4.67 & 9.64 & 4.09 & 3.28 & 3.29 & 2.51 & 1.54 & 0.64 & 1080.9 & 3.0 \\
\hline 50.0 & 0.90 & 2.01 & 3.58 & 4.44 & 4.56 & 4.51 & 4.00 & 3.24 & 3.32 & 2.59 & 1.62 & 0.67 & 1078.6 & 3.0 \\
\hline 60.0 & 0.96 & 2.08 & 3.59 & 4.27 & 4.27 & 4.18 & 3.74 & 3.10 & 3.32 & 2.70 & 1.75 & 0.73 & 1055.8 & 2.9 \\
\hline 70.0 & 0.99 & 2.10 & 3.51 & 4.02 & 3.92 & 3.81 & 3.43 & 2.91 & 3.24 & 2.74 & 1.83 & 0.77 & 1012.4 & 2.8 \\
\hline 80.0 & 1.00 & 2.06 & 3.35 & 3.69 & 3.50 & 3.37 & 3.07 & 2.67 & 3.08 & 2.70 & 1.86 & 0.79 & 946.8 & 2.6 \\
\hline 90.0 & 0.98 & 1.98 & 3.10 & 3.27 & 3.02 & 2.88 & 2.65 & 2.37 & 2.85 & 2.60 & 1.83 & 0.78 & 860.8 & 2.4 \\
\hline
\end{tabular}


AVERAGE DAILY TOTAL TERRESTRIAL INSOLATION ESTIMATES (KWH/SQ. M)

$\begin{aligned} \text { SITE: } & \text { JUNEAU } \\ \text { LATITUDE: } & 58 \text { DEGREES } 22 \text { MINUTES }\end{aligned}$

AVERAGE DAILY AMOUNTS BY MONTH

\begin{tabular}{|c|c|c|c|c|c|c|c|c|c|c|c|c|c|c|}
\hline ARRAY & J & $F$ & $M$ & A & $M$ & $J$ & $\mathrm{~J}$ & A & 5 & 0 & $N$ & D & $\begin{array}{l}\text { ANNUAL } \\
\text { AMOUNT }\end{array}$ & $\begin{array}{l}\text { AVERAGE } \\
\text { DAY }\end{array}$ \\
\hline 0.0 & 0.37 & 0.89 & 1.92 & 3.30 & 4.07 & 4.46 & 4.03 & 3.10 & 2.01 & 1.01 & 9.47 & 0.19 & 787.4 & 2.2 \\
\hline 10.0 & 0.55 & 1.13 & 2.15 & 3.52 & 4.20 & 4.55 & 4.16 & 3.30 & 2.26 & 1.23 & 0.68 & 0.32 & 855.6 & 2.3 \\
\hline 15.0 & 0.64 & 1.24 & 2.25 & 3.61 & 4.24 & 4.57 & 4.20 & 3.38 & 2.37 & 1.33 & 0.78 & 0.38 & 883.4 & 2.4 \\
\hline 20.0 & 0.72 & 1.34 & 2.34 & 3.68 & 4.25 & 4.56 & 4.21 & 3.44 & 2.47 & 1.42 & c. .88 & 0.44 & 906.5 & 2.5 \\
\hline 25.0 & 0.80 & 1.43 & 2.41 & $3.7 \overline{2}$ & 4.25 & 4.54 & 4.20 & 3.48 & 2.55 & 1.51 & C.97 & 0.50 & 924.9 & 2.5 \\
\hline 30.0 & 0.87 & 1.52 & 2.47 & 3.75 & 4.22 & 4.49 & 4.18 & 3.50 & 2.62 & 1.59 & 1.05 & 0.56 & 938.9 & 2.6 \\
\hline 35.0 & 0.94 & 1.59 & 2.52 & 3.76 & 4.18 & 4.43 & 4.14 & 3.50 & 2.67 & 1.65 & 1.13 & 0.61 & 948.0 & 2.6 \\
\hline 40.0 & 1.00 & 1.66 & 2.56 & 3.74 & 4.12 & 4.34 & 4.07 & 3.49 & 2.71 & 1.71 & 1.20 & 0.65 & 952.0 & 2.6 \\
\hline 45.0 & 1.06 & 1.71 & 2.58 & 3.71 & 4.03 & 4.24. & 3.99 & 3.45 & 2.73 & 1.76 & 1.27 & 0.69 & 950.8 & 2.6 \\
\hline 50.0 & 1.10 & 1.76 & 2.59 & 3.66 & 3.93 & 4.11 & 3.88 & 3.40 & 2.74 & 1.80 & 1.32 & 0.73 & 944.4 & 2.6 \\
\hline 60.0 & 1.18 & 1.81 & 2.56 & 3.49 & 3.66 & 3.80 & 3.62 & 3.25 & 2.71 & 1.84 & 1.40 & 0.79 & 916.7 & 2.5 \\
\hline 70.0 & 1.22 & 1.82 & 2.47 & 3.27 & 3.35 & 3.45 & 3.31 & 3.04 & 2.62 & 1.84 & 1.45 & 0.82 & 872.0 & 2.4 \\
\hline 80.0 & $1.22^{\circ}$ & 1.79 & 2.33 & 2.98 & 2.98 & 3.04 & 2.94 & 2.77 & 2.47 & 1.79 & $1 . .45$ & 0.84 & 808.9 & 2.2 \\
\hline 90.0 & 1.20 & 1.70 & 2.14 & 2.63 & 2.56 & 2.58 & 2.52 & 2.44 & 2.27 & 1.70 & 1.42 & 0.83 & 729.7 & 2.0 \\
\hline
\end{tabular}


AVERAGE DAILY TOTAL TERRESTRJAL INSOLATION ESTIMATES (KWH/SQ. M)

SITE: KING SALMON AK

AVERAGE JAILY AMOUNTS BY MONTH

\begin{tabular}{|c|c|c|c|c|c|c|c|c|c|c|c|c|c|c|}
\hline $\begin{array}{l}\text { ARRAY } \\
\text { TILT }\end{array}$ & -1 & $F$ & $M$ & A & $M$ & $\mathrm{~J}$ & 3 & A & 5 & 0 & N & D & $\begin{array}{l}\text { ANNUAL } \\
\text { ANOUNT }\end{array}$ & $\begin{array}{l}\text { AVERAGE } \\
\text { DAY }\end{array}$ \\
\hline 0.0 & 0.46 & 1.19 & 2.51 & 3.80 & 4.67 & 4.85 & 4.36 & 3.29 & 2.45 & 1.49 & $0 . \in 4$ & 0.28 & 915.2 & 2.5 \\
\hline 10.0 & 0.68 & 1.49 & 2.90 & 4.10 & 4.85 & 4.76 & $\dot{4} .51$ & 3.52 & 2.82 & 1.95 & 1.03 & 0.50 & 1015.4 & 2.8 \\
\hline 15.0 & 0.78 & 1.63 & 3.07 & 4.21 & 4.90 & 4.79 & 4.56 & 3.61 & 2.98 & 2.17 & 1.21 & 0.60 & 1057.6 & 2.9 \\
\hline 20.0 & 0.88 & 1.76 & 3.23 & 4.30 & 4.92 & 4.79 & +.57 & 3.68 & 3.12 & 2.37 & 1.39 & 0.70 & 1094.0 & 3.0 \\
\hline 25.0 & 0.97 & 1.88 & 3.36 & 4.37 & 4.92 & 4.96 & 4.57 & 3.73 & 3.25 & 2.55 & 1.56 & 0.79 & 1124.4 & 3.1 \\
\hline 30.0 & 1.05 & 1.99 & 3.48 & 4.41 & 4.91 & 4.91 & 4.55 & 3.76 & 3.36 & 2.72 & 1.71 & 0.88 & 1149.2 & 3.1 \\
\hline 35.0 & 1.13 & 2.09 & 3.57 & 4.43 & 4.86 & 4.85 & 4.51 & 3.76 & 3.44 & 2.87 & 1.86 & 0.96 & 1167.6 & 3.2 \\
\hline 40.0 & 1.20 & 2.17 & 3.65 & 4.42 & 4.79 & 4.76 & 4.44 & 3.75 & 3.51 & 3.00 & 1.99 & 1.04 & 1179.4 & 3.2 \\
\hline 45.0 & 1.27 & 2.24 & 3.70 & 4.39 & 4.70 & 4.64 & 4.35 & 3.72 & 3.56 & 3.12 & 2.11 & 1.11 & 1184.4 & 3.2 \\
\hline 50.0 & 1.32 & 2.30 & 3.73 & 4.34 & 4.58 & 4.50 & 4.24 & 3.67 & 3.58 & 3.21 & 2.21 & 1.17 & 1182.6 & 3.2 \\
\hline 60.0 & 1.40 & 2.37 & 3.72 & 4.15 & 4.27 & 4.17 & 3.95 & 3.51 & 3.57 & 3.33 & 2.38 & 1.26 & 1159.1 & 3.2 \\
\hline 70.0 & 1.45 & 2.38 & 3.62 & 3.89 & 3.91 & 3.78 & 3.62 & 3.28 & 3.47 & 3.37 & $2 .+7$ & 1.33 & 1112.2 & 3.0 \\
\hline 80.0 & 1.45 & 2.33 & 3.43 & 3.55 & 3.47 & 3.32 & 3.21 & 2.99 & 3.28 & 3.31 & 2.50 & 1.35 & 1040.4 & 2.9 \\
\hline 90.0 & 1.42 & 2.22 & 3.16 & 3.13 & 2.97 & 2.82 & 2.75 & 2.64 & 3.02 & 3.17 & 2.45 & 1.34 & 945.9 & 2.6 \\
\hline
\end{tabular}


AVERAGE DAILY TOTAL TERRESTRIAL INSOLATION ESTIMATES (KWH/SQ. M)

$\begin{array}{rlr}\text { SITE: } & \text { KODIAK } & \text { AK } \\ \text { LATITUDE: } & 57 \text { DEGREES } & 45 \text { MINUTES }\end{array}$

AVERAGE DAILY AMOUNTS BY MONTH

ARRAY

TILT

0.0

10.0

15.0

20.0

25.0

30.0

35.0

40.0

45.0

50.0

30.0

70.0

80.0

90.0

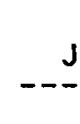

0.4

0.66

0.74

0.8

0.9

0.98

1.04

1. 10

$$
1.1
$$$$
1.2
$$$$
\text { 1. } 27
$$$$
1.30
$$

1. 30

1.27

F
1.12

1.. 12

1.36

1.36

2.81

A

$M$


AVERAGE DAILY TOTAL TEREESTRJAL INSOLATION ESTIMATES (KWH/SQ. M)

$\begin{aligned} \text { SITE: } & \text { MOME } \\ \text { ITUDE: } & 64 \text { DEGREES } 30 \text { MINUTES }\end{aligned}$

AVERAGE DAILY AMOUNTS BY MONTH

\begin{tabular}{|c|c|c|c|c|c|c|c|c|c|c|c|c|c|c|}
\hline ARRAY & $\int^{-1}$ & $F$ & $M$ & A & $M$ & $J$ & $J$ & A & $\mathrm{s}$ & 0 & $N$ & D & $\begin{array}{l}\text { ANNUAL } \\
\text { AMOUNT }\end{array}$ & $\begin{array}{c}\text { AVERAGE } \\
\text { DAY }\end{array}$ \\
\hline 0.0 & 0.09 & 0.71 & 1.99 & 3.74 & 4.96 & 5.53 & +.46 & 3.13 & 2.12 & j. 97 & 0.20 & 0.01 & 851.6 & 2.3 \\
\hline 10.0 & 0.15 & 0.93 & 2.37 & 4.11 & $5: 20$ & 5.70 & 4.66 & 3.40 & 2.54 & 1.38 & 0.43 & 0.01 & 942.1 & 2.6 \\
\hline 15.0 & 0.18 & 1.04 & 2.55 & 4.26 & 5.28 & 5.75 & 4.72 & 3.51 & 2.73 & 1.58 & 0.54 & 0.01 & 930.3 & 2.7 \\
\hline 20.0 & 0.21 & 1.13 & 2.71 & 4.39 & 5.34 & $5: 78$ & 4.77 & 3.61 & 2.90 & 1.76 & 0.55 & 0.01 & 1013.8 & 2.8 \\
\hline 25.0 & 0.24 & 1.23 & 2.85 & 4.49 & 5.36 & 5.78 & 4.79 & 3.68 & 3.06 & 1.94 & 0.75 & 0.01 & 1041.6 & 2.9 \\
\hline 30.0 & 0.26 & 1.31 & 2.97 & 4.57 & 3.36 & 5.74 & 4.78 & 3.73 & 3.19 & 2.10 & 0.35 & 0.01 & 1063.6 & 2.9 \\
\hline 35.0 & 0.29 & 1.39 & 3.08 & 4.62 & 5.35 & 5.69 & 4.76 & 3.77 & 3.31 & 2.25 & 0.94 & 0.01 & 1080.5 & 3.0 \\
\hline 40.0 & 0.31 & 1.46 & 3.17 & 4.65 & 5.30 & 5.61 & 4.72 & 3.78 & 3.41 & 2.38 & 1.02 & 0.01 & 1091.6 & 3.0 \\
\hline 45.0 & 0.33 & 1.52 & 3.24 & 4.65 & 5.23 & 5.51 & 4.65 & 3.77 & 3.49 & 2.50 & 1.10 & 0.01 & 1096.4 & 3.0 \\
\hline 50.0 & 0.35 & 1.57 & 3.29 & 4.62 & 5.13 & 5.38 & 4.55 & 3.74 & 3.54 & 2.60 & 1.17 & 0.01 & 1094.9 & 3.0 \\
\hline 60.0 & 0.37 & 1.64 & 3.33 & 4.49 & 4.84 & 5.03 & 4.29 & 3.62 & 3.58 & 2.75 & 1.28 & 0.01 & 1072.9 & 2.9 \\
\hline 70.0 & 0.39 & 1.66 & 3.29 & 4.26 & 4.47 & 4.60 & 3.96 & 3.43 & 3.54 & 2.82 & 1.36 & 0.01 & 1028.6 & 2.8 \\
\hline 80.0 & 0.40 & 1.65 & 3.16 & 3.94 & 4.03 & 4.10 & 3.57 & 3.16 & 3.40 & 2.82 & 1.39 & 0.01 & 963.0 & 2.6 \\
\hline 90.0 & 0.39 & 1.59 & 2.96 & 3.53 & 3.51 & 3.53 & 3.11 & 2.84 & 3.19 & 2.74 & 1.39 & 0.01 & 876.2 & 2.4 \\
\hline
\end{tabular}


AVERAGE DAILY TOTAL TERRESTRIAL INSOLATION ESTIMATES (KWH/SQ. M)

SITE: SUMMIT
LATITUDE: 63 DEGREES 20 MINUTES

AUERAGE DAILY AMOUNTS BY MONTH

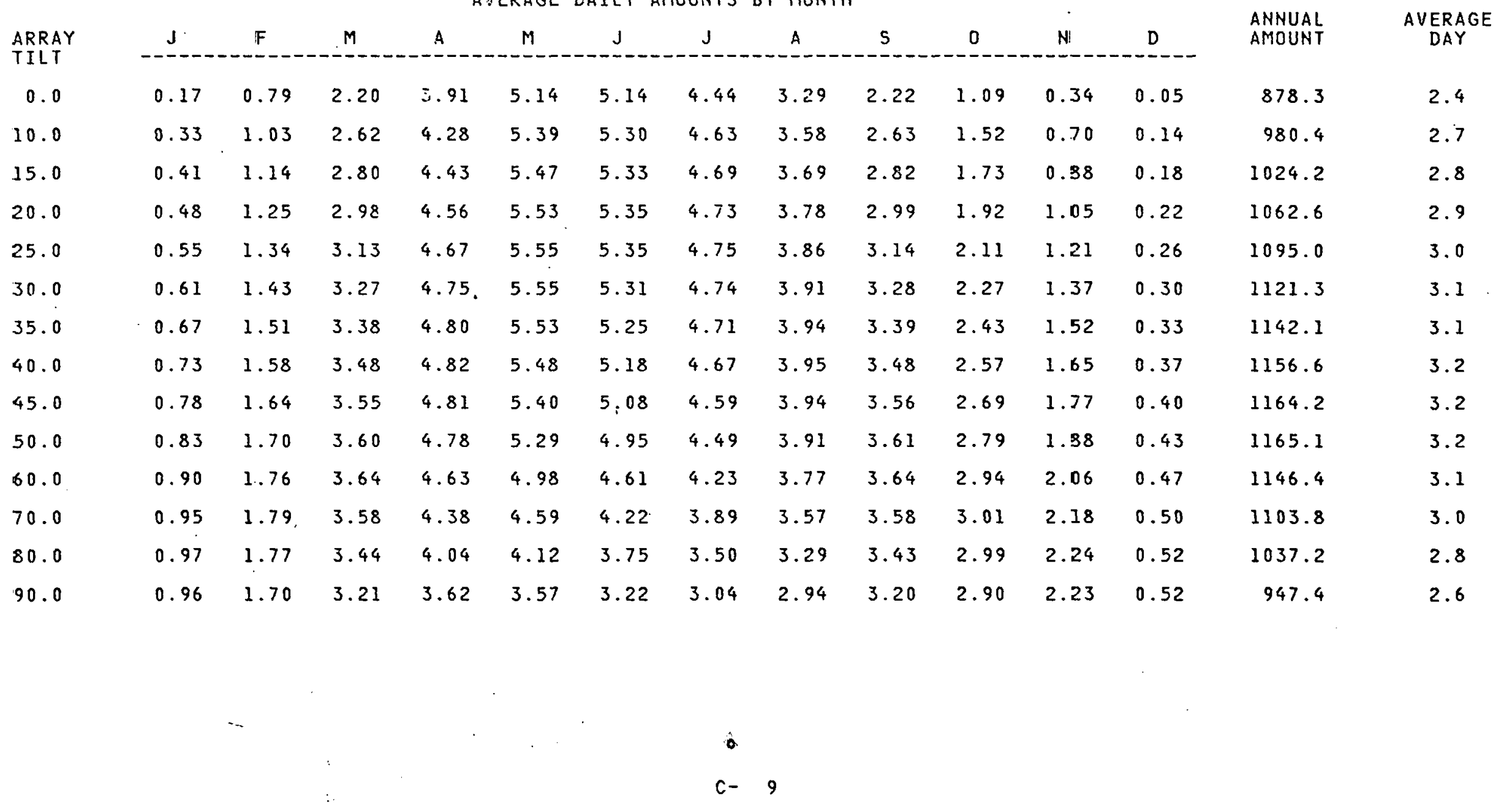


AVERAGE DAILY TOTAL TERRESTRIAL INSOLATION ESTIMATES (KWH/SQ. M)

SITE: YAKUTAT

LATITUDE: 59 DEGREES 31 MINUTES

AVERAGE DAILY AMOUNTS BY MONTH

\begin{tabular}{|c|c|c|c|c|c|c|c|c|c|c|c|c|c|c|}
\hline ARRAY & $\mathrm{J}$ & $F$ & $M$ & A & $M$ & 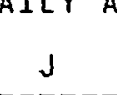 & $\mathrm{J}$ & A & $\subseteq$ & 0 & M & $D$ & $\begin{array}{l}\text { ANNUAL } \\
\text { AMOUNT }\end{array}$ & $\begin{array}{c}\text { AVERAGE } \\
\text { DAY }\end{array}$ \\
\hline 0.0 & 0.32 & 0.84 & 1.96 & 3.31 & 4.00 & 4.23 & 3.81 & 2.98 & 2.00 & 1.09 & 0.43 & 0.16 & 766.3 & 2.1 \\
\hline 10.0 & 0.49 & 1.08 & 2.23 & 3.55 & 4.13 & 4.32 & 3.93 & 3.17 & 2.26 & 1.38 & 0.65 & 0.29 & 838.1 & 2.3 \\
\hline 15.0 & 0.57 & 1.19 & 2.34 & 3.64 & 4.17 & 4.34 & 3.97 & 3.25 & 2.38 & 1.51 & 0.76 & 0.35 & 867.8 & 2.4 \\
\hline 20.0 & 0.65 & 1.29 & 2.45 & 3.72 & 4.19 & 4.34 & 3.98 & 3.30 & 2.48 & 1.64 & 0.86 & 0.41 & 893.0 & 2.4 \\
\hline 25.0 & 0.72 & 1.39 & 2.54 & 3.77 & 4.18 & $q .31$ & 3.97 & 3.34 & 2.57 & 1.76 & 0.96 & 0.46 & 913.5 & 2.5 \\
\hline 30.0 & 0.79 & 1.47 & 2.61 & 3.80 & 4.16 & +.27 & 3.95 & 3.37 & 2.65 & 1.86 & 1.05 & 0.52 & 929.5 & 2.5 \\
\hline 35.0 & 0.86 & 1.55 & 2.67 & 3.81 & 4.13 & 4.21 & 3.91 & 3.37 & 2.71 & 1.96 & 1.13 & 0.57 & 940.8 & 2.6 \\
\hline 40.0 & 0.92 & 1.62 & 2.72 & 3.81 & 4.07 & 4.14 & 3.86 & 3.36 & 2.76 & 2.04 & 1.20 & 0.61 & 947.0 & 2.6 \\
\hline 45.0 & 0.97 & 1.68 & 2.75 & 3.78 & 3.99 & 4.34 & 3.78 & 3.33 & 2.79 & 2.11 & 1.27 & 0.66 & 947.9 & 2.6 \\
\hline 50.0 & 1.03 & 1.72 & 2.76 & 3.73 & 3.89 & 3.72 & 3.68 & 3.28 & 2.80 & 2.16 & 1.33 & 0.69 & 943.6 & 2.6 \\
\hline 60.0 & 1.09 & 1.78 & 2.74 & 3.57 & 3.63 & 3.53 & 3.44 & 3.14 & 2.78 & 2.23 & 1.42 & 0.75 & 919.9 & 2.5 \\
\hline 70.0 & 1.13 & 1.80 & 2.66 & 3.35 & 3.33 & 3.31 & 3.15 & 2.94 & 2.70 & 2.25 & 1.47 & 0.79 & 878.8 & 2.4 \\
\hline 80.0 & 1.14 & 1.77 & 2.52 & 3.06 & 2.97 & 2.72 & 2.81 & $2: 68$ & 2.55 & 2.21 & 1.49 & 0.81 & 819.1 & 2.2 \\
\hline 90.0 & 1.12 & 1.69 & 2.32 & 2.71 & $2.56^{\circ}$ & 2.50 & 2.42 & 2.38 & 2.35 & 2.11 & 1.46 & 0.80 & 742.6 & 2.0 \\
\hline
\end{tabular}


AVERAGE DAILY TOTAL TERRESTRIAL INSOLATION ESTIMATES (KWH/SQ. M)

SITE:
LATITUDE: 33 DEGREES 34 MINUTES

AVERAGE DAILY AMOUNTS BY MONTH

\begin{tabular}{|c|c|c|c|c|c|c|c|c|c|c|c|c|c|c|}
\hline ARRAY & $J$ & $F$ & $M$ & $A$ & $M$ & $J$ & $J$ & A & S & 0 & $\mathbb{N}$ & $D$ & $\begin{array}{l}\text { ANNUAL } \\
\text { AMOUNT }\end{array}$ & $\begin{array}{c}\text { AVERAGE } \\
\text { DAY }\end{array}$ \\
\hline 0.0 & 2.22 & 3.05 & 4.08 & 5.27 & 5.85 & 6.05 & 5.70 & 5.44 & 4.58 & 3.82 & 2.70 & 2.09 & 1548.7 & 4.2 \\
\hline 10.0 & 2.53 & 3.34 & 4.32 & 5.39 & 5.83 & 5.98 & 5.69 & 5.56 & 4.86 & 4.25 & 3.13 & 2.44 & 1623.5 & 4.4 \\
\hline $1: .0$ & 2.66 & 3.46 & 4.40 & 5.40 & 5.78 & 5.90 & 5.63 & 5.58 & 4.96 & 4.43 & 3.32 & 2.60 & 1647.6 & 4.5 \\
\hline 20.0 & 2.78 & 3.56 & 4.45 & 5.38 & 5.69 & 5.79 & 5.55 & 5.56 & 5.03 & 4.59 & 3.49 & 2.74 & 1662.6 & 4.6 \\
\hline 25.0 & 2.89 & 3.64 & 4.49 & 5.33 & .5 .57 & 5.64 & 5.43 & 5.51 & 5.07 & 4.72 & 3.64 & 2.87 & 1668.3 & 4.6 \\
\hline 30.0 & 2.97 & 3.70 & 4.50 & 5.26 & 5.42 & 5.46 & 5.29 & 5.43 & 5.08 & 4.82 & 3.76 & 2.98 & 1664.6 & 4.6 \\
\hline 35.0 & 3.04 & 3.74 & 4.48 & 5.16 & 5.25 & 5.26 & 5.12 & 5.33 & 5.07 & 4.89 & 3.87 & 3.07 & 1652.1 & 4.5 \\
\hline 40.0 & 3.10 & 3.76 & 4.44 & 5.03 & 5.06 & 5.05 & 4.93 & 5.19 & 5.02 & 4.93 & 3.95 & 3.15 & .1631 .5 & 4.5 \\
\hline 45.0 & 3.13 & 3.76 & 4.37 & 4.87 & 4.84 & 4.80 & 4.72 & 5.03 & 4.95 & 4.94 & 4.01 & 3.20 & 1601.9 & 4.4 \\
\hline 50.0 & 3.15 & 3.73 & 4.28 & 4.69 & 4.60 & 4.54 & 4.49 & 4.84 & 4.85 & 4.93 & 4.04 & 3.24 & 1563.5 & 4.3 \\
\hline 60.0 & 3.12 & 3.62 & 4.03 & 4.26 & 4.05 & 3.95 & 3.95 & 4.39 & 4.57 & 4.80 & 4.03 & 3.25 & 1461.4 & 4.0 \\
\hline 70.0 & 3.03 & 3.43 & 3.70 & 3.74 & 3.44 & 3.32 & 3.36 & 3.85 & 4.19 & 4.57 & 3.92 & 3.19 & 1329.9 & 3.6 \\
\hline 80.0 & 2.86 & 3.16 & 3.29 & 3.16 & 2.81 & 2.66 & 2.75 & 3.24 & 3.71 & 4.22 & 3.71 & 3.05 & 1175.1 & 3.2 \\
\hline 90.0 & 2.63 & 2.83 & 2.82 & 2.55 & 2.17 & 2.05 & 2.13 & 2.60 & 3.17 & 3.78 & 3.42 & 2.83 & 1002.9 & 2.7 \\
\hline
\end{tabular}


AVERAGE DAILY TOTAL TEREESTRIAL INSOLATION ESTIMATES (KWH/SQ. M)

SITE: MOBILE

LATITUDE: 30 DEGREES 41 MINUTES

AVERAGE DAILY AMOUNTS BY MONTH

\section{ARRAY \\ TILT}

0.0

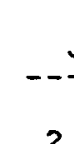

10.0

15.0

20.0

25.0

30.0

35.0

40.0

45:0

50.0

60.0

70.0

80.0

90.0

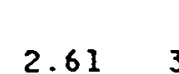

2.953 .79

3.22

3.91

4.73

4.78
4.81

3.344 .10

3.434 .16

4.80

3.50

3.55

$3.58 \quad 4.19$

$3.59 \quad 4.16$

3.55

3.42

4.01

3.22

3.46

$2.94 \quad 3.07$

2.8 .8

2.44 $M$ $\mathrm{J}$

5

5.9

5.89

5.86

5.41

5.80

5.41
5.37

5.17

4.57

$4.10 \quad 3.01 \quad 2.39$

4

4.80

4.53

4.53

3.44

$5.70 \quad 5.30 \quad 5.25$

4.88

$5.69 \quad 5.57$

$5.21 \quad 5.22$

4.93

4.70

2.76

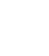

$\subseteq .96$

4.85

3.63

2.76

$3.80 \quad 3.07$

$4.97 \quad 3.94 \quad 3.20$

$\begin{array}{llll}4.95 & 5.06 & 4.05 & 3.31\end{array}$

4.92

$4.78 \quad 4.96$

$5.21 \quad 5.04$

$5.00 \quad 4.81$

$4.59 \quad 4.82$

4.86

5.12

5.14

4.15

$4.57 \quad 4.38 \quad 4.66$

$\begin{array}{llll}4.77 & 5.14 & 4.23 & 3.52\end{array}$

$\begin{array}{lllll}4.77 & 5.14 & 4.23 & 3.52\end{array}$

4.23

4.77

$\begin{array}{llll}4.66 & 5.11 & 4.30 \quad 3.55\end{array}$

4.51

$\begin{array}{lll}4.30 & 4.15 & 4.47 \\ 3.70 & 3.63 & 4.03\end{array}$

4.36

$\begin{array}{lll}5.11 & 4.30 & 3.55 \\ 4.95 & 4.25 & 3.55\end{array}$

$\begin{array}{llll}3.93 & 3.70 & 3.63 & 4.03 \\ 3.31 & 3.09 & 3.07 & 3.51\end{array}$

3.36

$\begin{array}{lll}4.95 & 4.25 & 3.55 \\ 4.67 & 4.12 & 3.45\end{array}$

$\begin{array}{llllllll}2.66 & 2.45 & 2.50 & 2.93 & 3.49 & 4.29 & 3.83 & 3.28\end{array}$

$\begin{array}{llllllll}2.66 & 2.45 & 2.50 & 2.93 & 3.49 & 4.29 & 3.83 & 3.28 \\ 2.04 & 1.88 & 1.93 & 2.34 & 2.95 & 3.81 & 3.54 & 3.03\end{array}$

ANNUAL
AMOUNT

AVERAGE
DAY

\section{5}

4.4

1665.3

4.6

4.6

4.7

4.7

4.6

4.6

4.5

4.4

4.3

4.0

3.7

3.2

2.7 
AVERAGE DAILY TOTAL TERRESTRIAL INSOLATION ESTIMATES (KWH:SQ. M)

SITE: MONTGOMERY
LATITUDE: 32 DEGREES 18 MINUTES

AVERAGE DAILY AMOUNTS BY MONTH
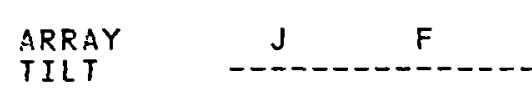

AVERAGE DAILY AMOUNTS BY MONTH

O. $\mathrm{C}$

10.0

$2.37 \quad 3.20 \quad 4.22$

$\begin{array}{lll}2.68 & 3.50 & 4.45\end{array}$

A

$M$

$--$

\section{0}

$2.82 \quad 3.62 \quad 4.53$

5.46
5.56

5.98

5.95

6.22

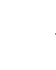

20.0

25.0

$2.94 \quad 3.72 \quad 4.58$

5.57

5.89

6.13

5.80

$3.05 \quad 3.80 \quad 4.61$

5.55

5.79

6

$5.49 \quad 5.66$

6.04

5.77
5.71

5.5

5.51

\section{0}

35.0

40.0

45.0

50.0

60.0

70.0

80.0

90.0

$3.13 \quad 3.86 \quad 4.62$

$\begin{array}{lll}5.66 & 5.76 \quad 5.49 & 5.55\end{array}$

$\begin{array}{lllll}5.41 & 5.51 & 5.57 & 5.34 & 5.46\end{array}$

$\begin{array}{lllll}5.30 & 5.33 & 5.36 & 5.16 & 5.35\end{array}$

5.16

$\begin{array}{llll}5.12 & 5.12 & 4.97 & 5.21\end{array}$

$3.25 \quad 3.91 \quad 4.55$

$3.29 \quad 3.90 \quad 4.47$

4.99

4.89

$4.87 \quad 4.75 \quad 5.04$

4

$\begin{array}{lllll}4.80 & 4.64 & 4.59 & 4.50 & 4.84\end{array}$

$\begin{array}{lllll}4.34 & 4.06 & 3.96 & 3.95 & 4.38\end{array}$

$3.27 \quad 3.75 \quad 4.11$

$3.16 \quad 3.54 \quad 3.76$

$2.98 \quad 3.26 \quad 3.33$

3.79

3.43

3.31

$3.34 \quad 3.82$

$2.54 \quad 2.13$

2.63
2.00

2.713 .20

2.092 .55 .

5
0

$\begin{array}{llll}4.62 & 3.98 & 2.88 & 2.26\end{array}$

ANNUAL AMIOUNT

AVERAGE DAY

4.4

4.6

4.6

4.7

4.7

4.7

4.6

4.6

4.5

4.4

4.1

3.7

3.3

2.8 
AVERAGE DAILY TOTAL TERRESTRIAL INSOLATION ESTIMATES (KWH/SQ. M)

$\begin{aligned} \text { SITE: } & \text { FORT SMITH } \\ \text { LATITUDE: } & 35 \text { DEGREES } 20 \text { MINUTES }\end{aligned}$

AVERAGE DAILY AMOUNTS BY MONTH

\begin{tabular}{|c|c|c|c|c|c|c|c|c|c|c|c|c|c|c|}
\hline $\begin{array}{l}\text { ARRAY } \\
\text { TILT }\end{array}$ & $\begin{array}{c}---m \\
J\end{array}$ & $F$ & $M$ & A & $M$ & 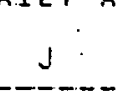 & $\int^{-1}$ & A & $S$ & 0 & $N$ & D. & $\begin{array}{l}\text { ANNUAL } \\
\text { AMOUNT }\end{array}$ & $\begin{array}{c}\text { AVERAGE } \\
\text { DAY }\end{array}$ \\
\hline 0.0 & 2.37 & 3.15 & 4.13 & 5.10 & 6.03 & 6.58 & 6.51 & 5.92 & 4.73 & 3.79 & 2.58 & 2.15 & 1617.4 & 4.4 \\
\hline $1 ? .0$ & 2.71 & 3.49 & 4.39 & 5.22 & 6.03 & 6.53 & 6.51 & 6.09 & 5.05 & 4.26 & 3.15 & 2.57 & 1705.7 & 4.7 \\
\hline 15.0 & 2.8 .3 & 3.63 & 4.48 & 5.24 & 5.98 & 6.45 & 6.46 & 6.12 & 5.17 & 4.46 & 3.36 & 2.76 & 1735.6 & 4.8 \\
\hline 20.0 & 3.03 & 3.75 & 4.55 & 5.23 & 5.90 & 6.33 & 6.37 & 6.11 & 5.26 & 4.63 & 3.55 & 2.93 & 1755.6 & 4.8 \\
\hline 25.0 & 3.15 & 3.85 & 4.60 & 5.20 & 5.79 & 6.18 & 6.25 & 6.08 & 5.32 & 4.77 & 3.71 & 3.08 & 1765.6 & 4.8 \\
\hline 30.0 & 3.27 & 3.92 & 4.6 .2 & 5.13 & 5.65 & 5.99 & 6.09 & 6.00 & 5.35 & 4.89 & 3.36 & 3.22 & 1765.6 & 4.8 \\
\hline 35.0 & 3.37 & 3.98 & 4.61 & 5.04 & 5.47 & 5.77 & 5.89 & 5.89 & 5.35 & 4.98 & 3.98 & 3.34 & 1755.4 & 4.8 \\
\hline 40.0 & 3.44 & 4.01 & 4.57 & 4.92 & 5.28 & $5.54^{\circ}$ & 5.68 & 5.76 & 5.32 & 5.03 & 4.08 & 3.43 & 1737.0 & 4.8 \\
\hline 45.0 & 3.49 & 4.02 & 4.52 & 4.78 & 5.06 & 5.28 & 5.44 & 5.59 & 5.25 & 5.06 & 4.15 & 3.51 & 1708.8 & 4.7 \\
\hline 50.0 & 3.52 & 4.00 & 4.43 & 4.61 & 4.82 & 4.99 & 5.17 & 5.39 & 5.16 & 5.05 & 4.20 & 3.56 & 1671.0 & 4.6 \\
\hline 60.0 & 3.52 & 3.90 & 4.19 & 4.21 & 4.26 & 4.34 & 4.55 & 4.90 & 4.88 & 4.95 & 4.21 & 3.60 & 1567.6 & 4.3 \\
\hline 70.0 & 3.43 & 3.71 & 3.86 & 3.72 & 3.62 & 3.64 & 3.85 & 4.31 & 4.49 & 4.73 & 4.12 & 3.55 & 1430.9 & 3.9 \\
\hline 80.0 & 3.27 & 3.44 & 3.45 & 3.16 & 2.98 & 2.93 & 3.14 & 3.64 & 4.00 & 4.39 & 3.92 & 3.41 & 1268.9 & 3.5 \\
\hline 90.0 & 3.02 & 3.09 & 2.97 & 2.57 & 2.30 & 2.23 & 2.40 & 2.91 & 3.43 & 3.95 & 3.53 & 3.19 & 1085.5 & 3.0 \\
\hline
\end{tabular}


AVERAGE DAILY TOTAL TERRESTRIAL INSOLATION ESTIMATES (KWH/SQ. M)

SITE: LITTLE ROCK

LATITUDE: 34 DEGREES 44 MINUTES

AVERAGE DAILY AMOUNTS BY MONTH

\begin{tabular}{|c|c|c|c|c|c|c|c|c|c|c|c|c|c|c|}
\hline ARRAY & $\mathrm{J}$ & $F$ & $M$ & $A$ & $M$ & $J$ & $J$ & A & 5 & 0 & $\mathbf{N}$ & D & $\begin{array}{l}\text { ANNUAL } \\
\text { AMOUNT }\end{array}$ & $\begin{array}{c}\text { AVERAGE } \\
\text { DAY }\end{array}$ \\
\hline 0.0 & 2.30 & 3.16 & 4.13 & 5.08 & 6.07 & 6.64 & 6.40 & 5.87 & 4.78 & 3.88 & 2.66 & 2.12 & 1617.3 & 4.4 \\
\hline 10.0 & 2.65 & 3.49 & 4.38 & 5.20 & 6.07 & 6.57 & 6.40 & 6.02 & 5.10 & 4.35 & 3.11 & 2.52 & 1701.8 & 4.7 \\
\hline 15.0 & 2.80 & 3.63 & 4.48 & 5.22 & 6.02 & 6.49 & 6.35 & 6.05 & 5.22 & 4.55 & 3.31 & 2.70 & 1729.8 & 4.7 \\
\hline 20.0 & 2.94 & 3.74 & 4.54 & 5.20 & 5.94 & 6.37 & 6.25 & 6.04 & 5.31 & 4.73 & 3.49 & 2.86 & 1748.0 & 4.8 \\
\hline 25.0 & 3.06 & 3.84 & 4.58 & 5.16 & 5.82 & 6.21 & 6.13 & 6.00 & 5.36 & 4.87 & 3.65 & 3.00 & 1756.3 & 4.8 \\
\hline 30.0 & 3.17 & 3.91 & 4.60 & 5.10 & 5.67 & 6.01 & 5.97 & 5.92 & 5.39 & 4.99 & 3.79 & 3.13 & 1754.6 & 4.8 \\
\hline 35.0 & 3.25 & 3.96 & 4.59 & 5.00 & 5.49 & 5.79 & 5.78 & 5.81 & 5.38 & 5.07 & 3.90 & 3.24 & 1743.0 & 4.8 \\
\hline 40.0 & 3.32 & 3.99 & 4.55 & 4.88 & 5.29 & 5.55 & 5.57 & 5.67 & 5.34 & 5.13 & .3 .99 & 3.32 & 1723.2 & 4.7 \\
\hline 45.0 & 3.36 & 3.99 & 4.49 & 4.74 & 5.07 & 5.29 & 5.33 & 5.50 & 5.28 & 5.15 & 4.06 & 3.39 & 1693.6 & 4.6 \\
\hline 50.0 & 3.39 & 3.98 & 4.40 & 4.57 & 4.82 & 4.99 & 5.06 & 5.30 & 5.18 & 5.14 & 4.10 & 3.44 & 1654.6 & 4.5 \\
\hline 60.0 & 3.38 & 3.87 & 4.16 & 4.16 & 4.25 & 4.34 & 4.45 & 4.81 & 4.89 & 5.03 & 4.10 & 3.47 & 1549.2 & 4.2 \\
\hline 70.0 & 3.29 & 3.68 & 3.83 & 3.67 & 3.61 & 3.63 & 3.76 & 4.23 & 4.49 & 4.80 & 4.00 & 3.41 & 1411.2 & 3.9 \\
\hline 80.0 & 3.12 & 3.40 & 3.41 & 3.12 & 2.96 & 2.90 & 3.06 & 3.56 & 4.00 & 4.45 & 3.81 & 3.27 & 1248.6 & 3.4 \\
\hline 90.0 & 2.88 & 3.05 & 2.93 & 2.53 & 2.28 & 2.20 & 2.34 & 2.85 & 3.42 & 4.00 & 3.52 & 3.05 & 1065.8 & 2.9 \\
\hline
\end{tabular}


AVERAGE DAILY TOTAL TERRESTRIAL INSOLATION ESTIMATES (KWH,SQ. M)

SITE: PHCENIX

LATITUDE: 33 DEGREES 26 MINUTES

AVERAGE DALLY AMOUNTS BY MONTH

\begin{tabular}{|c|c|c|c|c|c|c|c|c|c|c|c|c|c|c|}
\hline ARRAY & J & $F$ & $M$ & A & $M$ & J & J & A & s & $a$ & H & $D$ & $\begin{array}{l}\text { ANNUAL } \\
\text { AMOUNT }\end{array}$ & $\begin{array}{l}\text { AVERAGE } \\
\text { DAY }\end{array}$ \\
\hline 0.0 & 3.22 & 4.34 & 5.72 & 7.42 & 8.44 & 8.64 & 7.84 & 7.23 & 6.35 & 4.97 & 3.63 & 2.94 & 2153.9 & 5.9 \\
\hline 10.0 & 3.79 & 4.86 & 6.13 & 7.63 & 8.43 & 8.53 & 7.82 & $7 . .43$ & 6.85 & 5.65 . & 4.33 & 3.56 & 2283.7 & 6.3 \\
\hline 15.0 & 4.04 & 5.09 & 6.28 & 7.67 & 8.34 & 3.40 & 7.74 & 7.47 & 7.03 & 5.94 & 4.64 & 3.85 & 2327.8 & 6.4 \\
\hline 20.0 & 4.27 & 5.28 & 6.40 & 7.66 & 8.20 & 3.21 & 7.61 & 7.45 & 7.17 & 6.17 & 4.93 & 4.10 & 2357.7 & 6.5 \\
\hline 25.0 & 4.47 & 5.43 & 6.47 & 7.60 & 8.02 & $? .97$ & 7.44 & 7.40 & 7.26 & 6.40 & 5.18 & 4.34 & 2373.0 & 6.5 \\
\hline 30.0 & 4.64 & 5.56 & 6.51 & 7.49 & 7.78 & $? .69$ & 7.23 & 7.29 & 7.31 & 6.57 & 5.40 & 4.54 & 2373.7 & 6.5 \\
\hline 35.0 & 4.78 & 5.65 & 6.51 & 7.35 & 7.50 & ?.36 & 6.97 & 7.15 & 7.31 & 6.70 & 5.58 & 4.72 & 2360.6 & 6.5 \\
\hline 40.0 & 4.90 & 5.70 & 6.46 & 7.16 & 7.19 & $? .02$ & 6.69 & 6.97 & 7.27 & 6.78 & 5.73 & 4.86 & 2334.8 & 6.4 \\
\hline 45.0 & 4.98 & 5.72 & 6.38 & 6.93 & 6.85 & 6.63 & 6.38 & 6.74 & 7.18 & 6.82 & 5.84 & 4.97 & 2294.7 & 6.3 \\
\hline 50.0 & 5.03 & 5.71 & 6.26 & 6.66 & 6.46 & 6.21 & 6.02 & 6.48 & 7.04 & 6.82 & 5.91 & 5.06 & 2240.5 & 6.1 \\
\hline 60.0 & 5.04 & 5.57 & 5.91 & 6.00 & 5.57 & 5.26 & 5.22 & 5.84 & 6.64 & 6.68 & 5.94 & 5.12 & 2091.7 & 5.7 \\
\hline 70.0 & 4.91 & 5.30 & 5.41 & 5.20 & 4.58 & 4.26 & 4.32 & 5.07 & 6.08 & 6.37 & 5.81 & 5.05 & 1895.8 & 5.2 \\
\hline 80.0 & 4.67 & 4.90 & 4.79 & 4.29 & 3.58 & 5.23 & 3.41 & 4.18 & 5.37 & 5.90 & 5.53 & 4.85 & 1662.2 & 4.6 \\
\hline 90.0 & 4.30 & 4.38 & 4.07 & 3.34 & 2.56 & 2.27 & 2.49 & 3.26 & 4.53 & 5.28 & 5.11 & 4.52 & 1400.7 & 3.8 \\
\hline
\end{tabular}


AVERAGE DAZLY TOTAL TERRESTRIAL INSOLATION ESTIMATES (KWH/SQ. M)

\section{$\begin{aligned} \text { SITE: } & \text { PRESCOTT } \\ \text { LATITUDE: } & 34 \text { DEGREES } 39 \text { MINUTES }\end{aligned}$}

AVERAGE DAILY AMOUNTS BY MONTH

\begin{tabular}{|c|c|c|c|c|c|c|c|c|c|c|c|c|c|c|}
\hline ARRAY & $J$ & $F$ & $M$ & A & $M$ & $J$ & $\mathrm{~J}$ & $A$ & 5 & 0 & $\mathrm{~N}$ & D & $\begin{array}{l}\text { ANNUAL } \\
\text { ANIOUNT }\end{array}$ & $\begin{array}{l}\text { AVERAGE } \\
\text { DAY }\end{array}$ \\
\hline 0.0 & 3.20 & 4.21 & 5.60 & 7.18 & 8.28 & 8.71 & 7.27 & 6.60 & 6.16 & 4.86 & 3.59 & 2.92 & 2088.1 & 5.7 \\
\hline 10.0 & 3.81 & 4.74 & 6.03 & 7.40 & 8.29 & 8.62 & 7.28 & 6.79 & 6.66 & 5.56 & 4.34 & 3.60 & 2224.9 & 6.1 \\
\hline 15.0 & 4.08 & 4.97 & 6.19 & 7.44 & 3.22 & 8.50 & 7.21 & 6.82 & 6.85 & 5.86 & 9.67 & 3.90 & 2273.2 & 6.2 \\
\hline 20.0 & 4.33 & 5.16 & 6.31 & 7.44 & 8.10 & 8.32 & 7.10 & 6.82 & 6.99 & 6.12 & 9.97 & 4.18 & 2307.6 & 6.3 \\
\hline 25.0 & 4.54 & 5.33 & 6.39 & 7.39 & 7.92 & 8.09 & 6.96 & 6.77 & 7.09 & 6.34 & 5.24 & 4.43 & 2327.9 & 6.4 \\
\hline 30.0 & 4.73 & 5.46 & 6.44 & 7.30 & 7.70 & 7.82 & 6.77 & 6.69 & 7.15 & 6.52 & 5.48 & 4.66 & 2333.8 & 6.4 \\
\hline 35.0 & 4.89 & 5.55 & 6.45 & 7.16 & 7.44 & 7.49 & 6.54 & 6.56 & 7.16 & 6.65 & 5.68 & 4.85 & 2325.5 & 6.4 \\
\hline 40.0 & 5.02 & 5.62 & 6.41 & 6.99 & 7.15 & 7.16 & 6.30 & 6.41 & 7.12 & 6.75 & 5.85 & 5.02 & 2305.4 & 6.3 \\
\hline 45.0 & 5.12 & 5.64 & 6.34 & 6.78 & 6.82 & 6.78 & 6.01 & 6.21 & 7.05 & 6.80 & 5.97 & 5.15 & 2271.2 & 6.2 \\
\hline 50.0 & 5.18 & 5.64 & 6.23 & 6.53 & 6.45 & 6.36 & 5.70 & 5.98 & 6.92 & 6.81 & 6.06 & 5.24 & 2223.0 & 6.1 \\
\hline 60.0 & 5.21 & 5.52 & 5.89 & 5.91 & 5.60 & 5.41 & 4.98 & 5.42 & 6.55 & 6.69 & 6.11 & 5.33 & 2086.6 & 5.7 \\
\hline 70.0 & 5.10 & 5.26 & 5.42 & 5.15 & 4.63 & 4.40 & 4.16 & 4.74 & 6.01 & 6.40 & 6.00 & 5.28 & 1902.0 & 5.2 \\
\hline 80.0 & 4.86 & 4.88 & 4.82 & 4.29 & 3.66 & 3.37 & 3.34 & 3.96 & 5.33 & 5.94 & 5.73 & 5.08 & 1680.2 & 4.6 \\
\hline 90.0 & 4.50 & 4.38 & 4.11 & 3.37 & 2.66 & 2.39 & 2.50 & 3.13 & 4.53 & 5.34 & 5.31 & 4.76 & 1427.4 & 3.9 \\
\hline
\end{tabular}


AVERAGE DAILY TOTAL TERRESTRIAL INSOLATION ESTIMATES (KWH/SQ. M)

$$
\begin{aligned}
& \text { SITE: TUCSON } \\
& \text { LATITUDE: } 32 \text { DEGREES } 7 \text { MINUTES }
\end{aligned}
$$

AVERAGE DAILY AMOUNTS BY MONTH

\begin{tabular}{|c|c|c|c|c|c|c|c|c|c|c|c|c|c|c|}
\hline ARRAY & J & $F$ & $M$ & A & $M$ & J & $\mathrm{J}$ & A & $s$ & 0 & $N$ & D & $\begin{array}{l}\text { ANNUAL } \\
\text { AMOUNT }\end{array}$ & $\begin{array}{l}\text { AVERAGE } \\
\text { DAY }\end{array}$ \\
\hline 0.0 & 3.45 & 4.52 & 5.87 & 7.45 & 8.43 & 8.60 & 7.38 & 6.83 & 6.24 & 5.05 & 3.30 & 3.14 & 2156.0 & 5.9 \\
\hline 10.0 & 4.05 & 5.05 & 6.28 & 7.64 & 8.39 & 8.47 & 7.34 & 7.05 & 6.68 & 5.71 & 4.51 & 3.78 & 2280.4 & 6.2 \\
\hline 15.0 & 4.31 & 5.27 & 6.42 & 7.66 & 8.29 & 8.32 & 7.26 & 7.05 & 6.84 & 5.98 & 4.32 & 4.07 & 2322.1 & 6.4 \\
\hline 20.0 & 4.55 & 5.45 & 6.53 & 7.64 & 8.14 & 8.12 & 7.13 & 7.04 & 6.96 & 6.22 & 5.10 & 4.33 & 2349.5 & 6.4 \\
\hline 25.0 & 4.75 & 5.61 & 6.60 & 7.57 & 7.94 & 7.87 & 6.96 & 6.97 & 7.03 & 6.41 & 5.35 & 4.57 & 2362.5 & 6.5 \\
\hline 30.0 & 4.93 & 5.73 & 6.63 & 7.45 & 7.69 & 7.58 & 6.75 & 6.87 & 7.07 & 6.57 & 5.57 & 4.78 & 2361.0 & 6.5 \\
\hline 35.0 & 5.03 & 5.81 & 6.61 & 7.29 & 7.41 & 7.25 & 6.51 & 6.72 & 7.05 & 6.69 & 5.75 & 4.95 & 2346.5 & 6.4 \\
\hline 40.0 & 5.17 & 5.86 & 6.56 & 7.09 & 7.09 & 6.90 & 6.24 & 6.57 & 7.00 & 6.76 & 5.39 & 5.10 & 2318.6 & 6.4 \\
\hline 45.0 & 5.28 & 5.88 & 6.47 & 6.85 & 6.73 & 6.50 & 5.94 & $6.3 ?$ & 6.90 & 6.79 & 5.79 & 5.21 & 2276.6 & 6.2 \\
\hline 50.0 & 5.32 & 5.85 & 6.33 & 6.57 & 6.33 & 6.07 & 5.61 & 6.05 & 6.75 & 6.77 & 6.06 & 5.29 & 2220.7 & 6.1 \\
\hline 60.0 & 5.32 & 5.70 & 5.96 & 5.90 & 5.43 & 5.11 & 4.85 & 5.45 & 6.35 & 6.61 & $6.1) 6$ & 5.34 & 2069.2 & 5.7 \\
\hline 70.0 & 5.17 & 5.40 & 5.44 & 5.09 & 4.44 & 4.11 & 4.01 & 4.71. & 5.78 & 6.28 & 5.71 & 5.25 & 1872.6 & 5.1 \\
\hline 80.0 & 4.90 & 6.97 & 4.79 & 4.17 & 3.43 & 3.08 & 3.16 & 3.83 & 5.08 & 5.79 & 5.11 & 5.03 & 1637.7 & 4.5 \\
\hline 90.0 & 4.50 & 4.43 & 4.04 & 3.21 & 2.44 & 2.14 & 2.32 & 3.02 & 4.27 & 5.16 & 5.16 & 4.67 & 1378.0 & 3.8 \\
\hline
\end{tabular}


AVERAGE DAILY TOTAL TERRESTRIAL INSOLATION ESTIMATES (KWH/SQ. M)

SITE: WINSLOW

LATITUDE: 35 DEGREES 1 MINUTES

AVERAGE DAILY AMOUNTS BY MONTH

\begin{tabular}{|c|c|c|c|c|c|c|c|c|c|c|c|c|c|c|}
\hline ARRAY & $\mathrm{J}$ & $F$ & $M$ & $A$ & $M$ & $J$ & $J$ & $A$ & $S$ & 0 & $\mathbf{N}$ & $\mathrm{D}$ & $\begin{array}{l}\text { ANNUAL } \\
\text { AMOUNT }\end{array}$ & $\begin{array}{c}\text { AVERAGE } \\
\text { DAY }\end{array}$ \\
\hline 0.0 & 3.10 & 4.19 & 5.61 & 7.20 & 8.18 & 8.55 & 7.40 & 6.75 & 6.08 & 4.77 & 3.53 & 2.82 & 2075.4 & 5.7 \\
\hline 10.0 & 3.69 & 4.72 & 6.05 & 7.42 & 8.20 & 8.47 & 7.41 & 6.96 & 6.57 & 5.46 & 4.27 & 3.47 & 2212.2 & 6.1 \\
\hline 15.0 & 3.95 & 4.95 & 6.21 & 7.47 & 8.13 & 8.35 & 7.34 & 7.00 & 6.75 & 5.75 & 4.60 & 3.77 & 2260.6 & 6.2 \\
\hline 20.0 & 4.19 & 5.15 & 6.34 & 7.47 & 8.01 & 8.19 & 7.24 & 7.00 & 6.90 & 6.01 & 4.90 & 4.04 & 2295.2 & 6.3 \\
\hline 25.0 & 4.40 & 5.31 & 6.43 & 7.43 & 7.84 & 7.97 & 7.09 & 6.95 & 7.00 & 6.22 & 5.17 & 4.28 & 2315.7 & 6.3 \\
\hline 30.0 & 4.59 & 5.45 & 6.48 & 7.34 & 7.63 & 7.70 & 6.90 & 6.87 & 7.06 & 6.40 & 5.41 & 4.50 & 2322.0 & 6.4 \\
\hline 35.0 & 4.75 & 5.55 & 6.49 & 7.21 & 7.37 & 7.39 & 6.67 & 6.74 & 7.07 & 6.54 & 5.61 & 4.69 & 2314.0 & 6.3 \\
\hline 40.0 & 4.87 & 5.61 & 5.45 & 7.04 & 7.09 & 7.06 & 6.42 & 6.59 & 7.04 & 6.64 & 5.78 & 4.85 & 2294.4 & 6.3 \\
\hline 45.0 & 4.97 & 5.64 & 6.38 & 6.83 & 6.77 & 6.69 & 6.13 & 6.39 & 6.96 & 6.69 & 5.90 & 4.97 & 2260.8 & 6.2 \\
\hline 50.0 & 5.03 & 5.64 & 6.28 & 6.57 & 6.40 & 6.29 & 5.82 & 6.15 & 6.85 & 6.70 & 5.99 & 5.07 & 2213.4 & 6.1 \\
\hline 60.0 & 5.06 & 5.52 & 5.94 & 5.96 & 5.57 & 5.37 & 5.08 & 5.58 & 6.48 & 6.59 & 6.05 & 5.15 & 2078.6 & 5.7 \\
\hline 70.0 & 4.95 & 5.27 & 5.47 & 5.20 & 4.62 & 4.39 & 4.25 & 4.88 & 5.96 & 6.30 & 5.94 & 5.10 & 1895.5 & 5.2 \\
\hline 80.0 & 4.72 & 4.89 & 4.87 & 4.34 & 3.67 & 3.38 & 3.41 & 4.08 & $5.29^{\circ}$ & $5.86^{\circ}$ & 5.68 & 4.92 & 1675.6 & 4.6 \\
\hline 90.0 & 4.37 & 4.39 & 4.16 & 3.41 & 2.68 & 2.42 & 2.55 & 3.23 & 4.50 & 5.27 & 5.27 & 4.61 & 1424.1 & 3.9 \\
\hline
\end{tabular}


AUERAIE DAILY TOTAL TERRESTRIAL INSOLATION ESTIMATES (KWH/SQ. M)

SITE: YUUMA

LATITUDE: 52 DEGREES 40 MINUTES

AVERAGE DEILY AMOUNTS BY MONTH

\begin{tabular}{|c|c|c|c|c|c|c|c|c|c|c|c|c|c|c|}
\hline ARRAY & $j$ & $F$ & $y$ & A & $M$ & $\checkmark$ & $J$ & $A$ & $S$ & 0 & $N$ & D & $\begin{array}{l}\text { ANNUAL } \\
\text { ANIOUNT }\end{array}$ & $\begin{array}{c}\text { AVERAGE } \\
\text { DAY }\end{array}$ \\
\hline 0.0 & 3.45 & 4.55 & 6.04 & 7.60 & 8.61 & 8.87 & 7.74 & 7.35 & 6.46 & 5.12 & 3.83 & 3.15 & 2215.4 & 6.1 \\
\hline 10.0 & 4.06 & 5.10 & 6.48 & 7.81 & 8.58 & 8.74 & 7.70 & 7.54 & 6.95 & 5.81 & 4.56 & 3.83 & 2348.6 & 6.4 \\
\hline 15.0 & 4.33 & 5.33 & 6.64 & 7.84 & 3. 48 & 8.59 & 7.62 & 7.57 & 7.13 & 6.10 & 4.89 & 4.13 & 2393.8 & 6.6 \\
\hline 20.0 & 4.58 & 5.53 & 6.76 & 7.82 & 8.34 & 8.39 & 7.49 & 7.55 & 7.26 & 6.35 & 5.19 & 4.40 & 2424.1 & 6.6 \\
\hline 25.0 & 4.79 & 5.69 & 6.84 & 7.75 & 8.14 & 8.14 & 7.31 & 7.48 & 7.35 & 6.56 & 5.45 & 4.65 & 2439.5 & 6.7 \\
\hline 30.0 & 4.98 & 5.82 & 6.88 & 7.64 & 7.89 & $\therefore .84$ & 7.09 & 7.37 & 7.39 & 6.73 & 5.68 & 4.87 & 2439.7 & 6.7 \\
\hline 35.0 & 5.13 & 5.91 & 6.87 & 7.48 & 7.60 & . . 51 & 6.84 & 7.22 & 7.39 & 6.86 & 5.87 & 5.06 & 2426.0 & 6.6 \\
\hline 40.0 & 5.25 & 5.97 & 6.82 & 7.28 & 7.28 & . .14 & 6.56 & 7.03 & 7.34 & 6.94 & 6.02 & 5.21 & 2398.7 & 6.6 \\
\hline 45.0 & 5.34 & 5.99 & 6.73 & 7.04 & 6.92 & 6.74 & 6.24 & 6.80 & 7.24 & 6.98 & 6.13 & 5.33 & 2356.6 & 6.5 \\
\hline 50.0 & 5.39 & 5.97 & 6.60 & 6.76 & 6.51 & 6.29 & 5.89 & 6.52 & 7.10 & 6.97 & 6.20 & 5.42 & 2300.0 & 6.3 \\
\hline 60.0 & 5.39 & 5.82 & 6.21 & 6.07 & 5.59 & 5.30 & 5.09 & 5.86 & 6.68 & 6.82 & 6.22 & 5.48 & 2145.2 & 5.9 \\
\hline 70.0 & 5.25 & 5.53 & 5.68 & 5.24 & 4.58 & 9.27 & 4.21 & 5.07 & 6.10 & 6.49 & 6.08 & 5.40 & 1942.4 & 5.3 \\
\hline 80.0 & 4.98 & 5.10 & 5.01 & 4.30 & 3.55 & 3.20 & 3.31 & 4.16 & 5.37 & 6.00 & 5.78 & 5.18 & 1699.9 & 4.7 \\
\hline 90.0 & 4.59 & 4.55 & 4.23 & 3.31 & 2.52 & 2.22 & 2.41 & 3.22 & 4.51 & 5.35 & 5.32 & 4.82 & 1430.1 & 3.9 \\
\hline
\end{tabular}


AVERAGE DAILY TOTAL TERRESTRIAL INSOLATION ESTIMATES (KWH/SQ. M)

SITE : ARCATA

LATITUDE: 40 DEGREES 59 MINUTES

AVERAGE DAILY AMOUNTS BY MONTH

\begin{tabular}{|c|c|c|c|c|c|c|c|c|c|c|c|c|c|c|}
\hline ARRAY & $\mathrm{J}$ & $F$ & $M$ & $A$ & $M$ & $\mathrm{~J}$ & $J$ & A & 5 & 0 & $N$ & D & $\begin{array}{l}\text { ANNUAL } \\
\text { AMOUNT }\end{array}$ & $\begin{array}{c}\text { AVERAGE } \\
\text { DAY }\end{array}$ \\
\hline 0.0 & 1.67 & 2.50 & 3.57 & 5.00 & 5.81 & 6.19 & 5.70 & 4.98 & 4.23 & 2.95 & 1.87 & 1.48 & 1399.8 & 3.8 \\
\hline 10.0 & 1.97 & 2.81 & 3.84 & 5.18 & 5.88 & 6.20 & 5.76 & 5.17 & 4.58 & 3.36 & 2.25 & 1.82 & 1486.8 & 4.1 \\
\hline 15.0 & 2.11 & 2.94 & 3.95 & 5.23 & 5.86 & 6.15 & 5.74 & 5.22 & 4.72 & 3.54 & 2.42 & 1.97 & 1518.3 & 4.2 \\
\hline 20.0 & 2.24 & 3.05 & 4.03 & 5.25 & 5.82 & 6.08 & 5.69 & 5.24 & 4.84 & 3.70 & 2.57 & 2.11 & 1541.3 & 4.2 \\
\hline 25.0 & 2.35 & 3.15 & 4.09 & 5.24 & 5.74 & 5.97 & 5.61 & 5.23 & 4.92 & 3.83 & 2.72 & 2.24 & 1555.6 & 4.3 \\
\hline 30.0 & 2.45 & 3.23 & 4.13 & 5.20 & 5.63 & 5.82 & 5.51 & 5.19 & 4.98 & 3.95 & 2.84 & 2.36 & 1561.3 & 4.3 \\
\hline 35.3 & 2.53 & 3.29 & 4.14 & 5.14 & 5.48 & 5.65 & 5.37 & 5.12 & 5.01 & 4.04 & 2.95 & 2.46 & 1558.1 & 4.3 \\
\hline 40.0 & 2.60 & 3.34 & 4.14 & 5.04 & 5.31 & 5.45 & 5.20 & 5.03 & 5.00 & 4.10 & 3.04 & 2.55 & 1546.2 & 4.2 \\
\hline 45.0 & 2.66 & 3.36 & 4.11 & 4.93 & 5.13 & 5.23 & 5.02 & 4.91 & 4.97 & 4.15 & 3.11 & 2.62 & 1527.1 & 4.2 \\
\hline 50.0 & 2.69 & 3.36 & 4.05 & 4.78. & 4.92 & 4.99 & 4.81 & 4.77 & 4.91 & 4.16 & 3.16 & 2.67 & 1499.8 & 4.1 \\
\hline 60.0 & 2.72 & 3.31 & 3.88 & 4.42 & 4.42 & 4.44 & 4.33 & 4.41 & 4.71 & 4.12 & 3.20 & 2.73 & .1420 .6 & 3.9 \\
\hline 70.0 & 2.68 & 3.19 & 3.62 & 3.97 & 3.84 & 3.80 & 3.76 & 3.95 & 4.39 & 3.98 & 3.17 & 2.72 & 1310.6 & 3.6 \\
\hline 80.0 & 2.58 & 2.99 & 3.28 & 3.44 & 3.22 & 3.15 & 3.15 & 3.43 & 3.98 & 3.74 & 3.05 & 2.64 & 1176.0 & 3.2 \\
\hline 90.0 & 2.41 & 2.73 & 2.88 & 2.85 & 2.58 & 2.47 & 2.53 & 2.84 & 3.48 & 3.42 & 2.86 & 2.50 & 1020.4 & 2.8 \\
\hline
\end{tabular}


AVERAGE DAILY TOTAL TERRESTRIAL INSOLATION ESTIMATES (KWH/SQ. M)

SITE: 34 KERSFIELD

LATITUDE: 35 JEGREES 25 MINUTES

AVERAGE DAILY AMOUNTS BY MONTH

\begin{tabular}{|c|c|c|c|c|c|c|c|c|c|c|c|c|c|c|}
\hline ARRAY & $J$ & $F$ & $M$ & $A$ & $M$ & , & - & trum & $S$ & 0 & $N$ & D & $\begin{array}{l}\text { ANNUAL } \\
\text { AMOUNT }\end{array}$ & $\begin{array}{c}\text { AVERAGE } \\
\text { DAY }\end{array}$ \\
\hline 0.0 & 2.41 & 3.48 & 5.02 & 6.60 & 7.91 & 8.67 & 8.47 & 7.63 & 6.28 & 4.59 & 2.37 & 2.14 & 2016.1 & 5.5 \\
\hline 10.0 & 2.81 & 3.87 & 5.39 & 6.81 & 7.93 & 8.60 & 8.49 & 7.90 & 6.82 & 5.25 & 3.53 & 2.56 & 2130.8 & 5.8 \\
\hline 15.0 & 2.9 .3 & 4.04 & 5.52 & 6.85 & 7.87 & 8.48 & 8.42 & 7.96 & 7.02 & 5.53 & 3.79 & 2.75 & 2169.0 & 5.9 \\
\hline 20.0 & 3.14 & 4.18 & 5.63 & 6.85 & 7.76 & 8.32 & 8.30 & 7.97 & 7.18 & 5.78 & 4.02 & 2.92 & 2193.9 & 6.0 \\
\hline 25.0 & 3.28 & 4.30 & 5.70 & 6.81 & 7.60 & 8.10 & 8.13 & 7.93 & 7.30 & 5.99 & 4.22 & 3.07 & 2205.5 & 6.0 \\
\hline 30.0 & 3.40 & 4.39 & 5.74 & 6.73 & 7.40 & 7.83 & 7.90 & 7.84 & 7.36 & 6.16 & 4.40 & 3.21 & 2203.6 & 6.0 \\
\hline 35.0 & 3.50 & 4.46 & 5.74 & 6.61 & 7.15 & 7.51 & 7.63 & 7.70 & 7.39 & 6.29 & 4.55 & 3.32 & 2188.3 & 6.0 \\
\hline 40.0 & 3.58 & 4.50 & 5.71 & 6.46 & 6.89 & $\bar{i} .18$ & 7.34 & 7.52 & 7.36 & 6.39 & 4.67 & 3.42 & 2162.3 & 5.9 \\
\hline 45.0 & 3.63 & 4.52 & 5.65 & 6,27 & 6.58 & 6.8 .1 & 7.00 & 7.30 & 7.29 & 6.44 & 4.76 & 3.49 & 2123.3 & 5.8 \\
\hline 50.0 & 3.6 .7 & 4.50 & 5.55 & 6.04 & 6.24 & $6 .<0$ & 6.63 & 7.03 & 7.17 & 6.45 & 4.82 & 3.55 & 2071.5 & 5.7 \\
\hline 60.0 & 3.67 & 4.40 & 5.26 & 5.49 & 5.45 & 5.47 & 5.76 & 6.36 & 6.80 & 6.34 & 4.85 & 3.59 & 1931.1 & 5.3 \\
\hline 70.0 & 3.58 & 4.19 & 4.84 & 4.82 & 4.55 & 4.47 & 4.76 & 5.55 & 6.26 & 6.08 & 4.76 & 3.54 & 1746.4 & 4.8 \\
\hline 80.0 & 3.41 & 3.89 & 4.32 & 4.04 & 3.64 & 3.45 & 3.77 & 4.61 & 5.55 & 5.65 & 4.54 & 3.40 & 1529.6 & 4.2 \\
\hline 90.0 & 3.15 & 3.50 & 3.71 & 3.21 & 2.69 & 2.47 & 2.73 & 3.60 & 4.74 & 5.09 & 4.21 & 3.18 & 1285.2 & 3.5 \\
\hline
\end{tabular}


AVERAGE DAILY. TOTAL TERRESTRIAL INSOLATION ESTIMATES (KWH/SQ. M)

SITE: CHINA LAKE

LATITUDE: 35 DEGREES 41 MINUTES

AVERAGE DAILY AMOUNTS BY MONTH

\begin{tabular}{|c|c|c|c|c|c|c|c|c|c|c|c|c|c|c|}
\hline APRAY & $J$ & $F$ & $M$ & A & $M$ & $J$ & $J$ & A & $s$ & 0 & $N$ & D & $\begin{array}{l}\text { ANNUAL } \\
\text { AMOUNT }\end{array}$ & $\begin{array}{c}\text { AVERAGE } \\
\text { DAY }\end{array}$ \\
\hline 0.0 & 2.86 & 3.88 & 5.46 & 7.04 & 8.03 & 8.66 & 8.24 & 8.24 & 6.24 & 4.64 & 5.26 & 2.65 & 2109.0 & 5.8 \\
\hline 10.0 & 3.40 & 4.37 & 5.89 & 7.27 & 3.06 & 8.60 & 8.26 & 8.55 & 6.78 & 5.32 & 5.93 & 3.27 & 2245.0 & 6.2 \\
\hline 15.0 & 3.64 & 4.58 & 6.06 & 7.32 & 8.00 & 8.48 & 8.20 & 8.62 & 6.99 & 5.61 & $c .23$ & 3.54 & 2292.6 & 6.3 \\
\hline 20.0 & 3.86 & 4.76 & 6.18 & 7.33 & 7.89 & 8.32 & 8.08 & 8.63 & 7.15 & 5.87 & 4.51 & 3.80 & 2326.0 & 6.4 \\
\hline 25.0 & 4.05 & 4.91 & 6.27 & 7.29 & 7.73 & 8.10 & 7.92 & 8.59 & 7.26 & 6.08 & 4.76 & 4.03 & 2345.1 & 6.4 \\
\hline 30.0 & 4.22 & 5.03 & 6.32 & 7.21 & 7.53 & 7.844 & 7.71 & 8.50 & 7.34 & 6.26 & 4.97 & 4.24 & 2349.7 & 6.4 \\
\hline 35.0 & 4.37 & 5.12 & 6.34 & 7.08 & 7.28 & 7.53 & 7.45 & 8.35 & 7.36 & 6.40 & 5.16 & 4.41 & 2339.8 & 6.4 \\
\hline 40.0 & 4.48 & 5.18 & 6.31 & 6.92 & 7.01 & 7.19 & 7.17 & 8.16 & 7.34 & 6.50 & 5.31 & 4.56 & 2317.9 & 6.4 \\
\hline 45.0 & 4.57 & 5.21 & 6.25 & 6.72 & 6.70 & 6.83 & 6.85 & 7.92 & 7.27 & 6.56 & 5.43 & 4.68 & 2282.1 & 6.3 \\
\hline 50.0 & 4.62 & 5.20 & 6.14 & 6.48 & 6.35 & 6.42 & 6.48 & 7.63 & 7.15 & 6.57 & 5.51 & 4.77 & 2232.2 & 6.1 \\
\hline 60.0 & 4.65 & 5.10 & 5.83 & 5.88 & 5.55 & 5.50 & 5.65 & 6.91 & 6.79 & 6.47 & 5.56 & 4.86 & 2092.1 & 5.7 \\
\hline 70.0 & 4.56 & 4.87 & 5.37 & 5.16 & 4.63 & 4.49 & 4.70 & 6.03 & 6.25 & 6.20 & 5.46 & 4.82 & 1902.8 & 5.2 \\
\hline 80.0 & 4.35 & 6.53 & 4.80 & 4.32 & 3.70 & 3.48 & 3.74 & 5.01 & 5.56 & 5.77 & 5.23 & 4.65 & 1676.7 & 4.6 \\
\hline 90.0 & 4.04 & 4.07 & 4.11 & 3.41 & 2.73 & 2.49 & 2.73 & 3.91 & 4.74 & 5.20 & 4.85 & 4.36 & 1418.3 & 3.9 \\
\hline
\end{tabular}


AVERAGE DAILY TOTAL TERRESTRIAL INSOLATION ESTIMATES (KWH/SQ. M)

SITE:
LATITUDE : $3+$ DETT

$\begin{aligned} \text { SITE: } & \text { JAGGETT } \\ \text { LATITUDE: } & 3+\text { DEGREES } 52 \text { MINUTES }\end{aligned}$

AVERAGE DAILY AMOUNTS BY MONTH

\begin{tabular}{|c|c|c|c|c|c|c|c|c|c|c|c|c|c|c|}
\hline ARRAY & $\mathrm{J}$ & $F$ & $M$ & $A$ & $M$ & 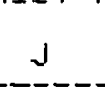 & $\mathrm{J}$ & A & $S$ & 0 & $N$ & $D$ & $\begin{array}{l}\text { ANNUAL } \\
\text { AMOUNT }\end{array}$ & $\begin{array}{l}\text { AVERAGE } \\
\text { DAY }\end{array}$ \\
\hline 0.0 & 3.01 & 4.04 & 5.59 & 7.17 & 8.17 & 8.72 & 8.20 & 7.52 & 6.33 & 4.78 & 3.42 & 2.76 & 2123.3 & 5.8 \\
\hline 10.0 & 3.57 & 4.54 & 6.02 & 7.39 & 8.18 & 8.64 & 8.21 & 7.76 & 6.86 & 5.46 & 4.11 & 3.38 & 2257.6 & 6.2 \\
\hline 15.0 & 3.82 & 4.75 & 6.18 & 7.44 & $8: 11$ & g. 52 & 8.14 & 7.82 & 7.06 & 5.75 & 4.42 & 3.67 & 2304.2 & 6.3 \\
\hline 20.0 & 4.04 & 4.94 & 6.30 & 7.44 & 7.99 & E. 34 & 8.02 & 7.82 & 7.21 & 6.01 & 4.71 & 3.92 & 2336.6 & 6.4 \\
\hline 25.0 & 4.24 & 5.09 & 6.39 & 7.39 & 7.83 & 8.12 & 7.85 & 7.77 & 7.32 & 6.22 & 4.96 & 4.16 & 2354.6 & 6.5 \\
\hline 30.0 & 4.42 & 5.21 & 6.43 & 7.30 & 7.61 & .. 84 & 7.63 & 7.68 & 7.39 & 6.40 & 5.18 & 4.36 & 2358.1 & 6.5 \\
\hline 35.0 & 4.56 & 5.30 & 6.44 & 7.17 & 7.35 & $? .52$ & 7.37 & 7.54 & 7.41 & 6.54 & 5.37 & 4.54 & 2347.0 & 6.4 \\
\hline 40.0 & 4.68 & 5.36 & 6.41 & 7.00 & 7.07 & 7.18 & 7.08 & 7.36 & 7.37 & 6.63 & 5.52 & 4.69 & 2324.2 & 6.4 \\
\hline 45.0 & 4.77 & 5.39 & 6.34 & 6.79 & 6.75 & 5.80 & 6.76 & 7.14 & 7.30 & 6.68 & 5.64 & 4.81 & 2287.0 & 6.3 \\
\hline 50.0 & 4.82 & 5.38 & 6.23 & 6.54 & 6.38 & 5.39 & 6.39 & 6.87 & 7.17 & 6.69 & 5.72 & 4.90 & 2235.8 & 6.1 \\
\hline 60.0 & 4.84 & 5.26 & 5.90 & 5.92 & 5.55 & 5.44 & 5.55 & 6.21 & 6.79 & 6.58 & 5.76 & 4.97 & 2092.9 & 5.7 \\
\hline 70.0 & 4.74 & 5.02 & 5.43 & 5.17 & 4.60 & 4.43 & 4.60 & 5.41 & 6.24 & 6.29 & 5.66 & 4.92 & 1901.4 & 5.2 \\
\hline 80.0 & 4.52 & 4.65 & 6.83 & 4.31 & 3.66 & 3.40 & 3.64 & 4.49 & 5.54 & 5.85 & 5.40 & 4.74 & 1673.0 & 4.6 \\
\hline 90.0 & 4.18 & 4,18 & 4.12 & 3.39 & 2.67 & 2.41 & 2.65 & 3.50 & 4.70 & 5.26 & 5.01 & 4.44 & 1413.8 & 3.9 \\
\hline
\end{tabular}


AVERAGE DAILY TOTAL TERRESTRIAL INSOLATION ESTIMATES (KWH/SP. M)

SITE: FRESNO

LATITUDE: 36 DEGREES 46 MINUTES

AVERAGE DAILY AMOUNTS BY MONTH

\begin{tabular}{|c|c|c|c|c|c|c|c|c|c|c|c|c|c|c|}
\hline ARRAY & $\mathrm{J}$ & $F$ & $M$ & A & $M$ & i & $J$ & A & 5 & 0 & $N$ & $D$ & $\begin{array}{l}\text { ANNUAL } \\
\text { AMOUNT }\end{array}$ & $\begin{array}{l}\text { AVERAGE } \\
\text { DAY }\end{array}$ \\
\hline 0.0 & 2.07 & 3.19 & 4.73 & 6.60 & 7.83 & 8.62 & g. 46 & 7.64 & 6.25 & 4.51 & 2.80 & 1.81 & 1972.2 & 5.4 \\
\hline 10.0 & 2.40 & 3.56 & 5.31 & 6.83 & 7.87 & 2.57 & E. 51 & 7.94 & 6.82 & 5.19 & 3.3 .6 & 2.16 & 2088.0 & 5.7 \\
\hline 15.0 & 2.55 & 3.72 & 5.45 & 6.88 & 7.82 & 8. 47 & 8.46 & 8.01 & 7.05 & 5.48 & 3.51 & 2.32 & 2127.2 & 5.8 \\
\hline 20.0 & 2.68 & 3.85 & 5.57 & 6.89 & 7.72 & 8. . 32 & 8.35 & 8.04 & 7.22 & 5.74 & 3.84 & 2.46 & 2153.4 & 5.9 \\
\hline 25.0 & 2.80 & 3.97 & 5.65 & 6.86 & 7.58 & 8.1I & 8.19 & 8.01 & 7.35 & 5.96 & 4.04 & 2.59 & 2166.5 & 5.9 \\
\hline 30.0 & 2.90 & 4.06 & 5.69 & 6.79 & 7.39 & 7.86 & 7.98 & 7.93 & 7.44 & 6.15 & 4.22 & 2.70 & 2166.3 & 5.9 \\
\hline 35.0 & 2.99 & 4.12 & 5.70 & 6.68 & 7.16 & 7.56 & 7.72 & 7.81 & 7.48 & 6.29 & 4.38 & 2.80 & 2152.9 & 5.9 \\
\hline 40.0 & 3.05 & 4.16 & 5.68 & 6.54 & 6.90 & 7.23 & 7.43 & 7.64 & 7.46 & 6.40 & 4.50 & 2.88 & 2128.2 & 5.8 \\
\hline 45.0 & 3.10 & 4.18 & 5.63 & 6.35 & 6.61 & 6.33 & 7.11 & 7.43 & 7.40 & 6.46 & 4.60 & 2.94 & 2091.7 & 5.7 \\
\hline 50.0 & 3.13 & 4.17 & 5.54 & 6.14 & 6.28 & 6.73 & 5.74 & 7.17 & 7.30 & 6.48 & 4.66 & 2.99 & 2042.4 & 5.6 \\
\hline 60.0 & 3.14 & 4.09 & 5.27 & 5.60 & 5.52 & 5.5 .3 & 5.89 & 6.52 & 6.95 & 6.40 & 4.71 & 3.02 & 1907.6 & 5.2 \\
\hline 70.0 & 3.06 & 3.90 & 4.87 & 4.93 & 4.64 & 4.59 & 4.91 & 5.71 & 6.42 & 6.15 & 4.63 & 2.98 & 1728.5 & 4.7 \\
\hline 80.0 & 2.92 & 3.63 & 4.36 & 4.16 & 3.74 & 3.59 & 3.91 & 4.78 & 5.73 & 5.74 & 4.43 & 2.87 & 1517.2 & 4.2 \\
\hline 90.0 & 2.71 & 3.28 & 3.76 & 3.33 & 2.79 & 2.59 & 2.86 & 3.76 & 4.91 & 5.19 & 4.12 & 2.69 & 1276.9 & 3.5 \\
\hline
\end{tabular}


AVERAGE DAILY TOTAL TERRESTRIAL INSOLATION ESTIMATES ( $K W H / S Q$. M)

SITE: LONG BEACH LATITUDE: 33 DEGREES 49 MINUTES

AVERAGE DAILY AMOUNTS BY MONTH

\begin{tabular}{|c|c|c|c|c|c|c|c|c|c|c|c|c|c|c|}
\hline ARRAY & 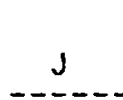 & $\mathbf{F}$ & $M$ & A & $M$ & (1) & 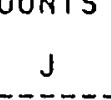 & grom. & $S$ & 0 & $N$ & $\mathrm{D}$ & $\begin{array}{l}\text { ANNUAL } \\
\text { AMOUNT }\end{array}$ & $\begin{array}{c}\text { AVERAGE } \\
\text { DAY }\end{array}$ \\
\hline 0.0 & 2.92 & 3.83 & 5.07 & 6.11 & 6.50 & 6.74 & 7.25 & 6.62 & 5.36 & 4.18 & 3.16 & 2.67 & 1840.3 & 5.0 \\
\hline 10.0 & 3.42 & 4.27 & 5.41 & 6.26 & 6.49 & 6.67 & 7.24 & 6.80 & 5.73 & 4.70 & 3.73 & 3.22 & 1946.9 & 5.3 \\
\hline 15.0 & 3.63 & 4.45 & 5.54 & 6.29 & 6.43 & 6.57 & 7.17 & 6.83 & 5.87 & 4.92 & 3.98 & 3.46 & 1983.3 & 5.4 \\
\hline 20.0 & 3.83 & 4.61 & 5.63 & 6.27 & 6.33 & 6.44 & 7.06 & 6.82 & 5.97 & 5.10 & 4.21 & 3.69 & 2008.1 & 5.5 \\
\hline 25.0 & 4.00 & 4.73 & 5.69 & 6.22 & 6.20 & 6.27 & 6.90 & 6.77 & 6.03 & 5.26 & 4.41 & 3.89 & 2021.1 & 5.5 \\
\hline 30.0 & 4.15 & 4.83 & 5.72 & 6.14 & 6.03 & 6.07 & 6.71 & $6: 67$ & 6.06 & 5.38 & 4.59 & 4.07 & 2022.2 & 5.5 \\
\hline 35.0 & 4.28 & 4.91 & 5.71 & 6.02 & 5.84 & 5.84 & 6.48 & 6.55 & 6.06 & 5.47 & 9.73 & 4.22 & 2011.8 & 5.5 \\
\hline 40.0 & 4.37 & 4.95 & 5.67 & 5.87 & 5.62 & 5.59 & 6.23 & 6.38 & 6.01 & 5.53 & $\dot{4} .85$ & 4.35 & 1991.1 & 5.5 \\
\hline 45.0 & 4.44 & 4.96 & 5.60 & 5.69 & 5.37 & 5.32 & 5.95 & 6.18 & 5.94 & 5.55 & 4.93 & 4.44 & 1958.8 & 5.4 \\
\hline 50.0 & 4.49 & 4.94 & 5.49 & $5.4 i$ & 5.10 & 5.01 & 5.63 & 5.94 & 5.82 & 5.54 & c. 99 & 4.51 & 1914.9 & 5.2 \\
\hline 60.0 & 4.49 & $4.82^{\circ}$ & 5.18 & 4.96 & 4.47 & 4.33 & 4.90 & 5.37 & 5.49 & 5.42 & 5.00 & 4.56 & 1794.4 & 4.9 \\
\hline 70.0 & 4.37 & 4.58 & 4.75 & 4.34 & 3.76 & 3.61 & 4.09 & 4.68 & 5.03 & 5.16 & 4.88 & 4.50 & 1634.9 & 4.5 \\
\hline 80.0 & 4.15 & 4.23 & 4.22 & 3.63 & 3.05 & 2.86 & 3.27 & 3.90 & 4.46 & 4.78 & 4.64 & 4.32 & 1444.4 & 4.0 \\
\hline 90.0 & 3.83 & 3.79 & 3.60 & 2.89 & 2.32 & 2.16 & 2.43 & 3.07 & 3.79 & 4.28 & 4.29 & 4.03 & 1229.9 & 3.4 \\
\hline
\end{tabular}


AVERAGE DAILY TOTAL TERRESTREAL INSOLATION ESTIMATES :KWH/SQ. M)

SITE: LOS ANGELES

LATITUDE $=33$ DEGREES 56 MINUTES

AVERAGE DAILY AMOUNTS BY MONTH

\begin{tabular}{|c|c|c|c|c|c|c|c|c|c|c|c|c|c|c|}
\hline ARRAY & $\mathrm{J}$ & $F$ & $m$ & A & $M$ & $\mathrm{~J}$ & $\mathrm{~J}$ & A & S & 0 & $\mathrm{~N}$ & D & $\begin{array}{l}\text { ANNUAL } \\
\text { AMOUNT }\end{array}$ & $\begin{array}{c}\text { A VERAGE } \\
\text { DAY }\end{array}$ \\
\hline 0.0 & 2.92 & 3.83 & 5.10 & 6.15 & 6.49 & 3.68 & 7.27 & 6.56 & 5.30 & 4.15 & 3.16 & 2.68 & 1836.5 & 5.0 \\
\hline 10.0 & $3.4 i$ & 4.27 & 5.45 & 6.31 & 6.48 & 5.61 & 7.26 & 6.73 & 5.67 & 4.66 & 3.74 & 3.23 & 1943.7 & 5.3 \\
\hline 15.0 & 3.63 & 4.45 & 5.58 & 6.33 & 6.42 & 6.52 & 7.19 & 6.76 & 5.80 & 4.88 & 3.79 & 3.48 & 1980.5 & 5.4 \\
\hline 20.0 & 3.83 & 9.61 & 5.67 & 6.32 & 6.33 & 6.39 & 7.08 & 6.75 & 5.90 & 5.07 & 4.23 & 3.71 & 2005.7 & 5.5 \\
\hline 25.0 & 4.01 & 4.74 & 5.73 & 6.27 & 6.19 & 6.23 & 6.93 & 6.70 & 5.97 & $5.2 ?$ & 4.43 & 3.92 & 2019.0 & 5.5 \\
\hline 30.0 & 4.16 & 4.84 & 5.76 & 6.18 & 6.03 & 6.03 & 6.74 & 6.61 & 5.00 & 5.35 & 4.61 & 4.10 & 2020.5 & 5.5 \\
\hline 35.0 & 4.23 & 4.91 & 5.75 & 6.06 & 5.83 & 5.80 & 6.51 & 6.48 & 5.99 & 5.44 & 4.76 & 4.25 & 2010.5 & 5.5 \\
\hline 40.0 & 4.3 .8 & 4.95 & 5.71 & 5.91 & 5.61 & 5.56 & 6.26 & 6.32 & 5.95 & 5.49 & 4.87 & 4.38 & 1990.3 & 5.5 \\
\hline 45.0 & 4.45 & 4.97 & 5.64 & 5.73 & 5.37 & 5.28 & 5.97 & 6.13 & 5.87 & 5.52 & 4.96 & 4.48 & 1958.3 & 5.4 \\
\hline 50.0 & 4.49 & 4.95 & 5.53 & 5.52 & 5.09 & 4.98 & 5.65 & 5.89 & 5.76 & 5.51 & 5.02 & 4.55 & 1915.0 & 5.2 \\
\hline 60.0 & 4.49 & 4.83 & 5.22 & 5.00 & 4.47 & 4.31 & 4.92 & 5.33 & 5.43 & 5.38 & 5.03 & 4.60 & 1795.3 & 4.9 \\
\hline 70.0 & 4.38 & 4.59 & 4.79 & 4.37 & 3.76 & 3.60 & 4.11 & 4.65 & 4.98 & 5.13 & 4.91 & 4.54 & 1636.5 & 4.5 \\
\hline 80.0 & 4.16 & 4.24 & 4.26 & 3.66 & 3.05 & 2.86 & 3.28 & 3.87 & 4.41 & 4.75 & 4.67 & 4.36 & 1446.7 & 4.0 \\
\hline 90.0 & 3.84 & 3.80 & 3.63 & 2.91 & 2.32 & 2.16 & 2.44 & 3.06 & 3.75 & 4.25 & 4.32 & 4.07 & 1232.6 & 3.4 \\
\hline
\end{tabular}


AVERAGE DA_LY TOTAL TERRESTRIAL INSOLATION ESTIMATES (KWH/SQ. M)

SITE: 1 OUNT SHASTA MAT CA
LATITUDE: 41 DEGREES 19 MINUTES

AVERAGE DAILY AMOUNTS BY MONTH

\begin{tabular}{|c|c|c|c|c|c|c|c|c|c|c|c|c|c|c|}
\hline ARRAY & $\mathrm{J}$ & $F$ & $M$ & A & $M$ & $J$ & $\mathbf{J}$ & A & 5 & 0 & $N$ & D & $\begin{array}{l}\text { ANNUAL } \\
\text { ANOUNT }\end{array}$ & $\begin{array}{c}\text { AVERAGE } \\
\text { DAY }\end{array}$ \\
\hline 0.0 & 1.77 & 2.71 & 3.94 & 5.54 & 6.89 & 7.68 & 8.13 & 6.98 & 5.47 & 3.64 & 2.08 & 1.59 & 1719.7 & 4.7 \\
\hline 10.0 & 2.12 & 3.06 & 4.26 & 5.77 & 6.99 & 7.70 & 8.26 & 7.32 & 6.03 & 4.24 & $z .54$ & 1.98 & 1837.1 & 5.0 \\
\hline 15.0 & 2.27 & 3.22 & 4.39 & 5.83 & 6.98 & 7.65 & 8.25 & 7.42 & 6.26 & 4.51 & 2.75 & 2.16 & 1880.0 & 5.2 \\
\hline $20: 0$ & 2.42 & 3.35 & 4.49 & 5.86 & 6.93 & 7.56 & 8.19 & 7.48 & 6.45 & 4.74 & 2.94 & 2.33 & 1911.6 & 5.2 \\
\hline 25.0 & 2.55 & 3.47 & 4.57 & 5.86 & 6.83 & 7.42 & 8.08 & 7.49 & 6.60 & 4.95 & 3.11 & 2.48 & 1931.9 & 5.3 \\
\hline 30.0 & 2.66 & 3.57 & 4.62 & 5.82 & 6.70 & 7.23 & 7.92 & 7.46 & 6.71 & 5.13 & 3.26 & 2.61 & 1940.6 & 5.3 \\
\hline 35.0 & 2.76 & 3.64 & 4.64 & 5.75 & 6.53 & 7.01 & 7.71 & 7.38 & 6.77 & 5.27 & 3.40 & 2.73 & 1937.6 & 5.3 \\
\hline 40.0 & 2.84 & 3.70 & 4.64 & 5.65 & 6.32 & 6.74 & 7.45 & 7.25 & 6.80 & 5.38 & $3 . .51$ & 2.84 & 1923.0 & 5.3 \\
\hline 45.0 & 2.91 & 3.73 & 4.61 & 5.52 & 6.10 & 6.46 & 7.17 & 7.09 & 6.77 & 5.46 & 3.60 & 2.92 & 1899.0 & 5.2 \\
\hline 50.0 & 2.95 & 3.74 & 4.55 & 5.36 & 5.84 & 6.15 & 6.86 & 6.89 & 6.71 & 5.50 & 3.67 & 2.99 & 1864.4 & 5.1 \\
\hline 60.0 & 2.99 & 3.70 & 4.37 & 4.96 & 5.23 & 5.43 & 6.11 & 6.36 & 6.46 & 5.48 & 3.73 & 3.07 & 1762.3 & 4.8 \\
\hline 70.0 & 2.96 & 3.56 & 4.08 & 4.45 & 4.52 & 4.59 & 5.22 & 5.68 & 6.04 & 5.32 & 3.70 & 3.06 & 1619.2 & 4.4 \\
\hline 80.0 & 2.85 & 3.35 & 3.71 & 3.84 & 3.74 & 3.74 & 4.27 & 4.87 & 5.48 & 5.02 & 357 & 2.98 & 1443.4 & 4.0 \\
\hline 90.0 & 2.67 & 3.06 & 3.25 & 3.17 & 2.95 & 2.85 & 3.29 & 3.95 & 4.79 & 4.59 & 3.36 & 2.83 & 1240.2 & 3.4 \\
\hline
\end{tabular}


AUERAGE DAILY TOTAL TERRESTREAL INSOLATION ESTIMATES (KWH/SQ. M)

\section{SITE:
PEEDLES
CA}

AVERAGE DAILY AMO'JNTS BY MONTH

\begin{tabular}{|c|c|c|c|c|c|c|c|c|c|c|c|c|c|c|}
\hline ARRAY & $J$ & $F$ & $M$ & A & $M$ & $\mathbf{J}$ & $J$ & A & s & 0 & $N$ & D & $\begin{array}{l}\text { ANNUAL } \\
\text { AMOUNT }\end{array}$ & $\begin{array}{l}\text { AVERAGE } \\
\text { DAY }\end{array}$ \\
\hline 0.0 & 3.10 & 4.27 & 5.75 & 7.31 & 8.36 & 3.80 & 3.01 & 7.18 & 6.35 & 4.84 & 3.54 & 2.88 & 2143.5 & 5.9 \\
\hline 10.0 & 3.68 & 4.82 & 6.20 & 7.54 & 8.37 & 8.72 & 8.02 & 7.40 & 6.87 & 5.54 & 4.27 & 3.54 & 2282.5 & 6.3 \\
\hline 15.0 & 3.94 & 5.05 & 6.37 & 7.58 & 8.30 & 8.59 & 7.95 & 7.45 & 7.07 & 5.84 & 4.60 & 3.84 & 2331.2 & 6.4 \\
\hline 20.0 & 4.17 & 5.25 & 6.50 & 7.58 & 8.18 & 8.41 & 7.83 & 7.45 & 7.23 & 6.10 & 4.90 & 4.12 & 2365.5 & 6.5 \\
\hline 25.0 & 4.38 & 5.42 & 6.59 & 7.54 & 8.00. & 8.18 & 7.66 & 7.40 & 7.34 & 6.32 & 5.17 & 4.37 & 2385.2 & 6.5 \\
\hline 30.0 & 4.56 & 5.56 & 6.64 & 7.45 & 7.78 & 7.91 & 7.45 & 7.31 & 7.40 & 6.50 & 5.40 & 4.59 & 2390.1 & 6.5 \\
\hline 35.0 & 4.71 & 5.66 & 6.65 & 7.31 & 7.51 & 7.58 & 7.19 & 7.18 & 7.42 & 6.64 & 5.60 & 4.78 & 2380.3 & 6.5 \\
\hline 40.0 & 4.83 & 5.73 & 6.62 & 7.13 & 7.22 & 7.24 & 6.92 & 7.01 & 7.39 & 6.73 & 5.76 & 4.94 & 2358.4 & 6.5 \\
\hline 45.0 & 4.93 & 5.76 & 6.54 & 6.92 & 6.89 & 6.86 & 6.60 & 6.79 & 7.31 & 6.78 & 5.88 & 5.07 & 2322.0 & 6.4 \\
\hline 50.0 & $4: 99$ & 5.75 & 6.43 & 6.66 & 6.51 & 6.43 & 6.25 & 6.54 & 7.18 & 6.79 & 5.97 & 5.16 & 2271.3 & 6.2 \\
\hline 60.0 & 5.01 & 5.53 & 6.09 & 6.03 & 5.66 & 5.48 & 5.43 & 5.92 & 6.80 & 6.68 & 6.02 & 5.25 & 2128.6 & 5.8 \\
\hline 70.0 & 4.90 & 5.38 & 5.60 & 5.26 & 4.68 & 4.46 & 4.51 & 5.16 & 6.25 & 6.37 & 5.91 & 5.20 & 1936.2 & 5.3 \\
\hline 80.0 & 4.67 & 4.99 & 4.98 & 4.37 & 3.70 & 3.41 & 3.58 & 4.29 & 5.54 & 5.94 & 5.65 & 5.01 & 1706.1 & 4.7 \\
\hline 90.0 & 4.32 & 4.48 & 4.25 & 3.43 & 2.68 & 2.42 & 2.62 & 3.37 & 4.70 & 5.33 & 5.23 & 4.69 & 1444.2 & 4.0 \\
\hline
\end{tabular}


AVERAGE DAILY TOTAL TERRESTRIAL INSOLATION ESTIMATES (KLH/SQ. M)

$\begin{array}{ll}\text { SITE: } & \text { OAKLAND } \\ \text { TTUDE: } & 37 \text { DEGREES } 44 \text { MINUTES }\end{array}$

AVERAGE DAILY AMOUNTS BY MONTH

\begin{tabular}{|c|c|c|c|c|c|c|c|c|c|c|c|c|c|c|}
\hline ARRAY & $\mathrm{J}$ & $F$ & $M$ & A & $M$ & $J$ & $\mathrm{~J}$ & A & $S$ & 0 & $N$ & $D$ & $\begin{array}{l}\text { ANNUAL } \\
\text { AMOUNT }\end{array}$ & $\begin{array}{c}\text { AVERAGE } \\
\text { DAY }\end{array}$ \\
\hline 0.0 & 2.23 & $3.2 i$ & 4.59 & 6.06 & 6.97 & 7.41 & 7.32 & 6.47 & 5.36 & 3.82 & 2.59 & 2.05 & 1769.4 & 4.8 \\
\hline 10.0 & 2.63 & 3.60 & 4.94 & 6.27 & 7.02 & 7.39 & 7.37 & 6.70 & 5.82 & 4.36 & 3.12 & 2.50 & 1879.7 & 5.1 \\
\hline 15.0 & 2.81 & 3.77 & 5.08 & 6.31 & 6.98 & 7.31 & 7.33 & 6.76 & 6.00 & 4.59 & 3.35 & 2.70 & 1918.6 & 5.3 \\
\hline 20.0 & 2.97 & 3.91 & 5.18 & 6.33 & $6.90^{\circ}$ & 7.19 & 7.24 & 6.78 & 6.14 & 4.80 & 3.56 & 2.89 & 1946.1 & 5.3 \\
\hline 25.0 & 3.12 & 4.03 & 5.26 & 6.30 & 6.79 & 7.03 & 7.12 & 6.76 & 6.24 & 4.97 & 3.76 & 3.06 & 1962.2 & 5.4 \\
\hline 30.0 & 3.25 & 4.13 & 5.30 & 6.24 & 6.63 & 6.83 & 6.95 & 6.70 & 6.31 & 5.12 & 3.93 & 3.21 & 1966.7 & 5.4 \\
\hline 35.0 & 3.35 & 4.21 & 5.32 & 6.15 & 6.43 & 6.59 & 6.74 & 6.59 & 6.33 & 5.23 & $c .07$ & 3.35 & 1959.5 & 5.4 \\
\hline 40.0 & 3.44 & 4.26 & 5.30 & 6.02 & 6.21 & 6.32 & 6.50 & 6.46 & 6.32 & 5.31 & $c .19$ & 3.46 & 1941.8 & 5.3 \\
\hline 45.0 & 3.51 & 4.28 & 5.25 & 5.86 & 5.96 & 6.04 & 6.24 & 6.28 & 6.27 & 5.36 & 4.28 & 3.55 & 1913.9 & 5.2 \\
\hline 50.0 & 3.55 & 4.28 & 5.17 & 5.67 & 5.68 & 5.72 & 5.94 & 6.08 & 6.18 & 5.37 & 4.35 & 3.62 & 1874.8 & 5.1 \\
\hline 60.0 & 3.57 & 4.20 & 4.92 & 5.19 & 5.03 & 4.99 & 5.25 & 5.56 & 5.89 & 5.30 & 4.39 & 3.68 & 1764.0 & 4.8 \\
\hline 70.0 & 3.51 & 4.02 & 4.56 & $i .60$ & 4.28 & 4.17 & 4.44 & 4.91 & 5.45 & 5.10 & 4.33 & 3.65 & 1613.2 & 4.4 \\
\hline 80.0 & 3.36 & 3.75 & 4.10 & 3.91 & 3.50 & 3.35 & 3.62 & 4.16 & 4.89 & 4.76 & 4.15 & 3.53 & 1432.3 & 3.9 \\
\hline 90.0 & 3.12 & 3.39 & 3.55 & 3.16 & 2.69 & 2.53 & 2.75 & 3.34 & 4.22 & 4.32 & 3.87 & 3.32 & 1224.1 & 3.4 \\
\hline
\end{tabular}


AVERAGE DAILY TOTAL TERRESTRIAL INSOLATION ESTIMATES (KWH/SQ. M)

SIIE: POINT MJGU
LATITUDE: 34 DEGREES 7 MINUTES

AVERAGE DAILY AMOUNTS BY MONTH

\begin{tabular}{|c|c|c|c|c|c|c|c|c|c|c|c|c|c|c|}
\hline $\begin{array}{l}\text { ARRAY } \\
\text { ARII }\end{array}$ & $\mathrm{J}$ & $F$ & $M$ & A & $M$ & $J$ & $\begin{array}{l}--2 \\
J\end{array}$ & A & $\mathrm{s}$ & 0 & $N$ & $D$ & $\begin{array}{l}\text { ANNUAL } \\
\text { AMOUNT }\end{array}$ & $\begin{array}{c}\text { AVERAGE } \\
\text { DAY }\end{array}$ \\
\hline $0: 0$ & 2.92 & 3.85 & 5.15 & 6.15 & 6.36 & 6.48 & 6.67 & 6.10 & 5.07 & 4.09 & 3.17 & 2.70 & 1787.2 & 4.9 \\
\hline 10.0 & 3.42 & 4.29 & 5.51 & 6.31 & 6.35 & 6.41 & 6.67 & 6.26 & 5.41 & 4.59 & 3.75 & 3.27 & 1894.6 & 5.2 \\
\hline 15.0 & 3.64 & 4.48 & 5.64 & 6.34 & 6.29 & 6.52 & 6.50 & 6.28 & 5.54 & 4.80 & 4.01 & 3.52 & 1932.0 & 5.3 \\
\hline 20.0 & 3.84 & 4.64 & 5.74 & 6.33 & 6.20 & 6.20 & 6.50 & 6.27 & 5.63 & 4.99 & 4.24 & 3.76 & 1958.1 & 5.4 \\
\hline 25.0 & 4.02 & 4.77 & 5.80 & 6.28 & 6.07 & 6.14 & 6.37 & 6.22 & 5.69 & 5.14 & 4.45 & 3.97 & 1972.8 & 5.4 \\
\hline 30.0 & 4.17 & 4.87 & 5.83 & 6.20 & 5.91 & 5.85 & 6.19 & 6.14 & 5.72 & 5.26 & 4.63 & 4.16 & 1976.0 & 5.4 \\
\hline 35.0 & 4.30 & 4.95 & 5.83 & 6.08 & 5.72 & 5.63 & 5.99 & 6.02 & 5.71 & 5.35 & 4.78 & 4.32 & 1967.9 & 5.4 \\
\hline 40.0 & 4.40 & 4.99 & 5.79 & 5.93 & 5.51 & 5.40 & 5.77 & 5.87 & 5.67 & 5.41 & 4.90 & 4.45 & 1949.8 & 5.3 \\
\hline 45.0 & 4.47 & 5.01 & 5.72 & 5.75 & 5.27 & 5.14 & 5.51 & 5.69 & 5.59 & 5.43 & 4.99 & 4.55 & 1920.4 & 5.3 \\
\hline 50.0 & 4.52 & 4.99 & 5.61 & 5.53 & 5.00 & 4.35. & 5.23 & 5.48 & 5.49 & 5.42 & 5.05 & 4.63 & 1879.8 & 5.2 \\
\hline 60.0 & 4.52 & 4.87 & 5.30 & 5.01 & 4.40 & 4.21 & 4.58 & 4.97 & 5.18 & 5.30 & 5.07 & 4.69 & 1766.4 & 4.8 \\
\hline 70.0 & 4.61 & 4.63 & 4.87 & 4.39 & 3.71 & 3.52 & 3.85 & 4.35 & 4.75 & 5.05 & 4.95 & 4.62 & 1614.6 & 4.4 \\
\hline 80.0 & 4.19 & 4.28 & 4.32 & 3.68 & 3.02 & 2.51 & 3.11 & 3.64 & 4.22 & 4.68 & 4.72 & 4.44 & 1432.1 & 3.9 \\
\hline 90.0 & 3.87 & 3.84 & 3.69 & 2.93 & 2.31 & 2.14 & 2.36 & 2.90 & 3.59 & 4.19 & 4.36 & 4.15 & 1225.2 & 3.4 \\
\hline
\end{tabular}


AVERAGE DAILY TOTAL TERRESTRIAL INSOLATION ESTIMATES (KINH/SQ. M)

SITE: RED BLUFF

LATITUDE: 40 DEGREES 9 MINUTES

AVERAGE DAILY AMOUNTS BY MONTH

\begin{tabular}{|c|c|c|c|c|c|c|c|c|c|c|c|c|c|c|}
\hline ARRAY & $J$ & $F$ & $M$ & $A$ & $M$ & $J$ & $J$ & $A$ & 5 & 0 & $\mathbf{N}$ & $\mathrm{D}$ & $\begin{array}{l}\text { ANNUAL } \\
\text { AMOUNT }\end{array}$ & $\begin{array}{c}\text { AUERAGE } \\
\text { DAY }\end{array}$ \\
\hline 0.0 & 1.80 & 2.81 & 4.27 & 6.02 & 7.49 & 8.20 & 8.42 & 7.29 & 5.81 & 3.87 & 2.23 & 1.61 & 1823.9 & 5.0 \\
\hline 10.0 & 2.13 & 3.17 & 4.62 & 6.27 & 7.58 & 8.21 & 8.54 & 7.63 & 6.41 & 4.50 & 2.70 & 1.98 & 1942.7 & 5.3 \\
\hline 15.0 & 2.28 & 3.32 & 4.75 & 6.33 & 7.56 & 8.15 & 8.52 & 7.74 & 6.65 & 4.78 & 2.91 & 2.14 & 1985.1 & 5.4 \\
\hline$\because 0.0$ & 2.41 & 3.46 & 4.86 & 6.36 & 7.50 & 8.03 & 8.44 & 7.79 & 6.84 & 5.02 & 3.11 & 2.30 & 2015.5 & 5.5 \\
\hline 25.0 & 2.53 & 3.57 & 4.95 & 6.36 & 7.39 & 7.87 & 8.32 & 7.79 & 7.00 & 5.24 & 3.29 & 2.44 & 2033.6 & 5.6 \\
\hline 30.0 & 2.64 & 5.67 & 5.00 & 6.31 & 7.24 & 7.66 & 8.14 & 7.75 & 7.10 & 5.42 & 3.45 & 2.56 & 2039.5 & 5.6 \\
\hline 35.0 & 2.73 & 3.74 & 5.02 & 6.23 & 7.04 & 7.41 & 7.91 & 7.66 & 7.17 & 5.56 & 3.58 & 2.67 & 2033.0 & 5.6 \\
\hline 40.0 & 2.80 & 3.79 & 5.02 & 6.11 & 6.80 & 7.11 & 7.64 & 7.52 & 7.19 & 5.68 & 3.69 & 2.76 & 2014.1 & 5.5 \\
\hline 45.0 & 2.86 & 3.82 & 4.98 & 5.97 & 6.55 & 6.81 & 7.34 & 7.34 & 7.16 & 5.75 & 3.78 & 2.84 & 1986.0 & 5.4 \\
\hline 50.0 & 2.90 & 3.83 & 4.92 & 5.79 & 6.26 & 6.46 & 7.00 & 7.12 & 7.08 & 5.79 & 3.85 & 2.90 & 1946.0 & 5.3 \\
\hline 60.0 & 2.92 & 3.77 & 4.71 & 5.33 & 5.57 & 5.66 & 6.20 & 6.55 & 6.80 & 5.75 & 3.91 & 2.96 & 1831.4 & 5.0 \\
\hline 70.0 & 2.88 & 3.63 & 4.39 & 4.76 & 4.77 & 4.74 & 5.26 & 5.82 & 6.34 & 5.57 & 3.87 & 2.95 & 1673.7 & 4.6 \\
\hline 80.0 & 2.77 & 3.40 & 3.97 & 4.09 & 3.91 & 3.82 & 4.26 & $4: 95$ & 5.73 & 5.24 & 3.73 & 2.86 & 1483.3 & 4.1 \\
\hline 94.0 & 2.59 & 3.09 & 3.47 & 3.34 & 3.03 & 2.86 & 3.23 & 3.98 & 4.98 & 4.79 & 3.49 & 2.70 & 1264.0 & 3.5 \\
\hline
\end{tabular}


AV ERAGE DAILY TOTAL TEREESTRIAL INSOLATION ESTIMATES (KWH/SQ. M)

SITE : SACRAMENTO

LATITUDE: $\$ 8$ DEGREES 31 MINUTES

AJERAGE DAZLY AMOUNTS BY MONTH

\begin{tabular}{|c|c|c|c|c|c|c|c|c|c|c|c|c|c|c|}
\hline $\begin{array}{l}\text { ARRAY } \\
\text { TILT }\end{array}$ & J & $F$ & $M$ & A & $M$ & 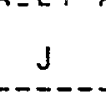 & 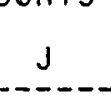 & $A$ & $S$ & 0 & N & $\begin{array}{c}D \\
---\end{array}$ & $\begin{array}{l}\text { ANNUAL } \\
\text { AMOUNT }\end{array}$ & $\begin{array}{c}\text { AVERAGE } \\
\text { DAY }\end{array}$ \\
\hline 0.0 & 1.88 & 2.97 & 4.59 & 6.32 & 7.67 & 3.46 & 3.48 & 7.47 & 6.01 & 4.14 & 2.47 & 1.70 & 1894.6 & 5.2 \\
\hline 10.0 & 2.20 & 3.32 & 4.96 & 6.55 & 7.74 & 3.44 & 3.56 & 7.79 & 6.59 & 4.79 & 2.97 & 2.05 & 2011.1 & 5.5 \\
\hline 15.0 & 2.34 & 3.48 & 5.10 & 6.61 & 7.71 & 3.35 & 3.53 & 7.88 & 6.82 & 5.07 & 3.20 & 2.21 & 2051.4 & 5.6 \\
\hline 20.0 & $2.4 ?$ & 3.61 & 5.21 & 6.64 & 7.63 & 3.23 & 3.44 & 7.92 & 7.01 & 5.32 & 3.41 & 2.36 & 2079.4 & 5.7 \\
\hline 25.0 & 2.58 & 3.72 & 5.29 & 6.62 & $7.50^{\circ}$ & 8.05 & 8.29 & 7.91 & 7.15 & 5.54 & 3.59 & 2.49 & 2094.7 & 5.7 \\
\hline 30.0 & 2.63 & 3.81 & 5.35 & 6.56 & 7.33 & 7.82 & 8.10 & 7.85 & 7.25 & 5.72 & 3.76 & 2.61 & 2097.2 & 5.7 \\
\hline 35.0 & 2.77 & 3.88 & 5.37 & 6.47 & 7.12 & 7.54 & 7.85 & 7.74 & 7.30 & 5.86 & 3.90 & 2.71 & 2087.0 & 5.7 \\
\hline 40.0 & 2.83 & 3.93 & 5.35 & 6.34 & 6.87. & 7.22 & 7.57 & 7.59 & 7.30 & 5.97 & 4.02 & 2.80 & 2065.0 & 5.7 \\
\hline 45.0 & 2.88 & 3.95 & 5.31 & 6.17 & 6.60 & 6.89 & 7.26 & 7.40 & 7.26 & 6.04 & 4.11 & 2.87 & 2032.5 & 5.6 \\
\hline 50.0 & 2.92 & 3.95 & 5.23 & 5.98 & 6.29 & 6.52 & 6.91 & 7.16 & 7.17 & 6.07 & 4.17 & 2.92 & 1987.8 & 5.4 \\
\hline 60.0 & $2.9: 3$ & 3.88 & 4.99 & E. 48 & 5.56 & 5.66 & 6.08 & 6.55 & 6.86 & 6.01 & 4.22 & 2.96 & 1863.0 & 5.1 \\
\hline 70.0 & 2.87 & 3.72 & 4.64 & 4.86 & 4.72 & 4.69 & 5.11 & 5.78 & 6.37 & 5.80 & 4.16 & 2.94 & 1694.5 & 4.6 \\
\hline 80.0 & 2.75 & 3.47 & 4.18 & 4.14 & 3.84 & 3.73 & 4.11 & 4.88 & 5.72 & 5.44 & 4.00 & 2.84 & 1494.1 & 4.1 \\
\hline 90.0 & 2.56 & 3.15 & 3.63 & 3.35 & 2.92 & 2.74 & 3.06 & 3.88 & 4.94 & 4.94 & 3.74 & 2.67 & 1264.6 & 3.5 \\
\hline
\end{tabular}


AVERAGE DAILY TOTAL TERRESTRIAL INSOLATION ESTIMATES (KWH/SQ. M)

SITE: SAN DIEGO

LATITUDE: 32 DEGREES 44 MINUTES

AVE.RAGE DAILY AMOUNTS BY MONTH

\begin{tabular}{|c|c|c|c|c|c|c|c|c|c|c|c|c|c|c|}
\hline ARRAY & $\mathrm{J}$ & $F$ & $M$ & $A$ & $M$ & $J$ & $J$ & A & $S$ & 0 & $N$ & D & $\begin{array}{l}\text { ANNUAL } \\
\text { AMOUNT }\end{array}$ & $\begin{array}{c}\text { AVERAGE } \\
\text { DAY }\end{array}$ \\
\hline 0.0 & 3.07 & 4.00 & 5.14 & $6.1 \mathrm{I}$ & 6.31 & $6: 50$ & 6.89 & 6.48 & 5.41 & 4.33 & 5.35 & 2.85 & 1840.5 & 5.0 \\
\hline 10.0 & 3.58 & 4.44 & 5.47 & 5.24 & 6.29 & 6.42 & 6.86 & 6.64 & 5.77 & 4.85 & 5.94 & 3.42 & 1945.7 & 5.3 \\
\hline 15.0 & 3.80 & 4.62 & 5.59 & 6.26 & 6.22 & 6.32 & 6.79 & 6.66 & 5.90 & 5.07 & C. 19 & 3.67 & 1981.5 & 5.4 \\
\hline 20.0 & 4.00 & 4.78 & 5.68 & 6.24 & 6.12 & 6.19 & 6.67 & 6.64 & 5.99 & 5.25 & 4.43 & 3.90 & 2005.8 & 5.5 \\
\hline 25.0 & 4.18 & 4.91 & 5.73 & 6.18 & 5.99 & 6.02 & 6.52 & 6.58 & 6.05 & 5.41 & 4.64 & 4.11 & 2018.3 & 5.5 \\
\hline 30.0 & 4.33 & 5.01 & 5.75 & 6.09 & 5.82 & 5.83 & 6.33 & 6.48 & 6.07 & 5.53 & 4.81 & 4.29 & 2018.9 & 5.5 \\
\hline 35.0 & 4.45 & 5.08 & 5.74 & 5.97 & 5.63 & 5.60 & 6.12 & 6.35 & 6.06 & 5.62 & 4.96 & 4.45 & 2008.5 & 5.5 \\
\hline 40.0 & 4.54 & 5.11 & 5.69 & 5.81 & 5.41 & 5.36 & 5.88 & 6.18 & 6.01 & 5.67 & 5.08 & 4.58 & 1987.4 & 5.4 \\
\hline 45.0 & 4.61 & 5.12 & 5.61 & 5.62 & 5.17 & 5.09 & 5.60 & 5.98 & 5.92 & 5.68 & 5.16 & 4.67 & 1954.6 & 5.4 \\
\hline 50.0 & 4.65 & 5.10 & 5.49 & 5.40 & 4.90 & 4.80 & 5.30 & 5.75 & 5.80 & 5.67 & 5.21 & 4.74 & 1910.5 & 5.2 \\
\hline 60.0 & 4.64 & 4.96 & 5.17 & 4.88 & 4.28 & 4.14 & 4.61 & 5.18 & 5.46 & 5.53 & 5.21 & 4.79 & 1789.5 & 4.9 \\
\hline 70.0 & 4.51 & 4.70 & 4.73 & 4.26 & 3.60 & 3.45 & 3.85 & 4.50 & 4.98 & 5.25 & 5.08 & 4.71 & 1630.5 & 4.5 \\
\hline 80.0 & 4.27 & 4.33 & 4.19 & 3.55 & 2.91 & 2.73 & 3.08 & 3.73 & 4.40 & 4.85 & 4.82 & 4.51 & 1439.7 & 3.9 \\
\hline 90.0 & 3.93 & 3.87 & 3.55 & 2.81 & 2.22 & 2.06 & 2.30 & 2.93 & 3.72 & 4.33 & 4.43 & 4.19 & 1226.3 & 3.4 \\
\hline
\end{tabular}


AVERAGE DAILY TOTAL TERRESTRIAL INSOLATION ESTIMATES (KWH/SQ. M)

$$
\begin{aligned}
& \text { SITE: WJAV FRANCISCO CAA } \\
& \text { LATITUDE: } 37 \text { DEGREES } 37 \text { MINUTES }
\end{aligned}
$$

AVERAGE DAILY AMOUNTS BY MONTH

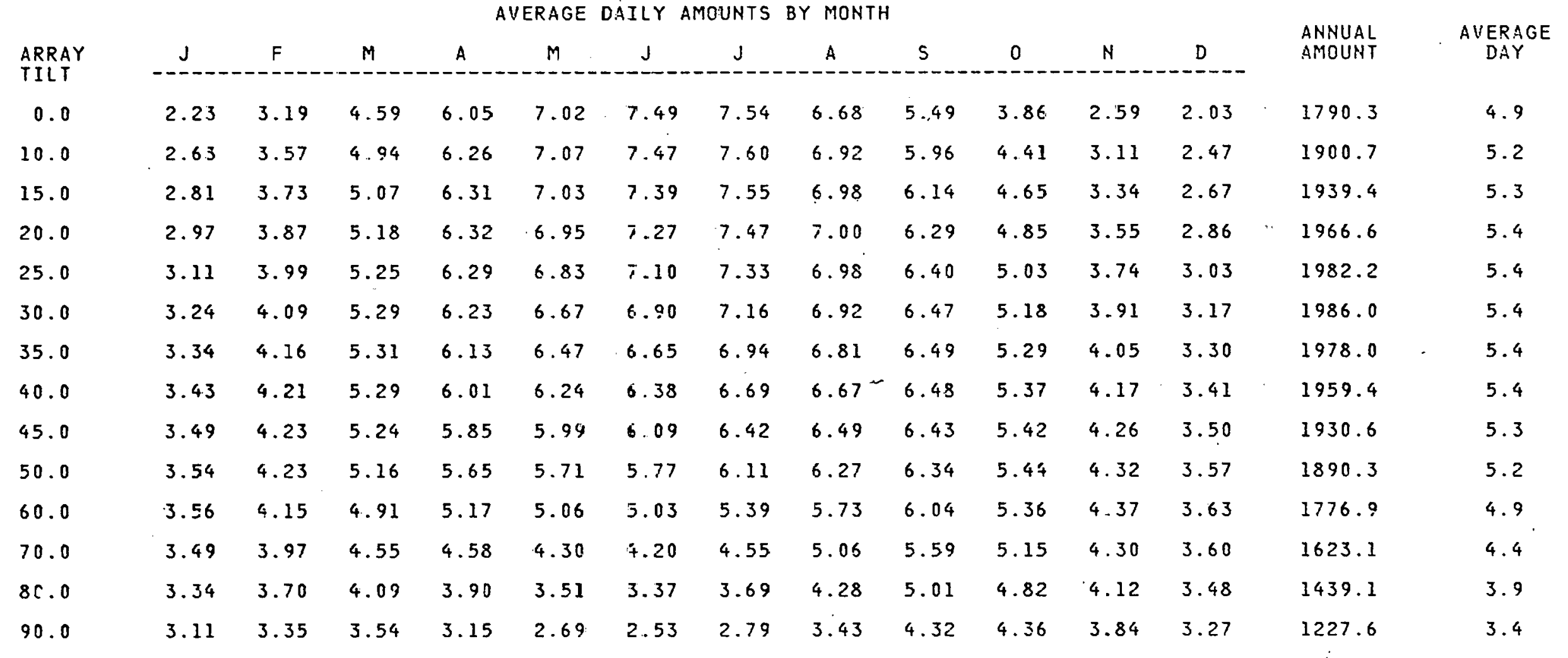


AVERAGE DA ILY TOTAL TÉRRESTRIAL INSOLATION ESTIMATES (KWH/SQ. M)

SITE: SANTA MARIA MA
LATITUDE: 34 DEGREES 54 MINUTES

AVERAGE DAILY AMOUNTS BY MONTH

\begin{tabular}{|c|c|c|c|c|c|c|c|c|c|c|c|c|c|c|}
\hline ARRAY & $\mathrm{J}$ & $F$ & $M$ & A & $M$ & $\mathrm{~J}$ & $\mathrm{~J}$ & A & ș & 0 & $N$ & D & $\begin{array}{l}\text { ANNUAL } \\
\text { ANOUNT }\end{array}$ & $\begin{array}{l}\text { AVERAGE } \\
\text { DAY }\end{array}$ \\
\hline 0.0 & 2.69 & 3.60 & 4.98 & 6.06 & 6.74 & 7.41 & 7.38 & 6.64 & 5.45 & 4.27 & 3.06 & 2.54 & 1852.3 & 5.1 \\
\hline 10.0 & 3.14 & 4.01 & 5.33 & 6.23 & 6.74 & 7.34 & 7.38 & 6.84 & 5.86 & 4.83 & 3.64. & 3.07 & 1962.0 & 5.4 \\
\hline 15.0 & 3.34 & $4.18:$ & 5.46 & 6.26 & 6.69 & 7.24 & 7.32 & 6.88 & 6.01 & 5.07 & 3.90 & 3.32 & 1999.7 & 5.5 \\
\hline 20.0 & 3.53 & 4.33 & 5.56 & 6.25 & 6.59 & 7.10 & 7.21 & 6.88 & 6.12 & 5.28 & 4.13 & 3.54 & 2025.7 & 5.5 \\
\hline 25.0 & 3.69 & 4.45 & 5.63 & 6.25 & 6.46 & 6.92 & 7.06 & 6.83 & 6.20 & 5.46 & $\dot{4} .34$ & 3.74 & 2039.7 & 5.6 \\
\hline 30.0 & -3.83 & 4.55 & 5.66 & 6.13 & 6.29 & 6.70 & 6.87 & 6.75 & 6.24 & 5.60 & i. 52 & 3.92 & 2041.6 & 5.6 \\
\hline 35.0 & 3.94 & 4.62 & 5.66 & 6.02 & 6.09 & 6.44 & 6.64 & 6.62 & 6.25 & 5.71 & 9.68 & 4.07 & 2031.4 & 5.6 \\
\hline 40.0 & 4.03 & 4.66 & 5.63 & 5.87 & 5.87 & 6.17 & 6.39 & 6.47 & 6.21 & 5.78 & 4.80 & 4.20 & 2011.4 & 5.5 \\
\hline 45.0 & 4.10 & 4.67 & 5.56 & 5.70 & 5.62 & 5.86 & 6.11 & 6.27 & 6.14 & 5.82 & 9.89 & 4.30 & 1979.5 & 5.4 \\
\hline 50.0 & 4.14 & 4.56 & 5.46 & 5.49 & 5.33 & 5.53 & 5.79 & 6.04 & 6.03 & 5.81 & 7. 95 & 4.37 & 1935.9 & 5.3 \\
\hline 60.0 & 4.15 & 4.55 & 5.17 & 4.99 & 4.69 & 4.77 & 5.06 & 5.48 & 5.70 & 5.70 & 9.98 & 4.43 & 1815.3 & 5.0 \\
\hline 70.0 & 4.05 & 4.33 & 4.75 & 4.38 & 3.95 & 3.95 & 4.23 & 4.79 & 5.24 & 5.45 & 4.88 & 4.38 & 1654.3 & 4.5 \\
\hline 80.0 & 3.85 & 4.01 & 4.24 & 3.69 & 3.21 & 3.12 & 3.40 & 4.01 & 4.66 & 5.06 & 9.65 & 4.21 & 1462.7 & 4.0 \\
\hline 90.0 & 3.56 & 3.60 & 3.63 & 2.95 & 2.43 & 2.32 & 2.53 & 3.17 & 3.97 & 4.55 & 4.31 & 3.94 & 1245.3 & 3.4 \\
\hline
\end{tabular}


AVERAGE DAILY TOTAL TEFFESTRIAL INSOLATION ESTIMATES (KWH/SQ. M)

$\begin{aligned} \text { SITE: } & \text { SUNNYVELE } \\ \text { LATITUDE: } & 3 \% \text { DEGR.EES } 25 \text { MINUTES }\end{aligned}$

AVERAGE DAILY AMOUNTS BY MONTH

\begin{tabular}{|c|c|c|c|c|c|c|c|c|c|c|c|c|c|c|}
\hline ARRAY & J & $F$ & $y$ & A & $M$ & 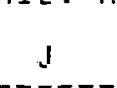 & J & A & $s$ & 0 & N & D & $\begin{array}{l}\text { ANNUAL } \\
\text { AMOUNT }\end{array}$ & $\begin{array}{l}\text { AVERAGE } \\
\text { DAY }\end{array}$ \\
\hline 0.0 & 2.32 & 3.27 & 4.67 & 6.13 & 7.18 & 7.74 & 7.69 & 6.83 & 5.54 & 3.93 & 2.66 & 2.08 & 1830.0 & 5.0 \\
\hline 10.0 & 2.74 & 3.67 & 5.03 & 6.34 & 7.22 & 7.21 & 7.74 & 7.09 & 6.02 & 4.49 & 3.19 & 2.54 & 1943.1 & 5.3 \\
\hline 15.0 & 2.93 & 3.84 & 5.17 & 6.38 & 7.18 & 7.62 & 7.70 & 7.15 & 6.21 & 4.73 & 3.43 & 2.75 & 1982.7 & 5.4 \\
\hline 20.0 & 3.10 & 3.98 & 5.28 & 6.40 & 7.10 & 7.29 & 7.61 & 7.17 & 6.35 & 4.94 & 3.65 & 2.94 & 2010.5 & 5.5 \\
\hline 25.0 & 3.25 & 4.11 & 5.35 & 6.37 & 6.98 & 7.32 & 7.47 & 7.15 & 6.46 & 5.12 & 3.84 & 3.11 & 2026.4 & 5.6 \\
\hline 30.0 & 3.39 & 4.21 & 5.40 & 6.31 & 6.81 & 7.11 & 7.29 & 7.08 & 6.53 & 5.27 & 4.01 & 3.26 & 2030.1 & 5.6 \\
\hline 35.0 & 3.50 & 4.28 & 5.41 & 6.21 & 6.61 & 6.85 & 7.06 & 6.97 & 6.55 & 5.39 & 4.16 & 3.40 & 2021.7 & 5.5 \\
\hline 40.0 & 3.59 & 4.33 & 5.39 & 6.08 & 6.37 & 6.57 & 6.81 & 6.82 & 6.54 & 5.47 & 4.28 & 3.51 & 2002.5 & 5.5 \\
\hline 45.0 & 3.66 & 4.35 & 5.34 & 5.91 & 6.12 & 6.27 & 6.53 & 6.64 & 6.49 & 5.52 & 4.38 & 3.60 & 1972.7 & 5.4 \\
\hline 50.0 & 3.70 & 4.35 & 5.26 & 5.72 & 5.82 & 5.93 & 6.21 & 6.42 & 6.39 & 5.54 & 4.44 & 3.66 & 1931.1 & 5.3 \\
\hline 60.0 & 3.73 & 4.27 & 5.01 & 5.23 & 5.15 & $5 . \pm 5$ & 5.47 & 5.86 & 6.09 & 5.46 & 4.49 & 3.73 & 1814.3 & 5.0 \\
\hline 70.0 & 3.66 & 4.39 & 4.64 & 4.63 & 4.36 & 4.29 & 4.61 & 5.16 & 5.64 & 5.24 & 4.42 & 3.70 & 1656.1 & 4.5 \\
\hline 80.0 & 3.50 & 3.81 & 4.16 & 3.93 & 3.56 & 3.42 & 3.73 & 4.36 & 5.05 & 4.90 & 4.23 & 3.57 & 1466.9 & 4.0 \\
\hline 90.0 & 3.25 & 3.44 & 3.60 & 3.17 & 2.71 & 2.55 & 2.80 & 3.48 & 4.35 & 4.44 & 3.94 & 3.36 & 1249.6 & 3.4 \\
\hline
\end{tabular}


AVERAGE DAELY TOTAL TERRESTRIAL INSOLATION ESTIMATES (KWH/SQ. M)

SITE: COLORADO SPRINGS CO

LATITUDE: 38 DEGREES 49 MINUTES

AVERAGE DAILY AMOUNTS BY MONTH

\begin{tabular}{|c|c|c|c|c|c|c|c|c|c|c|c|c|c|c|}
\hline $\begin{array}{l}\text { ARRAY } \\
\text { TILT }\end{array}$ & J & $F$ & $M$ & A & $M$ & 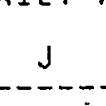 & ---1 & A & $s$ & 0 & $N$ & $\mathrm{D}$ & $\begin{array}{l}\text { ANNUAL } \\
\text { AMOUNT }\end{array}$ & $\begin{array}{c}\text { AVERAGE } \\
\text { DAY }\end{array}$ \\
\hline 0.0 & 2.81 & 3.72 & 4.88 & 6.09 & 6.71 & 7.47 & 6.97 & 6.39 & 5.54 & 4.28 & 2.98 & 2.47 & 1336.4 & 5.0 \\
\hline 10.0 & 3.45 & 4.25 & 5.29 & 6.32 & 6.77 & 7.46 & 7.03 & 6.64 & 6.05 & 4.98 & \pm .69 & 3.14 & 1980.9 & 5.4 \\
\hline 15.0 & 3.73 & 4.48 & 5.46 & 6.38 & 6.74 & 7.39 & 7.00 & 6.70 & 6.25 & 5.28 & 4.02 & 3.45 & 2035.8 & 5.6 \\
\hline 20.0 & 4.00 & 4.69 & 5.59 & 6.40 & 6.67 & 7.28 & 6.93 & 6.73 & 6.42 & 5.55 & 4.31 & 3.74 & 2078.5 & 5.7 \\
\hline 25.0 & 4.24 & 4.86 & 5.68 & 6.38 & 6.56 & 7.12 & 6.82 & 6.72 & 6.54 & 5.79 & 4.58 & 4.00 & 2108.7 & 5.8 \\
\hline 30.0 & 4.45 & 5.01 & 5.74 & 6.33 & 6.42 & 6.93 & 6.67 & 6.66 & 6.62 & 5.98 & 4.82 & 4.24 & 2126.1 & 5.8 \\
\hline 35.0 & 4.63 & 5.12 & 5.77 & 6.24 & 6.24 & 6.69 & 6.48 & 6.57 & 6.66 & 6.14 & 5.03 & 4.45 & 2130.6 & 5.8 \\
\hline 40.0 & 4.78 & 5.21 & 5.76 & 6.11 & 6.03 & 6.42 & 6.26 & 6.44 & 6.66 & 6.26 & 5.20 & 4.63 & 2122.8 & 5.8 \\
\hline 45.0 & 4.91 & 5.26 & 5.72 & 5.96 & 5.80 & 6.14 & 6.01 & 6.27 & 6.62 & 6.34 & 5.34 & 4.77 & 2103.9 & 5.8 \\
\hline 50.0 & 5.00 & 5.27 & 5.65 & 5.77 & 5.54 & 5.83 & 5.74 & 6.07 & 6.54 & 6.38 & 5.45 & 4.89 & 2072.3 & 5.7 \\
\hline 60.0 & 5.08 & 5.21 & 5.40 & 5.30 & 4.93 & 5.10 & 5.10 & 5.57 & 6.25 & 6.33 & 5.55 & 5.03 & 1971.9 & 5.4 \\
\hline $7 n .0$ & 5.03 & 5.02 & 5.02 & 4.71 & 4.22 & 4.28 & 4.35 & 4.94 & 5.81 & 6.11 & 5.50 & 5.03 & 1824.9 & 5.0 \\
\hline 80.0 & 4.84 & 4.70 & 4.52 & 4.02 & 3.48 & 3.46 & 3.57 & 4.21 & 5.22 & 5.74 & 5.31 & 4.89 & 1640.8 & 4.5 \\
\hline 90.0 & 4.53 & 6.27 & 3.93 & 3.26 & 2.71 & 2.62 & 2.76 & 3.40 & 4.52 & 5.22 & 4.97 & 4.63 & 1422.6 & 3.9 \\
\hline
\end{tabular}


AVERAGE DAILY TOTAL TERRESTRIAL INSOLATION ESTIMATES (KWH/SQ. M)

SITIE: DENVER
LATITUDE: 39 DEGREES 45 MINUTES

AVERAGE DAILY AMGUNTS BY MONTH

\begin{tabular}{|c|c|c|c|c|c|c|c|c|c|c|c|c|c|c|}
\hline ARRAY & $J$ & $F$ & $M$ & A & $M$ & 1 & $J$ & A & 5 & 0 & $\kappa$ & $D$ & $\begin{array}{l}\text { ANNUAL } \\
\text { AMOUNT }\end{array}$ & $\begin{array}{c}\text { AVERAGE } \\
\text { DAY }\end{array}$ \\
\hline 0.0 & 2.65 & 3.55 & 4.82 & 5.93 & 6.73 & 7.41 & 7.16 & 6.45 & 5.44 & 4.10 & 2.79 & 2.31 & 1807.2 & 5.0 \\
\hline 10.0 & 3.27 & 4.07 & 5.25 & 6.16 & 6.80 & 7.42 & 7.24 & 6.71 & 5.96 & 4.73 & 3.47 & 2.95 & 1950.9 & 5.3 \\
\hline 15.0 & 3.54 & 4.30 & 5.42 & 6.22 & 6.77 & 7.35 & 7.21 & 6.79 & 6.16 & 5.08 & 3.78 & 3.25 & 2005.7 & 5.5 \\
\hline 20.0 & 3.80 & 4.50 & 5.55 & 6.25 & 6.71 & 7.25 & 7.15 & 6.82 & 6.33 & 5.34 & 4.06 & 3.52 & 2048.5 & 5.6 \\
\hline 25.0 & 4.03 & 4.67 & 5.66 & 6.23 & 6.61 & 7.11 & 7.04 & 6.82 & 6.46 & 5.57 & 4.32 & 3.78 & 2078.9 & 5.7 \\
\hline 30.0 & 4.24 & 4.82 & 5.73 & 6.19 & 6.47 & 6.92 & 6.89 & 6.77 & 6.55 & 5.77 & 4.55 & 4.00 & 2096.8 & 5.7 \\
\hline 35.0 & 4.42 & 4.93 & 5.76 & 6.11 & 6.30 & 6.69 & 6.70 & 6.68 & 6.60 & 5.93 & 4.75 & 4.21 & 2102.0 & .5 .8 \\
\hline 40.0 & 4.57 & 5.01 & 5.76 & 5.99 & 6.09 & 6.43 & 6.47 & 6.56 & 6.60 & 6.05 & 4.92 & 4.38 & 2094.6 & 5.7 \\
\hline 45.0 & 4.69 & 5.07 & 5.72 & 5.84 & 5.86 & 6.15 & 6.23 & 6.40 & 6.57 & 6.13 & 5.05 & 4.53 & 2076.8 & 5.7 \\
\hline 50.0 & 4.78 & 5.09 & 5.65 & 5.67 & 5.61 & 5.85 & 5.95 & 6.20 & 6.49 & 6.17 & 5.16 & 4.64 & 2046.4 & 5.6 \\
\hline 60.0 & 4.37 & 5.03 & 5.41 & 5.21 & 5.00 & 5.14 & 5.29 & 5.70 & 6.22 & 6.13 & 5.26 & 4.78 & 1949.0 & 5.3 \\
\hline 70.0 & 4.33 & 4.86 & 5.05 & 4.65 & 4.30 & 4.33 & 4.53 & 5.07 & 5.80 & 5.94 & 5.22 & 4.79 & 1805.4 & 4.9 \\
\hline 80.0 & 4.56 & 4.56 & 4.56 & 3.99 & 3.55 & 3.52 & $3: 72$ & 4.34 & 5.23 & 5.59 & 5.05 & 4.67 & 1624.8 & 4.5 \\
\hline 30.0 & 4.37 & 4.15 & 3.97 & 3.25 & 2.78 & 2.67 & 2.88 & 3.51 & 4.54 & 5.09 & 4.74 & 4.42 & 1410.5 & 3.9 \\
\hline
\end{tabular}


AVERAGE DFILY TOTAL TERRESTRIAL INSOLATION ESTIMATES (KHH/SQ. M)

SITE: EAGLE
ITUDE: 39 DEGREES 39 MINUTES

AVERAGE DAILY AMOUNTS BY MONTH

\begin{tabular}{|c|c|c|c|c|c|c|c|c|c|c|c|c|c|c|}
\hline ARPAY & $\mathrm{J}$ & $F$ & $M$ & A & $M$ & " & - & 110m & s & 0 & $N$ & D & $\begin{array}{l}\text { ANNUAL } \\
\text { AMOUNT }\end{array}$ & $\begin{array}{c}\text { AVERAGE } \\
\text { DAY }\end{array}$ \\
\hline 0.0 & 2.38 & 3.413 & 4.73 & 6.09 & 7.11 & 7.90 & 7.52 & 6.57 & 5.57 & 4.12 & 2.74 & 2.18 & 1837.5 & 5.0 \\
\hline 10.0 & 2.89 & 3.88 & 5.14 & 6.33 & 7.19 & 7.91 & 7.61 & 6.85 & 6.11 & 4.80 & 3.40 & 2.76 & 1975.2 & 5.4 \\
\hline 15.0 & 3.12 & 4.07 & 5.30 & 6.40 & 7.16 & 7.84 & 7.58 & 6.92 & 6.32 & 5.10 & 3.69 & 3.03 & 2026.7 & 5.6 \\
\hline 20.0 & 3.33 & 4.27 & 5.43 & 6.42 & 7.10 & 7.73 & 7.51 & 6.96 & 6.49 & 5.37 & 3.96 & 3.28 & 2066.1 & 5.7 \\
\hline 25.0 & 3.52 & $4.4 \overline{3}$ & 5.53 & 6.41 & 6.99 & $7.5 ?$ & 7.39 & 6.95 & 6.63 & 5.60 & 7.21 & 3.50 & 2092.9 & 5.7 \\
\hline 30.0 & 3.69 & $4.5 \epsilon$ & 5.60 & 6.37 & 6.84 & 7.37 & 7.23 & 6.90 & 6.72 & 5.80 & 4.43 & 3.71 & 2107.1 & 5.8 \\
\hline 35.0 & 3.83 & 4.66 & 5.63 & 6.28 & 6.66 & 7.12 & 7.03 & 6.81 & 6.77 & 5.96 & 4.62 & 3.89 & 2108.5 & 5.8 \\
\hline 40.0 & 3.95 & 4.74 & 5.63 & 6.16 & 6.43 & 6.84 & 6.79 & 6.68 & 6.78 & 6.08 & 4.79 & 4.05 & 2097.2 & 5.7 \\
\hline 45.0 & 4.05 & 4.78 & 5.59 & 6.01 & 6.19 & 6.54 & 6.53 & 6.52 & 6.74 & 6.16 & 4.92 & 4.17 & 2075.8 & 5.7 \\
\hline 50.0 & 4.12 & $4.80^{\circ}$ & 5.52 & 5.85 & 5.91 & 6.20 & 6.23 & 6.32 & 6.66 & 6.20 & 5.01 & 4.28 & 2041.7 & 5.6 \\
\hline 60.0 & 4.18 & 4.74 & 5.28 & 5.36 & 5.27 & 5.44 & 5.54 & 5.81 & 6.39 & 6.16 & 5.11 & 4.39 & 1937.1 & 5.3 \\
\hline 70.0 & 4.13 & 4.57 & 4.92 & 4.78 & 4.51 & 4.55 & 4.72 & 5.17 & 5.95 & 5.96 & 5. .07 & 4.40 & 1786.6 & 4.9 \\
\hline 80.0 & 3.98 & 4.28 & 4.45 & 4.09 & 3.71 & 3.68 & 3.86 & 4.41 & 5.37 & 5.61 & 4.90 & 4.28 & 1600.2 & 4.4 \\
\hline 90.0 & 3.73 & 3.90 & 3.87 & 3.33 & 2.88 & 2.76 & 2.97 & 3.56 & 4.66 & 5.11 & 4.59 & 4.05 & 1381.0 & 3.8 \\
\hline
\end{tabular}


AVERAGE DAILY TOTAL TERRESTRIAL INSOLATION ESTIMATES (KWH/SQ. M)

SITE: GRAND JUNCTION CO
LATITUDE: 39 DEGREES 7 MINUTES

F.VERAGE DAILY AMOUNTS BY MONTH

\begin{tabular}{|c|c|c|c|c|c|c|c|c|c|c|c|c|c|c|}
\hline ARRAY & 7 & $F$ & $M$ & $A$ & $M$ & 2 & $J$ & $A$ & $s$ & 0 & $N$ & D & $\begin{array}{l}\text { ANNUAL } \\
\text { AMOUNT }\end{array}$ & $\begin{array}{c}\text { AVERAGE } \\
\text { DAY }\end{array}$ \\
\hline 0.0 & 2.50 & 3.52 & 4.89 & 6.26 & 7.50 & 8.19 & 7.77 & 6.88 & 5.78 & 4.24 & 2.90 & 2.31 & 1911.7 & 5.2 \\
\hline 10.0 & $3 . c .3$ & 4.02 & 5.31 & 6.51 & 7.58 & 8.19 & 7.85 & 7.17 & 6.34 & 4.93 & 3.59 & 2.92 & 2054.4 & 5.6 \\
\hline 15.0 & 3.27 & 4.24 & 5.48 & 6.57 & 7.55 & 8.12 & 7.82 & 7.25 & 6.56 & 5.24 & 3.90 & 3.21 & 2107.5 & 5.8 \\
\hline 20.0 & 3.49 & 4.43 & 5.61 & 6.60 & 7.47 & 7.99 & 7.75 & 7.29 & 6.74 & 5.51 & 4.19 & 3.47 & 2147.8 & 5.9 \\
\hline 25.0 & 3.69 & 4.59 & 5.71 & 6.58 & 7.36 & 7.82 & 7.62 & 7.28 & 6.88 & 5.74 & 4.46 & 3.71 & 2175.0 & 6.0 \\
\hline 30.0 & 3.86 & 4.72 & 5.78 & 6.53 & 7.19 & 7.61 & 7.45 & 7.22 & 6.97 & 5.94 & 4.69 & 3.92 & 2189.0 & 6.0 \\
\hline 35.0 & 4.02 & 4.83 & 5.81 & 6.44 & 6.99 & 7.35 & 7.24 & 7.13 & 7.02 & 6.10 & 4.89 & 4.11 & 2189.5 & 6.0 \\
\hline 40.0 & 4.14 & 4.91 & 5.80 & 6.32 & 6.75 & 7.05 & 6.99 & 6.99 & 7.03 & 6.22 & 5.06 & 4.28 & 2177.1 & 6.0 \\
\hline 45.0 & 4.24 & 4.95 & 5.77 & 6.16 & 6.49 & 6.73 & 6.71 & 6.81 & 6.99 & 6.30 & 5.20 & 4.41 & 2153.8 & 5.9 \\
\hline 50.0 & 4.31 & 4.97 & 5.69 & 5.97 & 6.19 & 6.38 & 6.40 & 6.60 & 6.91 & 6.34 & 5.30 & 4.52 & 2117.3 & 5.8 \\
\hline 60.0 & 4.38 & 4.90 & 5.44 & 5.48 & 5.50 & 5.57 & 5.67 & 6.05 & 6.61 & 6.29 & 5.40 & 4.64 & 2006.0 & 5.5 \\
\hline 70.0 & 4.32 & 4.72 & 5.06 & 4.87 & 4.68 & 4.64 & $4.8 i$ & 5.37 & 6.15 & 6.08 & 5.36 & 4.64 & 1846.9 & 5.1 \\
\hline 80.0 & 4.16 & 4.42 & 4.57 & 4.16 & 3.82 & 3.72 & 3.92 & 4.56 & 5.54 & 5.72 & 5.17 & 4.51 & 1650.8 & 4.5 \\
\hline 90.0 & 3.89 & 4.02 & 3.97 & 3.37 & 2.93 & 2.77 & 2.98 & 3.67 & 4.79 & 5.20 & 4.85 & 4.27 & 1420.6 & 3.9 \\
\hline
\end{tabular}


AVERAGE DAILY TOTAL TERRESTRIAL INSOLATION ESTIMATES (KWH/SQ. M)

$\begin{aligned} \text { SITE: } & \text { PUEBLO CO } \\ \text { LATITUDE: } & 38 \text { DEGREES } 17 \text { MINUTES }\end{aligned}$

AVERAGE DAILY AMOUNTS BY MONTH

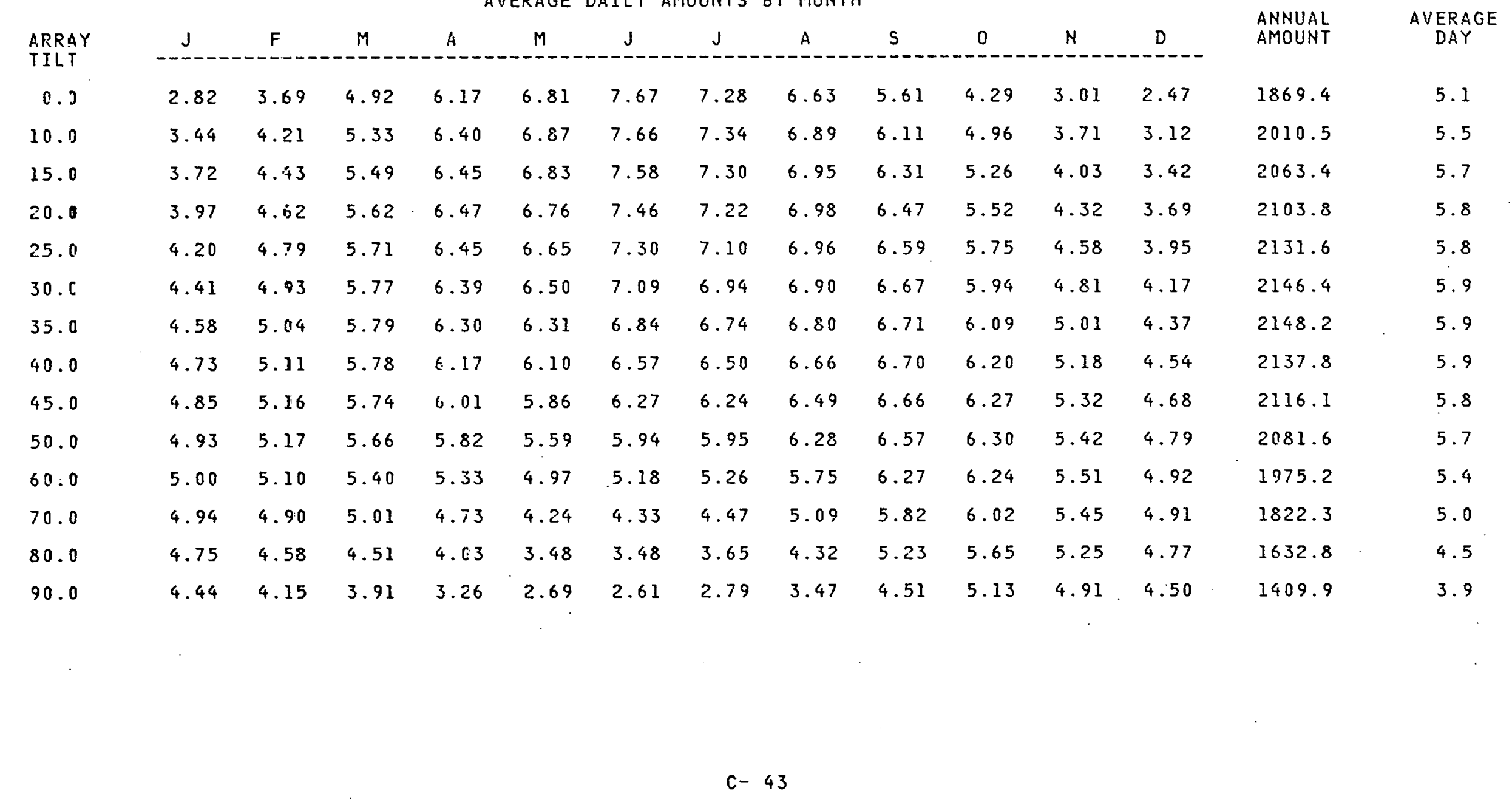


AVERAGE DAILY TOTAL TERRESTRIAL INSOLATION ESTIMATES (KHH/SQ. M)

SITE: HARTFORD

LATITUDE: 41 DEGREES 56 MINUTES

AVERAGE DAILY AMOUNTS BY MONTH

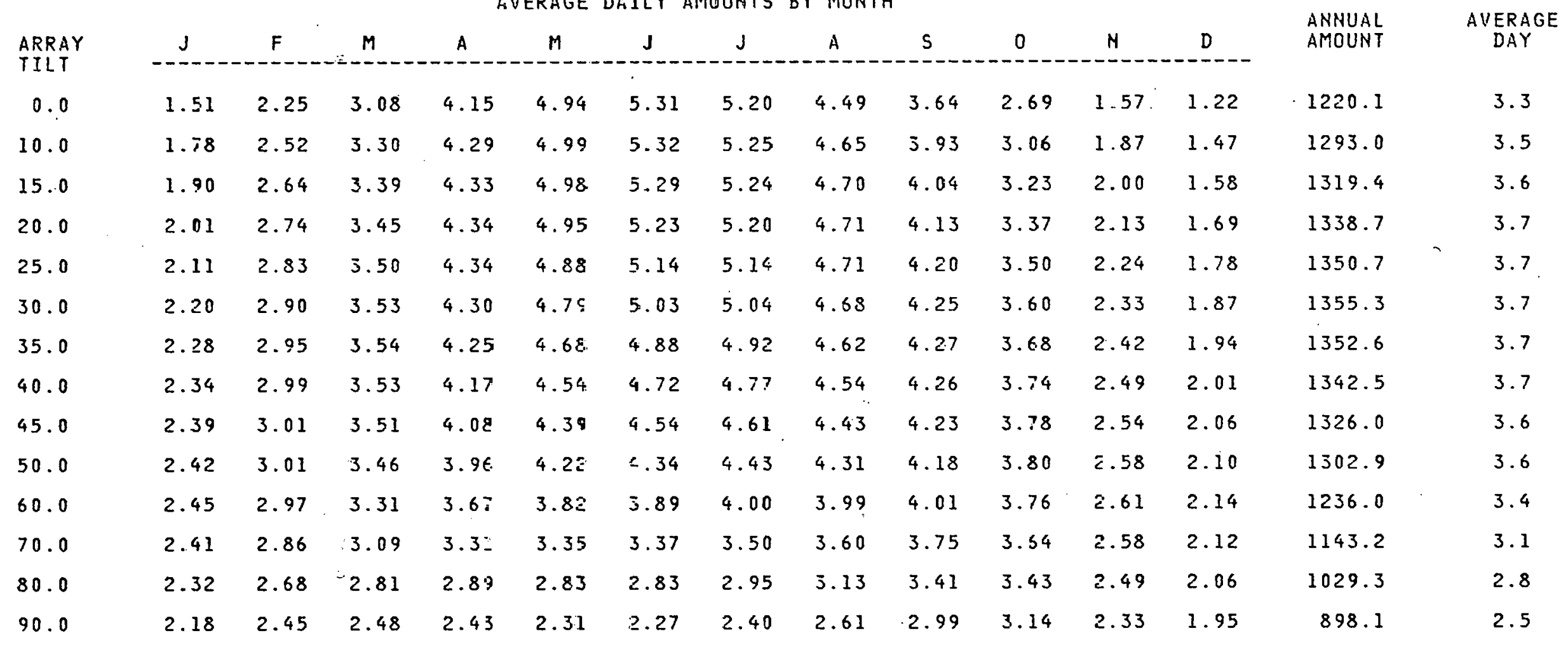


AVERAGE DAILY TOTAL TERRESTRIAL INSOLATION ESTIMATES (KLH/SQ. M)

SITE: WASHINGTON-STERLINDC

LATITUDE: 38 DEGREES 57 MINUTES

AVERAGE DAILY AMOUNTS BY MONTH

\begin{tabular}{|c|c|c|c|c|c|c|c|c|c|c|c|c|c|c|}
\hline ARRAY & $\mathrm{J}$ & $F$ & $M$ & $A$ & $M$ & $\mathrm{~J}$ & $J$ & A & $\mathrm{s}$ & 0 & $N$ & $\mathrm{D}$ & $\begin{array}{l}\text { ANNUAL } \\
\text { AMOUNT }\end{array}$ & $\begin{array}{c}\text { AUERAGE } \\
\text { DAY }\end{array}$ \\
\hline 0.0 & 1.80 & 2.57 & 3.54 & 4.6 .1 & 5.42 & 5.99 & 5.73 & 5.10 & 4.22 & 3.17 & 2.05 & 1.52 & 1392.8 & 3.8 \\
\hline 10.0 & 2.10 & 2.86 & 3.78 & 4.75 & 5.46 & 5.98 & 5.77 & 5.26 & 4.54 & 3.58 & 2.43 & 1.82 & 1472.4 & 4.0 \\
\hline 15.0 & 2.24 & 2.98 & 3.87 & 4.78 & 5.43 & 5.93 & 5.74 & 5.30 & 4.66 & 3.76 & 2.60 & 1.95 & 1500.3 & 4.1 \\
\hline 20.0 & 2.36 & 3.09 & 3.94 & 4.78 & 5.38 & 5.84 & 5.69 & 5.31 & 4.76 & 3.92 & 2.76 & 2.08 & $1519: 9$ & 4.2 \\
\hline 25.0 & 2.47 & 3.18 & 3.99 & 4.76 & 5.29 & 5.73 & 5.60 & 5.29 & 4.83 & 4.05 & 2.90 & 2.19 & 1531.1 & 4.2 \\
\hline 30.0 & 2.57 & 3.25 & 4.02 & 4.72 & .3 .18 & 5.58 & 5.48 & 5.25 & 4.87 & 4.16 & 3.02 & 2.29 & 1533.7 & 4.2 \\
\hline 35.0 & 2.65 & 3.33 & 4.02 & 4.65 & 5.04 & 5.40 & 5.33 & 5.17 & 4.88 & 4.25 & 3.13 & 2.38 & 1527.9 & 4.2 \\
\hline 40.0 & 2.71 & 3.33 & 4.01 & 4.55 & 4.88 & 5.20 & 5.15 & 5.06 & 4.86 & 4.31 & 3.21 & 2.45 & 1513.9 & 4.1 \\
\hline 45.0 & 2.76 & 3.35 & 3.97 & 4.44 & 4.70 & 4.99 & 4.96 & 4.94 & 4.82 & 4.34 & 3.28 & 2.50 & 1492.8 & 4.1 \\
\hline 50.0 & 2.79 & 3.34 & 3.91 & 4.33 & 4.50 & 4.75 & 4.75 & 4.78 & 4.75 & 4.35 & 3.32 & 2.55 & 1463.6 & 4.0 \\
\hline 60.0 & 2.80 & 3.28 & 3.72 & 3.95 & 4.04 & 4.20 & 4.25 & 4.40 & 4.53 & 4.29 & 3.35 & 2.58 & 1381.3 & 3.8 \\
\hline 70.0 & 2.75 & $3.1 c$ & 3.45 & 3.54 & 3.50 & 3.58 & 3.67 & 3.92 & 4.21 & 4.12 & 3.30 & 2.56 & 1269.7 & 3.5 \\
\hline 80.0 & 2.63 & 2.93 & 3.12 & 3.06 & 2.93 & 2.96 & 3.06 & 3.38 & 3.79 & 3.86 & 3.17 & 2.47 & 1135.7 & 3.1 \\
\hline 90.0 & 2.45 & 2.65 & 2.72 & 2.53 & 2.34 & 2.32 & 2.42 & 2.77 & 3.30 & 3.50 & 2.95 & 2.32 & 981.6 & 2.7 \\
\hline
\end{tabular}


AVERAGE DAILY TOTAL TERRESTRIAL INSOLATION ESTIMATES (KWH/SQ. M)

$\begin{aligned} \text { SITE: WILMINJTON } & \text { DE } \\ \text { LATITUDE: } & 39 \text { DEGPEES } 40 \text { MINUTES }\end{aligned}$

AUERAGE DAILY AMOUNTS BY MONTH

\begin{tabular}{|c|c|c|c|c|c|c|c|c|c|c|c|c|c|c|}
\hline ARRAY & $J$ & $\mathrm{~F}$ & $M$ & $A$ & M & $J$ & $\mathbf{J}$ & thom & $s$ & 0 & $N$ & D & $\begin{array}{l}\text { ANNUAL } \\
\text { AMOUNT }\end{array}$ & $\begin{array}{c}\text { AVERAGE } \\
\text { DAY }\end{array}$ \\
\hline 0.0 & 1.80 & 2.61 & 3.62 & 4.67 & 5.39 & 5.94 & 5.75 & 5.10 & 4.15 & 3.10 & 2.03 & 1.54 & 1392.2 & 3.8 \\
\hline 10.0 & 2.12 & 2.92 & 3.88 & 4.82 & 5.44 & 5.93 & 5.80 & 5.27 & 4.47 & 3.52 & 2.43 & 1.87 & 1475.9 & 4.0 \\
\hline 15.0 & 2.26 & 3.05 & 3.98 & 4.85 & 5.42 & 5.89 & 5.7 .8 & 5.31 & 4.60 & 3.70 & 2.60 & 2.01 & 1505.8 & 4.1 \\
\hline 20.0 & 2.39 & 3.16 & 4.06 & 4.86 & 5.36 & 5.81 & 5.72 & 5.33 & 4.70 & 3.85 & 2.77 & 2.15 & 1527.3 & 4.2 \\
\hline 25.0 & 2.51 & 3.26 & 4.11 & 4.85 & 5.28 & 5.69 & 5.63 & 5.31 & 4.77 & 3.99 & 2.91 & 2.27 & 1540.4 & 4.2 \\
\hline 30.0 & 2.61 & 3.33 & 4.15 & 4.80 & 5.18 & 5.55 & 5.52 & 5.27 & 4.81 & 4.10 & 3.04 & 2.38 & 1544.9 & 4.2 \\
\hline 35.0 & 2.69 & 3.39 & 4.16 & 4.74 & 5.04 & 5.38 & 5.37 & 5.19 & 4.83 & 4.19 & 3.15 & 2.48 & 1540.7 & 4.2 \\
\hline 40.0 & 2.76 & 3.43 & 4.14 & 4.64 & 4.83 & 5.18 & 5.20 & 5.09 & 4.82 & 4.25 & 3.24 & 2.56 & 1528.1 & 4.2 \\
\hline 45.0 & 2.82 & 3.45 & 4.11 & 4.53 & 4.71 & 4.97 & 5.01 & 4.97 & 4.78 & 4.29 & 3.31 & 2.62 & 1508.5 & 4.1 \\
\hline 50.0 & 2.85 & 3.45 & 4.05 & 4.39 & 4.51 & 4.74 & 4.80 & 4.82 & 4.71 & 4.30 & 3.36 & 2.67 & 1480.6 & 4.1 \\
\hline 60.0 & 2.88 & 3.39 & 3.86 & 4.05 & 4.05 & 4.21 & 4.30 & 4.44 & 4.50 & 4.24 & 3.40 & 2.72 & 1400.6 & 3.8 \\
\hline 70.6 & 2.83 & 3.25 & 3.59 & 3.63 & 3.52 & 3.59 & 3.72 & 3.97 & 4.19 & 4.09 & 3.35 & 2.70 & 1290.6 & 3.5 \\
\hline 80.0 & 2.71 & 3.06 & 3.25 & 3.14 & 2.95 & 2.98 & 3.11 & 3.42 & 3.78 & 3.83 & 3.22 & 2.62 & 1157.2 & 3.2 \\
\hline 90.0 & 2.53 & $2.7 i$ & 2.84 & 2.59 & 2.37 & 2.34 & 2.47 & 2.81 & 3.29 & 3.49 & 3.01 & 2.47 & 1002.9 & 2.7 \\
\hline
\end{tabular}


AVERAGE DAILY TOTAL TERRESTRIAL INSOLATION ESTIMATES (KI.HHSO. M)

SITE:
LATITUDE: 29 DEGREES 44 MINUTES

AVERAGE DAILY AMOUNTS BY MONTH

\begin{tabular}{|c|c|c|c|c|c|c|c|c|c|c|c|c|c|c|}
\hline ARRAY & $J$ & $F$ & $M$ & $A$ & $M$ & $J$ & $J$ & A & $S$ & 0 & n & $\mathrm{D}$ & $\begin{array}{l}\text { ANNUAL } \\
\text { AMOUNT }\end{array}$ & $\begin{array}{c}\text { AVERAGE } \\
\text { DAY }\end{array}$ \\
\hline 0.0 & 2.69 & 3.56 & 4.64 & 5.93 & 6.59 & 6.29 & 5.72 & 5.33 & 4.84 & 4.32 & 3.28 & 2.57 & 1697.1 & 4.6 \\
\hline 10.0 & 3.03 & 3.87 & 4.87 & 6.02 & 6.53 & 6.18 & 5.66 & 5.40 & 5.09 & 4.77 & 3.75 & 2.97 & 1769.4 & 4.8 \\
\hline 15.0 & 3.17 & 3.99 & 4.95 & 6.01 & 6.44 & 6.07 & 5.59 & 5.39 & 5.17 & 4.95 & 3.96 & 3.14 & 1790.8 & 4.9 \\
\hline 20.0 & 3.30 & 4.09 & 5.00 & 5.97 & 6.31 & 5.92 & 5.49 & 5.36 & 5.22 & 5.10 & 4.15 & 3.30 & 1802.1 & 4.9 \\
\hline 25.0 & 3.41 & $4.17^{\circ}$ & 5.02 & 5.90 & 6.15 & 5.75 & 5.35 & 5.29 & 5.24 & 5.23 & 4.30 & 3.44 & 1803.3 & 4.9 \\
\hline 30.0 & 3.50 & 4.23 & 5.01 & 5.80 & 5.96 & 5.54 & 5.19 & 5.20 & 5.24 & 5.32 & 4.44 & 3.55 & 1794.4 & 4.9 \\
\hline 35.0 & 3.57 & 4.26 & 4.98 & 5.66 & 5.75 & 5.32 & 5.01 & 5.08 & 5.20 & 5.37 & 4.54 & 3.65 & 1776.7 & 4.9 \\
\hline 40.0 & 3.62 & 4.27 & 4.91 & 5.50 & 5.50 & 5.07 & 4.81 & 4.93 & 5.14 & 5.40 & 4.62 & 3.72 & 1749.1 & 4.8 \\
\hline 45.0 & 3.65 & 4.25 & 4.82 & 5.30 & 5.23 & 4.80 & 4.58 & 4.76 & 5.04 & 5.39 & 4.67 & 3.77 & 1711.7 & 4.7 \\
\hline 50.0 & 3.65 & 4.21 & $4 \div 7$ & 5.08 & 4.93 & 4.50 & 4.33 & 4.56 & 4.92 & 5.36 & 4.69 & 3.80 & 1664.9 & 4.6 \\
\hline 60.0 & 3.60 & 4.06 & 4.40 & $4 . .55$ & $4.26^{\circ}$ & 3.84 & .3 .77 & 4.10 & 4.59 & 5.18 & 4.65 & 3.79 & 1544.3 & 4.2 \\
\hline 70.0 & 3.47 & 3.81 & 3.99 & 3.93 & 3.54 & 3.18 & 3.17 & 3.56 & 4.16 & $4 . .88$ & 4.49 & 3.69 & 1394.6 & 3.8 \\
\hline $50 \therefore 0$ & 3.25 & 3.48 & 3.51 & 3.24 & 2.79 & 2.49 & $2.54^{\circ}$ & 2.95 & 3.6 .5 & .4 .47$. & 4.22 & 3.49 & $1218: 9$ & 3.3 \\
\hline 90.0 & 2.96 & 3.08 & 2.96 & 2.54 & 2.08 & $1: 88$ & 1.95 & 2.34 & 3.07 & 3.96 & 3.85 & 3.22 & 1029.9 & 2.8 \\
\hline
\end{tabular}


AVERAGE DAILY TOTAL TERRESTRIAL INSOLATION ESTIMATES (KWH/SP. M)

SITE: DAYTONA BEACH LATITUDE: 29 DEGREES II MINUTES

AVERAGE I.AILY AMOUNTS BY MONTH

\begin{tabular}{|c|c|c|c|c|c|c|c|c|c|c|c|c|c|c|}
\hline ARRAY & $J$ & $F$ & $M$ & $A$ & $M$ & 等 & $\mathbf{J}$ & 年 & $S$ & 0 & $N$ & D & $\begin{array}{l}\text { ANNUAL } \\
\text { ARIOUNT }\end{array}$ & $\begin{array}{c}\text { AVERAGE } \\
\text { DAY }\end{array}$ \\
\hline 0.0 & 3.02. & 3.83 & 4.88 & 5.94 & 6.20 & 5.75 & 5.63 & 5.30 & 4.65 & 3.95 & 3.26 & 2.74 & 1678.6 & 4.6 \\
\hline 10.0 & 3.42 & 4.17 & 5.13 & 6.02 & 6.14 & 5.6 .5 & 5.57 & 5.37 & 4.87 & 4.32 & 3.72 & 3.16 & 1751.0 & 4.8 \\
\hline 15.0 & 3.59 & 4.31 & 5.21 & 6.01 & 6.05 & 5.55 & 5.49 & 5.36 & 4.95 & 4.47 & 3.72 & 3.35 & 1772.7 & 4.9 \\
\hline 20.0 & 3.74 & 4.42 & 5.26 & 5.97 & 5.93 & 5.42 & 5.39 & 5.32 & 4.99 & 4.59 & 4.10 & 3.52 & 1784.4 & 4.9 \\
\hline 25.0 & 3.87 & 4.51 & 5.28 & 5.89 & 5.78 & $E .26$ & $E .25$ & 5.25 & 5.01 & 4.69 & 4.25 & 3.67 & 1786.2 & 4.9 \\
\hline 30.0 & 3.98 & 4.57 & 5.27 & 5.79 & 5.60 & E. 08 & 5.09 & 5.16 & 5.00 & 4.76 & 4.37 & 3.79 & 1773.2 & 4.9 \\
\hline 35.0 & 4.06 & 4.60 & 5.23 & 5.65 & 5.40 & 4.88 & c. .92 & 5.04 & 4.96 & 4.80 & 4.47 & 3.89 & 1761.3 & 4.8 \\
\hline 40.0 & 4.12 & 4.61 & 5.17 & 5.48 & 5.17 & 4.66 & 6.71 & 4.89 & 4.89 & 4.81 & 4.55 & 3.97 & 1737.6 & 4.8 \\
\hline 45.0 & 4.16 & 4.59 & 5.07 & 5.28 & 4.92 & $c .<1$ & $c .49$ & 4.71 & 4.79 & 4.80 & 4.59 & 4.02 & 1698.3 & 4.7 \\
\hline 50.0 & 4.17 & 4.55 & 4.95 & 5.05 & 4.64 & c. .15 & 4.24 & 4.51 & 4.67 & 4.76 & $4 . E 1$ & 4.05. & 1652.6 & 4.5 \\
\hline 60.0 & 4.11 & 4.38 & 4.61 & 4.52 & 4.01 & $\overline{3} .56$ & 3.69 & 4.05 & 4.36 & 4.59 & 4.56 & 4.04 & 1534.7 & 4.2 \\
\hline 70.0 & 3.96 & 4.12 & 4.18 & 3.90 & 3.34 & 2.97 & 3.10 & 3.51 & 3.95 & 4.31 & 4.39 & 3.93 & 1388.0 & 3.8 \\
\hline 80.0 & 3.71 & 3.76 & 3.67 & 3.21 & 2.65 & $2.35^{\prime}$ & 2.49 & 2.91 & 3.46 & 3.94 & 4.12 & 3.72 & 1215.2 & 3.3 \\
\hline 90.0 & 3.38 & 3.32 & 3.08 & 2.50 & 2.00 & $=.81$ & 1.91 & 2.30 & 2.91 & 3.48 & 3.76 & 3.42 & 1029.1 & 2.8 \\
\hline
\end{tabular}


AVERAGE DAILY TOTAL TERRESTRIAL INSOLATION ESTIMATES (KWH/SQ. M)

SITE: JACKSONUILLE
LATITUDE: 30 DEGREES 30 MINUTES

AVERAGE DAILY AMOUNTS BY MONTH

\begin{tabular}{|c|c|c|c|c|c|c|c|c|c|c|c|c|c|c|}
\hline ARRAY & $J$ & $F$ & $M$ & A & $M$ & $j$ & $\mathrm{~J}$ & A & $S$ & 0 & $\mathbf{N}$ & $D$ & $\begin{array}{l}\text { ANNUAL } \\
\text { ANOUNT }\end{array}$ & $\begin{array}{c}\text { AUERAGE } \\
\text { DAY }\end{array}$ \\
\hline 0.0 & 2.83 & 3.67 & 4.79 & 5.85 & 6.17 & 5.93 & 5.68 & 5.34 & 4.54 & 3.86 & 3.14 & 2.57 & 1655.1 & 4.5 \\
\hline 10.0 & 3.22 & 4.02 & 5.05 & 5.95 & 6.12 & 5.84 & 5.63 & 5.43 & 4.77 & 4.24 & 3.60 & 2.99 & 1729.6 & 4.7 \\
\hline 15.0 & 3.39 & 4.15 & 5.13 & 5.95 & 6.04 & 5.74 & 5.56 & 5.42 & 4.84 & 4.39 & 3.80 & 3.17 & 1752.6 & 4.8 \\
\hline 20.0 & 3.53 & 4.27 & 5.19 & 5.91 & 5.93 & 5.61 & 5.46 & 5.39 & 4.89 & 4.52 & 3.98 & 3.33 & 1765.7 & 4.8 \\
\hline 25.0 & 3.66 & 4.36 & 5.22 & 5.85 & 5.79 & 5.45 & 5.33 & 5.33 & 4.92 & 4.63 & 4.14 & 3.48 & 1769.0 & 4.8 \\
\hline 30.0 & 3.77 & 4.42 & 5.22 & 5.75 & 5.61 & 5.27 & 5.18 & 5.24 & 4.91 & 4.70 & 4.27 & 3.60 & 1762.3 & 4.8 \\
\hline 35.0 & 3.85 & 4.46 & 5.19 & 5.62 & 5.42 & 5.06 & 5.00 & 5.12 & 4.88 & 4.75 & 4.37 & 3.71. & 1747.0 & 4.8 \\
\hline 40.0 & 3.91 & 4.47 & 5.13 & 5.46 & 5.20 & 4.84 & $4.8 \cdot 0$ & 4.98 & 4.82 & 4.77 & 4.45 & 3.79 & 1722.0 & 4.7 \\
\hline 45.0 & 3.95 & 4.46 & 5.04 & 5.27 & 4.95 & 4.59 & 4.58 & 4.81 & 4.73 & 4.76 & 4.50 & 3.84 & 1687.5 & 4.6 \\
\hline 50.0 & 3.96 & 4.43 & 4.92 & 5.05 & 4.68 & 4.32 & 4.34 & 4.61 & 4.62 & 4.73 & 4.52 & 3.88 & 1643.7 & 4.5 \\
\hline 50.0 & 3.92 & 4.27 & 4.61 & 4.54 & 4.07 & 3.72 & 3.78 & 4.15 & 4.32 & 4.57 & 4.48 & 3.87 & 1529.5 & 4.2 \\
\hline 70.0 & 3.78 & 4.03 & 4.19 & 3.93 & 3.41 & 3.10 & 3.19 & 3.61 & 3.93 & 4.31 & 4.34 & 3.78 & 1386.0 & 3.8 \\
\hline 30.0 & 3.56 & 3.69 & 3.59 & 3.26 & 2.73 & 2.45 & 2.58 & 3.01 & 3.46 & 3.95 & 4.08 & 3.59 & 1216.6 & 3.3 \\
\hline 30.0 & 3.25 & 3.27 & 3.12 & 2.56 & 2.07 & 1.88 & 1.98 & 2.39 & 2.92 & 3.51 & 3.73 & 3.31 & 1032.7 & 2.8 \\
\hline
\end{tabular}


AVERAGE DAILY TOQTAL TERRESTRIAL INSOLATION ESTIMATES (KWH/SQ. M)

SITE: MITMI

LATITUDE: 25 DEGREES 48 MINUTES

AVERAGE DAILY AMOUNTS. BY MONTH

\begin{tabular}{|c|c|c|c|c|c|c|c|c|c|c|c|c|c|c|}
\hline $\begin{array}{l}\text { ARRAY } \\
\text { TILT }\end{array}$ & $\mathrm{J}$ & $F$ & $M$ & A & $M-$ & {$[--1$} & $-\frac{-\pi}{3}$ & $A$ & $s^{\circ}$ & 0 & $N$ & $D$ & $\begin{array}{l}\text { ANNUAL } \\
\text { AMDUNT }\end{array}$ & $\begin{array}{c}\text { AVERAGE } \\
\text { DAY. }\end{array}$ \\
\hline 0.0 & 3.33 & 4.15 & 5.04 & 5.86 & 5.82 & 5.38 & 5.56 & 5.14 & 4.58 & 4.11 & 3.53 & 3.21 & 1695.3 & 4.6 \\
\hline 10.0 & 3.73 & 4.48 & 5.26 & 5.50 & 5.72 & 5.25 & 5.47 & 5.17 & 4.76 & 4.45 & 3.97 & 3.67 & 1759.2 & 4.8 \\
\hline 15.0 & $-3.8 \mathrm{~s}$ & 4.61 & 5.32 & 5.8 .7 & 5.53 & $5 .: 5$ & 5.38 & 5.15 & 4.81 & 4.58 & 4.15 & 3.87 & 1776.6 & 4.9 \\
\hline 20.0 & 4.04 & 4.72 & .5 .36 & 5.8 .1 & 5.50 & 5.83 & 5.26 & 5.09 & $\therefore 4.83$ & 4.68 & 4.32 & 4.04 & 1.784 .2 & 4.9 \\
\hline 25.0 & 4.16 & 4.79 & 5.36 & 5.i] & 5.34 & 4.85 & $5 . .11$ & $=5.01$ & 4.83 & 4.76 & 4.45 & 4.19 & 1781.9 & 4.9 \\
\hline 30.0 & $4.2 E$ & 4.84 & 5.33 & 5.5 .9 & 5.17 & 4.58 & 9.94 & 4.9 .1 & 4.80 & 4.81 & $4.5 ?$ & 4.32 & 1770.6 & 4.9 \\
\hline 35.0 & 4.33 & 4.86 & 5.28 & $5 .<4$ & 4.97 & 4.48 & 6.75 & 4.77 & 4.75 & 4.83 & 4.65 & 4.42 & 1749.6 & 4.8 \\
\hline 40.0 & 4.38 & 4.85 & 5.19 & 5.26 & 6.74 & 5.26 & $\dot{4} .54$ & 4.62 & 4.66 & 4.83 & 4.71 & 4.49 & 1719.0 & 4.7 \\
\hline 45.0 & 4.40 & 4.82 & 5.07 & 5.05 & 4.49 & 9.02 & 4.30 & 4.44 & 4.56 & 4.79 & 4.73 & 4.54 & 1679.0 & 4.6 \\
\hline 50.0 & 4.40 & 4.75 & 4.93 & $4.8 I$ & 4.22 & 3.77 & 4.05 & 4.24 & 4.43 & 4.73 & 4.73 & 4.56 & 1629.8 & 4.5 \\
\hline 60.0 & 4.31 & 4.55 & 4.56 & 4.26 & 3.62 & 3.22 & 3.48 & 3.77 & 4.09 & 4.53 & 4.65 & 4.51 & 1506.2 & 4.1 \\
\hline 70.0 & 4.12 & 4.24 & 4.10 & 3.6 .3 & 3.00 & 2.106 & 2.90 & 3.24 & 3.68 & 4.22 & 4.45 & 4.36 & 1355.3 & 3.7 \\
\hline 80.0 & $3.8 \bar{J}$ & 3.84 & 3.55 & 2.95 & 2.36 & 2.10 & 2.29 & 2.66 & 3.19 & 3.83 & 4.14 & 4.10 & 1180.4 & 3.2 \\
\hline 90.0 & 3.46 & 3.55 & 2.94 & 2.26 & 1.79 & 1.54 & 1.75 & 2.07 & 2.65 & 3.35 & 3.74 & 3.74 & 995.0 & 2.7 \\
\hline
\end{tabular}


AVERAGE DAILY TOTAL TERRESTRIAL INSOLATIOH ESTIMATES (KWH/SQ. M)

$\begin{aligned} \text { SITE: } & \text { ORLANDO } \\ \text { LF.TITUDE: } & 28 \text { DEGREES } 33 \text { MINUTES }\end{aligned}$

ALERAGE DAILY AMOUNTS BY MONTH

\begin{tabular}{|c|c|c|c|c|c|c|c|c|c|c|c|c|c|c|}
\hline ARRAY & $\mathrm{J}$ & $F$ & $M$ & $A$ & $M$ & $\mathrm{~J}$ & $J$ & $A$ & 5 & 0 & $N$ & D & $\begin{array}{l}\text { ANNUAL } \\
\text { AMOUNT }\end{array}$ & $\begin{array}{c}\text { AVERAGE } \\
\text { DAY }\end{array}$ \\
\hline 0.0 & 3.15 & 3.93 & 4.98 & 5.99 & 6.28 & 5.78 & 5.68 & 5.28 & 4.71 & 4.12 & $3 . \div 5$ & 2.91 & 1712.0 & 4.7 \\
\hline 10.0 & 3.56 & 4.27 & 5.23 & 6.07 & 6.20 & 5.66 & 5.61 & 5.34 & .4 .94 & 4.51 & 3.94 & 3.37 & 1785.9 & 4.9 \\
\hline 15.0 & 3.74 & 4.41 & 5.31 & 6.05 & 6.11 & 5.56 & 5.53 & 5.33 & 5.01 & 4.66 & $4 .: 5$ & 3.56 & 1808.0 & 5.0 \\
\hline 20.0 & 3.89 & 4.52 & 5.36 & 6.01 & 5.99 & 5.43 & 5.42 & 5.29 & 5.05 & 4.79 & 4.54 & 3.74 & 1820.0 & 5.0 \\
\hline 25.0 & 4.03 & 4.61 & 5.38 & 5.93 & 5.83 & 5.26 & 5.28 & 5.21 & 5.06 & 4.89 & 4.50 & 3.90 & 1821.7 & 5.0 \\
\hline 30.0 & 4.14 & 4.67 & 5.37 & 5.82 & 5.65 & 5.08 & 5.12 & 5.12 & 5.05 & 4.96 & 4.63 & 4.03 & 1813.6 & 5.0 \\
\hline 55.0 & 4.22 & 4.70 & 5.33 & 5.67 & 5.44 & 4.88 & 4.94 & 4.99 & 5.00 & 5.00 & $4 .: 4$ & 4.14 & 1796.2 & 4.9 \\
\hline 40.0 & 4.28 & 4.71 & 5.26 & 5.50 & 5.20 & 4.65 & 4.73 & 4.84 & 4.93 & 5.01 & 4.81 & 4.22 & 1768.7 & 4.8 \\
\hline 45.0 & 4.31 & 4.69 & 5.15 & 5.30 & 4.94 & 4.40 & 4.50 & 4.67 & 4.84 & 5.00 & 4.86 & 4.23 & 1731.4 & 4.7 \\
\hline 50.0 & 4.32 & 4.64 & 5.02 & 5.06 & 4.65 & 4.13 & 4.25 & 4.47 & 4.71 & 4.95 & 4.88 & 4.31 & 1684.5 & 4.6 \\
\hline 60.0 & 4.26 & 4.46 & 4.68 & 4.52 & 4.01 & 3.54 & 3.68 & 4.00 & 4.38 & 4.77 & 4.52 & 4.29 & 1563.6 & 4.3 \\
\hline$: 0.0$ & 4.10 & 4.18 & 4.24 & 3.89 & 3.33 & 2.94 & 3.09 & 3.46 & 3.97 & 4.48 & 4.65 & 4.17 & 1413.3 & 3.9 \\
\hline 80.0 & 3.84 & 3.81 & 3.70 & 3.19 & 2.63 & 2.32 & 2.47 & 2.87 & 3.47 & 4.09 & 4.36 & 3.94 & 1236.5 & 3.4 \\
\hline 90.0 & 3.49 & 3.36 & 3.10 & 2.48 & 1.97 & 1.79 & 1.88 & 2.26 & 2.90 & 3.61 & 3.36 & 3.62 & 1046.1 & 2.9 \\
\hline
\end{tabular}


AVERAGE DAILY TOTAL TERRESTRIAL INSOLATION ESTIMATES (KNH/SQ. M)

SITE: TALLAHASSEE
LATITUDE: 30 DEGREES 23 MINUTES

AVERAGE LAILY AMOUNTS BY MONTH

\begin{tabular}{|c|c|c|c|c|c|c|c|c|c|c|c|c|c|c|}
\hline \multirow{2}{*}{$\begin{array}{l}\text { ARRAY } \\
\text { TILT }\end{array}$} & \multicolumn{12}{|c|}{ 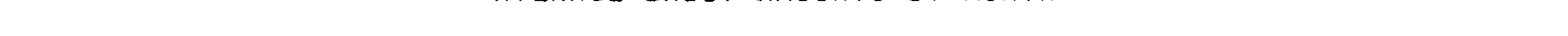 } & \multirow{2}{*}{$\begin{array}{l}\text { ANNUAL } \\
\text { AMOUNT }\end{array}$} & \multirow{2}{*}{$\begin{array}{l}\text { AVERAGE } \\
\text { DAY }\end{array}$} \\
\hline & $J$ & $F$ & $M$ & A & $M$ & $J$ & $J$ & A & $s$ & 0 & $\mathrm{~N}$ & D & & \\
\hline 0.0 & 2.76 & 3.59 & 4.6 .5 & 5.75 & 6.11 & 5.93 & 5.51 & 5.28 & 4.70 & 4.16 & 3.18 & 2.56 & 1649.4 & 4.5 \\
\hline 10.0 & 3.13 & 3.92 & 4.90 & 5.84 & 6.06 & 5.83 & 5.46 & 5.36 & 4.94 & 4.59 & 3.65 & 2.97 & 1724.3 & 4.7 \\
\hline 15.0 & 3.29 & 4.05 & 4.98 & 5.84 & 5.98 & 5.74 & 5.40 & 5.36 & 5.02 & 4.77 & 3.85 & 3.15 & 1747.5 & 4.8 \\
\hline 20.0 & 3.43 & 4.16 & 5.03 & 5.80 & 5.87 & 5.61 & 5.30 & 5.33 & 5.08 & 4.92 & 4.03 & 3.31 & 1760.9 & 4.8 \\
\hline 25.0 & 3.55 & 4.24 & 5.06 & 5.74 & 5.73 & 5.45 & 5.18 & 5.26 & 5.10 & 5.04 & 4.17 & 3.45 & 1764.5 & 4.8 \\
\hline 30.0 & 3.65 & 4.30 & 5.06 & 5.64 & 5.56 & 5.26 & 5.02 & 5.17 & 5.10 & 5.12 & 4.32 & 3.58 & 1758.1 & 4.8 \\
\hline 35.0 & 3.73 & 4.34 & 5.02 & 5.51 & 5.37 & 5.05 & 4.86 & 5.06 & 5.06 & 5.18 & 4.4 .3 & 3.68 & 1743.1 & 4.8 \\
\hline 40.0 & 3.79 & 4.35 & 4.97 & .5 .35 & 5.15 & 4.83 & 4.66 & 4.91 & 5.00 & 5.21 & 4.51 & 3.75 & 1718.6 & 4.7 \\
\hline 45.0 & 3.82 & 4.34 & 4.88 & 5.17 & 4.90 & $4.5 B$ & 4.45 & 4.75 & 4.91 & 5.21 & 4.55 & 3.81 & 1684.4 & 4.5 \\
\hline 50.0 & 3.83 & 4.30 & 4.76 & 4.96 & 4.63 & 4.31 & 4.21 & 4.55 & 4.80 & 5.17 & 4.53 & 3.84 & 1641.0 & 4.5 \\
\hline 60.0 & 3.79 & 4.15 & 4.46 & 4.45 & 4.03 & 3.71 & 3.68 & 4.10 & 4.48 & 5.01 & 4.54 & 3.84 & 1527.6 & 4.2 \\
\hline 70.0 & 3.65 & 3.91 & 4.05 & 3.86 & 3.38 & 3.07 & 3.11 & 3.57 & 4.07 & 4.73 & 4.39 & 3.74 & 1385.0 & 3.8 \\
\hline 80.0 & 3.43 & 3.58 & 3.57 & 3.20 & 2.70 & 2.45 & 2.51 & 2.97 & 3.58 & 4.33 & 4.16 & 3.55 & 1216.4 & 3.3 \\
\hline 90.0 & 3.13 & 3.17 & 5.02 & $\geq .52$ & 2.05 & 1.87 & 1.94 & 2.36 & 3.02 & 3.84 & 3.78 & 3.28 & 1033.2 & 2.8 \\
\hline
\end{tabular}


AVERAGE DAILY TOTAL TERRESTRIAL INSOLATION ESTIMATES (K!HH/SQ. M)

SITE: TAMPA

LATITUDE: 27 DEGREES 58 MINUTES

AVERAGE DAILY AMOUNTS BY MONTH

\begin{tabular}{|c|c|c|c|c|c|c|c|c|c|c|c|c|c|c|}
\hline ARRAY & $J$ & $F$ & $M$ & A & $M$ & $J$ & 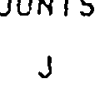 & i riors & 5 & 0 & $N$ & $D$ & $\begin{array}{l}\text { ANNUAL } \\
\text { AMIOUNT }\end{array}$ & $\begin{array}{c}\text { A VERAGE } \\
\text { DAY }\end{array}$ \\
\hline 0.0 & 3.18 & 3.97 & 5.02 & 6.02 & 6.30 & 5.82 & 5.53 & 5.21 & 4.70 & 4.25 & 3.49 & 2.94 & 1717.6 & 4.7 \\
\hline 10.0 & 3,59 & 4.31 & 5.26 & 6.09 & 6.22 & 5.70 & 5.46 & 5.27 & 4.91 & 4.64 & 3.97 & 3.39 & 1789.8 & 4.9 \\
\hline 15.0 & 3.76 & 4.45 & 5.34 & 6.07 & 6.13 & 5.59 & 5.38 & 5.25 & 4.98 & 4.80 & 4.18 & 3.59 & 1811.0 & 5.0 \\
\hline 20.0 & 3.92 & 4.56 & 5.39 & 6.02 & 6.00 & 5.46 & 5.27 & 5.21 & 5.02 & 4.93 & 4.36 & 3.76 & 1822.0 & 5.0 \\
\hline 25.0 & 4.05 & 4.64 & 5.40 & 5.94 & 5.84 & 5.29 & 5.13 & 5.13 & 5.03 & 5.03 & 4.52 & 3.91 & 1822.9 & 5.0 \\
\hline 30.0 & 4.15 & 4.70 & 5.39 & 5.82 & 5.65 & 5.10 & 4.97 & 5.03 & 5.01 & 5.10 & 4.65 & 4.04 & 1813.9 & 5.0 \\
\hline 35.0 & 4.24 & 4.73 & 5.34 & 5.68 & 5.44 & 4.89 & 4.80 & 4.91 & 4.96 & 5.15 & 4.75 & 4.14 & 1795.6 & 4.9 \\
\hline 40.0 & 4.29 & .4 .73 & 5.27 & 5.50 & 5.20 & 4.66 & 4.59 & 4.76 & 4.89 & 5.16 & 4.83 & 4.22 & 1767.2 & 4.8 \\
\hline 45.0 & 4.32 & $c .71$ & 5.16 & 5.29 & 4.93 & 4.41 & 4.37 & 4.59 & 4.79 & 5.14 & 4.87 & 4.28 & 17.29 .0 & 4.7 \\
\hline 50.0 & 4.33 & 4.66 & 5.03 & 5.05 & 4.64 & 4.13 & 4.12 & 4.39 & 4.66 & 5.09 & 4.88 & 4.30 & 1681.2 & 4.6 \\
\hline 60.0 & 4.26 & 6.48 & 4.68 & 4.51 & 3.99 & 3.53 & 3.57 & 3.93 & 4.34 & 4.90 & 4.82 & 4.28 & 1558.8 & 4.3 \\
\hline 70.0 & 4.09 & 4.19 & 4.23 & 3.87 & 3.31 & 2.92 & 3.00 & 3.39 & 3.92 & 4.59 & 4.64 & 4.15 & 1407.3 & 3.9 \\
\hline 80.0 & 3.83 & 3.82 & 3.6 .9 & 3.16 & 2.60 & 2.30 & 2.39 & 2.81 & 3.42 & 4.19 & 434 & 3.92 & 1229.6 & 3.4 \\
\hline 90.0 & 3.48 & 3.36 & 3.08 & 2.45 & 1.94 & 1.77 & 1.83 & 2.21 & 2.86 & 3.68 & 3.95 & 3.59 & 1038.8 & 2.8 \\
\hline
\end{tabular}


AVERAGE DAILY TOTAL TERRESTRIAL INSOLATION ESTIMATES (KWH/SQ. M)

SITE: JEST PALM BEACH FL

LATITUDE: 26 DEGREES 41 MINUUTES

AVERAGE DATLY AMOJNTS BY MONTH

\begin{tabular}{|c|c|c|c|c|c|c|c|c|c|c|c|c|c|c|}
\hline ARRAY & $J$ & $F$ & $M$ & A & $M$ & $\mathrm{~J}$ & $\cdot J$ & A & s & 0 & $N$ & D & $\begin{array}{l}\text { ANNUAL } \\
\text { AMOUNT }\end{array}$ & $\begin{array}{c}\text { AVERAGE } \\
\text { DAY }\end{array}$ \\
\hline 0.0 & 3.15 & 3.89 & 4.90 & 5.72 & 5.82 & 5.38 & 5.61 & 5.24 & 4.46 & 3.87 & 3.34 & 3.02 & 1655.7 & 4.5 \\
\hline 10.0 & 3.55 & 4.21 & 5.12 & $5.7 ?$ & 5.73 & 5.26 & 5.53 & 5.28 & 4.64 & 4.18 & 3.76 & 3.45 & 1717.7 & 4.7 \\
\hline 15.0 & 3.69 & 4.33 & 5.18 & 5.74 & 5.64 & 5.16 & 5.44 & 5.26 & 4.69 & 4.30 & 3.94 & 3.64 & 1734.7 & 4.8 \\
\hline 20.0 & 3.83 & 4.42 & 5.22 & 5.6 .9 & 3.52 & 5.03 & 5.33 & 5.21 & 4.72 & 4.40 & 4.09 & 3.80 & 1742.0 & 4.8 \\
\hline 25.0 & 3.94 & 4.50 & 5.23 & 5.6 .0 & 5.37 & 9.87 & 5.18 & 5.13 & 4.72 & 4.47 & 4.22 & 3.95 & 1739.8 & 4.8 \\
\hline 30.0 & 4.04 & 4.54 & 5.20 & $5 .<9$ & 5.19 & 4.70 & 5.02 & 5.02 & 4.70 & 4.52 & 4.33 & 4.07 & 1728.6 & 4.7 \\
\hline 35.0 & 4.11 & 4.56 & 5.15 & 5.54 & 5.00 & 4.50 & 4.83 & 4.89 & 4.65 & 4.55 & 4.41 & 4.16 & 1708.3 & 4.7 \\
\hline 40.0 & $4.1 \epsilon$ & 4.56 & 5.07 & 5.17 & 4.78 & 4.29 & 4.61 & 4.74 & 4.57 & 4.54 & 4.47 & 4.23 & 1678.6 & 4.6 \\
\hline 45.0 & 4.18 & 4.53 & 4.96 & 4.97 & 4.53 & 4.05 & 4.38 & 4.56 & 4.47 & 4.51 & 4.50 & 4.28 & 1639.8 & 4.5 \\
\hline 50.0 & 4.18 & 4.47 & 4.83 & 4.76 & 4.26 & 3.80 & 4.12 & 4.35 & 4.35 & 4.46 & 4.50 & 4.30 & 1592.1 & 4.4 \\
\hline 60.0 & 4.10 & 4.28 & 4.48 & 4.22 & 3.67 & 3.26 & 3.55 & 3.88 & 4.03 & 4.27 & 4.42 & 4.26 & 1472.0 & 4.0 \\
\hline 70.0 & 3.92 & 4.00 & 4.03 & $3.6 I$ & 3.05 & 2.70 & 2.97 & 3.34 & 3.63 & 3.99 & 4.23 & 4.12 & 1325.4 & 3.6 \\
\hline 80.0 & 3.66 & 3.63 & 3.51 & 2.95 & 2.41 & 2.14 & 2.35 & 2.75 & 3.16 & 3.63 & 3.95 & 3.88 & 1155.0 & 3.2 \\
\hline 90.0 & 3.31 & 3.18 & 2.92 & 2.28 & 1.83 & ]. 56 & ]. 80 & 2.15 & 2.63 & 3.18 & 3.58 & 3.54 & 974.2 & 2.7 \\
\hline
\end{tabular}


AVERAGE DAILY TOTAL TERRESTRIAL INSOLATION ESTIMATES (KWH/SQ. M)

SITE: ATLANTA
L.ATITUDE: 33 DEGREES 39 MINUTES

A:IERAGE DAILY AMOUNTS BY MONTH

\begin{tabular}{|c|c|c|c|c|c|c|c|c|c|c|c|c|c|c|}
\hline ARRAY & $J$ & $=$ & $M$ & A & $M$ & $J$ & $J$ & $A$ & $s$ & 0 & $N$ & D & $\begin{array}{l}\text { ANNUAL } \\
\text { AMIOUNT }\end{array}$ & $\begin{array}{c}\text { AVERAGE } \\
\text { DAY }\end{array}$ \\
\hline 0.0 & 2.26 & 3.06 & 4.10 & 5.31 & 5.85 & 6.03 & 5.71 & 5.39 & 4.48 & 3.78 & 2.78 & 2.12 & 1549.2 & 4.2 \\
\hline 10.0 & 2.57 & 3.36 & 4.34 & 5.43 & 5.83 & 5.96 & 5.70 & 5.51 & 4.75 & 4.21 & 3.23 & 2.49 & 1625.3 & 4.5 \\
\hline 15.0 & 2.71 & 3.48 & 4.42 & 5.44 & 5.78 & 5.88 & 5.64 & 5.52 & 4.84 & 4.39 & $3 . \div 3$ & 2.66 & 1650.0 & 4.5 \\
\hline 20.0 & 2.83 & 3.58 & 4.48 & 5.43 & 5.69 & 5.77 & 5.56 & 5.50 & 4.91 & 4.55 & 3.51 & 2.81 & 1665.5 & 4.6 \\
\hline 25.0 & 2.94 & 3.66 & 4.52 & 5.38 & 5.57 & 5.62 & 5.44 & 5.46 & 4.95 & 4.67 & 3.76 & 2.94 & 1671.8 & 4.6 \\
\hline 30.0 & 3.03 & 3.72 & 4.53 & 5.30 & 5.43 & 5.45 & 5.30 & 5.38 & 4.96 & 4.77 & 3.70 & 3.05 & 1668.7 & 4.6 \\
\hline 35.0 & 3.11 & 3.76 & 4.51 & 5.20 & 5.25 & 5.25 & 5.13 & 5.28 & 4.95 & 4.84 & 4.01 & 3.15 & 1656.7 & $4: 5$ \\
\hline$\div 0.0$ & 3.16 & 3.78 & 4.47 & 5.07 & 5.06 & 5.03 & 4.94 & 5.14 & 4.90 & 4.88 & 4.10 & 3.23 & 1536.5 & 4.5 \\
\hline$\mp 5.0$ & 3.20 & 3.78 & 4.40 & 4.91 & 4.85 & 4.79 & 4.73 & 4.98 & 4.83 & 4.90 & 4.16 & 3.29 & 1607.3 & 4.4 \\
\hline 50.0 & 3.21 & 3.75 & 4.31 & 4.73 & 4.60 & 4.53 & 4.50 & 4.80 & 4.73 & 4.88 & 4.19 & 3.33 & 1569.3 & 4.3 \\
\hline 30.0 & 3.19 & 3.64 & 4.06 & 4.30 & 4.06 & 3.94 & 3.96 & 4.35 & 4.46 & 4.76 & 4.19 & 3.34 & 1467.9 & 4.0 \\
\hline 70.0 & 3.10 & 3.45 & 3.73 & 3.78 & 3.44 & 3.31 & 3.37 & 3.82 & 4.09 & 4.52 & 4.08 & 3.28 & 1336.8 & 3.7 \\
\hline 30.0 & 2.93 & 3.18 & 3.32 & 3.19 & 2.82 & 2.66 & 2.76 & 3.22 & 3.63 & 4.18 & 3.37 & 3.13 & 1182.2 & 3.2 \\
\hline 30.0 & 2.69 & 2.85 & 2.84 & 2.57 & 2.18 & 2.05 & 2.14 & 2.59 & 3.10 & 3.74 & 3.56 & 2.91 & 1010.1 & 2.8 \\
\hline
\end{tabular}


AVERAGE DAILY TOTAL TEFRESTRIAL INSOLATION ESTIMATES (XLH/SQ. M)

SITE: ALEUSTA
LATITUDE: $3 Z$ DEGREES 22 MINUTES

AVERAGE DAILY AMOLNTS BY MONTH

\begin{tabular}{|c|c|c|c|c|c|c|c|c|c|c|c|c|c|c|}
\hline ARRAY & $\begin{array}{l}--2 \\
\mathrm{~J}\end{array}$ & $F$ & $M$ & A & $M$ & $\sqrt{J}$ & $\mathrm{~J}$ & A & 5 & 0 & $N$ & $D$ & $\begin{array}{l}\text { ANNUAL } \\
\text { AMOUNT }\end{array}$ & $\begin{array}{c}\text { AVERAGE } \\
\text { DAY }\end{array}$ \\
\hline 0.0 & 2.36 & 3.21 & 4.22 & 5.46 & 5.88 & 6.00 & 5.68 & 5.26 & 4.44 & 3.85 & 2.89 & 2.27 & 1568.3 & 4.3 \\
\hline 10.0 & 2.70 & 3.52 & 4.46 & 5.57 & 5.86 & 5.93 & 5.66 & $5: 37$ & 4.70 & 4.28 & 3.36 & 2.68 & 1647.1 & 4.5 \\
\hline 15.0 & 2.85 & 3.65 & 4.54 & 5.59 & 5.81 & 5.85 & 5.61 & 5.38 & 4.79 & 4.46 & 3.57 & 2.86 & 1672.9 & 4.6 \\
\hline 20.0 & 2.98 & 3.76 & 4.60 & 5.57 & 5.72 & 5.73 & 5.52 & 5.36 & 4.86 & 4.62 & 3.76 & 3.02 & 1689.5 & 4.6 \\
\hline 25.0 & 3.10 & 3.85 & 4.64 & 5.52 & 5.60 & 5.59 & 5.40 & 5.32 & 4.89 & 4.75 & 3.92 & 3.17 & 1696.5 & 4.6 \\
\hline 30.0 & 3.19 & 3.91 & 4.65 & 5.44 & 5.45 & 5.41 & 5.26 & 5.24 & 4.90 & 4.85 & 4.06 & 3.30 & 1694.1 & 4.6 \\
\hline 35.0 & 3.27 & 3.96 & 4.63 & 5.33 & 5.27 & 5.21 & 5.09 & 5.13 & 4.89 & 4.92 & 4.18 & 3.40 & 1632.7 & 4.6 \\
\hline 40.0 & 3.33 & 3.98 & 4.59 & 5.20 & 5.08 & 5.00 & 4.91 & 5.01 & 4.84 & 4.96 & 4.27 & 3.49 & 1662.9 & 4.6 \\
\hline 45.0 & 3.37 & 3.98 & 4.52 & 5.04 & 4.86 & 4.76 & 4.69 & 4.85 & 4.77 & 4.97 & 4.34 & 3.55 & 1633.8 & 4.5 \\
\hline 50.0 & 3.39 & 3.95 & 4.43 & 4.85 & 4.62 & 4.50 & 4.46 & 4.67 & 4.67 & 4.98 & 4.37 & 3.60 & 1595.7 & 4.4 \\
\hline 60.0 & 3.37 & 3.83 & $\$ .17$ & 4.40 & 4.06 & 3.91 & 3.93 & 4.23 & 4.39 & 4.83 & 4.37 & 3.62 & 1493.7 & 4.1 \\
\hline 70.0 & 3.26 & 3.63 & 3.82 & 3.86 & 3.44 & 3.29 & 3.33 & 3.72 & 4.02 & 4.59 & 4.25 & 3.55 & 1361.5 & 3.7 \\
\hline 80.0 & 3.09 & 3.35 & 3.40 & 3.25 & 2.81 & 2.64 & 2.73 & 3.13 & 3.57 & 4.24 & 4.03 & 3.39 & 1204.9 & 3.3 \\
\hline 90.0 & 2.84 & 2.99 & 2.91 & 2.60 & 2.17 & 2.03 & 2.12 & 2.52 & 3.05 & 3.79 & 3.71 & 3.15 & 1030.4 & 2.8 \\
\hline
\end{tabular}


AVERAGE DAILY TOTAL TERRESTRIAL INSOLATION ESTIMATES (KWH SQ. M)

SITE: MACON
LATITUDE: 32 DEGREES 42 MINUTES

A:ERAGE DAILY AMOUNTS BY MONTH

\begin{tabular}{|c|c|c|c|c|c|c|c|c|c|c|c|c|c|c|}
\hline ARRAY & $\lambda^{-1}$ & $=$ & $M$ & A & M & $\mathrm{J}$ & $\mathrm{J}$ & A & $\mathrm{s}$ & 0 & $\mathbf{H}$ & D & $\begin{array}{l}\text { ANNUAL } \\
\text { AMOUNT }\end{array}$ & $\begin{array}{c}\text { AVERAGE } \\
\text { DAY }\end{array}$ \\
\hline $0.0^{\circ}$ & 2.42 & 3.22 & 4.29 & 5.48 & 5.94 & 6.05 & 5.62 & 5.42 & 4.53 & 3.93 & 2.36 & 2.29 & 1588.2 & 4.4 \\
\hline 10.0 & 2.75 & 3.53 & 4.53 & 5.59 & 5.91 & 5.97 & 5.60 & 5.53 & 4.79 & 4.37 & 3.74 & 2.69 & 1665.6 & 4.6 \\
\hline 15.0 & 2.90 & 3.65 & 4.62 & 5.60 & 5.85 & 5.89 & 5.54 & 5.54 & 4.89 & 4.55 & 3.55 & 2.86 & 1690.6 & 4.6 \\
\hline 20.0 & 3.03 & 3.76 & 4.68 & 5.58 & 5.76 & 5.77 & 5.45 & 5.52 & 4.95 & 4.71 & 3.33 & 3.02 & 1706.1 & 4.7 \\
\hline 25.0 & 3.14 & 3.84 & 4.71 & 5.53 & 5.63 & 5.62 & 5.33 & 5.46 & 4.98 & 4.84 & 4.10 & 3.16 & 1712.1 & 4.7 \\
\hline 30.0 & 3.24 & 3.90 & 4.71 & 5.44 & 5.48 & 5.43 & 5.19 & 5.38 & 4.99 & 4.94 & 4.14 & 3.29 & 1708.5 & 4.7 \\
\hline 35.0 & 3.32 & 3.94 & 4.69 & 5.33 & 5.30 & $5: 23$ & 5.02 & 5.27 & 4.97 & 5.01 & 4.25 & 3.39 & 1695.0 & 4.6 \\
\hline 40.0 & 3.37 & 3.96 & 4.65 & 5.20 & 5.10 & 5.01 & 4.83 & 5.14 & 4.92 & 5.04 & 4.34 & 3.47 & 1674.8 & 4.6 \\
\hline 45.0 & 3.41 & 3.95 & 4.58 & 5.03 & 4.88 & 4.77 & 4.62 & 4.97 & 4.85 & 5.05 & 4.80 & 3.53 & 1644.3 & 4.5 \\
\hline 50.0 & 3.43 & 3.93 & 4.48 & 4.84 & 4.63 & 4.50 & 4.39 & 4.78 & 4.74 & 5.03 & 4.84 & 3.57 & 1604.8 & 4.4 \\
\hline 60.0 & 3.40 & 3.30 & 4.21 & 4.38 & 4.06 & 3.90 & 3.86 & 4.33 & 4.46 & 4.90 & 4.93 & 3.58 & 1499.7 & 4.1 \\
\hline 70.0 & 3.29 & 3.60 & 3.86 & 3.83 & 3.43 & 3.27 & 3.27 & 3.79 & 4.07 & 4.65 & 4.30 & 3.51 & 1364.6 & 3.7 \\
\hline 80.0 & 3.11 & 3.31 & 3.42 & 3.22 & 2.79 & 2.61 & 2.68 & 3.18 & 3.61 & 4.29 & 4.07 & 3.35 & 1204.9 & 3.3 \\
\hline 30.0 & 2.85 & 2.96 & 2.92 & 2.57 & 2.15 & 2.00 & 2.08 & 2.54 & 3.07 & 3.83 & 3.75 & 3.11 & 1028.1 & 2.8 \\
\hline
\end{tabular}


AVERAGE DAILY TOTAL TEREESTRIAL INSOLATION ESTIMATES (KWH/SQ. M)

SITE: SAUANAAT

LATITUDE: Z2 DEGREES \& MINUTES

AVERAGE IAILY AMOLNTS BY MONTH

\section{ARRAY
TILT}

0.0

10.0

15.0

20.0

25.0

30.0

35.0

40.0

45.0

50.0

60.0

70.0

80.0

90.0

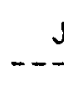

$2.50 \quad 3.29 \quad 4.4$

$2.85 \quad 3.60 \quad 4.54$

3.00

3.73

3.13

3.2 .5

3.83

4.73

3.91

3.34

3.42

3.98

4.01

3.48 .4 .03$$
3.5
$$

3.5

3.50

3.38

3.1

4.02

3.99

3.87

3.87

2.93

\begin{abstract}
3.65
3.36
\end{abstract}
3.36
2.99

4.79
4.82

4.82

$--1$

$M$

J

J

$\mathrm{S}$

0

D ANNUAL
AMOUNT

ANNUAL
AMOUNT

AVERAGE

$\begin{array}{lllllll}21 & 5.62 & 5.12 & 4.29 & 3.84 & 2.96 & 2.37\end{array}$

5.66

5.84

$=.81$
$=.73$

$.62 \quad 5.12$

1571.4

4.3

5.67

5.75

5. 65

$5.53 \quad 5.21$

4.52

4.25

$3.42 \quad 2.78$

1646.1

1670.0

4.5

4.6

4.6

4.6

4.6

4.6

4.5

4.4

4.3

4.0

3.7

3.2

2.8 
AVERAGE DAILY TOTAL TERRESTRIAL INSOLATION ESTIMATES (KWH/SQ. M) SITE: BARBERS POINT MUT HI
LATITUDE:

AUERAGE DAILY AMOUNTS BY MONTH

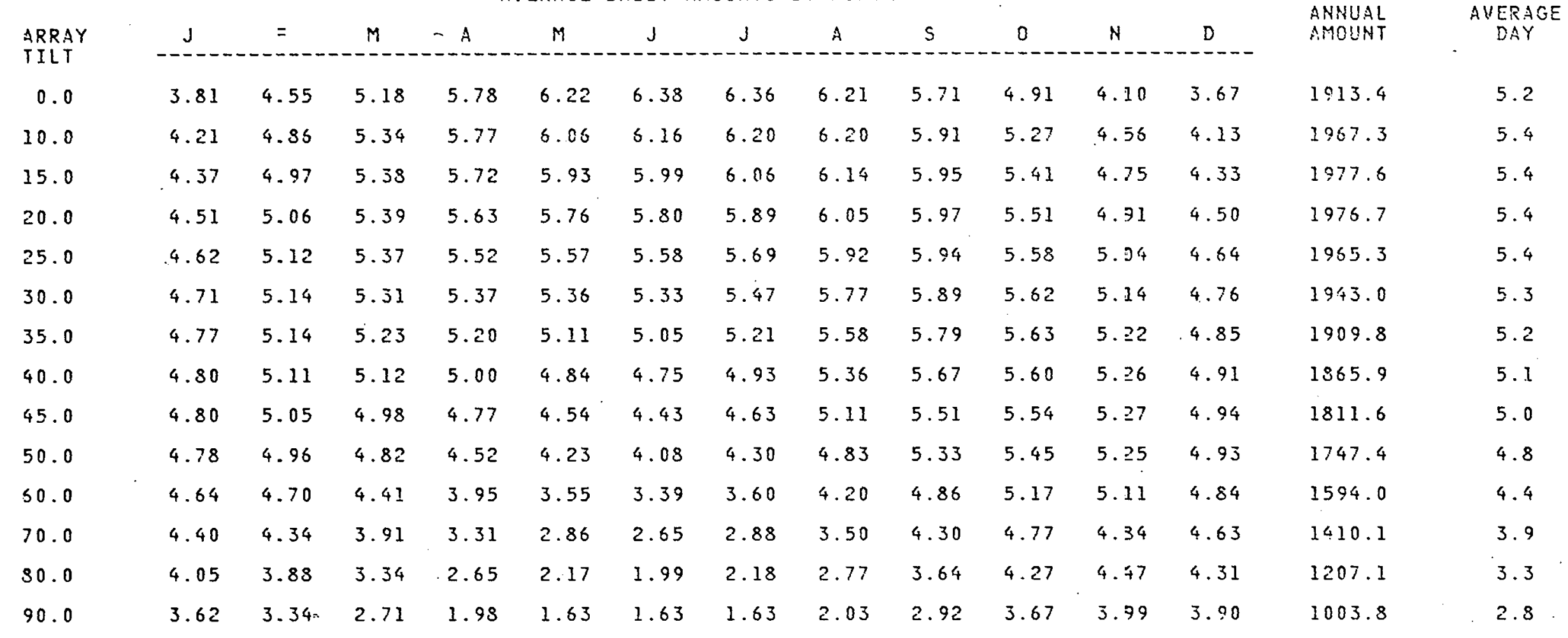


AVERAGE DAILY TOTAL TERRESTRIAL INSOLATION ESTIMATES (KWH/SQ. M)

SITE: HILO
LATITUDE:
I9 DEGREES 43 MINUTES

AVERAGE DAILY AMOUNTS EY MONTH

\begin{tabular}{|c|c|c|c|c|c|c|c|c|c|c|c|c|c|c|}
\hline ARRAY & $\mathrm{J}$ & $F$ & $M$ & $A$ & $M$ & $J$ & $J$ & $A$ & $s$ & 0 & $N$ & $\mathrm{D}$ & $\begin{array}{l}\text { ANNUAL } \\
\text { AMOUNT }\end{array}$ & $\begin{array}{c}\text { AVERAGE } \\
\text { DAY }\end{array}$ \\
\hline 0.0 & 3.53 & 3.93 & 4.24 & 4.52 & 4.89 & 5.23 & 5.12 & 5.02 & 4.87 & 4.33 & 3.48 & 3.21 & 1594.2 & 4.4 \\
\hline 10.0 & 3.86 & 4.16 & 4.34 & 4.49 & $\therefore .77$ & 5.05 & 4.99 & 5.00 & 4.99 & 4.60 & 3.81 & 3.56 & 1631.2 & 4.5 \\
\hline 15.0 & 3.99 & 4.24 & 9.36 & 4.45 & 4.67 & 4.93 & 4.88 & 4.94 & 5.02 & 4.69 & 3.94 & 3.70 & 1636.7 & 4.5 \\
\hline 20.0 & 4.1 .0 & 4.29 & 4.35 & 4.38 & 4.55 & 4.77 & 4.75 & 4.87 & 5.01 & 4.76 & 4.04 & 3.82 & 1633.4 & 4.5 \\
\hline 25.0 & 4.19 & 4.32 & 4.32 & 4.29 & 4.40 & 4.60 & 4.60 & 4.76 & 4.98 & 4.81 & 4.13 & 3.92 & 1622.0 & 4.4 \\
\hline 30.0 & 4.26 & 4.33 & 4.27 & 4.18 & 4.24 & 4.40 & 4.42 & 4.63 & 4.92 & 4.82 & 4.19 & 3.99 & 1501.9 & 4.4 \\
\hline 35.0 & 4.30 & 4.32 & 4.20 & 4.04 & 4.06 & 4.13 & 4.22 & 4.48 & 4.83 & 4.81 & 4.23 & 4.05 & 1573.3 & 4.3 \\
\hline 40.0 & 4.31 & 4.28 & 4.10 & 3.89 & 3.85 & 3.95 & 4.01 & 4.31 & 4.72 & 4.77 & 4.25 & 4.08 & 1536.4 & 4.2 \\
\hline 45.0 & 4.30 & 4.22 & 3.98 & 3.72 & 3.63 & 3.57 & 3.77 & 4.11 & 4.58 & 4.71 & 4.24 & 4.09 & 1491.5 & 4.1 \\
\hline 50.0 & 4.27 & 4.13 & 3.85 & 3.53 & 3.39 & $3 .+2$ & 3.52 & 3.90 & 4.42 & 4.62 & 4.20 & 4.07 & 1438.9 & 3.9 \\
\hline 60.0 & 4.12 & 3.90 & 3.52 & 3.11 & 2.90 & 2.33 & 2.99 & 3.41 & 4.03 & 4.36 & 4.06 & 3.97 & 1315.1 & 3.6 \\
\hline 70.0 & 3.89 & 3.58 & 5.12 & 2.63 & 2.38 & 2.31 & 2.44 & 2.87 & 3.56 & 4.01 & 3.83 & 3.78 & 2167.5 & 3.2 \\
\hline 80.0 & 3.57 & 3.19 & 2.67 & 2.15 & 1.87 & 1.31 & 1.91 & 2.31 & 3.03 & 3.57 & 3.52 & 3.50 & 1005.2 & 2.8 \\
\hline 90.0 & 3.18 & 2.74 & 2.18 & 1.66 & 1.51 & 1.56 & 1.54 & 1.76 & 2.44 & 3.07 & 3.13 & 3.15 & 848.2 & 2.3 \\
\hline
\end{tabular}


AVERAGE DAILY TOTAL TERRESTRIAL INSOLATION ESTIMATES (KWH/SP. M)

SITE: HONOLULU

LATITUDE: 21 DEGREES 20 MINUTES

AVERAGE DAILY AMOUNTS BY MONTH

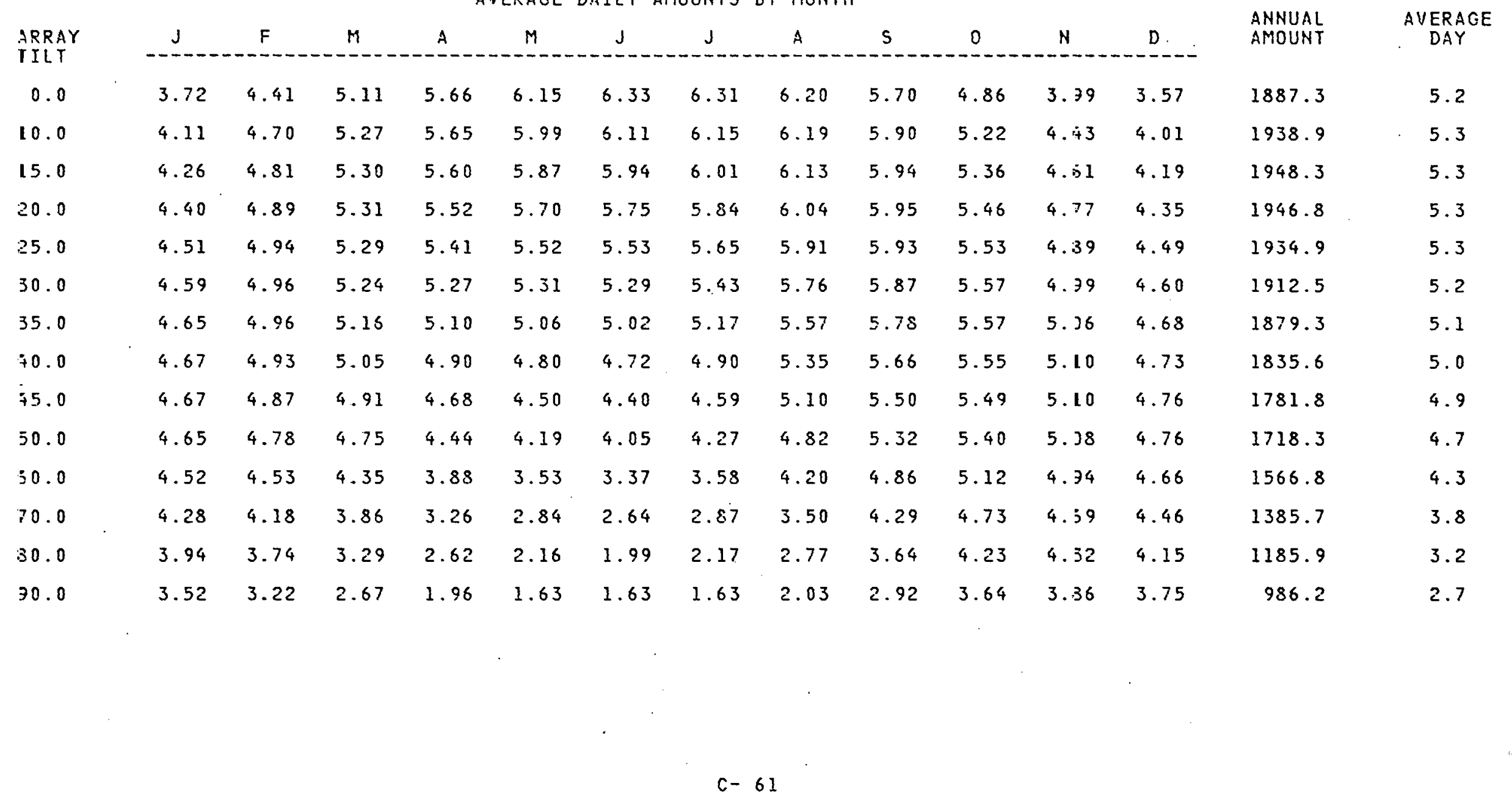


AVERAGE DAILY TOTAL TERRESTRIAL INSOLATION ESTIMATES (KWH/SQ. M)

SITE: LIYHUE

LATITUDE: $2 I$ DEGREES 59 NINUTES

QVERAGE TIAILY AMOUNTS BY MONTH

\begin{tabular}{|c|c|c|c|c|c|c|c|c|c|c|c|c|c|c|}
\hline $\begin{array}{l}\text { ARRAY } \\
\text { TILT }\end{array}$ & 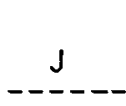 & $F$ & $M$ & A & $M$ & 3 & 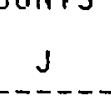 & A & 5 & 0 & $N$ & D & $\begin{array}{l}\text { ANNUAL } \\
\text { AMOUNT }\end{array}$ & $\begin{array}{c}\text { AVERAGE } \\
\text { DAY }\end{array}$ \\
\hline 0.0 & 3.48 & 4.11 & 4.54 & 5.17 & 5.76 & 5.89 & 5.87 & 5.73 & 5.48 & 4.57 & 3.64 & 3.32 & 1754.7 & 4.8 \\
\hline 10.0 & 3.84 & 4.38 & 4.78 & 5.16 & 5.62 & 5.70 & 5.73 & 5.73 & 5.67 & 4.90 & 4.02 & 3.72 & 1803.1 & 4.9 \\
\hline 15.0 & 3.98 & 4.48 & 4.32 & 5.12 & 5.51 & 5.56 & 5.61 & 5.68 & 5.72 & 5.03 & 4.18 & 3.88 & 1812.4 & 5.0 \\
\hline 20.0 & 4.11 & 4.55 & 4.32 & 5.05 & 5.36 & 5.39 & 5.47 & 5.60 & 5.73 & 5.12 & 4.31 & 4.03 & 1811.7 & 5.0 \\
\hline 25.0 & 4.21 & 4.60 & 4.30 & 4.95 & 5.20 & 5.19 & $E .29$ & 5.49 & 5.71 & 5.19 & 4.42 & 4.15 & 1801.6 & 4.9 \\
\hline 30.0 & 4.29 & 4.62 & 4.76 & 4.83 & 5.01 & 4.97 & 5.09 & 5.35 & 5.66 & 5.23 & 4.51 & 4.25 & 1781.8 & 4.9 \\
\hline 35.0 & 4.34 & 4.62 & 4.59 & 4.68 & 4.79 & 4.73 & 4.87 & 5.18 & 5.58 & 5.23 & 4.57 & 4.33 & 1752.2 & 4.8 \\
\hline 40.0 & 4.36 & 4.59 & 4.59 & 4.51 & 4.55 & 4.47 & 4.62 & 4.98 & 5.46 & 5.21 & 4.60 & 4.38 & 1713.1 & 4.7 \\
\hline 45.0 & 4.37 & 4.54 & 4.47 & 4.32 & 4.28 & 4.18 & 4.35 & 4.76 & 5.32 & 5.15 & 4.60 & 4.40 & 1664.7 & 4.6 \\
\hline 50.0 & 4.34 & 4.46 & 4.32 & 4.10 & 4.00 & 3.87 & 4.06 & 4.51 & 5.14 & 5.07 & 4.58 & 4.40 & 1607.3 & 4.4 \\
\hline 60.0 & 4.22 & 4.23 & 3.37 & 3.61 & 3.39 & 3.25 & 3.43 & 3.95 & 4.70 & 4.81 & 4.45 & 4.31 & 1469.9 & 4.0 \\
\hline 70.0 & 4.00 & 3.90 & 3.54 & 3.06 & 2.77 & 2.59 & 2.79 & 3.32 & 4.17 & 4.45 & 4.23 & 4.13 & 1305.6 & 3.6 \\
\hline 80.0 & 3.69 & 3.50 & 3.04 & 2.49 & 2.15 & 1.99 & 2.15 & 2.66 & 3.55 & 3.99 & 3.90 & 3.84 & 1123.0 & 3.1 \\
\hline 90.0 & 3.30 & 3.03 & 2.89 & 3. .90 & 1.64 & 1.62 & 1.63 & 1.99 & 2.87 & 3.45 & 3.49 & 3.48 & 933.1 & 2.6 \\
\hline
\end{tabular}


AVERAGE DAILY TOTAL TERRESTRIAL INSOLATION ESTIMATES (KLH/SQ. M)

SITE: BUPLINGTON
LATITUDE: 40 DEGREES 47 MINUTES

AVERAGE DAILY AMOUNTS BY MONTH

\begin{tabular}{|c|c|c|c|c|c|c|c|c|c|c|c|c|c|c|}
\hline ARRAY & $J$ & $F$ & $M$ & $A$ & $M$ & $J$ & $J$ & $A$ & $s$ & 0 & $N$ & $D$ & $\begin{array}{l}\text { ANNUAL } \\
\text { ANOUNT }\end{array}$ & $\begin{array}{c}\text { AVERAGE } \\
\text { DAY }\end{array}$ \\
\hline 0.0 & 1.83 & 2.71 & 3.67 & 4.85 & $\vdots .92$ & 6.68 & 6.58 & 5.77 & 4.46 & 3.34 & 2.10 & 1.52 & 1505.7 & 4.1 \\
\hline 10.0 & 2.18 & 3.05 & 3.95 & 5.02 & 5.98 & 6.69 & 6.66 & 6.00 & 4.85 & 3.85 & 2.54 & 1.86 & 1603.9 & 4.4 \\
\hline 15.0 & 2.34 & 3.20 & 4.06 & 5.06 & 5.97 & 6.64 & 6.64 & 6.07 & 5.01 & 4.07 & 2.74 & 2.02 & 1639.7 & 4.5 \\
\hline 20.0 & 2.49 & 3.34 & 4.15 & 5.08 & 5.92 & 6.56 & 6.58 & 6.10 & 5.13 & 4.27 & 2.93 & 2.17 & 1666.1 & 4.6 \\
\hline 25.0 & 2.62 & 3.45 & 4.21 & $5.0 \%$ & 5.83 & 6.44 & 6.49 & 6.10 & 5.23 & 4.44 & 5.10 & 2.30 & 1683.1 & 4.6 \\
\hline 30.0 & 2.74 & 3.54 & 4.25 & $5.0 \geq$ & 5.72 & 6.28 & 6.36 & 6.06 & 5.29 & 4.58 & $\Xi .25$ & 2.42 & 1690.3 & 4.6 \\
\hline 35.0 & 2.84 & 3.61 & 4.27 & 4.96 & 5.57 & 6.08 & 6.20 & 5.98 & 5.32 & 4.70 & $\Xi .38$ & 2.52 & 1687.9 & 4.6 \\
\hline 40.0 & 2.92 & 3.66 & 4.26 & 4.87 & 5.40 & 5.86 & 6.00 & 5.87 & 5.32 & 4.78 & $\equiv .48$ & 2.61 & 1675.7 & 4.6 \\
\hline 45.0 & 2.98 & 3.69 & 4.23 & 4.76 & 5.21 & 5.62 & 5.78 & 5.74 & 5.29 & 4.84 & \pm .57 & $2: 68$ & 1655.7 & 4.5 \\
\hline 50.0 & 3.03 & 3.70 & 4.17 & 4.62 & 4.99 & 5.36 & 5.53 & 5.57 & 5.23 & 4.87 & 3.63 & 2.74 & 1626.5 & 4.5 \\
\hline 60.0 & 3.06 & 3.65 & 3.99 & 4.27 & 4.49 & 4.74 & 4.95 & 5.14 & 5.01 & 4.83 & 3.69 & 2.80 & 1540.8 & 4.2 \\
\hline 70.0 & 3.03 & 3.52 & 3.72 & 3.83 & 3.89 & 4.04 & 4.27 & 4.60 & 4.67 & 4.67 & 3.65 & 2.79 & 1420.8 & 3.9 \\
\hline 80.0 & 2.91 & 3.30 & 3.38 & 3.32 & 3.26 & 3.33 & 3.55 & 3.96 & 4.23 & 4.40 & 3.53 & 2.71 & 1273.6 & 3.5 \\
\hline 90.0 & 2.73 & 3.01 & 2.96 & 2.75 & 2.60 & 2.58 & 2.80 & 3.25 & 3.70 & 4.02 & 3.31 & 2.56 & 1102.8 & 3.0 \\
\hline
\end{tabular}


AVERAGE DAIL $`$ TOTAL TERRESTRIAL INSOLATION ESTIMATES (KWH/SQ. M)

$\begin{aligned} \text { SITE: } & \text { DES MOINES } \\ \text { LATITUDE: } & 41 \text { DEGREES } 32 \text { MINUTES }\end{aligned}$

AUERAGE DAI:Y AMOIJNTS BY MONTH

\begin{tabular}{|c|c|c|c|c|c|c|c|c|c|c|c|c|c|c|}
\hline ARRAY & J. & $F$ & $r$ & $A$ & $M$ & 5 & $\mathrm{~J}$ & $A$ & $\mathrm{~S}$ & 0 & $N$ & $\mathrm{D}$ & $\begin{array}{l}\text { ANNUAL } \\
\text { AMOUNT }\end{array}$ & $\begin{array}{c}\text { AVERAGE } \\
\text { DAY }\end{array}$ \\
\hline 0.0 & 1.83 & 2.71 & 3.72 & 4.91 & 5.89 & 5.70 & 5.60 & 5.77 & 4.52 & 3.36 & 2.38 & 1.54 & 1512.3 & 4.1 \\
\hline 10.0 & 2.23 & 3.08 & 4.01 & 5.10 & 5.96 & 5.71 & 6.69 & 6.02 & 4.93 & 3.90 & 2.54 & 1.91 & 1616.6 & 4.4 \\
\hline 15.0 & 2.38 & 3.24 & 4.13 & 5.15 & 5.95 & 6.67 & 6.68 & 8.09 & 5.10 & 4.13 & 2.75 & 2.08 & 1655.3 & 4.5 \\
\hline 20.0 & 2.53 & 3.37 & 4.22 & 5.17 & 5.90 & 6.59 & 6.63 & 6.13 & 5.23 & 4.34 & 2.95 & 2.24 & 1684.6 & 4.6 \\
\hline 25.0 & 2.67 & 3.49 & 4.29 & 5.16 & 5.83 & 6.47 & 6.54 & 6.13 & 5.33 & 4.53 & 3.12 & 2.39 & 1704.2 & 4.7 \\
\hline 30.0 & 2.89 & 3.59 & 4.34 & 5.13 & 5.72 & 6.32 & 6.42 & 6.09 & 5.41 & 4.68 & 3.28 & 2.52 & 1713.9 & 4.7 \\
\hline 35.0 & 2.91 & 3.67. & 4.36 & 5.06 & 5.58 & 6.13 & 6.26 & 6.02 & 5.44 & 4.81 & 3.41 & 2.63 & 1713.7 & 4.7 \\
\hline 40.0 & 3.00 & 3.73 & 4.36 & 4.97 & 5.40 & 5.91 & 6.06 & 5.92 & 5.45 & 4.90 & 3.53 & 2.73 & 1703.6 & 4.7 \\
\hline 45.0 & 3.07 & 3.76 & 4.33 & 4.86 & 5.22 & 5.67 & 5.84 & $5.78^{\circ}$ & 5.43 & 4.97 & 3.62 & 2.81 & 1685.1 & 4.6 \\
\hline 50.0 & 3.12 & 3.77 & 4.28 & 4.72 & 5.00 & c. .41 & 5.60 & 5.62 & 5.37 & 5.00 & 3.69 & 2.88 & 1657.6 & 4.5 \\
\hline 60.0 & 3.17 & 3.73 & 4.10 & 4.37 & 4.51. & 4.80 & 5.02 & 5.19 & 5.15 & 4.98 & 3.76 & 2.95 & 1574.4 & 4.3 \\
\hline 70.0 & 3.13 & 3.60 & 3.83 & 3.93 & 3.92 & $c .10$ & 4.35 & 4.66 & 4.82 & 4.82 & 3.73 & 2.95 & 1455.8 & $4.0 \ldots$ \\
\hline 80.0 & 3.02 & 3.39 & 3.48 & 3.41 & 3.29 & $\overline{3} .39$ & 3.61 & 4.02 & 4.37 & 4.55 & 3.61 & 2.87 & 1308.4 & 3.6 \\
\hline 90.0 & 2.84 & 3.09 & 3.06 & 2.84 & 2.64 & 2.64 & 2.86 & 3.31 & 3.83 & 4.17 & 3.39 & 2.72 & 1136.8 & 3.1 \\
\hline
\end{tabular}


AVERAGE DAILY TOTAL TERRESTRIAL INSOLATION ESTIMATES (KWH/SQ. M)

$$
\begin{aligned}
& \text { SITE: MASON CITY } \\
& \text { ITUDE: } 43 \text { DEGREES } 9 \text { MINUTES }
\end{aligned}
$$

AVERAGE DAILY AMOUNTS BY MONTH

\begin{tabular}{|c|c|c|c|c|c|c|c|c|c|c|c|c|c|c|}
\hline ARRAY & $\lambda^{--2}$ & $F$ & $M$ & A & $M$ & $\mathrm{j}$ & 3 & $A$ & $s$ & 0 & $N$ & D & $\begin{array}{l}\text { ANNUAL } \\
\text { ANOUITT }\end{array}$ & $\begin{array}{c}\text { A VERAGE } \\
\text { DAY }\end{array}$ \\
\hline 0.0 & 1.75 & 2.64 & 3.68 & 4.79 & 5.97 & 6.66 & 6.57 & 5.78 & 4.42 & 3.18 & $\therefore .90$ & 1.40 & 1485.3 & 4.1 \\
\hline 10.0 & 2.14 & 3.02 & 4.00 & 4.98 & 6.07 & 8.70 & 6.67 & 6.06 & 4.86 & 3.72 & 2.34 & 1.76 & 1593.6 & 4.4 \\
\hline 15.0 & 2.31 & 3.18 & 4.12 & 5.04 & 6.06 & 6.66 & 6.67 & 6.14 & 5.03 & 3.96 & c.. 54 & 1.93 & 1634.6 & 4.5 \\
\hline 20.0 & 2.48 & 3.33 & 4.23 & 5.07 & 6.03 & 6.59 & 6.63 & 6.19 & 5.18 & 4.17 & 2.73 & 2.08 & 1666.2 & 4.6 \\
\hline 25.0 & 2.62 & 3.46 & 4.30 & 5.07 & 5.96 & 6.49 & 6.56 & 6.20 & 5.29 & 4.35 & 2.90 & 2.22 & 1688.3 & 4.6 \\
\hline 30.0 & 2.76. & 3.57 & 4.36 & 5.04 & 5.85 & 6.34 & 6.44 & 6.18 & 5.37 & 4.51 & 3.06 & 2.35 & 1700.6 & 4.7 \\
\hline 35.0 & 2.87 & 3.65 & 4.39 & 4.99 & 5.72 & 6.16 & 6.29 & 6.12 & 5.42 & 4.64 & 3.19 & 2.47 & 1703.0 & 4.7 \\
\hline 40.0 & 2.97 & 3.72 & 4.40 & 4.91 & 5.55 & 5.95 & 6.10 & 6.03 & 5.44 & 4.75 & 3.31 & 2.57 & 1695.5 & 4.6 \\
\hline 45.0 & 3.05 & 3.76 & 4.38 & 4.80 & 5.36 & 5.72 & 5.89 & 5.90 & 5.42 & 4.82 & 3.40 & 2.65 & 1678.9 & 4.6 \\
\hline 50.0 & 3.11 & 3.78 & 4.33 & 4.67 & 5.16 & 5.46 & 5.66 & 5.74 & 5.37 & 4.86 & 3.47 & 2.72 & 1654.0 & 4.5 \\
\hline 60.0 & 3.17 & 3.75 & 4.17 & 4.34 & 4.66 & 4.88 & 5.10 & 5.33 & 5.18 & 4.85 & 3.55 & 2.79 & 1576.0 & 4.3 \\
\hline 70.0 & 3.15 & 3.64 & 3.91 & 3.92 & 4.07 & 4.19 & 4.44 & 4.80 & 4.87 & 4.72 & 3.53 & 2.80 & 1462.2 & 4.0 \\
\hline 80.0 & 3.06 & 3.43 & 3.57 & $\geq .42$ & 3.42 & 3.48 & 3.71 & 4.17 & 4.44 & 4.47 & 3.43 & 2.74 & 1318.4 & 3.6 \\
\hline 90.0 & 2.88 & 3.15 & 3.15 & 2.87 & 2.77 & $2.7 \dot{4}$ & 2.97 & 3.45 & 3.91 & 4.11 & 3.24 & 2.61 & 1150.7 & 3.2 \\
\hline
\end{tabular}


AVERAGE DAILY TOTAL TERRESTRIAL INSOLATION ESTIMATES (KWH/SQ. M)

SITE: SICUX CITY

LATITUDE: 42 DEGREES 24 MINUTES

AVERAGE DAILY AMOUNTS BY MONTH

\begin{tabular}{|c|c|c|c|c|c|c|c|c|c|c|c|c|c|c|}
\hline ARRAY & $J$ & $F$ & $M$ & $A$ & $M$ & 舟 & 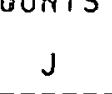 & ' trom & $\mathrm{s}$ & 0 & $N$ & $D$ & $\begin{array}{l}\text { ANNUAL } \\
\text { AMOUNT }\end{array}$ & $\begin{array}{c}\text { AVERAGE } \\
\text { DAY }\end{array}$ \\
\hline 0.0 & 1.80 & 2.65 & 3.68 & 4.97 & 5.99 & 6.70 & 6.69 & 5.81 & 4.48 & 3.27 & 2.03 & 1.48 & 1510.0 & 4.1 \\
\hline 10.0 & 2.18 & 3.02 & 3.99 & 5.17 & 6.08 & 6.72 & 6.79 & 6.08 & 4.90 & 3.80 & 2.50 & 1.86 & 1617.7 & 4.4 \\
\hline 15.0 & 2.36 & 3.18 & 4.11 & 5.23 & 6.07 & 6.59 & 6.78 & 6.16 & 5.07 & 4.04 & 2.72 & 2.03 & 1658.1 & 4.5 \\
\hline 20.0 & 2.52 & 3.32 & 4.21 & 5.25 & 6.03 & 6.51 & 6.74 & 6.20 & 5.21 & 4.25 & 2.92 & 2.19 & 1689.1 & 4.6 \\
\hline 25.0 & 2.56 & 3.44 & 4.28 & 5.25 & 5.96 & 6.50 & 6.65 & 6.21 & 5.32 & 4.44 & 3.10 & 2.34 & 1710.3 & 4.7 \\
\hline 30.0 & 2.79 & 3.55 & 4.33 & 5.22 & 5.85 & 6.35 & 6.53 & 6.18 & 5.40 & 4.59 & 3.26 & 2.47 & 1721.6 & 4.7 \\
\hline 35.0 & 2.91 & 3.63 & 4.36 & 5.16 & 5.71 & 6.17 & 6.37 & 6.12 & 5.44 & 4.72 & 3.40 & 2.59 & 1722.8 & 4.7 \\
\hline 40.0 & 3.00 & 3.69 & 4.36 & 5.07 & 5.54 & 5.95 & 6.18 & 6.02 & 5.46 & 4.82 & 3.52 & 2.69 & 1714.1 & 4.7 \\
\hline 45.0 & 3.08 & 3.72 & 4.34 & 4.96 & 5.35 & 5.71 & 5.96 & 5.89 & 5.43 & 4.89 & 3.62 & 2.77 & 1696.6 & 4.6 \\
\hline 50.0 & 3.14 & 3.74 & 4.29 & 4.83 & 5.18 & 5.45 & 5.72 & 5.72 & 5.38 & .4 .93 & 3.70 & 2.84 & 1670.3 & 4.6 \\
\hline 60.0 & 3.19 & 3.71 & 4.12 & 4.48 & 4.63 & 4.86 & 5.14 & 5.30 & 5.18 & 4.91 & 3.77 & 2.92 & 1589.1 & 4.4 \\
\hline 70.0 & 3.17 & 3.58 & 3.86 & 4.03 & $4.0 \div$ & 4.16 & 4.45 & 4.77 & 4.85 & 4.77 & 3.75 & 2.92 & 1472.0 & 4.0 \\
\hline 80.0 & 3.06 & 3.37 & 3.51 & 3.51 & 3.39 & 3.44 & 3.71 & 4.13 & 4.42 & 4.51 & 3.64 & 2.85 & 1325.0 & 3.6 \\
\hline 90.0 & 2.88 & 3.09 & 3.09 & 2.93 & 2.73 & 2.70 & 2.95 & 3.41 & 3.85 & 4.14 & 3.43 & 2.71 & 1153.8 & 3.2 \\
\hline
\end{tabular}


AVERAGE DAILY TOTAL TERRESTRIAL INSOLATION ESTIMATES (KLAH/SQ. M)

SITE: BOISE

LATITUDE: 43 DEGREES 34 MINUTES

AVERAGE DAILY AMOUNTS BY MONTH

\begin{tabular}{|c|c|c|c|c|c|c|c|c|c|c|c|c|c|c|}
\hline ARRAY & $\mathrm{J}$ & $F$ & $M$ & A & $M$ & $J$ & $J$ & $A$ & $S$ & 0 & $N$ & $D$ & $\begin{array}{l}\text { ANNUAL } \\
\text { AMOUNT }\end{array}$ & $\begin{array}{c}\text { AVERAGE } \\
\text { DAY }\end{array}$ \\
\hline 0.0 & 1.53 & 2.65 & 4.11 & 5.75 & 7.18 & 7.76 & 8.23 & 6.93 & 5.48 & 3.58 & 1.99 & 1.33 & 1724.7 & 4.7 \\
\hline 10.0 & 1.85 & 3.04 & 4.50 & 6.04 & 7.32 & 7.82 & 8.41 & 7.32 & 6.11 & 4.26 & 2.48 & 1.74 & 1855.6 & 5.1 \\
\hline 15.0 & 2.00 & 3.21 & 4.66 & 6.12 & 7.33 & 7.78 & 8.42 & 7.14 & 6.37 & 4.55 & 2.71 & 1.91 & 1904.9 & 5.2 \\
\hline 20.0 & 2.13 & 3.36 & 4.79 & 6.17 & 7.29 & 7.71 & 8.39 & 7.52 & 6.59 & 4.82 & 2.92 & 2.07 & 1942.9 & $5 . j$ \\
\hline 25.0 & 2.25 & 3.49 & 4.90 & 6.19 & 7.21 & 7.58 & 8.29 & 7.56 & 6.77 & 5.06 & 3.11 & 2.21 & 1969.1 & 5.4 \\
\hline 30.0 & 2.36 & 3.61 & 4.97 & $6.1 \%$ & 7.09 & 7.41 & 8.15 & 7.54 & 6.91 & 5.27 & 3.28 & 2.34 & 1983.4 & 5.4 \\
\hline 35.0 & 2.45 & 3.70 & 5.02 & 6.11 & 6.92 & 7.20 & 7.96 & 7.48 & 7.00 & 5.44 & 5.44 & 2.46 & 1735.6 & 5.4 \\
\hline 40.0 & 2.53 & 3.77 & 5.03 & $6.0 \bar{z}$ & 6.72 & 6.95 & 7.72 & 7.38 & 7.05 & 5.58 & 3.57 & 2.56 & 1975.8 & 5.4 \\
\hline 45.0 & 2.60 & 3.81 & 5.02 & 5.89 & 6.49 & 6.67 & 7.44 & 7.23 & 7.05 & 5.68 & $\equiv .67$ & 2.64 & 1954.8 & 5.4 \\
\hline 50.0 & 2.64 & 3.83 & 4.97 & 5.74 & 6.23 & 6.36 & 7.13 & 7.04 & .7 .01 & 5.74 & $\equiv .76$ & 2.71 & 1924.1 & 5.3 \\
\hline 60.0 & 2.69 & 3.81 & 4.80 & 5.33 & 5.61 & 5.65 & 6.40 & 6.54 & 6.79 & 5.76 & 3.85 & 2.79 & 1828.2 & 5.0 \\
\hline 70.0 & 2.67 & 3.70 & 4.51 & 4.81 & 4.88 & 4.83 & 5.52 & 5.88 & 6.39 & 5.62 & 3.85 & 2.80 & 1688.8 & 4.6 \\
\hline 80.0 & 2.59 & 3.49 & 4.12 & 4.19 & 4.06 & 3.97 & 4.54 & 5.09 & 5.84 & 5.34 & 3.74 & 2.74 & 1512.7 & 4.1 \\
\hline 90.0 & 2.44 & 3.21 & 3.54 & 3.48 & 3.23 & 3.07 & 3.55 & 4.18 & 5.14 & 4.92 & 3.54 & 2.61 & 1308.6 & 3.6 \\
\hline
\end{tabular}


AVERAGE DAILY TOTAL TERRESTRIAL INSOLATION ESTIMATES (KWH/SQ. M)

SITE: LEWISTON

LATITUDE: 46 DEGREES 23 MINUTES

AVERAGE DAILY AMCUNTS BY MONTH

\begin{tabular}{|c|c|c|c|c|c|c|c|c|c|c|c|c|c|c|}
\hline ARRAY & $J$ & $F$ & $M$ & A & $M$ & $\therefore$ & 3 & A & $S$ & 0 & $N$ & $\mathrm{D}$ & $\begin{array}{l}\text { ANNUAL } \\
\text { AMOUNT }\end{array}$ & $\begin{array}{c}\text { AVERAGE } \\
\text { DAY }\end{array}$ \\
\hline 0.0 & 1.07 & 1.92 & 3.21 & 4.52 & 5.81 & 6.35 & 7.36 & 6.09 & 4.52 & 2.71 & 1.30 & 0.90 & 1396.5 & 3.8 \\
\hline 10.0 & 1.28 & 2.18 & 3.51 & 4.74 & 5.93 & 6.42 & 7.54 & 6.45 & 5.05 & 3.20 & 1.61 & 1.12 & 1495.6 & 4.1 \\
\hline 15.0 & 1.37 & 2.30 & 3.63 & 4.81 & 5.94 & 6.40 & 7.57 & 6.58 & 5.27 & 3.42 & 1.75 & 1.22 & 1532.7 & 4.2 \\
\hline 20.0 & 1.46 & 2.40 & 3.73 & 4.85 & 5.93 & $\dot{0} .35$ & 7.56 & 6.66 & 5.46 & 3.62 & 1.88 & 1.32 & 1561.5 & 4.3 \\
\hline 25.0 & 1.53 & 2.49 & 3.81 & 4.86 & 5.87 & 5.27 & 7.50 & 6.70 & 5.61 & 3.79 & 2.00 & 1.91 & 1581.2 & 4.3 \\
\hline 30.0 & 1.60 & 2.57 & 3.87 & 4.85 & 5.79 & 5.15 & 7.39 & 6.71 & 5.73 & 3.95 & 2.11 & 1.48 & 1591.8 & 4.4 \\
\hline 35.0 & 1.66 & 2.63 & 3.91 & 4.81 & 5.68 & 6.00 & 7.24 & 6.67 & 5.82 & 4.08 & 2.20 & 1.55 & 1593.1 & 4.4 \\
\hline 40.0 & 1.71 & 2.68 & 3.92 & 4.75 & 5.53 & 5.81 & 7.05 & 6.59 & 5.86 & 4.18 & 2.28 & 1.62 & 1585.1 & 4.3 \\
\hline 45.0 & 1.75 & 2.71 & 3.91 & 4.66 & 5.35 & 5.60 & 6.82 & 6.48 & 5.88 & 4.26 & 2.35 & 1.67 & 1568.0 & 4.3 \\
\hline 50.0 & 1.78 & 2.72 & 3.88 & 4.55 & 5.16 & 5.37 & 6.57 & 6.33 & 5.85 & 4.31 & 2.40 & 1.71 & 1543.5 & 4.2 \\
\hline 60.0 & 1.31 & 2.71 & 3.76 & 4.26 & 4.71 & 4.85 & 5.96 & 5.92 & 5.69 & 4.33 & 2.46 & 1.76 & 1469.5 & 4.0 \\
\hline 70.0 & 1.30 & 2.63 & 3.55 & 5.88 & 4.15 & 4.22 & 5.22 & 5.38 & 5.39 & 4.24 & 2.46 & 1.77 & 1362.3 & 3.7 \\
\hline 80.0 & 1.74 & 2.49 & 3.27 & 3.42 & 3.54 & 3.55 & 4.38 & 4.72 & 4.97 & 4.05 & 2.40 & 1.73 & 1225.7 & 3.4 \\
\hline 90.0 & 1.64 & 2.30 & 2.91 & $2.9 C$ & 2.90 & 2.86 & 3.52 & 3.95 & 4.42 & 3.75 & 2.28 & 1.65 & 1068.5 & 2.9 \\
\hline
\end{tabular}


AVERAGE DAILY TOTAL TERRESTRIAL INSOLATION ESTIMATES (KHH/SQ. M)

SITE: POCATELLO
LATITUDE: 42 DEGREES 55 MINUTES

AVERAGE DAILY AMOUNTS BY MONTH

\begin{tabular}{|c|c|c|c|c|c|c|c|c|c|c|c|c|c|c|}
\hline ARRAY & $J$ & $F$ & $M$ & $A$ & $M$ & $J$ & $\mathrm{~J}$ & $A$ & $s$ & 0 & $N$ & D & $\begin{array}{l}\text { ANNUAL } \\
\text { AMOUHT }\end{array}$ & $\begin{array}{c}\text { A VERAGE } \\
\text { DAY }\end{array}$ \\
\hline 0.0 & 1.70 & 2.75 & 4.32 & 5.75 & 7.19 & 7.82 & 8.20 & 7.06 & 5.58 & 3.79 & 2.17 & 1.50 & 1764.1 & 4.8 \\
\hline 10.0 & 2.07 & 3.19 & 4.73 & 6.01 & 7.32 & 7.87 & 8.36 & 7.45 & 6.22 & 4.50 & 2.73 & 1.90 & 1900.3 & 5.2 \\
\hline 15.0 & 2.23 & 3.37 & 4.90 & 6.09 & 7.32 & 7.83 & 8.36 & 7.57 & 6.48 & 4.82 & 2.98 & 2.09 & 1951.8 & 5.3 \\
\hline 20.0 & 2.39 & 3.53 & 5.04 & 6.14 & 7.28 & 7.75 & 8.32 & 7.65 & 6.70 & 5.10 & .3 .22 & 2.26 & 1991.6 & 5.5 \\
\hline 25.0 & 2.53 & 3.65 & 5.15 & 6.15 & 7.19 & 7.62 & 8.22 & 7.68 & 6.87 & 5.35 & 3.43 & 2.42 & 2019.3 & 5.5 \\
\hline 30.0 & $2.65^{\circ}$ & 3.78 & 5.22 & 6.12 & 7.07 & 7.44 & 8.07 & 7.66 & 7.01 & 5.57 & 3.63 & 2.56 & 2034.8 & 5.6 \\
\hline 35.0 & 2.76 & 3.87 & 5.27 & 6.05 & 6.90 & 7.22 & 7.88 & 7.59 & 7.09 & 5.75 & 3.80 & 2.69 & 2037.8 & 5.6 \\
\hline 40.0 & 2.85 & 3.96 & 5.29 & 5.97 & 6.69 & 6.96 & 7.63 & 7.48 & 7.14 & 5.90 & 3.94 & 2.80 & 2028.3 & 5.6 \\
\hline 45.0 & 2.92 & 3.99 & 5.27 & $5.8 \%$ & 6.45 & 6.67 & 7.35 & 7.32 & 7.13 & 6.00 & $\therefore .06$ & 2.89 & 2007.7 & 5.5 \\
\hline 50.0 & 2.98 & 4.01 & 5.22 & 5.68 & 6.19 & 6.37 & 7.05 & 7.13 & 7.09 & 6.07 & 5.16 & 2.97 & 1976.7 & 5.4 \\
\hline 60.0 & 3.03 & 3.99 & 5.03 & 5.27. & 5.57 & 5.65 & 6.31 & 6.61 & 6.85 & 6.08 & 4.26 & 3.06 & 1879.0 & 5.1 \\
\hline 70.0 & 3.01 & 3.86 & 4.73 & 4.75 & 4.83 & 4.81 & 5.43 & 5.93 & 6.44 & 5.93 & 4.26 & 3.07 & 1736.4 & 4.8 \\
\hline 80.0 & 2.91 & 3.65 & 4.31 & 4.12 & 4.01 & 3.93 & 4.46 & 5.11 & 5.87 & 5.63 & c. 14 & 3.00 & 1556.3 & 4.3 \\
\hline 90.0 & 2.74 & 3.34 & 3.80 & $3.4 \vec{c}$ & 3.18 & 3.03 & 3.47 & 4.19 & 5.16 & 5.18 & 5.91 & 2.85 & 1346.5 & 3.7 \\
\hline
\end{tabular}


AVERAJE DAILY TOTAL TERRESTRIAL INSOLATION ESTIMATES (KWH/SQ. M)

SITE: CHICAGO
ATITUDE: 4 I DEGREES 47 MINUTES

AVERAGE DAILY AMOUNTS BY MONTH

\begin{tabular}{|c|c|c|c|c|c|c|c|c|c|c|c|c|c|c|}
\hline ARRAY & $J$ & $F$ & $M$ & A & $M$ & $J$ & $a^{-1}$ & $A$ & $S$ & 0 & $N$ & $D$ & $\begin{array}{l}\text { ANNUAL } \\
\text { AMOUNT }\end{array}$ & $\begin{array}{c}\text { AVER AGE } \\
\text { DAY }\end{array}$ \\
\hline 0.0 & 1.50 & 2.39 & 3.49 & 4.61 & 5.64 & 6.33 & 6.15 & 5.42 & 4.26 & 3.06 & 1.79 & 1.27 & 1401.4 & 3.8 \\
\hline 10.0 & 1.90 & 2.69 & 3.76 & 4.77 & 5.71 & 6.35 & 6.21 & 5.65 & 4.64 & 3.52 & 2.16 & 1.54 & 1489.7 & 4.1 \\
\hline 15.0 & 2.03 & 2.82 & 3.86 & 4.82 & 5.70 & 6.31 & 6.20 & 5.71 & 4.79 & 3.72 & 2.32 & 1.66 & 1521.8 & 4.2 \\
\hline 20.0 & 2.15 & 2.93 & 3.95 & 4.84 & 5.65 & 6.24 & 6.15 & 5.75 & 4.92 & 3.90 & 2.48 & 1.78 & 1545.3 & 4.2 \\
\hline 25.0 & 2.27 & 3.03 & 4.01 & 4.83 & 5.58 & 6.13 & 6.07 & 5.75 & 5.01 & 4.05 & 2.61 & 1.88 & 1560.2 & 4.3 \\
\hline 30.0 & 2.37 & 3.11 & 4.05 & $4.8 C$ & 5.48 & 5.98 & 5.96 & 5.71 & 5.07 & 4.18 & 2.74 & 1.97 & 1566.2 & 4.3 \\
\hline 35.0 & 2.45 & 3.17 & 4.07 & $4.7 c$ & 5.34 & 5.81 & 5.81 & 5.65 & 5.11 & 4.29 & 2.84 & 2.05 & 1563.4 & 4.3 \\
\hline 40.0 & 2.52 & 3.21 & 4.07 & 4.65 & 5.18 & 5.60 & 5.63 & 5.55 & 5.11 & 4.37 & 2.93 & 2.12 & 1551.7 & 4.3 \\
\hline 45.0 & 2.58 & 3.24 & 4.04 & 4.55 & 5.00 & 5.38 & 5.43 & 5.42 & 5.08 & 4.42 & 3.00 & 2.18 & 1532.5 & 4.2 \\
\hline 50.0 & 2.62 & 3.24 & 3.99 & 4.42 & 4.83 & 5.14 & 5.21 & 5.27 & 5.03 & 4.45 & 3.06 & 2.22 & 1505.4 & 4.1 \\
\hline 60.0 & 2.65 & 3.20 & 3.82 & 4.07 & 4.37 & 4.57 & 4.69 & 4.88 & 4.83 & 4.42 & 3.10 & 2.26 & 1426.3 & 3.9 \\
\hline 70.0 & 2.61 & 3.08 & 3.57 & 3.57 & 3.78 & 3.92 & 4.07 & 4.38 & 4.52 & 4.28 & 3.07 & 2.25 & 1316.0 & 3.6 \\
\hline 80.0 & 2.52 & 2.90 & 3.25 & 3.21 & 3.13 & 3.26 & 3.40 & 3.79 & 4.10 & 4.03 & 2.97 & 2.19 & 1180.6 & 3.2 \\
\hline 96.0 & 2.36 & 2.64 & 2.86 & 2.65 & 2.57 & 2.56 & 2.72 & 3.14 & 3.60 & 3.69 & 2.79 & 2.07 & 1024.3 & 2.8 \\
\hline
\end{tabular}


AVERAGE IAALY TOTAL TERRESTRIAL INSOLATION ESTIMATES ( $\%$ WH/SQ. M)

SITE: MOLINE
LATITUDE: 41 DEGREES 27 MINUTES

AVERAGE DAILY AMOUNTS BY MONTH

\begin{tabular}{|c|c|c|c|c|c|c|c|c|c|c|c|c|c|c|}
\hline ARRAY & $\mathrm{J}$ & $\mathrm{F}$ & $M$ & $A$ & $M$ & J & J & A & 5 & 0 & $N$ & $D$ & $\begin{array}{l}\text { ANINUAL } \\
\text { APIOUNT }\end{array}$ & $\begin{array}{c}\text { AVERAGE } \\
\text { DAY }\end{array}$ \\
\hline 0.0 & 1.69 & 2.56 & 3.52 & 4.51 & 5.53 & 6.21 & 6.11 & 5.41 & 4.28 & 3.14 & 1.88 & 1.37 & 1410.5 & 3.9 \\
\hline 10.0 & 2.01 & 2.39 & 3.79 & 4.77 & 5.59 & 6.23 & 6.19 & 5.62 & 4.65 & 3.61 & 2.27 & 1.67 & 1501.5 & 4.1 \\
\hline $15 . \mathrm{c}$ & 2.16 & 3.03 & 3.90 & 4.31 & 5.58 & 6.19 & 6.17 & 5.69 & 4.80 & 3.81 & 2.45 & 1.81 & 1534.8 & 4.2 \\
\hline $20 . \mathrm{C}$ & 2.29 & 3.15 & 3.98 & 4.33 & 5.54 & 6.11 & 6.12 & 5.72 & 4.92 & 4.00 & 2.61 & 1.94 & 1559.5 & 4.3 \\
\hline 25.0 & 2.41 & 3.26 & 4.04 & 4.32 & 5.46 & 6.00 & 6.04 & 5.71 & 5.01 & 4.16 & 2.76 & 2.05 & 1575.5 & 4.3 \\
\hline 30.0 & 2.52 & 3.35 & 4.08 & 4.79 & 5.36 & 5.86 & 5.93 & 5.68 & 5.07 & 4.29 & 2.89 & 2.16 & 1582.5 & 4.3 \\
\hline 35.0 & 2.61 & $3 .<2$ & 4.10 & 4.73 & 5.23 & 5.69 & 5.78 & 5.61 & 5.10 & 4.40 & 3.00 & 2.25 & 1530.5 & 4.3 \\
\hline 40.0 & 2.68 & 3.46 & 4.10 & 4.64 & 5.07 & 5.49 & 5.60 & 5.51 & 5.11 & 4.48 & 3.09 & 2. 32 & 1569.6 & 4.3 \\
\hline 45.0 & 2.74 & 3.49 & 4.07 & 4.53 & 4.89 & 5.27 & 5.40. & 5.39 & 5.08 & 4.54 & 3.17 & 2.39 & 1551.2 & 4.2 \\
\hline 50.0 & 2.79 & 3.50 & 4.01 & $4 .<0$ & 4.70 & 5.03 & 5.18 & 5.23 & 5.02 & 4.58 & 3.23 & 2.44 & 1524.5 & 4.2 \\
\hline 60.0 & 2.82 & 3.46 & 3.85 & 4.18 & 4.24 & 4.48 & 4.65 & 4.84 & 4.82 & 4.53 & 3.28 & 2.49 & 1446.0 & 4.0 \\
\hline 70.0 & 2.78 & 3.33 & 3.59 & 3.67 & 3.70 & 3.84 & 4.04 & 4.34. & 4.50 & 4.38 & 3.24 & 2.48 & 1335.7 & 3.7 \\
\hline 80.0 & 2.68 & 3.13 & 3.26 & 3.19 & 3.11 & 3.19 & 3.37 & 3.78 & 4.08 & 4.13 & 3.13 & 2.41 & 1199.9 & 3.3 \\
\hline 90.0 & 2.51 & 2.86 & 2.87 & 2.66 & 2.51 & 2.51 & 2.69 & 3.10 & 3.58 & 3.78 & 2.94 & 2.28 & 1042.6 & 2.9 \\
\hline
\end{tabular}


LVERAGE DAILY TOTAL TERRESTRIAL INSOLATION ESTIMATES (KWH/SQ. M)

SITE: SPRINGFIELD

LATITUEE: 39. DEGREES 50 MINUTES

AVERAGE DAILY AMOUNTS BY MONTH

\begin{tabular}{|c|c|c|c|c|c|c|c|c|c|c|c|c|c|c|}
\hline ARRAY & $\frac{-1}{7}$ & $F$ & $M$ & $A$ & $M$ & J & $\mathbf{J}$ & r. & $s$ & 0 & $N$ & $D$ & $\begin{array}{l}\text { ANNUAL } \\
\text { ANOUNT }\end{array}$ & $\begin{array}{c}\text { AVERAGE } \\
\text { DAY }\end{array}$ \\
\hline 0.0 & 1.84 & 2.72 & 3.60 & 4.77 & 5.88 & 6.61 & 6.48 & 5.69 & 4.58 & 3.57 & 2.14 & 1.55 & 1499.8 & 4.1 \\
\hline 10.0 & 2.18 & 3.05 & 3.86 & 4.93 & 5.93 & E.61 & 6.55 & 5.91 & 4.97 & 3.86 & 2.57 & 1.88 & 1592.7 & 4.4 \\
\hline 15.0 & 2.33 & 3.19 & 3.96 & 4.97 & 5.91 & 6.55 & 6.52 & 5.97 & 5.12 & 4.07 & 2.76 & 2.03 & 1625.9 & 4.5 \\
\hline 20.0 & 2.47 & 3.31 & 4.03 & 4.98 & $5.8 E$ & 5.46 & 6.45 & 5.99 & 5.24 & 4.26 & $=.94$ & 2.17 & 1650.0 & 4.5 \\
\hline 25.0 & 2.59 & 3.42 & 4.09 & 4.97 & $5.7 \pi$ & 6.34 & 6.37 & 5.98 & 5.34 & 4.42 & 3.10 & 2.30 & 1664.6 & 4.6 \\
\hline 30.0 & 2.70 & 3.51 & 4.12 & 4.92 & 5.65 & 6.17 & 6.23 & 5.93 & 5.40 & 4.55 & 5.24 & 2.41 & 1669.8 & 4.6 \\
\hline 35.0 & 2.79 & 3.57 & 4.13 & 4.85 & 5.50 & 5.98 & 6.06 & 5.85 & 5.42 & 4.56 & 5.36 & 2.51 & 1665.4 & 4.6 \\
\hline 40.0 & 2.86 & 3.62 & 4.12 & 4.76 & 5.33 & 5.75 & 5.86 & 5.74 & 5.42 & 4.74 & 3.47 & 2.59 & 1651.6 & 4.5 \\
\hline 45.0 & 2.92 & 3.64 & 4.08 & 4.64 & 5.13 & 5.52 & 5.65 & 5.60 & 5.38 & 4.79 & 3.55 & ) 2.66 & 1630.2 & 4.5 \\
\hline 50.0 & 2.96 & 3.64 & 4.02 & 4.53 & 4.92 & 5.25 & 5.40 & 5.43 & 5.31 & 4.81 & 3.60 & 2.71 & 1599.7 & 4.4 \\
\hline 60.0 & 2.99 & 3.58 & 3.84 & 4.15 & 4.41 & 4.64 & 4.82 & 5.00 & 5.08 & 4.76 & 3.65 & 2.76 & 1511.8 & 4.1 \\
\hline 70.0 & 2.94 & 3.44 & 3.57 & 3.72 & 3.81 & 3.94 & 4.15 & 4.46 & 4.73 & 4.59 & 3.60 & 2.74 & 1390.7 & 3.8 \\
\hline 30.0 & 2.82 & 3.22 & 3.23 & 3.22 & 3.13 & 3.24 & 3.44 & 3.83 & 4.27 & 4.31 & 3.47 & 2.66 & 1243.8 & 3.4 \\
\hline 90.0 & 2.64 & 2.93 & 2.83 & 2.66 & 2.53 & 2.51 & 2.70 & 3.13 & 3.72 & 3.93 & 3.25 & 2.50 & 1074.1 & 2.9 \\
\hline
\end{tabular}


AVERAGE DAILY TOTAL TERRESTRIAL INSOLATION ESTIMATES (KWH/SQ. M)

SITE: EVANSVILLE
LATITUDE: 38 DEGREES 3 MINUTES

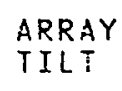

0.0

10.0

15.0

20.0

25.0

30.0

35.0

40.0

45.0

50.0

60.0

70.0

$8 C .0$

90.0

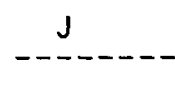

$1.81 \quad 2.59 \quad 3.62$

$2.09 \quad 2.37 \quad 3.85$

$2.22 \quad 2.99 \cdot 3.95$

2.33

3.09

2.43

3.17

4.02

4.06

4.0

2.523 .24

$2.59 \quad 3.29$

4.09

$2.65 \quad 3.12$

4.07

4.02

3.96

3.7

$2.73 \quad 3.25$

3.76

3.49

3.14

2.73

2. 36

2.61

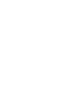

\section{AVERAGE DAILY AMOUNTS BY MONTH}


AVERAGE DAILY TOTAL TERRES-RIAL INSOLATION ESTIMATES (KWH/SQ. M) SITE: FORT'HAYNE
LATITUDE: I DEGREES O MINUTES

A $Z$ ERAGE JAILY AMOUNTS BY MONTH

\begin{tabular}{|c|c|c|c|c|c|c|c|c|c|c|c|c|c|c|}
\hline ARRAY & 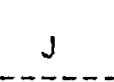 & $F$ & $M$ & A & $M$ & $\mathrm{~J}$ & $\jmath$ & $A$ & $s$ & 0 & $N$ & $D$ & $\begin{array}{l}\text { ANNUAL } \\
\text { ANOUNT }\end{array}$ & $\begin{array}{c}\text { AVERAGE } \\
\text { DAY }\end{array}$ \\
\hline 0.0 & 1.44 & 2.20 & 3.05 & 4.29 & 5.27 & 5.80 & 5.63 & 5.02 & 4.01 & 2.91 & 1.63 & 1.17 & 1294.2 & 3.5 \\
\hline 10.0 & 1.66 & 2.44 & $3.3 C$ & 4.43 & 5.33 & 5.81 & 5.69 & 5.21 & 4.33 & 3.32 & 1.92 & 1.38 & 1365.9 & 3.7 \\
\hline 15.0 & 1.77 & 2.55 & $3.3 \varepsilon$ & 4.46 & 5.51 & 5.77 & 5.67 & 5.26 & 4.46 & 3.49 & 2.06 & 1.47 & 1390.9 & 3.8 \\
\hline 20.0 & 1.86 & 2.64 & 3.45 & 4.48 & 5.27 & 5.70 & 5.6 .3 & 5.28 & 4.56 & 3.65 & 2.18 & 1.56 & 1408.4 & 3.9 \\
\hline 25.0 & 1.94 & 2.71 & 3.49 & 4.46 & 5.20 & 5.59 & 5.55 & 5.27 & 4.64 & 3.78 & 2.29 & 1.64 & 1418.2 & 3.9 \\
\hline 30.0 & 2.01 & 2.77 & $3.5 \mathrm{a}$ & 4.43 & 5.10 & 5.46 & 5.44 & 5.23 & 4.69 & 3.89 & 2.38 & 1.71 & 1420.2 & 3.9 \\
\hline 35.0 & 2.07 & 2.82 & 3.52 & 4.37 & 4.97 & 5.30 & 5.30 & 5.17 & 4.71 & 3.98 & 2.46 & 1.77 & 1414.4 & 3.9 \\
\hline 40.0 & 2.12 & 2.85 & $3.5 !$ & 4.29 & 4.82 & 5.11 & 5.14 & 5.07 & 4.70 & 4.04 & 2.53 & 1.82 & 1400.8 & 3.8 \\
\hline 45.0 & 2.15 & 2.86 & 3.43 & 4.19 & 4.65 & 4.91 & 4.96 & 4.95 & 4.67 & 4.08 & 2.58 & 1.36 & 1381.0 & 3.8 \\
\hline 50.0 & 2.18 & 2.86 & 3.43 & 4.07 & 4.46 & 4.69 & 4.75 & 4.81 & 4.61 & 4.10 & 2.62 & 1.89 & 1353.9 & 3.7 \\
\hline 60.0 & 2.19 & 2.81 & 3.23 & 3.76 & $4 \cdot[2$ & 4.18 & 4.28 & 4.44 & 4.42 & 4.06 & 2.64 & 1.91 & 1278.2 & 3.5 \\
\hline 70.0 & 2.15 & 2.69 & 3.05 & 3.38 & 3.51 & 3.60 & 3.72 & 3.99 & 4.12 & 3.92 & 2.61 & 1.89 & 1175.8 & 3.2 \\
\hline 80.0 & 2.06 & 2.52 & 2.77 & 2.94 & 2.96 & 3.00 & 3.12 & 3.46 & 3.73 & 3.68 & 2.51 & 1.83 & 1052.2 & 2.9 \\
\hline 90.0 & 1.92 & 2.30 & 2.44 & 2.46 & 2.39 & 2.37 & 2.51 & 2.86 & 3.27 & 3.36 & 2.35 & 1.72 & 910.9 & 2.5 \\
\hline
\end{tabular}


AVERAGE DAILY TOTAL TERRESTRIAL INSOLATION ESTIMATES (KWH'SR. M)

SITE: INDIANAFOLIS M IN
LATITUDE: 39 DEGPEES 44 MINUTES

AVERAGE DAILY AMOUNTS BY MONTH

\begin{tabular}{|c|c|c|c|c|c|c|c|c|c|c|c|c|c|c|}
\hline ARRAY & $\mathrm{J}$ & $F$ & $M$ & A & $M$ & J & $\mathrm{J}$ & A & $s$ & 0 & 1 & $D$ & $\begin{array}{l}\text { ANNUAL } \\
\text { AMIOUNT }\end{array}$ & $\begin{array}{c}\text { AVERAGE } \\
\text { DAY }\end{array}$ \\
\hline 0.0 & 1.56 & 2.35 & 3.26 & 4.41 & 5.33 & 5.89 & 5.69 & 5.18 & 4.17 & 3.08 & 1.83 & 1.32 & 1343.4 & 3.7 \\
\hline 10.0 & 1.81 & 2.61 & 3.48 & 4.55 & 5.37 & 5.89 & 5.74 & 5.35 & 4.50 & 3.50 & 2.16 & 1.56 & 1417.3 & 3.9 \\
\hline 15.0 & 1.91 & 2.72 & 3.57 & 4.58 & 5.35 & 5.84 & 5.72 & 5.41 & 4.63 & 3.67 & 2.31 & 1.66 & 1442.9 & 4.0 \\
\hline 20.0 & 2.01 & 2.81 & 3.63 & 4.59 & 5.30 & 5.76 & 5.66 & 5.43 & 4.73 & 3.83 & 2.44 & 1.76 & 1460.6 & 4.0 \\
\hline 25.0 & 2.10 & 2.89 & 3.67 & 4.57 & 5.22 & 5.65 & 5.58 & 5.41 & 4.80 & 3.97 & 2.56 & 1.85 & 1470.3 & 4.0 \\
\hline 30.0 & 2.17 & 2.95 & 3.70 & 4.53 & 5.11 & 5.51 & 5.46 & 5.37 & 4.84 & 4.08 & 2.67 & 1.93 & 1471.8 & 4.0 \\
\hline 35.0 & 2.24 & 3.00 & 3.70 & 4.47 & 4.98 & 5.34 & 5.32 & 5.29 & 4.86 & 4.16 & 2.76 & 2.00 & 1465.3 & 4.0 \\
\hline 40.0 & 2.29 & 3.03 & 3.68 & 4.38 & 4.82 & 5.14 & 5.15 & 5.19 & 4.85 & 4.23 & 2.83 & 2.06 & 1450.7 & 4.0 \\
\hline 45.0 & 2.32 & 3.04 & 3.65 & 4.27 & 4.65 & 4.94 & 4.96 & 5.06 & 4.81 & 4.26 & 2.89 & 2.10 & 1429.8 & 3.9 \\
\hline 50.0 & 2.35 & 3.04 & 3.59 & 4.14 & 4.46 & 4.71 & 4.75 & 4.91 & 4.75 & 4.27 & 2.93 & 2.13 & 1401.1 & 3.8 \\
\hline 60.0 & 2.35 & 2.98 & 3.42 & 3.82 & 4.01 & 4.18 & 4.26 & 4.52 & 4.53 & 4.22 & 2.95 & 2.16 & 1321.1 & 3.6 \\
\hline 70.0 & 2.31 & 2.85 & 3.18 & 3.43 & 3.48 & 3.58 & 3.69 & 4.04 & 4.22 & 4.06 & 2.91 & 2.13 & 1213.4 & 3.3 \\
\hline 80.0 & 2.20 & 2.66 & 2.88 & 2.97 & 2.92 & 2.97 & 3.08 & 3.48 & 3.81 & 3.81 & 2.79 & 2.06 & 1084.3 & 3.0 \\
\hline 90.0 & 2.05 & 2.42 & 2.52 & 2.46 & 2.35 & 2.34 & 2.46 & 2.86 & 3.32 & 3.47 & 2.61 & 1.94 & 936.5 & 2.6 \\
\hline
\end{tabular}


AVERAGE DAILY TOTAL TERRESTRIAL INSOLATION ESTIMATES (KWH/SQ. M)

SITE: SOUTH BEND

LATITUDE: 41 DEGREES 42 MINUTES

AVERAEE DAILY AMOUNTS BY MONTH

\begin{tabular}{|c|c|c|c|c|c|c|c|c|c|c|c|c|c|c|}
\hline $\begin{array}{l}\text { ARRAY } \\
\text { TILT }\end{array}$ & $-J^{-}$ & $F$ & $M$ & A & $M$ & $J$ & $J$ & A & $\mathrm{S}$ & 0 & $N$ & D & $\begin{array}{l}\text { ANNUAL } \\
\text { AMOUNT }\end{array}$ & $\begin{array}{c}\text { AVERAGE } \\
\text { DAY }\end{array}$ \\
\hline 0.0 & 1.31 & 2.08 & 3.12 & 4.38 & 5.43 & 6.06 & 5.84 & 5.26 & 4.06 & 2.86 & 1.57 & 1.08 & 1312.4 & 3.6 \\
\hline 10.0 & 1.51 & 2.30 & 3.35 & 4.53 & 5.49 & 6.68 & 5.91 & 5.47 & 4.41 & 3.27 & 1.85 & 1.26 & 1385.2 & 3.8 \\
\hline 15.0 & 1.60 & 2.40 & 3.43 & 4.57 & 5.48 & 6.04 & 5.90 & 5.53 & 4.54 & 3.45 & 1.97 & 1.35 & 1410.6 & 3.9 \\
\hline 20.0 & 1.68 & 2.48 & 3.50 & 4.59 & 5.44 & 5.97 & 5.85 & 5.56 & 4.65 & 3.61 & 2.11 & 1.43 & 1428.2 & 3.9 \\
\hline 25.0 & 1.75 & 2.55 & 3.55 & 4.58 & 5.36 & 5.85 & 5.78 & 5.56 & 4.74 & 3.75 & 2.22 & 1.50 & 1438.0 & 3.9 \\
\hline 30.0 & 1.81 & 2.61 & 3.58 & 4.55 & 5.26 & 5.73 & 5.67 & 5.52 & 4.79 & $3: 86$ & $2.3 ?$ & 1.56 & 1439.8 & 3.9 \\
\hline 35.0 & 1.87 & 2.65 & 3.59 & 4.49 & 5.14 & 5.55 & 5.53 & 5.46 & 4.82 & 3.95 & 2.43 & 1.61 & 1433.7 & 3.9 \\
\hline 40.0 & 1.91 & 2.68 & 3.58 & 4.41 & 4.98 & 5.35 & 5.36 & 5.36 & 4.82 & 4.02 & 2.47 & 1.66 & 1419.7 & 3.9 \\
\hline 45.0 & 1.94 & 2.69 & 3.55 & 4.31 & 4.81 & 5.15 & 5.17 & 5.24 & 4.79 & 4.06 & 2.52 & 1.69 & 1399.0 & 3.8 \\
\hline 50.0 & 1.96 & 2.69 & 3.50 & 4.18 & 4.62 & 4.92 & 4.96 & 5.09 & 4.74 & 4.08 & $2.5 i$ & 1.72 & 1371.4 & 3.8 \\
\hline 60.0 & 1.96 & 2.64 & .3 .35 & 3.88 & 4.17 & 4.39 & 4.47 & 4.71 & 4.55 & 4.05 & 2.53 & 1.74 & 1293.9 & 3.5 \\
\hline $7 n .0$ & 1.93 & 2.54 & 3.13 & 3.49 & 3.64 & 3.73 & 3.89 & 4.23 & 4.25 & 3.91 & 2.55 & 1.72 & 1189.2 & 3.3 \\
\hline 80.0 & 1.85 & 2.38 & 2.85 & -3.04 & 3.07 & 3.14 & 3.26 & 3.67 & 3.86 & 3.68 & 2.45 & 1.66 & 1062.7 & 2.9 \\
\hline 90.0 & 1.72 & 2.17 & 2.51 & 2.54 & $\therefore .48$ & 2.4 .3 & 2.62 & 3.04 & 3.39 & 3.37 & 2.33 & 1.56 & 918.6 & 2.5 \\
\hline
\end{tabular}


AUERAGE DAILY TOTAL TERRESTRIAL INSOLATION ESTIMATES (KIUH/SQ. M)

SITE: DODGE CITY KS

LATITUDE: 37 DEGREES 46 MINUTES

AVERAGE DAILY AMOUNTS BY MONTH

\begin{tabular}{|c|c|c|c|c|c|c|c|c|c|c|c|c|c|c|}
\hline ARRAY & $J$ & $F$ & $M$ & $A$ & KAGE & 11 L A & $\mathrm{J}$ & merr & $S$ & 0 & $N$ & $D$ & $\begin{array}{l}\text { ANNUAL } \\
\text { ANOUNT }\end{array}$ & $\begin{array}{c}\text { AVERAGE } \\
\text { DAY }\end{array}$ \\
\hline 0.0 & 2.61 & 3.54 & 4.65 & 5.94 & 6.59 & 7.44 & 7.24 & 6.43 & 5.32 & 4.10 & 2.82 & 2.31 & 1797.8 & 4.9 \\
\hline 10.0 & 3.13 & 4.01 & 5.02 & 6.15 & 6.63 & 7.41 & 7.29 & 6.71 & 5.77 & 4.71 & 3.42 & 2.87 & 1921.7 & 5.3 \\
\hline 15.0 & 3.36 & 4.21 & 5.16 & 6.19 & 6.60 & 7.33 & 7.25 & 6.77 & 5.94 & 4.98 & 3.69 & 3.12 & 1966.9 & 5.4 \\
\hline 20.0 & 3.58 & 4.38 & 5.26 & 6.21 & 6.52 & 7.22 & 7.16 & 6.79 & 6.08 & 5.21 & $3 . \subseteq 4$ & 3.36 & 2000.5 & 5.5 \\
\hline 25.0 & 3.77 & 4.53 & 5.34 & 6.18 & 6.41 & 7.05 & 7.04 & 6.77 & 6.19 & 5.41 & 4.16 & 3.57 & 2022.1 & 5.5 \\
\hline 30.0 & 3.94 & 4.65 & 5.39 & 6.12 & 6.26 & 6.85 & 6.87 & 6.71 & 6.25 & 5.58 & $4 . \pm 6$ & 3.76 & 2031.7 & 5.6 \\
\hline 35.0 & 4.08 & 4.74 & 5.40 & 6.03 & 6.08 & 6.61 & 6.67 & 6.61 & 6.28 & 5.71 & $4 \cdot 5 \cdot 3$ & 3.93 & 2029.2 & 5.6 \\
\hline 40.0 & 4.20 & 4.81 & 5.39 & 5.90 & 5.87 & 6.34 & 6.43 & 6.47 & 6.26 & 5.81 & $4 . \in 7$ & 4.07 & 2015.6 & 5.5 \\
\hline 45.0 & 4.29 & 4.84 & 5.34 & 5.75 & 5.64 & 6.06 & 6.17 & 6.30 & 6.21 & 5.87 & 4.78 & 4.19 & 1991.3 & 5.5 \\
\hline 50.0 & 4.36 & 4.85 & 5.26 & 5.56 & 5.38 & 5.74 & 5.88 & 6.09 & 6.12 & 5.89 & $4.8: 6$ & 4.28 & 1955.1 & 5.4 \\
\hline 60.0 & 4.40 & 4.77 & 5.01 & 5.09 & 4.78 & 5.00 & 5.20 & 5.57 & 5.83 & 5.82 & 4.92 & 4.37 & 1848.5 & 5.1 \\
\hline 70.0 & 4.34 & 4.57 & 4.64 & 4.51 & 4.08 & 4.18 & 4.41 & 4.92 & 5.40 & 5.60 & 4.86 & 4.35 & 1699.3 & 4.7 \\
\hline 80.0 & 4.16 & 4.27 & 4.17 & 3.84 & 3.35 & 3.36 & 3.59 & 4.17 & 4.85 & 5.24 & 4.6 .6 & 4.22 & 1517.2 & 4.2 \\
\hline 90.0 & 3.88 & 3.86 & 3.61 & 3.11 & 2.60 & $2.5 j$ & 2.73 & 3.35 & 4.18 & 4.75 & 4.35 & 3.97 & 1305.2 & 3.6 \\
\hline
\end{tabular}


AVERAGE DAILY TOTAL TERRESTRIAL INSOLATION ESTIMATES (KWH/SQ. M)

SITE: GOODLAND $\quad K S$

AVERAGE DAILY AMOUNTS BY MONTH

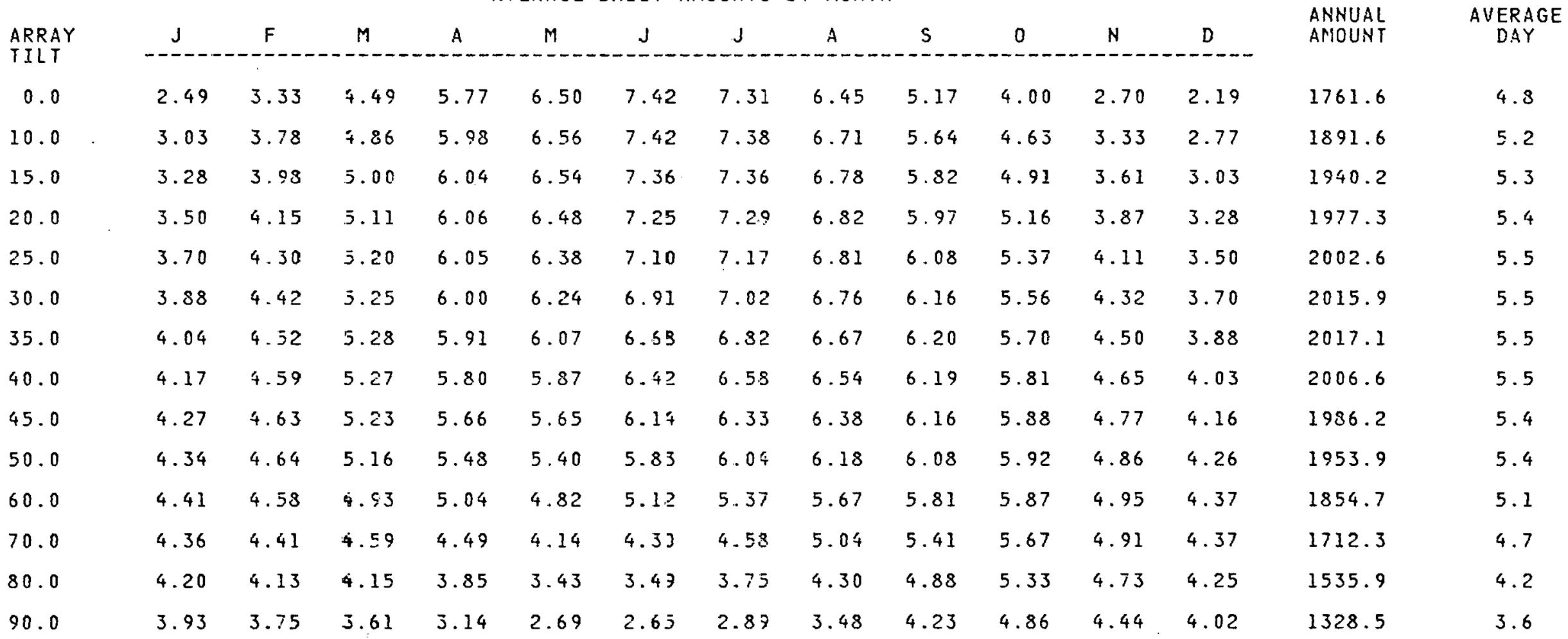


AVERAGE DAILY TOTAL TERRESTRIAL INSOLATION ESTIMATES (KIUH/SQ. N)

SITE: TOPEKA LATITUDE: 39 DEGREES 4 MINUTES

AUERAGE DAILY AMOUNTS BY MONTH

\begin{tabular}{|c|c|c|c|c|c|c|c|c|c|c|c|c|c|c|}
\hline ARRAY & $\mathrm{J}$ & $F$ & $M$ & A & $M$ & $J$ & $\mathrm{~J}$ & A & $s$ & 0 & $N$ & $D$ & $\begin{array}{l}\text { ANNUAL } \\
\text { AMOUNT }\end{array}$ & $\begin{array}{c}\text { AVERAGE } \\
\text { DAY }\end{array}$ \\
\hline 0.0 & 2.15 & 2.96 & 3.96 & 5.18 & 6.04 & 6.71 & 6.71 & 6.02 & 4.78 & 3.61 & $2 . \mp 4$ & 1.84 & 1596.6 & 4.4 \\
\hline 10.0 & 2.56 & 3.33 & 4.25 & 5.35 & 6.09 & 6.70 & 6.77 & 6.25 & 5.18 & 4.14 & 2.75 & 2.26 & 1700.8 & 4.7 \\
\hline 15.0 & 2.74 & 3.49 & 4.36 & 5.39 & 6.06 & 6.64 & 6.74 & 6.30 & 5.34 & 4.37 & 3.18 & 2.46 & 1738.6 & 4.8 \\
\hline 20.0 & 2.91 & 3.63 & 4.45 & 5.41 & 6.00 & 6.54 & 6.68 & 6.33 & 5.46 & 4.57 & 3.39 & 2.63 & 1766.4 & 4.8 \\
\hline 25.0 & 3.06 & 3.75 & 4.51 & 5.39 & 5.91 & 6.41 & 6.57 & 6.31 & 5.55 & 4.75 & 3.58 & 2.79 & 1784.0 & 4.9 \\
\hline 30.0 & 3.19 & 3.84 & 4.55 & $5.3^{\prime}$ & 5.78 & 6.24 & 6.43 & 6.26 & 5.61 & 4.89 & 3.75 & 2.94 & 1791.3 & 4.9 \\
\hline 35.0 & 3.31 & 3.91 & 4.56 & 5.26 & 5.63 & 6.04 & 6.25 & 6.17 & 5.64 & 5.01 & 3.90 & 3.06 & 1788.1 & 4.9 \\
\hline 40.0 & 3.40 & 3.96 & 4.55 & 5.16 & 5.44 & 5.80 & 6.04 & 6.05 & 5.63 & 5.09 & 4.02 & 3.17 & 1775.0 & 4.9 \\
\hline 45.0 & 3.47 & 3.99 & 4.51 & 5.03 & 5.24 & 5.56 & 5.81 & 5.90 & 5.59 & 5.14 & 4.11 & 3.25 & 1753.3 & 4.8 \\
\hline 50.0 & 3.52 & 3.99 & 4.44 & 4.87 & 5.01 & 5.28 & 5.54 & 5.71 & 5.51 & 5.16 & 4.18 & 3.32 & 1721.5 & 4.7 \\
\hline 60.0 & 3.56 & 3.93 & 4.23 & 4.48 & 4.48 & 4.65 & 4.9 .4 & 5.25 & 5.26 & 5.11 & 4.24 & 3.39 & 1628.7 & 4.5 \\
\hline 70.0 & 3.50 & 3.77 & 3.94 & 4.00 & 3.86 & 3.94 & 4.23 & 4.67 & 4.89 & 4.92 & 4.19 & 3.37 & 1499.3 & 4.1 \\
\hline 80.0 & 3.36 & 3.52 & 3.55 & 3.44 & 3.21 & 3.22 & 3.48 & 3.99 & 4.41 & 4.62 & 4.03 & 3.27 & 1341.8 & 3.7 \\
\hline 90.0 & 3.14 & 3.20 & 3.10 & 2.83 & 2.53 & 2.48 & 2.71 & 3.24 & 3.83 & 4.20 & 3.77 & 3.08 & 1158.7 & 3.2 \\
\hline
\end{tabular}


AVERAGE DAILY TOTAL TERRESTRIAL INSOLATION ESTIMATES (KWH/SQ. M)

$$
\begin{gathered}
\text { SITE: } \\
\text { LATITUDE: } \\
37 \text { DEGTA } \\
\text { DERES } 39 \text { MINUTES }
\end{gathered}
$$

AUERAGE IAILY AMOUNTS EY MONTH

\begin{tabular}{|c|c|c|c|c|c|c|c|c|c|c|c|c|c|c|}
\hline ARRAY & 1 & $F$ & $M$ & $A$ & $M$ & $J$ & $\int^{-2}$ & A & $s$ & 0 & $N$ & $D$ & $\begin{array}{l}\text { ANNUAL } \\
\text { AMOUNT }\end{array}$ & $\begin{array}{c}\text { AVERAGE } \\
\text { DAY }\end{array}$ \\
\hline 0.0 & 2.47 & 3.34 & $4 .<2$ & 5.62 & 6.42 & 7.14 & 7.06 & 6.41 & 5.09 & 3.94 & 2.75 & 2.18 & 1731.4 & 4.7 \\
\hline 10.0 & 2.94 & 3.76 & 4.75 & 5.80 & 6.46 & 7.12 & 7.10 & 6.64 & 5.51 & 4.50 & $3.3 ?$ & 2.68 & 1845.0 & 5.1 \\
\hline 15.0 & 3.15 & 3.93 & $\dot{4} .88$ & 5.84 & 6.42 & 7.04 & 7.06 & 6.70 & 5.67 & 4.75 & 3.53 & 2.91 & 1885.9 & 5.2 \\
\hline 20.0 & 3.35 & 4.09 & 4.58 & 5.85 & 6.35 & 6.93 & 6.93 & 6.71 & 5.79 & 4.96 & 3.81 & 3.11 & 1915.8 & 5.2 \\
\hline 25.0 & 3.52 & 4.22 & 5.04 & 5.83 & 6.24 & 6.77 & 6.86 & 6.69 & 5.88 & 5.15 & $4.0 ?$ & 3.31 & 1934.4 & 5.3 \\
\hline 30.0 & 3.67 & 4.33 & 5.68 & 5.77 & 6.10 & 6.53 & 6.70 & 6.63 & 5.94 & 5.30 & 4.21 & 3.48 & 1941.7 & 5.3 \\
\hline 35.0 & 3.80 & 4.41 & 5. C9 & 5.68 & 5.92 & 6.35 & 6.50 & 6.53 & 5.96 & 5.42 & 4.37 & 3.62 & 1937.5 & 5.3 \\
\hline 40.0 & 3.90 & 4.46 & 5.88 & 5.56 & 5.72 & 6.10 & 6.27 & 6.39 & 5.94 & 5.51 & $4.5)$ & 3.75 & 1322.9 & 5.3 \\
\hline 45.0 & 3.98 & 4.49 & 5.03 & 5.41 & 5.50 & 5.33 & 6.02 & 6.22 & 5.89 & 5.56 & 4.61 & 3.85 & 1398.2 & 5.2 \\
\hline 50.0 & 4.04 & 4.49 & 4.55 & 5.23 & 5.24 & 5.52 & 5.73 & 6.01 & 5.80 & 5.57 & 4.63 & 3.93 & 1862.3 & 5.1 \\
\hline 60.0 & 4.07 & 4.41 & 4.71 & 4.79 & 4.66 & 4.32 & 5.07 & 5.50 & 5.53 & 5.50 & 4.73 & 4.00 & 1758.4 & 4.8 \\
\hline 70.0 & 4.00 & 4.22 & 4.36 & 4.25 & 3.98 & 4.05 & 4.30 & 4.86 & 5.12 & 5.29 & $4.6 ?$ & 3.98 & 1614.7 & 4.4 \\
\hline 80.0 & 3.83 & 3.94 & 3.92 & 3.63 & 3.28 & 3.27 & 3.51 & 4.12 & 4.59 & 4.94 & 4.48 & 3.85 & 1440.4 & 3.9 \\
\hline 90.0 & 3.57 & 3.56 & 3.40 & 2.95 & 2.54 & 2.43 & 2.68 & 3.31 & 3.96 & 4.48 & 4.18 & 3.62 & 1238.4 & 3.4 \\
\hline
\end{tabular}


AYERAGE DAIL TOTAL TERRESTRIAL INSOLATION ESTIMATES (KWH/SQ. M)

$\begin{array}{rlr}\text { SITE: } & \text { LEXINGTON } \\ \text { LATETUDE: } & 38 \text { DEGREES } 2 \text { MINUTES }\end{array}$

A EERAGE DAILY AMOUNTS BY MONTH

\begin{tabular}{|c|c|c|c|c|c|c|c|c|c|c|c|c|c|c|}
\hline ARRAY & 3 & $F$ & $M$ & A & $M$ & $J$ & $\mathrm{~J}$ & A & $s$ & 0 & $N$ & $D$ & $\begin{array}{l}\text { ANNUAL } \\
\text { AMOUNT }\end{array}$ & $\begin{array}{c}\text { AVERAGE } \\
\text { DAY }\end{array}$ \\
\hline 0.0 & 1.72 & 2.46 & 3.46 & 4.66 & 5.50 & 5.98 & 5.84 & 5.32 & 4.29 & 3.29 & 2.37 & 1.53 & 1405.2 & 3.8 \\
\hline 10.0 & 1.98 & 2.71 & 3.68 & 4.79 & 5.53 & 5.96 & 5.87 & 5.48 & 4.60 & 3.72 & 2.74 & 1.81 & 1479.9 & 4.1 \\
\hline 15.0 & 2.09 & 2.82 & 3.76 & 4.82 & 5.50 & 5.90 & 5.84 & 5.52 & 4.72 & 3.90 & 2.50 & 1.94 & 1505.3 & 4.1 \\
\hline 20.0 & 2.19 & 2.91 & 3.83 & 4.82 & 5.44 & 5.81 & 5.77 & 5.53 & 4.81 & 4.06 & 2.75 & 2.06 & 1522.4 & 4.2 \\
\hline 25.0 & 2.28 & 2.98 & 3.87 & 4.80 & 5.35 & 5.69 & 5.68 & 5.51 & 4.88 & 4.20 & 2.38 & 2.16 & 1531.1 & 4.2 \\
\hline 30.0 & 2.36 & 3.04 & 3.89 & 4.75 & 5.24 & 5.54 & 5.55 & 5.45 & 4.91 & 4.31 & 3.00 & 2.25 & 1531.3 & 4.2 \\
\hline 35.0 & 2.43 & 3.03 & 3.89 & 4.68 & 5.09 & 5.36 & 5.39 & 5.37 & 4.92 & 4.39 & 3.09 & 2.33 & 1523.0 & 4.2 \\
\hline 40.0 & 2.48 & 3.10 & 3.86 & 4.58 & 4.92 & 5.15 & 5.21 & 5.26 & 4.90 & 4.45 & 3.17 & 2.40 & 1507.0 & 4.1 \\
\hline 45.0 & 2.51 & 3.11 & 3.82 & 4.46. & 4.74 & 4.94 & 5.01 & 5.12 & 4.85 & 4.48 & 3.23 & 2.45 & 1483.6 & 4.1 \\
\hline 50.0 & 2.53 & 3.10 & 3.76 & 4.31 & 4.53 & 4.70 & 4.79 & 4.95 & 4.78 & 4.48 & 3.27 & 2.48 & 1452.1 & 4.0 \\
\hline 60.0 & 2.53 & 3.03 & 3.57 & 3.96 & 4.05 & 4.14 & 4.27 & 4.54 & 4.55 & 4.41 & 3.29 & 2.51 & 1365.8 & 3.7 \\
\hline 70.0 & 2.47 & 2.89 & 3.31 & 3.54 & 3.50 & 3.53 & 3.67 & 4.04 & 4.21 & 4.23 & 3.23 & 2.48 & 1250.8 & 3.4 \\
\hline 90.0 & 2.36 & 2.69 & 2.98 & 3.04 & 2.92 & 2.90 & 3.05 & 3.46 & 3.79 & 3.96 & 3.09 & 2.39 & 1114.3 & 3.1 \\
\hline 90.0 & 2.19 & 2.43 & 2.60 & 2.51 & 2.32 & 2.27 & 2.40 & 2.82 & 3.28 & 3.59 & 2.88 & 2.24 & 958.6 & 2.6 \\
\hline
\end{tabular}


AVERAGE DAILY TOTAL TERRESTRIAL INSOLATION ESTIMATES (KWH/SQ. M)

SITE: LOUISUILLE
LATITUDE: 38 DEGREES 12 MINUTES

AVERAGE DAILY AMOUNTS BY MONTH

\begin{tabular}{|c|c|c|c|c|c|c|c|c|c|c|c|c|c|c|}
\hline ARRAY & $\mathrm{J}$ & $F$ & $M$ & $A$ & $M$ & $J$ & $\mathrm{~J}$ & A & 5 & 0 & $N$ & $\dot{D}$ & $\begin{array}{l}\text { ANNUAL } \\
\text { AMOUNT }\end{array}$ & $\begin{array}{c}\text { AVERAGE } \\
\text { DAY }\end{array}$ \\
\hline 0.0 & 1.72 & 2.49 & 3.47 & 4.63 & 5.42 & 6.00 & 5.73 & 5.30 & 4.29 & 3.28 & 2.06 & 1.54 & 1401.1 & 3.8 \\
\hline 10.0 & 1.98 & 2.75 & 3.69 & 4.76 & 5.45 & 5.98 & 5.82 & 5.47 & 4.60 & 3.71 & 2.42 & 1.83 & 1476.6 & 4.0 \\
\hline 15.0 & 2.10 & 2.86 & 3.78 & 4.79 & 5.42 & 5.93 & 5.79 & 5.51 & 4.72 & 3.89 & 2.58 & 1.96 & 1502.4 & 4.1 \\
\hline 20.0 & 2.20 & 2.95 & 3.34 & 4.79 & 5.37 & 5.84 & 5.73 & 5.52 & 4.82 & 4.05 & 2.73 & 2.07 & 1520.0 & 4.2 \\
\hline 25.0 & 2.29 & 3.03 & 3.88 & 4.77 & 5.28 & 5.72 & 5.63 & 5.50 & 4.88 & 4.19 & 2.86 & 2.18 & 1529.1 & 4.2 \\
\hline 30.0 & 2.37 & 3.09 & 3.90 & 4.72 & 5.16 & 5.56 & 5.51 & 5.44 & 4.92 & 4.30 & 2.98 & 2.28 & 1529.8 & 4.2 \\
\hline 35.0 & 2.44 & 3.13 & 3.90 & 4.64 & 5.02 & 5.38 & 5.36 & 5.36 & 4.93 & 4.38 & 3.08 & 2.36 & 1521.9 & 4.2 \\
\hline 40.0 & 2.49 & 3.16 & 3.88 & 4.55 & 4.86 & 5.18 & 5.18 & 5.25 & 4.91 & 4.44 & 3.15 & 2.42 & 1506.3 & 4.1 \\
\hline 45.0 & 2.53 & 3.17 & 3.84 & 4.43 & 4.68 & 4.96 & 4.98 & 5.11 & 4.86 & 4.47 & 3.21 & 2.48 & 1483.4 & 4.1 \\
\hline 50.0 & 2.55 & 3.16 & 3.78 & 4.29 & 4.47 & 4.72 & 4.76 & 4.95 & 4.79 & 4.48 & 3.25 & 2.51 & 1452.3 & 4.0 \\
\hline 60.0 & 2.55 & 3.09 & 3.59 & 3.94 & 4.00 & 4.17 & 4.25 & 4.54 & 4.56 & 4.41 & 3.28 & 2.54 & 1366.9 & 3.7 \\
\hline 70.0 & 2.49 & 2.95 & 3.33 & 3.52 & 3.46 & 3.55 & 3.66 & 4.04 & 4.22 & 4.23 & 3.22 & 2.51 & 1252.7 & 3.4 \\
\hline 80.0 & 2.38 & 2.74 & 3.00 & 3.03 & 2.89 & 2.92 & 3.04 & 3.46 & 3.30 & 3.95 & 3.08 & 2.42 & 1116.9 & 3.1 \\
\hline 90.0 & 2.21 & 2.48 & 2.61 & 2.50 & 2.30 & 2.28 & 2.40 & 2.82 & 3.29 & 3.59 & 2.87 & 2.27 & 961.7 & 2.6 \\
\hline
\end{tabular}


AVERAGE DAILY TOTAL TERRESTRIAL INSOLATIOH ESTIMATES (KWH/SQ. M)

SITE: BATON ROUGE

LATITUDE: 30 DEGREES 32 MINUTES

AVERAGE DAILY AMOUNTS BY MONTH

\begin{tabular}{|c|c|c|c|c|c|c|c|c|c|c|c|c|c|c|}
\hline ARRAY & $J$ & $F$ & $M$ & A & $M$ & $J$ & 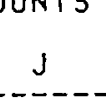 & rom & 5 & 0 & $\boldsymbol{k}$ & D & $\begin{array}{l}\text { ANNUAL } \\
\text { ANOUNT }\end{array}$ & $\begin{array}{c}\text { AVERAGE } \\
\text { DAY }\end{array}$ \\
\hline 0.0 & 2.47 & 3.53 & 4.35 & 5.30 & 5.89 & 6.07 & 5.50 & 5.29 & 4.61 & 4.11 & 2.89 & 2.32 & 1597.4 & 4.3 \\
\hline 10.0 & 2.78 & 3.62 & 4.56 & 5.38 & 5.85 & 5.97 & 5.45 & 5.37 & 4.85 & 4.53 & 3.30 & 2.67 & 1654.3 & 4.5 \\
\hline 15.0 & 2.91 & 3.74 & 4.64 & 5.38 & 5.78 & 5.87 & 5.37 & 5.37 & 4.93 & 4.70 & 3.47 & 2.82 & 1674.1 & 4.6 \\
\hline 20.0 & 3.03 & 3.83 & 4.68 & 5.35 & 5.67 & 5.74 & 5.29 & 5.34 & 4.98 & 4.85 & 3.53 & 2.96 & 1684.8 & 4.5 \\
\hline 25.0 & 3.13 & 3.91 & 4.71 & 5.28 & 5.54 & 5.58 & 5.17 & 5.28 & 5.00 & 4.97 & 3.76 & 3.08 & 1686.1 & 4.6 \\
\hline 30.0 & 3.21 & 3.96 & 4.70 & 5.19 & 5.37 & 5.38 & 5.02 & 5.19 & 5.00 & 5.05 & 3.87 & 3.19 & 1678.0 & 4.6 \\
\hline 35.0 & 3.28 & 3.99 & 4.67 & 5.08 & 5.19 & 5.18 & 4.85 & 5.07 & 4.96 & 5.11 & 3.96 & 3.27 & 1661.9 & 4.6 \\
\hline 40.0 & 3.32 & 4.00 & 4.61 & c. .94 & 4.98 & 4.94 & 4.66 & 4.93 & 4.90 & 5.14 & 4.03 & 3.34 & 1636.7 & 4.5 \\
\hline 45.0 & 3.34 & 3.98 & 4.53 & 4.77 & 4.75 & 4.69 & 4.45 & 4.76 & 4.82 & 5.13 & 4.07 & 3.38 & 1602.6 & 4.4 \\
\hline 50.0 & 3.35 & 3.95 & 4.42 & 4.58 & 4.49 & 4.41 & 4.21 & 4.57 & $4.70^{\circ}$ & 5.10 & 4.08 & 3.41 & 1559.8 & 4.3 \\
\hline 60.0 & 3.30 & 3.81 & 4.14 & 4.12 & 3.92 & 3.79 & 3.68 & 4.11 & 4.40 & 4.94 & 4.04 & 3.40 & 1449.3 & 4.0 \\
\hline 70.0 & 3.18 & 3.58 & 3.77 & 3.59 & 3.29 & 3.15 & 3.11 & 3.58 & 4.00 & 4.66 & 3.90 & 3.31 & 1311.7 & 3.6 \\
\hline 30.0 & 2.99 & 3.28 & 3.32 & 3.00 & 2.65 & 2.48 & 2.52 & 2.98 & 3.52 & 4.28 & 3.67 & 3.14 & 1150.2 & 3.2 \\
\hline 90.0 & 2.73 & 2.91 & 2.82 & 2.39 & 2.03 & 1.89 & 1.95 & 2.37 & 2.97 & 3.79 & 3.36 & 2.89 & 975.7 & 2.7 \\
\hline
\end{tabular}


AVERAGE DAILY TOTAL TERRESTRIAL INSOLATION ESTIMATES :KWH/SQ. M)

$$
\begin{aligned}
& \text { SITE: LARE CHARLES } \\
& \text { LATITUDE: } 50 \text { DEGREES } 7 \text { MINUTES }
\end{aligned}
$$

ALERAGE DAIL: AMOUNTS BY MONTH

\section{ARRAY
TILT}

0.0

10.0

15.0

20.0

25.0

30.0

35.0

40.0

45.0

50.0

60.0

70.0

80.0

90.0

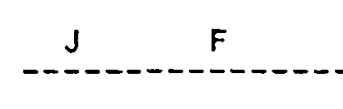

2.56. $3.45 \quad 4.33$

$2.67 \quad 3.56 \quad 4.39$

2.77

3.64

2.85

3.71

4.43

4.45

$2.91 \quad 3.75 \quad 4.44$

2.96

3.00

3.78

4.41

$\begin{array}{lll}3.01 & 3.76 & 4.27\end{array}$

3.76
3.72

$4.27 \quad 4.43$

$4.17 \quad 4.26$

$\begin{array}{llll}2.96 & 3.58 & 3.90 & 3.84\end{array}$

$2.85 \quad 3.37$

$2.85 \quad 3.37$

3.55

3.34

$\begin{array}{llll}2.67 & 3.08 & 3.13 & 2.80\end{array}$

$2.43 \quad 2.73$

2.65

2.24

MAGE DAILY AMOUNTS BY MONTH 
AUER.AGE DA_LY TOTAL TERRESTRIAL INSOLATION ESTIMATES (KWH/SQ. M)

SITE: NEW ORLEANS
ITUDE: 29 DEGREES 59 MINUTES

AVERAGE DAILY AMOUNTS BY MONTH

\begin{tabular}{|c|c|c|c|c|c|c|c|c|c|c|c|c|c|c|}
\hline ARRAY & $J$ & $F$ & $M$ & $A$ & $M$ & J & $J$ & ' & $s$ & 0 & $N$ & $D$ & $\begin{array}{l}\text { ANNUAL } \\
\text { AMOUNT }\end{array}$ & $\begin{array}{c}\text { AVERAGE } \\
\text { DAY }\end{array}$ \\
\hline 0.0 & 2.63 & 3.51 & 4.45 & $5.6 \bar{c}$ & 6.21 & 6.32 & 5.72 & 5.42 & 4.77 & 4.21 & $\equiv .06$ & 2.45 & 1655.1 & 4.5 \\
\hline 10.0 & 2.96 & 3.82 & 4.67 & 5.70 & 6.15 & 6.20 & 5.67 & 5.50 & 5.02 & 4.65 & $\equiv .49$ & 2.83 & 1724.3 & 4.7 \\
\hline 15.0 & 3.10 & 3.94 & 4.75 & 5.70 & 6.07 & 6.10 & 5.60 & $5.49^{\circ}$ & 5.10 & 4.82 & $\Xi .67$ & 2.99 & 1744.6 & 4.8 \\
\hline 20.0 & 3.23 & 4.04 & 4.79 & 5.66 & 5.96 & 5.95 & 5.49 & 5.45 & 5.15 & 4.97 & 3.84 & 3.14 & 1755.2 & 4.8 \\
\hline 25.0 & 3.33 & 4.12 & 4.81 & 5.59 & 5.81 & 5.78 & 5.36 & 5.39 & 5.17 & 5.09 & 3.98 & 3.27 & 1755.9 & 4.8 \\
\hline 30.0 & 3.42 & 4.18 & 4.80 & 5.49 & 5.83 & 5.57 & 5.20 & 5.29 & 5.17 & 5.18 & 4.10 & 3.37 & 1746.8 & 4.8 \\
\hline 35.0 & 3.49 & 4.21 & 4.77 & 5.37 & 5.44 & 5.35 & 5.02 & 5.17 & 5.13 & 5.23 & 4.19 & 3.46 & 1729.4 & 4.7 \\
\hline 40.0 & 3.54 & 4.22 & 4.71 & 5.21 & 5.21 & 5.10 & 4.82 & 5.02 & 5.07 & 5.26 & 4.26 & 3.53 & 1702.4 & 4.7 \\
\hline 45.0 & 3.56 & 4.20 & 4.63 & 5.03 & 4.96 & 4.83 & 4.59 & 4.85 & 4.98 & 5.25 & 4.30 & 3.58 & 1665.9 & 4.6 \\
\hline 50.0 & 3.57 & 4.16 & 4.51 & 4.82 & 4.68 & 4.53 & 4.34 & 4.65 & 4.86 & 5.21 & 4.32 & 3.61 & 1620.3 & 4.4 \\
\hline 60.0 & 3.52 & 4.01 & 4.22 & 4.33 & $\therefore .06$ & 3.87 & 3.78 & 4.18 & 4.54 & 5.05 & 4.27 & 3.59 & 1503.0 & 4.1 \\
\hline 70.0 & 3.39 & 3.77 & 3.84 & 3.76 & 3.39 & 3.21 & 3.18 & 3.63 & 4.12 & 4.76 & 4.12 & 3.50 & 1357.7 & 3.7 \\
\hline 80.0 & 3.18 & 3.45 & 3.38 & 3.11 & 2.70 & 2.51 & 2.56 & 3.01 & 3.61 & 4.306. & 3.88 & 3.31 & 1187.3 & 3.3 \\
\hline 90.0 & 2.90 & 3.06 & 2.85 & 2.46 & 2.04 & 1.89 & 1.96 & 2.38 & 3.04 & 3.86 & 3.54 & 3.05 & 1004.0 & 2.8 \\
\hline
\end{tabular}


AVERAGE DAILY TOTAL TERRESTRIAL INSDLATION ESTIMATES (KWH/SQ. M)

SITE: SHREVEPDRT

LATITUDE: 32 DEGREES 28 MINUTES

AVERAGE JAILY AMOJNTS BY MONTH

\begin{tabular}{|c|c|c|c|c|c|c|c|c|c|c|c|c|c|c|}
\hline ARRAY & $\mathrm{J}$ & $F$ & $M$ & A & $M$ & $\mathrm{~J}$ & $\mathrm{~J}$ & A & s & 0 & $\mathrm{~N}$ & $\mathrm{D}$ & $\begin{array}{l}\text { ANNUAL } \\
\text { ANIOUNT }\end{array}$ & $\begin{array}{c}\text { AVERAGE } \\
\text { DAY }\end{array}$ \\
\hline 0.0 & 2.40 & 3.28 & 4.22 & 5.09 & 5.95 & 6.51 & 6.34 & 5.92 & 4.89 & 4.11 & 2.92 & 2.30 & 1642.9 & 4.5 \\
\hline 10.0 & 2.73 & 3.59 & 4.45 & 5.18 & 5.92 & 6.42 & 6.31 & 6.05 & 5.19 & 4.58 & 3.38 & 2.69 & 1720.7 & 4.7 \\
\hline 15.0 & 2.87 & 3.72 & 4.53 & 5.19 & 5.86 & 6.32 & 6.25 & 6.06 & 5.29 & 4.77 & 3.58 & 2.87 & 1745.2 & 4.8 \\
\hline 20.0 & 3.00 & 3.82 & 4.59 & 5.17 & 5.76 & 6.19 & 6.14 & 6.04 & 5.37 & 4.94. & 3.76 & 3.02 & 1759.9 & 4.8 \\
\hline 25.0 & 3.11 & 3.91 & 4.62 & 5.11 & 5.64 & 6.02 & 6.00 & 5.98 & 5.41 & 5.08 & 3.92 & 3.16 & 1764.7 & 4.8 \\
\hline 30.0 & 3.20 & 3.97 & 4.62 & 5.04 & 5.48 & 5.82 & 5.83 & 5.89 & 5.42 & 5.18 & 4.06 & 3.28 & 1759.5 & 4.8 \\
\hline 35.0 & 3.27 & 4.01 & 4.60. & 4.93 & 5.30 & 5.60 & 5.64 & 5.77 & 5.40 & 5.26 & 4.16 & 3.38 & 1745.2 & 4.8 \\
\hline 40.0 & 3.32 & 4.03 & 4.56 & 4.80 & 5.10 & 5.36 & 5.42 & 5.62 & 5.35 & 5.30 & 4.25 & 3.47 & 1721.8 & 4.7 \\
\hline 45.0 & 3.36 & 4.02 & $4.48 \backslash$ & 4.65 & 4.87 & 5.08 & 5.17 & 5.43 & 5.27 & 5.31 & 4.31 & 3.52 & 1688.8 & 4.6 \\
\hline 50.0 & 3.37 & 4.00 & 4.39 & 4.47 & 4.62 & 4.79 & 4.90 & 5.22 & 5.16 & 5.29 & 4.34 & 3.56 & 1646.4 & 4.5 \\
\hline 60.0 & 3.34 & 3.87 & 4.12 & 4.05 & 4.05 & 4.12 & 4.27 & 4.71 & 4.85 & 5.15 & 4.32 & 3.57 & 1534.7 & 4.2 \\
\hline 70.0 & 3.24 & 3.66 & 3.77 & 3.56 & 3.42 & 3.43 & 3.59 & 4.11 & 4.43 & 4.88 & 4.20 & 3.50 & 1392.4 & 3.8 \\
\hline 80.0 & 3.0 .5 & 3.37 & 3.34 & 2.99 & 2.78 & 2.71 & 2.89 & 3.42 & 3.91 & 4.50 & 3.97 & 3.33 & 1224.8 & 3.4 \\
\hline 90.0 & 2.80 & 3.00 & 2.85 & 2.41 & 2.14 & 2.04 & 2.19 & 2.71 & 3.32 & 4.02 & 3.65 & 3.09 & 1040.6 & 2.9 \\
\hline
\end{tabular}


AVERAGE DAJLY OOTAL TERRESTRIAL INSOLATION ESTIMATES (KWH/SQ. M)

SITE: BOSTON MA
LATITUDE: 42 DEGREES 22 MINUTES

AVERAGE DAILY ATOUNTS BY MONTH

\begin{tabular}{|c|c|c|c|c|c|c|c|c|c|c|c|c|c|c|}
\hline ARRAY & $J$ & $F$ & $M$ & I & $M$ & $J$ & $J$ & $A$ & 5 & 0 & $N$ & $D$ & $\begin{array}{l}\text { ANNUAL } \\
\text { AMOUNT }\end{array}$ & $\begin{array}{c}\text { AVERAGE } \\
\text { DAY }\end{array}$ \\
\hline 0.0 & 1.50 & 2.24 & 3.20 & 4.18 & 5.11 & 5.72 & 5.52 & 4.69 & 3.97 & 2.80 & 1.59 & 1.27 & 1273.4 & 3.5 \\
\hline 10.0 & 1.78 & 2.51 & 3.44 & 4.33 & 5.17 & 5.74 & 5.59 & 4.87 & 4.31 & 3.21 & 1.90 & 1.56 & 1353.0 & 3.7 \\
\hline 15.0 & 1.90 & 2.63 & 3.54 & 4.37 & 5.16 & 5.71 & 5.58 & 4.92 & 4.44 & 3.39 & 2.04 & 1.69 & 1382.1 & 3.8 \\
\hline 20.0 & 2.02 & 2.73 & 3.61 & 4.38 & 5.12 & 5.64 & 5.54 & 4.95 & 4.56 & 3.55 & 2.17 & 1.81 & 1403.7 & 3.8 \\
\hline 25.0 & 2.12 & 2.82 & 3.67 & 4.38 & 5.06 & 5.55 & 5.47 & 4.94 & 4.64 & 3.69 & 2.29 & 1.92 & 1417.6 & 3.9 \\
\hline 30.0 & 2.22 & 2.89 & 3.70 & 4.35 & 4.97 & 5.43 & 5.37 & 4.91 & 4.70 & 3.80 & 2.39 & 2.02 & 1423.6 & 3.9 \\
\hline 35.0 & 2.29 & 2.95 & 3.72 & 4.29 & 4.85 & 5.27 & 5.24 & 4.86 & 4.73 & 3.90 & 2.48 & 2.11 & 1421.8 & 3.9 \\
\hline 40.0 & 2.36 & 2.99 & 3.71 & 4.22 & 4.71 & 5.09 & 5.09 & 4.77 & 4.73 & 3.97 & 2.56 & 2.18 & 1412.1 & 3.9 \\
\hline 45.0 & 2.41 & 3.01 & 3.69 & 4.12 & 4.55 & 4.90 & 4.91 & 4.67 & 4.70 & 4.01 & 2.62 & 2.24 & 1395.5 & 3.8 \\
\hline 50.0 & 2.45 & 3.02 & 3.64 & 4.01 & 4.37 & 4.69 & 4.72 & 4.54 & 4.65 & 4.03 & 2.65 & 2.29 & 1372.0 & 3.8 \\
\hline 60.0 & 2.48 & 2.98 & 3.49 & 3.72 & 3.96 & 4.19 & 4.26 & 4.21 & 4.47 & 4.00 & 2.70 & 2.34 & 1302.8 & 3.6 \\
\hline 70.0 & 2.44 & 2.87 & 3.27 & 3.36 & 3.47 & 3.63 & 3.73 & 3.79 & 4.18 & 3.88 & 2.67 & 2.34 & 1205.9 & 3.3 \\
\hline 80.0 & 2.36 & 2.69 & 2.97 & 2.93 & 2.94 & 3.04 & 3.14 & 3.30 & 3.81 & 3.66 & 2.58 & 2.27 & 1096.1 & 3.0 \\
\hline 90.0 & 2.21 & 2.46 & 2.62 & 2.46 & 2.40 & 2.42 & 2.54 & 2.76 & 3.35 & 3.35 & 242 & 2.15 & 947.8 & 2.6 \\
\hline
\end{tabular}


AVERAGE DAILY TOTAL TERRESTRIAL INSOLATION ESTIMATES (KWH/SQ. M)

$\begin{aligned} \text { SITE: } & \text { BALTIMORE } \\ \text { LATITUDE: } & 39 \text { DEGREES } 11 \text { MINUTES }\end{aligned}$

AVERAGE DAILY AMOUNTS BY MONTH

\begin{tabular}{|c|c|c|c|c|c|c|c|c|c|c|c|c|c|c|}
\hline ARRAY & 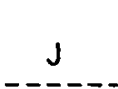 & $F$ & $M$ & $A$ & $M$ & $J$ & $\mathrm{~J}$ & $A$ & $S$ & 0 & $N$ & $\mathrm{D}$ & $\begin{array}{l}\text { ANNUAL } \\
\text { AMOUNT }\end{array}$ & $\begin{array}{l}\text { AVERAGE } \\
\text { DAY }\end{array}$ \\
\hline 0.0 & 1.85 & 2.65 & 3.65 & 4.69 & 5.40 & 5.93 & 5.74 & 5.05 & 4.19 & 3.14 & 2.08 & 1.58 & 1399.7 & 3.8 \\
\hline 10.0 & 2.17 & 2.96 & 3.91 & 4.84 & 5.44 & 5.92 & 5.78 & 5.22 & 4.51 & 3.56 & 2.48 & 1.90 & 1482.7 & 4.1 \\
\hline 15.0 & 2.32 & 3.09 & 4.01 & 4.87 & 5.41 & 5.86 & 5.76 & 5.26 & 4.63 & 3.74 & 2.66 & 2.05 & 1512.1 & 4.1 \\
\hline 20.0 & 2.45 & 3.20 & 4.09 & 4.88 & 5.36 & 5.78 & 5.70 & 5.27 & 4.73 & 3.89 & 2.82 & 2.18 & 1533.2 & 4.2 \\
\hline 25.0 & 2.57 & 3.30 & 4.14 & 4.86 & 5.28 & 5.67 & 5.61 & 5.25 & 4.80 & 4.03 & 2.97 & 2.31 & 1545.8 & 4.2 \\
\hline 30.0 & 2.67 & 3.38 & 4.17 & 4.82 & 5.17 & 5.52 & 5.49 & 5.20 & 4.84 & 4.14 & 3.10 & 2.42 & 1549.8 & 4.2 \\
\hline 35.0 & 2.76 & 3.44 & 4.18 & 4.74 & 5.03 & 5.35 & 5.35 & 5.13 & 4.85 & 4.23 & 3.21 & 2.51 & 1545.1 & 4.2 \\
\hline 40.0 & 2.83 & 3.47 & 4.16 & 4.65 & 4.87 & 5.15 & 5.17 & 5.02 & 4.84 & $4.2 \%$ & 3.30 & 2.59 & 1532.1 & 4.2 \\
\hline 45.0 & 2.88 & 3.49 & 4.12 & 4.53 & 4.69 & 4.94 & 4.98 & 4.90 & 4.80 & 4.32 & 3.37 & 2.65 & 1511.9 & 4.1 \\
\hline 50.0 & 2.92 & 3.49 & 4.06 & 4.39 & 4.49 & 9.71 & 4.77 & 4.75 & 4.73 & 4.33 & 3.42 & 2.70 & 1483.4 & 4.1 \\
\hline 60.0 & 2.93 & 3.43 & 3.87 & 4.05 & 4.03 & 4.17 & 4.27 & 4.37 & 4.51 & 4.27 & 3.45 & 2.75 & 1402.3 & 3.8 \\
\hline 70.0 & 2.8 .8 & 3.28 & 3.60 & 3.62 & 3.49 & 3.56 & 3.69 & 3.90 & 4.19 & 4.11 & 3.40 & 2.72 & 1291.1 & 3.5 \\
\hline 80.0 & 2.76 & 3.07 & 3.25 & 3.13 & 2.93 & 2.95 & 3.08 & 3.36 & 3.78 & 3.84 & 3.26 & 2.63 & 1156.8 & 3.2 \\
\hline 90.0 & 2.57 & 2.78 & 2.84 & 2.58 & 2.34 & 2.32 & 2.44 & 2.76 & 3.29 & 3.49 & 3.05 & 2.48 & 1001.8 & 2.7 \\
\hline
\end{tabular}


AVERAGE DAILY TOTAL TERRESTRIAL INSOLATION ESTIMATES (KWH.SQ. M)

SITE: PATUXENT RIVER MD

LATITUDE: 38 DEGREES 17 MINUTES

aVERAGE DAILY AMDUNTS BY MONTH

\begin{tabular}{|c|c|c|c|c|c|c|c|c|c|c|c|c|c|c|}
\hline ARRAY & $J$ & $F$ & $M$ & A & $M$ & $J$ & 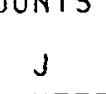 & trum & $s$ & 0 & $\theta$ & $D$ & $\begin{array}{l}\text { ANNUAL } \\
\text { AMOUNT }\end{array}$ & $\begin{array}{c}\text { AVERAGE } \\
\text { DAY }\end{array}$ \\
\hline 0.0 & 1.92 & 2.72 & 3.72 & 4.85 & 5.56 & 5.97 & 5.72 & 5.14 & 4.27 & 3.22 & 2.23 & 1.70 & 1431.6 & 3.9 \\
\hline 10.0 & 2.24 & 5.03 & 3.97 & 4.97 & 5.59 & 5.95 & 5.76 & 5.30 & 4.59 & 3.63 & 2.65 & 2.04 & 1514.5 & 4.1 \\
\hline 15.0 & 2.38 & 3.16 & 4.07 & 5.03 & 5.56 & 5.89 & 5.72 & 5.33 & 4.71 & 3.81 & 2.83 & 2.20 & 1543.6 & 4.2 \\
\hline 20.0 & 2.51 & 3.27 & 4.14 & 5.03 & 5.50 & 5.80 & 5.66 & 5.34 & 4.80 & 3.96 & 3.00 & 2.34 & 1564.1 & 4.3 \\
\hline 25.0 & 2.63 & 3.37 & 4.19 & 5.01 & 5.41 & 5.69 & 5.57 & 5.32 & 4.87 & 4.09 & 3.16 & 2.47 & 1576.0 & 4.3 \\
\hline 30.0 & 2.73 & 3.44 & 4.22 & 4.96 & 5.30 & 5.54 & 5.45 & 5.27 & 4.91 & 4.20 & 3.29 & 2.59 & 1579.0 & 4.3 \\
\hline 35.0 & 2.81 & 3.50 & 4.22 & 4.88 & 5.15 & 5.36 & 5.30 & 5.19 & 4.92 & 4.28 & 3.41 & 2.69 & 1573.3 & 4.3 \\
\hline 40.0 & 2.88 & 3.53 & 4.20 & 4.78 & 4.98 & 5.16 & 5.12 & 5.08 & 4.90 & 4.34 & 3.50 & 2.77 & 1559.3 & 4.3 \\
\hline 45.0 & 2.93 & 3.55 & 4.16 & 4.65 & 4.80 & 4.94 & 4.93 & 4.95 & 4.85 & 4.37 & 3.57 & 2.84 & 1537.7 & 4.2 \\
\hline 50.0 & 2.97 & 3.54 & 4.09 & 4.51 & 4.59 & 4.70 & 4.71 & 4.79 & 4.78 & 4.37 & 3.62 & 2.88 & 1507.6 & 4.1 \\
\hline 60.0 & 2.98 & 3.47 & 3.89 & 4.14 & 4.10 & 4.15 & 4.21 & 4.40 & 4.55 & 4.30 & 3.65 & 2.93 & 1422.9 & 3.9 \\
\hline 70.0 & 2.92 & 3.32 & 3.61 & 3.69 & 3.54 & 3.53 & 3.62 & 3.92 & 4.22 & 4.13 & 3.59 & 2.90 & 1307.9 & 3.6 \\
\hline 80.0 & 2.79 & 3.09 & 3.25 & $3: 18$ & 2.96 & 2.91 & 3.02 & 3.36 & 3.79 & 3.86 & 3.44 & 2.80 & 1169.6 & 3.2 \\
\hline $90.0^{\circ}$ & 2.60 & 2.80 & 2.83 & 2.61 & 2.35 & 2.28 & 2.38 & 2.75 & 3.29 & 3.50 & 3.21 & 2.63 & 1010.6 & 2.8 \\
\hline
\end{tabular}


AVERAGE DAILY TOTAL TERRESTRIAL INSOLATION ESTIMATES (KWH/SQ. M)

$$
\text { SITE: } \quad \text { BANGOR }
$$

LATITUDE: 44 DEGREES 48 MINUTES

AVERAGE DAILY AMOUNTS BY MONTH

\begin{tabular}{|c|c|c|c|c|c|c|c|c|c|c|c|c|c|c|}
\hline ARRAY & $\int^{-1}$ & $F$ & $M$ & $A$ & $M$ & $J$ & $\mathrm{~J}$ & $A$ & 5 & 0 & $N$ & D & $\begin{array}{l}\text { ANNUAL } \\
\text { AMOUNT }\end{array}$ & $\begin{array}{c}\text { AVERAGE } \\
\text { DAY }\end{array}$ \\
\hline 0.0 & 1.44 & 2.29 & 3.44 & 4.54 & 5.45 & 5.85 & 5.86 & 5.08 & 3.95 & 2.64 & 1.49 & 1.19 & 1317.7 & 3.6 \\
\hline 10.0 & 1.75 & 2.62 & 3.75 & 4.74 & 5.54 & 5.89 & 5.97 & 5.32 & 4.34 & 3.07 & 1.83 & 1.51 & 1411.7 & 3.9 \\
\hline 15.0 & 1.89 & 2.76 & 3.38 & 4.80 & 5.55 & 5.87 & 5.97 & 5.40 & 4.49 & 3.26 & 1.98 & 1.66 & 1447.3 & 4.0 \\
\hline 20.0 & 2.03 & 2.89 & 3.98 & 4.83 & 5.52 & 5.81 & E. 54 & 5.44 & 4.62 & 3.43 & 2.12 & 1.79 & 1475.1 & 4.0 \\
\hline 25.0 & 2.15 & 3.00 & 4.06 & 4.84 & 5.46 & 5.73 & $E_{.} .88$ & 5.46 & 4.73 & 3.58 & 2.25 & 1.92 & 1494.5 & 4.1 \\
\hline 30.0 & 2.26 & 3.10 & 4.12 & 4.82 & 5.38 & 5.61 & 5.79 & 5.44 & 4.80 & 3.71 & 2.37 & 2.03 & 1505.5 & 4.1 \\
\hline 35.0 & 2.35 & 3.17 & 4.15 & 4.78 & 5.26 & 5.47 & 5.67 & 5.40 & 4.85 & 3.81 & 2.47 & 2.14 & 1508.0 & 4.1 \\
\hline 40.0 & 2.43 & 3.23 & 4.16 & 4.71 & 5.12 & 5.30 & 5.51 & 5.32 & 4.87 & 3.89 & 2.56 & 2.23 & 1502.0 & 4.1 \\
\hline 45.0 & 2.50 & 3.27 & 4.15 & 4.61 & 4.95 & 5.10 & 5.32 & 5.21 & 4.86 & 3.95 & 2.63 & 2.30 & 1487.5 & 4.1 \\
\hline 50.0 & 2.55 & 3.29 & 4.11 & 4.49 & 4.77 & 4.89 & 5.13 & 5.08 & 4.82 & 3.99 & 2.69 & 2.36 & 1466.4 & 4.0 \\
\hline 60.0 & 2.60 & 3.27 & 3.97 & 4.19 & 4.34 & 4.40 & 9.66 & 4.74 & 4.66 & 3.98 & 2.75 & 2.44 & 1399.9 & 3.8 \\
\hline 70.0 & 2.59 & 3.17 & 3.74 & 3.80 & 3.82 & 3.83 & 4.09 & 4.29 & 4.39 & 3.88 & 2.74 & 2.45 & 1302.5 & 3.6 \\
\hline 80.0 & 2.51 & 3.00 & 3.43 & 3.34 & 3.24 & 3.22 & 3.45 & 3.76 & 4.02 & 3.68 & 2.66 & 2.40 & 1178.1 & 3.2 \\
\hline 90.0 & 2.37 & 2.77 & 3.04 & 2.82 & 2.66 & 2.60 & 2.81 & 3.15 & 3.56 & 3.40 & 2.52 & 2.29 & 1033.8 & 2.8 \\
\hline
\end{tabular}


AVERAGE DAILY TOTAL TERRESTRIAL INSOLATION ESTIMATES (KLH/SQ. M)

SITE: CARIBOU
ITUDE: 46 DEGREES 52 MINUTES

AVERAGE DAILY AMOUNTS BY MONTH

\begin{tabular}{|c|c|c|c|c|c|c|c|c|c|c|c|c|c|c|}
\hline ARRAY & $J$ & $F$ & $M$ & $A$ & $M$ & $\jmath$ & $J$ & $A$ & S & 0 & $N$ & $D$ & $\begin{array}{l}\text { ANNUAL } \\
\text { AMOUNT }\end{array}$ & $\begin{array}{c}\text { AVERAGE } \\
\text { DAY }\end{array}$ \\
\hline 0.0 & 1.32 & 2.28 & 3.57 & $4.4{ }^{\circ}$ & 4.98 & 5.54 & 5.56 & 4.73 & 3.47 & 2.17 & 1.16 & 0.98 & 1225.1 & 3.4 \\
\hline 10.0 & 1.65 & 2.65 & 3.93 & 4.67 & 5.07 & 5.59 & 5.67 & 4.97 & 3.81 & 2.52 & 1.41 & 1.25 & 1315.9 & 3.6 \\
\hline 15.0 & 1.80 & 2.81 & 4.08 & 4.79 & 5.08 & 5.58 & 5.68 & 5.05 & 3.95 & 2.67 & !. 53 & 1.38 & 1350.8 & 3.7 \\
\hline 20.0 & 1.94 & 2.96 & 4.21 & 4.78 & 5.07 & 5.53 & 5.67 & 5.10 & 4.07 & 2.81 & -.63 & 1.50 & 1378.5 & 3.8 \\
\hline 25.0 & 2.06 & 3.05 & 4.31 & 4.80 & 5.02 & 5.46 & 5.62 & 5.12 & 4.17 & 2.94 & $=.73$ & 1.61 & 1398.6 & 3.8 \\
\hline 30.0 & 2.18 & 3.21 & 4.39 & 4.79 & 4.95 & 5.37 & 5.54 & 5.11 & 4.24 & 3.04 & i. .82 & 1.71 & 1410.9 & 3.9 \\
\hline 35.0 & 2.28 & 3.30 & 4.44 & 4.76 & 4.86 & 5.24 & 5.43 & 5.07 & 4.29 & 3.13 & 1. 90 & 1.80 & 1415.2 & 3.9 \\
\hline $4 C .0$ & 2.37 & 3.37 & 4.46 & 4.69 & 4.73 & 5.08 & 5.29 & 5.01 & 4.31 & 3.20 & 1.97 & 1.88 & 1411.5 & $3 . ?$ \\
\hline 45.0 & 2.44 & 3.43 & 4.46 & 4.61 & 4.59 & 4.90 & 5.13 & 4.92 & 4.30 & 3.25 & $\approx .02$ & 1.95 & 1400.0 & 3.8 \\
\hline 50.0 & 2.50 & 3.46 & 4.44 & 4.50 & 1.43 & 4.71 & 4.95 & 4.80 & 4.28 & 3.29 & 2.06 & 2.00 & 1381.7 & 3.8 \\
\hline 60.0 & 2.57 & 3.46 & 4.31 & 4.21 & 4.05 & 4.27 & 4.52 & 4.50 & 4.15 & 3.29 & 2.11 & 2.08 & 1323.5 & 3.6 \\
\hline 70.0 & 2.57 & 3.38 & 4.08 & 3.84 & 3.60 & 3.74 & 4.00 & 4.10 & 3.92 & 3.21 & 2.10 & 2.09 & 1236.3 & 3.4 \\
\hline 80.0 & 2.51 & 3.22 & 3.76 & 3.39 & 3.08 & 3.17 & 3.41 & 3.62 & 3.61 & 3.06 & 2.05 & 2.06 & 1123.1 & 3.1 \\
\hline 90.0 & 2.38 & 2.98 & 3.35 & 2.88 & 2.56 & 2.59 & 2.81 & 3.07 & 3.22 & 2.83 & 1.94 & 1.97 & 990.8 & 2.7 \\
\hline
\end{tabular}


AVERAGE DAILY TOTAL TERRESTRIAL INSOLATION ESTIMATES (KWH/S?. M)

SITE:
LATITUDE: 43 METLAND

ALERAGE DAILY AMOLNTS BY MONTH

\begin{tabular}{|c|c|c|c|c|c|c|c|c|c|c|c|c|c|c|}
\hline ARRAY & $\mathrm{J}$ & $F$ & $M$ & A & $M$ & $\therefore$ & $\mathrm{J}$ & A & S & 0 & $N$ & $\mathrm{D}$ & $\begin{array}{l}\text { ANNUAL } \\
\text { AMOUNT }\end{array}$ & $\begin{array}{c}\text { AVERAGE } \\
\text { DAY }\end{array}$ \\
\hline 0.0 & 1.42 & 2.15 & 3.05 & 4.11 & 4.94 & $5 .<0$ & 5.23 & 4.61 & 3.65 & 2.59 & 1.45 & 1.14 & 1211.0 & 3.3 \\
\hline 10.0 & 1.70 & 2.42 & 3.29 & 4.27 & 5.01 & 5.42 & 5.30 & 4.80 & 3.97 & 2.98 & 1.75 & 1.41 & 1289.0 & 3.5 \\
\hline 15.0 & 1.83 & 2.54 & 3.38 & 4.31 & 5.00 & 5.39 & 5.30 & 4.86 & 4.09 & 3.15 & 1.88 & 1.53 & 1317.9 & 3.6 \\
\hline 20.0 & 1.95 & 2.65 & 3.46 & 4.33 & 4.97 & 5.34 & 5.26 & 4.88 & 4.20 & 3.30 & 2.00 & 1.64 & 1339.7 & 3.7 \\
\hline 25.0 & 2.05 & 2.74 & 3.51 & 4.33 & 4.91 & 5.26 & 5.20 & 4.89 & 4.28 & 3.43 & 2.1 & 1.74 & 1354.2 & 3.7 \\
\hline 30.0 & 2.15 & 2.81 & 3.55 & 4.30 & 4.83 & 5.15 & 5.12 & 4.86 & 4.33 & 3.55 & 2.21 & 1.84 & 1361.2 & 3.7 \\
\hline 35.0 & 2.23 & 2.87 & 3.57 & 4.26 & 4.72 & 5.01 & 5.00 & 4.81 & 4.36 & 3.64 & 2.30 & 1.92 & 1360.8 & 3.7 \\
\hline 40.0 & 2.30 & 2.91 & 3.57 & 4.19 & 4.59 & 4.85 & 4.86 & 4.73 & 4.37 & 3.71 & 2.37 & 1.99 & 1352.9 & 3.7 \\
\hline 45.0 & 2.35 & 2. 94 & 3.55 & 4.10 & 4.44 & 4.66 & 4.70 & 4.63 & 4.35 & 3.75 & 2.43 & 2.04 & 1338.0 & 3.7 \\
\hline 50.0 & 2.37 & 2.95 & 3.51 & 3.99 & 4.27 & 4.47 & 4.52 & 4.51 & 4.31 & 3.78 & 2.47 & 2.09 & 1316.9 & 3.6 \\
\hline 60.0 & 2.43 & 2.92 & 3.37 & 3.71 & 3.88 & 4.02 & 4.10 & 4.20 & 4.15 & 3.76 & 2.51 & 2.14 & 1253.5 & 3.4 \\
\hline 70.0 & 2.41 & 2.82 & 3.16 & 3.35 & 3.42 & $\Xi .50$ & 3.60 & 3.79 & 3.89 & 3.65 & 2.49 & 2.14 & 1163.6 & 3.2 \\
\hline 80.0 & 2.32 & 2.66 & 2.89 & 2.95 & 2.91 & $\bar{c} .95$ & 3.05 & 3.32 & 3.55 & 3.45 & 2.41 & 2.09 & 1051.0 & 2.9 \\
\hline 90.0 & 2.19 & 2.44 & 2.55 & 2.49 & 2.39 & c. 38 & 2.50 & 2.78 & 3.14 & 3.17 & 2.27 & 1.98 & 921.0 & 2.5 \\
\hline
\end{tabular}


AVERAGE DAILY TOTAL TERRESTRIAL INSOLATION ESTIMATES (KL:H/SQ. M)

$$
\begin{aligned}
& \text { SITE: ALPENA } \\
& \text { LATITUDE: } 45 \text { DEGREES } 4 \text { MINUTES }
\end{aligned}
$$

AVERAGE DAILY AMOUNTS BY MONTH

\begin{tabular}{|c|c|c|c|c|c|c|c|c|c|c|c|c|c|c|c|}
\hline ARRAI & & $J$ & $F$ & $M$ & A & $M$ & $J$ & $J$ & A & $S$ & 0 & $N$ & $D$ & $\begin{array}{l}\text { ANNUAL } \\
\text { AMOUNT }\end{array}$ & $\begin{array}{c}\text { AVERAGE } \\
\text { DAY }\end{array}$ \\
\hline 0.0 & & 1.15 & 1.94 & 3.24 & 4.44 & 5.43 & 5.92 & 5.94 & 5.00 & 3.64 & 2.34 & 1.21 & 0.85 & 1252.7 & 3.4 \\
\hline 10.0 & & 1.35 & 2.19 & 3.52 & 4.63 & 5.52 & 5.97 & 6.05 & 5.23 & 3.98 & 2.70 & 1.44 & 1.09 & 1331.1 & 3.6 \\
\hline 15.0 & & 1.44 & 2.36 & 3.63 & 4.69 & 5.52 & 5.94 & 6.05 & 5.31 & .4 .12 & 2.86 & i. 54 & 1.20 & 1359.8 & 3.7 \\
\hline 20.0 & - & 1.53 & 2.39 & 3.73 & 4.72 & 5.50 & 5.89 & 6.03 & 5.35 & 4.23 & 3.00 & j. .64 & 1.31 & 1381.1 & 3.8 \\
\hline 25.0 & & 1.60 & 2.48 & 3.80 & 4.73 & 5.44 & 5.80 & 5.97 & 5.37 & 4.32 & 3.12 & 1.73 & 1.41 & 1394.8 & 3.8 \\
\hline 30.0 & & 1.67 & 2.55 & 3.85 & 4.71 & 5.36 & 5.69 & 5.88 & 5.35 & 4.39 & 3.23 & 1.81 & 1.49 & 1400.7 & 3.8 \\
\hline 35.0 & & 1.73 & 2.60 & 3.88 & 4.67 & 5.25 & 5.54 & 5.75 & 5.30 & 4.43 & $3.3 i$ & 1.87 & 1.57 & 1398.9 & 3.8 \\
\hline 40.0 & & 1.78 & 2.64 & 3.89 & 4.60 & 5.11 & 5.37 & 5.59 & 5.23 & 4.44 & 3.38 & 1.93 & 1.64 & 1389.2 & 3.8 \\
\hline 45.0 & & 1.82 & 2.66 & 3.88 & 4.50 & 4.94 & 5.17 & 5.41 & 5.12 & 4.43 & 3.42 & 1.98 & 1.70 & 1371.8 & 3.8 \\
\hline 50.0 & & 1.85 & 2.67 & 3.84 & 4.39 & 4.76 & 4.96 & 5.21 & 5.00 & 4.39 & 3.45 & 2.01 & 1.75 & 1348.6 & 3.7 \\
\hline 60.0 & & 1.87 & 2.65 & 3.7 .1 & 4.10 & 4.33 & 4.46 & 4.73 & 4.66 & 4.24 & 3.44 & 2.04 & 1.81 & 1280.5 & 3.5 \\
\hline 70.0 & & 1.85 & 2.56 & 3.49 & 3.72 & 3.82 & 3.89 & 4.16 & 4.23 & 3.99 & 3.35 & 2.02 & 1.82 & 1184.7 & 3.2 \\
\hline 80.0 & & 1.79 & 2.42 & 3.20 & 3.27 & 3.24 & 3.27 & 3.51 & 3.71 & 3.66 & 3.17 & 1.96 & 1.79 & 1065.2 & 2.9 \\
\hline 90.0 & & 1.68 & 2.23 & 2.84 & 2.76 & 2.66 & 2.64 & 2.86 & 3.12 & 3.25 & 2.93 & 185 & 1.71 & 929.0 & 2.5 \\
\hline
\end{tabular}


AVERAGE DAILY TOTAL TERRESTRIAL INSOLATION ESTIMATES (KAH/SQ. M)

$\begin{aligned} \text { SITE: } & \text { IETROIT } \\ \text { LATITUDE: } & \text { M2 DEGREES } 25 \text { MINUTES }\end{aligned}$

AVERAGE DAZLY AMOUNTS BY MONTH

\begin{tabular}{|c|c|c|c|c|c|c|c|c|c|c|c|c|c|c|}
\hline ARRAY & J & $F$ & $M$ & A & $M$ & $r-2.5$ & 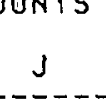 & A & 5 & 0 & $\mathrm{~N}$ & $D$ & $\begin{array}{l}\text { ANNUAL } \\
\text { AMOUNT }\end{array}$ & $\begin{array}{c}\text { AVERAGE } \\
\text { DAY }\end{array}$ \\
\hline 0.0 & 1.32 & 2.14 & 3.14 & 4.42 & 5.41 & 5.39 & 5.79 & 4.97 & 3.95 & 2.76 & 1.51 & 1.08 & 1291.6 & 3.5 \\
\hline 10.0 & 1.53 & 2.40 & 3.38 & 4.58 & 5.48 & 5.91 & 5.87 & 5.17 & 4.29 & 3.17 & 1.79 & 1.29 & 1366.4 & 3.7 \\
\hline 15.0 & 1.63 & 2.50 & 3.47 & 4.62 & 5.47 & 5.87 & 5.86 & 5.22 & $4 . .42$ & 3.34 & 1.92 & 1.39 & 1393.0 & 3.8 \\
\hline 20.0 & 1.72 & 2.60 & 3.54 & 4.64 & 5.43 & 5.81 & 5.82 & 5.25 & 4.53 & 3.50 & 2.04 & 1.47 & 1412.0 & 3.9 \\
\hline 25.0 & 1.80 & 2.68 & 3.60 & 4.64 & 5.36 & 5.71 & 5.74 & 5.25 & 4.62 & 3.63 & 2.15 & 1.55 & 1423.2 & 3.9 \\
\hline 30.0 & 1.87 & 2.74 & 3.63 & 4.61 & 5.27 & 5.58 & 5.64 & 5.22 & 4.67 & 3.74 & 2.24 & 1.62 & 1426.6 & 3.9 \\
\hline $3 E .0$ & $1.9: 2$ & 2.79 & 3.64 & 4.55 & 5.14 & 5.43 & 5.50 & 5.16 & 4.70 & 3.84 & 2.32 & 1.68 & 1422.1 & 3.9 \\
\hline 40.0 & 1.97 & 2.83 & 3.64 & 4.47 & 4.99 & 5.24 & 5.34 & 5.07 & $4.7 I$ & 3.91 & 2.39 & 1.73 & 1409.7 & 3.9 \\
\hline 45.0 & 2.01 & 2.85 & 3.61 & 4.37 & 4.82 & 5.04 & 5.15 & 4.96 & 4.68 & 3.95 & 2.44 & 1.77 & 1390.5 & 3.8 \\
\hline 50.0 & 2.03 & 2.35 & 3.57 & 4.25 & 4.64 & 4.82 & 4.95 & 4.82 & 4.63 & 3.97 & 2.48 & 1.80 & 1364.6 & 3.7 \\
\hline 60.0 & 2.04 & 2.81 & 3.42 & 3.95 & 4.19 & 4.31 & 4.47 & 4.47 & 4.45 & 3.94 & 2.51 & 1.83 & 1290.7 & 3.5 \\
\hline 70.0 & 2.01 & 2.70 & 3.20 & 3.56 & 3.67 & 3.72 & 3.90 & 4.03 & 4.16 & 3.82 & 2.48 & 1.82 & 1189.5 & 3.5 \\
\hline 80.0 & 1.93 & 2.54 & 2.91 & 3.11 & 3.10 & 3.11 & 3.28 & 3.51 & 3.79 & 3.60 & 2.40 & 1.76 & 1066.0 & 2.9 \\
\hline 90.0 & 1.81 & 2.32 & 2.57 & 2.60 & 2.52 & 2.48 & $2.6 \dot{4}$ & 2.92 & 3.33 & 3.30 & 2.25 & 1.67 & 925.0 & 2.5 \\
\hline
\end{tabular}


AVERAGE DAILY TOTAL TERRESTRIAL INSOLATION ESTIMATES (KLH/SQ. M)

SITE: FLINT LATITUDE: 42 DEGREES 58 MINUTES

AVERAGE DAILY AMOUNTS BY MIONTH

\begin{tabular}{|c|c|c|c|c|c|c|c|c|c|c|c|c|c|c|}
\hline ARRAY & $\mathrm{J}$ & $F$ & $M$ & $A$ & $M$ & $J$ & J & $A$ & $s$ & 0 & H & $D$ & $\begin{array}{l}\text { ANNUAL } \\
\text { ANIOUNT }\end{array}$ & $\begin{array}{c}\text { AVERAGE } \\
\text { DAY }\end{array}$ \\
\hline 0.0 & 1.21 & 2.01 & 3.01 & 4.22 & 5.22 & 5.71 & 5.67 & 4.91 & 3.77 & 2.61 & 1.36 & 0.97 & 1239.8 & 3.4 \\
\hline 10.0 & 1.40 & 2.24 & 3.23 & 4.38 & 5.29 & 5.74 & 5.75 & 5.11 & 4.09 & 2.99 & 1.60 & 1.23 & 1311.7 & 3.6 \\
\hline 15.0 & 1.48 & 2.34 & 3.32 & 4.42 & 5.29 & 5.70 & 5.74 & 5.17 & 4.22 & 3.16 & 171 & 1.34 & 1337.3 & 3.7 \\
\hline 20.0 & 1.55 & 2.42 & 3.39 & 4.44 & 5.25 & 5.64 & 5.71 & 5.20 & 4.32 & 3.31 & 1.81 & 1.45 & 1355.7 & 3.7 \\
\hline 25.0 & 1.62 & 2.49 & 3.44 & 4.44 & 5.19 & 5.55 & 5.64 & 5.20 & 4.40 & 3.43 & 1.90 & 1.55 & 1356.7 & 3.7 \\
\hline 30.0 & 1.68 & 2.55 & 3.47 & 4.41 & 5.10 & 5.43 & 5.54 & 5.17 & 4.46 & 3.54 & 1.98 & 1.64 & 1370.2 & 3.8 \\
\hline 35.0 & 1.73 & 2.60 & 3.48 & 4.36 & 4.98 & 5.28 & 5.41 & 5.12 & 4.49 & 3.63 & 2.05 & 1.72 & 1366.1 & 3.7 \\
\hline 40.0 & 1.77 & 2.63 & 3.48 & 4.28 & 4.84 & 5.11 & 5.25 & 5.03 & 4.49 & 3.69 & 2.10 & $\because 1.79$ & 1354.5 & 3.7 \\
\hline 45.0 & 1.80 & 2.65 & 3.45 & $4: 19$ & 4.68 & 4.91 & 5.07 & 4.92 & 4.47 & 3.74 & 2.15 & 1.85 & 1336.3 & 3.7 \\
\hline 50.0 & 1.82 & 2.65 & 3.41 & 4.07 & 4.50 & 4.70 & 4.88 & 4.79 & 4.42 & 3.76 & 2.18 & 1.90 & 1311.7 & 3.6 \\
\hline 60.0 & 1.83 & 2.51 & 3.27 & 3.78 & 4.08 & 4.21 & 4.41 & 4.45 & 4.25 & 3.73 & 2.20 & 1.95 & 1241.6 & 3.4 \\
\hline 70.0 & 1.80 & 2.51 & 3.06 & 3.42 & 3.58 & 3.65 & 3.86 & 4.01 & 3.98 & 3.62 & 2.17 & 1.95 & 1145.4 & 3.1 \\
\hline 80.0 & 1.72 & 2.36 & 2.79 & 2.99 & 3.03 & 3.06 & 3.25 & 3.50 & 3.63 & 3.41 & 2.09 & 1.91 & 1027.6 & 2.8 \\
\hline 90.0 & 1.61 & 2.16 & 2.47 & 2.52 & 2.47 & 2.45 & 2.63 & 2.92 & 3.20 & 3.13 & 1.97 & 1.82 & 893.2 & 2.4 \\
\hline
\end{tabular}


AVERAGE DAILY TOTAL TERRESTRIAL INSOLATION ESTIMATES (KWH/SQ. M)

SITE: GRIND RAPIDS MUI
TUDE: $\angle 2$ DEGREES 53 MINUTES

AVERAGE DAZLY AMOUNTS BY MONTH

\begin{tabular}{|c|c|c|c|c|c|c|c|c|c|c|c|c|c|c|}
\hline ARRAY & $J$ & $F$ & $M$ & A & $M$ & $J$ & $J$ & $A$ & 5 & 0 & $N$ & $D$ & $\begin{array}{l}\text { ANNUAL } \\
\text { ANIOUNT }\end{array}$ & $\begin{array}{c}\text { AVERAGE } \\
\text { DAY }\end{array}$ \\
\hline 0.0 & 1.17 & 2.04 & 3.19 & 4.45 & 5.54 & 0.17 & b. 03 & 5.28 & 3.97 & 2.71 & 1.41 & 0.98 & 1309.0 & 3.6 \\
\hline 10.0 & 1.42 & 2.28 & 3.44 & 4.62 & 5.62 . & b. 20 & 5. 12 & 5.51 & 4.33 & 3.10 & 1.67 & 1.23 & 1387.9 & 3.8 \\
\hline 15.0 & $1.5 j$ & 2.38 & 3.54 & 4.67 & 5.61 & 5.16 & 5.11 & 5.58 & 4.47 & 3.28 & 1.78 & 1.35 & 1416.1 & 3.9 \\
\hline 20.0 & 1.64 & 2.47 & 3.61 & 4.69 & 5.57 & 5.10 & 3.08 & 5.62 & 4.58 & 3.43 & 1.89 & 1.46 & 1436.6 & 3.9 \\
\hline 25.0 & 1.73 & 2.54 & 3.67 & 4.69 & 5.51 & 5.00 & 5.00 & 5.63 & 4.67 & 3.57 & 1.79 & 1.56 & 1449.1 & 4.0 \\
\hline 30.0 & 1.82 & 2.60 & 3.71 & 4.66 & 5.41 & 5.36 & 5.90 & 5.60 & 4.73 & 3.68 & 2.97 & 1.65 & 1453.4 & 4.0 \\
\hline 35.0 & 1.89 & 2.65 & 3.73 & 4.61 & 5.28 & 5.70 & 5.76 & 5.54 & 4.77 & 3.77 & 2.15 & 1.73 & 1449.6 & 4.0 \\
\hline 40.0 & 1.95 & 2.68 & 3.72 & 4.53 & 5.13 & 5.51 & 5.59 & 5.45 & 4.77 & 3.84 & 2.21 & 1.80 & 1437.7 & 3.9 \\
\hline 45.0 & 2.01 & 2.70 & 3.70 & 4.43 & 4.96 & 5.29 & 5.39 & 5.33 & 4.75 & 3.89 & 2.26 & 1.85 & 1418.6 & 3.9 \\
\hline 50.0 & 2.04 & 2.70 & 3.66 & 4.31 & 4.77 & 5.06 & 5.18 & 5.19 & 4.70 & 3.91 & 2.29 & 1.90 & 1392.6 & 3.8 \\
\hline 60.0 & 2.08 & 2.66 & 3.51 & 4.00 & 4.31 & 4.53 & 4.68 & 4.81 & 4.52 & 3.89 & 2.32 & 1.96 & 1318.0 & 3.6 \\
\hline 70.0 & 2.07 & 2.56 & 3.29 & 3.61 & 3.78 & 3.90 & 4.08 & 4.34 & 4.24 & 3.77 & 2.29 & 1.96 & 1215.0 & 3.3 \\
\hline 80.0 & 2.03 & 2.41 & 3.00 & 3.16 & 3.19 & 3.26 & 3.42 & 3.77 & 3.86 & 3.56 & 2.21 & 1.92 & 1088.6 & 3.0 \\
\hline 90.0 & 1.83 & 2.20 & 2.65 & 2.65 & 2.59 & 2.58 & 2.76 & 3.14 & 3.40 & 3.26 & 2.08 & 1.82 & 944.1 & 2.6 \\
\hline
\end{tabular}


AVERAGE DAILY TOTAL TERRESTRIAL INSOLATION ESTIMATES (KWH $/ S Q .{ }^{\circ} M$ )

SITE: HOUGHTON

LATITUDE: 47 DEGREES 10 MINUTES

AVERAGE DAILY AMOUNTS BY MONTH

\begin{tabular}{|c|c|c|c|c|c|c|c|c|c|c|c|c|c|c|}
\hline ARRAY & $\mathrm{J}$ & $F$ & $M$ & $A$ & $M$ & J & $J$ & A & 5 & 0 & $\mathbf{N}$ & $D$ & $\begin{array}{l}\text { ANNUAL } \\
\text { AMOUNT }\end{array}$ & $\begin{array}{c}\text { AVERAGE } \\
\text { DAY }\end{array}$ \\
\hline 0.0 & 0.77 & 1.53 & 2.94 & 4.30 & 5.23 & 5.79 & 5.80 & 4.80 & 3.18 & 2.11 & 0.92 & 0.60 & 1158.3 & 3.2 \\
\hline 10.0 & 0.94 & 1.70 & 3.20 & 4.51 & 5.34 & 5.85 & 5.92 & 5.04 & 3.49 & 2.45 & 1.16 & 0.76 & 1231.0 & 3.4 \\
\hline 15.0 & 1.01 & 1.77 & 3.31 & 4.57 & 5.35 & 5.83 & 5.94 & 5.12 & 3.61 & 2.61 & 1.27 & 0.84 & 1257.6 & 3.4 \\
\hline 20.0 & 1.09 & 1.84 & 3.40 & 4.62 & 5.33 & 5.79 & 5.93 & 5.18 & 3.71 & 2.74 & 1.37 & 0.90 & 1277.7 & 3.5 \\
\hline 25.0 & 1.15 & 1.89 & 3.47 & 4.63 & 5.29 & 5.71 & 5.88 & 5.20 & 3.80 & 2.86 & 1.46 & 0.97 & $1290 . ?$ & 3.5 \\
\hline 30.0 & 1.21 & 1.94 & 3.53 & 4.62 & 5.22 & 5.61 & 5.80 & 5.20 & 3.86 & 2.97 & 1.55 & 1.02 & 1296.7 & 3.6 \\
\hline 35.0 & 1.26 & \pm .98 & 3.56 & 4.59 & 5.12 & 5.48 & 5.69 & 5.16 & 3.90 & 3.05 & 162 & 1.08 & 1295.4 & 3.5 \\
\hline 40.0 & 1.30 & 2.00 & 3.57 & 4.53 & 1.99 & 5.32 & 5.54 & 5.10 & 3.91 & 3.12 & 1.69 & 1.12 & 1286.9 & 3.5 \\
\hline 45.0 & 1.34 & 2.02 & 3.57 & 4.45 & 4.84 & 5.13 & 5.37 & 5.01 & 3.91 & 3.17 & 1.74 & 1.16 & 1271.4 & 3.5 \\
\hline 50.0 & 1.36 & 2.02 & 3.54 & $4 . .34$ & 4.67 & 4.93 & 5.18 & 4.89 & 3.88 & 3.20 & 1.79 & 1.19 & 1249.9 & 3.4 \\
\hline 60.0 & 1.39 & 1.99 & 3.43 & 4.07 & 4.27 & 4.47 & 4.73 & 4.58 & 3.76 & 3.21 & 1.84 & 1.23 & 1188.3 & 3.3 \\
\hline 70.0 & 1.39 & 1.93 & 3.24 & 3.71 & 3.79 & 3.92 & 4.19 & 4.18 & 3.56 & 3.13 & 1.85 & 1.24 & 1101.2 & 3.0 \\
\hline 80.0 & 1.35 & 1.82 & 2.99 & 3.29 & 3.25 & 3.31 & 3.57 & 3.69 & 3.27 & 2.99 & 1.80 & 1.22 & 991.8 & 2.7 \\
\hline 90.0 & 1.28 & 1.68 & 2.67 & 2.80 & 2.69 & 2.71 & 2.93 & 3.13 & 2.92 & 2.77 & 1.72 & 1.16 & 866.9 & 2.4 \\
\hline
\end{tabular}


AVERAGE DAILY TOTAL TERRESTRIAL INSOLATION ESTIMATES (KWH/SQ. M)

SITE: SAULT S-E. MARIE MI

LATITUDE: 46 DEGREES 28 MINUTES

AVERAGE DAILY AMOUNTS BY MONTH

\begin{tabular}{|c|c|c|c|c|c|c|c|c|c|c|c|c|c|c|}
\hline ARRAY & $\mathrm{J}$ & $\mathbf{F}$ & $M_{i}$ & $A$ & $M$ & $J$ & $J$ & A & $\mathrm{S}$ & 0 & $N$ & $D$ & $\begin{array}{l}\text { ANNUAL } \\
\text { AMOUNT }\end{array}$ & $\begin{array}{c}\text { AVERAGE } \\
\text { DAY }\end{array}$ \\
\hline 0.0 & 1.03 & 1.90 & 3.24 & 4.36 & 5.32 & 5.71 & 5.79 & 4.80 & 3.30 & 2.12 & 1.05 & 0.80 & 1201.5 & 3.3 \\
\hline 10.0 & 1.21 & 2.17 & 3.54 & 4.56 & 5.42 & 5.76 & 5.90 & 5.03 & 3.61 & 2.45 & 1.25 & 1.05 & 1278.9 & 3.5 \\
\hline 15.0 & 1.29 & 2.28 & 3.67 & 4.62 & 5.43 & 5.74 & $5.92^{\circ}$ & 5.11 & 3.74 & 2.59 & 1.34 & 1.16 & 1307.5 & 3.6 \\
\hline 20.0 & 1.37 & 2.38 & 3.77 & 4.66 & 5.41 & 5.69 & 5.90 & 5.16 & 3.85 & 2.73 & 1.42 & 1.27 & 1329.2 & 3.6 \\
\hline 25.0 & 1.44 & 2.47 & 3.86 & 4.68 & 5.37 & 5.62 & 5.85 & 5.18 & 3.93 & 2.84 & 1.49 & 1.37 & 1343.7 & 3.7 \\
\hline 30.0 & 1.50 & 2.55 & 3.92 & 4.66 & 5.29 & 5.52 & 5.76 & 5.17 & 3.99 & 2.94 & 1.56 & 1.46 & 1350.6 & 3.7 \\
\hline 35.0 & 1.55 & 2.61 & 3.96 & 4.63 & 5.18 & 5.38 & 5.65 & 5.13 & 4.03 & 3.02 & 1.62 & 1.54 & 1350.1 & 3.7 \\
\hline 40.0 & 1.60 & 2.66 & 3.97 & 4.56 & 5.05 & 5.22 & 5.50 & 5.06 & 4.05 & 3.08 & 1.67 & 1.62 & 1342.0 & 3.7 \\
\hline 45.0 & 1.64 & 2.69 & 3.97 & 4.48 & 4.89 & 5.03 & 5.33 & 4.97 & 4.04 & 3.13 & 1.71 & $1.68^{\circ}$ & 1326.5 & 3.6 \\
\hline 50.0 & 1.66 & 2.70 & 3.94 & 4.37 & 4.72 & 4.83 & 5.14 & 4.85 & 4.01 & 3.16 & 1.74 & 1.73 & 1305.1 & 3.6 \\
\hline 60.0 & 1.69 & 2.69 & 3.81 & 4.09 & 4.31 & 4.37 & 4.68 & 4.54 & 3.88 & 3.15 & 1.76 & 1.80 & 1242.0 & 3.4 \\
\hline 70.0 & 1.67 & 2.61 & 3.60 & 3.73 & 3.82 & 3.83 & 4.14 & 4.13 & 3.67 & 3.07 & 1.75 & 1.82 & 1152.2 & 3.2 \\
\hline 80.0 & 1.62 & 2.47 & 3.31 & 3.29 & 3.26 & 3.24 & 3.51 & 3.64 & 3.37 & 2.92 & $1: 70$ & 1.79 & 1038.9 & 2.8 \\
\hline 90.0 & 1.53 & 2.28 & 2.95 & 2.79 & 2.69 & 2.64 & 2.88 & 3.08 & 3.00 & 2.70 & 1.60 & 1.72 & 909.3 & 2.5 \\
\hline
\end{tabular}


AVERAGE DAILY TOTAL TERRESTRIAL INSOLATION ESTIMATES (KWH:SQ. M)

SITE: TRAVERSE CITY MII
LATITUDE: 44 DEGREES 44 MINUTES

BVERAGE DAILY AMOUNTS BY MONTH

\begin{tabular}{|c|c|c|c|c|c|c|c|c|c|c|c|c|c|c|c|}
\hline ARRAY & & $J$ & $F$ & $M$ & $A$ & $M$ & $\mathrm{~J}$ & $J$ & $A$ & 5 & 0 & H & $D$ & $\begin{array}{l}\text { ANNUAL } \\
\text { AHOUNT }\end{array}$ & $\begin{array}{c}\text { AVERAGE } \\
\text { DAY }\end{array}$ \\
\hline 0.0 & & 0.98 & 1.79 & 3.15 & 4.43 & 5.45 & 6.03 & 6.02 & 5.08 & 3.67 & 2.38 & 1.19 & 0.81 & 1249.4 & 3.4 \\
\hline 10.0 & & 1.19 & 1.99 & 3.42 & 4.62 & 5.54 & 6.07 & 6.13 & 5.32 & 4.01 & 2.74 & 1.41 & 1.02 & 1324.9 & 3.6 \\
\hline 15.0 & & 1.29 & 2.08 & 3.52 & 4.67 & 5.55 & 6.04 & 6.14 & 5.39 & 4.14 & 2.89 & 1.51 & 1.12 & 1352.1 & 3.7 \\
\hline 20.0 & - & 1.38 & 2.16 & 3.61 & 4.70 & 5.52 & 5.99 & 6.11 & 5.43 & 4.26 & 3.03 & 1.60 & 1.21 & 1372.0 & 3.8 \\
\hline 25.0 & & 1.46 & 2.22 & 3.68 & 4.71 & 5.46 & 5.90 & 6.04 & 5.45 & 4.34 & 3.16 & 1.68 & 1.30 & 1384.3 & 3.8 \\
\hline 30.0 & & 1.54 & 2.27 & 3.72 & 4.69 & 5.38 & 5.78 & 5.95 & 5.43 & 4.41 & 3.26 & 1.75 & 1.37 & 1388.8 & 3.8 \\
\hline 35.0 & & 1.60 & 2.32 & 3.75 & 4.65 & 5.26 & 5.63 & 5.82 & 5.38 & 4.45 & 3.35 & 1.81 & 1.44 & 1385.7 & 3.8 \\
\hline 40.0 & & 1.66 & 2.34 & 3.75 & 4.58 & 5.12 & 5.45 & 5.66 & 5.31 & 4.46 & 3.41 & 1.86 & 1.50 & 1374.8 & 3.8 \\
\hline 45.0 & & 1.70 & 2.36 & 3.74 & 4.48 & 4.95 & 5.24 & 5.47 & 5.20 & 4.45 & 3.46 & 1.91 & 1.55 & 1356.4 & 3.7 \\
\hline 50.0 & & 1.73 & 2.36 & 3.70 & 4.37 & 4.77 & 5.03 & 5.26 & 5.07 & 4.41 & 3.48 & 1.94 & 1.59 & 1332.3 & 3.7 \\
\hline 60.0 & & 1.77 & 2.33 & 3.57 & 4.07 & 4.34 & 4.52 & 4.78 & 4.72 & 4.25 & 3.47 & 1.96 & 1.64 & 1262.4 & 3.5 \\
\hline 70.0 & & 1.76 & 2.25 & 3.36 & 3.69 & 3.82 & 3.93 & 4.19 & 4.28 & 4.00 & 3.37 & 1.94 & 1.65 & 1165.5 & 3.2 \\
\hline 80.0 & & 1.71 & 2.12 & 3.07 & 3.25 & 3.24 & 3.30 & 3.53 & 3.75 & 3.66 & 3.20 & 1.88 & 1.62 & 1045.4 & 2.9 \\
\hline 90.0 & & 1.61 & 1.94 & 2.73 & 2.74 & 2.66 & 2.65 & 2.87 & 3.14 & 3.25 & 2.95 & 1.77 & 1.54 & 909.1 & 2.5 \\
\hline
\end{tabular}


AVERAGE DAILY TOTAL TERRESTRIAL INSOLATION ESTIMATES (KWH/SQ. M)

SITE: DULUTH
LATITUDE:

AVERAGE DAILY AMOUNTS BY MONTH ;

\begin{tabular}{|c|c|c|c|c|c|c|c|c|c|c|c|c|c|c|}
\hline ARRAY & J & $F$ & $M$ & $A$ & $M$ & 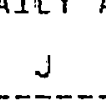 & 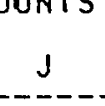 & A & $S$ & 0 & $N$ & D & $\begin{array}{l}\text { ANNUAL } \\
\text { APIOUNT }\end{array}$ & $\begin{array}{c}\text { AVERAGE } \\
\text { DAY }\end{array}$ \\
\hline 0.0 & 1.23 & 2.12 & 3.25 & 4.33 & 5.18 & 5.57 & 5.85 & 4.88 & 3.45 & 2.29 & 1.20 & 0.92 & 1227.1 & 3.4 \\
\hline 10.0 & 1.53 & 2.45 & 3.57 & 4.53 & 5.28 & 5.62 & 5.97 & 5.13 & 3.79 & 2.67 & 1.48 & 1.16 & 1314.9 & 3.6 \\
\hline 15.0 & 1.63 & 2.60 & 3.70 & 4.59 & 5.29 & 5.60 & 5.99 & $5: 21$ & 3.93 & 2.84 & 1.60 & 1.28 & 1348.3 & 3.7 \\
\hline 20.0 & 1.75 & 2.73 & 3.80 & 4.63 & 5.27 & 5.56 & 5.97 & 5.26 & 4.05 & 2.99 & 1.72 & 1.38 & 1374.5 & 3.8 \\
\hline 25.0 & 1.86 & 2.84 & 3.89 & 4.65 & 5.23 & 5.49 & 5.92 & 5.29 & 4.14 & 3.13 & 1.83 & 1.48 & 1393.2 & 3.8 \\
\hline 30.0 & 1.96 & 2.94 & 3.95 & 4.64 & 5.16 & 5.39 & 5.84 & 5.28 & 4.21 & 3.24 & 1.92 & 1.57 & 1404.0 & 3.8 \\
\hline 35.0 & 2.05 & 3.02 & 3.99 & 4.60 & 5.06 & 5.26 & 5.72 & 5.24 & 4.26 & 3.34 & 2.01 & 1.65 & 1406.9 & 3.9 \\
\hline 40.0 & 2.12 & 3.08 & 4.01 & 4.54 & 4.93 & 5.10 & 5.58 & 5.18 & 4.28 & 3.42 & 2.08 & 1.71 & 1402.0 & 3.8 \\
\hline 45.0 & 2.18 & 3.13 & 4.01 & 4.46 & 4.78 & 4.92 & 5.40 & 5.08 & .4 .27 & 3.48 & 2.14 & 1.77 & 1389.2 & 3.8 \\
\hline 50.0 & 2.23 & 3.15 & 3.98 & 4.35 & 4.61 & 4.73 & 5.21 & 4.96 & 4.25 & 3.51 & 2.39 & 1.82 & 1369.8 & 3.8 \\
\hline 60.0 & 2.28 & 3.15 & 3.86 & 4.08 & 4.22 & +.28 & 9.75 & 4.65 & 4.12 & 3.52 & 2.24 & 1.88 & 1309.7 & 3.6 \\
\hline 70.0 & 2.28 & 3.07 & 3.65 & 3.72 & 3.74 & 3.76 & 4.20 & 4.24 & 3.89 & 3.44 & 2.24 & 1.90 & 1221.0 & 3.3 \\
\hline 80.0 & 2.22 & 2.92 & 3.36 & 3.28 & 3.20 & 3.18 & 3.57 & 3.74 & 3.58 & 3.28 & 2.18 & 1.86 & 1106.7 & 3.0 \\
\hline 90.0 & 2.11 & 2.70 & 3.00 & 2.79 & 2.65 & 2.50 & 2.93 & 3.16 & 3.19 & 3.04 & 2.07 & 1.78 & 974.0 & 2.7 \\
\hline
\end{tabular}


AVERAGE DAILY TOTAL TERRESTRIAL INSOLATION ESTIMATES (KWH/SQ. M)

SITE: INTERNATIONAL FALLMN

LATITUDE: 48 DEGREES 34 MINUTES

AVERAGE DAILY AMOUNTS BY MONTH

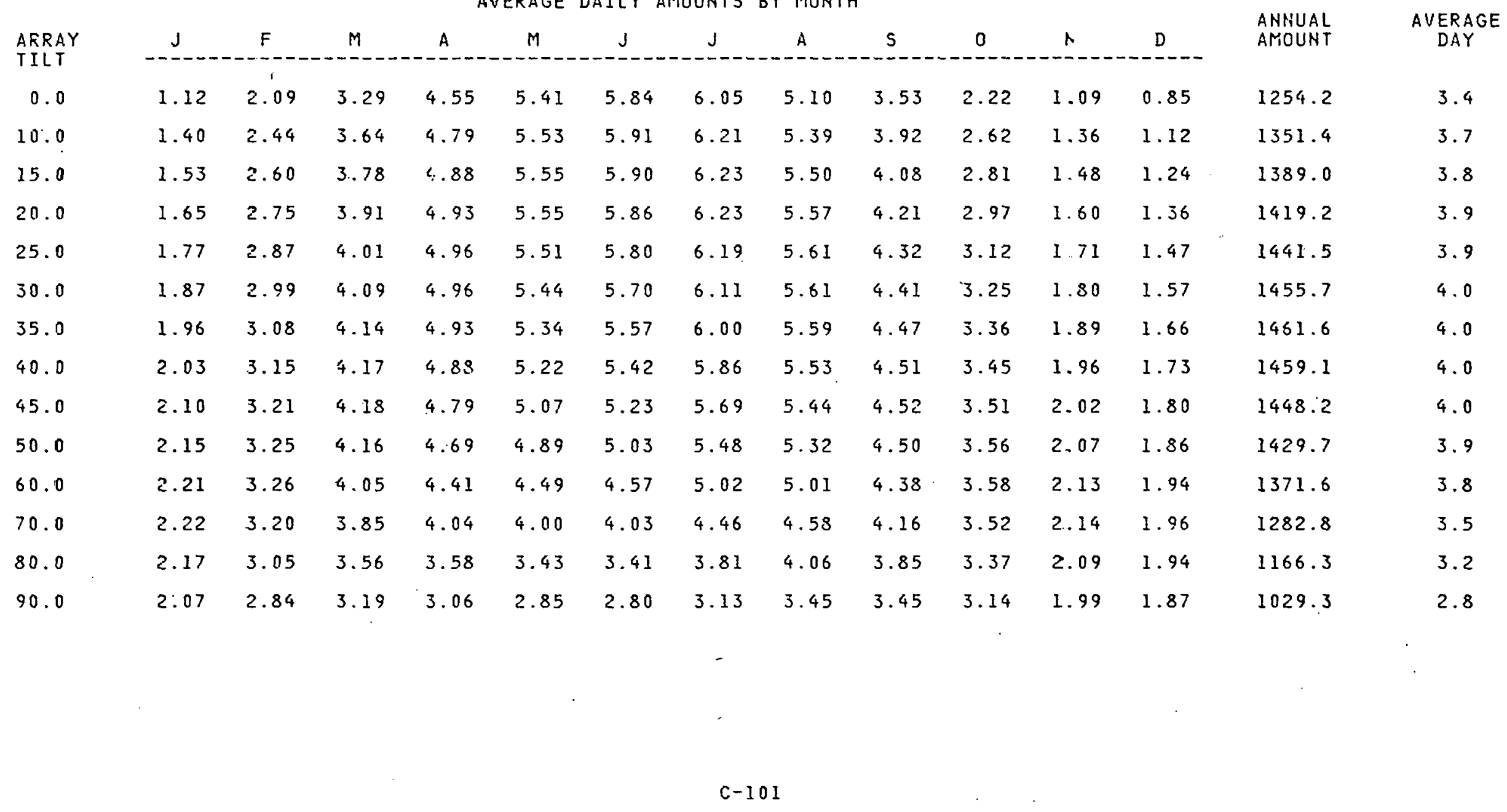


AVERAGE DAI:Y TOTAL TERRESTRIAL INSOLATION ESTIMATES (KWHISQ. M)

SITE: MINNEAPOLIS-ST.PAUNIN

LATITUDE: 44 DEGREES 53 MINUTES

aVERAGE DAILY AMOUNTS BY MONTH

\begin{tabular}{|c|c|c|c|c|c|c|c|c|c|c|c|c|c|c|}
\hline $\begin{array}{l}\text { ARRAY } \\
\text { TILT }\end{array}$ & $\mathbf{J}$ & $F$ & $M$ & $A$ & if & $\mathbf{J}$ & $j$ & A & $S$ & 0 & $N$ & D & $\begin{array}{l}\text { ANNUAL } \\
\text { ANIOUNT }\end{array}$ & $\begin{array}{c}\text { AVERAGE } \\
\text { DAY }\end{array}$ \\
\hline 0.0 & 1.47 & 2.41 & 3.47 & 4.55 & 5.47 & 6.08 & 6.21 & 5.32 & 3.95 & 2.71 & 1.52 & 1.11 & 1349.1 & 3.7 \\
\hline 10.0 & 1.80 & 2.77 & 3.79 & 4.75 & 5.57 & 6.12 & 6.33 & 5.59 & 4.34 & 3.15 & 1.87 & 1.40 & 1445.9 & 4.0 \\
\hline 15.0 & 1.95 & 2.92 & 3.92 & 4.81 & 5.57 & 6.09 & 6.33 & 5.67 & 4.50 & 3.35 & 2.03 & 1.53 & 1482.6 & 4.1 \\
\hline 20.0 & 2.03 & 3.07 & 4.02 & 4.34 & 5.54 & 6.04 & 6.31 & 5.72 & 4.63 & 3.53 & 2.18 & 1.65 & 1511.2 & 4.1 \\
\hline 25.0 & 2.21 & 3.19 & 4.11 & 4.35 & 5.49 & 5.95 & 6.24 & 5.74 & 4.74 & 3.69 & 2.32 & 1.76 & 1531.2 & 4.2 \\
\hline 30.0 & 2.33 & 3.30 & 4.17 & 4.33 & 5.40 & 5.83 & 6.15 & 5.72 & 4.81 & 3.82 & 2.44 & 1.86 & 1542.5 & 4.2 \\
\hline 35.0 & 2.42 & 3.38 & 4.20 & 4.79 & 5.29 & 5.68 & 6.01 & 5.68 & 4.86 & 3.93 & 2.55 & 1.95 & 1545.0 & 4.2 \\
\hline 40.0 & 2.51 & 3.45 & 4.21 & 4.72 & 5.14 & 5.50 & 5.85 & 5.60 & 4.88 & 4.02 & 2.64 & 2.03 & 1538.7 & 4.2 \\
\hline 45.0 & 2.58 & 3.49 & 4.20 & 4.62 & 4.97 & 5.29 & 5.65 & 5.49 & 4.87 & 4.08 & 2.71 & 2.09 & 1523.7 & 4.2 \\
\hline 50.0 & 2.63 & 3.52 & 4.17 & 4.50 & 4.79 & 5.07 & 5.44 & 5.35 & 4.83 & 4.12 & 2.77 & 2.15 & 1501.9 & 4.1 \\
\hline 60.0 & 2.69 & 3.50 & 4.02 & 4.20 & 4.36 & 4.56 & 4.93 & 4.99 & 4.67 & 4.12 & 2.84 & 2.21 & 1433.2 & 3.9 \\
\hline 70.0 & 2.68 & 3.40 & 3.79 & $3.8 I$ & 3.84 & 3.97 & 9.33 & 4.52 & 4.40 & 4.01 & 2.83 & 2.22 & 1332.7 & 3.7 \\
\hline 80.0 & 2.60 & 3.22 & 3.47. & $3 . \geq 5$ & 3.26 & 3.33 & 3.64 & 3.95 & 4.03 & 3.81 & 2.75 & 2.17 & 1204.4 & 3.3 \\
\hline 90.0 & 2.46 & 2.97 & 3.08 & 2.82 & 2.67 & 2.68 & 2. 95 & 3.31 & 3.57 & 3.52 & 2.60 & 2.07 & 1055.6 & 2.9 \\
\hline
\end{tabular}


AVERAGE DAILY TOTAL TERRESTRIAL INSOLATION ESTIMATES (KWH:SQ. M)

SITE: ROCHESTER

LATITUDE: 43 DEGREES 55 MINUTES

AVERAGE DAILY AMOUNTS BY MONTH

\begin{tabular}{|c|c|c|c|c|c|c|c|c|c|c|c|c|c|c|}
\hline ARRAY & $\mathbf{J}$ & $F$ & $M$ & $A$ & $M$ & $\mathrm{~J}$ & 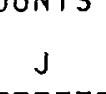 & 100. & $\mathrm{s}$ & 0 & $N$ & $D$ & $\begin{array}{l}\text { ANNUAL } \\
\text { AMOUNT }\end{array}$ & $\begin{array}{c}\text { AVERAGE } \\
\text { DAY }\end{array}$ \\
\hline 0.0 & 1.51 & 2.37 & 3.41 & 4.45 & 5.35 & 5.99 & 6.02 & 5.25 & 3.94 & 2.74 & 1.56 & 1.17 & 1333.4 & 3.7 \\
\hline 10.0 & 1.83 & 2.71 & 3.70 & 4.63 & 5.43 & 6.03 & 6.12 & 5.49 & 4.31 & 3.17 & 1.90 & 1.45 & 1424.6 & 3.9 \\
\hline 15.0 & 1.97 & 2.85 & 3.82 & 4.68 & 5.43 & 6.00 & 6.12 & 5.56 & 4.45 & 3.36 & 2.06 & 1.58 & 1458.7 & 4.0 \\
\hline 20.0 & 2.11 & 2.98 & 3.91 & 4.71 & 5.40 & 5.94 & 6.09 & 5.60 & 4.58 & 3.53 & 2.21 & 1.70 & 1484.8 & 4.1 \\
\hline 25.0 & 2.23 & 3.09 & 3.99 & 4.71 & 5.34 & 5.85 & 6.02 & 5.61 & 4.67 & 3.68 & 2.34 & 1.81 & 1502.5 & 4.1 \\
\hline 30.0 & 2.34 & 3.19 & 4.04 & 4.69 & .3 .25 & 5.73 & 5.92 & 5.59 & 4.74 & 3.81 & 2.45 & 1.91 & 1511.8 & 4.1 \\
\hline 35.0 & 2.43 & 3.26 & 4.06 & 4.64 & 5.13 & 5.57 & 5.79 & 5.54 & 4.78 & 3.91 & c. .56 & 2.00 & 1512.5 & 4.1 \\
\hline 40.0 & 2.51 & 3.32 & 4.07 & $4.5 t$ & 4.99 & 5.39 & 5.62 & 5.46 & 4.79 & 3.99 & 2.64 & 2.07 & 1504.7 & 4.1 \\
\hline 45.0 & 2.58 & 3.36 & 4.05 & 4.47 & 4.82 & 5.18 & 5.43 & 5.34 & 4.78 & 4.05 & 2.71 & 2.13 & 1488.8 & 4.1 \\
\hline 50.0 & 2.62 & 3.37 & 4.01 & 4.35 & 4.64 & 4.97 & 5.22 & 5.21 & 4.73 & 4.08 & 2.77 & 2.18 & 1466.1 & 4.0 \\
\hline 60.0 & 2.67 & 3.35 & 3.86 & 4.05 & 4.22 & 4.46 & 4.73 & 4.84 & 4.57 & 4.06 & 2.82 & 2.24 & 1396.2 & 3.8 \\
\hline 70.0 & 2.66 & 3.25 & 3.63 & $3.6 !$ & 3.71 & 3.87 & 4.14 & 4.38 & 4.29 & 3.95 & 2.81 & 2.25 & 1295.7 & 3.5 \\
\hline 80.0 & 2.57 & 3.07 & 3.32 & 3.21 & 3.14 & 3.24 & 3.48 & 3.82 & 3.92 & 3.74 & $\geq .72$ & 2.19 & 1169.0 & 3.2 \\
\hline 90.0 & 2.43 & 2.82 & 2.94 & $2.7 !$ & 2.57 & 2.59 & 2.82 & 3.19 & 3.46 & 3.44 & 2.57 & 2.08 & 1022.4 & 2.8 \\
\hline
\end{tabular}


AVERAGE DAILY TOTAL TERRESTRIAL INSOLATION ESTIMATES (KWH/SQ. M)

$\begin{aligned} \text { SITE: } & \text { COLUMBIA } \\ \text { LATITUDE: } & 38 \text { DEGREES } 49 \text { MINUTES }\end{aligned}$

AVERAGE DAILY AMJUNTS BY MONTH

\begin{tabular}{|c|c|c|c|c|c|c|c|c|c|c|c|c|c|c|}
\hline \multirow{2}{*}{$\begin{array}{l}\text { ARRAY } \\
\text { TILT }\end{array}$} & & \multirow{2}{*}{$\begin{array}{l}\text { ANNUAL } \\
\text { AMOUNT }\end{array}$} & \multirow{2}{*}{$\begin{array}{l}\text { AVERAGE } \\
\text { DAY }\end{array}$} \\
\hline & $J$ & $F$ & $M$ & A & $M$ & $J$ & $J$ & A & $s$ & 0 & $\mathrm{H}$ & $D$ & & \\
\hline 0.0 & 1.93 & 2.76 & 3.71 & 4.81 & 5.93 & 6.59 & 6.67 & 5.93 & 4.57 & 3.47 & 2.22 & 1.65 & 1530.8 & 4.2 \\
\hline 10.0 & 2.27 & 3.08 & 3.98 & 4.96 & 5.97 & 6.58 & 6.72 & 6.14 & 4.93 & 3.96 & 2.65 & 1.99 & 1621.9 & 4.4 \\
\hline 15.0 & 2.42 & 3.22 & 4.07 & 5.00 & 5.95 & 6.52 & 6.69 & 5.20 & 5.07 & 4.17 & 2.84 & 2.15 & 1653.9 & 4.5 \\
\hline 20.0 & 2.55 & 3.34 & 4.15 & 5.00 & 5.89 & 6.42 & 6.62 & 6.22 & 5.18 & 4.36 & 3.02 & 2.29 & 1676.6 & 4.6 \\
\hline 25.0 & 2.68 & 3.44 & 4.20 & 4.98 & 5.79 & 6.29 & 6.52 & 6.20 & 5.27 & 4.51 & 3.17 & 2.42 & 1689.7 & 4.6 \\
\hline 30.0 & 2.78 & 3.52 & 4.23 & 4.94 & 5.67 & 6.12 & 6.37 & 6.15 & 5.32 & 4.65 & 3.31 & 2.53 & 1693.2 & 4.6 \\
\hline 35.0 & 2.87 & 3.58 & 4.24 & 4.86 & 5.51 & 5.92 & 6.19 & 6.06 & 5.34 & 4.75 & 3.43 & 2.63 & 1687.0 & 4.6 \\
\hline 40.0 & 2.95 & 3.62 & 4.22 & 4.76 & 5.33 & 5.69 & 5.98 & 5.94 & 5.32 & 4.82 & 3.53 & 2.72 & 1671.6 & 4.6 \\
\hline 45.0 & 3.00 & 3.64 & 4.18 & 4.64 & 5.13 & 5.45 & 5.75 & 5.79 & 5.28 & 4.87 & $3.51^{-}$ & 2.78 & 1648.2 & 4.5 \\
\hline 50.0 & 3.04 & 3.64 & 4.12 & 4.50 & 4.91 & 5.18 & 5.49 & 5.61 & 5.20 & 4.88 & 3.56 & 2.83 & 1615.5 & 4.4 \\
\hline 60.0 & 3.06 & 3.57 & 3.92 & 4.14 & 4.39 & 4.57 & 4.89 & 5.15 & 4.96 & 4.82 & 3.70 & 2.88 & 1523.1 & 4.2 \\
\hline 70.0 & 3.00 & 3.42 & 3.64 & 3.70 & 3.78 & 3.85 & 4.18 & 4.58 & 4.61 & 4.64 & 3.64 & 2.86 & 1397.3 & 3.8 \\
\hline 80.0 & 2.88 & 3.19 & 3.29 & 3.19 & 3.15 & 3.16 & 3.45 & 3.91 & 4.15 & 4.35 & 3.50 & 2.76 & 1246.3 & 3.4 \\
\hline 90.0 & 2.68 & 2.90 & 2.86 & 2.62 & 2.49 & 2.44 & 2.68 & 3.17 & 3.60 & 3.95 & 3.26 & 2.60 & 1072.5 & 2.9 \\
\hline
\end{tabular}


AVERAGE DAILY TOTAL TERRESTRIAL INSOLATION ESTIMATES (KLH/SQ. M)

$\begin{aligned} \text { SITE: } & \text { KANSAS CITY M MO } \\ \text { LATITUDE: } & 39 \text { DEGREES } 18 \text { MINUTES }\end{aligned}$

AVERAGE DAILY AMOUNTS BY MONTH

\begin{tabular}{|c|c|c|c|c|c|c|c|c|c|c|c|c|c|c|}
\hline ARRAY & $\mathrm{J}$ & $F$ & $M$ & A & $M$ & $\mathrm{~J}$ & $\mathrm{~J}$ & A & $S$ & 0 & $N$ & $D$ & $\begin{array}{l}\text { ANNUAL } \\
\text { AMOUNT }\end{array}$ & $\begin{array}{c}\text { AVERAGE } \\
\text { DAY }\end{array}$ \\
\hline 0.0 & 2.05 & 2.82 & 3.79 & 4.97 & 5.90 & 6.56 & 6.62 & 5.87 & 4.58 & 3.44 & 2.32 & 1.78 & 1544.8 & 4.2 \\
\hline 10.0 & 2.43 & 3.17 & 4.06 & 5.13 & 5.95 & 6.55 & 6.68 & 6.09 & 4.95 & 3.94 & 2.80 & 2.18 & 1643.3 & 4.5 \\
\hline 15.0 & 2.60 & 5.31 & 4.17 & 5.17 & 5.93 & 6.49 & 6.65 & 6.15 & 5.10 & 4.15 & 3.02 & 2.36 & 1678.8 & 4.6 \\
\hline 20.0 & 2.76 & 3.44 & 4.25 & 5.18 & 5.87 & 6.40 & 6.59 & 6.17 & 5.22 & 4.34 & 3.22 & 2.53 & 1704.7 & 4.7 \\
\hline 25.0 & 2.90 & 3.55 & 4.31 & 5.17 & 5.78 & 6.27 & 6.49 & 6.16 & 5.30 & 4.50 & 3.40 & 2.68 & 1720.9 & 4.7 \\
\hline 30.0 & 3.03 & 3.64 & 4.34 & 5.12 & 5.66 & 6.11 & 6.35 & 6.11 & 5.36 & 4.64 & 355 & 2.82 & 1727.2 & 4.7 \\
\hline 35.0 & 3.13 & 3.70 & 4.35 & 5.05 & 5.51 & 5.91 & 6.17 & 6.02 & 5.38 & 4.74 & 3.69 & 2.94 & 1723.6 & 4.7 \\
\hline 40.0 & 3.22 & 3.75 & 4.34 & 4.95 & 5.33 & 5.69 & 5.96 & 5.91 & 5.37 & 4.82 & 3.80 & 3.04 & 1710.3 & 4.7 \\
\hline 45.0 & 3.29 & 3.77 & 4.30 & 4.82 & 5.13 & 5.45 & 5.74 & 5.76 & 5.33 & 4.87 & 3.89 & 3.12 & 1689.0 & 4.6 \\
\hline 50.0 & 3.34 & 3.78 & 4.24 & 4.67 & 4.91 & 5.18 & 5.48 & 5.58 & 5.26 & 4.89 & 3.95 & 3.18 & 1658.0 & 4.5 \\
\hline 60.0 & 3.37 & 3.71 & 4.04 & 4.30 & 4.40 & 4.57 & 4.89 & 5.13 & 5.02 & 4.83 & .4 .01 & 3.25 & 1568.1 & 4.3 \\
\hline 70.0 & 3.32 & 3.56 & 3.76 & 3.85 & 3.80 & 3.88 & 4.19 & 4.57 & 4.67 & 4.65 & 3.96 & 3.23 & 1443.4 & 4.0 \\
\hline 80.0 & 3.18 & 3.33 & 3.40 & 3.32 & 3.16 & 3.18 & 3.46 & 3.91 & 4.21 & 4.37 & 3.81 & 3.13 & 1291.9 & 3.5 \\
\hline 90.0 & 2.97 & 3.03 & 2.96 & 2.73 & 2.51 & 2.46 & 2.70 & 3.18 & 3.66 & 3.97 & 3.56 & 2.96 & 1116.1 & 3.1 \\
\hline
\end{tabular}


AVERAGE DAILY TOTAL TERRESTRIAL INSOLATION ESTIMATES (KWH/SQ. M)

$\begin{aligned} \text { SITE: } & \text { SPRINGFIELD } \\ \text { LATITUDE: } & \text { jT DEGREES } 14 \text { MINUTES }\end{aligned}$

$\begin{aligned} \text { SITE: } & \text { 'PPRINGFIELD } \\ \text { LATITUDE: } & \text { jT DEGREES } 14 \text { MINUTES }\end{aligned}$

AVERAGE DAELY AMOUNTS BY MONTH

\begin{tabular}{|c|c|c|c|c|c|c|c|c|c|c|c|c|c|c|}
\hline ARRAY & $J$ & $F$ & $M$ & $A$ & $M$ & $J$ & $J$ & $A$ & $S$ & 0 & $N$ & D & $\begin{array}{l}\text { ANNUAL } \\
\text { AMOUNT }\end{array}$ & $\begin{array}{c}\text { AVERAGE } \\
\text { DAY }\end{array}$ \\
\hline 0.0 & 2.15 & 2.92 & 3.89 & 5.06 & 5.94 & 6.54 & 6.51 & 5.91 & 4.66 & 3.60 & 2.44 & 1.90 & 1569.9 & 4.3 \\
\hline 10.0 & 2.52 & 3.25 & 4.15 & 5.20 & 5.96 & e. 51 & 6.54 & 6.10 & 5.01 & 4.08 & 2.90 & 2.29 & 1660.7 & 4.5 \\
\hline 15.0 & 2.69 & 3.39 & 4.24 & 5.23 & 5.92 & $\epsilon .44$ & 6.50 & 6.14 & 5.14 & 4.29 & 3.10 & 2.47 & 1692.2 & 4.6 \\
\hline 20.0 & 2.83 & 3.51 & 4.32 & 5.23 & 5.86 & E. 34 & E. 42 & 6.15 & 5.25 & 4.46 & 3.28 & 2.63 & 1714.1 & 4.7 \\
\hline 25.0 & 2.97 & 3.61 & 4.37 & 5.21 & 5.75 & 6.20 & 6.31 & 6.12 & 5.32 & 4.62 & 3.45 & 2.78 & 1726.3 & 4.7 \\
\hline 30.0 & 3.08 & 3.68 & 4.39 & 5.15 & 5.62 & 6.02 & 6.16 & 6.06 & 5.36 & 4.74 & 3.59 & 2.91 & 1728.6 & 4.7 \\
\hline 35.0 & 3.18 & 3.74 & 4.39 & 5.07 & 5.46 & 5.82 & 5.97 & 5.97 & 5.37 & 4.84 & 3.71 & 3.02 & 1721.0 & 4.7 \\
\hline 40.0 & 3.25 & 3.78 & 4.37 & 4.96 & 5.28 & 5.59 & 5.77 & 5.84 & 5.35 & 4.90 & 3.81 & 3.11 & 1704.7 & 4.7 \\
\hline 45.0 & 3.31 & 3.79 & 4.32 & 4.82 & 5.07 & 5.34 & 5.54 & 5.68 & 5.29 & 4.94 & 3.89 & 3.18 & 1679.5 & 1.4 .6 \\
\hline 50.0 & 3.35 & 3.78 & 4.25 & 4.66 & 4.84 & 5.07 & 5.27 & 5.49 & 5.21 & 4.94 & 3.94 & 3.24 & 1644.9 & 4.5 \\
\hline 60.0 & 3.36 & 3.70 & 4.03 & 4.27 & 4.31 & 4.44 & 4.67 & 5.02 & 4.95 & 4.86 & 3.97 & 3.29 & 1548.1 & 4.2 \\
\hline 70.0 & 3.29 & 3.54 & 3.73 & 3.80 & 3.69 & 3.74 & 3.98 & 4.44 & 4.58 & 4.66 & 3.89 & 3.25 & 1417.9 & 3.9 \\
\hline 80.0 & 3.14 & 3.29 & 3.35 & 3.25 & 3.06 & 3.04 & 3.27 & 3.77 & 4.10 & 4.35 & 3.73 & 3.14 & 1262.2 & 3.5 \\
\hline 90.0 & 2.92 & 2.97 & 2.91 & 2.66 & 2.39 & 2.34 & 2.52 & 3.05 & 3.54 & 3.94 & 3.46 & 2.94 & 1083.8 & 3.0 \\
\hline
\end{tabular}


AVERAGE DAILY TOTAL TERRESTRIAL INSOLATION ESTIMATES (KWH:SQ. M)

SITE: ST. LOUIS MO

LATITUDE: 38 DEGREES 45 MINUTES

aVERAGE DAILY AMOUNTS BY MONTH

\begin{tabular}{|c|c|c|c|c|c|c|c|c|c|c|c|c|c|c|}
\hline ARRAY & $J$ & $F$ & $M$ & $A$ & $M$ & $\begin{array}{ll}2 \\
0\end{array}$ & ש & trom & $S$ & 0 & $H$ & $\mathrm{D}$ & $\begin{array}{l}\text { ANHUAL } \\
\text { AMOUNT }\end{array}$ & $\begin{array}{c}\text { AUERAGE } \\
\text { DAY }\end{array}$ \\
\hline 0.0 & 1.98 & 2.80 & 3.79 & 4.94 & 5.90 & 6.60 & 6.46 & 5.73 & 4.59 & 3.47 & 226 & 1.67 & 1528.9 & 4.2 \\
\hline 10.0 & 2.33 & 5.12 & 4.06 & 5.09 & 5.94 & 6.59 & 6.51 & 5.93 & 4.96 & 3.95 & 2.71 & 2.02 & 1620.9 & 4.4 \\
\hline 15.0 & 2.49 & 5.26 & 4.16 & 5.13 & 5.91 & 6.53 & 6.47 & 5.98 & 5.11 & 4.16 & 2.91 & 2.18 & 1653.5 & 4.5 \\
\hline 20.0 & 2.63 & 5.39 & 4.24 & 5.13 & 5.85 & 6.43 & 6.41 & 6.00 & 5.22 & $4: 34$ & 3.09 & 2.33 & 1676.7 & 4.6 \\
\hline 25.0 & 2.76 & 5.49 & 4.30 & 5.11 & 5.76 & 6.30 & 6.30 & 5.98 & 5.30 & 4.50 & 3.25 & 2.46 & 1690.3 & 4.6 \\
\hline 30.0 & 2.87 & $\Xi .57$ & 4.33 & 5.07 & 5.63 & 6.13 & 6.17 & 5.93 & 5.35 & 4.63 & 3.39 & 2.58 & 1694.3 & 4.6 \\
\hline 35.0 & 2.97 & 3.63 & 4.34 & 4.99 & 5.48 & 5.93 & 5.99 & 5.84 & 5.37 & 4.73 & 3.52 & 2.68 & 1688.7 & 4.6 \\
\hline 40.0 & 3.04 & 3.67 & 4.32 & 4.89 & 5.30 & 5.70 & 5.79 & 5.73 & 5.36 & 4.81 & 3.62 & 2.76 & 1673.8 & 4.6 \\
\hline 45.0 & 3.10 & 3.69 & 4.28 & 4.76 & 5.10 & 5.46 & 5.57 & 5.58 & 5.31 & 4.85 & 3.69 & 2.83 & 1651.0 & 4.5 \\
\hline 50.0 & 3.14 & 3.69 & 4.21 & 4.61 & 4.88 & 5.19 & 5.32 & 5.40 & 5.24 & 4.86 & 3.75 & 2.88 & 1618.7 & 4.4 \\
\hline 60.0 & 3.16 & $\equiv .62$ & 4.01 & 4.24 & 4.36 & 4.57 & 4.74 & 4.96 & 5.00 & 4.80 & 3.79 & 2.93 & 1527.2 & 4.2 \\
\hline 70.0 & $3.11^{\circ}$ & 3.47 & 3.73 & 3.79 & 3.76 & 3.87 & 4.06 & 4.41 & 4.64 & 4.62 & 3.73 & 2.91 & 1402.3 & 3.8 \\
\hline 80.0 & 2.97 & I. 24 & 3.36 & 3.26 & 3.13 & 3.16 & 3.35 & 3.77 & 4.18 & 4.33 & 3.58 & 2.81 & 1251.9 & 3.4 \\
\hline 90.0 & 2.77 & 2. 94 & 2.33 & 2.68 & 2.47 & 2.44 & 2.61 & 3.07 & 3.62 & 3.93 & 3.35 & 2.65 & 1078.6 & 3.0 \\
\hline
\end{tabular}


AVERAGE DAILY TOTAL TERRESTRIAL INSOLATION ESTIMATES (KWH/SQ. M)

SITE: JACKSON

NS

LATITUDE: 32 DEGREES 19 MINUTES

AVERAGE CAILY AMOLNTS BY MONTH

\begin{tabular}{|c|c|c|c|c|c|c|c|c|c|c|c|c|c|c|}
\hline $\begin{array}{l}\text { ARRAY } \\
\text { TILT }\end{array}$ & $\mathbf{J}$ & $F$ & $M$ & A & $M$ & $\therefore$ & $\mathrm{J}$ & $A$ & 5 & 0 & $N$ & $D$ & $\begin{array}{l}\text { ANNUAL } \\
\text { AMOUNT }\end{array}$ & $\begin{array}{c}\text { AVERAGE } \\
\text { DAY }\end{array}$ \\
\hline 0.0 & 2.37 & 3.24 & 4.31 & 5.38 & 6.12 & 6.38 & 6.02 & $5.6 I$ & 4.75 & 4.01 & 2.84 & 2.23 & 1622.0 & 4.4 \\
\hline 10.0 & 2.69 & 3.54 & 4.55 & 5.49 & 6.09 & 6.29 & E.99 & 5.73 & 5.03 & 4.45 & 3.27 & 2.60 & 1696.4 & 4.6 \\
\hline 15.0 & 2.83 & 3.66 & 4.53 & 5.49 & 6.02 & 6.20 & 5.92 & 5.73 & 5.12 & 4.64 & 3.46 & 2.76 & 1719.5 & 4.7 \\
\hline 20.0 & 2.95 & 3.77 & 4.59 & 5.47 & 5.92 & 6.07 & 5.82 & 5.71 & 5.19 & 4.80 & 3.63 & 2.91 & 1733.0 & 4.7 \\
\hline 25.0 & 3.05 & 3.85 & 4.72 & 5.42 & 5.79 & 5.90 & 5.69 & 5.66 & 5.23 & 4.93 & 3.77 & 3.04 & 1736.8 & 4.8 \\
\hline 30.0 & 3.14 & 3.91 & 4.72 & 5.33 & 5.63 & 5.71 & 5.53 & 5.57 & 5.24 & 5.03 & 3.90 & 3.15 & 1730.8 & 4.7 \\
\hline 35.0 & 3.21 & 3.95 & 4.70 & 5.22 & 5.44 & 5.49 & 5.35 & 5.45 & 5.22 & 5.10 & 4.00 & 3.25 & 1715.9 & 4.7 \\
\hline 40.0 & 3.26 & 3.96 & 4.55 & 5.09 & 5.23 & $=.25$ & 5.14 & 5.31 & 5.17 & 5.14 & 4.08 & 3.32 & 1692.2 & 4.6 \\
\hline 45.0 & 3.30 & 3.95 & 4.58 & 4.92 & 5.00 & 4.98 & 6.91 & 5.14 & 5.09 & 5.15 & 4.13 & 3.38 & 1659.0 & 4.5 \\
\hline 50.0 & 3.31 & 3.93 & 4.48 & 4.73 & 4.74 & 4.69 & c. 65 & 4.94 & 4.98 & 5.12 & 4.16 & 3.41 & 1616.6 & 4.4 \\
\hline 60.0 & 3.28 & 3.80 & 4.21 & 4.28 & 4.14 & 4.05 & $c .07$ & 4.46 & 4.67 & 4.98 & $4: 13$ & 3.42 & 1505.7 & 4.1 \\
\hline 70.0 & 3.17 & 3.59 & 3.35 & 3.75 & 3.49 & 3.37 & 3.43 & 3.89 & 4.27 & 4.72 & 4.01 & 3.34 & 1365.1 & 3.7 \\
\hline 80.0 & 2.99 & 3.30 & 3.41 & 3.14 & 2.82 & 2.67 & 2.78 & 3.25 & 3.77 & 4.35 & 3.79 & 3.18 & 1199.9 & 3.3 \\
\hline 90.0 & 2.74 & 2.94 & 2.91 & 2.52 & 2.16 & 2.02 & 2.13 & 2.59 & 3.20 & 3.88 & 3.48 & 2.95 & 1018.8 & 2.8 \\
\hline
\end{tabular}


AVERAGE DAILY TOTAL TERRESTRIAL INSOLATION ESTIMATES (KWHASQ. M)

SITE: MERIDIAN

LATITUDE: 32 DEGREES 20 MINUTES

AVERAGE DAILY AMOUNTSS BY MONTH

\begin{tabular}{|c|c|c|c|c|c|c|c|c|c|c|c|c|c|c|}
\hline ARRAY & $J$ & $F$ & $M$ & $A$ & M & $\mathrm{J}$ & J & A & 5 & 0 & $N$ & $D$ & $\begin{array}{l}\text { ANNUAL } \\
\text { APIOUNT }\end{array}$ & $\begin{array}{c}\text { AVERAGE } \\
\text { DAY }\end{array}$ \\
\hline 0.0 & 2.34 & 3.20 & 4.18 & 5.24 & 5.86 & 6.18 & 5.74 & 5.49 & 4.58 & 3.97 & 2.82 & 2.20 & 1577.5 & 4.3 \\
\hline 10.0 & 2.65 & 3.50 & 4.41 & 5.34 & 5.83 & 6.10 & 5.72 & 5.60 & 4.84 & 4.41 & 3.25 & 2.57 & 1650.0 & 4.5 \\
\hline 15.0 & 2.79 & 3.62 & 4.49 & 5.34 & 5.77 & 6.01 & 5.65 & 5.60 & 4.93 & 4.59 & 3.43 & 2.73 & 1672.6 & 4.6 \\
\hline 20.0 & 2.91 & 3.72 & 4.54 & 5.32 & 5.67 & 5.38 & 5.56 & 5.58 & 4.99 & 4.74 & 3.60 & 2.87 & 1686.0 & 4.6 \\
\hline 25.0 & 3.01 & 3.80 & 4.57 & 5.26 & 5.55 & 5.73 & 5.44 & 5.53 & 5.03 & 4.87 & 3.75 & 3.00 & 1690.0 & 4.6 \\
\hline 30.0 & 3.10 & 3.86 & $4.57^{\circ}$ & 5.18 & 5.40 & 5.54 & 5.29 & 5.44 & 5.03 & 4.97 & 3.37 & 3.11 & 1684.5 & 4.6 \\
\hline 35.0 & 3.16 & 3.89 & 4.55 & 5.08 & 5.22 & 5.33 & 5.12 & 5.33 & 5.01 & 5.03 & 3.97 & 3.20 & 1670.5 & 4.6 \\
\hline 40.0 & 3.21 & 3.91 & 4.50 & 4.94 & 5.02 & 5.10 & 4.92 & 5.19 & 4.96 & 5.07 & 4.05 & 3.27 & 1647.8 & 4.5 \\
\hline 45.0 & 3.24 & 3.90 & 4.43 & 4.78 & 4.80 & 4.85 & 4.70 & 5.02 & 4.88 & 5.08 & 4.10 & 3.33 & 1616.1 & 4.4 \\
\hline 50.0 & 3.26 & 3.87 & 4.34 & 4.60 & 4.55 & 4.57 & 4.46 & 4.82 & 4.77 & 5.05 & 4.12 & 3.36 & 1575.5 & 4.3 \\
\hline 50.0 & 3.22 & 3.75 & 4.07 & 4.17 & 3.99 & 3.95 & 3.91 & 4.36 & 4.48 & 4.91 & 4.10 & 3.36 & 1468.9 & 4.0 \\
\hline 70.0 & 3.12 & 3.54 & 3.73 & 3.65 & 3.37 & 3.30 & 3.31 & 3.81 & 4.09 & 4.66 & 3.98 & 3.29 & 1333.6 & 3.7 \\
\hline .80 .0 & 2.94 & 3.26 & 3.30 & 3.07 & 2.74 & 2.62 & 2.70 & 3.19 & 3.62 & 4.29 & 3.76 & 3.13 & 1174.4 & 3.2 \\
\hline 90.0 & 2.69 & 2.90 & 2.82 & 2.46 & 2.11 & 2.00 & 2.08 & 2.55 & 3.08 & 3.83 & 3.45 & 2.90 & 999.5 & 2.7 \\
\hline
\end{tabular}


AVERAGE DAILY TOTAL TERRESTRIAL IHSOLATION ESTIMATES (KWH/SQ. M)

SITE: BILLINGS

MT

LATITUDE: 45 DEGREES 48 MINUTES

AVERAGE DAILY AMOLNTS BY MONTH

\begin{tabular}{|c|c|c|c|c|c|c|c|c|c|c|c|c|c|c|}
\hline ARRAY & $J^{-1}$ & $F$ & $M$ & A & $M$ & $J$ & $J$ & A & $S$ & 0 & $N$ & $D$ & $\begin{array}{l}\text { ANNUAL } \\
\text { AMOUNT }\end{array}$ & $\begin{array}{c}\text { AVERAGE } \\
\text { DAY }\end{array}$ \\
\hline 0.0 & 1.53 & 2.40 & 3.75 & 4.81 & 6.03 & 6.86 & 7.51 & 6.38 & 4.64 & 3.11 & 1.77 & 1.33 & 1528.4 & 4.2 \\
\hline 10.0 & 1.92 & 2.78 & 4.12 & 5.04 & 6.15 & 6.53 & 7.69 & 6.76 & 5.17 & 3.71 & 2.26 & 1.74 & 1654.8 & 4.5 \\
\hline 15.0 & 2.09 & 2.95 & 4.27 & 5.11 & 6.17 & 6.50 & 7.72 & 6.89 & 5.39 & 3.9 .8 & 2.49 & 1.93 & 1704.0 & 4.7 \\
\hline 20.0 & 2.25 & 3.10 & 4.40 & 5.15 & 6.14 & E.25 & 7.70 & 6.98 & 5.58 & 4.22 & 2.70 & 2.12 & 1743.5 & 4.8 \\
\hline 25.0 & 2.40 & 3.23 & 4.51 & 5.17 & 6.09 & 6.76 & 7.64 & 7.02 & 5.74 & 4.44 & 2.89 & 2.28 & 1772.8 & 4.9 \\
\hline 30.0 & 2.54 & 3.35 & 4.58 & 5.15 & 6.00 & $E . E 3$ & $\pi .52$ & 7.02 & 5.86 & 4.63 & 3.06 & 2.44 & 1791.5 & 4.9 \\
\hline 35.0 & 2.66 & 3.44 & 4.63 & 5.11 & 5.88 & 6.26 & 7.37 & 6.98 & 5.94 & 4.79 & 3.22 & 2.57 & 1799.5 & 4.9 \\
\hline 40.0 & 2.76 & 3.51 & 4.66 & 5.04 & 5.72 & 6.25 & 7.17 & 6.90 & 5.99 & 4.92 & 3.35 & 2.70 & 1796.9 & 4.9 \\
\hline 45.0 & 2.84 & 3.57 & 4.65 & 4.95 & 5.54 & $\dot{\epsilon} \cdot E 1$ & 6.93 & 6.77 & 5.99 & 5.02 & 3.47 & 2.80 & 1783.4 & 4.9 \\
\hline 50.0 & 2.91 & 3.60 & 4.62 & 4.83 & 5.34 & 5.76 & 6.67 & 6.61 & 5.97 & 5.08 & 3.56 & 2.89 & 1761.6 & 4.8 \\
\hline 60.0 & 2.99 & 3.59 & 4.48 & 4.51 & 4.86 & 5.18 & 6.04 & 6.18 & 5.80 & 5.11 & 3.67 & 3.00 & 1687.6 & 4.6 \\
\hline 70.0 & 2.99 & 3.50 & 4.23 & 4.10 & 4.28 & c. $<9$ & 5.28 & 5.60 & 5.49 & 5.01 & 3.68 & 3.03 & 1574.2 & 4.3 \\
\hline 80.0 & 2.92 & 3.32 & 3.39 & 3.61 & 3.62 & 5.75 & 4.41 & 4.90 & 5.04 & 4.78 & 3.60. & 2.99 & 1425.5 & 3.9 \\
\hline 90.0 & 2.77 & 3.07 & 3.46 & 3.05 & 2.96 & c. 99 & 3.53 & 4.09 & 4.48 & 4.43 & 3.42 & 2.86 & 1251.1 & 3.4 \\
\hline
\end{tabular}


AVERAGE DAILY TOTAL TERRESTRIAL INSOLATION ESTIMATES (KWH/SQ. M)

SITE: CUT BANK

$M T$

IATITUDE: 48 DEGREES 36 MINUTES

AVERAGE DAILY AMOUNTS BY MONTH

\begin{tabular}{|c|c|c|c|c|c|c|c|c|c|c|c|c|c|c|}
\hline ARRAY & $J$ & $F$ & $M$ & A & $M$ & $J$ & $\mathrm{~J}$ & A & 5 & 0 & $i v$ & $D$ & ANTOUNT & $\begin{array}{c}\text { AVERAGE } \\
\text { DAY }\end{array}$ \\
\hline 0.0 & 1.27 & 2.17 & 3.55 & 4.68 & 5.94 & 6.45 & 7.21 & 5.98 & 4.26 & 2.75 & 1.51 & 1.05 & 1427.7 & 3.9 \\
\hline 10.0 & 1.62 & 2.55 & 3.95 & 4.94 & 6.09 & 6.54 & 7.42 & 6.37 & 4.79 & 3.33 & 1.99 & 1.43 & 1555.2 & 4.3 \\
\hline 15.0 & 1.78 & 2.72 & 4.11 & 5.02 & 6.12 & 6.53 & 7.46 & 6.51 & 5.01 & 3.59 & 221 & 1.61 & 1605.9 & 4.4 \\
\hline 20.0 & 1.93 & 2.87 & 4.25 & 5.08 & 6.11 & 6.49 & 7.46 & 6.61 & 5.21 & 3.83 & 2.42 & 1.78 & 1647.6 & 4.5 \\
\hline 25.0 & 2.08 & 5.01 & 4.37 & 5.11 & 6.07 & 6.42 & 7.42 & 6.57 & 5.37 & 4.05 & 2.61 & 1.94 & 1679.9 & 4.6 \\
\hline 30.0 & 2.20 & 2.13 & 4.46 & 5.11 & 6.00 & 6.31 & 7.33 & 6.69 & 5.50 & 4.24 & 2.78 & 2.08 & 1702.2 & 4.7 \\
\hline 35.0 & 2.32 & 3.23 & 4.52 & 5.09 & 5.89 & 6.17 & 7.20 & 6.67 & 5.60 & 4.41 & 2.94 & 2.21 & 1714.4 & 4.7 \\
\hline 40.0 & 2.42 & 3.31 & 4.56 & 5.03 & 5.76 & 6.00 & 7.03 & 6.61 & 5.66 & 4.54 & 3.08 & 2.33 & 1716.3 & 4.7 \\
\hline 45.0 & 2.50 & 3.37 & 4.57 & 4.95 & 5.59 & 5.79 & 6.82 & 6.51 & 5.68 & 4.65 & 3.20 & 2.43 & 1708.1 & 4.7 \\
\hline 50.0 & 2.57 & 3.41 & 4.55 & 4.84 & 5.39 & 5.56 & 6.57 & 6.38 & 5.67 & 4.73 & 3.29 & 2.52 & 1690.3 & 4.6 \\
\hline 60.0 & 2.65 & 3.43 & 4.44 & 4.55 & 4.94 & 5.04 & 6.01 & 6.00 & 5.55 & $4: 79$ & 3.42 & 2.64 & 1628.7 & 4.5 \\
\hline 70.0 & 2.67 & 3.36 & 4.22 & 4.17 & 4.39 & 4.42 & 5.31 & 5.49 & 5.29 & 4.73. & 3.46 & 2.69 & 1528.8 & 4.2 \\
\hline 80.0 & 2.62 & 3.22 & 3.91 & 3.70 & 3.76 & 3.73 & 4.50 & 4.85 & 4.89 & 4.54 & 3.40 & 2.67 & 1393.9 & 3.8 \\
\hline 90.0 & 2.50 & 2.99 & 3.51 & 3.15 & 3.10 & 3.04 & 3.66 & 4.10 & 4.38 & 4.24 & 3.25 & 2.58 & 1232.9 & 3.4 \\
\hline
\end{tabular}


AVERAGE DAILY TOTAL TERRESTRIAL INSOLATION ESTIMATES (KWH/SQ. M)

SITE: DILLON
LATITUDE:

$\begin{array}{cl}\text { SITE: } & \text { DILLON } \\ \text { LATITUDE: } & 4.5 \text { DEGREES } 15 \text { MINUTES }\end{array}$

AVERAGE DAILY AMOLNTS BY MONTH

\begin{tabular}{|c|c|c|c|c|c|c|c|c|c|c|c|c|c|c|}
\hline ARRAY & $J$ & $F$ & $M$ & A & $M$ & $\therefore$ & $\mathrm{J}$ & A & 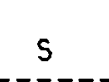 & 0 & $\mathrm{~N}$ & $D$ & $\begin{array}{l}\text { ANNUAL } \\
\text { AMOUNT }\end{array}$ & $\begin{array}{c}\text { AVERAGE } \\
\text { DAY }\end{array}$ \\
\hline 0.0 & 1.66 & 2.66 & 4.33 & 5.17 & 6.27 & 6.75 & 7.54 & 6.38 & 4.79 & 3.23 & 1.90 & 1.42 & 1579.6 & 4.3 \\
\hline $1 C .0$ & 2.08 & 3.10 & 4.45 & 5.42 & 6.40 & $E \cdot \varepsilon l$ & 7.72 & 6.75 & 5.34 & 3.85 & 2.43 & 1.86 & 1712.7 & 4.7 \\
\hline 15.0 & 2.27 & 3.29 & 4.52 & 5.50 & 6.41 & 6.79 & 7.74 & 6.87 & 5.57 & 4.12 & 2.67 & 2.06 & 1764.8 & 4.8 \\
\hline 20.0 & 2.45 & 3.46 & 4.76 & 5.55 & $\therefore .39$ & 6.73 & 7.72 & 6.95 & 5.77 & 4.37 & 2.8 .9 & 2.25 & 1806.7 & 4.9 \\
\hline 25.0 & 2.61 & 3.61 & 4.87 & 5.57 & 6.33 & 664 & ?.65 & 6.99 & 5.92 & 4.60 & 3.10 & 2.43 & 1837.8 & 5.0 \\
\hline 30.0 & 2.76 & 3.74 & 4.96 & 5.56 & 6.23 & 6.50 & 7.53 & 6.99 & 6.05 & 4.79 & 3.29 & 2.59 & 1858.0 & 5.1 \\
\hline 35.0 & 2.89 & 3.85 & 5.02 & 5.51 & 6.10 & 6.34 & 7.37 & 6.94 & 6.13 & 4.95 & 3.46 & 2.73 & 1867.0 & 5.1 \\
\hline 40.0 & 3.00 & 3.94 & 5.04 & 5.44 & 5.93 & 6.13 & 7.16 & 6.86 & 6.17 & 5.08 & 3.60 & 2.86 & 1864.8 & 5.1 \\
\hline 45.0 & 3.09 & 4.00 & 5.04 & 5.33 & 5.74 & 5.89 & 5.92 & 6.73 & 6.18 & 5.18 & 3.72 & 2.97 & 1851.4 & 5.1 \\
\hline 50.0 & 3.16 & 4.03 & 5.01 & 5.20 & 5.53 & 5.55 & 5.65 & $6.5 ?$ & 6.15 & 5.25 & 3.82 & 3.06 & 1829.4 & 5.0 \\
\hline 60.0 & 3.25 & 4.03 & 4.85 & 4.85 & 5.02 & 5.37 & 5.02 & 6.13 & 5.97 & 5.28 & 3.94 & 3.18 & 1753.1 & 4.8 \\
\hline 70.0 & 3.25 & 3.93 & 4.58 & 4.40 & 4.41 & 4.39 & 5.25 & 5.5 .5 & 5.64 & 5.17 & 3.35 & 3.21 & 1635.5 & 4.5 \\
\hline 80.0 & 3.17 & 3.73 & 4.20 & 3.86 & 3.71 & 3.56 & 4.37 & 4.87 & 5.18 & 4.93 & 3.36 & 3.16 & 1481.0 & 4.1 \\
\hline 90.0 & 3.08 & 3.44 & 3.73 & 3.25 & 3.02 & 2.92 & 3.49 & 4.03 & 4.59 & 4.56 & 3.57 & 3.03 & 1299.5 & 3.6 \\
\hline
\end{tabular}


AVERAGE DAILY TOTAL TERRESTRIAL INSOLATION ESTIMATES (KINH/SQ. M)

SITE: GLASGOW
LATITUDE: 48 DEGREES 13 MINUTES

AUERAGE DAILY AMOUNTS BY MONTH

\begin{tabular}{|c|c|c|c|c|c|c|c|c|c|c|c|c|c|c|}
\hline ARRAY & $j$ & $F$ & $M$ & A & $M$ & $\mathrm{~J}$ & $J$ & A & $s$ & 0 & N & D & $\begin{array}{l}\text { ANNUAL } \\
\text { AMOUNT }\end{array}$ & $\begin{array}{c}\text { AVERAGE } \\
\text { DAY }\end{array}$ \\
\hline 0.0 & 1.22 & 2.12 & 3.48 & 4.69 & 5.76 & 6.45 & 6.91 & 5.88 & 4.22 & 2.76 & 1.51 & 1.05 & 1404.5 & 3.8 \\
\hline 10.0 & 1.54 & 2.47 & 3.85 & 4.94 & 5.90 & $6.54^{\circ}$ & 7.10 & 6.25 & 4.74 & 3.34 & 1.96 & 1.42 & 1526.0 & 4.2 \\
\hline 15.0 & 1.69 & 2.63 & 4.01 & 5.02 & 5.92 & 6.52 & 7.14 & 6.38 & 4.96 & 3.60 & 2.17 & 1.59 & 1573.9 & 4.3 \\
\hline 20.0 & 1.83 & 2.78 & 4.14 & 5.08 & 5.91 & 6.48 & 7.14 & 6.47 & 5.14 & 3.83 & 2.37 & 1.75 & 1613.2 & 4.4 \\
\hline 25.0 & 1.95 & 2.90 & 4.25 & 5.11 & 5.87 & 6.41 & 7.09 & 6.53 & 5.30 & 4.05 & 2.55 & 1.90 & 1643.2 & 4.5 \\
\hline 30.0 & 2.07 & 3.01 & 4.33 & 5.11 & 5.80 & 6.30 & 7.01 & 6.55 & 5.42 & 4.24 & 2.72 & 2.04 & 1663.6 & 4.6 \\
\hline 35.0 & 2.17 & 3.11 & 4.39 & 5.08 & 5.69 & 6.16 & 6.88 & 6.52 & 5.51 & 4.40 & 2.37 & 2.16 & 1674.2 & 4.6 \\
\hline 40.0 & 2.26 & 3.18 & 4.42 & 5.02 & 5.56 & 5.98 & 6.71 & 6.46 & 5.57 & 4.53 & 3.00 & 2.28 & 1674.8 & 4.6 \\
\hline 45.0 & 2.33 & 3.24 & 4.43 & 4.93 & 5.39 & 5.77 & 6.51 & 6.36 & 5.59 & 4.63 & 3.11 & 2.37 & 1665.5 & 4.6 \\
\hline 50.0 & 2.39 & 3.27 & 4.41 & 4.82 & 5.20 & 5.54 & 6.27 & 6.22 & 5.58 & 4.71 & $3 . \geq 0$ & 2.45 & 1647.3 & 4.5 \\
\hline 60.0 & 2.47 & 3.28 & 4.30 & 4.53 & 4.77 & 5.02 & 5.73 & 5.85 & 5.45 & 4.76 & 3.32 & 2.57 & 1585.1 & 4.3 \\
\hline 70.0 & 2.48 & 3.22 & 4.08 & 4.15 & 4.24 & 4.40 & 5.07 & 5.34 & 5.18 & 4.69 & 3.35 & 2.61 & 1486.1 & 4.1 \\
\hline 80.0 & 2.43 & 3.07 & 3.77 & 3.67 & 3.62 & 3.71 & 4.29 & 4.72 & 4.79 & 4.50 & 3.29 & 2.59 & 1353.4 & 3.7 \\
\hline 90.0 & 2.31 & 2.85 & 3.38 & 3.13 & 2.99 & 3.01 & 3.50 & 3.99 & 4.29 & 4.20 & 3.15 & 2.49 & 1195.8 & 3.3 \\
\hline
\end{tabular}


AVERAGE DAILY TOTAL TERRESTRIAL INSOLATION ESTIMATES (KWH/SQ. M)

SITE: GREAT FA-LS

LATITUDE: 47 DEGREES 29 MINUTES

AVERAGE DAI:Y AMOUNTS BY MONTH

\begin{tabular}{|c|c|c|c|c|c|c|c|c|c|c|c|c|c|c|}
\hline ARRAY & $\mathrm{J}$ & $F$ & $M$ & A & $M$ & $J$ &.$J$ & A & $S$ & 0 & $N$ & D & $\begin{array}{l}\text { ANNUAL } \\
\text { AMOUNT }\end{array}$ & $\begin{array}{c}\text { AVERAGE } \\
\text { DAY }\end{array}$ \\
\hline 0.0 & 1.33 & 2.27 & 3.69 & 4.69 & 5.83 & 6.63 & 7.34 & 6.09 & 4.35 & 2.91 & 1.57 & 1.06 & 1456.0 & 4.0 \\
\hline 10.0 & 1.67 & 2.65 & 4.08 & 4.93 & 5.96 & 6.71 & 7.54 & 6.48 & 4.87 & 3.51 & 2.02 & 1.40 & 1579.9 & 4.3 \\
\hline 15.0 & 1.83 & 2.82 & 4.25 & 5.01 & 5.98 & 6.70 & 7.57 & 6.61 & 5.09 & 3.78 & 2.23 & 1.56 & 1628.6 & 4.5 \\
\hline 20.0 & 1.97 & 2.98 & 4.39 & 5.06 & 5.97 & 6.65 & 7.57 & 6.70 & 5.27 & 4.02 & 2.43 & 1.70 & 1668.1 & 4.6 \\
\hline 25.0 & 2.11 & 3.11 & 4.50 & 5.09 & 5.92 & 6.57 & 7.52 & 6.76 & 5.43 & 4.24 & 2.61 & 1.84 & 1698.0 & 4.7 \\
\hline 30.0 & 2.23 & 3.23 & 4.59 & 5.08 & 5.84 & 6.46 & 7.42 & 6.77 & 5.55 & 4.44 & 2.78 & 1.97 & 1717.8 & 4.7 \\
\hline 35.0 & 2.34 & 3.33 & 4.65 & 5.05 & 5.73 & 6.30 & 7.28 & 6.74 & 5.64 & 4.60 & 2.93 & 2.09 & 1727.5 & 4.7 \\
\hline 40.0 & 2.44 & 3.41 & 4.69 & 4.99 & 5.59 & 6.12 & 7.10 & 6.67 & 5.69 & 4.74 & 3.06 & 2.19 & 1726.8 & 4.7 \\
\hline 45.0 & 2.52 & 3.46 & 4.69 & 4.90 & 5.42 & 5.90 & 6.83 & 6.56 & 5.71 & 4.84 & 3.16 & 2.27 & 1715.9 & 4.7 \\
\hline 50.0 & 2.58 & 3.50 & 4.67 & 4.79 & 5.23 & 5.66 & 6.62 & 6.42 & 5.69 & 4.92 & 3.25 & 2.35 & 1696.1 & 4.6 \\
\hline 60.0 & 2.66 & 3.51 & 4.54 & 4.49 & 4.78 & 5.11 & 6.03 & 6.03 & 5.55 & 4.97 & 3.37 & 2.44 & 1629.1 & 4.5 \\
\hline 70.0 & 2.66 & 3.44 & 4.31 & 4.10 & 4.24 & 4.47 & 5.31 & 5.49 & 5.27 & 4.89 & 3.39 & 2.48 & 1524.2 & 4.2 \\
\hline 80.0 & 2.61 & 3.28 & 3.98 & 3.63 & 3.62 & 3.75 & 4.47 & 4.83 & 4.87 & 4.69 & 3.32 & 2.45 & 1384.7 & 3.8 \\
\hline 90.0 & 2.48 & 3.04 & 3.56 & 3.08 & 2.98 & 3.03 & 3.62 & 4.07 & 4.35 & 4.36 & 3.17 & 2.35 & 1219.9 & 3.3 \\
\hline
\end{tabular}


AVERAGE DAILY TOTAL TERRESTRIAL INSOLATION ESTIMATES (KWHハSQ. M)

SITE: HELENA
LATITUDE:

ASERAGE DAILY AMOUNTS BY MONTH

\begin{tabular}{|c|c|c|c|c|c|c|c|c|c|c|c|c|c|c|}
\hline ARRAY & $J$ & $F$ & $M$ & $A$ & $M$ & $J$ & $J$ & $A$ & $S$ & 0 & $N$ & $D$ & $\begin{aligned} & \text { ANNUAL } \\
- & \text { AMOUNT }\end{aligned}$ & $\begin{array}{c}\text { AVERAGE } \\
\text { DAY }\end{array}$ \\
\hline C. 0 & $1: 32$ & 2.23 & 3.61 & 4.69 & 5.86 & 6.43 & 7.35 & 6.08 & 4.45 & 2.92 & $1 . \dot{6} 4$ & 1.15 & 1456.3 & 4.0 \\
\hline 10.0 & 1.64 & 2.58 & 3.97 & 4.92 & 5.99 & 6.50 & 7.54 & 6.45 & 4.97 & 3.49 & 2.10 & 1.50 & 1575.2 & 4.3 \\
\hline 15.0 & 1.79 & 2.74 & 4.12 & 4.99 & $\therefore .00$ & 6.48 & 7.57 & 6.57 & 5.19 & 3.74 & 2.31 & 1.67 & 1621.4 & 4.4 \\
\hline 20.0 & 1.92 & 2.88 & 4.25 & 5.04 & 5.99 & 6.43 & 7.56 & 6.66 & 5.37 & 3.98 & 2.51 & 1.82 & 1658.6 & 4.5 \\
\hline 25.0 & 2.04 & 3.00 & 4.35 & 5.06 & 5.94 & 6.35 & 7.50 & 6.70 & 5.53 & 4.18 & 2.109 & 1.97 & 1686.1 & 4.6 \\
\hline 30.0 & 2.16 & 3.11 & 4.43 & 5.05 & 5.85 & 6.24 & 7.40 & 6.71 & 5.65 & 4.36 & 2.86 & 2.10 & 1703.7 & 4.7 \\
\hline 35.0 & 2.25 & 3.20 & 4.48 & 5.01 & 5.74 & 6.08 & 7.25 & 6.67 & 5.73 & 4.52 & 3.30 & 2.22 & 1711.2 & 4.7 \\
\hline 40.0 & 2.34 & 3.26 & 4.50 & 4.95 & 5.59 & 5.90 & 7.06 & 6.60 & 5.78 & 4.64 & 3.13 & 2.32 & 1708.6 & 4.7 \\
\hline 45.0 & 2.41 & 3.31 & 4.50 & 4.85 & 5.42 & 5.68 & 6.83 & 6.48 & 5.79 & 4.74 & 3.24 & 2.41 & 1695.9 & 4.6 \\
\hline 50.0 & 2.46 & 3.34 & 4.48 & 4.74 & 5.22 & 5.45 & 6.58 & 6.33 & 5.77 & 4.80 & 3.32 & 2.48 & 1674.9 & 4.6 \\
\hline 60.0 & 2.53 & 3.34 & 4.34 & 4.44 & 4.76 & 4.92 & 5.98 & 5.93 & 5.61 & 4.84 & 3.93 & 2.58 & 1605.2 & 4.4 \\
\hline 70.0 & 2.53 & 3.26 & 4.11 & 4.04 & 4.21 & 4.29 & 5.24 & 5.39 & 5.32 & 4.75 & 3.45 & 2.61 & 1498.3 & 4.1 \\
\hline 80.0 & 2.46 & 3.10 & 3.78 & 3.57 & 3.58 & 3.60 & 4.40 & 4.73 & 4.90 & 4.54 & 3.38 & 2.57 & 1358.0 & 3.7 \\
\hline 90.0 & 2.34 & 2.87 & 3.37 & 3.02 & 2.94 & 2.90 & 3.54 & 3.97 & 4.36 & 4.21 & 3.21 & 2.47 & 1193.4 & 3.3 \\
\hline
\end{tabular}


AVERAGE DAILY TOTAL TERRESTRIAL INSSOLATION ESTIMATES (KIJH/S?. M)

$\begin{aligned} \text { SITE: } & \text { LEWISTOWN } \\ \text { LATITUDE: } & 47 \text { DEGREES } 3 \text { MINUTES }\end{aligned}$

AVERAGE DAILY AMOUNTS BY MONTH

\begin{tabular}{|c|c|c|c|c|c|c|c|c|c|c|c|c|c|c|}
\hline ARRAY & $J$ & $F$ & $M$ & $A$ & $M$ & 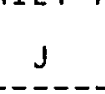 & $\lambda^{-a}$ & $A$ & $s$ & 0 & $N$ & D & $\begin{array}{l}\text { ANNUAL } \\
\text { AMOUNT }\end{array}$ & $\begin{array}{c}\text { AVERAGE } \\
\text { DAY }\end{array}$ \\
\hline 0.0 & 1.32 & 2.18 & 3.56 & 4.56 & 5.70 & 6.49 & 7.21 & 5.99 & 4.32 & 2.85 & 1.53 & 1.14 & 1430.8 & 3.9 \\
\hline 10.0 & 1.65 & 2.53 & 3.92 & 4.78 & 5.82 & 6.57 & 7.40 & 6.36 & 4.83 & 3.42 & 2.0 .3 & 1.51 & 1549.4 & 4.2 \\
\hline 15.0 & 1.81 & 2.68 & 4.67 & 4.85 & 5.84 & 6.55 & 7.43 & 6.48 & 5.04 & 3.67 & 2.24 & 1.68 & 1595.7 & 4.4 \\
\hline 20.0 & 1.95 & 2.82 & 4.20 & 4.90 & 5.82 & 6.50 & 7.42 & 6.57 & 5.22 & 3.90 & 2.4 .3 & 1.85 & 1633.2 & 4.5 \\
\hline 25.0 & 2.08 & 2.94 & 4.30 & 4.92 & 5.78 & 6.42 & 7.37 & 6.62 & 5.37 & 4.10 & 2.61 & 2.00 & 1661.2 & 4.6 \\
\hline 30.0 & 2.19 & 3.05 & 4.38 & 4.91 & 5.70 & 6.31 & 7.27 & 6.62 & 5.48 & 4.28 & 2.77 & 2.13 & 1679.5 & 4.6 \\
\hline 35.0 & 2.30 & 3.14 & 4.43 & 4.88 & 5.59 & 6.15 & 7.13 & 6.59 & 5.57 & 4.44 & 2.91 & 2.26 & 1687.8 & 4.6 \\
\hline 40.0 & 2.39 & 3.20 & 4.46 & 4.81 & 5.45 & 5.97 & 6.95 & 6.52 & 5.61 & 4.56 & $3.0 \%$ & 2.37 & 1686.2 & 4.6 \\
\hline 45.0 & 2.46 & 3.25 & 4.46 & 4.73 & 5.28 & 5.75 & 6.72 & 6.41 & 5.63 & 4.66 & 3.14 & 2.46 & 1674.7 & 4.6 \\
\hline 50.0 & 2.52 & 3.28 & 4.43 & 4.62 & 5.09 & 5.52 & 6.48 & 6.27 & 5.61 & 4.72 & 3.23 & 2.54 & 1654.7 & 4.5 \\
\hline 60.0 & 2.59 & 3.29 & 4.21 & 4.33 & 4.65 & 4.99 & 5.89 & 5.87 & 5.46 & 4.76 & 3.34 & 2.65 & 1587.8 & 4.4 \\
\hline 70.0 & 2.60 & 3.21 & $\$ .08$ & 3.95 & 4.12 & 4.35 & 5.18 & 5.35 & 5.18 & 4.68 & 3.35 & 2.68 & 1484.3 & 4.1 \\
\hline 80.0 & 2.54 & 3.05 & 3.76 & 3.49 & 3.52 & 3.65 & 4.36 & 4.70 & 4.78 & 4.48 & 3.27 & 2.65 & 1347.5 & 3.7 \\
\hline 90.0 & 2.41 & 2.83 & 3.36 & 2.96 & 2.89 & 2.95 & 3.52 & 3.95 & 4.26 & 4.16 & 3.13 & 2.54 & 1186.5 & 3.3 \\
\hline
\end{tabular}


AVERAGE DAILY TOTAL TERRESTRIAL INSOLATION ESTIMATES (KWH/SQ. M)

SITE: MILES CITY
LATITUDE: 46 DEGREES 26 MINUTES

A'IERAGE DAILY AMOUNTS BY MONTH

\begin{tabular}{|c|c|c|c|c|c|c|c|c|c|c|c|c|c|c|}
\hline ARRAY & $\mathrm{J}$ & $F$ & $M$ & $A$ & M & $\begin{array}{ll}2 \\
0\end{array}$ & $J$ & row & $\mathrm{s}$ & 0 & $N$ & $D$ & $\begin{array}{l}\text { ANNUAL } \\
\text { ANOUNT }\end{array}$ & $\begin{array}{c}\text { AVERAGE } \\
\text { DAY }\end{array}$ \\
\hline 0.0 & 1.44 & 2.35 & 3.73 & 4.86 & 5.98 & 6.76 & 7.22 & 6.23 & 4.55 & 3.03 & 1.74 & 1.26 & 1498.5 & 4.1 \\
\hline 10.0 & 1.80 & 2.73 & 4.11 & 5.10 & 6.10 & 6.84 & 7.40 & 6.61 & 5.08 & 3.63 & 2.24 & 1.66 & 1624.9. & 4.5 \\
\hline 15.0 & 1.97 & 2.89 & 4.27 & 5.18 & 6.12 & 6.82 & 7.43 & 6.74 & 5.31 & 3.89 & 2.47 & 1.85 & 1674.4 & 4.6 \\
\hline 20.0 & 2.13 & 3.04 & 4.40 & 5.23 & 6.10 & 6.77 & 7.41 & 6.83 & 5.50 & 4.14 & 2.68 & 2.02 & 1714.4 & 4.7 \\
\hline$\ddot{25.0}$ & 2.27 & 3.18 & 4.51 & 5.25 & 6.05 & 6.68 & 7.36 & 6.88 & 5.65 & 4.35 & 2.88 & 2.19 & 1744.5 & 4.8 \\
\hline 30.0 & 2.40 & 3.29 & 4.59 & 5.24 & 5.97 & 6.55 & 7.25 & 6.88 & 5.77 & 4.54 & 3.06 & 2.34 & 1764.1 & 4.8 \\
\hline $35: 0$ & 2.51 & 3.39 & 4.64 & 5.20 & 5.85 & 6.39 & 7.11 & $6 . .85$ & 5.86 & 4.71 & 3.22 & 2.47 & 1773.3 & 4.9 \\
\hline 40.0 & 2.61 & 3.46 & 4.67 & 5.13 & 5.70 & 6.19 & 6.92 & 6.77 & 5.91 & 4.84 & 3.36 & 2.59 & 1771.9 & 4.9 \\
\hline 45.0 & 2.69 & 3.52 & 4.67 & 5.04 & 5.52 & 5.96 & 6.69 & 6.65 & 5.92 & 4.94 & 3.48 & 2.70 & 1760.0 & 4.8 \\
\hline 50.0 & 2.75 & 3.55 & 4.64 & 4.92 & 5.32 & 5.72 & 6.45 & 6.50 & 5.90 & 5.01 & 3.57 & 2.78 & 1739.4 & 4.8 \\
\hline 60.0 & 2.83 & 3.55 & 4.51 & 4.60 & 4.85 & 5.15 & 5.86 & 6.08 & 5.74 & 5.04 & 3.69 & 2.90 & 1668.9 & 4.6 \\
\hline 70.0 & 2.83 & 3.46 & 4.26 & 4.19 & 4.28 & 4.48 & 5.14 & 5.53 & 5.44 & 4.95 & 3.71 & 2.93 & 1559.5 & 4.3 \\
\hline 80.0 & 2.77 & 3.29 & 3.92 & 3.69 & 3.63 & 3.74 & 4.31 & 4.84 & 5.01 & 4.73 & 3.64 & 2.89 & 1415.0 & 3.9 \\
\hline 90.0 & 2.63 & 3.05 & 3.50 & 3.13 & 2.98 & 3.00 & 3.47 & 4.06 & 4.46 & 4.39 & 3.46 & 2.78 & 1244.7 & 3.4 \\
\hline
\end{tabular}


AVERAGE DAILY TOTAL TERRESTRIAL INSOLATION ESTIMATES (KWH/SQ. M)

SITE: MISSOULA

MT

LATITUDE: 46 DEGREES 55 MINUTES

AVERAGE DAZLY AMOUNTS BY MONTH

\begin{tabular}{|c|c|c|c|c|c|c|c|c|c|c|c|c|c|c|}
\hline ARRAY & $\mathrm{J}$ & $F$ & $M$ & A & $M$ & ( & $J$ & tratr & $S$ & 0 & $N$ & D & $\begin{array}{l}\text { ANNUAL } \\
\text { AMOUNT }\end{array}$ & $\begin{array}{c}\text { AVERAGE } \\
\text { DAY }\end{array}$ \\
\hline 0.0 & 0.99 & 1.81 & 3.09 & 4.36 & 5.62 & 6.09 & :. 34 & 5.93 & 4.28 & 2.56 & 1.29 & 0.84 & 1348.9 & 3.7 \\
\hline 10.0. & 1.16 & 2.06 & 3.38 & 4.57 & 5.74 & 6.16 & 7.53 & 6.29 & 4.77 & 3.02 & 1.61 & 1.05 & 1443.8 & 4.0 \\
\hline 15.0 & 1.24 & 2.16 & 3.49 & 4.63 & 5.76 & $\epsilon .14$ & 7.56 & 6.41 & 4.97 & 3.23 & 1.76 & 1.14 & 1479.4 & 4.1 \\
\hline 20.0 & 1.32 & 2.26 & 3.59 & 4.67 & 5.74 & 6.09 & 7.55 & 6.49 & 5.15. & 3.42 & 1.89 & 1.23 & 1506.9 & 4.1 \\
\hline 25.0 & 1.38 & 2.34 & 3.67 & 4.69 & 5.69 & $E .02$ & 7.49 & 6.54 & 5.29 & 3.58 & 2.02 & 1.31 & 1525.9 & 4.2 \\
\hline 30.0 & 1.44 & 2.41 & 3.73 & 4.68 & 5.61 & E. $\$ I$ & 7.39 & 6.54 & 5.40 & 3.73 & 2.13 & 1.38 & 1536.1 & 4.2 \\
\hline 35.0 & 1.50 & 2.47 & 3.76 & 4.6 .5 & 5.51 & 5.77 & 7.25 & 6.51 & 5.48 & 3.85 & 2.23 & 1.45 & 1537.4 & 4.2 \\
\hline 40.0 & 1.54 & 2.51 & 3.78 & 4.58 & 5.37 & 5.60 & 7.06 & 6.44 & 5.53 & 3.95 & 2.31 & 1.51 & 1529.8 & 4.2 \\
\hline 45.0 & 1.57 & 2.54 & 3.77 & 4.50 & 5.20 & 5.39 & 6.84 & 6.33 & 5.54 & 4.02 & 2.38 & 1.55 & 1513.4 & 4.1 \\
\hline 50.0 & 1.60 & 2.56 & 3.75 & 4.39 & 5.02 & 5.18 & 6.58 & 6.18 & 5.52 & 4.07 & 2.44 & 1.59 & 1489.8 & 4.1 \\
\hline 60.0 & 1.62 & 2.54 & 3.63 & 4.12 & 4.58 & 4.68 & 5.99 & 5.80 & 5.37 & 4.09 & 2.51 & 1.64 & 1419.1 & 3.9 \\
\hline 70.0 & 1.61 & 2.47 & 3.43 & 3.75 & 4.06 & 4.10 & 5.26 & 5.28 & 5.09 & 4.01 & 2.51 & 1.65 & 1316.5 & 3.6 \\
\hline 80.0 & 1.55 & 2.34 & 3.16 & 3.32 & 3.46 & 3.45 & 4.42 & 4.64 & 4.69 & 3.82 & 2.45 & 1.61 & 1185.6 & 3.2 \\
\hline 90.0 & 1.47 & 2.16 & 2.82 & 2.82 & 2.85 & 2.80 & 3.56 & 3.90 & 4.18 & 3.55 & 2.33 & 1.54 & 1035.0 & 2.8 \\
\hline
\end{tabular}


AVERAGE DAILY TOTAL TERRESTRIAL INSOLATION ESTIMATES (KWH_SQ. M)

SITE: ASHEVILLE 2 NC

AVERAGE DAILY AMOUNTS BY MONTH

\begin{tabular}{|c|c|c|c|c|c|c|c|c|c|c|c|c|c|c|}
\hline ARRAY & $J_{---}^{J}$ & $F$ & $M$ & A & $M$ & 3 & $J$ & $A$ & $s$ & 0 & $H$ & $D$ & $\begin{array}{l}\text { ANNUAL } \\
\text { ANIOUNT }\end{array}$ & $\begin{array}{c}\text { AVERAGE } \\
\text { DAY }\end{array}$ \\
\hline 0.0 & 2.27 & 3.07 & 4.12 & 5.26 & 5.69 & 5.85 & 5.59 & 5.13 & 4.28 & 3.62 & 2.67 & 2.07 & 1510.8 & 4.1 \\
\hline 10.0 & 2.63 & 3.39 & 4.38 & 5.39 & 5.69 & 5.81 & 5.59 & 5.26 & 4.56 & 4.05 & 3.15 & 2.47 & 1594.1 & 4.4 \\
\hline 15.0 & 2.78 & 3.53 & 4.47 & 5.42 & 5.65 & 5.74 & 5.55 & 5.28 & 4.65 & 4.24 & 3.36 & 2.65 & 1622.7 & 4.4 \\
\hline 20.0 & 2.93 & 3.64 & 4.54 & 5.41 & 5.57 & 5.64 & 5.47 & 5.27 & 4.73 & 4.40 & 3.55 & 2.81 & 1642.3 & 4.5 \\
\hline 25.0 & 3.05 & 3.74 & 4.58 & 5.37 & 5.47 & 5.50 & 5.37 & 5.23 & 4.77 & 4.53 & 3.72 & 2.96 & 1652.7 & 4.5 \\
\hline 30.0 & 3.16 & 3.81 & 4.60 & 5.31 & 5.33 & 5.35 & 5.24 & 5.17 & 4.79 & 4.64 & 3.86 & 3.09 & 1653.9 & 4.5 \\
\hline 35.0 & 3.25 & 3.86 & 4.60 & 5.21 & 5.17 & 5.16 & 5.08 & 5.07 & 4.79 & 4.71 & 3.99 & 3.20 & 1645.8 & 4.5 \\
\hline 40.0 & 3.32 & 3.89 & 4.56 & 5.09 & 4.99 & 4.96 & 4.90 & 4.96 & 4.75 & 4.76 & 4.08 & 3.29 & 1629.9 & 4.5 \\
\hline 45.0 & 3.37 & 3.90 & 4.50 & 4.94 & 4.79 & 4.74 & 4.70 & 4.81 & 4.69 & 4.79 & 4.16 & 3.36 & 1605.1 & 4.4 \\
\hline 50.0 & 3.40 & 3.88 & 4.42 & 4.77 & 4.57 & 4.49 & 4.48 & 4.64 & 4.60 & 4.78 & 4.20 & 3.41 & 1571.3 & 4.3 \\
\hline 60.0 & 3.39 & 5.78 & 4.18 & 4.35 & 4.05 & 3.93 & 3.97 & 4.24 & 4.35 & 4.68 & 422 & 3.44 & 1478.3 & 4.1 \\
\hline 70.0 & 3.31 & 5.60 & 3.85 & 3.84 & 3.46 & 3.33 & 3.59 & 3.74 & 4.01 & 4.46 & 413 & 3.39 & 1354.3 & 3.7 \\
\hline 80.0 & 3.15 & 3.34 & 3.45 & 3.27 & 2.86 & 2.72 & 2.81 & 3.18 & 3.58 & 4.15 & 393 & 3.26 & 1206.5 & 3.3 \\
\hline 90.0 & 2.91 & 5.00 & 2.97 & 2.65 & 2.23 & 2.12 & 2.20 & 2.59 & 3.08 & 3.73 & 364 & 3.05 & 1038.2 & 2.8 \\
\hline
\end{tabular}


AVERAGE DAILY TOTAL TERRESTRIAL INSOLATION ESTIMATES (KWH/SQ. M)

\section{SITE: OAPE HATTERAS NC
LATITUDE: 35 DEGREES 16 MINUTES}

AVERAGE JAILY AMOUNTS BY MONTH

\begin{tabular}{|c|c|c|c|c|c|c|c|c|c|c|c|c|c|c|}
\hline ARRAY & $\int^{--2}$ & $\mathrm{~F}$ & $M$ & $A$ & $M$ & $\mathbf{J}$ & $\mathrm{J}$ & A & $s$ & 0 & $\mathrm{H}$ & $D$ & $\begin{array}{l}\text { ANNUAL } \\
\text { AMOUNT }\end{array}$ & $\begin{array}{c}\text { AVERAGE } \\
\text { DAY }\end{array}$ \\
\hline 0.0 & 2.15 & 3.00 & 4.18 & 5.60 & 6.18 & 5.42 & 6.05 & 5.38 & 4.63 & 3.59 & 2.74 & 2.08 & 1583.8 & 4.3 \\
\hline 10.0 & 2.48 & 3.31 & 4.44 & 5.74 & 6.19 & 5.37 & 6.06 & 5.52 & 4.94 & 4.01 & 3.24 & 2.47 & 1667.7 & 4.6 \\
\hline 15.0 & 2.62 & 3.44 & 4.54 & 5.77 & 6.14 & 5.29 & 6.01 & 5.54 & 5.06 & 4.19 & 3.45 & 2.65 & 1695.8 & 4.6 \\
\hline 20.0 & 2.75 & 3.55 & 4.61 & 5.76 & 6.06 & 5.17 & 5.92 & 5.53 & 5.14 & 4.35 & 3.65 & 2.81 & 1714.3 & 4.7 \\
\hline 25.0 & 2.86 & 3.64 & 4.65 & 5.72 & 5.94 & 5.03 & 5.81 & 5.50 & 5.20 & 4.48 & 3.83 & 2.96 & 1723.2 & 4.7 \\
\hline 30.0 & 2.96 & 3.71 & 4.67 & 5.65 & 5.79 & 5.84 & 5.66 & 5.43 & 5.22 & 4.58 & 3.98 & 3.08 & 1722.3 & 4.7 \\
\hline 35.0 & 3.04 & 3.76 & 4.66 & 5.55 & 5.61 & 5.63 & 5.49 & 5.33 & 5.22 & 4.66 & 4.11 & 3.19 & 1711.6 & 4.7 \\
\hline 40.0 & 3.10 & 3.78 & 4.63 & 5.42 & 5.41 & 5.41 & 5.29 & 5.20 & 5.18 & 4.71 & 4.21 & 3.28 & 1692.9 & 4.6 \\
\hline 45.0 & $: 3.14$ & 3.79 & 4.57 & 5.26 & 5.19 & 5.15 & 5.07 & 5.05 & 5.12 & 4.73 & 4.29 & 3.35 & 1664.7 & 4.6 \\
\hline 50.0 & 3.17 & 3.77 & 4.48 & 5.08 & 4.93 & 4.88 & 4.82 & 4.87 & 5.03 & 4.72 & 4.33 & 3.40 & 1627.3 & 4.5 \\
\hline 60.0 & 3.16 & 3.67 & 4.24 & 4.62 & 4.36 & 4.25 & 4.26 & 4.44 & 4.75 & 4.61 & 4.55 & 3.43 & 1525.6 & 4.2 \\
\hline 70.0 & 3.07 & 3.49 & 3.91 & 4.08 & 3.70 & 3.57 & 3.62 & 3.92 & 4.37 & 4.40 & 4.26 & 3.38 & 1391.7 & 3.8 \\
\hline 80.0 & 2.92 & 3.23 & 3.49 & 3.45 & 3.03 & 2.87 & 2.97 & 3.32 & 3.90 & 4.08 & 4.06 & 3.25 & 1233.6 & 3.4 \\
\hline 90.0 & 2.69 & 2.91 & 3.00 & ż. 78 & 2.33 & 2.20 & 2.29 & 2.68 & 3.34 & 3.67 & 3.76 & 3.03 & 1054.9 & 2.9 \\
\hline
\end{tabular}


AVERAGE DAILY TOTAL TERRESTRIAL INSOLATION ESTIMATES (KWH.'SQ. M)

$\begin{aligned} \text { SITE: } & \text { CHARLOTTE NC } \\ \text { LATITUDE: } & 35 \text { DEGREES } 13 \text { MINUTES }\end{aligned}$

AVERAGE DAILY AMOUNTS BY MONTH

\begin{tabular}{|c|c|c|c|c|c|c|c|c|c|c|c|c|c|c|}
\hline AERAY & $\mathrm{J}$ & $F$ & $M$ & $A$ & $M$ & $J$ & oris & $A$ & $s$ & 0 & $H$ & $D$ & $\begin{array}{l}\text { ANNUAL } \\
\text { AMOUNT }\end{array}$ & $\begin{array}{c}\text { AVERAGE } \\
\text { DAY }\end{array}$ \\
\hline 0.0 & 2.26 & 3.07 & 4.14 & 5.35 & 5.85 & 6.06 & 5.77 & 5.35 & 4.46 & 3.70 & 272 & 2.12 & 1548.5 & 4.2 \\
\hline 10.0 & 2.61 & 3.39 & 4.40 & 5.48 & 5.85 & 6.01 & 5.77 & 5.49 & 4.75 & 4.15 & 321 & 2.53 & 1633.1 & 4.5 \\
\hline 15.0 & 2.76 & 3.52 & 4.50 & 5.51 & 5.80 & 5.93 & 5.72 & 5.51 & 4.85 & 4.34 & 342 & 2.71 & 1661.9 & 4.6 \\
\hline 20.0 & 2.90 & 3.63 & 4.57 & 5.50 & 5.72 & 5.83 & 5.65 & 5.50 & 4.93 & 4.50 & 3.61 & 2.88 & 1681.5 & 4.6 \\
\hline 25.0 & 3.03 & 3.73 & 4.61 & 5.46 & 5.61 & 5.69 & 5.54 & 5.46 & 4.98 & 4.64 & 3.79 & 3.03 & 1691.5 & 4.6 \\
\hline 30.0 & 3.13 & 3.80 & 4.63 & 5.39 & 5.47 & 5.52 & 5.40 & 5.39 & 5.01 & 4.75 & 3.94 & 3.16 & 1692.1 & 4.6 \\
\hline 35.0 & 3.22 & 3.85 & 4.62 & 5.29 & 5.30 & 5.32 & 5.23 & 5.30 & 5.00 & 4.83 & 4.06 & 3.27 & 1683.1 & 4.6 \\
\hline 40.0 & 3.28 & 3.88 & 4.59 & 5.17 & 5.12 & 5.12 & 5.05 & 5.17 & 4.96 & 4.88 & 4.16 & 3.37 & 1666.2 & 4.6 \\
\hline 45.0 & 3.33 & 3.88 & 4.53 & 5.02 & 4.91 & 4.88 & 4.84 & 5.02 & 4.90 & 4.91 & 4.23 & 3.44 & 1640.0 & 4.5 \\
\hline 50.0 & 3.36 & 3.87 & 4.44 & 4.84 & 4.67 & 4.62 & 4.61 & 4.84 & 4.81 & 4.90 & 4.28 & 3.49 & 1604.7 & 4.4 \\
\hline 60.0 & 3.35 & 3.77 & 4.20 & 4.41 & 4.14 & 4.04 & 4.08 & 4.41 & 4.55 & 4.80 & 4.29 & 3.53 & 1507.8 & 4.1 \\
\hline 70.0 & 3.27 & 5.58 & 3.87 & 3.89 & 3.52 & 3.41 & 3.47 & 3.89 & 4.18 & 4.58 & 4.20 & 3.47 & 1379.2 & 3.8 \\
\hline 80.0 & 3.10 & 3.32 & 3.46 & 3.30 & 2.90 & 2.76 & 2.86 & 3.30 & 3.73 & 4.25 & 4.00 & 3.34 & 1226.3 & 3.4 \\
\hline 90.0 & 2.87 & 2.98 & 2.98 & 2.67 & 2.26 & 2.14 & 2.23 & 2.67 & 3.20 & 3.82 & 3.70 & 3.12 & 1052.8 & 2.9 \\
\hline
\end{tabular}


AVERAGE DAILY TOTAL TERSESTRIAL INSOLATION ESTIMATES (KWH/SQ. M)

$\begin{aligned} \text { SITE: } & \text { CHERRY POINT M NC } \\ \text { LATITUDE: } & 54 \text { DEGREES } 54 \text { MINUTES }\end{aligned}$

AVERAGE DAELY AMOUNTS BY MONTH

\begin{tabular}{|c|c|c|c|c|c|c|c|c|c|c|c|c|c|c|}
\hline ARRAY & 3 & $F$ & $M$ & $A$ & ERAGE & $A=L ð A$ & DUNTS & BY MONTH & $s$ & 0 & $N$ & $D$ & $\begin{array}{l}\text { ANNUAL } \\
\text { AMOUNT }\end{array}$ & $\begin{array}{c}\text { AVERAGE } \\
\text { DAY }\end{array}$ \\
\hline 0.0 & 2.38 & 3.23 & 4.37 & $5 . \epsilon \cdot 6$ & 6.06 & $6 . ? 1$ & 5.76 & 5.15 & 4.49 & 3.69 & 2.85 & 2.27 & 1584.1 & 4.3 \\
\hline 10.0 & 2.76 & 3.58 & 4.65 & 5.80 & 6.06 & 6.06 & 5.76 & 5.28 & 4.78 & 4.13 & 3.36 & 2.71 & 1672.0 & 4.6 \\
\hline 15.0 & 2.92 & 3.72 & 4.75 & 5.83 & 6.01 & 5.98 & 5.71 & 5.29 & 4.89 & 4.32 & 3.59 & 2.91 & 1702.1 & 4.7 \\
\hline 20.0 & 3.07 & 3.84 & 4.82 & 5.82 & 5.93 & 5.87 & 5.63 & 5.28 & 4.96 & 4.48 & 3.80 & 3.09 & 1722.6 & 4.7 \\
\hline 25.0 & 3.20 & 3.94 & 4.87 & $5 .: 8$ & 5.81 & 5.73 & 5.52 & 5.24 & 5.01 & 4.61 & 3.98 & 3.25 & 1733.4 & 4.7 \\
\hline 30.0 & 3.32 & 4.02 & 4.89 & 5.70 & 5.66 & 5.56 & 5.38 & 5.18 & 5.03 & 4.72 & 4.14 & 3.39 & 1734.4 & 4.8 \\
\hline 35.0 & 3.41 & 4.08 & 4.88 & 5.60 & 5.49 & 5.36 & 5.21 & 5.08 & 5.03 & 4.79 & 4.27 & 3.52 & 1725.6 & 4.7 \\
\hline 40.0 & 3.48 & 4.11 & 4.85 & 5.47 & 5.29 & 5.15 & 5.03 & 4.96 & 4.99 & 4.84 & 4.38 & 3.62 & 1708.6 & 4.7 \\
\hline 45.0 & 3.53 & 4.11 & 4.78 & 5.31 & 5.07 & 4.71 & $\mp .82$ & 4.81 & 4.92 & 4.86 & 4.46 & 3.70 & 1682.0 & 4.6 \\
\hline 50.0 & 3.56 & 4.10 & 4.69 & 5.11 & 4.82 & +.55 & 4.59 & 4.64 & 4.83 & 4.85 & 4.51 & 3.75 & 1646.0 & 4.5 \\
\hline 60.0 & 3.56 & 3.99 & 4.44 & 4.155 & 4.26 & 4.05 & 4.05 & 4.23 & 4.56 & 4.75 & 4.52 & 3.79 & 1546.8 & 4.2 \\
\hline 70.0 & 3.47 & 3.80 & 4.08 & 4.39 & 3.61 & 3.42 & 3.45 & 3.73 & 4.19 & 4.52 & 4.43 & 3.74 & 1414.9 & 3.9 \\
\hline 80.0 & 3.29 & 3.51 & 3.64 & 3.46 & 2.96 & 2.76 & 2.84 & 3.17 & 3.74 & 4.20 & 4.22 & 3.59 & 1257.9 & 3.4 \\
\hline 90.0 & 3.04 & 3.15 & 3.13 & 2.78 & 2.29 & 2.13 & 2.21 & 2.57 & 3.20 & 3.77 & 3.90 & 3.35 & 1079.8 & 3.0 \\
\hline
\end{tabular}


AVERAGE DAILY TOTAL TERRESTRIAL INSOLATION ESTIMATES (KWH/SQ. M)

SITE:
LATITUDE :
36 DEGRERS 5 MINUTES

AVERAGE DAILY AMOUNTS BY MONTH

\begin{tabular}{|c|c|c|c|c|c|c|c|c|c|c|c|c|c|c|}
\hline ARRAY & $\mathrm{J}$ & $F$ & $M$ & A & $M$ & 至 & 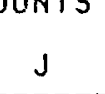 & 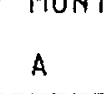 & 5 & 0 & $N$ & $D$ & $\begin{array}{l}\text { ANNUAL } \\
\text { AMOUNT }\end{array}$ & $\begin{array}{c}\text { AVERAGE } \\
\text { DAY }\end{array}$ \\
\hline 0.0 & 2.25 & 3.06 & 4.13 & 5.31 & 5.88 & 6.16 & 5.87 & 5.35 & 4.46 & 3.60 & 2.64 & 2.08 & 1546.8 & 4.2 \\
\hline 10.0 & 2.62 & 3.39 & 4.40 & 5.45 & 5.89 & 6.12 & 5.88 & 5.50 & 4.77 & 4.05 & 3.13 & 2.49 & 1634.7 & 4.5 \\
\hline 15.0 & 2.78 & 3.53 & 4.50 & 5.48 & 5.85 & 6.05 & 5.84 & 5.52 & 4.88 & 4.24 & 3.34 & 2.68 & 1665.2 & 4.6 \\
\hline 20.0 & 2.92 & 3.65 & 4.58 & 5.47 & 5.78 & 5.94 & 5.77 & 5.52 & 4.97 & 4.40 & 3.54 & 2.85 & 1686.3 & 4.6 \\
\hline 25.0 & 3.05 & 3.75 & 4.63 & 5.44 & 5.67 & 5.81 & 5.66 & 5.49 & 5.02 & 4.54 & 3.71 & 3.01 & 1697.9 & 4.7 \\
\hline 30.0 & 3.17 & 3.83 & 4.65 & 5.37 & 5.53 & 5.64 & 5.52 & 5.42 & 5.05 & 4.65 & 3.87 & 3.14 & 1699.9 & 4.7 \\
\hline 35.0 & 3.26 & 3.88 & 4.65 & 5.28 & 5.37 & 5.44 & 5.35 & 5.33 & 5.05 & 4.74 & 3.99 & 3.26 & 1692.2 & 4.6 \\
\hline 40.0 & 3.33 & 3.92 & 4.62 & 5.16 & 5.18 & 5.23 & 5.17 & 5.21 & 5.02 & 4.79 & 4.10 & 3.36 & 1676.4 & 4.6 \\
\hline 45.0 & 3.39 & 3.93 & 4.56 & 5.02 & 4.98 & 5.00 & 4.96 & 5.06 & 4.96 & 4.82 & 4.18 & 3.43 & 1651.4 & 4.5 \\
\hline 50.0 & 3.42 & 3.91 & 4.48 & 4.84 & 4.74 & 4.74 & 4.73 & 4.88 & 4.87 & 4.81 & 4.23 & 3.49 & 1617.2 & 4.4 \\
\hline 60.0 & 3.42 & 3.82 & 4.25 & 4.42 & 4.21 & 4.15 & 4.19 & 4.46 & 4.62 & 4.72 & 4.25 & 3.53 & 1522.2 & 4.2 \\
\hline 70.0 & 3.34 & 3.64 & 3.92 & 3.92 & 3.59 & 3.50 & 3.58 & 3.94 & 4.26 & 4.51 & 4.17 & 3.49 & 1394.8 & 3.8 \\
\hline 80.0 & 3.18 & 3.38 & 3.51 & 3.33 & 2.97 & 2.85 & 2.95 & 3.35 & 3.80 & 4.20 & 3.98 & 3.36 & 1242.5 & 3.4 \\
\hline 90.0 & 2.95 & 3.04 & 3.03 & 2.71 & 2.31 & 2.20 & 2.30 & 2.72 & 3.27 & 3.78 & 3.69 & 3.14 & 1068.5 & 2.9 \\
\hline
\end{tabular}


AVERAGE DAILY TOTAL TERRESTRIAL INSOLATION ESTIMATES (KWH/SQ. M)

SITE: RALEIGH-DURHAM NC

LATITUDE: 35 DEGREES 52 MINUTES

AVERAGE EAILY AMOUNTS BY MONTH

\begin{tabular}{|c|c|c|c|c|c|c|c|c|c|c|c|c|c|c|}
\hline ARRAY & $J$ & $F$ & $M$ & A & M & $J$ & $J$ & A & $S$ & 0 & $N$ & $D$ & $\begin{array}{l}\text { ANNUAL } \\
\text { AMOUNT }\end{array}$ & $\begin{array}{c}\text { AVERAGE } \\
\text { DAY }\end{array}$ \\
\hline 0.0 & 2.18 & 2.98 & 4.02 & 5.19 & 5.70 & 5.88 & 5.59 & 5.08 & 4.34 & 3.49 & 2.55 & 2.00 & 1491.8 & 4.1 \\
\hline 10.0 & 2.53 & 3.29 & 4.27 & 5.32 & 5.71 & 5.84 & 5.60 & 5.21 & 4.62 & 3.91 & 3.00 & 2.39 & 1573.6 & 4.3 \\
\hline 15.0 & 2.68 & 3.42 & 4.36 & 5.35 & 5.67 & 5.77 & 5.55 & 5.23 & 4.73 & 4.08 & 3.20 & 2.57 & 1601.7 & 4.4 \\
\hline 20.0 & 2.81 & 3.54 & 4.43 & 5.34 & 5.59 & 5.67 & 5.48 & 5.23 & 4.81 & 4.24 & 3.38 & 2.72 & 1620.8 & 4.4 \\
\hline 25.0 & 2.94 & 3.63 & 4.48 & 5.31 & 5.49 & 5.54 & 5.38 & 5.19 & 4.86 & 4.36 & 3.54 & 2.87 & 1630.9 & 4.5 \\
\hline 30.0 & 3.04 & 3.70 & 4.50 & 5.24 & 5.36 & 5.38 & 5.25 & 5.13 & 4.88 & 4.47 & 3.68 & 2.99 & 1631.9 & 4.5 \\
\hline 35.0 & 3.12 & 3.75 & 4.49 & 5.15 & 5.20 & 5.20 & 5.09 & 5.04 & 4.88 & 4.54 & 3.80 & 3.10 & 1623.8 & 4.4 \\
\hline 40.0 & 3.19 & 3.78 & 4.46 & 5.03 & 5.02 & 5.00 & 4.92 & 4.92 & 4.85 & 4.59 & 3.89 & 3.19 & 1607.9 & 4.4 \\
\hline 45.0 & 3.24 & 3.79 & 4.41 & 4.89 & 4.82 & 4.77 & 4.72 & 4.78 & 4.79 & 4.61 & 3.96 & 3.26 & 1583.4 & 4.3 \\
\hline 50.0 & 3.27 & 3.77 & 4.33 & 4.72 & 4.59 & 4.53 & $千 .50$ & 4.62 & 4.70 & 4.61 & 4.01 & 3.31 & 1550.1 & 4.2 \\
\hline 60.0 & 3.27 & 3.68 & 4.10 & 4.31 & 4.08 & 3.97 & +.00 & 4.22 & 4.45 & 4.51 & 4.02 & 3.34 & 1458.4 & 4.0 \\
\hline 70.0 & 3.19 & 3.50 & 3.78 & 3.82 & 3.49 & 3.37 & 3.42 & 3.73 & 4.10 & 4.31 & 3.93 & 3.30 & 1336.1 & 3.7 \\
\hline 80.0 & 3.03 & 3.25 & 3.38 & 3.25 & 2.89 & 2.75 & 2.83 & 3.18 & 3.66 & 4.00 & 3.75 & 3.17 & 1190.4 & 3.3 \\
\hline 90.0 & 2.81 & 2.93 & 2.92 & 2.64 & 2.26 & 2.14 & 2.22 & 2.59 & 3.15 & 3.61 & 3.48 & 2.97 & 1024.4 & 2.8 \\
\hline
\end{tabular}


AVERAGE DAILY TOTAL TERRESTRIAL INSOLATION ESTIMATES (KWH/SQ. M)

$\begin{aligned} \text { SITE: } & \text { BISMARCK } \\ \text { LATITUDE: } & 46 \text { DEGREES } 46 \text { MINUTES }\end{aligned}$

aVERAge DAILY AMOUNTS BY MONTH

\begin{tabular}{|c|c|c|c|c|c|c|c|c|c|c|c|c|c|c|}
\hline ARRAY & 5 & $F$ & $M$ & A & KAUE & ILt & soms & Fum & $s$ & 0 & $N$ & $D$ & $\begin{array}{l}\text { ANNUAL } \\
\text { AMOUNT }\end{array}$ & $\begin{array}{c}\text { AVERAGE } \\
\text { DAY }\end{array}$ \\
\hline 0.0 & 1.47 & 2.44 & 3.68 & 4.60 & 5.83 & 6.49 & 6.88 & 5.92 & 4.27 & 2.86 & 1.60 & 1.17 & 1439.4 & 3.9 \\
\hline 10.0 & 1.86 & 2.86 & 4.06 & 4.82 & 5.95 & 6.56 & 7.05 & 6.27 & 4.75 & 3.42 & 2.05 & 1.55 & 1560.2 & 4.3 \\
\hline 15.0 & 2.04 & 3.04 & 4.21 & 4.89 & 5.97 & 6.54 & 7.08 & 6.39 & 4.95 & 3.66 & 2.25 & 1.72 & 1607.6 & 4.4 \\
\hline 20.0 & 2.20 & 3.21 & 4.35 & 4.94 & 5.95 & 6.50 & 7.06 & 6.47 & 5.13 & 3.89 & 2.44 & 1.89 & 1646.1 & 4.5 \\
\hline 25.0 & 2.35 & 3.35 & 4.45 & 4.96 & 5.90 & 6.41 & 7.01 & 6.52 & 5.27 & 4.09 & 2.62 & 2.04 & 1675.1 & 4.6 \\
\hline 30.0 & 2.49 & 3.48 & 4.53 & 4.95 & 5.82 & 6.30 & 6.91 & 6.52 & 5.38 & 4.27 & 2.78 & 2.18 & 1694.1 & 4.6 \\
\hline 35.0 & 2.61 & 3.59 & 4.59 & 4.91 & 5.71 & 6.14 & 6.78 & 6.49 & 5.46 & 4.42 & 2.92 & 2.31 & 1703.3 & 4.7 \\
\hline 40.0 & 2.72 & 3.67 & 4.52 & 4.85 & 5.56 & 5.96 & 6.60 & 6.41 & 5.50 & 4.54 & 3.05 & 2.42 & 1702.3 & 4.7 \\
\hline 45.0 & 2.81 & 3.73 & 4.62 & 4.76 & 5.39 & 5.74 & 6.39 & 6.30 & 5.51 & 4.63 & 3.15 & 2.51 & 1691.3 & 4.6 \\
\hline 50.0 & 2.88 & 3.77 & 4.59 & 4.64 & 5.20 & 5.51 & 6.16 & 6.16 & 5.49 & 4.69 & 3.23 & 2.59 & 1671.9 & 4.6 \\
\hline 60.0 & 2.96 & 3.78 & 4.46 & 4.35 & 4.74 & 4.97 & 5.60 & 5.77 & 5.34 & 4.73. & 3.34 & 2.70 & 160.5 .6 & 4.4 \\
\hline 70.0 & 2.97 & 3.69 & 4.22 & 3.97 & 4.20 & 4.33 & 4.93 & 5.25 & 5.06 & 4.64 & 3.35 & 2.73 & 1502.1 & 4.1 \\
\hline 80.0 & 2.91 & 3.52 & 3.89 & 3.50 & $3: 57$ & 3.64 & 4.15 & 4.61 & 4.66 & 4.44 & 3.28 & 2.70 & 1365.1 & 3.7 \\
\hline 90.0 & 2.76 & 3.26 & 3.47 & 2.97 & 2.93 & 2.93 & 3.37 & 3.87 & 4.15 & 4.12 & 3.13 & 2.59 & 1203.3 & 3.3 \\
\hline
\end{tabular}


AVERAGE DAILY TOTAL TERRESTRIAL INSOLATION ESTIMATES (KWH/SQ. M)

$\begin{aligned} \text { SITE: } & \text { FARGO } \\ \text { LATITUDE: } & 46 \text { DEGREES } 54 \text { MINUTES }\end{aligned}$

AVERAGE CAILY AMOL'NTS BY MONTH

\begin{tabular}{|c|c|c|c|c|c|c|c|c|c|c|c|c|c|c|}
\hline ARRAY & -1 & $F$ & $M$ & A & $M$ & $\mathrm{~J}$ & $\mathrm{~J}$ & A & $s$ & 0 & $\mathrm{~N}$ & D & $\begin{array}{l}\text { ANNUAL } \\
\text { AMOUNT }\end{array}$ & $\begin{array}{l}\text { AVERAGE } \\
\text { DAY }\end{array}$ \\
\hline 0.0 & 1.31 & 2.22 & 3.46 & 4.65 & 5.78 & 6.28 & 6.68 & 5.75 & 4.11 & 2.75 & 1.44 & 1.06 & 1387.3 & 3.8 \\
\hline 10.0 & 1.63 & 2.58 & 3.80 & 4.88 & 5.90 & 6.35 & 6.84 & 6.09 & 4.57 & 3.28 & 1.83 & 1.38 & 1497.5 & 4.1 \\
\hline 15.0 & 1.77 & 2.74 & 3.94 & 4.95 & 5.92 & 6.33 & 6.87 & 6.20 & 4.76 & 3.51 & 2.00 & 1.53 & 1540.2 & 4.2 \\
\hline 20.0 & 1.91 & 2.88 & 4.06 & 5.00 & 5.90 & 6.28 & 6.85 & 6.28 & 4.92 & 3.72 & 2.16 & 1.67 & 1574.5 & 4.3 \\
\hline 25.0 & 2.04 & 3.01 & 4.16 & 5.02 & 5.86 & 6.21 & 6.80 & 6.32 & 5.06 & 3.91 & 2.32 & 1.80 & 1599.7 & 4.4 \\
\hline 30.0 & 2.15 & 3.12 & 4.23 & 5.01 & 5.78 & 6.09 & 6.71 & 6.32 & 5.16 & 4.08 & 2.45 & 1.92 & 1615.6 & 4.4 \\
\hline 35.0 & 2.25 & 3.21 & 4.28 & 4.98 & 5.67 & 5.95 & 6.58 & 6.29 & 5.23 & 4.22 & 2.57 & 2.02 & 1622.2 & 4.4 \\
\hline 40.0 & 2.33 & 3.28 & 4.31 & 4.91 & 5.52 & 5.77 & 6.41 & 6.22 & 5.27 & 4.34 & 2.68 & 2.12 & 1619.2 & 4.4 \\
\hline 45.0 & 2.41 & 3.33 & 4.30 & 4.82 & 5.35 & 5.56 & 6.20 & 6.11 & 5.28 & 4.42 & 2.77 & 2.20 & 1606.8 & 4.4 \\
\hline 50.0 & 2.46 & 3.36 & 4.28 & 4.71 & 5.16 & 5.33 & 5.98 & 5.97 & 5.26 & 4.48 & 2.84 & 2.26 & 1586.5 & 4.3 \\
\hline 60.0 & 2.53 & 3.36 & 4.15 & 4.41 & 4.71 & 4.82 & 5.44 & 5.60 & 5.11 & 4.51 & 2.92 & 2.35 & 1520.3 & 4.2 \\
\hline 70.0 & 2.53 & 3.28 & 3.93 & 4.02 & 4.17 & 4.21 & 4.80 & 5.09 & 4.85 & 4.43 & 2.93 & 2.38 & 1419.5 & 3.9 \\
\hline 80.0 & 2.47 & 3.12 & 3.52 & 3.55 & 3.55 & ?. 54 & 9.05 & 4.48 & 4.46 & 4.23 & 2.87 & 2.34 & 1287.4 & 3.5 \\
\hline 90.0 & 2.35 & 2.89 & 3.23 & 3.02 & 2.92 & 2.87 & 3.29 & 3.77 & 3.98 & 3.93 & 2.73 & 2.25 & 1132.6 & 3.1 \\
\hline
\end{tabular}


AVERAGE DAILY TOTAL TERRESTRIAL INSOLATION ESTIMATES (KWH/SQ. M)

SITE: MINOT

ND

LATITUDE: 48 DEGREES 16 MINUTES

AVERAGE DAILY AMOUNTS BY MONTH

\begin{tabular}{|c|c|c|c|c|c|c|c|c|c|c|c|c|c|c|}
\hline ARRAY & $\mathbf{J}$ & $F$ & $M$ & A & $M$ & $J$ & $\mathrm{~J}$ & A & S & 0 & $\underline{N}$ & $D$ & $\begin{array}{l}\text { ANNUAL } \\
\text { AMOUNT }\end{array}$ & $\begin{array}{c}\text { AVERAGE } \\
\text { DAY }\end{array}$ \\
\hline 0.0 & 1.21 & 2.07 & 3.29 & 4.60 & 5.83 & 6.23 & 6.61 & $5: 67$ & 4.02 & 2.68 & 1.38 & 0.98 & 1359.2 & 3.7 \\
\hline 10.0 & 1.53 & 2.41 & 3.63 & 4.85 & 5.96 & 6.31 & 6.79 & 6.03 & 4.50 & 3.23 & 1.78 & 1.30 & 1472.8 & 4.0 \\
\hline 15.0 & 1.67 & 2.57 & 3.77 & 4.93 & 5.99 & 6.30 & 6.82 & 6.15 & 4.70 & 3.47 & 1.76 & 1.45 & 1517.3 & 4.2 \\
\hline 20.0 & 1.81 & 2.71 & 3.90 & 4.98 & 5.98 & 6.25 & 6.82 & 6.24 & 4.87 & 3.70 & 2.13 & 1.59 & 1553.6 & 4.3 \\
\hline 25.0 & 1.93 & 2.83 & 3.99 & 5.01 & 5.94 & 6.18 & 6.77 & 6.29 & 5.01 & 3.90 & 2.29 & 1.73 & 1581.1 & 4.3 \\
\hline 30.0 & 2.04 & 2.94 & 4.07 & 5.01 & 5.87 & 6.08 & 6.69 & 6.30 & 5.12 & 4.08 & 2.74 & 1.85 & 1599.3 & 4.4 \\
\hline 35.0 & 2.15 & 3.02 & 4.12 & 4.98 & 5.76 & 5.94 & 6.57 & 6.28 & 5.20 & 4.23 & 2.57 & 1.96 & 1608.2 & 4.4 \\
\hline 40.0 & 2.23 & 3.10 & 4.15 & 4.92 & 5.62 & 5.77 & 6.41 & 6.21 & 5.25 & 4.35 & 2.58 & 2.06 & 1607.7 & 4.4 \\
\hline 45.0 & 2.31 & 3.15 & 4.15 & 4.84 & 5.46 & 5.57 & 6.22 & 6.12 & 5.27 & 4.45 & 2.78 & 2.14 & 1597.8 & 4.4 \\
\hline 50.0 & 2.36 & 3.18 & 4.13 & 4.73 & 5.27 & 5.35 & 5.99 & 5.98 & 5.26 & 4.52 & 2.35 & 2.21 & 1579.4 & 4.3 \\
\hline 60.0 & 2.44 & 3.19 & 4.02 & 4.44 & 4.82 & 4.85 & 5.48 & 5.63 & 5.13 & 4.57 & 2.95 & 2.31 & 1518.1 & 4.2 \\
\hline 70.0 & 2.45 & 3.12 & 3.82 & 4.07 & 4.29 & 4.26 & 4.85 & 5.14 & 4.88 & 4.50 & 2.98 & 2.35 & 1422.0 & 3.9 \\
\hline 30.0 & 2.40 & 2.98 & 3.53 & 3.60 & 3.67 & 3.60 & 4.12 & 4.54 & 4.51 & 4.32 & 2.92 & 2.32 & 1294.2 & 3.5 \\
\hline 90.0 & 2.28 & 2.77 & 3.16 & 3.07 & 3.02 & 2.93 & 3.37 & 3.85 & 4.04 & 4.03 & 2.79 & 2.23 & 1142.8 & 3.1 \\
\hline
\end{tabular}


AVERAGE DAILY TOTAL TERRESTRIAL INSOLATION ESTIMATES (KWH/SQ. M)

$\begin{aligned} \text { SITE: } & \text { GRAND ISLAND N NE } \\ \text { LATITUDE: } & 40 \text { DEGREES } 58 \text { MINUTES }\end{aligned}$

AVERAGE CAILY AMOUNTS BY MONTH

\begin{tabular}{|c|c|c|c|c|c|c|c|c|c|c|c|c|c|c|}
\hline ARRAY & $\begin{array}{l}---2 \\
J\end{array}$ & $\mathbf{F}$ & $M$ & A & $M$ & 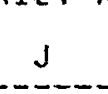 & 3 & A & $s$ & 0 & $N$ & D & $\begin{array}{l}\text { ANNUAL } \\
\text { AMOUNT }\end{array}$ & $\begin{array}{c}\text { AVERAGE } \\
\text { DAY }\end{array}$ \\
\hline 0.0 & 2.09 & 2.89 & 3.98 & 5.34 & 6.22 & 7.07 & 6.98 & 6.12 & 4.76 & 3.59 & 2.33 & 1.80 & 1620.0 & 4.4 \\
\hline 10.0 & 2.53 & 3.29 & 4.50 & 5.55 & 6.29 & 7.08 & 7.07 & 6.38 & 5.20 & 4.16 & 2.88 & 2.26 & 1736.4 & 4.8 \\
\hline 15.0 & 2.73 & 3.45 & 4.43 & 5.61 & 6.28 & 7.03 & 7.06 & 6.46 & 5.37 & 4.42 & 3.12 & 2.47 & 1780.0 & 4.9 \\
\hline 20.0 & 2.92 & 3.60 & 4.53 & 5.63 & 6.23 & 6.94 & 7.00 & 6.50 & 5.51 & 4.64 & 3.35 & 2.66 & 1813.1 & 5.0 \\
\hline 25.0 & 3.08 & 3.73 & $\dot{4} . \in 1$ & 5.62 & 6.14 & 6.81 & 6.90 & 6.50 & 5.62 & 4.84 & 3.56 & 2.84 & 1835.7 & 5.0 \\
\hline 30.0 & 3.23 & 3.84 & $\dot{4} . \in 6$ & 5.59 & 6.02 & 6.65 & 6.77 & 6.46 & 5.70 & E.01 & 3.74 & 3.01 & 1847.5 & 5.1 \\
\hline 35.0 & 3.36 & 3.92 & 4. $\leqslant 8$ & 5.52 & 5.87 & 6.44 & 6.59 & 6.38 & 5.74 & 5.14 & 3.90 & $3 . .15$ & 1848.4 & 5.1 \\
\hline 40.0 & 3.47 & 3.98 & 6.68 & 5.42 & 5.69 & 6.20 & 6.38 & 6.27 & 5.74 & 5.25 & 4.04 & 3.27 & 1838.4 & 5.0 \\
\hline 45.0 & 3.55 & 4.02 & 4.65 & 5.29 & 5.48 & 5.94 & 6.14 & 6.13 & 5.71 & 5.32 & 4.15 & 3.37 & 1819.5 & 5.0 \\
\hline 50.0 & 3.62 & 4.03 & 4.59 & 5.14 & 5.26 & 5.55 & 5.88 & 5.95 & 5.65 & 5.35 & 4.23 & 3.45 & 1790.3 & 4.9 \\
\hline 60.0 & 3.67 & 3.99 & 4.40 & 4.75 & 4.72 & 5.01 & 5.26 & 5.49 & 5.42 & 5.32 & 4.32 & 3.55 & 1701.0 & 4.7 \\
\hline 70.0 & 3.64 & 3.84 & 4.11 & 4.26 & 4.09 & 4.25 & 4.53 & 4.91 & 5.06 & 5.16 & 4.29 & 3.55 & 1572.7 & 4.3 \\
\hline 80.0 & 3.51 & 3.61 & 3.73 & 3.68 & 3.41 & 3.49 & 3.74 & 4.22 & 4.59 & 4.86 & 4.15 & 3.46 & 1413.0 & 3.9 \\
\hline 90.0 & 3.30 & 3.29 & 3.27 & 3.04 & 2.71 & 2.57 & 2.93 & 3.45 & 4.01 & 4.45 & 3.90 & 3.28 & 1226.0 & 3.4 \\
\hline
\end{tabular}


AVERAGE DAILY TOTAL TERRESTRIAL INSOLATION ESTIMATES (KWH/SQ. M)

SITE: NORTH OMAHA

LATITUDE: 41 DEGREES 22 MINUTES

A?ERAGE DAILY AMOUNTS BY MONTH

\begin{tabular}{|c|c|c|c|c|c|c|c|c|c|c|c|c|c|c|}
\hline ARRAY & J & $F$ & $M$ & A & $M$ & $J^{-1}$ & 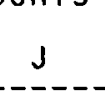 & A & $s$ & 0 & $N$ & D & $\begin{array}{l}\text { ANNUAL } \\
\text { AMOUNT }\end{array}$ & $\begin{array}{c}\text { AVERAGE } \\
\text { DAY }\end{array}$ \\
\hline 0.0 & 2.00 & 2.81 & 3.85 & 4.92 & 5.90 & 6.69 & 6.64 & 5.86 & 4.32 & 3.31 & 2.33 & 1.61 & 1522.4 & 4.2 \\
\hline 10.0 & 2.43 & $3.19^{\circ}$ & 4.17 & 5.10 & 5.97 & 6.71 & 6.73 & 6.12 & 4.70 & 3.82 & $2 .+7$ & 2.01 & 1627.8 & 4.5 \\
\hline 15.0 & 2.62 & 3.36 & 4.29 & 5.15 & 5.96 & 6.67 & 6.72 & 6.19 & 4.85 & 4.05 & 2.57 & 2.20 & 1666.9 & 4.6 \\
\hline 20.0 & 2.80 & 3.50 & 4.39 & 5.17 & 5.91 & 6.58 & 6.66 & 6.23 & 4.97 & 4.25 & 2.36 & 2.37 & 1696.6 & 4.6 \\
\hline 25.0 & $2: 96$ & 3.63 & 4.46 & 5.16 & 5.83 & 6.47 & 6.57 & 6.23 & 5.07 & 4.43 & 3.02 & 2.52 & 1716.4 & 4.7 \\
\hline 30.0 & 3.11 & 3.73 & 4.51 & 5.13 & 5.72 & 6.31 & 6.45 & 6.19 & 5.13 & 4.57 & 3.17 & 2.66 & 1726.4 & 4.7 \\
\hline 35.0 & 3.23 & 3.82 & 4.53 & 5.06 & 5.58 & 6.12 & 6.28 & 6.12 & 5.16 & 4.69 & 3.30 & 2.78 & 1726.3 & 4.7 \\
\hline 40.0 & 3.33 & 3.87 & 4.53 & 4.97 & 5.41 & 5.90 & 6.08 & 6.01 & 5.16 & 4.78 & 3.41 & 2.89 & 1716.2 & 4.7 \\
\hline 45.0 & 3.42 & 3.91 & 4.50 & 4.86 & 5.22 & 5.66 & 5.86 & 5.88 & 5.14 & 4.85 & 3.49 & 2.98 & 1697.8 & 4.7 \\
\hline 50.0 & 3.48 & 3.92 & 4.45 & 4.72 & 5.01 & 5.40 & 5.62 & 5.71 & 5.08 & 4.88 & 3.56 & 3.05 & 1670.2 & 4.6 \\
\hline 60.0 & 3.54 & 3.88 & 4.26 & 4.37 & 4.51 & 4.79 & 5.04 & 5.27 & 4.87 & 4.85 & 3.62 & 3.13 & 1586.5 & 4.3 \\
\hline 70.0 & 3.50 & 3.74 & 3.99 & 5.92 & 3.92 & 4.09 & 4.35 & 4.73 & 4.55 & 4.69 & 3.59 & 3.13 & 1467.2 & 4.0 \\
\hline 80.0 & 3.38 & 3.52 & 3.62 & 3.41 & 3.28 & 3.37 & 3.62 & 4.08 & 4.13 & 4.43 & 3.47 & 3.05 & 1318.9 & 3.6 \\
\hline 90.0 & 3.18 & 3.22 & 3.18 & 2.83 & 2.63 & 2.62 & 2.86 & 3.35 & 3.62 & 4.05 & 3.26 & 2.89 & 1146.0 & 3.1 \\
\hline
\end{tabular}


AVERAGE DAILY TOTAL TERRESTRJAL INSOLATION ESTIMATES (KWH/SQ. M)

SITE: NGRTH PLATTE M NE
LATITUDE: 4] DEGREES 8 MINUTES

AVE??AGE CAILY AMOLNTS BY MONTH

\begin{tabular}{|c|c|c|c|c|c|c|c|c|c|c|c|c|c|c|}
\hline $\begin{array}{l}\text { ARRAY } \\
\text { TILT }\end{array}$ & J & $F$ & $M$ & A & $M$ & $\therefore$ & J & A & 5 & 0 & N & $D$ & $\begin{array}{l}\text { ANNUAL } \\
\text { AMOUNT }\end{array}$ & $\begin{array}{c}\text { AVERAGE } \\
\text { DAY }\end{array}$ \\
\hline 0.0 & 2.18 & 3.02 & 4.20 & 5.44 & 6.26 & 7.15 & 7.18 & 6.27 & 4.93 & 3.71 & 2.40 & 1.91 & 1665.1 & 4.6 \\
\hline 10.0 & 2.67 & 3.44 & 4.56 & 5.65 & 6.34 & 7.17 & 7.28 & 6.55 & 5.40 & 4.33 & 2.97 & 2.43 & 1790.9 & 4.9 \\
\hline 15.0 & 2.89 & 3.63 & 4.70 & 5.71 & 6.33 & 7.12 & 7.27 & 6.63 & 5.59 & 4.60 & 3.24 & 2.66 & 1838.6 & 5.0 \\
\hline 20.0 & 3.09 & 3.79 & 4.81 & 5.74 & 6.28 & 7.03 & 7.21 & 6.68 & 5.74 & 4.84 & 3.48 & 2.89 & 1875.4 & 5.1 \\
\hline 25.0 & 3.27 & 3.93 & 4.90 & 5.74 & 6.19 & 6.90 & 7.11 & 6.68 & 5.86 & 5.05 & 3.70 & 3.09 & 1901.2 & 5.2 \\
\hline 30.0 & 3.44 & 4.04 & 4.96 & 5.70 & 6.07 & 6.73 & 6.97 & 6.64 & 5.94 & 5.23 & 3.90 & 3.27 & 1915.7 & 5.2 \\
\hline 35.0 & 3.58 & 4.14 & 7.99 & 5.63 & 5.92 & 6.52 & 6.79 & 6.57 & 5.99 & 5.38 & 4.07 & 3.44 & 1918.7 & 5.3 \\
\hline 40.0 & 3.70 & 4.20 & 7.99 & 5.53 & 5.73 & 6.28 & 6.57 & 6.45 & 6.00 & 5.49 & 4.21 & 3.58 & 1910.4 & 5.2 \\
\hline 45.0 & 3.80 & 4.25 & 7.96 & 5.43 & 5.53 & 6.02 & 6.33 & 6.30 & 5.98 & 5.57 & 4.33 & 3.70 & 1892.5 & 5.2 \\
\hline 50.0 & 3.87 & 4.26 & 4.90 & 5.27 & 5.30 & 5.73 & 6.06 & 6.12 & 5.91 & 5.62 & 4.42 & 3.79 & 1864.0 & 5.1 \\
\hline 60.0 & 3.94 & 4.22 & 4.70 & 4.8 .5 & 4.76 & 5.07 & 5.41 & 5.65 & 5.68 & 5.59 & 4.52 & 3.90 & 1774.2 & 4.9 \\
\hline 70.0 & 3.90 & 4.07 & 4.39 & 4.35 & 4.13 & 4.31 & 4.66 & 5.05 & 5.31 & 5.42 & 4.49 & 3.91 & 1643.3 & 4.5 \\
\hline 80.0 & 3.77 & 3.83 & 3.99 & 3.75 & 3.44 & 3.53 & 3.84 & 4.35 & 4.81 & 5.12 & 4.35 & 3.82 & 1478.7 & 4.1 \\
\hline 90.0 & 3.54 & 3.50 & 3.50 & 3.10 & 2.74 & 2.72 & 3.00 & 3.55 & 4.20 & 4.68 & 4.09 & 3.63 & 1285.1 & 3.5 \\
\hline
\end{tabular}


AVERAGE DAILY TOTAL TERRESTRIAL INSOLATION ESTIMATES (KWH;SQ. M)

SITE:
SCOTTSBLUFF NE

AVERAGE DAILY AMOUNTS BY MONTH

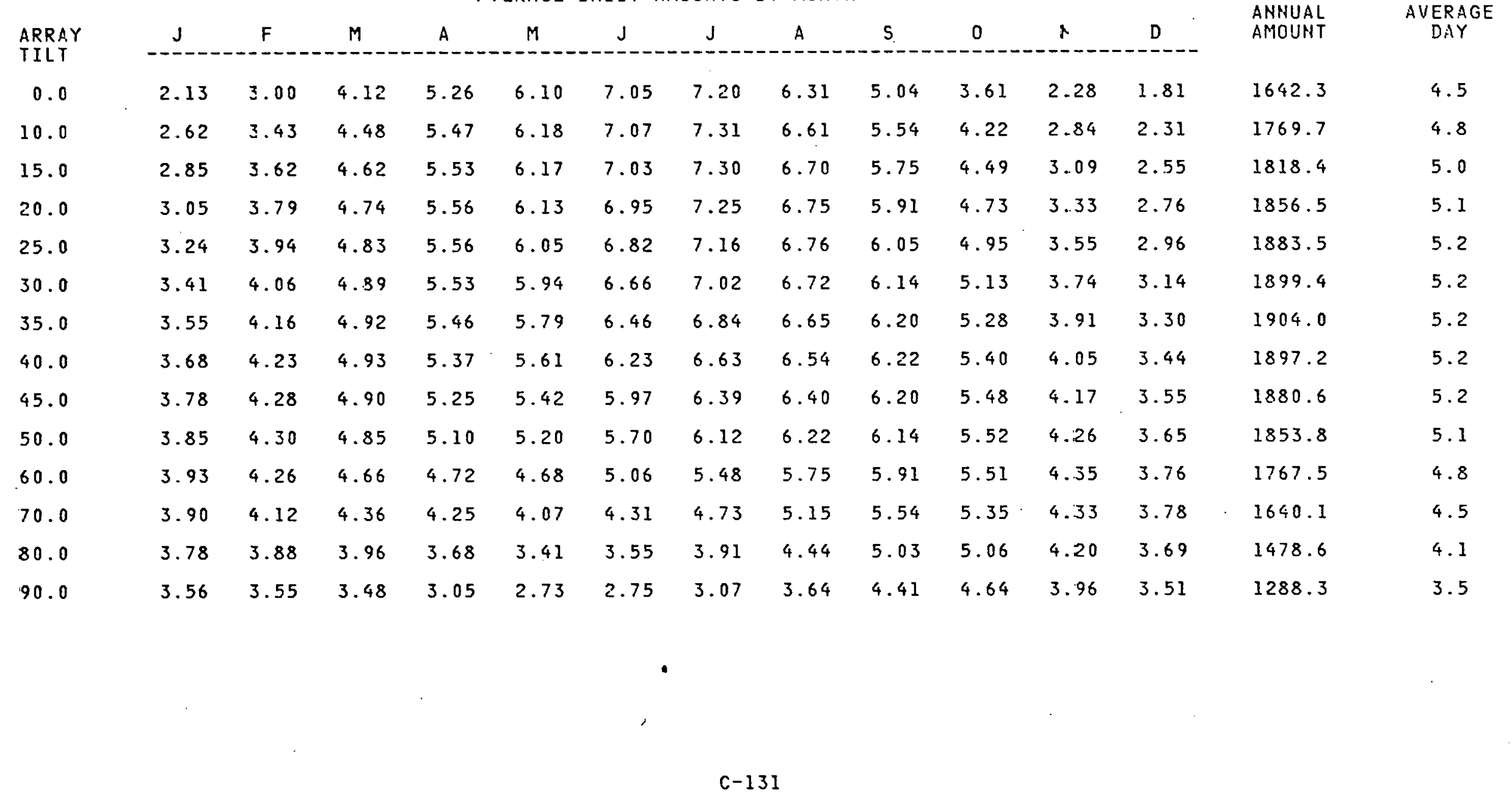


AVERAGE DAILY TOTAL TERRESTRIAL INSOLATION ESTIMATES (KWH/SQ. M)

SITE: CONCORD

LATITUDE: 43 DEGREES 12 MINUTES

A'VERAGE DAILY AMOUNTS BY MONTH

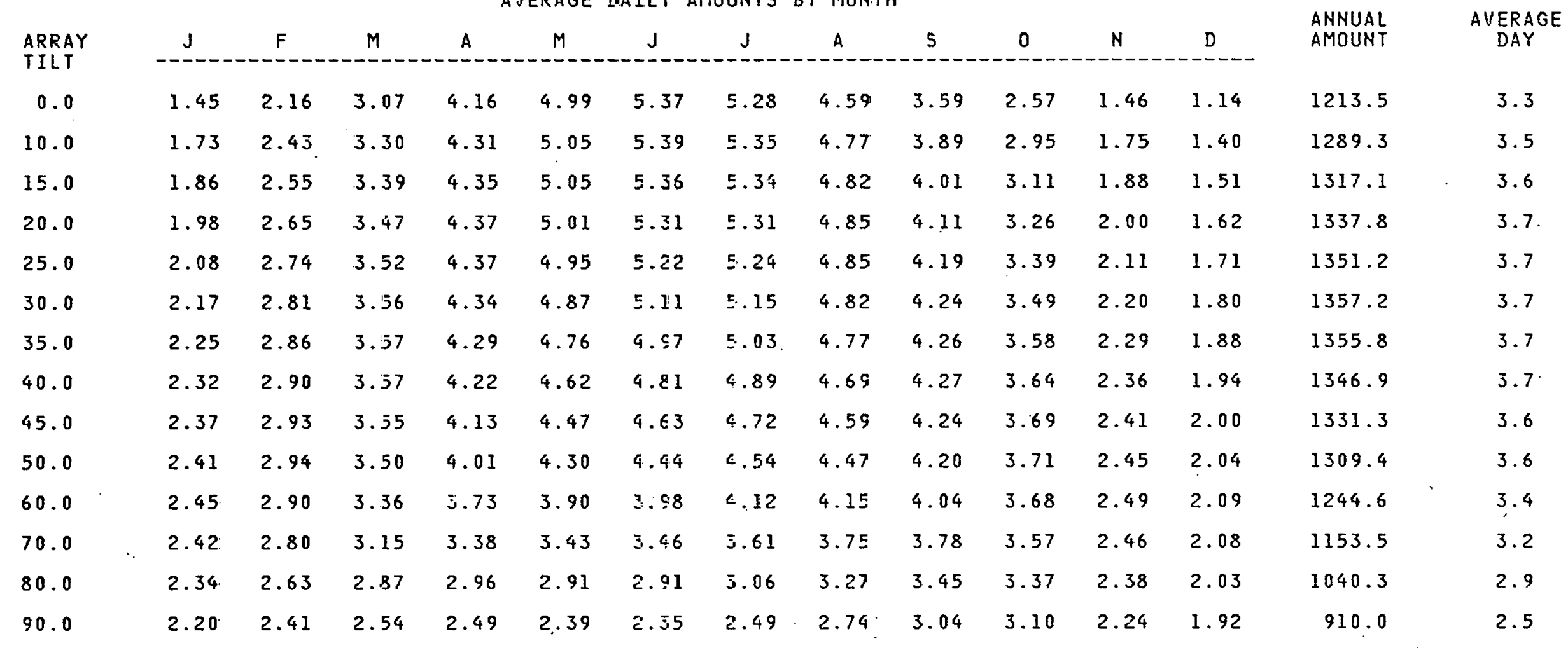


AVERAGE DAILY TOTAL TERRESTRIAL INSOLATION ESTIMATES (KWH'SQ. M)

SITE:
LATITUDE: 40 DEGRERT

AVERAGE DAILY ANOUNTS BY MONTH

\begin{tabular}{|c|c|c|c|c|c|c|c|c|c|c|c|c|c|c|c|}
\hline ARRAY & & $J$ & $F$ & $M$ & A & $M$ & $J$ & $J$ & $A$ & $S$ & 0 & $N$ & $D$ & $\begin{array}{l}\text { ANNUAL } \\
\text { AMOUNT }\end{array}$ & $\begin{array}{c}\text { AVERAGE } \\
\text { DAY }\end{array}$ \\
\hline 0.0 & & 1.77 & 2.51 & 3.49 & 4.60 & 5.27 & 5.60 & 5.37 & 4.83 & 3.97 & 3.01 & 1.96 & 1.50 & 1336.4 & 3.7 \\
\hline 10.0 & & 2.08 & 2.80 & 3.74 & 4.75 & 5.31 & 5.60 & 5.41 & 5.00 & 4.27 & 3.42 & 2.34 & 1.82 & 1417.4 & 3.9 \\
\hline 15.0 & & 2.22 & 2.93 & 3.83 & 4.78 & 5.29 & 5.55 & 5.39 & 5.04 & 4.39 & 3.59 & 2.51 & 1.96 & 1446.5 & 4.0 \\
\hline 20.0 & & 2.35 & 3.04 & 3.91 & 4.79 & 5.24 & 5.48 & 5.34 & 5.05 & 4.48 & 3.74 & 2.67 & 2.10 & 1467.7 & 4.0 \\
\hline 25.0 & & 2.47 & 3.13 & 3.96 & 4.78 & 5.17 & 5.38 & 5.26 & 5.04 & 4.55 & 3.88 & 2.81 & 2.22 & 1480.8 & 4.1 \\
\hline 30.0 & & 2.57 & 3.20 & 3.99 & 4.74 & 5.06 & 5.25 & 5.15 & 5.00 & 4.59 & 3.99 & 2.93 & 2.33 & 1485.7 & 4.1 \\
\hline 35.0 & & 2.66 & 3.26 & 4.00 & 4.67 & 4.93 & 5.09 & 5.02 & 4.93 & 4.61 & 4.07 & 3.04 & 2.42 & 1482.4 & 4.1 \\
\hline 40.0 & & 2.73 & 3.30 & 3.99 & 4.58 & 4.78 & 4.90 & 4.86 & 4.83 & 4.60 & 4.13 & 3.13 & 2.50 & 1470.9 & 4.0 \\
\hline 45.0 & & 2.78 & 3.32 & 3.95 & 4.47 & 4.61 & 4.71 & 4.69 & 4.71 & 4.56 & 4.17 & 3.20 & 2.57 & 1452.9 & 4.0 \\
\hline 50.0 & & 2.82 & 3.32 & 3.89 & 4.34 & 4.42 & 4.50 & 4.49 & 4.57 & 4.50 & 4.18 & 3.25 & 2.61 & 1426.8 & 3.9 \\
\hline 60.0 & $-\rightarrow$ & 2.84 & 3.26 & 3.72 & 4.00 & 3.98 & 4.00 & 4.04 & 4.22 & 4.29 & 4.13 & 3.29 & 2.66 & 1351.6 & 3.7 \\
\hline 70.0 & & 2.79 & 3.13 & 3.46 & 3.59 & 3.46 & 3.43 & 3.51 & 3.78 & 4.00 & 3.98 & 3.24 & $2.65-$ & 1247.7 & 3.4 \\
\hline 80.0 & & 2.68 & 2.93 & 3.13 & 3.11 & 2.91 & 2.86 & 2.95 & 3.27 & 3.61 & 3.73 & 3.12 & 2.56 & 1121.0 & 3.1 \\
\hline 90.0 & & 2.51 & 2.66 & 2.74 & 2.58 & 2.34 & 2.27 & 2.36 & 2.70 & 3.15 & 3.40 & 2.92 & 2.42 & 974.4 & 2.7 \\
\hline
\end{tabular}


AVERAGE DAILY TOTAL TERRESTRIAL INSOLATION ESTIMATES (KWH/SQ. M)

SITE: NENARK

LATITUDE: 40 DEGREES 42 MINUTES

AVERAGE DAILY AMOUNTS BY MONTH

\begin{tabular}{|c|c|c|c|c|c|c|c|c|c|c|c|c|c|c|}
\hline ARRAY & $J$ & $\mathrm{~F}$ & $M$ & $A$ & $M$ & 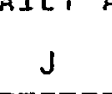 & 知 & chom & $s$ & 0 & $N$ & D & $\begin{array}{l}\text { ANNUAL } \\
\text { AMOUNT }\end{array}$ & $\begin{array}{c}\text { AVERAGE } \\
\text { DAY }\end{array}$ \\
\hline 0.0 & 1.74 & 2.50 & 3.49 & 4.57 & 5.32 & 5.66 & 5.55 & 4.93 & 4.01 & 2.99 & 1.88 & 1.43 & 1342.9 & 3.7 \\
\hline 10.0 & 2.0 .5 & 2.80 & 3.74 & 4.72 & 5.37 & 5.66 & 5.61 & 5.11 & 4.33 & 3.41 & 2.25 & 1.74 & 1426.1 & 3.9 \\
\hline 15.0 & 2.21 & 2.93 & 3.84 & 4.76 & 5.36 & 5.62 & 5.59 & 5.16 & 4.46 & 3.59 & 2.42 & 1.88 & 1456.3 & 4.0 \\
\hline 20.0 & 2.34 & 3.04 & 3.92 & 4.78 & 5.31 & 5.55 & 5.54 & 5.17 & 4.55 & 3.75 & 2.57 & 2.01 & 1478.4 & 4.1 \\
\hline 25.0 & 2.45 & 3.14 & 3.98 & 4.76 & 5.24 & 5.45 & 5.46 & 5.16 & 4.63 & 3.89 & 2.71 & 2.13 & 1492.4 & 4.1 \\
\hline 30.0 & 2.56 & 3.22 & 4.01 & 4.73 & 5.13 & 5.32 & 5.36 & 5.12 & 4.67 & 4.00 & 2.83 & 2.24 & 1498.0 & 4.1 \\
\hline 35.0 & 2.65 & 3.28 & 4.03 & 4.66 & 5.00 & 5.16 & 5.22 & 5.06 & 4.69 & 4.09 & 2.94 & 2.33 & 1495.4 & 4.1 \\
\hline 40.0 & 2.73 & 3.32 & 4.02 & 4.57 & 4.85 & 4.98 & 5.06 & 4.96 & 4.69 & 4.16 & 3.03 & 2.41 & 1484.4 & 4.1 \\
\hline 45.0 & 2.78 & 3.34 & 3.98 & 4.47 & 4.68 & 4.79 & 4.88 & 4.85 & 4.65 & 4.20 & 3.10 & 2.47 & 1466.6 & 4.0 \\
\hline 50.0 & 2.82 & 3.34 & 3.93 & 4.33 & 4.49 & 4.57 & 4.68 & 4.70 & 4.59 & 4.21 & 3.15 & 2.52 & 1440.9 & 3.9 \\
\hline 60.0 & 2.85 & 3.29 & 3.76 & 4.01. & 4.05 & 4.07 & 4.21 & 4.34 & 4.39 & 4.17 & 3.19 & 2.57 & 1366.2 & 3.7 \\
\hline 70.0 & 2.81 & 3.16 & 3.50 & 3.60 & 3.53 & 3.50 & 3.66 & 3.90 & 4.10 & 4.02 & 3.15 & 2.55 & 1262.1 & 3.5 \\
\hline 80.0 & 2.70 & 2.97 & 3.18 & 3.12 & 2.97 & 2.92 & 3.07 & 3.37 & 3.71 & 3.78 & 3.03 & 2.48 & 1134.7 & 3.1 \\
\hline 90.0 & 2.53 & 2.70 & 2.79 & 2.60 & 2.39 & 2.32 & 2.47 & 2.79 & 3.25 & 3.45 & 2.84 & 2.34 & 987.1 & 2.7 \\
\hline
\end{tabular}


AVERAGE DAILY TOTAL TERRESTRIAL INSOLATION ESTIMATES (KWHISQ. M)

$\begin{aligned} \text { SITE: } & \text { ALBUQUERQUE } \\ \text { LATITUDE: } & 35 \text { DEGREES } 3 \text { MINUTES }\end{aligned}$

AVERAGE DAILY AMOUNTS BY MONTH

\begin{tabular}{|c|c|c|c|c|c|c|c|c|c|c|c|c|c|c|}
\hline ARRAY & 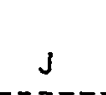 & $F$ & $M$ & A & $M$ & $J$ & $J$ & A & 5 & 0 & $\checkmark$ & $D$ & $\begin{array}{l}\text { ANNUAL } \\
\text { AMOUNT }\end{array}$ & $\begin{array}{c}\text { AVERAGE } \\
\text { DAY }\end{array}$ \\
\hline 0.0 & 3.20 & 4.23 & 5.57 & 7.03 & 8.00 & 8.44 & 7.84 & 7.22 & 6.21 & 4.88 & 3.57 & 2.93 & 2104.8 & 5.8 \\
\hline 10.0 & 3.82 & 4.78 & 5.00 & 7.25 & 8.01 & 8.37 & 7.85 & 7.45 & 6.73 & 5.59 & 4.33 & 3.62 & 2246.8 & 5.2 \\
\hline 15.0 & 4.10 & 5.01 & 6.16 & 7.29 & 7.95 & 8.25 & 7.78 & 7.50 & 6.92 & 5.90 & 4.67 & 3.93 & 2297.4 & 5.3 \\
\hline 20.0 & 4.35 & 5.21 & 6.28 & 7.29 & 7.83 & 8.09 & 7.67 & 7.51 & 7.07 & 6.17 & 4.98 & 4.22 & 2334.0 & 5.4 \\
\hline 25.0 & 4.58 & 5.38 & 6.37 & 7.25 & 7.67 & 7.87 & 7.51 & 7.46 & 7.18 & 6.40 & 5.25 & 4.49 & 2356.2 & 5.5 \\
\hline 30.0 & 4.77 & 5.52 & 6.42 & 7.16 & 7.46 & 7.61 & 7.31 & 7.37 & 7.24 & 6.58 & 5.50 & 4.72 & 2363.8 & 5.5 \\
\hline 35.0 & 4.94 & 5.62 & 6.43 & 7.03 & 7.21 & 7.30 & 7.06 & 7.24 & 7.26 & 6.73 & 5.70 & 4.92 & 2356.9 & 6.5 \\
\hline 40.0 & 5.07 & 5.69 & 6.40 & 6.87 & 6.94 & 6.98 & 6.79 & 7.07 & 7.23 & 6.83 & 5.87 & 5.09 & 2338.0 & 6.4 \\
\hline 45.0 & 5.17 & 5.72 & 6.33 & 6.66 & 6.63 & 6.62 & 6.49 & 6.86 & 7.16 & 6.89 & 6.00 & 5.23 & 2304.8 & 6.3 \\
\hline 50.0 & 5.24 & 5.72 & 6.22 & 6.42 & 6.28 & 6.22 & 6.14 & 6.60 & 7.04 & 6.90 & 6.09 & 5.33 & 2257.4 & 6.2 \\
\hline 60.0 & 5.27 & 5.61 & 5.89 & 5.82 & 5.47 & 5.33 & 5.36 & 5.98 & 6.66 & 6.79 & 6.15 & 5.43 & 2121.6 & 5.8 \\
\hline 70.0 & 5.17 & 5.35 & 5.42 & 5.09 & 4.55 & 4.36 & 4.46 & 5.22 & 6.13 & 6.50 & 6.05 & 5.38 & 1936.3 & 5.3 \\
\hline 80.0 & 4.93 & 4.97 & 4.83 & 4.25 & 3.63 & 3.37 & 3.56 & 4.35 & 5.44 & 6.05 & 5.78 & 5.19 & 1712.9 & 4.7 \\
\hline 90.0 & 4.57 & 4.46 & 4.13 & 3.35 & 2.67 & 2.42 & 2.63 & 3.42 & 4.63 & 5.44 & 5.36 & 4.86 & 1456.8 & 4.0 \\
\hline
\end{tabular}


AVERAGE DAI:Y TOTAL TERRESTRIAL INSOLATION ESTIMATES (KWH,SQ. M)

SITE: CLAYTON LATITUDE: 36 DEGREES 27 MINUTES

AVERAGE DAILY AMDUNTS BY MONTH

\begin{tabular}{|c|c|c|c|c|c|c|c|c|c|c|c|c|c|c|}
\hline ARRAY & $J$ & $F$ & $M$ & A & M & $\mathfrak{J}$ & $J$ & A & $s$ & 0 & N & D & $\begin{array}{l}\text { ANNUAL } \\
\text { AMOUNT }\end{array}$ & $\begin{array}{c}\text { AVERAGE } \\
\text { DAY }\end{array}$ \\
\hline 0.0 & 3.03 & 3.91 & 5.20 & 6.43 & 7.00 & 7.62 & 7.20 & 6.61 & 5.68 & 4.52 & 3.24 & 2.72 & 1923.3 & 5.3 \\
\hline 10.0 & 3.6 .5 & 4.43 & 5.62 & 6.64 & 7.03 & 7.58 & 7.22 & 6.84 & 6.15 & 5.19 & 3.94 & 3.39 & 2060.2 & 5.6 \\
\hline 15.0 & 3.92 & 4.65 & 5.77 & 6.68 & 6.98 & 7.49 & 7.17 & 6.89 & 6.34 & 5.48 & 4.26 & 3.69 & 2110.4 & 5.8 \\
\hline 20.0 & 4.18 & 4.84 & 5.90 & 6.69 & 6.89 & 7.35 & 7.08 & 6.90 & 6.48 & 5.74 & 4.55 & 3.98 & 2148.0 & 5.9 \\
\hline 25.0 & 4.40 & 5.00 & 5.98 & 6.66 & 6.77 & 7.18 & 6.95 & 6.86 & 6.58 & 5.96 & 4.81 & 4.23 & 2172.6 & 6.0 \\
\hline 30.0 & 4.60 & 5.13 & 6.03 & 6.59 & 6.60 & 6.96 & 6.78 & 6.79 & 6.64 & 6.14 & 5.04 & 4.46 & 2184.0 & 6.0 \\
\hline 35.0 & 4.77 & 5.23 & 6.05 & 6.48 & 6.39 & 6.70 & 6.56 & 6.68 & 6.66 & 6.28 & 5.23 & 4.66 & 2182.1 & 6.0 \\
\hline 40.0 & 4.91 & 5.30 & 6.03 & 6.33 & 6.17 & 6.42 & 6.33 & 6.53 & 6.64 & 6.38 & 5.40 & 4.83 & 2168.8 & 5.9 \\
\hline 45.0 & 5.02 & 5.34 & 5.97 & 6.16 & 5.91 & 6.12 & 6.06 & 6.35 & 6.58 & 6.44 & 5.52 & 4.97 & 2142.9 & 5.9 \\
\hline 50.0 & 5.09 & 5.34 & 5.87 & 5.94 & 5.62 & 5.78 & $5.76^{\circ}$ & 6.13 & 6.48 & 6.46 & 5.61 & 5.08 & 2104.2 & 5.8 \\
\hline 60.0 & 5.14 & 5.24 & 5.58 & 5.42 & 4.96 & 5.01 & 5.07 & 5.58 & 5.15 & 6.37 & 5.68 & 5.18 & 1989.1 & 5.4 \\
\hline 70.0 & 5.06 & 5.02 & 5.16 & 4.77 & 4.19 & 4.16 & 4.27 & 4.91 & 5.68 & 6.12 & 5.60 & 5.15 & 1827.8 & 5.0 \\
\hline 80.0 & 4.84 & 4.67 & 4.61 & 4.03 & 3.41 & 3.31 & 3.47 & 4.13 & 5.07 & 5.71 & 5.37 & 4.99 & 1630.3 & 4.5 \\
\hline 90.0 & 4.50 & 4.22 & 3.97 & 3.22 & 2.59 & 2.46 & 2.61 & 3.30 & 4.35 & 5.16 & 5.00 & 4.69 & 1400.3 & 3.8 \\
\hline
\end{tabular}


A.VERAGE DAILY TOTAL TERRESTRIAL INSOLATION ESTIMATES (KINH/SQ. M)

SITE: FARMINGTON

LATITUDE: 36 DEGREES 45 MINUTES

AVERAGE DAILY AMOUNTS BY MONTH

\begin{tabular}{|c|c|c|c|c|c|c|c|c|c|c|c|c|c|c|}
\hline ARRAY & $\mathbf{J}$ & $F$ & $M$ & $A$ & $M$ & $J$ & $\mathrm{~J}$ & A & $\mathrm{S}$ & 0 & $\mathbf{N}$ & D & $\begin{array}{l}\text { ANNUAL } \\
\text { AMOUNT }\end{array}$ & $\begin{array}{c}\text { AVERAGE } \\
\text { DAY }\end{array}$ \\
\hline 0.0 & 2.98 & 4.04 & 5.33 & 6.73 & 7.73 & 8.41 & 7.81 & 7.10 & 6.09 & 4.66 & 3.30 & 2.64 & 2034.4 & 5.6 \\
\hline 10.0 & 3.59 & 4.59 & 5.77 & 6.96 & 7.77 & 8.36 & 7.85 & 7.36 & 6.64 & 5.38 & 4.04 & 3.29 & 2179.5 & 6.0 \\
\hline 15.0 & 3.87 & 4.83 & 5.93 & 7.01 & 7.72 & 8.26 & 7.80 & 7.42 & 6.85 & 5.69 & 4.37 & 3.59 & 2232.5 & 6.1 \\
\hline 20.0 & 4.12 & 5.03 & 6.07 & 7.03 & 7.62 & 8.12 & 7.70 & 7.44 & 7.01 & 5.96 & 4.67 & 3.87 & 2271.8 & 6.2 \\
\hline 25.0 & 4.34 & 5.21 & 6.16 & 7.00 & 7.48 & 7.92 & 7.55 & 7.41 & 7.14 & 6.20 & 4.95 & 4.12 & 2297.1 & 6.3 \\
\hline 30.0 & 4.54 & 5.35 & 6.22 & 6.92 & 7.29 & 7.67 & 7.36 & 7.34 & 7.22 & 6.40 & 5.19 & 4.34 & 2308.3 & 6.3 \\
\hline 35.0 & 4.71 & 5.47 & 6.24 & 6.81 & 7.07 & 7.38 & 7.13 & 7.22 & 7.25 & 6.55 & 5.40 & 4.54 & 2305.2 & 6.3 \\
\hline 40.0 & 4.85 & 5.54 & 6.22 & 6.66 & 6.81 & 7.07 & 6.87 & 7.06 & 7.23 & 6.67 & 5.57 & 4.70 & 2289.8 & 6.3 \\
\hline 45.0 & 4.96 & 5.58 & 6.16 & 6.48 & 6.52 & 6.72 & 6.58 & 6.86 & 7.17 & 6.74 & 5.71 & 4.84 & 2261.1 & 6.2 \\
\hline 50.0 & 5.03 & 5.59 & 6.07 & 6.26 & 6.20 & 6.34 & 6.25 & 6.63 & 7.07 & 6.76 & 5.30 & 4.94 & 2218.7 & 6.1 \\
\hline 60.0 & 5.08 & 5.50 & 5.77 & 5.70 & 5.45 & 5.47 & 5.48 & 6.03 & 6.73 & 6.68 & 5.38 & 5.05 & 2093.5 & 5.7 \\
\hline 70.0 & 5.00 & 5.27 & 5.34 & 5.02 & 4.58 & 4.51 & 4.60 & 5.30 & 6.22 & 6.42 & 5.30 & 5.02 & 1918.7 & 5.3 \\
\hline 30.0 & 4.79 & 4.91 & 4.78 & 4.24 & 3.70 & 3.54 & 3.71 & 4.46 & 5.55 & 6.00 & 5.57 & 4.86 & 1705.8 & 4.7 \\
\hline 90.0 & $4: 46$ & 4.44 & 4.11 & 3.38 & 2.77 & 2.58 & 2.76 & 3.54 & 4.76 & 5.42 & 5.19 & 4.57 & 1458.2 & 4.0 \\
\hline
\end{tabular}


AVERAGE DAILY TOTAL TERFESTRIAL INSOLATION ESTIMATES (KWH/SQ. M)

SITE: ROSWELL
ITUDE: 33 LEGREES 24 MINUTES

AVERAGE DAIL: AMOLNTS BY MONTH

\begin{tabular}{|c|c|c|c|c|c|c|c|c|c|c|c|c|c|c|}
\hline ARRAY & $\mathrm{J}$ & $F$ & $M$ & A & $M$ & $J$ & J & A & $\mathrm{s}$ & 0 & $N$ & D & $\begin{array}{l}\text { ANNUAL } \\
\text { AMOUNT }\end{array}$ & $\begin{array}{c}\text { AVERAGE } \\
\text { DAY }\end{array}$ \\
\hline 0.0 & 3.29 & 4.33 & 5.59 & 7.00 & 7.75 & c. 23 & 7.69 & 7.07 & 6.03 & 4.81 & 3.56 & 3.00 & 2084.6 & 5.7 \\
\hline 10.0 & 3.88 & 4.86 & 6.10 & 7.18 & 7.73 & a.:-3 & 7.67 & 7.26 & 6.48 & 5.45 & 4.25 & 3.65 & 2211.6 & 6.1 \\
\hline 15.0 & 4.14 & 5.08 & 6.25 & 7.21 & 7.66 & a. 10 & 7.59 & 7.29 & 6.64 & 5.73 & 4.55 & 3.94 & 2255.3 & 6.2 \\
\hline 20.0 & 4.38 & 5.27 & 6.37 & 7.20 & 7.53 & 7.23 & 7.47 & 7.28 & 6.77 & 5.95 & 4.82 & 4.21 & 2285.3 & 6.3 \\
\hline 25.0 & 4.59 & 5.43 & 6.44 & 7.14 & 7.36 & 7.61 & 7.30 & 7.22 & 6.85 & 6.15 & 5.07 & 4.45 & 2301.3 & 6.3 \\
\hline 30.0 & 4.77 & 5.55 & 6.48 & 7.05 & 7.15 & 7.34 & 7.09 & 7.12 & 6.89 & 6.32 & 5.28 & 4.66 & 2303.3 & 6.3 \\
\hline 35.0 & 4.92 & 5.64 & 6.47 & 6.91 & 6.90 & 7.04 & 6.84 & 6.98 & 6.89 & 6.44 & 5.46 & 4.84 & 2292.0 & 6.3 \\
\hline 40.0 & 5.04 & 5.69 & 6.43 & 6.73 & 6.63 & 6.72 & 6.57 & 6.80 & 6.84 & 6.52 & 5.60 & 4.99 & 2268.5 & 6.2 \\
\hline 45.0 & 5.13 & 5.71 & 6.35 & 6.51 & 6.32 & 6.35 & 6.26 & 6.58 & 6.75 & 6.55 & 5.71 & 5.11 & 2231.1 & 6.1 \\
\hline 50.0 & 5.18 & 5.70 & 6.23 & 6.26 & 5.97 & 5.36 & 5.91 & 6.32 & 6.62 & 6.55 & 5.77 & 5.20 & 2180.2 & 6.0 \\
\hline 60.0 & 5.19 & 5.56 & 5.87 & 5.65 & 5.18 & 5.37 & 5.13 & 5.70 & 6.24 & 6.41 & 5.80 & 5.26 & 2039.5 & 5.6 \\
\hline 70.0 & 5.06 & 5.29 & 5.38 & 4.91 & 4.29 & 4.13 & 4.2 .5 & 4.95 & 5.71 & 6.11 & 5.67 & 5.19 & 1853.3 & 5.1 \\
\hline 80.0 & 4.81 & 4.89 & 4.77 & 4.07 & 3.40 & 3.17 & 3.36 & 4.10 & 5.05 & 5.65 & 5.39 & 4.99 & 1630.3 & 4.5 \\
\hline 90.0 & 4.43 & 4.37 & 4.04 & 3.18 & 2.50 & 2.27 & 3.47 & 3.20 & 4.27 & 5.06 & 4.98 & 4.65 & 1379.9 & 3.8 \\
\hline
\end{tabular}


AVERAGE DAILY TOTAL TERRESTRIAL INSOLATION ESTIMATES (KWH/SQ. M)

SITE: TRUTH OR CONSEQUENNM

-ATITUDE: 33 DEGREES 14 MINUTES

AVERAGE DAILY AMOUNTS BY MONTH

\begin{tabular}{|c|c|c|c|c|c|c|c|c|c|c|c|c|c|c|}
\hline ARRAY & $\mathrm{J}$ & $F$ & $M$ & $A$ & - & 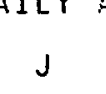 & ל & 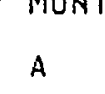 & $S$ & 0 & W & $D$ & $\begin{array}{l}\text { ANNUAL } \\
\text { AMOUNT }\end{array}$ & $\begin{array}{c}\text { AVERAGE } \\
\text { DAY }\end{array}$ \\
\hline 0.0 & 3.52 & 4.58 & 5.94 & 7.37 & 8.07 & 8.36 & 7.45 & 6.99 & 6.11 & 4.98 & 3.83 & 3.16 & 2141.8 & 5.9 \\
\hline 10.0 & 4.18 & 5.15 & 6.38 & 7.57 & 8.05 & 8.25 & 7.43 & 7.17 & 6.56 & 5.65 & 4.59 & 3.86 & 2278.2 & 6.2 \\
\hline 15.0 & 4.47 & 5.39 & 6.54 & 7.60 & 7.96 & 8.12 & 7.36 & 7.20 & 6.73 & 5.94 & 4.93 & 4.18 & 2325.8 & 6.4 \\
\hline 20.0 & 4.73 & 5.60 & 6.67 & 7.59 & 7.83 & 7.94 & 7.23 & 7.19 & 6.86 & 6.18 & 5.24 & 4.47 & 2359.2 & 6.5 \\
\hline 25.0 & 4.97 & 5.77 & 6.75 & 7.53 & 7.65 & 7.71 & 7.07 & 7.13 & 6.94 & 6.39 & 552 & 4.73 & 2378.0 & 6.5 \\
\hline $30.0^{\prime}$ & 5.17 & 5.91 & 6.79 & 7.42 & 7.43 & 7.44 & 6.87 & 7.03 & 6.98 & 6.56 & 5.75 & 4.96 & 2382.2 & 6.5 \\
\hline 35.0 & 5.34 & 6.01 & 6.78 & 7.28 & 7.17 & 7.13 & 6.63 & 6.89 & 6.98 & 6.68 & 5.95 & 5.16 & 2372.6 & 6.5 \\
\hline 40.0 & 5.47 & 6.07 & 6.74 & 7.09 & 6.88 & 6.80 & 6.37 & 6.71 & 6.93 & 6.77 & 6.11 & 5.33 & 2350.0 & 6.4 \\
\hline 45.0 & 5.57 & 6.10 & 6.65 & 6.86 & 6.55 & 6.43 & 6.07 & 6.50 & 6.84 & 6.81 & 6.24 & 5.45 & 2313.0 & 6.3 \\
\hline 50.0 & 5.63 & 6.09 & 6.53 & 6.59 & 6.18 & 6.02 & 5.73 & 6.24 & 6.71 & 6.80 & 6.32 & 5.55 & 2261.9 & 6.2 \\
\hline 60.0 & 5.65 & 5.94 & 6.16 & 5.93 & 5.35 & 5.11 & 4.98 & 5.62 & 6.32 & 6.66 & 6.35 & 5.62 & 2118.7 & 5.8 \\
\hline 70.0 & 5.52 & 5.65 & 5.64 & 5.14 & 4.41 & 4.15 & 4.13 & 4.88 & 5.78 & 6.35 & 6.21 & 5.55 & 1927.8 & 5.3 \\
\hline 80.0 & 5.24 & 5.22 & 4.98 & 4.24 & 3.47 & 3.17 & 3.28 & 4.04 & 5.10 & 5.87 & 5.91 & 5.33 & 1697.7 & 4.7 \\
\hline 90.0 & 4.83 & 4.67 & 4.22 & 3.30 & 2.52 & 2.25 & 2.42 & 3.15 & 4.31 & 5.25 & 5.46 & 4.97 & 1438.6 & 3.9 \\
\hline
\end{tabular}


AVERAGE DAILY TOTAL TERRESTRIAL INSOLATION ESTIMATES (KHH/SQ. M)

SITE: TUCUMCARI
LATITUDE:
35 DEGREES 12 MINUTES

AVERAGE DAILY AMOUNTS BY MONTH

\begin{tabular}{|c|c|c|c|c|c|c|c|c|c|c|c|c|c|c|}
\hline ARRAY & $\begin{array}{l}\mathrm{J} \\
---\end{array}$ & $F$ & $M$ & A & $M$ & $j$ & $\mathrm{~J}$ & A & 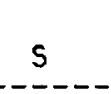 & 0 & $N$ & D & $\begin{array}{l}\text { ANNUAL } \\
\text { AMOUNT. }\end{array}$ & $\begin{array}{c}\text { AVERAGE } \\
\text { DAY }\end{array}$ \\
\hline 0.0 & 3.17 & 4.09 & 5.39 & 6.62 & 7.30 & 7.83 & 7.40 & 6.83 & 5.77 & 4.55 & 3.38 & 2.87 & 1985.2 & 5.4 \\
\hline 10.0 & 3.79 & 4.61 & 5.30 & 6.82 & 7.31 & 7.76 & 7.41 & 7.04 & 6.22 & 5.19 & 4.07 & 3.55 & 2118.2 & 5.8 \\
\hline 15.0 & 4.07 & 4.83 & 5.76 & 6.86 & 7.25 & 7.66 & 7.35 & 7.08 & 6.40 & 5.46 & 4.38 & 3.85 & 2165.9 & 5.9 \\
\hline 20.0 & 4.32 & 5.02 & 6.37 & 6.86 & 7.15 & 7.51 & 7.24 & 7.09 & 6.53 & 5.70 & 4.67 & 4.14 & 2200.5 & 6.0 \\
\hline 25.0 & 4.55 & 5.18 & 6.16 & 6.82 & 7.00 & 7.32 & 7.10 & 7.04 & 6.62 & 5.90 & 4.92 & 4.39 & 2221.8 & 6.1 \\
\hline 30.0 & 4.74 & 5.31 & 6.20 & 6.74 & 6.82 & 7.68 & $\epsilon .91$ & 6.96 & 6.67 & 6.07 & 5.14 & 4.62 & 2229.6 & 6.1 \\
\hline 35.0 & 4.91 & 5.41 & 6.21 & 6.62 & 6.60 & 6.81 & $\epsilon .68$ & 6.83 & 6.68 & 6.20 & 5.33 & 4.82 & 2223.8 & 6.1 \\
\hline 40.0 & 5.04 & 5.47 & 6.18 & 6.46 & 6.35 & $6: \equiv 2$ & $\epsilon .43$ & 6.67 & 6.65 & 6.28 & 5.49 & 4.98 & 2206.8 & 6.0 \\
\hline 45.0 & 5.14 & 5.50 & 6.11 & 6.27 & 6.08 & 6.19 & $\epsilon .15$ & 6.47 & 6.58 & 6.33 & 5.61 & 5.12 & 2176.6 & 6.0 \\
\hline 50.0 & 5.21 & 5.49 & 6.31 & 6.04 & 5.76 & 5.8 .3 & 5.83 & 6.24 & 6.47 & 6.34 & 5.69 & 5.22 & 2133.2 & 5.8 \\
\hline 60.0 & 5.24 & 5.38 & 5.59 & 5.49 & 5.05 & 5. 52 & 5.10 & 5.66 & 6.12 & 6.23 & 5.74 & 5.31 & 2008.3 & 5.5 \\
\hline 70.0 & 5.14 & 5.14 & 5.24 & 4.81 & 4.23 & 4.14 & c. .26 & 4.95 & 5.63 & 5.96 & 5.64 & 5.26 & 1837.2 & 5.0 \\
\hline 80.0 & 4.91 & 4.77 & 4.57 & 4.03 & .5 .41 & 3.25 & 5.43 & 4.14 & 5.01 & 5.54 & 5.39 & 5.08 & 1630.3 & 4.5 \\
\hline 90.0 & 4.55 & 4.29 & 3.99 & 3.20 & 2.56 & 2.59. & c. 56 & 3.27 & 4.27 & 4.99 & 5.00 & 4.76 & 1392.5 & 3.8 \\
\hline
\end{tabular}


AUERAGE DAILY TOTAL TERRESTRIAL INSOLATION ESTIMATES (KWH/SQ. M)

SITE: ZUNI
LATITUDE: 35 DEGREES 6 MINUTES

ALERAGE DAILY AMOUNTS BY MONTH

\begin{tabular}{|c|c|c|c|c|c|c|c|c|c|c|c|c|c|c|}
\hline ARRAY & $J$ & $F$ & $M$ & $A$ & $M$ & 7tor & $\mathrm{J}$ & Tाuा & $s$ & 0 & $N$ & D & $\begin{array}{l}\text { ANNUAL } \\
\text { AMOUNT }\end{array}$ & $\begin{array}{c}\text { AUERAGE } \\
\text { DAY }\end{array}$ \\
\hline 0.0 & 3.10 & 4.09 & 5.32 & 6.83 & 7.80 & 8.20 & 7.14 & 6.55 & 5.97 & 4.71 & $3 .<3$ & 2.82 & 2008.2 & 5.5 \\
\hline 10.0 & 3.70 & 4.61 & 5.72 & 7.04 & 7.81 & 8.13 & 7.14 & 6.75 & 6.45 & 5.39 & $4 .: 4$ & 3.47 & 2141.2 & 5.9 \\
\hline 15.0 & 3.96 & 4.83 & 5.87 & 7.08 & 7.74 & 8.02 & 7.09 & 6.79 & 6.64 & 5.68 & 4.46 & 3.77 & 2188.5 & 6.0 \\
\hline 20.0 & 4.20 & 5.02 & 5.98 & 7.08 & 7.63 & 7.86 & 6.98 & 6.79 & 6.78 & 5.93 & 4.75 & 4.04 & 2222.7 & 6.1 \\
\hline 25.0 & 4.42 & 5.18 & 6.06 & 7.04 & 7.48 & 7.65 & 6.84 & 6.74 & 6.88 & 6.15 & 5.00 & 4.28 & 2243.3 & 6.1 \\
\hline 30.0 & 4.60 & 5.30 & 6.10 & 6.96 & 7.28 & 7.40 & 6.66 & 6.66 & 6.93 & 6.32 & 5.23 & 4.50 & 2250.2 & 6.2 \\
\hline 35.0 & 4.76 & 5.40 & 6.11 & 6.83 & 7.03 & 7.11 & 6.44 & 6.54 & 6.94 & 6.46 & 5.82 & 4.69 & 2243.4 & 6.1 \\
\hline 40.0 & 4.89 & 5.46 & 6.08 & 6.67 & 6.77 & 6.80 & 6.20 & 6.39 & 6.91 & 6.55 & 5.58 & 4.85 & 2225.5 & 6.1 \\
\hline 45.0 & 4.98 & 5.49 & 6.01 & 6.47 & 6.47 & 6.46 & 5.93 & 6.20 & 6.84 & 6.60 & 5.70 & 4.98 & 2194.0 & 6.0 \\
\hline 50.0 & 5.05 & 5.49 & 5.90 & 6.23 & 6.13 & 6.07 & 5.63 & 5.97 & 6.72 & 6.61 & 5.79 & 5.07 & 2149.3 & 5.9 \\
\hline 60.0 & 5.07 & 5.37 & $5.5 s$ & 5.66 & 5.35 & 5.21 & 4.93 & 5.42 & 6.37 & 6.50 & 5.34 & 5.16 & 2021.4 & 5.5 \\
\hline 70.0 & 4.97 & 5.13 & 5.15 & 4.95 & 4.46 & 4.28 & 4.13 & 4.75 & 5.85 & 6.22 & 5.73 & 5.11 & 1846.9 & 5.1 \\
\hline 80.0 & 4.74 & 4.76 & 4.59 & $4.14^{\circ}$ & 3.57 & 3.33 & 3.34 & 3.98 & 5.20 & 5.79 & 5.78 & 4.93 & 1636.6 & 4.5 \\
\hline 90.0 & 4.39 & 4.28 & 3.92 & 3.28 & 2.64 & 2.42 & 2.50 & 3.15 & 4.43 & 5.21 & 5.D 8 & 4.62 & 1395.6 & 3.8 \\
\hline
\end{tabular}


AVERAG DAILY TOTAL TERRESTRIAL INSOLATION ESTIMATES (KWH/SQ. M)

SITE: ELKO NV

AVERAGE DAILY AMOLNTS BY MONTH

\begin{tabular}{|c|c|c|c|c|c|c|c|c|c|c|c|c|c|c|}
\hline $\begin{array}{l}\text { ARRAY } \\
\text { TILT }\end{array}$ & J & $F$ & $M$ & A & $M$ & $\mathrm{~J}$ & 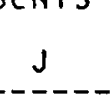 & A & 5 & 0 & $N$ & D & $\begin{array}{l}\text { ANNUAL } \\
\text { AMOUNT }\end{array}$ & $\begin{array}{c}\text { AVERAGE } \\
\text { DAY }\end{array}$ \\
\hline 0.0 & 2.18 & 3.26 & 4.61 & 5.99 & 7.26 & 7.98 & 8.27 & 7.30 & 5.97 & 4.17 & 2.56 & 1.95 & 1873.7 & 5.1 \\
\hline 10.0 & 2.65 & 3.74 & 5.03 & 6.24 & 7.36 & 8.00 & 8.39 & 7.66 & 6.61 & 4.91 & 3.19 & 2.47 & 2018.4 & 5.5 \\
\hline 15.0 & 2.86 & 3.95 & 5.19 & 6.31 & 7.34 & 7.94 & 8.38 & 7.77 & 6.87 & 5.23 & 3.48 & 2.71 & 2072.9 & 5.7 \\
\hline 20.0 & 3.06 & 4.13 & 5.33 & 6.34 & 7.28 & 7.84 & 8.31 & 7.83 & 7.09 & 5.52 & 3.75 & 2.94 & 2114.8 & 5.8 \\
\hline 25.0 & 3.23 & 4.29 & 5.43 & 6.34 & 7.18 & 7.69 & 8.19 & 7.84 & 7.26 & 5.78 & 3.99 & 3.14 & 2143.7 & 5.9 \\
\hline 30.0 & 3.39 & 4.42 & 5.50 & 6.30 & 7.04 & 7.49 & 8.03 & 7.80 & 7.39 & 6.00 & 4.21 & 3.33 & 2159.5 & 5.9 \\
\hline 35.0 & 3.53 & 4.53 & 5.54 & 6.23 & 6.86 & 7.25 & 7.81 & 7.72 & 7.47 & 6.18 & 4.39 & $3.49^{\circ}$ & 2162.0 & 5.9 \\
\hline 40.0 & 3.64 & 4.61 & 5.55 & 6.11 & 6.63 & 6.97 & 7.55 & 7.58 & 7.50 & 6.32 & 4.55 & 3.63 & 2151.2 & 5.9 \\
\hline 45.0 & 3.73 & 4.66 & 5.52 & 5.97 & 6.39 & 6.67 & 7.26 & 7.41 & 7.48 & 6.42 & 4.68 & 3.75 & 2129.6 & 5.8 \\
\hline 50.0 & 3.80 & 4.58 & 5.46 & 5.80 & 6.11 & 6.34 & 6.93 & 7.19 & 7.41 & 6.47 & 4.78 & 3.85 & 2095.5 & 5.7 \\
\hline 60.0 & 3.86 & 4.64 & 5.24 & 5.35 & 5.46 & 5.58 & 6.16 & 6.63 & 7.13 & 6.46 & 4.89 & 3.96 & 1989.2 & 5.4 \\
\hline 70.0 & 3.83 & 4.48 & 4.89 & 4.79 & 4.69 & 4.70 & 5.25 & 5.90 & 6.67 & 6.27 & 4.86 & 3.97 & 1835.0 & 5.0 \\
\hline 80.0 & 3.69 & 4.21 & 4.44 & 4.12 & 3.87 & 3.81 & 4.27 & 5.04 & 6.04 & 5.92 & 4.71 & 3.87 & 1642.5 & 4.5 \\
\hline 90.0 & 3.47 & 3.85 & 3.88 & 3.38 & 3.02 & 2.87 & 3.27 & 4.07 & 5.26 & 5.42 & 4.43 & 3.67 & 1416.3 & 3.9 \\
\hline
\end{tabular}


AVERIGE DAILY TOTAL TERRESTRIAL INSOLATION ESTIMATES (KWH/SQ. M)

$\begin{aligned} \text { SITE: } & \text { ELY } \\ \text { LATITUDE: } & 39 \text { DEGREES } 17 \text { MINUTES }\end{aligned}$

AVERAGE DAILY AMOUNTS BY MONTH

\begin{tabular}{|c|c|c|c|c|c|c|c|c|c|c|c|c|c|c|}
\hline ARRAY & $J$ & $\mathbf{F}$ & $M$ & $A$ & $M$ & $J$ & $\mathrm{~J}$ & $A$ & s & 0 & $N$ & $\mathrm{D}$ & $\begin{array}{l}\text { ANNUAL } \\
\text { ANOUNT }\end{array}$ & $\begin{array}{c}\text { AVERAGE } \\
\text { DAY }\end{array}$ \\
\hline 0.0 & 2.59 & 3.60 & 5.06 & 6.34 & 7.28 & 7.93 & 7.72 & 7.04 & 6.10 & 4.43 & 2.32 & 2.28 & 1927.6 & 5.3 \\
\hline 10.0 & 3.16 & 4.12 & 5.52 & 6.59 & 7.36 & 7.92 & 7.80 & 7.34 & 6.72 & 5.19 & 3.53 & 2.89 & 2078.1 & 5.7 \\
\hline 15.0 & 3.42 & 4.34 & 5.59 & 6.66 & 7.33 & 7.85 & 7.77 & 7.42 & 6.97 & 5.52 & 3.75 & 3.17 & 2134.8 & 5.8 \\
\hline 20.0 & 3.66 & 4.54 & 5.84 & 6.69 & 7.26 & 7.74 & 7.69 & 7.46 & 7.17 & 5.82 & 4.25 & 3.43 & 2178.5 & 6.0 \\
\hline 25.0 & 3.87 & 4.71 & 5.95 & 6.68 & 7.15 & 7.58 & 7.57 & 7.46 & 7.33 & 6.08 & 4.52 & 3.67 & 2208.8 & 6.1 \\
\hline 30.0 & 4.06 & 4.86 & 6.02 & 6.62 & 6.99 & 7.37 & 7.41 & 7.40 & 7.44 & 6.30 & 4.76 & 3.88 & 2225.6 & 6.1 \\
\hline 35.0 & 4.23 & 4.97 & 6.06 & 6.53 & 6.80 & 7.12 & 7.19 & 7.31 & 7.50 & 6.47 & 4.77 & 4.07 & 2228.7 & 6.1 \\
\hline 40.0 & 4.36 & 5.05 & 6.05 & 6.41 & 6.57 & 6.83 & 6.94 & 7.17 & 7.52 & 6.61 & 5.14 & 4.23 & 2218.4 & 6.1 \\
\hline 45.0 & 4.47 & 5.10 & 6.02 & 6.25 & 6.32 & 6.53 & 6.67 & 6.99 & 7.48 & 6.70 & 5.29 & 4.37 & 2196.9 & 6.0 \\
\hline $5 C .0$ & 4.55 & 5.12 & 5.94 & 6.05 & 6.03 & 6.19 & 6.36 & 6.77 & 7.40 & 6.75 & 5.39 & 4.47 & 2161.8 & 5.9 \\
\hline 60.0 & 4.62 & 5.061 & 5.69 & 5.56 & 5.36 & 5.42 & 5.64 & 6.21 & 7.09 & 6.71 & 5.50 & 4.60 & 2052.5 & 5.6 \\
\hline 70.0 & 4.58 & 4.87 & 5.29 & 4.95 & $\dot{4} .58$ & 4.53 & 4.80 & 5.51 & 6.60 & 6.50 & 5.46 & 4.60 & 1893.8 & 5.2 \\
\hline 80.0 & 4.41 & 4.57 & 4.78 & 4.23 & 3.75 & 3.65 & 3.91 & 4.68 & 5.95 & 6.11 & 5.27 & 4.47 & 1696.2 & 4.6 \\
\hline 90.0 & 4.13 & 4.16 & 4.15 & 3.42 & 2.89 & 2.74 & 2.98 & 3.76 & 5.14 & 5.57 & 4.94 & 4.23 & 1463.0 & 4.0 \\
\hline
\end{tabular}


AVERAGE DAILY TOTAL TERRESTRIAL INSOLATION ESTIMATES (KWH/SZ. M)

SITE: LAS VEGAS
LATITUDE: 36 DEGREES 5 MINUTES

AVERAGE DAILY AMOUNTS BY MONTH

\begin{tabular}{|c|c|c|c|c|c|c|c|c|c|c|c|c|c|c|}
\hline ARRAY & $\mathbf{J}$ & $F$ & $M$ & $A$ & $M$ & $J$ & J & A & 5 & 0 & $N$ & D & $\begin{array}{l}\text { ANNUAL } \\
\text { ANOUNT }\end{array}$ & $\begin{array}{c}\text { AUERAGE } \\
\text { DAY }\end{array}$ \\
\hline 0.0 & 3.08 & 4.22 & 5.75 & 7.31 & 8.34 & 8.75 & 8.16 & 7.42 & 6.42 & 4.85 & 3.42 & 2.78 & 2147.3 & 5.9 \\
\hline 10.0 & 3.70 & 4.80 & 6.23 & 7.57 & 8.38 & 8.73 & 8.19 & 7.69 & 7.00 & 5.61 & 4.17 & 3.46 & 2298.2 & 6.3 \\
\hline 15.0 & 3.98 & 5.04 & 6.41 & 7.63 & 8.32 & 8.57 & 8.13 & 7.75 & 7.22 & 5.93 & 4.51 & 3.77 & 2352.5 & 6.4 \\
\hline 20.0 & 4.23 & 5.26 & 6.55 & 7.64 & 8.21 & $8 .+3$ & 8.02 & 7.77 & 7.40 & 6.21 & 4.82 & 4.05 & 2392.3 & 6.6 \\
\hline 25.0 & 4.46 & 5.44 & 6. 66 & 7.61 & 8.05 & $8 . \geq \mathrm{l}$ & 7.86 & 7.73 & 7.53 & 6.46 & 5.09 & 4.31 & 2417.1 & 6.6 \\
\hline 30.0 & 4.66 & 5.59 & 6.72 & 7.53 & 7.84 & 7.75 & 7.66 & 7.65 & 7.61 & 6.66 & 5.34 & 4.54 & 2426.9 & 6.6 \\
\hline 35.0 & 4.83 & 5.70 & 6.74 & 7.40 & 7.58 & 7.53 & 7.41 & 7.52 & 7.64 & 6.82 & 5.55 & 4.75 & 2421.4 & 6.6 \\
\hline 40.0 & 4.97 & 5.78 & 6.72 & 7.23 & 7.29 & 7.30 & 7.13 & 7.35 & 7.62 & 6.94 & 5.72 & 4.92 & 2403.2 & 6.6 \\
\hline 45.0 & 5.07 & 5.82 & 5.66 & 7.03 & 6.97 & 6.73 & 6.81 & 7.14 & 7.56 & 7.01 & 5.85 & 5.06 & 2370.6 & 6.5 \\
\hline 50.0 & 5.15 & 5.83 & c. 5.6 & $\dot{b .78}$ & 6.61 & 6.52 & 6.46 & 6.88 & 7.44 & 7.03 & 5.95 & 5.16 & 2323.2 & 6.4 \\
\hline 60.0 & 5.19 & 5.73 & 5.23 & 6. 16 & 5.77 & 5.59 & 5.64 & 6.25 & 7.07 & 6.94 & 6.02 & 5.27 & 2185.8 & 6.0 \\
\hline 70.0 & 5.11 & 5.49 & $\Xi .75$ & 5.40 & 4.81 & 4.57 & 4.70 & 5.47 & 6.52 & 6.67 & 5.93 & 5.23 & 1996.1 & 5.5 \\
\hline 80.0 & 4.88 & 5.11 & $\equiv .14$ & 4.52 & 3.83 & 3.54 & 3.75 & 4.57 & 5.81 & 6.22 & 5.69 & 5.06 & 1766.7 & 4.8 \\
\hline 90.0 & 4.54 & 4.60 & 4.40 & 3.57 & 2.80 & 2.54 & 2.76 & 3.60 & 4.96 & 5.61 & 5.29 & 4.75 & 1501.5 & 4.1 \\
\hline
\end{tabular}


AVERAGE DAIL :OTAL TERRESTRIAL INSOLATION ESTINATES (KWH/SQ. M)

SITE:
LATITUDE:
40 DEGREES 4 MINUTES

AYERAGE DAILY AMOUNTS BY MONTH

\begin{tabular}{|c|c|c|c|c|c|c|c|c|c|c|c|c|c|c|}
\hline ARRAY & $\mathrm{J}$ & $=$ & $M$ & A & $M$ & 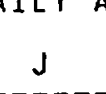 & 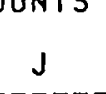 & 170 & $s$ & 0 & $N$ & D & $\begin{array}{l}\text { ANNUAL } \\
\text { AMOUNT }\end{array}$ & $\begin{array}{c}\text { AVERAGE } \\
\text { DAY }\end{array}$ \\
\hline 0.0 & 2.54 & 3.67 & 5.22 & 6.83 & 8.05 & 8.67 & 8.78 & 7.83 & 6.39 & 4.57 & 2.73 & 2.25 & 2064.3 & 5.7 \\
\hline 10.0 & 3.12 & 4.23 & 5.72 & 7.13 & 8.16 & 8.68 & 8.90 & 8.21 & 7.07 & 5.41 & 3.59 & 2.89 & 2230.7 & 6.1 \\
\hline 15.0 & 3.39 & 4.48 & 5.91 & 7.22 & 8.14 & 8.61 & 8.88 & 8.33 & 7.35 & 5.78 & 4.33 & 3.18 & 2293.6 & 6.3 \\
\hline 20.0 & 3.63 & 4.69 & 6.08 & 7.26 & 8.07 & 8.49 & 8.80 & 8.39 & 7.58 & 6.11 & 4.35 & 3.45 & 2342.3 & 6.4 \\
\hline 25.0 & 3.85 & 4.88 & 6.20 & 7.26 & 7.95 & 8.32 & 8.67 & 8.40 & 7.76 & 6.40 & 4.34 & 3.70 & 2376.2 & 6.5 \\
\hline 30.0 & 4.05 & 5.04 & 6.29 & 7.22 & 7.79 & 8.09 & 8.49 & 8.35 & 7.88 & 6.65 & 4.99 & 3.92 & 2395.2 & 6.6 \\
\hline 35.0 & 4.22 & 5.16 & 6.33 & 7.13 & 7.57 & 7.82 & 8.25 & 8.25 & 7.96 & 6.86 & 5.12 & 4.12 & 2399.2 & 6.6 \\
\hline 40.0 & 4.37 & 5.26 & 6.34 & 7.00 & 7.31 & 7.50 & 7.96 & 8.10 & 7.99 & 7.02 & 5.31 & 4.29 & 2388.0 & 6.5 \\
\hline 45.0 & 4.48 & 5.32 & 6.31 & 6.83 & 7.03 & 7.17 & 7.65 & 7.91 & 7.96 & 7.13 & 5.47 & 4.43 & 2365.1 & 6.5 \\
\hline 50.0 & 4.57 & 5.35 & 6.24 & 6.62 & 6.71 & 6.79 & 7.29 & 7.67 & 7.88 & 7.19 & 5.59 & 4.55 & 2327.4 & 6.4 \\
\hline 60.0 & 4.65 & 5.30 & 5.99 & 6.1 .0 & 5.96 & 5.93 & 6.45 & 7.05 & 7.57 & 7.18 & 5.71 & 4.69 & 2208.7 & 6.1 \\
\hline 70.0 & 4.61 & 5.12 & 5.59 & .5 .43 & 5.08 & 4.94 & 5.47 & 6.25 & 7.07 & 6.97 & 5.68 & 4.70 & 2035.5 & 5.6 \\
\hline 80.0 & 4.45 & 4.81 & 5.06 & 4.64 & 4.14 & 3.95 & 4.42 & 5.31 & 6.38 & 6.57 & 5.50 & 4.58 & 1819.3 & 5.0 \\
\hline 90.0 & 4.18 & 4.38 & 4.40 & 3.75 & 3.16 & 2.91 & 3.34 & 4.25 & 5.54 & 6.00 & 5.17 & 4.34 & 1564.2 & 4.3 \\
\hline
\end{tabular}


AVERIIGE DAILY TOTAL TERRESTRIAL INSOLATION ESTIMATES (KWH/SQ. M)

SITE: FENO

NV

LATITUDE: 39 DEGREES 30 MINUTES

AVERAGE CAILY AMOLINTS BY MONTH

\begin{tabular}{|c|c|c|c|c|c|c|c|c|c|c|c|c|c|c|}
\hline ARRAY & 2 & $F$ & $M$ & A & $M$ & J & $\mathrm{J}$ & A & $s$ & 0 & $\mathrm{~N}$ & D & $\begin{array}{l}\text { ANNUAL } \\
\text { AMOUNT }\end{array}$ & $\begin{array}{c}\text { AVERAGE } \\
\text { DAY }\end{array}$ \\
\hline 0.0 & 2.53 & 3.62 & 5.19 & 6.81 & 7.96 & 8.51 & d. 48 & 7.59 & 6.29 & 4.51 & 2.88 & 2.23 & 2029.2 & 5.6 \\
\hline 10.0 & 3.08 & 4.16 & 5.57 & 7.09 & 8.05 & 8.52 & 8.59 & 7.94 & 6.95 & 5.30 & 3.58 & 2.82 & 2186.0 & 6.0 \\
\hline 15.0 & 3.34 & 4.39 & 5.36 & 7.18 & 8.03 & 8.44 & 8.56 & 8.05 & 7.21 & 5.65 & 3.90 & 3.10 & 2244.5 & 6.1 \\
\hline 20.0 & 3.57 & 4.59 & 6.02 & 7.21 & 7.95 & 8.32 & 8.48 & 8.10 & 7.43 & 5.96 & 4.20 & 3.35 & 2289.2 & 6.3 \\
\hline 25.0 & 3.78 & 4.77 & 6.14 & 7.21 & 7.83 & 8.14 & 8.35 & 8.10 & 7.60 & 6.23 & 4.46 & 3.58 & 2319.6 & 6.4 \\
\hline 30.0 & 3.96 & 4.91 & 6.22 & 7.16 & 7.66 & 7.92 & 8.16 & 8.05 & 7.71 & 6.46 & 4.70 & 3.79 & 2335.6 & $6: 4$ \\
\hline 35.0 & 4.12 & 5.03 & 6.26 & 7.06 & 7.44 & 7.65 & 7.93 & 7.95 & 7.78 & 6.65 & 4.91 & 3.98 & 2336.9 & 6.4 \\
\hline 40.0 & 4.26 & 5.11 & 5.26 & 6.93 & 7.19 & 7.33 & 7.64 & 7.80 & 7.80 & 6.79 & 5.08 & 4.14 & 2323.9 & 6.4 \\
\hline 45.0 & 4.36 & 5.17 & 5.22 & 6.76 & 6.91 & 7.00 & 7.34 & 7.61 & 7.77 & 6.89 & 5.23 & 4.27 & 2299.3 & 6.3 \\
\hline 50.0 & 4.44 & 5.19 & 5.15 & 6.55 & 6.59 & 6.63 & 7.00 & 7.37 & 7.69 & 6.95 & 5.33 & 4.37 & 2260.4 & 6.2 \\
\hline 60.0 & 4.51 & 5.13 & 5.89 & 6.02 & 3.85 & 5.79 & 6.18 & 6.76 & 7.37 & 6.92 & 5.44 & 4.49 & 2140.8 & 5.9 \\
\hline 70.0 & 4.47 & 4.95 & 5.49 & 5.35 & 4.97 & 4.81 & 5.23 & 5.99 & 6.87 & 6.70 & 5.40 & 4.49 & 1968.8 & 5.4 \\
\hline 80.0 & 4.30 & 4.64 & 4.96 & 4.56 & 4.04 & 3.85 & 4.22 & 5.08 & 6.19 & 6.31 & 5.22 & 4.37 & 1756.2 & 4.8 \\
\hline 90.0 & 4.03 & 4.22 & $\dot{4} .31$ & 3.68 & 3.09 & 2.84 & 3.17 & 4.05 & 5.36 & 5.75 & 4.90 & 4.14 & 1506.4 & 4.1 \\
\hline
\end{tabular}


AVERAGE DAI_Y TOTAL TERRESTRIAL INSOLATION ESTIMATES (KWH/SQ. M)

SITE: TONOPAH

-ATITUDE: 38 DEGREES 4 MINUTES

¿VERAGE DAILY AMOUNTS BY MONTH

\begin{tabular}{|c|c|c|c|c|c|c|c|c|c|c|c|c|c|c|}
\hline ARRAY & $J$ & $F$ & $M$ & $A$ & $M$ & $J$ & $J$ & $A$ & 5 & 0 & $\checkmark$ & $D$ & $\begin{array}{l}\text { ANNUAL } \\
\text { AMOUNT }\end{array}$ & $\begin{array}{c}\text { AVERAGE } \\
\text { DAY }\end{array}$ \\
\hline 0.0 & 2.90 & 4.02 & 5.60 & 7.10 & 8.13 & 8.78 & 8.53 & 7.69 & 6.44 & 4.79 & 3.25 & 2.61 & 2127.1 & 5.8 \\
\hline 10.0 & 3.53 & 4.60 & 6.11 & 7.38 & 8.20 & 8.76 & 8.60 & 8.02 & 7.08 & 5.60 & 4.04 & 3.31 & 2291.1 & 6.3 \\
\hline 15.0 & 3.82 & 4.86 & 6.31 & 7.45 & 8.16 & 8.67 & 8.56 & 8.11 & 7.33 & 5.96 & 4.39 & 3.63 & 2352.2 & 6.4 \\
\hline 20.0 & 4.08 & 5.08 & 6.47 & 7.48 & 8.07 & 8.53 & 8.47 & 8.15 & 7.53 & 6.28 & 4.72 & 3.93 & 2398.5 & 6.6 \\
\hline 25.0 & 4.32 & 5.27 & 6.59 & 7.46 & 7.93 & 8.33 & 8.32 & 8.13 & 7.68 & 6.55 & 5.01 & 4.21 & 2429.8 & 6.7 \\
\hline 30.0 & 4.53 & 5.43 & 6.67 & 7.40 & 7.75 & 8.08 & 8.12 & 8.07 & 7.79 & 6.78 & 5.28 & 4.45 & 2445.8 & 6.7 \\
\hline 35.0 & 4.71 & 5.56 & 6.70 & 7.29 & 7.51 & 7.79 & 7.87 & 7.95 & 7.84 & 6.97 & 5.50 & 4.67 & 2446.4 & 6.7. \\
\hline 40.0 & 4.86 & 5.65 & 6.70 & 7.14 & 7.24 & 7.45 & 7.58 & 7.79 & 7.85 & 7.11 & 5.69 & 4.85 & 2432.8 & 6.7 \\
\hline 45.0 & 4.98 & 5.70 & 6.65 & 6.96 & 6.95 & 7.10 & 7.26 & 7.59 & 7.80 & 7.21 & 585 & 5.01 & 2405.9 & 6.6 \\
\hline 50.0 & 5.07 & 5.72 & 6.57 & 6.73 & 6.61 & 6.70 & 6.91 & $7.33^{\circ}$ & 7.70 & 7.25 & 596 & 5.13 & 2363.9 & 6.5 \\
\hline 60.0 & 5.14 & 5.65 & 6.27 & 6.15 & 5.83 & 5.80 & 6.07 & 6.70 & 7.36 & 7.20 & 6.07 & 5.26 & 2235.9 & 6.1 \\
\hline 70.0 & 5.08 & 5.43 & 5.82 & 5.44 & 4.91 & 4.78 & 5.09 & 5.90. & 6.83 & 6.96 & 6.01 & 5.25 & 2053.2 & 5.6 \\
\hline $80: 0$ & 4.88 & 5.08 & 5.23 & 4.60 & 3.96 & 3.76 & 4.08 & 4.97 & 6.12 & 6.53 & 5.79 & 5.11 & 1828.3 & 5.0 \\
\hline 90.0 & 4.56 & 4.61 & 4.52 & 3.67 & 2.97 & 2.73 & 3.02 & 3.93 & 5.27 & 5.93 & 5.42 & 4.82 & 1564.4 & 4.3 \\
\hline
\end{tabular}


AVERAGE DAILY TOTAL TERRESTRIAL INSOLATION ESTIMATES (KWH/SQ. M)

SITE: WINNEMUCCA
LATITUDE:
SO DEGREES 54 MINUTES

AVERAGE DAILY AMOUNTS BY MONTH

\begin{tabular}{|c|c|c|c|c|c|c|c|c|c|c|c|c|c|c|}
\hline $\begin{array}{l}\text { ARRAY } \\
\text { TILT }\end{array}$ & 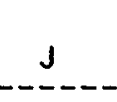 & $F$ & $M$ & $A$ & $M$ & J & J & A & $S$ & 0 & $N$ & $D$ & $\begin{array}{l}\text { ANNUAL } \\
\text { AMOUNT }\end{array}$ & $\begin{array}{c}\text { AVERAGE } \\
\text { DAY }\end{array}$ \\
\hline 0.0 & 2.18 & 3.24 & 4.64 & 6.21 & 7.45 & 8.10 & 8.45 & 7.40 & 6.01 & 4.16 & 2.56 & 1.95 & 1900.0 & 5.2 \\
\hline 10.0 & 2.65 & 3.71 & 5.06 & 6.97 & 7.55 & 8.12 & 8.58 & 7.78 & 6.67 & 4.91 & 3.19 & 2.48 & 2046.8 & 5.6 \\
\hline 15.0 & 2.87 & 3.92 & 5.23 & 6.55 & 7.54 & 8.06 & 8.57 & 7.89 & 6.93 & 5.23 & 3.48 & 2.72 & 2102.0 & 5.8 \\
\hline 20.0 & 3.07. & 4.10 & 5.37 & 6.59 & 7.48 & 7.96 & 8.50 & 7.95 & 7.15 & 5.53 & 3.74 & 2.95 & 2144.4 & 5.9 \\
\hline 25.0 & 3.24 & 4.26 & 5.47 & 6.59 & 7.38 & 7.81 & 3.38 & 7.97 & 7.33 & 5.78 & 3.98 & 3.16 & 2173.5 & 6.0 \\
\hline 30.0 & 3.40 & 4.39 & 5.55 & 6.55 & 7.23 & $? .61$ & 3.21 & 7.93 & 7.46 & 6.00 & 4.20 & 3.35 & 2189.3 & 6.0 \\
\hline 35.0 & 3.54 & 4.50 & 5.58 & 6.48 & 7.04 & 7.36 & 7.99 & 7.85 & 7.54. & 6.19 & 4.39 & 3.51 & 2191.5 & 6.0 \\
\hline 40.0 & 3.66 & 4.57 & 5.59 & 6.36 & 6.81 & 7.08 & 7.72 & 7.71 & 7.57 & 6.33 & 4.55 & 3.66 & 2180.2 & 6.0 \\
\hline 45.0 & 3.75 & 4.62 & 5.56 & 6.21 & 6.56 & 6.77 & 7.42 & 7.54 & 7.55 & 6.43 & 4.68 & 3.78 & 2158.0 & 5.9 \\
\hline 50.0 & 3.82 & 4.64 & 5.50 & 6.03 & 6.28 & 5.44 & 7.09 & 7.31 & 7.48 & 6.48 & 4.78 & 3.87 & 2123.0 & 5.8 \\
\hline 60.0 & 3.88 & 4.60 & 5.28 & 5.57 & 5.60 & 5.66 & 3.30 & 6.74 & 7.20 & 6.47 & 4.88 & 3.99 & 2014.3 & 5.5 \\
\hline 70.0 & 3.85 & 4.45 & 4.94 & 4.98 & 4.81 & 4.76 & 5.36 & 6.00 & 6.74 & 6.28 & 4.86 & 3.99 & 1856.9 & 5.1 \\
\hline 80.0 & 3.71 & 4.18 & 4.48 & 4.28 & 3.96 & 3.86 & 4.35 & 5.12 & 6.10 & 5.93 & 4.70 & 3.90 & 1660.5 & 4.5 \\
\hline 90.0 & 3.49 & 3.82 & 3.92 & 3.50 & 3.08 & 2.90 & 3.32 & 4.13 & 5.31 & 5.43 & $4 . \dot{4} 3$ & 3.70 & 1430.1 & 3.9 \\
\hline
\end{tabular}


AVERAGE DAILY TOTAL TERRESTRIAL INSOLATION ESTIMATES (KWH/SQ. M)

SITE: YUCCA FLATS
LATITUDE:
36 DEGREES 57 MINUTES

AVERAGE DAILY AMOUNTS BY MONTH

\begin{tabular}{|c|c|c|c|c|c|c|c|c|c|c|c|c|c|c|}
\hline ARRAY & $\mathbf{J}$ & $F$ & $M$ & A & $M$ & $\mathrm{~J}$ & $\mathrm{~J}$ & $A$ & 5 & 0 & $N$ & D & $\begin{array}{l}\text { ANNUAL } \\
\text { AMOUNT }\end{array}$ & $\begin{array}{c}\text { AVERAGE } \\
\text { DAY }\end{array}$ \\
\hline 0.0 & 3.00 & 4.01 & 5.56 & 7.09 & 8.12 & 8.62 & 8.36 & 7.51 & 6.38 & 4.77 & 3.28 & 2.69 & 2114.0 & 5.8 \\
\hline 10.0 & 3.63 & 4.57 & 6.03 & 7.35 & 8.17 & 8.58 & 8.41 & 7.80 & 6.97 & 5.54 & 4.02 & 3.38 & 2267.4 & 6.2 \\
\hline 15.0 & 3.92 & 4.80 & 6.21 & 7.41 & 8.12 & 8.48 & $8, .36$ & 7.88 & 7.20 & 5.87 & 4.36 & 3.69 & 2323.4 & 6.4 \\
\hline 20.0 & 4.17 & 5.01 & 6.36 & 7.43 & 8.02 & 8.33 & 8.26 & 7.90 & 7.39 & 6.16 & 4.66 & 3.98 & 2365.0 & 6.5 \\
\hline 25.0 & 4.41 & 5.19 & 6.46 & 7.40 & 7.87 & 8.12 & 8.10 & 7.88 & 7.53 & 6.41 & 4.94 & 4.24 & 2391.8 & 6.6 \\
\hline 30.0 & 4.61 & 5.33 & 6.53 & 7.33 & 7.68 & 7.87 & 7.90 & 7.80 & 7.62 & 6.63 & 5.18 & 4.48 & 24.03 .7 & 6.6 \\
\hline 35.0 & 4.79 & 5.44 & 6.55 & 7.21 & 7.44 & 7.57 & 7.64 & 7.68 & 7.66 & 6.79 & 5.39 & 4.69 & 2400.5 & 6.6 \\
\hline 40.0 & 4.93 & 5.52 & 6.54 & 7.06 & 7.17 & 7.25 & 7.36 & 7.51 & 7.65 & 6.92 & 5.57 & 4.86 & 2384.2 & 6.5 \\
\hline 45.0 & 5.04 & 5.56 & 6.48 & 6.86 & 6.86 & 6.89 & 7.04 & 7.31 & 7.59 & 6.99 & 5.70 & 5.01 & 2354.2 & 6.4 \\
\hline 50.0 & 5.12 & 5.57 & 6.39 & 6.63 & i. 52 & 6.50 & 6.68 & 7.05 & 7.49 & 7.03 & 5.80 & 5.12 & 2309.6 & 6.3 \\
\hline 60.0 & 5.18 & 5.48 & 6.08 & 6.04 & 5.72 & 5.60 & 5.85 & 6.42 & 7.13 & 6.95 & 5.89 & 5.23 & 2177.7 & 6.0 \\
\hline 70.0 & 5.10 & 5.26 & 5.63 & 5.32 & 4.80 & 4.60 & 4.88 & 5.63 & 6.60 & 6.69 & 5.81 & 5.21 & 1993.5 & 5.5 \\
\hline 80.0 & 4.89 & 4.90 & 5.04 & 4.48 & 3.85 & 3.61 & 3.90 & 4.72 & 5.89 & 6.26 & 5.58 & 5.05 & 1769.2 & 4.8 \\
\hline 90.0 & 4.56 & 4.43 & 4.34 & 3.56 & 2.86 & 2.61 & 2.87 & 3.73 & 5.05 & 5.66 & 5.20 & 4.76 & 1508.2 & 4.1 \\
\hline
\end{tabular}


AUERAGE DAILY TOTAL TERRESTRIAL INSOLATION ESTIMATES (KWH/SQ. M)

SITE:
LATITUDE:
42 DEG NY

AVERAGE DAILY AMOUNTS BY MONTH

\begin{tabular}{|c|c|c|c|c|c|c|c|c|c|c|c|c|c|c|}
\hline ARRAY & $J$ & $F$ & $M$ & A & $M$ & 3 & $\mathrm{~J}$ & $A$ & $s$ & 0 & $N$ & $\mathrm{D}$ & $\begin{array}{l}\text { ANNUAL } \\
\text { AMOUNT }\end{array}$ & $\begin{array}{c}\text { AVERAGE } \\
\text { DAY }\end{array}$ \\
\hline 0.0 & 1.44 & 2.17 & 3.10 & 4.21 & 4.94 & 5.46 & 5.44 & 4.73 & 3.69 & 2.57 & $\cdot 1.44$ & 1.12 & 1228.4 & 3.4 \\
\hline 10.0 & 1.71 & 2.43 & 3.34 & 4.36 & 5.01 & 5.47 & 5.51 & 4.92 & 4.00 & 2.94 & 1.71 & 1.35 & 1302.2 & 3.6 \\
\hline 15.0 & 1.83 & 2.55 & 3.43 & 4.40 & 5.00 & 5.44 & 5.50 & 4.97 & 4.12 & 3.10 & 1.84 & 1.46 & 1328.9 & 3.6 \\
\hline 20.0 & 1.94 & 2.65 & 3.50 & 4.42 & 4.96 & 5.38 & 5.46 & 5.00 & 4.22 & 3.24 & 1.95 & 1.56 & 1348.4 & 3.7 \\
\hline 25.0 & 2.03 & 2.73 & 3.56 & 4.42 & 4.90 & 5.29 & 5.40 & 4.99 & 4.29 & 3.36 & 2.05 & 1.64 & 1360.5 & 3.7 \\
\hline 30.0 & 2.12 & 2.80 & 3.59 & 4.39 & 4.82 & 5.18 & 5.30 & 4.97 & 4.34 & 3.46 & 2.14 & 1.72 & 1365.3 & 3.7 \\
\hline 35.0 & 2.20 & 2.86 & 3.60 & 4.34 & 4.71 & 5.04 & 5.18 & 4.91 & 4.37 & 3.55 & 2.21 & 1.79 & 1362.5 & 3.7 \\
\hline 40.0 & 2.26 & 2.89 & 3.60 & 4.26 & 4.57 & 4.87 & 5.02 & 4.83 & 4.37 & 3.61 & 2.28 & 1.85 & 1352.3 & 3.7 \\
\hline 45.0 & 2.31 & 2.92 & 3.58 & 4.17 & 4.42 & 4.68 & 4.85 & 4.72 & 4.35 & 3.65 & 2.33 & 1.90 & 1335.4 & 3.7 \\
\hline 50.0 & 2.34 & 2.92 & 3.53 & 4.05 & 4.25 & 4.48 & 4.66 & 4.59 & 4.30 & 3.67 & 2.36 & 1.94 & 1312.2 & 3.6 \\
\hline 60.0 & 2.37 & 2.88 & 3.39 & 3.76 & 3.85 & 4.02 & 4.22 & 4.26 & 4.13 & 3.64 & 2.39 & 1.97 & 1244.7 & 3.4 \\
\hline 70.0 & 2.34 & 2.78 & 3.17 & 3.40 & 3.39 & 3.49 & 3.69 & 3.84 & 3.87 & 3.52 & 2.36 & 1.96 & 1151.0 & 3.2 \\
\hline 80.0 & 2.26 & 2.61 & 2.89 & 2.97 & 2.87 & 2.93 & 3.12 & 3.35 & 3.52 & 3.32 & 2.28 & 1.91 & 1035.6 & 2.8 \\
\hline 90.0 & 2.12 & 2.39 & 2.55 & 2.50 & 2.35 & 2.35 & 2.53 & 2.80 & 3.10 & 3.05 & 2.14 & 1.80 & 903.2 & 2.5 \\
\hline
\end{tabular}


AVERAGE DAILY TOTAL TERRESTRIAL INSOLATION ESTIMATES (KWHISQ. M)

SITE: BINGHAMTON
ITY
ITUE: 42 DEGREES 13 MINUTES

EVERAGE DAILY AMOUNTS BY MONTH

\begin{tabular}{|c|c|c|c|c|c|c|c|c|c|c|c|c|c|c|}
\hline ARRAY & $J$ & $F$ & $M$ & A & $M$ & $J$ & (2. & trom & 5 & 0 & $M$ & D & $\begin{array}{l}\text { ANNUAL } \\
\text { AMOUNT }\end{array}$ & $\begin{array}{c}\text { AVERAGE } \\
\text { DAY }\end{array}$ \\
\hline 0.0 & 1.22 & ]. 82 & 2.71 & 3.92 & 4.71 & 5.30 & 5.23 & 4.49 & 3.56 & 2.46 & 1.31 & 0.94 & 1147.8 & 3.1 \\
\hline 10.0 & 1.48 & 1.99 & 2.89 & 4.04 & 4.77 & 5.31 & 5.29 & 4.66 & 3.84 & 2.78 & 1.52 & 1.16 & 1210.9 & 3.3 \\
\hline 15.0 & 1.60 & 2.06 & 2.95 & 4.08 & 4.75 & 5.28 & 5.28 & 4.70 & 3.95 & 2.93 & 1.61 & 1.26 & 1233.1 & 3.4 \\
\hline 20.0 & 1.70 & 2.13 & 3.00 & 4.09 & 4.72 & 5.22 & 5.24 & 4.72 & 4.04 & 3.05 & 1.70 & 1.36 & 1248.7 & 3.4 \\
\hline 25.0 & $1.80^{\circ}$ & 2.18 & 3.04 & 4.08 & 4.66 & 5.14 & 5.17 & 4.72 & 4.11 & 3.16 & 1.77 & 1.44 & 1257.6 & 3.4 \\
\hline 30.0 & 1.89 & 2.22 & 3.06 & 4.05 & 4.58 & 5.02 & 5.08 & 4.69 & 4.15 & 3.25 & 1.84 & 1.52 & 1259.7 & 3.5 \\
\hline 35.0 & 1.97 & 2.25 & 3.06 & 4.00 & 4.47 & 4.88 & 4.96 & 4.63 & 4.18 & 3.32 & 1.89 & 1.59 & 1255.1 & 3.4 \\
\hline 40.0 & 2.03 & 2.26 & 3.05 & 3.93 & 4.34 & 4.72 & 4.81. & 4.55 & 4.17 & 3.37 & 1.94 & 1.65 & 1243.7 & 3.4 \\
\hline 45.0 & 2.08 & 2.27 & 3.02 & 3.84 & 4.19 & 4.54 & 4.65 & 4.45 & 4.15 & 3.40 & 1.97 & 1.70 & 1226.4 & 3.4 \\
\hline 50.0 & 2.12 & 2.26 & 2.98 & 3.73 & 4.03 & 4.34 & 4.46 & 4.32 & 4.10 & 3.42 & 2.00 & 1.73 & 1203.3 & 3.3 \\
\hline 60.0 & 2.15 & 2.22 & 2.85 & 3.46 & 3.66 & 3.89 & 4.04 & 4.01 & 3.93 & 3.38 & 2.01 & 1.78 & 1138.2 & 3.1 \\
\hline 70.0 & 2.14 & 2.12 & 2.66 & 3.13 & 3.21 & 3.37 & 3.53 & 3.61 & 3.67 & 3.26 & 1.97 & 1.78 & 1049.8 & 2.9 \\
\hline 80.0 & 2.06 & 1.99 & 2.42 & 2.74 & 2.73 & 2.84 & 2.98 & 3.15 & 3.34 & 3.07 & 1.90 & 1.73 & 942.4 & 2.6 \\
\hline 90.0 & 1.94 & 1.81 & 2.13 & 2.30 & 2.24 & 2.28 & 2.42 & 2.63 & 2.94 & 2.81 & 1.77 & 1.64 & 819.9 & 2.2 \\
\hline
\end{tabular}


AVERAGE DAILY TOTAL TERRESTRIAL INSOLATION ESTIMATES (KWH/SQ. M)

$\begin{aligned} \text { SITE: } & \text { BUFFALO } \\ \text { LATITUDE: } & 42 \text { DEGREES } 56 \text { MINUTES }\end{aligned}$

AYERAGE DAILY AMOUNTS BY MONTH

\begin{tabular}{|c|c|c|c|c|c|c|c|c|c|c|c|c|c|c|}
\hline ARRAY & J & $F$ & $M$ & A & $M$ & J & $J$ & A & s & 0 & $N$ & D & $\begin{array}{l}\text { ANNUAL } \\
\text { AMOUNT }\end{array}$ & $\begin{array}{c}\text { AVERAGE } \\
\text { DAY }\end{array}$ \\
\hline 0.0 & 1.10 & 1.72 & 2.30 & 4.14 & 5.03 & 5.59 & 5.60 & 4.77 & 3.63 & 2.47 & 1.27 & 0.89 & 1193.1 & 3.3 \\
\hline 10.0 & 1.33 & 1.96 & 2.99 & 4.29 & 5.10 & 5.71 & 5.68 & 4.96 & 3.93 & 2.81 & 1.49 & 1.11 & 1261.3 & 3.5 \\
\hline 15.0 & 1.43 & 2.07 & 3.07 & 4.33 & 5.09 & 5.68 & 5.67 & 5.02 & 4.05 & 2.96 & 1.58 & 1.21 & 1285.4 & 3.5 \\
\hline 20.0 & 1.53 & 2.16 & 3.12 & 4.35 & 5.06 & 5.02 & 5.64 & 5.05 & 4.15 & 3.10 & 1.67 & 1.30 & 1302.7 & 3.6 \\
\hline 25.0 & 1.61 & 2.24 & 3.17 & 4.35 & 5.00 & 5.53 & 5.57 & 5.05 & 4.23 & 3.21 & 1.75 & 1.39 & 1312.8 & 3.6 \\
\hline 30.0 & 1.69 & 2.30 & 3.19 & 4.32 & 4.91 & 5.41 & 5.47 & 5.02 & 4.28 & 3.31 & 1.82 & 1.46 & 1315.7 & 3.6 \\
\hline 35.0 & 1.75 & 2.36 & 3.20 & 4.27 & 4.80 & 5.26 & 5.34 & 4.96 & 4.30 & 3.39 & 1.87 & 1.53 & 1311.4 & 3.6 \\
\hline 40.0 & 1.81 & 2.40 & 3.19 & 4.20 & 4.66 & 5.38 & 5.19 & 4.88 & 4.30 & 3.44 & 1.92 & 1.59 & 1299.9 & 3.6 \\
\hline 45.0 & 1.86 & 2.42 & 3.17 & 4.10 & 4.50 & 4.89 & 5.01 & 4.77 & 4.28 & 3.48 & 1.96 & 1.64 & 1282.0 & 3.5 \\
\hline 50.0 & 1.89 & 2.43 & 3.13 & 3.99 & 4.33 & 4.58 & 4.82 & 4.65 & 4.23 & 3.50 & 1.98 & 1.67 & 1258.2 & 3.4 \\
\hline 60.0 & 1.92 & 2.41 & 2.99 & 3.71 & 3.93 & 4.20 & 4.36 & 4.31 & 4.07 & 3.47 & 2.00 & 1.72 & 1190.3 & 3.3 \\
\hline 70.0 & 1.90 & 2.33 & 2.80 & 3.35 & 3.45 & 3.53 & 3.81 & 3.89 & 3.81 & 3.36 & 1.97 & 1.72 & 1097.5 & 3.0 \\
\hline 80.0 & 1.84 & 2.20 & 2.55 & 2.94 & 2.93 & 3.35 & 3.22 & 3.40 & 3.47 & 3.17 & 1.89 & 1.68 & 984.2 & 2.7 \\
\hline 90.0 & 1.73 & 2.02 & 2.26 & 2.47 & 2.39 & 2.44 & 2.61 & 2.84 & 3.06 & 2.90 & 1.78 & 1.59 & 855.0 & 2.3 \\
\hline
\end{tabular}


AVERAGE DAILY TOTAL TERRESTRIAL INSOLATION ESTIMATES (KWH/SQ. M)

SITE: MASSENA

LATITUDE: 44 DEGREES 56 MINUTES

AVERAGE DAILY AMOUNTS BY MONTH

\begin{tabular}{|c|c|c|c|c|c|c|c|c|c|c|c|c|c|c|}
\hline $\begin{array}{l}\text { ARRAY } \\
\text { TILT }\end{array}$ & $J$ & $F$ & $M$ & A & $M$ & $J$ & J & A & $s$ & 0 & $N$ & D & $\begin{array}{l}\text { ANNUAL } \\
\text { AMOUNT }\end{array}$ & $\begin{array}{l}\text { AVERAGE } \\
\text { DAY }\end{array}$ \\
\hline 0.0 & 1.24 & 1.96 & 3.08 & 4.24 & 5.09 & 5.61 & 5.52 & 4.68 & 3.54 & 2.32 & 1.22 & 0.93 & 1201.3 & 3.3 \\
\hline 10.0 & 1.47 & 2.21 & 3.34 & 4.41 & 5.17 & 5.64 & 5.61 & 4.89 & 3.86 & 2.67 & 1.46 & 1.12 & 1275.5 & 3.5 \\
\hline 15.0 & 1.58 & 2.31 & 3.44 & 4.47 & 5.18 & 5.62 & 5.61 & 4.96 & 3.99 & 2.82 & 1.57 & 1.21 & 1302.7 & 3.6 \\
\hline 20.0 & 1.68 & 2.41 & 3.52 & 4.49 & 5.15 & 5.57 & 5.59 & 4.99 & 4.09 & 2.96 & 1.67 & 1.29 & 1322.9 & 3.6 \\
\hline 25.0 & 1.77 & 2.49 & 3.59 & 4.50 & 5.10 & 5.49 & 5.53 & 5.00 & 4.18 & 3.08 & 1.76 & 1.37 & 1335.9 & 3.7 \\
\hline 30.0 & 1.85 & 2.56 & 3.63 & 4.48 & 5.02 & 5.38 & 5.44 & 4.98 & 4.24 & 3.18 & 1.84 & 1.44 & 1341.6 & 3.7 \\
\hline 35.0 & 1.92 & 2.62 & 3.66 & 4.44 & 4.91 & 5.24 & 5.32 & 4.94 & 4.27 & 3.26 & 1.90 & 1.50 & 1339.9 & 3.7 \\
\hline 40.0 & 1.98 & 2.65 & 3.66 & 4.37 & 4.78 & 5.08 & 5.18 & 4.87 & 4.29 & 3.33 & 1.96 & 1.55 & 1330.8 & 3.6 \\
\hline 45.0 & 2.03 & 2.68 & 3.65 & 4.28 & 4.62 & 4.89 & 5.01 & 4.77 & 4.27 & 3.37 & $2: 101$ & 1.59 & 1314.5 & 3.6 \\
\hline 50.0 & 2.06 & 2.69 & 3.61 & 4.17 & 4.46 & 4.69 & 4.82 & 4.65 & 4.24 & 3.40 & 2.104 & 1.62 & 1292.7 & 3.5 \\
\hline 60.0 & 2.09 & 2.66 & 3.48 & 3.89 & 4.06 & 4.23 & 4.39 & 4.34 & 4.09 & 3.38 & 2.07 & 1.66 & 1228.3 & 3.4 \\
\hline 70.0 & 2.07 & 2.58 & 3.28 & 3.53 & 3.59 & 3.69 & 3.86 & 3.93 & 3.85 & 3.29 & 2.06 & 1.65 & 1137.9 & 3.1 \\
\hline 80.0 & 2.01 & 2.43 & 3.00 & 3.11 & 3.05 & 3.11 & 3.27 & 3.45 & 3.52 & 3.12 & 1.99 & 1.61 & 1025.0 & 2.8 \\
\hline 90.0 & 1.89 & 2.24 & 2.67 & 2.63 & 2.51 & 2.52 & 2.68 & 2.91 & 3.12 & 2.87 & 1.88 & 1.53 & 896.1 & 2.5 \\
\hline
\end{tabular}


AVERAGE DAILY TOTAL TERRESTRIAL INSOLATION ESTIMATES (KWH/SQ. M)

SITE: CENTRAL PARK NY NY
LATITUDE: 40 DEGREES 47 MINUTES

AVERAGE [AILY AMOLNTS BY MONTH

\begin{tabular}{|c|c|c|c|c|c|c|c|c|c|c|c|c|c|c|}
\hline ARRAY & $\frac{-\pi-}{2}$ & $F$ & $M$ & A & $M$ & 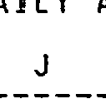 & J & trum & $S$ & 0 & $N$ & $D$ & $\begin{array}{l}\text { ANNUAL } \\
\text { AMOUNT }\end{array}$ & $\begin{array}{c}\text { AVERAGE } \\
\text { DAY }\end{array}$ \\
\hline 0.0 & 1.58 & 2.27 & 3.26 & 4.30 & 5.16 & 5.39 & 5.32 & 4.68 & 3.82 & 2.82 & 1.68 & 1.28 & 1266.8 & 3.5 \\
\hline 10.0 & 1.85 & 2.53 & 3.49 & 4.44 & 5.21 & 5.40 & 5.37 & 4.84 & 4.12 & 3.20 & 1.99 & 1.52 & 1339.5 & 3.7 \\
\hline 15.0 & 1.97 & 2.64 & 3.58 & 4.48 & 5.20 & 5.36 & 5.35 & 4.89 & 4.23 & 3.37 & 2.13 & 1.64 & 1365.3 & 3.7 \\
\hline 20.0 & 2.08 & 2.73 & 3.65 & 4.49 & 5.15 & 5.29 & 5.30 & 4.90 & 4.33 & 3.51 & 2.25 & 1.74 & 1383.8 & 3.8 \\
\hline 25.0 & 2.18 & 2.81 & 3.70 & 4.48 & 5.08 & 5.19 & 5.23 & 4.89 & 4.39 & 3.64 & 2.37 & 1.83 & 1394.7 & 3.8 \\
\hline 30.0 & 2.26 & 2.88 & 3.73 & 4.44 & 4.98 & 5.07 & 5.13 & 4.85 & 4.43 & 3.74 & 2.47 & 1.92 & 1397.9 & 3.8 \\
\hline 35.0 & 2.34 & 2.92 & 3.74 & 4.3 .8 & 4.86 & 4.92 & 5.00 & 4.79 & 4.45 & 3.82 & 2.55 & 1.99 & 1393.5 & 3.8 \\
\hline 40.0 & 2.40 & 2.95 & 3.73 & 4.30 & 4.71 & 4.75 & 4.84 & 4.70 & 4.44 & 3.88 & 2.62 & 2.05 & 1381.5 & 3.8 \\
\hline 45.0 & 2.44 & 2.97 & 3.70 & 4.20 & 4.55 & 4.57 & 4.67 & 4.59 & 4.41 & 3.91 & 2.68 & 2.10 & 1363.3 & 3.7 \\
\hline 50.0 & 2.47 & 2.97 & 3.64 & 4.07 & 4.36 & 4.36 & 9.48 & 4.45 & 4.35 & 3.93 & 2.71 & 2.13 & 1338.0 & 3.7 \\
\hline 60.0 & 2.49 & 2.92 & 3.48 & 3.77 & 3.93 & 3.89 & 9.04 & 4.12 & 4.16 & 3.88 & 2.74 & 2.17 & 1265.9 & 3.5 \\
\hline 70.0 & 2.45 & 2.80 & 3.25 & 3.59 & 3.43 & 3.36 & 3.52 & 3.70 & 3.88 & 3.74 & 2.70 & 2.15 & 1167.3 & 3.2 \\
\hline 80.0 & 2.35 & 2.62 & 2.94 & 2.95 & 2.89 & 2.81 & 2.96 & 3.20 & 3.52 & 3.52 & $2 . \in 0$ & 2.08 & 1047.8 & 2.9 \\
\hline 90.0 & 2.20 & 2.39 & 2.58 & 2.46 & 2.34 & 2.24 & 2.38 & $2.66^{\circ}$ & 3.08 & 3.21 & 2.43 & 1.96 & 910.2 & 2.5 \\
\hline
\end{tabular}


AVERAGE DAILY TOTAL TERRESTRIAL INSOLATION ESTIMATES (KLH/SQ. M)

SITE:
LATITUDE: 40 GUARDIA

AVERAGE DAILY AMOUNTS BY MONTH

\begin{tabular}{|c|c|c|c|c|c|c|c|c|c|c|c|c|c|c|}
\hline ARRAY & $J$ & $F$ & $M$ & A & $M$ & $J$ & $J$ & ( & $S$ & 0 & $\checkmark$ & D & $\begin{array}{l}\text { ANNUAL } \\
\text { AMOUNT }\end{array}$ & $\begin{array}{c}\text { AVERAGE } \\
\text { DAY }\end{array}$ \\
\hline 0.0 & 1.73 & 2.51 & 3.52 & 4.60 & 5.33 & 5.69 & 5.62 & 4.99 & 4.03 & 3.00 & 1.87 & 1.44 & 1350.1 & 3.7 \\
\hline 10.0 & 2.05 & 2.81 & 3.78 & 4.75 & 5.38 & 5.69 & 5.68 & 5.17 & 4.36 & 3.41 & 2.24 & 1.76 & 1434.0 & 3.9 \\
\hline 15.0 & 2.19 & 2.94 & 3.88 & 4.79 & 5.37 & 5.65 & 5.66 & 5.22 & 4.48 & 3.59 & 2.41 & 1.90 & 1464.4 & 4.0 \\
\hline 20.0 & $2: 32$ & 3.06 & 3.96 & 4.81 & 5.32 & 5.58 & 5.61 & 5.24 & 4.58 & 3.75 & 2.56 & 2.03 & 1486.8 & 4.1 \\
\hline 25.0 & 2.44 & $3: 15$ & 4.02 & 4.80 & 5.25 & 5.48 & 5.53 & 5.23 & 4.66 & 3.89 & 2.70 & 2.15 & 1501.0 & 4.1 \\
\hline 30.0 & 2.54 & 3.23 & 4.05 & 4.76 & 5.15 & 5.35 & 5.42 & 5.19 & 4.70 & 4.01 & 2.82 & 2.26 & 1506.7 & 4.1 \\
\hline 35.0 & 2.63 & 3.29 & 4.07 & 4.69 & 5.02 & 5.19 & 5.29 & 5.12 & 4.73 & 4.10 & 2.93 & 2.36 & 1504.1 & 4.1 \\
\hline 40.0 & 2.70 & 3.34 & 4.06 & 4.61 & 4.86 & 5.00 & 5.12 & 5.03 & 4.72 & 4.16 & 3.01 & 2.44 & 1493.1 & 4.1 \\
\hline 45.0 & 2.76 & 3.36 & 4.02 & 4.50 & 4.69 & 4.81 & 4.94 & 4.91 & 4.69 & 4.21 & 3.08 & 2.50 & 1475.3 & 4.0 \\
\hline 50.0 & 2.80 & 3.36 & 3.97 & 4.37 & 4.50 & 4.59 & 4.74 & 4.76 & 4.63 & 4.22 & 3.13 & 2.55 & 1449.5 & 4.0 \\
\hline 60.0 & 2.83 & 3.31 & 3.80 & 4.04 & 4.06 & 4.09 & 4.26 & 4.40 & 4.43 & 4.18 & 3.17 & 2.60 & 1374.3 & 3.8 \\
\hline 70.0 & 2.79 & 3.18 & 3.54 & 3.62 & 3.54 & 3.52 & 3.70 & 3.95 & 4.13 & 4.03 & 3.13 & 2.59 & 1269.7 & 3.5 \\
\hline 80.0 & 2.68 & 2.98 & 3.21 & 3.15 & 2.98 & 2.94 & 3.11 & 3.42 & 3.74 & 3.79 & 3.02 & 2.51 & 1141.4 & 3.1 \\
\hline 90.0 & 2.51 & 2.72 & 2.82 & 2.62 & 2.40 & 2.33 & 2.49 & 2.83 & 3.27 & 3.46 & 2.83 & 2.37 & 992.7 & 2.7 \\
\hline
\end{tabular}


AVERAGE DAILY TOTAL TERRESTRIAL INSOLATION ESTIMATES (KWH/S?. M)

SITE: ROCHESTER
LATITUDE:

AVERAGE DAILY AMOUNTS BY MONTH

\begin{tabular}{|c|c|c|c|c|c|c|c|c|c|c|c|c|c|c|}
\hline ARRAY & J & $F$ & $M$ & A & $M$ & J & $J$ & rivi & $s$ & 0 & $N$ & D : & $\begin{array}{l}\text { ANNUAL } \\
\text { ANIOUNT }\end{array}$ & $\begin{array}{c}\text { AVERAGE } \\
\text { DAY }\end{array}$ \\
\hline 0.0 & 1.15 & 1.77 & 2.85 & 4.23 & 5.07 & 5.73 & 5.61 & 4.80 & 3.65 & 2.46 & 1.23 & 0.89 & 1203.1 & 3.3 \\
\hline 10.0 & 1.40 & 1.94 & 3.05 & 4.33 & 5.13 & 5.75 & 5.69 & 4.99 & 3.96 & 2.81 & 1.49 & 1.10 & 1271.3 & 3.5 \\
\hline 15.0 & 1.51 & 2.01 & 3.12 & 4.43 & 5.13 & 5.72 & 5.69 & 5.05 & 4.08 & 2.96 & 1.59 & 1.20 & 1295.4 & 3.5 \\
\hline 20.0 & 1.62 & 2.08 & 3.19 & 4.45 & 5.09 & 5.66 & 5.65 & 5.08 & 4.18 & 3.09 & 1.63 & 1.29 & 1312.5 & 3.6 \\
\hline 25.0 & 1.71 & 2.13 & 3.23 & 4.44 & 5.03 & 5.57 & 5.58 & 5.08 & 4.26 & 3.21 & 1.76 & 1.38 & 1322.5 & 3.6 \\
\hline 30.0 & 1.80 & 2.17 & 3.26 & 4.42 & 4.95 & 5.45 & 5.49 & 5.05 & 4.31 & 3.31 & 1.83 & 1.45 & 1325.3 & 3.6 \\
\hline 35.0 & 1.87 & 2.20 & 3.27 & 4.37 & 4.83 & 5.30 & 5.36 & 5.00 & 4.34 & 3.39 & 1.89 & 1.52 & 1320.8 & 3.6 \\
\hline 40.0 & 1.94 & 2.22 & 3.26 & 4.29 & 4.70 & 5.12 & 5.20 & 4.91 & 4.34 & 3.44 & 1.94 & 1.58 & 1309.1 & 3.6 \\
\hline 45.0 & 1.99 & 2.23 & 3.24 & 4.20 & 4.54 & 4.93 & 5.03 & 4.81 & 4.32 & 3.48 & 1.98 & 1.63 & 1290.9 & 3.5 \\
\hline 50.0 & 2.02 & 2.22 & 3.20 & 4.09 & 4.37 & 4.72 & 4.83 & 4.68 & 4.27 & 3.50 & 2.00 & 1.67 & 1266.8 & 3.5 \\
\hline 60.0 & 2.06 & 2.18 & 3.06 & 3.80 & 3.96 & 4.23 & 4.37 & 4.35 & 4.11 & 3.47 & 2.02 & 1.71 & 1198.4 & 3.3 \\
\hline 70.0 & 2.05 & 2.09 & 2.87 & 3.43 & 3.48 & 3.67 & 3.83 & 3.92 & 3.85 & 3.36 & 1.99 & 1.71 & 1104.8 & 3.0 \\
\hline 80.0 & 1.98 & 1.96 & 2.62 & 3.01 & 2.95 & 3.07 & 3.23 & 3.42 & 3.51 & 3.17 & 1.92 & 1.67 & 990.7 & 2.7 \\
\hline 90.0 & 1.87 & 1.79 & 2.31 & 2.53 & 2.42 & 2.46 & 2.62 & 2.86 & 3.09 & 2.91 & 1.80 & 1.59 & 860.8 & 2.4 \\
\hline
\end{tabular}


AVERAGE DAIL TOTAL TERRESTRIAL INSOLATION ESTIMATES (KWH/SQ. M)

$\begin{aligned} \text { SITE: } & \text { SYRACUSE } \\ \text { LATITUDE: } & 43 \text { DEGREES } 7 \text { MINUTES }\end{aligned}$

AUERAGE DAILY AMOUNTS BY MONTH

\begin{tabular}{|c|c|c|c|c|c|c|c|c|c|c|c|c|c|c|}
\hline ARRAY & $J$ & $F$ & $M$ & A & $M$ & $J$ & $\mathrm{~J}$ & A & 5 & 0 & $N$ & D & $\begin{array}{l}\text { ANNUAL } \\
\text { AMOUNT }\end{array}$ & $\begin{array}{c}\text { AVERAGE } \\
\text { DAY }\end{array}$ \\
\hline 0.0 & 1.22 & 1.80 & 2.80 & 4.18 & 4.93 & 5.61 & 5.55 & 4.75 & 3.67 & 2.45 & 1.26 & 0.90 & 1193.6 & 3.3 \\
\hline 10.0 & 1.41 & 1.98 & 2.99 & 4.33 & 5.04 & 5.63 & 5.62 & 4.94 & 3.99 & 2.80 & 1.47 & 1.12 & 1259.6 & 3.5 \\
\hline 15.0 & 1.50 & 2.06 & 3.07 & 4.37 & 5.03 & 5.60 & 5.62 & 4.99 & 4.11 & 2.95 & 1.57 & 1.22 & 1282.8 & 3.5 \\
\hline 20.0 & 1.57 & 2.13 & 3.13 & 4.39 & 5.00 & 5.54 & 5.58 & 5.02 & 4.21 & 3.08 & 1.65 & 1.32 & 1299.2 & 3.6 \\
\hline 25.0 & 1.64 & 2.18 & 3.17 & 4.39 & 4.94 & 5.45 & 5.51 & 5.02 & 4.29 & 3.19 & 1.73 & 1.40 & 1308.5 & 3.6 \\
\hline 30.0 & 1.70 & 2.23 & 3.20 & 4.36 & 4.86 & 5.33 & 5.42 & 5.00 & 4.34 & 3.29 & 1.80 & 1.48 & 1310.8 & 3.6 \\
\hline 35.0 & 1.76 & 2.26 & 3.21 & 4.31 & 4.75 & 5.19 & 5.29 & 4.94 & 4.37 & 3.37 & 1.86 & 1.55 & 1305.8 & 3.6 \\
\hline 40.0 & 1.80 & 2.28 & $3.20^{-}$ & 4.24 & 4.61 & 5.02 & 5.14 & 4.86 & 4.37 & 3.43 & 1.90 & 1.61 & 1293.9 & 3.5 \\
\hline 45.0 & 1.83 & 2.29 & 3.17 & 4.15 & 4.46 & 4.83 & 4.97 & 4.76 & 4.35 & 3.46 & 1.94 & 1.66 & 1275.5 & 3.5 \\
\hline 50.0 & 1.85 & $\approx .29$ & 3.13 & 4.03 & 4.29 & 4.62 & 4.77 & 4.63 & 4.30 & 3.48 & 1.97 & 1.70 & 1251.3 & 3.4 \\
\hline 60.0 & 1.86 & 2.24 & 3.00 & 3.75 & 3.89 & 4.15 & 4.32 & 4.30 & 4.14 & 3.45 & 1.98 & 1.75 & 1183.1 & 3.2 \\
\hline 70.0 & 1.83 & 2.15 & 2.81 & 3.39 & 3.42 & 3.60 & 3.79 & 3.88 & 3.88 & 3.34 & 1.95 & 1.75 & 1090.4 & 3.0 \\
\hline 80.0 & 1.76 & 2.02 & 2.56 & 2.97 & 2.90 & 3.02 & 3.19 & 3.39 & 3.54 & 3.16 & 1.88 & 1.71 & 977.5 & 2.7 \\
\hline 90.0 & 1.65 & 1.84 & 2.27 & 2.50 & 2.38 & 2.42 & 2.60 & 2.83 & 3.12 & 2.89 & 1.76 & 1.62 & 849.2 & 2.3 \\
\hline
\end{tabular}


AVERAGE DAILY TOTAL TERRESTRIAL INSOLATION ESTIMATES (KWH/SQ. M)

SITE: AKRON-CANTON

LATITUDE: 40 DEGREES 55 MINUTES

AVERAGE DAILY AMOUNTS BY MONTH

\begin{tabular}{|c|c|c|c|c|c|c|c|c|c|c|c|c|c|c|}
\hline $\begin{array}{l}\text { ARRAY } \\
\text { TILT }\end{array}$ & $J$ & $F$ & $M$ & A & $M$ & $J$ & 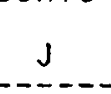 & A & 5 & 0 & $N$ & $D$ & $\begin{array}{l}\text { ANNUAL } \\
\text { AMOUNT }\end{array}$ & $\begin{array}{c}\text { AVERAGE } \\
\text { DAY }\end{array}$ \\
\hline 0.0 & 1.35 & 2.05 & 3.03 & 4.23 & 5.26 & 5.8 .0 & 5.63 & 5.04 & 4.01 & 2.86 & 1.59 & 1.11 & 1280.7 & 3.5 \\
\hline 10.0 & 1.55 & 2.26 & 3.23 & 4.42 & 5.32 & 5.81 & 5.69 & 5.22 & 4.33 & 3.25 & 1.87 & 1.30 & 1348.5 & 3.7 \\
\hline 15.0 & 1.64 & 2.34 & 3.31 & 4.46 & 5.30 & 5.77 & 5.67 & 5.27 & 4.45 & 3.42 & 2.00 & 1.39 & 1371.8 & 3.8 \\
\hline 20.0 & 1.71 & 2.42 & 3.37 & $4 .<7$ & 5.26 & 5.70 & 5.62 & 5.29 & 4.56 & 3.57 & 2.11 & 1.46 & 1387.6 & 3.8 \\
\hline 25.0 & 1.78 & 2.48 & 3.41 & $4 .<5$ & 5.18 & 5.59 & 5.55 & 5.28 & 4.63 & 3.70 & 2.22 & 1.53 & 1395.9 & 3.8 \\
\hline 30.0 & 1.84 & 2.53 & 3.43 & 4.42 & 5.08 & 5.46 & 5.44 & 5.25 & 4.68 & 3.80 & 2.31 & 1.59 & 1396.5 & 3.8 \\
\hline 35.0 & 1.89 & 2.57 & 3.44 & 4.36 & 7.96 & 5.30 & 5.30 & 5.18 & 4.70 & 3.89 & 2.38 & 1.65 & 1389.5 & 3.8 \\
\hline 40.0 & 1.93 & 2.59 & 3.43 & $4 . \equiv 8$ & 4.80 & 5.11 & 5.14 & 5.08 & 4.69 & 3.95 & 2.45 & 1.69 & 1375.0 & 3.8 \\
\hline 45.0 & 1.96 & 2.60 & 3.39 & 4.18 & 4.64 & $c .91$ & c. .95 & 4.96 & 4.66 & 3.99 & 2.49 & 1.72 & 1354.3 & 3.7 \\
\hline 50.0 & 1.98 & 2.59 & 3.35 & 4.135 & 4.45 & 4.69 & $c .75$ & 4.82 & 4.60 & 4.00 & 2.53 & 1.75 & 1326.7 & 3.6 \\
\hline 60.0 & 1.98 & 2.54 & 3.19 & 3.75 & 4.01 & 4.18 & 4.28 & 4.45 & 4.40 & 3.96 & 2.55 & 1.76 & 1250.4 & 3.4 \\
\hline 70.0 & 1.94 & 2.43 & 2.78 & 3.57 & 3.50 & 3.59 & 3.72 & 3.99 & 4.11 & 3.82 & 2.51 & 1.74 & 1148.0 & 3.1 \\
\hline 80.0 & 1.86 & 2.27 & 2.70 & 2.94 & 2.95 & 3.00 & 3.12 & 3.46 & 3.72 & 3.59 & 2.41 & 1.68 & 1025.5 & 2.8 \\
\hline 90.0 & 1.73 & 2.07 & 2.37 & 2.45 & 2.38 & 2.37 & 2.51 & 2.86 & 3.26 & 3.28 & 2.26 & 1.58 & 885.9 & 2.4 \\
\hline
\end{tabular}


AVERAGE DAILY TOTAL TERRESTRIAL INSOLATION ESTIMATES (KLH/SQ. M)

SITE:
LATITUDE: 39 DEINRATI

AVERAGE DAILY AMOUNTS - BY MONTH

\begin{tabular}{|c|c|c|c|c|c|c|c|c|c|c|c|c|c|c|}
\hline ARRAY & $J$ & $F$ & $M$ & $A$ & 年 & 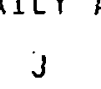 & - & nom & 5 & 0 & r & $D$ & $\begin{array}{l}\text { ANNUAL } \\
\text { ANOUNT }\end{array}$ & $\begin{array}{c}\text { AVERAGE } \\
\text { DAY }\end{array}$ \\
\hline 0.0 & 1.58 & 2.33 & 3.23 & 4.41 & 5.27 & 5.80 & 5.58 & 5.15 & 4.13 & 3.12 & 1.85 & 1.37 & 1335.4 & 3.7 \\
\hline 10.0 & 1.81 & 2.57 & 3.44 & 4.54 & 5.31 & 5.79 & 5.62 & 5.32 & 4.44 & 3.53 & 2.18 & 1.61 & 1406.3 & 3.9 \\
\hline 15.0 & 1.92 & 2.67 & 3.51 & 4.57 & 5.28 & 5.74 & 5.60 & 5.37 & 4.56 & 3.70 & 2.32 & 1.72 & 1430.6 & 3.9 \\
\hline 20.0 & 2.01 & 2.76 & 3.57 & 4.57 & 5.23 & 5.65 & 5.54 & 5.38 & 4.65 & 3.86 & 2.45 & 1.82 & 1447.0 & 4.0 \\
\hline 25.0 & 2.09 & 2.83 & 3.61 & 4.56 & 5.15 & 5.54 & 5.45 & 5.36 & 4.72 & 3.99 & 2.57 & 1.91 & 1455.5 & 4.0 \\
\hline 30.0 & 2.17 & 2.89 & 3.63 & 4.51 & 5.04 & 5.40 & 5.34 & 5.31 & 4.75 & 4.10 & 2.67 & 1.99 & 1456.1 & 4.0 \\
\hline 35.0 & 2.22 & 2.93 & 3.63 & 4.44 & 4.91 & 5.23 & 5.19 & 5.23 & 4.77 & 4.18 & 2.76 & 2.06 & 1448.6 & 4.0 \\
\hline 40.0 & 2.27 & 2.95 & 3.61 & 4.35 & 4.75 & 5.04 & 5.03 & 5.13 & 4.75 & 4.24 & 2.83 & 2.12 & 1433.5 & 3.9 \\
\hline 45.0 & 2.30 & 2.96 & 3.57 & 4.24 & 4.58 & 4.83 & 4.84 & 5.00 & 4.71 & 4.27 & 2.89 & 2.16 & 1411.9 & 3.9 \\
\hline 50.0 & 2.33 & 2.95 & 3.52 & 4.11 & 4.39 & 4.61 & 4.63 & 4.84 & 4.64 & 4.28 & 2.92 & 2.20 & 1382.6 & 3.8 \\
\hline 60.0 & 2.33 & 2.89 & 3.35 & 3.79 & 3.94 & 4.08 & 4.15 & 4.46 & 4.42 & 4.22 & 2.94 & 2.22 & 1302.1 & 3.6 \\
\hline 70.0 & 2.27 & 2.76 & 3.11 & 3.39 & 3.41 & 3.49 & 3.59 & 3.98 & 4.11 & 4.05 & 2.89 & 2.19 & 1194.4 & 3.3 \\
\hline 80.0 & 2.17 & 2.57 & 2.81 & 2.93 & 2.86 & 2.89 & 3.00 & 3.42 & 3.70 & 3.79 & 2.77 & 2.11 & 1066.2 & 2.9 \\
\hline 90.0 & 2.02 & 2.33 & 2.45 & 2.43 & 2.29 & 2.28 & 2.39 & 2.81 & 3.22 & 3.45 & 2.58 & 1.98 & 919.7 & 2.5 \\
\hline
\end{tabular}


AVERAGE DAILY TOTAL TEREESTRIAL INSOLATION ESTIMATES (KWH/SQ. M)

SITE: CLEVELAHD

LATITUDE: GI DEGREES 24 MINUTES

AVERAGE EAILY AMOUNTS BY MONTH

\begin{tabular}{|c|c|c|c|c|c|c|c|c|c|c|c|c|c|c|}
\hline ARRAY & $\mathrm{J}$ & $F$ & M & A & $M$ & $\therefore$ & $\mathrm{J}$ & A & s & 0 & N & 0 & $\begin{array}{l}\text { ANNUAL } \\
\text { AROUNT }\end{array}$ & $\begin{array}{l}\text { AVERAGE } \\
\text { DAY }\end{array}$ \\
\hline 0.0 & 1.23 & 1.90 & 2.90 & 4.26 & 5.30 & 5.82 & 5.76 & 4.99 & 3.91 & 2.73 & 1.47 & 1.00 & 1258.2 & 3.4 \\
\hline 10.0 & 1.47 & 2.08 & 3.109 & 4.40 & 5.36 & 5.83 & 5.83 & 5.18 & 4.22 & 3.11 & 1.72 & 1.24 & 1327.0 & 3.6 \\
\hline 15.0 & 1.59 & 2.15 & 3.16 & 4.44 & 5.35 & 5.79 & 5.81 & 5.23 & 4.35 & 3.27 & 1.84 & 1.34 & 1350.9 & 3.7 \\
\hline 20.0 & 1.69 & 2.22 & 3.22 & 4.45 & 5.31 & 5.72 & 5.77 & 5.25 & 4.45 & 3.41 & 1.94 & 1.44 & 1367.4 & 3.7 \\
\hline 25.0 & 1.78 & 2.27 & 3.25 & 4.44 & 5.24 & 5.62 & 5.69 & 5.25 & 4.52 & 3.54 & 2.03 & 1.53 & 1376.5 & 3.8 \\
\hline 30.0 & 1.86 & 2.32 & 3.28 & 4.41 & 5.14 & 5.49 & 5.58 & 5.21 & 4.57 & 3.64 & 2.11 & 1.61 & 1377.9 & 3.8 \\
\hline 35.0 & 1.93 & 2.35 & 3.28 & 4.35 & 5.01 & 5.33 & 5.44 & 5.15 & 4.59. & 3.72 & 2.18 & 1.69 & 1371.9 & 3.8 \\
\hline 40.0 & 1.99 & 2.36 & 3.27 & 4.27 & 4.86 & 5.1 .4 & 5.28 & 5.05 & 4.59 & 3.78 & 2.23 & 1.75 & 1358.3 & 3.7 \\
\hline 45.0 & 2.04 & 2.37 & 3.24 & 4.17 & 4.69 & 4.9 .4 & 5.09 & 4.94 & 4.56 & 3.82 & 2.28 & 1.80 & 1338.5 & 3.7 \\
\hline 50.0 & 2.07 & 2.36 & 3.19 & 4.05 & 4.51 & 4.72 & 4.88 & 4.80 & 4.50 & 3.83 & 2.31 & 1.84 & 1311.9 & 3.6 \\
\hline 60.0 & 2.10 & 2.31 & 3.05 & 3.75 & 4.07 & 4.21 & 4.40 & 4.44 & 4.31 & 3.79 & 2.32 & 1.88 & 1237.8 & 3.4 \\
\hline 70.0 & 2.08 & 2.21 & 2.84 & 3.38 & 3.55 & 3.63 & 3.83 & 3.99 & 4.03 & 3.66 & 2.29 & 1.88 & 1137.7 & 3.1 \\
\hline 80.0 & 2.00 & 2.06 & 2.58 & 2.94 & 2.99 & 3.0 .3 & 3.21 & 3.46 & 3.66 & 3.44 & 2.20 & 1.82 & 1017.2 & 2.8 \\
\hline 90.0 & 1.88 & 1.88 & 2.27 & 2.46 & 2.42 & 2.40 & 2.58 & 2.87 & 3.21 & 3.15 & 2.05 & 1.73 & 879.9 & 2.4 \\
\hline
\end{tabular}


AVERAGE DAILY TOTAL TERRESTRIAL INSOLATION ESTIMATES (KWH,SQ. M)

SITE: COLUMBUS
LATITUDE: 40 DEGREES O MINUTES

AVERAGE DAILY AMOUNTS BY MONTH

\begin{tabular}{|c|c|c|c|c|c|c|c|c|c|c|c|c|c|c|}
\hline ARRAY & $\mathrm{J}$ & $F$ & $M$ & A & $M$ & $\mathrm{~J}$ & $J^{-2-2}$ & A & $S$ & 0 & -- & $D$ & $\begin{array}{l}\text { ANNUAL } \\
\text { AMOUNT }\end{array}$ & $\begin{array}{c}\text { AVERAGE } \\
\text { DAY }\end{array}$ \\
\hline 0.0 & 1.45 & 2.13 & 3.08 & 4.27 & 5.19 & 5.72 & 5.54 & 5.18 & 4.03 & 2.98 & 1.70 & 1.22 & 1294.9 & 3.5 \\
\hline 10.0 & 1.66 & 2.35 & 3.28 & 4.40 & 5.23 & 5.72 & 5.58 & 5.36 & 4.35 & 3.38 & 1.99 & 1.44 & 1363.1 & 3.7 \\
\hline 15.0 & 1.75 & 2.44 & 3.35 & 4.43 & 5.21 & 5.67 & 5.56 & 5.41 & 4.47 & 3.55 & 2.13 & 1.53 & 1386.4 & 3.8 \\
\hline 20.0 & 1.84 & 2.51 & 3.41 & 4.43 & 5.16 & 5.60 & 5.51 & 5.42 & 4.56 & 3.70 & 2.25 & 1.62 & 1402.2 & 3.8 \\
\hline 25.0 & 1.91 & 2.58 & 3.45 & 4.42 & 5.09 & 5.49 & 5.43 & 5.41 & 4.63 & 3.83 & 2.35 & 1.70 & 1410.4 & 3.9 \\
\hline 30.0 & 1.98 & 2.63 & 3.47 & 4.38 & 4.99 & 5.36 & 5.32 & 5.37 & 4.67 & 3.94 & 2.45 & 1.76 & 1410.8 & 3.9 \\
\hline 35.0 & 2.03 & 2.66 & 3.47 & 4.32 & 4.86 & 5.19 & 5.18 & 5.30 & 4.69 & 4.02 & 2.53 & 1.82 & 1403.6 & 3.8 \\
\hline 40.0 & 2.07 & 2.68 & 3.46 & 4.23 & 4.71 & 5.00 & 5.01 & 5.19 & 4.68 & 4.08 & 2.59 & 1.87 & 1388.7 & 3.8 \\
\hline 45.0 & 2.10 & 2.69 & 3.42 & 4.13 & 4.54 & 4.81 & 4.84 & 5.07 & 4.64 & 4.12 & 2.64 & 1.91 & 1367.8 & 3.7 \\
\hline 50.0 & 2.12 & 2.68 & 3.37 & 4.00 & $4: 35$ & 4.58 & 4.63 & 4.91 & 4.58 & 4.13 & 2.68 & 1.94 & 1339.6 & 3.7 \\
\hline 60.0 & 2.12 & 2.63 & 3.21 & 3.70 & 3.92 & 4.08 & 4.16 & 4.53 & 4.37 & 4.08 & 2.70 & 1.96 & 1262.0 & 3.5 \\
\hline 70.0 & 2.08 & 2.51 & 2.99 & 3.32 & 3.41 & 3.50 & 3.61 & 4.05 & 4.07 & 3.93 & 2.65 & 1.93 & 1158.1 & 3.2 \\
\hline 80.0 & 1.98 & 2.34 & 2.70 & 2.88 & 2.87 & 2.91 & 3.02 & 3.50 & 3.68 & 3.68 & 2.54 & 1.86 & 1034.2 & 2.8 \\
\hline 90.0 & 1.85 & 2.13 & 2.37 & 2.40 & 2.31 & 2.30 & 2.42 & 2.88 & 3.21 & 3.35 & 2.37 & 1.75 & 892.8 & 2.4 \\
\hline
\end{tabular}


AVERAGE DAILY TOTAL TERPESTRIAL INSOLATION ESTIMATES (KWH/SQ. M) $\begin{aligned} \text { SITE: } & \text { DAYTON } \\ \text { LATITUDE: } & \text { I9 DEGREES } \\ & 54 \text { MINUTES }\end{aligned}$

ALERAGE IIAILY AMOUNTS $3 Y$ MONTH

\begin{tabular}{|c|c|c|c|c|c|c|c|c|c|c|c|c|c|c|}
\hline ARRAY & $J$ & $F$ & $M$ & $A$ & $M$ & $\mathrm{~J}$ & $\mathrm{~J}$ & A & 5 & 0 & $N$ & D & $\begin{array}{l}\text { ANNUAL } \\
\text { AMOUNT }\end{array}$ & $\begin{array}{c}\text { AVERAGE } \\
\text { DAY }\end{array}$ \\
\hline 0.0 & 1.54 & 2.29 & 3.22 & 4.43 & 5.36 & 5.91 & 5.70 & 5.19 & 4.15 & 3.06 & 1.78 & 1.29 & 1338.2 & 3.7 \\
\hline 10.0 & 1.78 & 2.53 & 3.44 & 4.57 & 5.40 & 5.90 & 5.75 & 5.37 & 4.47 & 3.47 & 2.10 & 1.52 & 1411.3 & 3.9 \\
\hline 15.0 & 1.89 & 2.64 & 3.52 & 4.60 & 5.38 & 5.86 & 5.73 & 5.42 & $4.60^{\circ}$ & 3.65 & 2.24 & 1.63 & 1436.5 & 3.9 \\
\hline 20.0 & 1.99 & 2.73 & 3.58 & 4.61 & 5.33 & 5.78 & 5.68 & 5.44 & 4.70 & 3.80 & 2.37 & 1.72 & 1453.9 & 4.0 \\
\hline 25.0 & 2.07 & 2.80 & 3.63 & 4.59 & 5.26 & $5: \in 7$ & 5.59 & 5.42 & 4.77 & 3.94 & 2.49 & 1.81 & 1463.3 & 4.0 \\
\hline 30.0 & 2.15 & 2.86 & 3.65 & 4.55 & 5.15 & 5.53 & 5.48 & 5.38 & 4.82 & 4.05 & 2.59 & 1.88 & 1464.6 & 4.0 \\
\hline 35.0 & 2.21 & 2.90 & 3.65 & 4.49 & 5.02 & 5.56 & 5.34 & 5.31 & 4.84 & 4.14 & 2.68 & 1.95 & 1457.9 & 4.0 \\
\hline 40.0 & 2.26 & 2.93 & 3.64 & 4.40 & 4.86 & 5.16 & 5.16 & 5.20 & 4.83 & 4.20 & 2.75 & 2.00 & 1443.2 & 4.0 \\
\hline 45.0 & 2.30 & 2.94 & 3.50 & 4.29 & 4.69 & 4.95 & c. .98 & 5.08 & 4.79 & 4.23 & 2.81 & 2.05 & 1422.2 & 3.9 \\
\hline 50.0 & 2.32 & 2.94 & 3.55 & 4.16 & 4.49 & 4.72 & 6.77 & 4.92 & 4.72 & 4.25 & 2.84 & 2.08 & 1393.4 & 3.8 \\
\hline 60.0 & 2.33 & 2.88 & 3.38 & 3.84 & 4.04 & 4.20 & 6.28 & 4.54 & 4.51 & 4.19 & 2.87 & 2.10 & 1313.6 & 3.6 \\
\hline 70.0 & 2.28 & 2.76 & 3.15 & .3 .45 & 3.51 & 3.59 & 3.71 & 4.06 & 4.20 & 4.04 & 2.82 & 2.08 & 1206.1 & 3.3 \\
\hline 80.0 & 2.18 & 2.57 & 2.35 & 2.99 & 2.95 & 2.58 & 3.10 & 3.50 & 3.79 & 3.79 & 2.71 & 2.01 & 1077.5 & 3.0 \\
\hline 90.0 & 2.03 & 2.34 & 2.50 & 2.48 & 2.37 & 2.35 & 2.47 & 2.88 & 3.31 & 3.45 & 2.53 & 1.89 & 930.3 & 2.5 \\
\hline
\end{tabular}


AVERAGE DAILY TOTAL TERRESTRIAL INSOLATION ESTIMATES (KWH SQ. M)

SITE:
LATITUDE:

A.VERAGE DAILY AMOUNTS BY MONTH

\begin{tabular}{|c|c|c|c|c|c|c|c|c|c|c|c|c|c|c|}
\hline ARRAY & J & $F$ & M & A & $M$ & J & $\mathrm{J}$ & A & 5 & 0 & H & D & $\begin{array}{l}\text { ANNUAL } \\
\text { AMOUNT }\end{array}$ & $\begin{array}{c}\text { AVERAGE } \\
\text { DAY }\end{array}$ \\
\hline 0.0 & 1.37 & 2.14 & 3.14 & 4.36 & 5.42 & 5.92 & 5.83 & 5.09 & 4.01 & 2.87 & 1.57 & 1.12 & 1306.2 & 3.6 \\
\hline 10.0 & 1.59 & 2.38 & 3.36 & 4.51 & 5.48 & 5.93 & 5.90 & 5.29 & 4.35 & 3.28 & 1.86 & 1.33 & 1379.6 & 3.8 \\
\hline 15.0 & 1.69 & 2.48 & 3.45 & 4.55 & 5.46 & 5.90 & 5.89 & 5.35 & 4.48 & 3.46 & 1.99 & 1.42 & 1405.3 & 3.9 \\
\hline 20.0 & 1.78 & 2.57 & 3.51 & 4.57 & 5.42 & 5.83 & 5.84 & 5.37 & 4.59 & 3.62 & 2. 11 & 1.51 & 1423.3 & 3.9 \\
\hline 25.0 & 1.85 & 2.65 & 3.56 & 4.56 & 5.35 & 5.72 & 5.76 & 5.37 & 4.67 & 3.75 & 2.22 & 1.58 & 1433.5 & 3.9 \\
\hline 30.0 & 1.92 & 2.71 & 3.59 & 4.52 & 5.25 & 5.59 & 5.65 & 5.33 & 4.72 & 3.87 & 231 & 1.65 & 1435.8 & 3.9 \\
\hline 35.0 & 1.98 & 2.75 & 3.60 & 4.47 & 5.12 & 5.43 & 5.51 & 5.27 & 4.75 & 3.96 & 2.40 & 1.71 & 1430.2 & 3.9 \\
\hline 40.0 & 2.03 & 2.78 & 3.59 & 4.39 & 4.97 & 5.24 & 5.34 & 5.18 & 4.75 & 4.02 & 2.46 & 1.76 & 1416.7 & 3.9 \\
\hline 45.0 & 2.06 & 2.80 & 3.56 & 4.28 & 4.80 & 5.03 & 5.16 & 5.06 & 4.72 & 4.07 & $2.51^{\circ}$ & 1.80 & 1396.6 & 3.8 \\
\hline 50.0 & 2.09 & 2.80 & 3.52 & 4.16 & 4.61 & 4.81 & 4.95 & 4.91 & 4.66 & 4.09 & 2.55 & 1.83 & 1369.4 & 3.8 \\
\hline 60.0 & 2.10 & 2.75 & 3.36 & 3.86 & 4.16 & 4.29 & 4.46 & 4.55 & 4.47 & 4.05 & 2.58 & 1.85 & 1293.1 & 3.5 \\
\hline 70.0 & 2.06 & 2.64 & 3.14 & 3.47 & 3.63 & 3.69 & 3.88 & 4.09 & 4.18 & 3.91 & 2.54 & 1.84 & 1189.5 & 3.3 \\
\hline 80.0 & 1.97 & 2.47 & 2.85 & 3.03 & 3.06 & 3.08 & 3.25 & 3.54 & 3.79 & 3.69 & 2.45 & 1.78 & 1064.2 & 2.9 \\
\hline 90.0 & 1.84 & 2.26 & 2.51 & 2.53 & 2.48 & 2.44 & 2.61 & 2.94 & 3.33 & 3.37 & 2.30 & 1.67 & 921.1 & 2.5 \\
\hline
\end{tabular}


AVERAGE DAILY TOTAL TERRESTRIAL INSOLATION ESTIMATES (KWH/SQ. M)

SITE: YOUNGSTOWN
LATITUDE:

AVERAGE DAILY AMOUNTS BY MONTH

\begin{tabular}{|c|c|c|c|c|c|c|c|c|c|c|c|c|c|c|}
\hline ARRAY & 1 & $F$ & $M$ & A & $M$ & $J$ & $J$ & A & $s$ & 0 & $N$ & D & $\begin{array}{l}\text { ANNUAL } \\
\text { AMOUNT }\end{array}$ & $\begin{array}{l}\text { AVERAGE } \\
\text { DAY }\end{array}$ \\
\hline 0.0 & 1.22 & 1.85 & 2.30 & 4.03 & 5.00 & 5.55 & 5.47 & 4.75 & 3.76 & 2.69 & 1.44 & 1.00 & 1205.7 & 3.3 \\
\hline 10.0 & 1.46 & 2.02 & 2.98 & 4.16 & 5.05 & 5.55 & 5.53 & 4.92 & 4.06 & 3.05 & 1.68 & 1.22 & 1270.4 & 3.5 \\
\hline 15.0 & 1.57 & 2.09 & 3.34 & 4.19 & 5.04 & 5.52 & 5.51 & 4.96 & 4.17 & 3.20 & 1.79 & 1.33 & 1292.8 & 3.5 \\
\hline 26.0 & 1.67 & 2.15 & 3.39 & 4.20 & 5.00 & 5.45 & 5.47 & 4.98 & 4.27 & 3.34 & 1.88 & 1.42 & 1308.2 & 3.6 \\
\hline 25.0 & 1.76 & 2.20 & 3.13 & 4.19 & 4.93 & 5.35 & 5.39 & 4.97 & 4.34 & 3.46 & 1.97 & 1.51 & 1316.6 & 3.6 \\
\hline 30.0 & 1.83 & 2.24 & 3.14 & 4.15 & 4.84 & 5.23 & 5.29 & 4.94 & 4.38 & 3.56 & 2.0 .5 & 1.59 & 1317.8 & 3.6 \\
\hline 35.0 & 1.90 & 2.26 & 3.15 & 4.10 & 4.72 & 5.08 & E.16 & 4.88 & 4.40 & 3.63 & 2.11 & 1.66 & 1311.9 & 3.6 \\
\hline 40.0 & 1.96 & 2.28 & 3.13 & 4.02 & 4.58 & 4.90 & 5.00 & 4.79 & 4.39 & 3.69 & 2.16 & 1.72 & 1298.8 & 3.6 \\
\hline 45.0 & 2.00 & 2.28 & 3.10 & 3.93 & 4.42 & 4.71 & 4.83 & 4.68 & 4.36 & 3.72 & 2.20 & 1.77 & 1280.0 & 3.5 \\
\hline 50.0 & 2.04 & 2.27 & 3.05 & 3.82 & 4.24 & 4.50 & 4.63 & 4.54 & 4.31 & 3.73 & 2.23 & 1.81 & 1254.6 & 3.4 \\
\hline 60.0 & 2.06 & 2.22 & 2.91 & 3.53 & 3.83 & 4.02 & 4.17 & 4.20 & 4.12 & 3.69 & 2.24 & 1.85 & 1184.1 & 3.2 \\
\hline 70.0 & 2.04 & $2: 12$ & 2.72 & 3.18 & 3.35 & 3.47 & 3.64 & 3.78 & 3.85 & 3.56 & 2.20 & 1.84 & 1089.2 & 3.0 \\
\hline 80.0 & 1.97 & 1.98 & 2.47 & 2.78 & 2.83 & 2.90 & 3.06 & 3.28 & 3.49 & 3.35 & 2.11 & 1.79 & 974.9 & 2.7 \\
\hline 90.0 & 1.84 & 1.80 & $2 .: 7$ & 2.33 & 2.30 & 2.31 & 2.47 & 2.72 & 3.06 & 3.06 & 1.98 & 1.69 & 844.7 & 2.3 \\
\hline
\end{tabular}


AVERAGE DAILY TOTAL TERRESTRIAL INSOLATION ESTIMATES (KWH/SQ. M)

SITE: OKLAHOMA CITY OK

LATITUDE: 35 DEGREES 24 MINUTES

AVERAGE DAILY AMOUNTS BY MONTH

\begin{tabular}{|c|c|c|c|c|c|c|c|c|c|c|c|c|c|c|}
\hline ARRAY & $\mathrm{J}$ & $F$ & $M$ & A & ond & $\begin{array}{ll}2 \\
\end{array}$ & 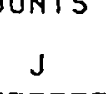 & . & 5 & 0 & H & $D$ & $\begin{array}{l}\text { ANNUAL } \\
\text { AMOUNT }\end{array}$ & $\begin{array}{c}\text { AVERAGE } \\
\text { DAY }\end{array}$ \\
\hline 0.0 & 2.52 & 3.33 & 4.41 & 5.44 & 6.04 & 6.76 & 6.71 & 6.16 & 4.90 & 3.89 & 2.84 & 2.29 & 1683.5 & 4.6 \\
\hline 10.0 & 2.94 & 3.70 & 4.70 & 5.58 & 6.04 & 6.71 & 6.72 & 6.33 & 5.24 & 4.38 & 3.36 & 2.75 & 1780.6 & 4.9 \\
\hline $15.0^{\circ}$ & 3.13 & 3.86 & 4.81 & 5.60 & 6.00 & 6.62 & 6.66 & 6.37 & 5.37 & 4.59 & 3.60 & 2.96 & 1814.0 & 5.0 \\
\hline 20.0 & 3.30 & 3.99 & $4.80^{\circ}$ & 5.60 & 5.92 & 6.50 & 6.57 & 6.37 & 5.47 & 4.78 & 3.81 & 3.15 & 1837.1 & 5.0 \\
\hline 25.0 & 3.45 & 4.10 & 4.94 & 5.56 & 5.80 & 6.34 & 6.44 & 6.33 & 5.54 & 4.93 & 4.00 & 3.32 & 1849.5 & 5.1 \\
\hline 30.0 & 3.58 & 4.18 & 4.97 & 5.49 & 5.66 & 6.15 & 6.28 & 6.25 & 5.57 & 5.05 & 4.16 & 3.47 & 1851.3 & 5.1 \\
\hline 35.0 & 3.68 & 4.25 & 4.96 & 5.39 & 5.48 & 5.9 .2 & 6.08 & 6.14 & 5.57 & 5.15 & 4.30 & 3.61 & 1842.3 & 5.0 \\
\hline 40.0 & 3.77 & 4.28 & 4.93 & 5.27 & 5.29 & 5.68 & 5.86 & 6.00 & 5.54 & 5.21 & 4.41 & 3.71 & 1824.5 & $5: 0$ \\
\hline 45.0 & 3.83 & 4.30 & 4.87 & 5.11 & 5.07 & 5.41 & 5.61 & 5.82 & 5.47 & 5.24 & 4.49. & 3.80 & 1796.3 & 4.9 \\
\hline 50.0 & 3.87 & 4.28 & 4.78 & 4.93 & 4.83 & 5.12 & 5.33 & 5.61 & 5.37 & 5.23 & 4.55 & 3.86 & 1757.9 & 4.8 \\
\hline 60.0 & 3.88 & 4.18 & 4.52 & 4.50 & 4.27 & 4.45 & 4.69 & 5.10 & 5.08 & 5.13 & 4.57 & 3.91 & 1651.4 & 4.5 \\
\hline 70.0 & 3.79 & 3.9 .8 & 4.17 & 3.97 & 3.63 & 3.72 & 3.95 & 4.48 & 4.68 & 4.90 & $4 . .48$ & 3.86 & 1509.1 & 4.1 \\
\hline 80.0 & 3.60 & 3.69 & 3.72 & 3.37 & 2.98 & 2.98 & 3.22 & 3.78 & 4.17 & 4.55 & 4.27 & 3.71 & 1339.5 & 3.7 \\
\hline 90.0 & 3.33 & 3.32 & $3 . \geq 0$ & 2.72 & 2.31 & 2.26 & 2.45 & 3.02 & 3.57 & 4.10 & 3.96 & 3.47 & 1146.6 & 3.1 \\
\hline
\end{tabular}


AlERAGE DAILY TOTAL TERRESTRIAL INSOLATION ESTIMATES (KWH/SQ. M)

SITE: TULSA

OK

LATITUDE: 36 DEGREES 12 MINUTES

AYERAGE DAILY AMO JNTS BY MONTH

\begin{tabular}{|c|c|c|c|c|c|c|c|c|c|c|c|c|c|c|}
\hline ARRAY & $J$ & $F$ & $M$ & A & $M$ & $J$ & $J$ & A & $S$ & 0 & $N$ & D & $\begin{array}{l}\text { ANNUAL } \\
\text { AMOUNT }\end{array}$ & $\begin{array}{c}\text { AVERAGE } \\
\text { DAY }\end{array}$ \\
\hline 0.0 & 2.30 & 3.08 & 4.11 & 5.06 & 5.74 & 6.37 & $\dot{0} .39$ & 5.89 & 4.64 & 3.67 & 2.60 & 2.08 & 1582.2 & 4.3 \\
\hline 10.0 & 2.69 & 3.42 & 4.38 & 5.19 & 5.76 & 6.33 & 0.41 & 6.06 & 4.97 & 4.14 & 3.018 & 2.50 & 1672.7 & 4.6 \\
\hline 15.0 & 2.86 & 3.57 & 4.48 & 5.21 & 5.72 & 6.26 & 6.36 & 6.10 & 5.09 & 4.33 & 3.29 & 2.69 & 1704.0 & 4.7 \\
\hline 20.0 & 3.01 & 3.69 & 4.55 & 5.21 & 5.64 & $6 . \Sigma 5$ & 6.28 & 6.10 & 5.18 & 4.51 & 3.48 & 2.86 & 1725.7 & 4.7 \\
\hline 25.0 & 3.15 & 3.79 & 4.60 & 5.18 & 5.54 & 6.01 & 6.16 & 6.07 & 5.24 & 4.65 & 3.65 & 3.02 & 1737.6 & 4.8 \\
\hline 30.0 & 3.26 & 3.87 & 4.63 & 5.11 & 5.41 & 5.83 & 6.01 & 6.00 & 5.28 & 4.77 & 3.80 & 3.16 & 1739.6 & 4.8 \\
\hline 35.0 & 3.36 & 3.93 & 4.62 & 5.02 & 5.25 & 5.63. & 5.83 & $5: 90$ & 5.28 & 4.86 & 3.93 & 3.28 & 1731.7 & 4.7 \\
\hline 40.0 & 3.44 & 3.96 & 4.59 & 4.91 & 5.07 & 5.41 & 5.62 & 5.76 & 5.25 & 4.92 & 4.03 & 3.38 & 1715.2 & 4.7 \\
\hline 45.0 & 3.50 & 3.97 & 4.54 & 4.77 & 4.87 & 5.16 & 5.39 & 5.60 & 5.19 & 4.95 & 4.10 & 3.45 & 1689.5 & 4.6 \\
\hline 50.0 & 3.53 & 3.96 & 4.46 & 4.61 & 4.64 & 4.89 & 5.13 & 5.41 & 5.10 & 4.95 & 4.15 & 3.51 & 1654.2 & 4.5 \\
\hline 60.0 & 3.54 & 3.87 & 4.23 & 4.22 & 4.12 & 4.28 & 4.53 & 4.93 & 4.84 & 4.85 & 4.18 & 3.56 & 1556.2 & 4.3 \\
\hline 70.0 & 3.46 & 3.69 & 3.90 & 3.74 & 3.53 & 3.60 & ?. 85 & 4.35 & 4.46 & 4.64 & 4.09 & 3.51 & 1424.8 & 3.9 \\
\hline 80.0 & 3.30 & 3.43 & 3.49 & 3.19 & 2.92 & 2.92 & ป. 15 & 3.68 & 3.99 & 4.32 & 3.9 .1 & 3.38 & 1267.9 & 3.5 \\
\hline 90.0 & 3.06 & 3.09 & 3.32 & 2.60 & 2.28 & 2.25 & 2.42 & 2.96 & 3.43 & 3.90 & 3.63 & 3.17 & 1088.6 & 3.0 \\
\hline
\end{tabular}


AVERAGE DAILY TOTAL TERRESTRIAL INSOLATION ESTIMATES (KWH/SQ. M)

SITE:
ITUDE: 46 DSORIA
DEGRES 9 MINUTES

AVERAGE DAILY AMOUNTS BY MONTH

\begin{tabular}{|c|c|c|c|c|c|c|c|c|c|c|c|c|c|c|}
\hline ARRAY & $\jmath$ & $F$ & $M$ & $A$ & $M$ & $\mathrm{~J}$ & $J$ & $A$ & $s$ & 0 & $\mathbf{N}$ & D & $\begin{array}{l}\text { ANNUAL } \\
\text { AMOUNT }\end{array}$ & $\begin{array}{c}\text { AVERAGE } \\
D A Y\end{array}$ \\
\hline 0.0 & 0.99 & 1.72 & 2.73 & 3.95 & 5.07 & 5.12 & 5.51 & 4.72 & 3.73 & 2.25 & 1.22 & 0.82 & 1153.8 & 3.2 \\
\hline 10.0 & 1.24 & 1.93 & 2.94 & 4.12 & 5.16 & 5.16 & 5.62 & 4.95 & 4.10 & 2.61 & 1.49 & 1.00 & 1229.0 & 3.4 \\
\hline 15.0 & 1.35 & 2.02 & 3.03 & 4.17 & 5.16 & 5.14 & 5.63 & 5.02 & 4.25 & 2.76 & 1.61 & 1.08 & 1256.9 & 3.4 \\
\hline 20.0 & 1.46 & 2.10 & 3.10 & 4.20 & 5.14 & 5.10 & 5.60 & 5.07 & 4.38 & 2.91 & 1.72 & 1.15 & 1278.2 & 3.5 \\
\hline 25.0 & 1.56 & 2.16 & 3.16 & 4.21 & 3.10 & 5.03 & 5.55 & 5.08 & 4.48 & 3.03 & 1.82 & 1.22 & 1292.6 & 3.5 \\
\hline 30.0 & 1.64 & 2.22 & 3.20 & 4.19 & 5.02 & 4.94 & 5.47 & 5.07 & 4.56 & 3.14 & 1.92 & 1.28 & 1299.9 & 3.6 \\
\hline 35.0 & 1.72 & 2.26 & 3.22 & 4.16 & 4.92 & 4.82 & 5.36 & 5.03 & 4.61 & 3.23 & 2.00 & 1.33 & 1300.0 & 3.6 \\
\hline 40.0 & 1.79 & 2.30 & 3.22 & 4.10 & 4.80 & 4.68 & 5.22 & 4.97 & 4.63 & 3.30 & 2.06 & 1.38 & 1293.0 & 3.5 \\
\hline 45.0 & 1.84 & 2.32 & 3.21 & 4.01 & 4.64 & 4.51 & 5.05 & 4.87 & 4.63 & 3.35 & 2.12 & 1.41 & 1278.8 & 3.5 \\
\hline 50.0 & 1.89 & 2.32 & 3.18 & 3.92 & 4.48 & 4.33 & 4.87 & 4.75 & 4.60 & 3.37 & 2.16 & 1.44 & 1259.0 & 3.4 \\
\hline 60.0 & 1.94 & 2.30 & 3.06 & 3.66 & 4.09 & 3.92 & 4.44 & 4.45 & 4.46 & 3.37 & 2.21 & 1.48 & 1199.7 & 3.3 \\
\hline 70.0 & 1.94 & 2.22 & 2.89 & 3.34 & 3.63 & 3.44 & 3.93 & 4.04 & 4.21 & 3.29 & 2.20 & 1.48 & 1115.0 & 3.1 \\
\hline 80.0 & 1.89 & 2.10 & 2.65 & 2.95 & 3.10 & 2.92 & 3.34 & 3.56 & 3.87 & 3.13 & 2.14 & 1.44 & 1007.7 & 2.8 \\
\hline 90.0 & 1.79 & 1.94 & 2.36 & 2.51 & 2.56 & 2.40 & 2.75 & 3.01 & 3.44 & 2.89 & 2.03 & 1.37 & 884.3 & 2.4 \\
\hline
\end{tabular}


AVERAGE DAILY TOTA: TERRESTRIAL INSOLATION ESTIMATES (KWH/SQ. M)

$\begin{array}{ll}\text { SITE: } & \text { BURNS } \\ \text { LATITUDE: } & 43 \text { DEGREES } 35 \text { MINUTES }\end{array}$

$\begin{array}{rlr}\text { SITE: } & \text { BURNS } & \text { OR } \\ \text { LATITUDE: } & 43 \text { DEGREES } & 35 \text { MINUTES }\end{array}$

AVERAGE DKILY AMOUNTS BY MONTH

\begin{tabular}{|c|c|c|c|c|c|c|c|c|c|c|c|c|c|c|}
\hline ARRAY & $\mathbf{J}$ & $F$ & $M$ & A & $M$ & \rfloor & $J$ & $A$ & $s$ & 0 & $\kappa$ & D & $\begin{array}{l}\text { ANNUAL } \\
\text { ArIOUNT }\end{array}$ & $\begin{array}{c}\text { AVERAGE } \\
\text { DAY }\end{array}$ \\
\hline 0.0 & 1.55 & 2.50 & 3.74 & 5.20 & 6.47 & 7.19 & 7.75 & 6.57 & 5.10 & 3.28 & 1.87 & 1.36 & 1603.0 & 4.4 \\
\hline 10.0 & 1.87 & 2.85 & 4.07 & 5.43 & 6.59 & 7.24 & 7.90 & 6.52 & 5.67 & 3.86 & 2.33 & 1.71 & 1720.4 & 4.7 \\
\hline 15.0 & 2.02 & 3.01 & 4.20 & 5.50 & 6.59 & 7.20 & 7.91 & 7.03 & 5.90 & 4.12 & 2.53 & 1.88 & 1764.5 & 4.8 \\
\hline 20.0 & 2.16 & 3.15 & 4.31 & 5.54 & 6.55 & 7.13 & 7.88 & 7.10 & 6.09 & 4.35 & 2.72 & 2.03 & 1798.4 & 4.9 \\
\hline 25.0 & 2.28 & 3.27 & 4.40 & 5.55 & 6.48 & 7.02 & 7.79 & 7.13 & 6.25 & 4.55 & 2.90 & 2.17 & 1821.6 & 5.0 \\
\hline 30.0 & 2.39 & 3.37 & 4.46 & 5.52 & 6.37 & 6.86 & 7.66 & 7.12 & 6.37 & 4.73 & 3.05 & 2.30 & 1834.0 & 5.0 \\
\hline 35.0 & 2.49 & 3.45 & 4.49 & 5.47 & 6.23 & 6.67 & 7.48 & 7.06 & 6.45 & 4.87 & 3.19 & 2.41 & 1835.5 & 5.0 \\
\hline 40.0 & 2.57 & 3.51 & 4.50 & 5.38 & 6.05 & 6.44 & 7.25 & 6.95 & 6.48 & 4.98 & 3.31 & 2.51 & 1826.0 & 5.0 \\
\hline 45.0 & 2.63 & 3.55 & 4.48 & 5.27 & 5.84 & 6.18 & 6.99 & 6.81 & 6.48 & 5.07 & 3.40 & 2.59 & 1806.5 & 4.9 \\
\hline 50.0 & 2.63 & 3.57 & 4.44 & 5.13 & 5.61 & 5.91 & 6.71 & 6.64 & 6.43 & 5.12 & 3.48 & 2.66 & 1778.1 & 4.9 \\
\hline 60.0 & 2.73 & 3.54 & 4.28 & 4.77 & 5.07 & 5.27 & 6.04 & 6.17 & 6.22 & 5.12 & 3.56 & 2.74 & 1690.2 & 4.6 \\
\hline 70.0 & 2.71 & 3.43 & 4.02 & 4.31 & 4.43 & 4.52 & 5.22 & 5.5 .5 & 5.86 & 4.99 & 3.55 & 2.75 & 1563.1 & 4.3 \\
\hline 80.0 & 2.62 & 3.24 & 3.57 & 3.76 & 3.71 & 3.73 & 4.32 & 4.81 & 5.35 & 4.74 & 3.45 & 2.69 & 1402.7 & 3.8 \\
\hline 90.0 & 2.47 & 2.98 & 3.25 & 3.14 & 2.98 & 2.92 & 3.41 & 3.97 & 4.71 & 4.36 & 3.26 & 2.56 & 1217.1 & 3.3 \\
\hline
\end{tabular}


AVERAGE DAILY TOTAL TERRESTRIAL INSOLATION ESTIMATES (KWHVSC. M)

SITE: MEDFORD

LATITUDE: 42 DEGREES 22 MINUTES

AVERAGE DAILY AMOUNTS BY MONTH

\begin{tabular}{|c|c|c|c|c|c|c|c|c|c|c|c|c|c|c|}
\hline ARRAY & $J$ & $F$ & $M$ & A & $M$ & 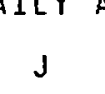 & 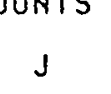 & $170 \mathrm{~T}$ & $s$ & 0 & $y$ & D & $\begin{array}{l}\text { ANNUAL } \\
\text { AMOUNT }\end{array}$ & $\begin{array}{c}\text { AVERAGE } \\
\text { DAY }\end{array}$ \\
\hline 0.0 & 1.29 & 2.33 & 3.57 & 5.17 & 6.41 & 7.18 & 7.80 & 6.68 & 5.01 & 3.09 & 1.59 & 1.06 & 1560.5 & 4.3 \\
\hline 10.0 & 1.49 & 2.62 & 3.86 & 5.39 & 6.51 & 7.21 & 7.94 & 7.02 & 5.52 & 3.58 & 1.91 & 1.26 & 1655.1 & 4.5 \\
\hline 15.0 & 1.58 & 2.75 & 3.97 & 5.45 & 6.50 & 7.17 & 7.93 & 7.13 & 5.73 & 3.79 & 2.05 & 1.35 & 1688.5 & 4.6 \\
\hline 20.0 & 1.66 & 2.86 & 4.06 & 5.48 & 6.46 & 7.09 & 7.89 & 7.19 & 5.90 & 3.98 & 2.18 & 1.43 & 1712.2 & 4.7 \\
\hline 25.0 & 1.73 & 2.95 & 4.13 & 5.48 & 6.38 & 6.97 & 7.79 & 7.21 & 6.04 & 4.15 & 2.30 & 1.51 & 1725.8 & 4.7 \\
\hline 30.0 & 1.80 & 3.03 & 4.18 & 5.45 & 6.26 & 6.80 & 7.64 & 7.18 & 6.14 & 4.29 & 2.40 & 1.57 & 1729.4 & 4.7 \\
\hline 35.0 & 1.85 & 3.09 & 4.20 & 5.39 & 6.11 & 6.60 & 7.45 & 7.11 & 6.20 & 4.41 & 2.49 & 1.63 & 1722.8 & 4.7 \\
\hline 40.0 & 1.89 & 3.14 & 4.20 & 5.30 & 5.93 & 6.37 & 7.22 & 7.00 & 6.22 & 4.50 & 257 & 1.68 & 1706.2 & 4.7 \\
\hline 45.0 & 1.93 & 3.16 & 4.18 & 5.18 & 5.72 & 6.11 & $6 .-96$ & 6.85 & 6.20 & 4.56 & 2.63 & 1.72 & 1681.0 & 4.6 \\
\hline 50.0 & 1.95 & 3.17 & 4.13 & 5.04 & 5.49 & 5.83 & 6.66 & 6.66 & 6.15 & 4.59 & 2.67 & 1.75 & 1647.3 & 4.5 \\
\hline 60.0 & 1.96 & 3.13 & 3.96 & 4.67 & 4.94 & 5.18 & 5.97 & 6.17 & 5.93 & 4.57 & 2.71 & 1.77 & 1551.6 & 4.3 \\
\hline 70.0 & 1.92 & 3.02 & 3.71 & 4.20 & 4.30 & 4.42 & 5.14 & 5.53 & 5.56 & 4.43 & 2.68 & 1.76 & 1421.0 & 3.9 \\
\hline 80.0 & 1.85 & 2.84 & 3.38 & 3.66 & 3.59 & 3.64 & 4.23 & 4.77 & 5.06 & 4.18 & 2.59 & 1.70 & 1262.3 & 3.5 \\
\hline 90.0 & 1.73 & 2.60 & 2.98 & 3.04 & 2.87 & 2.82 & 3.31 & 3.90 & 4.44 & 3.84 & 2.43 & 1.60 & 1081.8 & 3.0 \\
\hline
\end{tabular}


AVERAGE DAILY TOTAL TERRESTRIAL INSOLATION ESTIMATES (KWH/SQ. M)

SITE: NORTH BEND

LATITUDE: 43 DEGREES 25 MINUTES

AVERAGE DAILY AMQUNTS BY MONTH

\begin{tabular}{|c|c|c|c|c|c|c|c|c|c|c|c|c|c|c|}
\hline $\begin{array}{l}\text { ARRAY } \\
\text { TILT }\end{array}$ & J & $F$ & $\mathbf{M}$ & A & $M$ & 7 & J & $A$ & 5 & 0 & $N$ & $D$ & $\begin{array}{l}\text { ANNUAL } \\
\text { AMOUNT }\end{array}$ & $\begin{array}{c}\text { AVERAGE } \\
\text { DAY }\end{array}$ \\
\hline 0.0 & 1.39 & 2.22 & 3.33 & 4.76 & 5.86 & 6.29 & 6.64 & 5.64 & 4.34 & 2.81 & 1.66 & 1.20 & 1406.3 & 3.9 \\
\hline $\ln .0$ & 1.65 & 2.51 & 3.60 & 4.96 & 5.95 & 6.32 & 6.76 & 5.90 & 4.76 & 3.25 & 2.02 & 1.48 & 1498.5 & 4.1 \\
\hline 15.0 & 1.77 & 2.63 & 3.71 & $5.0 \mathrm{I}$ & 5.95 & 6.29 & 6.76 & 5.98 & 4.93 & 3.44 & 2.19 & 1.61 & 1532.3 & 4.2 \\
\hline 20.0 & 1.88 & 2.74 & 3.80 & 5.04 & 3.91 & 6.22 & 6.72 & 6.03 & 5.07 & 3.62 & 2.34 & 1.73 & 1557.6 & 4.3 \\
\hline 25.0 & 1.97 & 2.84 & 3.86 & 5.04 & 5.85 & 6.13 & 6.64 & 6.05 & 5.18 & 3.77 & 2.48 & 1.84 & 1574.0 & 4.3 \\
\hline 30.0 & 2.06 & 2.92 & 3.91 & 5.02 & 5.75 & 5.99 & 6.53 & 6.02 & 5.26 & 3.89 & 2.61 & 1.94 & 1581.4 & 4.3 \\
\hline 35.0 & 2.14 & 2.98 & 3.93 & 4.97 & 5.62 & 5.83 & 6.38 & 5.97 & 5.31 & 4.00 & 2.72 & 2.03 & 1579.8 & 4.3 \\
\hline 40.0 & 2.20 & 3.02 & 3.93 & 4.89 & 5.46 & 5.63 & 6.19 & 5.87 & 5.33 & 4.08 & 2.81 & 2.10 & 1569.2 & 4.3 \\
\hline 45.0 & 2.25 & 3.05 & 3.91 & 4.78 & 5.27 & 5.41 & 5.97 & 5.75 & 5.32 & 4.13 & 2.89 & 2.17 & 1550.3 & 4.2 \\
\hline 50.0 & 2.28 & 3.16 & 3.87 & 4.65 & 5.07 & 5.18 & 5.74 & 5.60 & 5.27 & 4.16 & 2.94 & 2.22 & 1524.1 & 4.2 \\
\hline 60.0 & 2.31 & 3.03 & 3.72 & 4.33 & 4.59 & 4.63 & 5.18 & 5.20 & 5.08 & 4.15 & 3.00 & 2.27 & 1446.1 & 4.0 \\
\hline 70.0 & 2.29 & 2.93 & 3.49 & 3.91 & 4.02 & 4.00 & 4.51 & 4.69 & 4.78 & 4.03 & 2.98 & 2.27 & 1336.2 & 3.7 \\
\hline 80.0 & 2.21 & 2.76 & 3.19 & 3.42 & 3.38 & 3.34 & 3.76 & 4.08 & 4.36 & 3.81 & 2.89 & 2.22 & 1199.4 & 3.3 \\
\hline 90.0 & 2.08 & 2.53 & 2.82 & 2.86 & 2.74 & 2.65 & 3.01 & 3.39 & 3.84 & 3.50 & 2.73 & 2.10 & 1042.3 & 2.9 \\
\hline
\end{tabular}


AVERAGE DAIEY TOTAL TERRESTRIAL INSOLATION ESTIMATES (KWH/SQ. M)

SITE: PENDLETON OR

ATITUDE: 45 DEGREES 41 MINUTES

AVERAGE DAILY AMOUNTS BY MONTH

\begin{tabular}{|c|c|c|c|c|c|c|c|c|c|c|c|c|c|c|}
\hline ARRAY & J & $\mathrm{F}$ & $M$ & A & $M$ & $J$ & $J$ & A & $S$ & 0 & N & D & $\begin{array}{l}\text { ANNUAL } \\
\text { AMOUNT }\end{array}$ & $\begin{array}{c}\text { AVERAGE } \\
\text { DAY }\end{array}$ \\
\hline 0.0 & 1.10 & 1.93 & 3.29 & 4.74 & 6.07 & 6.77 & 7.55 & 6.29 & 4.73 & 2.86 & 1.38 & 0.92 & 1453.1 & 4.0 \\
\hline 10.0 & 1.30 & 2.19 & 3.59 & 4.96 & 6.19 & 6.83 & 7.73 & 6.66 & 5.29 & 3.38 & 1.70 & 1.14. & 1554.0 & 4.3 \\
\hline 15.0 & 1.39 & 2.30 & 3.71 & 5.03 & 6.20 & 6.81 & 7.76 & 6.78 & 5.52 & 3.61 & 1.85 & 1.24 & 1591.5 & 4.4 \\
\hline 20.0 & 1.48 & 2.40 & 3.81 & 5.07 & 6.18 & 6.75 & 7.73 & 6.87 & 5.71 & 3.82 & 1.99 & 1.33 & 1620.2 & 4.4 \\
\hline 25.0 & 1.55 & 2.49 & 3.89 & 5.08 & 6.12 & 6.66 & 7.67 & 6.91 & 5.87 & 4.01 & 2.11 & 1.41 & 1639.4 & 4.5 \\
\hline 30.0 & 1.62 & 2.56 & 3.94 & 5.07 & .6 .03 & 6.53 & 7.56 & 6.91 & 5.99 & 4.17 & 2.22 & 1.49 & 1649.0 & 4.5 \\
\hline 35.0 & 1.67 & 2.62 & 3.98 & 5.03 & 5.91 & 6.36 & 7.40 & 6.87 & 6.08 & 4.30 & 2.32 & 1.55 & 1649.0 & 4.5 \\
\hline 40.0 & 1.72 & 2.66 & 3.99 & 4.96 & 5.75 & 6.16 & 7.20 & 6.78 & 6.13 & 4.41 & 2.40 & 1.61 & 1639.4 & 4.5 \\
\hline 45.0 & 1.76 & 2.69 & 3.98 & 4.86 & 5.56 & 5.93 & 6.95 & 6.66 & 6.14 & 4.49 & 2.47 & 1.66 & 1620.1 & 4.4 \\
\hline 50.0 & 1.79 & 2.70 & 3.95 & 4.74 & 5.36 & 5.68 & 6.69 & 6.50 & 6.11 & 4.54 & 2.53 & 1.70 & 1593.6 & 4.4 \\
\hline 60.0 & 1.81. & 2.68 & 3.82 & 4.43 & 4.88 & 5.11 & 6.06 & 6.07 & 5.94 & 4.56 & 2.59 & 1.74 & 1514.0 & 4.1 \\
\hline 70.0 & 1.80 & 2.60 & 3.60 & 4.03 & 4.30 & 4.43 & 5.29 & 5.50 & 5.62 & 4.46 & 2.58 & 1.74 & 1399.9 & 3.8 \\
\hline 80.0 & 1.74 & 2.46 & 3.31 & 3.54 & 3.63 & 3.70 & 4.41 & 4.81 & 5.16 & 4.25 & 2.51 & 1.70 & 1255.7 & 3.4 \\
\hline 90.0 & 1.64 & 2.26 & 2.94 & 2.99 & 2.96 & 2.95 & 3.53 & 4.01 & 4.58 & 3.93 & 2.38 & 1.62 & 1090.7 & 3.0 \\
\hline
\end{tabular}


AVERAGE DAILY TOTAL TERRESTRIAL INSOLATION ESTIMATES (KWH/SQ. M)

$\begin{array}{cl}\text { SITE: } & \text { PORTLAND } \\ \text { LATITUDE: } & \text { 45. DEGREES } 36 \text { MINUTES }\end{array}$

AVERAGE JAILY AMOUNTS BY MONTH

\begin{tabular}{|c|c|c|c|c|c|c|c|c|c|c|c|c|c|c|}
\hline ARRAY & $\lambda^{-1}$ & $F$ & $M$ & A & $M$ & $J$ & $J$ & $A$ & $\varsigma$ & 0 & $N$ & $D$ & $\begin{array}{l}\text { ANNUAL } \\
\text { AMOUNT }\end{array}$ & $\begin{array}{c}\text { AVERAGE } \\
\text { DAY }\end{array}$ \\
\hline 0.0 & 0.98 & 1.75 & 2.82 & 4.13 & 5.24 & 5.59 & 5.42 & 5.27 & 3.83 & 2.28 & 1.22 & 0.82 & 1231.0 & 3.4 \\
\hline 10.0 & 1.21 & 1.95 & 3.05 & 4.30 & 5.33 & 5.64 & 5.56 & 5.54 & 4.21 & 2.63 & 1.58 & 1.06 & 1310.0 & 3.6 \\
\hline 15.0 & 1.31 & 2.04 & 3.14 & 4.36 & 5.34 & 5.61 & 5.57 & 5.63 & 4.37 & 2.79 & 1.59 & 1.17 & 1339.1 & 3.7 \\
\hline 20.0 & 1.41 & 2.12 & 3.21 & 4.39 & 5.32 & 5.57 & 5.55 & 5.68 & 4.50 & 2.92 & 1.70 & 1.27 & 1361.0 & 3.7 \\
\hline 25.0 & 1.50 & 2.19 & 3.27 & 4.39 & 5.27 & 5.49 & 5.49 & 5.71 & 4.60 & 3.05 & 1.79 & 1.37 & 1375.3 & 3.8 \\
\hline 30.0 & 1.58 & 2.24 & 3.31 & 4.57 & 5.19 & 5.33 & 5.39 & 5.70 & 4.68 & 3.15 & $1 . .88$ & 1.45 & 1381.9 & 3.8 \\
\hline 35.0 & 1.65 & 2.29 & 3.33 & 4.53 & 5.08 & 5.25 & 5.26 & 5.65 & 4.73 & 3.24 & 1.95 & 1.53 & 1380.8 & 3.8 \\
\hline 40.0 & 1.71 & 2.32 & 3.33 & 4.27 & 4.95 & 5.09 & 6.09 & 5.58 & 4.75 & 3.30 & 2.02 & 1.60 & 1371.9 & 3.8 \\
\hline 45.0 & 1.76 & 2.34 & 3.32 & 4.19 & 4.79 & 4.90 & 5.89 & 5.47 & 4.74 & 3.35 & 2.07 & 1.66 & 1355.4 & 3.7 \\
\hline 50.0 & 1.80 & 2.34 & 3.28 & 4.18 & 4.62 & 4.70 & 5.67 & 5.34 & 4.71 & 3.38 & 2.11 & 1.70 & 1333.1 & 3.7 \\
\hline 60.0 & 1.84 & 2.32 & 3.16 & $3 . \pm 1$ & 4.21 & 4.25 & 5.15 & 4.99 & 4.56 & 3.37 & 2.15 & 1.77 & 1266.9 & 3.5 \\
\hline 70.0 & 1.84 & 2.24 & 2.98 & 3.47 & 3.72 & 3.71 & 4.52 & 4.53 & 4.30 & 3.28 & 2.13 & 1.78 & 1173.4 & 3.2 \\
\hline 80.0 & 1.79 & 2.11 & 2.73 & 3.16 & 3.17 & 5.14 & 3.81 & 3.97 & 3.95 & 3.12 & 2.17 & 1.75 & 1055.8 & 2.9 \\
\hline 90.0 & 1.70 & 1.94 & 2.43 & $2 . \equiv 9$ & 2.61 & 2. 55 & 3.09 & 3.34 & 3.51 & 2.88 & 1.96 & 1.67 & 921.7 & 2.5 \\
\hline
\end{tabular}


AVERAGE DAILY TOTAL TERRESTRIAL INSOLATION ESTIMATES (KWH/SQ. M)

SITE: REDMOND

LATITUDE: 44 DEGREES 16 MINUTES

AUERAGE DAILY AMOUNTS BY MONTH

\begin{tabular}{|c|c|c|c|c|c|c|c|c|c|c|c|c|c|c|}
\hline ARRAY & $J$ & $\mathbf{F}$ & $M$ & $A$ & $M$ & $\mathrm{~J}$ & $J$ & A & $s$ & 0 & $\mathbf{N}$ & $D$ & $\begin{array}{l}\text { ANNUAL } \\
\text { AMOUNT }\end{array}$ & $\begin{array}{c}\text { AVERAGE } \\
\text { DAY }\end{array}$ \\
\hline 0.0 & 1.55 & 2.44 & 3.75 & 5.31 & 6.55 & 7.21 & 7.72 & 6.53 & 4.99 & 3.15 & 1.81 & 1.34 & 1596.1 & 4.4 \\
\hline 10.0 & 1.90 & 2.80 & 4.09 & 5.56 & 6.68 & 7.27 & 7.88 & 6.89 & 5.56 & 3.71 & 2.25 & 1.71 & 1716.0 & 4.7 \\
\hline 15.0 & 2.05 & 2.96 & 4.23 & 5.64 & 5.69 & 7.24 & 7.90 & 7.01 & 5.79 & 3.96 & 2.46 & 1.88 & 1761.5 & 4.8 \\
\hline 20.0 & 2.20 & 3.10 & 4.35 & 5.68 & 6.66 & 7.17 & 7.86 & 7.08 & 5.98 & 4.18 & 2.65 & 2.04 & 1796.7 & 4.9 \\
\hline 25.0 & 2.33 & 3.22 & 4.44 & 5.70 & 6.59 & 7.06 & 7.78 & 7.11 & 6.14 & 4.38 & 2.82 & 2.19 & 1821.2 & 5.0 \\
\hline 30.0 & $2.45^{\prime}$ & 3.32 & 4.51 & 5.68 & 6.48 & 6.91 & 7.66 & 7.10 & 6.26 & 4.55 & 2.98 & 2.32 & 1835.0 & 5.0 \\
\hline 35.0 & 2.55 & 3.40 & 4.54 & 5.63 & 6.34 & 6.72 & 7.48 & 7.05 & 6.34 & 4.69 & 3.11 & 2.44 & 1837.7 & 5.0 \\
\hline 40.0 & 2.64 & 3.47 & 4.56 & 5.54 & 6.16 & 6.50 & 7.27 & 6.95 & 6.39 & 4.81 & 3.23 & 2.54 & 1829.5 & 5.0 \\
\hline 45.0 & 2.71 & 3.51 & 4.54 & 5.43 & 5.95 & 6.24 & 7.01 & 6.82 & 6.39 & 4.89 & 3.33 & 2.63 & 1810.8 & 5.0 \\
\hline 50.0 & 2.77 & 3.53 & 4.50 & 5.29 & 5.72 & 5.97 & 6.74 & 6.65 & 6.35 & 4.94 & 3.40 & 2.70 & 1783.7 & 4.9 \\
\hline 60.0 & 2.83 & 3.51 & 4.35 & 4.93 & 5.18 & 5.33 & 6.07 & 6.19 & 6.15 & 4.95 & 3.49 & 2.79 & 1698.0 & 4.7 \\
\hline 70.0 & 2.81 & 3.41 & 4.09 & 4.46 & 4.53 & 4.59 & 5.27 & 5.58 & 5.80 & 4.83 & 3.49 & 2.81 & 1572.8 & 4.3 \\
\hline 80.0 & 2.73 & 5.22 & 3.74 & 3.90 & 3.79 & 3.79 & 4.37 & 4.85 & 5.31 & 4.59 & 3.39 & 2.75 & 1413.4 & 3.9 \\
\hline 90.0 & 2.58 & 2.96 & 3.32 & 3.26 & 3.06 & 2.98 & 3.46 & 4.01 & 4.69 & 4.23 & 3.21 & 2.62 & 1228.9 & 3.4 \\
\hline
\end{tabular}


AVERAGE DAILY TOTAL TERRESTRIAL INSOLATION ESTIMATES (KWH/SQ. M)

SITE:
LATITUDE:
L4 DEGREES 55 MINUTES

AVERAGE DAILY AMOJNTS BY MONTH

\begin{tabular}{|c|c|c|c|c|c|c|c|c|c|c|c|c|c|c|}
\hline ARRAY & $\mathrm{J}$ & $F$ & $M i$ & A & $M$ & $J$ & $J$ & A & s & 0 & $\mathrm{~N}$ & D & $\begin{array}{l}\text { ANNUAL } \\
\text { AMOUNT }\end{array}$ & $\begin{array}{l}\text { AVERAGE } \\
\text { DAY }\end{array}$ \\
\hline 0.0 & 1.05 & 1.86 & 2.98 & 4.33 & 5.48 & 5.33 & 5.76 & 5.59 & 4.19 & 2.42 & $1 . \equiv 0$ & 0.87 & 1300.9 & 3.6 \\
\hline 10.0 & 1.29 & 2.08 & 3.22 & 4.51 & 5.58 & 5.87 & 6.89 & 5.88 & 4.62 & 2.80 & 1.56 & 1.12 & 1385.2 & 3.8 \\
\hline 15.0 & 1.41 & 2.17 & 3.32 & 4.56 & 5.58 & 5.84 & 0.91 & 5.97 & 4.79 & 2.96 & 1.68 & 1.24 & 1416.2 & 3.9 \\
\hline 20.0 & 1.51 & 2.26 & 3.40 & 4.59 & 5.56 & 5.79 & 6.88 & 6.03 & 4.94 & 3.11 & 1.79 & 1.35 & 1439.3 & 3.9 \\
\hline 25.0 & 1.60 & 2.33 & 3.46 & 4.60 & 5.50 & 5.71 & 6.81 & 6.05 & 5.06 & 3.24 & 1.89 & 1.45 & 1454.4 & 4.0 \\
\hline 30.0 & 1.69 & 2.39 & 3.50 & 4.58 & 5.41 & 5.59 & 6.70 & 6.04 & 5.14 & 3.35 & 1.98 & 1.54 & 1461.3 & 4.0 \\
\hline 35.0 & 1.76 & 2.44 & 3.52 & 4.54 & 5.30 & $5 .<5$ & $\epsilon .56$ & 5.99 & 5.20 & 3.44 & 2.0 .6 & 1.62 & 1459.8 & 4.0 \\
\hline 40.0 & 1.83 & 2.47 & 3.53 & 4.47 & 5.15 & 5.28 & $\epsilon .38$ & 5.91 & 5.23 & 3.51 & 2.13 & 1.69 & 1450.1 & 4.0 \\
\hline 45.0 & 1.88 & 2.49 & 3.51 & 4.37 & 4.99 & 5.08 & 6.16 & 5.79 & 5.22 & 3.56 & 2.18 & 1.75 & 1432.2 & 3.9 \\
\hline 50.0 & 1.92 & 2.50 & 3.48 & 4.26 & 4.80 & 4.87 & 5.93 & 5.65 & 5.18 & 3.59 & 2.22 & 1.80 & 1408.3 & 3.9 \\
\hline 60.0 & 1.97 & 2.47 & 3.35 & 3.98 & 4.37 & 4.39 & 5.37 & 5.27 & 5.02 & 3.58 & 2.25 & 1.86 & 1337.0 & 3.7 \\
\hline 70.0 & 1.96 & 2.39 & 3.15 & 3.61 & 3.85 & 3.82 & 4.69 & 4.77 & 4.73 & 3.48 & 2.25 & 1.87 & 1236.5 & 3.4 \\
\hline 80.0 & 1.91 & 2.25 & 2.88 & 3.18 & 3.27 & 3.22 & 3.93 & 4.17 & 4.33 & 3.30 & 2.13 & 1.84 & 1110.5 & 3.0 \\
\hline 90.0 & 1.81 & 2.07 & 2.56 & 2.68 & 2.68 & 2.60 & 3.17 & 3.49 & 3.84 & 3.04 & 2.05 & 1.76 & 966.8 & 2.6 \\
\hline
\end{tabular}


AVERAGE DAILY TOTAL TERRESTRIAL INSOLATION ESTIMATES (KWH/SQ. M)

SITE: ALLENTOINN

EATITUDE: 40 DEGREES 39 MINUTES

AUERAGE DAILY AMOUNTS BY MONTH

\begin{tabular}{|c|c|c|c|c|c|c|c|c|c|c|c|c|c|c|}
\hline ARRAY & $J$ & $F$ & $M$ & A & $M$ & $J$ & $\mathrm{~J}$ & $A$ & 5 & 0 & $N$ & D & $\begin{array}{l}\text { ANNUAL } \\
\text { AlIOUNT }\end{array}$ & $\begin{array}{c}\text { AVERAGE } \\
\text { DAY }\end{array}$ \\
\hline 0.0 & 1.66 & 2.41 & 3.39 & 4.45 & 5.16 & 5.60 & 5.57 & 4.88 & 3.90 & 2.92 & 1.79 & 1.36 & 1313.1 & 3.6 \\
\hline 10.0 & 1.96 & 2.69 & 3.64 & 4.60 & 5.21 & 5.61 & 5.62 & 5.05 & 4.21 & 3.32 & 2.14 & 1.64 & 1391.2 & 3.8 \\
\hline 15.0 & 2.09 & 2.81 & 3.73 & 4.63 & 5.19 & 5.56 & 5.60 & 5.09 & 4.32 & 3.49 & 2.29 & 1.77 & 1419.2 & 3.9 \\
\hline 20.0 & 2.22 & 2.92 & 3.80 & 4.64 & 5.15 & 5.49 & 5.55 & 5.11 & 4.42 & 3.64 & 2.43 & 1.88 & 1439.4 & 3.9 \\
\hline 25.0 & 2.32 & 3.01 & 3.86 & 4.63 & 5.08 & 5.39 & 5.47 & 5.10 & 4.49 & 3.78 & 2.56 & 1.99 & 1451.8 & 4.0 \\
\hline 30.0 & 2.42 & 3.08 & 3.89 & 4.59 & 4.98 & 5.27 & 5.37 & 5.06 & 4.53 & 3.88 & 2.67 & 2.08 & 1456.1 & 4.0 \\
\hline 35.0 & 2.50 & 3.13 & 3.90 & 4.53 & 4.85 & 5.11 & 5.23 & 4.99 & 4.55 & 3.97 & 2.76 & 2.16 & 1452.4 & 4.0 \\
\hline 40.0 & 2.57 & 3.17 & 3.89 & 4.45 & 4.70 & 4.93 & 5.06 & 4.90 & 4.54 & 4.03 & 2.84 & 2.23 & 1440.6 & 3.9 \\
\hline 45.0 & 2.62 & 3.19 & 3.86 & 4.34 & 4.54 & 4.74 & 4.89 & 4.78 & 4.51 & 4.07 & 2.90 & 2.29 & 1422.3 & 3.9 \\
\hline 50.0 & 2.65 & 3.19 & 3.80 & 4.21 & 4.36 & 4.52 & 4.69 & 4.64 & 4.45 & 4.08 & 2.95 & 2.33 & 1396.5 & 3.8 \\
\hline 60.0 & 2.68 & 3.14 & 3.63 & 3.89 & 3.93 & 4.03 & 4.21 & 4.29 & 4.25 & 4.04 & 2.98 & 2.37 & 1322.3 & 3.6 \\
\hline 70.0 & 2.63 & 3.01 & 3.39 & 3.50 & 3.43 & 3.47 & 3.66 & 3.85 & 3.96 & 3.89 & 2.94 & 2.36 & 1220.0 & 3.3 \\
\hline 80.0 & 2.53 & 2.82 & 3.07 & 3.04 & 2.89 & 2.90 & 3.07 & 3.33 & 3.59 & 3.66 & 2.83 & 2.28 & 1095.5 & 3.0 \\
\hline 90.0 & 2.37 & 2.57 & 2.69 & 2.53 & 2.33 & 2.30 & 2.47 & 2.76 & 3.14 & 3.34 & 265 & 2.15 & 951.9 & 2.6 \\
\hline
\end{tabular}


AVERAGE DAILY TOTAL TERRESTRIAL INSOLATION ESTIMATES (KWH/SQ. M)

SITE: ERIE
LATITUDE: 22 DEGREES 5 MINUTES

AVERAGE EAILY AMOLNTS BY MONTH

\begin{tabular}{|c|c|c|c|c|c|c|c|c|c|c|c|c|c|c|}
\hline $\begin{array}{l}\text { ARRAY } \\
\text { TILT }\end{array}$ & -2 & $F$ & $M$ & $A$ & $M$ & 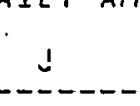 & $J$ & A & $S$ & 0 & N & $D$ & $\begin{array}{l}\text { ANNUAL } \\
\text { AMOUNT }\end{array}$ & $\begin{array}{c}\text { AVERAGE } \\
\text { DAY }\end{array}$ \\
\hline 0.0 & 1.09 & 1.82 & 2.89 & 4.29 & 5.19 & 5.82 & 5.78 & 4.59 & 3.78 & 2.61 & 1.31 & 0.88 & 1220.9 & 3.3 \\
\hline 10.0 & $1.30^{\circ}$ & 2.00 & 3.09 & 4.44 & 5.25 & 5.83 & 5.85 & 4.76 & 4.09 & 2.97 & 1.52 & 1.07 & 1285.5 & 3.5 \\
\hline 15.0 & 1.39 & 2.07 & 3.16 & 4.48 & 5.24 & 5.80 & 5.84 & 4.81 & 4.21 & 3.13 & 1.62 & 1.16 & 1307.8 & 3.6 \\
\hline 20.0 & 1.48 & 2.13 & 3.22 & 4.50 & 5.20 & 5.73 & 5.80 & 4.83 & 4.31 & 3.26 & 1.70 & 1.24 & 1323.0 & 3.6 \\
\hline 25.0 & 1.56 & 2.18 & 3.26 & 4.49 & 5.13 & 5.63 & 5.73 & 4.83 & 4.39 & 3.38 & 1.73 & 1.31 & 1331.0 & 3.6 \\
\hline 30.0 & 1.63 & 2.22 & 3.29 & 4.46 & 5.04 & 5.51 & 5.62 & 4.79 & 4.44 & 3.48 & 1.84 & 1.38 & 1331.7 & 3.6 \\
\hline 35.0 & 1.69 & 2.25 & 3.30 & 4.40 & 4.92 & $5.35 \ldots$ & 5.48 & 4.74 & 4.46 & 3.56 & 1.90 & 1.44 & 1325.2 & 3.6 \\
\hline 40.0 & 1.74 & 2.27 & 3.28 & 4.32 & 4.77 & 5.17 & 5.32 & 4.65 & 4.46 & 3.62 & 1.95 & 1.49 & 1311.5 & 3.6 \\
\hline 45.0 & 1.78 & 2.27 & 3.26 & 4.23 & 4.61 & 4.77 & 5.13 & 4.55 & 4.43 & 3.66 & 1.98 & 1.53 & 1291.6 & 3.5 \\
\hline 50.0 & 1.80 & 2.27 & 5.21 & 4.11 & 4.43 & 4.75 & 4.93 & 4.42 & 4.38 & 3.67 & 2.00 & 1.56 & 1265.5 & 3.5 \\
\hline 60.0 & 1.83 & 2.22 & $\Xi .07$ & 3.81 & 4.01 & 4.24 & 4.44 & 4.10 & 4.20 & 3.64 & 2.01 & 1.59 & 1193.3 & 3.3 \\
\hline 70.0 & 1.81 & 2.12 & 2.87 & 3.43 & 3.51 & 3.66 & 3.88 & 3.69 & 3.93 & 3.52 & 1.98 & 1.59 & 1096.2 & 3.0 \\
\hline 80.0 & 1.74 & 1.99 & 2.61 & 3.00 & 2.97 & 3.86 & 3.25 & 3.22 & 3.57 & 3.31 & $1.9 C$ & 1.54 & 979.5 & 2.7 \\
\hline 90.0 & 1.63 & 1.81 & 2.30 & 2.51 & 2.41 & 2.44 & 2.62 & 2.68 & 3.14 & 3.03 & 1.78 & 1.46 & 847.2 & 2.3 \\
\hline
\end{tabular}


AVERAGE DAILY TOTAL TERRESTRIAL INSOLATION ESTIMATES (KWH/SQ. M)

SITE: HARRISBURG
LATITUDE: 40 DEGREES 13 MINUTES

AVERAGE DAILY AMOUNTS BY MONTH

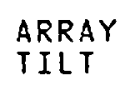

\begin{tabular}{|c|c|c|c|c|c|c|c|c|c|c|c|}
\hline $\mathbf{J}$ & $F$ & $M$ & A & $M$ & $\jmath$ & $\jmath$ & A & $S$ & 0 & $\mathbf{N}$ & D \\
\hline 1.69 & 2.43 & 3.41 & 4.45 & 5.21 & 5.70 & 5.56 & 4.89 & 3.99 & 2.95 & 1.83 & 1.41 \\
\hline 1.98 & 2.71 & 3.65 & 4.59 & 5.26 & 5.69 & 5.61 & 5.06 & 4.30 & 3.34 & 2.17 & 1.70 \\
\hline 2.11 & 2.83 & 3.74 & 4.63 & 5.24 & 5.65 & 5.59 & 5.10 & 4.42 & 3.51 & 2.33 & 1.83 \\
\hline 2.23 & 2.93 & 3.81 & 4.64 & 5.19 & 5.58 & 5.53 & 5.11 & 4.51 & 3.66 & 2.47 & 1.96 \\
\hline 2.34 & 3.02 & 3.86 & 4.62 & 5.12 & 5.47 & 5.45 & 5.10 & 4.58 & 3.79 & 2.59 & 2.07 \\
\hline 2.44 & 3.09 & 3.89 & 4.58 & 5.01 & 5.34 & 5.34 & 5.06 & 4.63 & 3.90 & 2.70 & 2.16 \\
\hline 2.52 & 3.14 & 3.90 & 4.52 & 4.89 & 5.18 & $5: 21$ & 4.99 & 4.64 & 3.98 & 2.80 & 2.25 \\
\hline 2.58 & 3.18 & 3.89 & 4.43 & 4.73 & 4.99 & 5.04 & 4.90 & 4.63 & 4.04 & 2.38 & 2.32 \\
\hline 2.63 & 3.20 & 3.85 & 4.33 & 4.57 & 4.80 & 4.86 & 4.78 & 4.60 & 4.08 & 2.94 & 2.38 \\
\hline 2.66 & 3.20 & 3.30 & 4.20 & 4.38 & 4.58 & 4.66 & 4.63 & 4.54 & 4.09 & 2.98 & 2.42 \\
\hline 2.68 & 3.14 & 3.53 & 3.88 & 3.94 & 4.07 & 4.19 & 4.28 & 4.33 & 4.04 & 3.01 & 2.46 \\
\hline 2.64 & 3.01 & 3.38 & 3.48 & 3.43 & 3.50 & 3.63 & 3.83 & 4.04 & 3.89 & 2.97 & 2.44 \\
\hline 2.53 & 2.82 & 3.06 & 3.02 & 2.89 & 2.91 & 3.05 & 3.31 & 3.65 & 3.65 & 2.85 & 2.37 \\
\hline 2.37 & 2.56 & 2.68 & 2.51 & 2.33 & 2.30 & 2.44 & 2.74 & 3.19 & 3.33 & 2.67 & 2.23 \\
\hline
\end{tabular}

ANNUAL

AMOUNT

AVERAGE

TILT

0.0

10.0

15.0

20.0

25.0

30.0

35.0

40.0

45.0

50.0

60.0

70.0

80.0

90.0

2.3

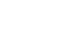

$\begin{array}{ll}1325.5 & 3.6 \\ 1403.2 & 3.8 \\ 1430.8 & 3.9 \\ 1450.7 & 4.0 \\ 1462.5 & 4.0 \\ 1466.3 & 4.0 \\ 1462.0 & 4.0 \\ 1449.6 & 4.0 \\ 1430.8 & 3.9 \\ 1404.2 & 3.8 \\ 1328.4 & 3.6 \\ 1224.5 & 3.4 \\ 1098.5 & 3.0 \\ 953.3 & 2.6\end{array}$


AVERAGE DAILY TOTAL TERRESTRIAL INSOLATION ESTIMATES (KWH/SQ. M)

SITE: PHILADELPHIA

LATITUDE: 39 DEGREES 53 MINUTES

AVERAGE DAILY AMOUNTS BY MONTH

\begin{tabular}{|c|c|c|c|c|c|c|c|c|c|c|c|c|c|c|}
\hline ARRAY & $\mathrm{J}$ & $F$ & $M$ & A & $M$ & $\mathrm{~J}$ & $J$ & $A$ & $s$ & 0 & $N$ & $D$ & $\begin{array}{l}\text { ANNUAL } \\
\text { AMOUNT }\end{array}$ & $\begin{array}{c}\text { AVERAGE } \\
\text { DAY }\end{array}$ \\
\hline 0.0 & 1.75 & 2.51 & 3.49 & 4.52 & 5.24 & 5.71 & 5.55 & 4.97 & 4.03 & 3.02 & 1.95 & 1.49 & 1347.0 & 3.7 \\
\hline 10.0 & 2.06 & 2.80 & 3.73 & 4.66 & 5.28 & 5.70 & 5.59 & 5.14 & 4.34 & 3.43 & 2.33 & 1.79 & 1427.2 & 3.9 \\
\hline 15.0 & 2.19 & 2.92 & 3.83 & 4.70 & 5.26 & 5.66 & 5.57 & 5.18 & 4.46 & 3.60 & 2.50 & 1.93 & 1455.8 & 4.0 \\
\hline 20.0 & 2.32 & 3.03 & 3.90 & 4.71 & 5.21 & 5.58 & 5.52 & 5.19 & 4.56 & 3.75 & 2.65 & 2.06 & 1476.5 & 4.0 \\
\hline 25.0 & 2.43 & 3.12 & 3.95 & 4.69 & 5.13 & 5.48 & 5.44 & 5.18 & 4.63 & 3.88 & 2.79 & 2.18 & 1489.0 & 4.1 \\
\hline 30.0 & 2.53 & 3.19 & 3.98 & 4.65 & 5.03 & 5.34 & 5.33 & 5.14 & 4.67 & 3.99 & 2.91 & 2.28 & 1493.3 & 4.1 \\
\hline 35.0 & 2.61 & 3.25 & 3.99 & 4.59 & 4.90 & 5.18 & 5.19 & 5.07 & 4.68 & 4.08 & 3.02 & 2.37 & 1489.3 & 4.1 \\
\hline 40.0 & 2.68 & 3.28 & 3.98 & 4.49 & 4.74 & 4.99 & 5.02 & 4.97 & 4.67 & 4.14 & 3.13 & 2.45 & 1477.0 & 4.0 \\
\hline 45.0 & 2.73 & 3.30 & 3.94 & 4.39 & 4.58 & 4.79 & 4.84 & 4.85 & 4.63 & 4.17 & 3.17 & 2.51 & 1458.2 & 4.0 \\
\hline 50.0 & 2.77 & 3.30 & 3.89 & 4.25 & 4.39 & 4.57 & 4.64 & 4.70 & 4.57 & 4.19 & $3.2 ?$ & 2.56 & 1431.4 & 3.9 \\
\hline 60.0 & 2.79 & 3.24 & 3.71 & 3.92 & 3.95 & 4.06 & 4.16 & 4.33 & 4.36 & 4.13 & 3.25 & 2.60 & 1354.6 & 3.7 \\
\hline 70.0 & 2.74 & 3.11 & 3.45 & 3.52 & 3.43 & 3.48 & 3.61 & 3.88 & 4.06 & 3.98 & 3.21 & 2.58 & 1249.0 & 3.4 \\
\hline 80.0 & 2.63 & 2.91 & 3.12 & 3.05 & 2.89 & 2.90 & 3.02 & 3.35 & 3.67 & 3.73 & 3.03 & 2.50 & 1120.9 & 3.1 \\
\hline 90.0 & 2.45 & 2.64 & 2.73 & 2.53 & 2.32 & 2.29 & 2.42 & 2.76 & 3.20 & 3.40 & 2.83 & 2.36 & 972.9 & 2.7 \\
\hline
\end{tabular}


AVERAGE DAILY TOTAL TERRESTRIAL INSOLATION ESTIMATES (KWH/SQ. M)

SITE: PITTSBURGH

LATITUDE: 40 DEGREES 30. MINUTES

AVERAGE DAILY AMOUNTS BY MONTH

\begin{tabular}{|c|c|c|c|c|c|c|c|c|c|c|c|c|c|c|}
\hline APRAY & $J$ & $F$ & $M$ & A & $M$ & 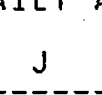 & J & A & 5 & 0 & $\mathrm{~N}$ & $\mathrm{D}$ & $\begin{array}{l}\text { ANNUAL } \\
\text { AMOUNT }\end{array}$ & $\begin{array}{c}\text { AVERAGE } \\
\text { DAY }\end{array}$ \\
\hline 0.0 & 1.34 & 1.97 & 2.97 & 4.15 & 5.05 & 5.56 & 5.33 & 4.76 & 3.80 & 2.82 & 1.59 & 1.10 & 1232.7 & 3.4 \\
\hline 10.0 & 1.52 & 2.16 & 3.16 & 4.28 & 5.10 & 5.56 & 5.38 & 4.92 & 4.10 & 3.20 & 1.36 & 1.35 & 1297.8 & 3.6 \\
\hline 15.0 & 1.60 & 2.24 & 3.23 & 4.31 & 5.08 & 5.52 & 5.36 & 4.97 & 4.21 & 3.36 & 1.98 & 1.47 & 1320.2 & 3.6 \\
\hline 20.0 & 1.68 & 2.30 & 3.28 & 4.32 & 5.03 & 5.44 & 5.31 & 4.98 & 4.30 & 3.50 & 2.10 & 1.57 & 1335.5 & 3.7 \\
\hline 25.0 & 1.74 & 2.36 & 3.32 & 4.30 & 4.96 & 5.35 & 5.24 & 4.97 & 4.36 & 3.63 & 2.19 & 1.67 & 1343.6 & 3.7 \\
\hline 30.0 & 1.80 & 2.40 & 3.34 & 4.27 & 4.87 & 5.22 & 5.13 & 4.93 & 4.40 & 3.73 & 2.28 & 1.76 & 1344.4 & 3.7 \\
\hline 35.0 & 1.84 & 2.43 & 3.34 & 4.21 & 4.74 & 5.06 & 5.00 & 4.86 & 4.42 & 3.81 & 2.35 & 1.84 & 1337.9 & 3.7 \\
\hline 40.0 & 1.88 & 2.45 & 3.33 & 4.13 & 4.60 & 4.88 & 4.84 & 4.77 & 4.41 & 3.86 & 2.41 & 1.90 & 1324.1 & 3.6 \\
\hline 45.0 & 1.91 & 2.45 & 3.30 & 4.03 & 4.44 & 4.69 & 4.67 & 4.66 & 4.37 & 3.90 & 2.46 & 1.96 & 1304.6 & 3.6 \\
\hline 50.0 & 1.92 & 2.44 & 3.24 & 3.91 & 4.26 & 4.48 & 4.48 & 4.52 & 4.31 & 3.91 & 2.49 & 2.00 & 1278.3 & 3.5 \\
\hline 60.0 & 1.92 & 2.38 & 3.09 & 3.61 & 3.84 & 3.99 & 4.03 & 4.17 & 4.12 & 3.86 & 2.51 & 2.04 & 1205.5 & 3.3 \\
\hline 70.0 & 1.87 & 2.28 & 2.88 & 3.25 & 3.35 & 3.43 & 3.51 & 3.74 & 3.84 & 3.72 & 2.46 & 2.04 & 1107.9 & 3.0 \\
\hline 80.0 & 1.79 & 2.13 & 2.61 & 2.83 & 2.82 & 2.87 & 2.95 & 3.24 & 3.48 & 3.49 & 2.36 & 1.98 & 991.1 & 2.7 \\
\hline 90.0 & 1.66 & 1.93 & 2.29 & 2.36 & 2.29 & 2.27 & 2.37 & 2.69 & 3.04 & 3.18 & 2.21 & 1.87 & 857.7 & 2.3 \\
\hline
\end{tabular}


AVERAGE DAILY TOTAL TERRESTRIAL INSOLATION ESTIMATES (KWH/SQ. M)

SITE: WILKES-EARRE-SCRANPA

LATITUDE: 41 IEEGRES 20 MINUTES

ALERAGE DAILY AMOLNTS BY MONTH

\begin{tabular}{|c|c|c|c|c|c|c|c|c|c|c|c|c|c|c|}
\hline $\begin{array}{l}\text { ARRAY } \\
\text { TILT }\end{array}$ & $\mathrm{J}$ & $F$ & $M$ & A & $M$ & J & 7 & A & 5 & 0 & $\mathrm{~N}$ & $D$ & $\begin{array}{l}\text { ANNUAL } \\
\text { AMOUNT }\end{array}$ & $\begin{array}{l}\text { AVERAGE } \\
\text { DAY }\end{array}$ \\
\hline 0.0 & 1.44 & 2.17 & 3.12 & 4.22 & 5.01 & 5.55 & 5.50 & 4.77 & 3.77 & 2.83 & 1.55 & 1.16 & 1252.4 & 3.4 \\
\hline 10.0 & 1.67 & 2.41 & 3.34 & 4.36 & 5.06 & 5.55 & 5.56 & 4.95 & 4.07 & 3.22 & 1.82 & 1.38 & 1322.6 & 3.6 \\
\hline 15.0 & 1.78 & 2.51 & 3.42 & 4.39 & 5.05 & 5.52 & 5.55 & 5.00 & 4.19 & 3.39 & 1.95 & 1.48 & 1347.3 & 3.7 \\
\hline 20.0 & 1.87 & 2.60 & 3.48 & 4.41 & 5.01 & 5.45 & 5.50 & 5.01 & 4.28 & 3.54 & 2.06 & 1.57 & 1364.7 & 3.7 \\
\hline 25.0 & 1.96 & 2.68 & 3.53 & 4.40 & 4.94 & 5.35 & 5.43 & 5.01 & 4.35 & 3.67 & 2.16 & 1.65 & 1374.7 & 3.8 \\
\hline 30.0 & 2.03 & 2.74 & 3.56 & 4.36 & 4.85 & 5.23 & 5.33 & 4.97 & 4.40 & 3.78 & 2.25 & 1.72 & 1377.2 & 3.8 \\
\hline 35.0 & 2.10 & 2.78 & 3.57 & 4.30 & 4.73 & 5.08 & 5.19 & 4.91 & 4.42 & 3.87 & 2.33 & 1.78 & 1372.2 & 3.8 \\
\hline 40.0 & 2.15 & 2.81 & 3.56 & 4.23 & -4.59 & 4.90 & 5.03 & 4.82 & 4.41 & 3.93 & 2.39 & 1.83 & 1359.8 & 3.7 \\
\hline 45.0 & 2.18 & 2.83 & 3.53 & 4.13 & 4.43 & 4.71 & 4.86 & 4.71 & 4.38 & 3.97 & 2.44 & 1.87 & 1341.1 & 3.7 \\
\hline 50.0 & 2.21 & 2.83 & 3.48 & 4.01 & 4.26 & 4.50 & 4.66 & 4.57 & 4.33 & 3.99 & 2.47 & 1.91 & 1315.5 & 3.6 \\
\hline 60.0 & 2.22 & 2.78 & 3.32 & 3.71 & 3.84 & 4.02 & 4.20 & 4.23 & 4.14 & 3.95 & 2.49 & 1.93 & 1243.6 & 3.4 \\
\hline 70.0 & 2.19 & 2.67 & 3.10 & 3.34 & 3.36 & 3.47 & 3.66 & 3.80 & 3.87 & 3.81 & 2.45 & 1.91 & 1145.8 & 3.1 \\
\hline 80.0 & 2.10 & 2.50 & 2.82 & 2.91 & 2.84 & 2.90 & 3.08 & 3.30 & 3.51 & 3.59 & 2.35 & 1.85 & 1027.3 & 2.8 \\
\hline 90.0 & 1.96 & 2.28 & $2 .<8$ & 2.44 & 2.31 & 2.31 & 2.48 & 2.74 & 3.08 & 3.28 & 2.21 & 1.74 & 891.6 & 2.4 \\
\hline
\end{tabular}


AVERAGE DAILY TOTAL TERRESTRIAL INSOLATION ESTIMATES (KWH/SQ. M)

$\begin{array}{rrr}\text { SITE: } & \text { PROVIDENCE } & \text { RI } \\ \text { LATITUDE: } & 4 I \text { DEGREES } 44 \text { MINUTES }\end{array}$

AVERAGE DAILY AMOUNTS BY MONTH

\begin{tabular}{|c|c|c|c|c|c|c|c|c|c|c|c|c|c|c|}
\hline ARRAY & $J$ & $F$ & $M$ & A & $M$ & $\mathrm{~J}$ & $J$ & A & 5 & 0 & $N$ & $D$ & $\begin{array}{l}\text { ANNUAL } \\
\text { AMOUNT }\end{array}$ & $\begin{array}{c}\text { AVERAGE } \\
\text { DAY }\end{array}$ \\
\hline 0.0 & 1.60 & 2.33 & 3.25 & 4.34 & 5.22 & 5.59 & 5.34 & 4.72 & 3.81 & 2.86 & 1.70 & 1.32 & 1282.0 & 3.5 \\
\hline 10.0 & 1.90 & 2.61 & 3.49 & 4.49 & 5.28 & 5.61 & 5.40 & 4.90 & 4.12 & 3.26 & 2.03 & 1.61 & 1361.6 & 3.7 \\
\hline 15.0 & 2.03 & 2.73 & 3.58 & 4.53 & 5.27 & 5.57 & 5.39 & 4.95 & 4.24 & 3.44 & 2.18 & 1.74 & 1390.6 & 3.8 \\
\hline 20.0 & 2.16 & 2.83 & 3.66 & 4.54 & 5.23 & 5.50 & 5.35 & 4.97 & 4.34 & 3.60 & 2.32 & 1.87 & 1412.0 & 3.9 \\
\hline 25.0 & 2.27 & 2.93 & $3.7 \mathrm{i}$ & 4.54 & 5.16 & 5.41 & 5.28 & 4.96 & 4.41 & 3.73 & 2.45 & 1.98 & 1425.7 & 3.9 \\
\hline 30.0 & 2.36 & 3.00 & 3.74 & 4.50 & 5.07 & 5.29 & 5.18 & 4.93 & 4.46 & 3.85 & 2.56 & 2.08 & 1431.5 & 3.9 \\
\hline 35.0 & 2.45 & 3.06 & 3.76 & 4.45 & 4.94 & 5.13 & 5.05 & 4.87 & 4.48 & 3.94 & 2.66 & 2.17 & 1429.4 & 3.9 \\
\hline 40.0 & 2.52 & 3.10 & 3.75 & 4.37 & 4.80 & 4.96 & 4.90 & 4.78 & 4.48 & 4.01 & 2.74 & 2.24 & 1419.4 & 3.9 \\
\hline 45.0 & 2.57 & 3. 12 & 3.72 & 4.26 & 4.63 & 4.77 & 4.73 & 4.67 & 4.45 & 4.05 & 2.80 & 2.30 & 1402.7 & 3.8 \\
\hline 50.0 & 2.61 & 3.12 & 3.67 & 4.14 & 4.45 & 4.56 & 4.54 & 4.54 & 4.39 & 4.07 & 2.84 & 2.35 & 1378.8 & 3.8 \\
\hline 60.0 & 2.64 & 3.08 & 3.52 & 3.84 & 4.02 & 4.07 & 4.10 & 4.20 & 4.21 & 4.03 & 2.88 & 2.40 & 1308.8 & 3.6 \\
\hline 70.0 & 2.61 & 2.96 & 3.29 & 3.46 & 3.52 & 3.52 & 3.58 & 3.78 & 3.94 & 3.90 & 2.85 & 2.39 & 1210.9 & 3.3 \\
\hline 80.0 & 2.51 & 2.78 & 2.99 & 3.02 & 2.97 & 2.95 & 3.02 & 3.29 & 3.57 & 3.68 & 2.75 & 2.32 & 1090.3 & 3.0 \\
\hline 90.0 & .2 .35 & 2.54 & 2.63 & 2.52 & 2.41 & 2.35 & 2.44 & 2.74 & 3.14 & 3.36 & 2.58 & 2.20 & 950.8 & 2.6 \\
\hline
\end{tabular}


AVERAGE DAILY TOTAL TERRESTRIAL INSOLATION ESTIMATES (KWH/SQ. M)

SITE: CHARLESTON
LATITUDE: 32 DEGREES 54 MINUTES

AVERAGE EAILY AMOUNTS BY MONTH

\begin{tabular}{|c|c|c|c|c|c|c|c|c|c|c|c|c|c|c|}
\hline \multirow[b]{2}{*}{$\begin{array}{l}\text { ARRAY } \\
\text { TILT }\end{array}$} & \multicolumn{12}{|c|}{ 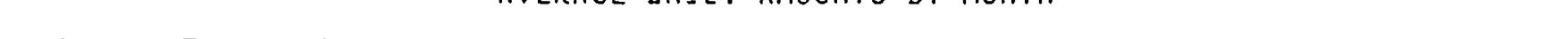 } & \multirow{2}{*}{$\begin{array}{l}\text { ANNUAL } \\
\text { AMOUNT }\end{array}$} & \multirow{2}{*}{$\begin{array}{l}\text { A VERAGE } \\
\text { DAY }\end{array}$} \\
\hline & 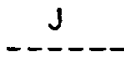 & $F$ & $M$ & A & $M$ & 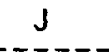 & $J^{2}$ & A & 5 & 0 & N & $D$ & & \\
\hline 0.0 & 2.34 & 3.14 & 4.21 & 5.47 & 5.86 & 5.81 & 5.67 & 5.00 & 4.39 & 3.76 & 2.94 & 2.27 & 1548.5 & 4.2 \\
\hline 10.0 & 2.66 & 3.44 & 4.45 & 5.58 & 5.84 & 5.74 & 5.64 & 5.10 & 4.64 & 4.17 & 3.42 & 2.66 & 1623.7 & 4.4 \\
\hline 15.0 & 2.80 & 3.56 & 4.53 & 5.59 & 5.78 & 5.66 & 5.59 & 5.10 & 4.73 & 4.34 & 3.63 & 2.84 & 1648.0 & 4.5 \\
\hline 20.0 & 2.93 & 3.66 & 4.59 & 5.57 & 5.69 & 5.54 & 5.50 & 5.08 & 4.79 & 4.48 & 3.82 & 2.99 & 1663.2 & 4.6 \\
\hline 25.0 & 3.04 & 3.74 & 4.52 & 5.52 & .5 .56 & 5.40 & 5.38 & 5.03 & 4.82 & 4.60 & 3.98 & 3.13 & 1669.1 & 4.6 \\
\hline 30.0 & 3.13 & 3.80 & 4.53 & 5.44 & 5.41 & $E .23$ & 5.24 & 4.96 & 4.83 & 4.69 & 4.12 & 3.26 & 1665.7 & 4.6 \\
\hline 35.0 & 3.20 & 3.84 & 4.51 & 5.33 & 5.24 & 5.04 & 5.07 & 4.86 & 4.81 & 4.76 & 4.24 & 3.36 & 1653.7 & 4.5 \\
\hline 40.0 & 3.25 & 3.86 & 4.56 & 5.19 & 5.04 & 4.83 & 4.88 & 4.73 & 4.76 & 4.79 & 4.33 & 3.44 & 1633.3 & 4.5 \\
\hline 45.0 & 3.29 & 3.85 & 4.49 & 5.03 & 4.82 & 4.60 & 4.67 & 4.59 & 4.69 & 4.80 & 4.39 & 3.50 & 1603.9 & 4.4 \\
\hline 50.0 & 3.31 & 3.83 & 4.40 & 4.83 & 4.58 & 4.35 & 4.43 & 4.41 & 4.59 & 4.78 & 4.43 & 3.54 & 1565.7 & 4.3 \\
\hline 60.0 & 3.28 & 3.71 & 4.14 & 4.38 & 4.02 & 3.78 & 3.90 & 4.00 & 4.31. & 4.65 & 4.42 & 3.56 & 1464.2 & 4.0 \\
\hline 70.0 & 3.17 & 3.51 & 3.79 & 3.84 & 3.41 & 3.18 & 3.31 & 3.51 & 3.94 & 4.41 & 4.30 & 3.48 & 1333.4 & 3.7 \\
\hline 80.0 & 3.00 & 3.23 & 3.36 & 3.22 & 2.78 & 2.56 & 2.70 & 2.96 & 3.50 & 4.07 & 4.07 & 3.33 & 1178.9 & 3.2 \\
\hline 90.0 & 2.75 & 2.88 & 2.87 & 2.58 & 2.14 & 1.98 & 2.10 & 2.39 & 2.98 & 3.63 & 3.75 & 3.09 & 1007.6 & 2.8 \\
\hline
\end{tabular}


AVERAGE DAILY TOTAL TERRESTRIAL INSOLATION ESTIMATES (KWH/SQ. M)

SITE: COLUNBIA
ITUDE: 33 DEEREES 57 MINUTES

AVERAGE DAILY AMOUNTS BY MONTH

\begin{tabular}{|c|c|c|c|c|c|c|c|c|c|c|c|c|c|c|}
\hline ARRAY & J & $F$ & $M$ & A & $M$ & $J$ & J & $A$ & $\mathrm{~s}$ & 0 & $N$ & $D$ & $\begin{array}{l}\text { ANNUAL } \\
\text { AMOUNT }\end{array}$ & $\begin{array}{c}\text { AVERAGE } \\
\text { DAY }\end{array}$ \\
\hline 0.0 & 2.39 & 3.22 & 4.27 & 5.50 & 5.97 & 6.14 & 5.80 & 5.37 & 4.53 & 3.82 & 2.90 & 2.28 & 1589.4 & 4.4 \\
\hline 10.0 & 2.75 & 3.55 & 4.52 & 5.63 & 5.96 & 6.07 & 5.79 & 5.49 & 4.81 & 4.27 & 3.40 & 2.70 & 1672.7 & 4.6 \\
\hline 15.0 & 2.91 & 3.68 & 4.61 & 5.65 & 5.91 & 5.99 & 5.74 & 5.51 & 4.91 & 4.45 & 3.62 & 2.88 & 1700.5 & 4.7 \\
\hline 20.0 & 3.05 & 3.79 & 4.68 & 5.63 & 5.82 & 5.88 & 5.65 & 5.49 & 4.98 & 4.61 & 3.81 & 3.06 & 1718.7 & 4.7 \\
\hline 25.0 & 3.17 & 3.89 & 4.72 & 5.59 & 5.70 & 5.73 & 5.54 & 5.45 & 5.03 & 4.75 & 3.99 & 3.21 & 1727.3 & 4.7 \\
\hline 30.0 & 3.28 & 3.96 & 4.73 & 5.51 & 5.55 & 5.55 & 5.39 & 5.37 & 5.04 & 4.85 & 4.14 & 3.34 & 1726.1 & 4.7 \\
\hline 35.0 & 3.36 & 4.00 & 4.72 & 5.40 & 5.37 & 5.35 & 5.22 & 5.27 & 5.03 & 4.93 & 4.27 & 3.46 & 1715.5 & 4.7 \\
\hline 40.0 & 3.43 & 4.03 & 4.58 & 5.27 & 5.18 & 5.13 & 5.03 & 5.14 & 4.99 & 4.97 & 4.36 & 3.55 & $16 \% 6.5$ & 4.6 \\
\hline 45.0 & 3.47 & 4.03 & 4.51 & 5.11 & 4.96 & 4.88 & 4.82 & 4.98 & 4.92 & 4.99 & 4.44 & 3.62 & 1668.0 & 4.8 \\
\hline 50.0 & 3.49 & 4.01 & 4.52 & 4.92 & 4.71 & 4.62 & 4.58 & 4.80 & 4.82 & 4.97 & 4.48 & 3.67 & 1630.2 & 4.5 \\
\hline 60.0 & 3.48 & 3.90 & 4.26 & 4.47 & 4.15 & 4.01 & 4.03 & 4.36 & 4.54 & 4.85 & 4.48 & 3.69 & 1528.0 & 4.2 \\
\hline 70.0 & 3.38 & 3.70 & 3.91 & 3.93 & 3.52 & 3.37 & 3.42 & 3.83 & 4.17 & 4.62 & 4.37 & 3.63 & 1394.2 & 3.8 \\
\hline 80.0 & 3.20 & 3.41 & 3.48 & 3.31 & 2.87 & 2.71 & 2.80 & 3.23 & 3.70 & 4.27 & 4.15 & 3,48 & 1235.4 & 3.4 \\
\hline 90.0 & 2.95 & 3.06 & 2.98 & 2.66 & 2.22 & 2.08 & 2.17 & 2.60 & 3.16 & 3.83 & 3.83 & 3.24 & 1057.4 & 2.9 \\
\hline
\end{tabular}


AVERAGE DAILY TOTAL TERRESTRIAL INSOLATION ESTIMATES (KWH/SQ. M)

SITE: SREENVI!LE-SPARTANSC

L.ATITUDE: 34 DEGREES 54 MINUTES

AYERAGE DAIIY AMOUNTS BY MOHTH

\begin{tabular}{|c|c|c|c|c|c|c|c|c|c|c|c|c|c|c|}
\hline ARRAY. & 3 & $F$ & $M$ & A & $M$ & $J$ & $\mathrm{~J}$ & A & $s$ & 0 & $\mathrm{~N}$ & $D$ & $\begin{array}{l}\text { ANNUAL } \\
\text { AMOUNT }\end{array}$ & $\begin{array}{c}\text { AVERAGE } \\
\text { DAY }\end{array}$ \\
\hline 0.0 & 2.29 & 3.10 & 4.19 & 5.36 & 5.79 & b. 04 & 5.77 & 5.38 & 4.42 & 3.72 & 2.77 & 2.12 & 1550.8 & 4.2 \\
\hline 10.0 & 2.65 & 3.42 & 4.45 & 5.49 & 5.79 & 5.99 & 5.77 & 5.49 & 4.70 & 4.17 & 3.26 & 2.51 & 1634.4 & 4.5 \\
\hline 15.0 & 2.80 & 3.56 & 4.54 & 5.51 & 5.74 & 5.92 & 5.72 & $5.5 \mathrm{i}$ & 4.81 & 4.35 & $3 .<8$ & 2.69 & 1662.8 & 4.6 \\
\hline 20.0 & 2.94 & 3.67 & 4.61 & 5.50 & 5.66 & 5.81 & 5.64 & 5.50 & 4.88 & 4.52 & 3.6 .7 & 2.85 & 1681.8 & 4.6 \\
\hline 25.0 & 3.06 & 3.76 & 4.65 & 5.46 & 5.55 & 5.67 & 5.53 & $5.4 t$ & 4.93 & 4.65 & 3.85 & 3.00 & 1691.4 & 4.6 \\
\hline 30.0 & 3.17 & 3.83 & 4.67 & 5.39 & 5.41 & 5.50 & 5.39 & 5.39 & .4 .95 & 4.76 & 4. 8.0 & 3.13 & 1691.5 & 4.6 \\
\hline 35.0 & 3.25 & 3.88 & 4.66 & 5.29 & 5.24 & 5.30 & 5.22 & 5.29 & 4.94 & 4.84 & 4.12 & 3.23 & 1682.1 & 4.6 \\
\hline 40.0 & 3.32 & 3.91 & .4 .62 & 5.16 & 5.06 & 5.09 & 5.04 & 5.17 & 4.90 & 4.89 & 4.22 & 3.32 & 1664.8 & 4.6 \\
\hline 45.0 & 3.37 & 3.91 & 4.56 & 5.01 & 4.85 & c. .86 & 4.83 & 5.01 & 4.84 & 4.91 & 4.30 & 3.39 & 1638.1 & 4.5 \\
\hline 50.0 & 3.39 & 3.90 & 4.48 & 4.83 & 4.62 & $c .60$ & 4.59 & 4.83 & 4.74 & 4.90 & 4.34 & 3.44 & 1602.4 & 4.4 \\
\hline 60.0 & 3.38 & 3.79 & 4.23 & 4.40 & 4.09 & $c .01$ & 4.06 & 4.40 & 4.48 & 4.79 & 4.35 & 3.47 & 1504.8 & 4.1 \\
\hline 70.0 & 3.30 & 3.61 & 3.59 & 3.88 & 3.48 & 3.59 & 3.46 & 3.89 & 4.12 & 4.57 & 4.26 & 3.41 & 1375.6 & 3.8 \\
\hline 80.0 & 3.13 & 3.34 & 3.47 & 3.29 & 2.87 & 2.74 & 2.85 & 3.28 & 3.67 & 4.24 & 4.05 & 3.28 & 1222.3 & 3.3 \\
\hline 90.0 & 2.89 & 3.00 & 2.99 & 2.66 & 2.23 & 2.12 & 2.21 & 2.65 & 3.15 & 3.81 & 3.75 & 3.06 & 1048.8 & 2.9 \\
\hline
\end{tabular}


AVERAGE DAILY TOTAL TERRESTRIAL INSOLATION ESTIMATES (KLH/SQ. M)

$\begin{aligned} \text { SITE: } & \text { HURON } \\ \text { LATITUDE: } & 44 \text { DEGREES } 23 \text { MINUTES }\end{aligned}$

AYERAGE DAILY AMOUNTS BY MONTH

\begin{tabular}{|c|c|c|c|c|c|c|c|c|c|c|c|c|c|c|}
\hline ARRAY & $J$ & ' $F$ & $M$ & A & $M$ & $J$ & $\mathrm{~J}$ & A & $s$ & 0 & $N$ & D & $\begin{array}{l}\text { ANNUAL } \\
\text { AMOUNT }\end{array}$ & $\begin{array}{c}\text { AVERAGE } \\
\text { DAY }\end{array}$ \\
\hline 0.0 & 1.54 & 2.35 & 3.50 & 4.82 & 5.90 & 6.63 & 6.89 & 5.97 & 4.47 & 3.12 & 1.82 & 1.28 & 1471.9 & 4.0 \\
\hline 10.0 & 1.89 & 2.68 & 3.82 & 5.03 & 6.01 & 6.68 & 7.02 & 6.28 & 4.93 & 3.67 & 2.28 & 1.62 & 1582.1 & 4.3 \\
\hline 15.0 & 2.04 & 2.83 & 3.94 & 5.10 & 6.01 & 6.65 & 7.03 & 6.38 & 5.12 & 3.92 & 2.49 & 1.78 & 1624.1 & 4.4 \\
\hline 20.0 & 2.19 & 2.96 & 4.05 & 5.14 & 5.98 & 6.58 & 7.00 & 6.44 & 5.28 & 4.14 & 2.58 & 1.93 & 1656.9 & 4.5 \\
\hline 25.0 & 2.32 & 3.08 & 4.13 & 5.14 & 5.92 & 6.49 & 6.93 & 6.46 & 5.41 & 4.34 & 2.36 & 2.06 & 1680.1 & 4.6 \\
\hline 30.0 & 2.44 & 3.17 & 4.18 & 5.12 & 5.82 & 6.35 & 6.82 & 6.45 & 5.51 & 4.50 & 3.02 & 2.19 & 1693.5 & 4.8 \\
\hline 35.0 & 2.55 & 3.25 & 4.22 & 5.07 & 5.70 & 6.18 & 6.66 & 6.40 & 5.57 & 4.65 & 3.16 & 2.30 & 1697.0 & 4.6 \\
\hline 40.0 & 2.63 & 3.31 & 4.23 & 5.00 & 5.54 & 5.98 & 6.47 & 6.31 & 5.60 & 4.76 & 3.28 & 2.39 & 1690.6 & 4.6 \\
\hline 45.0 & 2.71 & 3.35 & 4.21 & 4.89 & 5.35 & 5.74 & 6.25 & 6.18 & 5.59 & 4.84 & 3.38 & 2.47 & 1674.6 & 4.6 \\
\hline 50.0 & 2.76 & 3.37 & 4.17 & 4.77 & 5.15 & 5.50 & 6.01 & 6.03 & 5.55 & 4.89 & 3.76 & 2.54 & 1650.9 & 4.5 \\
\hline 50.0 & 2.82 & 3.34 & 4.03 & 4.44 & 4.68 & 4.93 & 5.43 & 5.61 & 5.37 & 4.90 & 3.55 & 2.62 & 1575.1 & 4.3 \\
\hline 70.0 & 2.81 & $3: 24$ & 3.79 & 4.02 & 4.10 & 4.26 & 4.74 & 5.07 & 5.06 & 4.78 & 3.55 & 2.64 & 1463.3 & 4.0 \\
\hline 30.0 & 2.72 & 3.07 & 3.47 & 3.53 & 3.46 & 3.55 & 3.96 & 4.42 & 4.63 & 4.55 & 3.75 & 2.58 & 1320.5 & 3.6 \\
\hline 70.0 & 2.57 & 2.82 & 3.07 & 2.96 & 2.82 & 2.82 & 3.17 & 3.68 & 4.09 & 4.19 & 3.27 & 2.46 & 1154.4 & 3.2 \\
\hline
\end{tabular}


AVERAGE DAILY TOTAL TERRESTRIAL INSOLATION ESTIMATES (KWH/S?. M)

SITE: PIERRE
LATITUDE: 44 DEGREES 23 MINUTES

AVERAGE DAILY AMOUNTS BY MONTH

\begin{tabular}{|c|c|c|c|c|c|c|c|c|c|c|c|c|c|c|}
\hline ARRAY & $\mathbf{J}$ & $F$ & $M$ & A & $M$ & $\mathrm{~J}$ & $J$ & A & 5 & 0 & $N$ & $D$ & $\begin{array}{l}\text { ANNUAL } \\
\text { AMOUNT }\end{array}$ & $\begin{array}{c}\text { AUERAGE } \\
\text { DAY }\end{array}$ \\
\hline 0.0 & 1.67 & 2.51 & 3.80 & 5.09 & 6.19 & 6.92 & 7.18 & 6.29 & 4.72 & 3.31 & 1.95 & 1.39 & 1555.8 & 4.3 \\
\hline 10.0 & 2.07 & 2.88 & 4.16 & 5.32 & 6.31 & 6.97 & 7.32 & 6.63 & 5.23 & 3.93 & 2.49 & 1.79 & 1679.3 & 4.6 \\
\hline 15.0 & 2.25 & 3.05 & 4.30 & 5.39 & 6.31 & 6.94 & 7.34 & 6.74 & 5.44 & 4.20 & 2.73 & 1.98 & 1726.9 & 4.7 \\
\hline 20.0 & 2.41 & 3.19 & 4.42 & 5.43 & 6.28 & 6.88 & 7.30 & 6.81 & 5.62 & 4.45 & 2.95 & 2.15 & 1764.5 & 4.8 \\
\hline 25.0 & 2.57 & 3.32 & 4.32 & 5.45 & 6.22 & 6.78 & 7.23 & 6.84 & 5.76 & 4.66 & 3.15 & 2.31 & 1791.8 & 4.9 \\
\hline 30.0 & 2.70 & 3.43 & 4.58 & 5.43 & 6.12 & 6.63 & 7.11 & 6.83 & 5.87 & 4.85 & 3.34 & 2.45 & 1808.4 & 5.0 \\
\hline 35.0 & 2.83 & 3.52 & 4.52 & 5.38 & 5.99 & 6.46 & 6.95 & 6.78 & 5.94 & 5.01 & 3.50 & 2.58 & 1814.3 & 5.0 \\
\hline 40.0 & 2.93 & 3.59 & 4.34 & 5.30 & 5.82 & 6.24 & 6.76 & 6.68 & 5.98 & 5.14 & 3.64 & 2.69 & 1809.5 & 5.0 \\
\hline 45.0 & 3.01 & 3.63 & 4.53 & 5.19 & 5.62 & 5.99 & 6.52 & 6.55 & 5.97 & 5.23 & 3.76 & 2.79 & 1794.2 & 4.9 \\
\hline 50.0 & 3.08 & 3.66 & 4.59 & 5.06 & 5.41 & 5.74 & 6.27 & 6.39 & 5.93 & 5.29 & 3.85 & 2.87 & 1770.4 & 4.9 \\
\hline 60.0 & 3.15 & 3.64 & 4.43 & 4.71 & 4.90 & 5.13 & 5.66 & 5.95 & 5.75 & 5.31 & 3.96 & 2.96 & 1691.8 & 4.6 \\
\hline 70.0 & 3.14 & 3.54 & 4.17 & 4.26 & 4.30 & 4.43 & 4.93 & 5.37 & 5.42 & 5.19 & 3.96 & 2.99 & 1573.9 & 4.3 \\
\hline 80.0 & 3.05 & 3.35 & 3.32 & 3.73 & 3.61 & 3.68 & 4.11 & 4.67 & 4.96 & 4.94 & 3.86 & 2.93 & 1421.7 & 3.9 \\
\hline 90.0 & 2.89 & 3.08 & 3.38 & 3.13 & 2.93 & $z . \subseteq 1$ & I. 28 & 3.88 & 4.38 & 4.56 & 3.66 & 2.80 & 1243.7 & 3.4 \\
\hline
\end{tabular}


AVERAGE DAILY TOTAL TERRESTRIAL INSOLATION ESTIMATES (XWH/SQ. M)

SITE: RAPID CITY
LATITUDE: 44 DEGREES 3 MINUTES

AVERAGE DAILY AMOUNTS BY MONTH

\begin{tabular}{|c|c|c|c|c|c|c|c|c|c|c|c|c|c|c|}
\hline ARRAY & $\mathrm{J}$ & $F$ & $M$ & A & $M$ & $J$ & $J$ & A & $s$ & 0 & $\mathrm{~N}$ & $D$ & $\begin{array}{l}\text { ANNUAL } \\
\text { AMOUNT }\end{array}$ & $\begin{array}{c}\text { AVERAGE } \\
\text { DAY }\end{array}$ \\
\hline 0.0 & 1.71 & 2.60 & 3.87 & 5.01 & 5.95 & 6.72 & 7.00 & 6.18 & 4.78 & 3.35 & 2.04 & 1.50 & 1546.3 & 4.2 \\
\hline 10.0 & 2.11 & 3.00 & 4.23 & 5.23 & 6.05 & 6.77 & 7.14 & 6.51 & 5.30 & 3.96 & 2.58 & 1.94 & 1670.7 & 4.6 \\
\hline 15.0 & 2.30 & 3.17 & 4.38 & 5.30 & 6.06. & 6.74 & 7.15 & 6.62 & 5.51 & 4.24 & 2.83 & 2.14 & 1718.8 & 4.7 \\
\hline 20.0 & 2.47 & 3.32 & 4.50 & 5.34 & 6.02 & 3.67 & 7.11. & 6.68 & 5.69 & 4.48 & 3.05 & 2.32 & 1757.1 & 4.8 \\
\hline 25.0 & 2.62 & 3.45 & 4.59 & 5.35 & 5.96 & 6.57 & 7.04 & 6.71 & 5.83 & 4.70 & 3.28 & 2.50 & 1785.0 & 4.9 \\
\hline 30.0 & 2.76 & 3.57 & 4.66 & 5.33 & 5.86 & 6.43 & 6.92 & 6.69 & 5.94 & 4.89 & 3.47 & 2.66 & 1802.4 & 4.9 \\
\hline 35.0 & 2.88 & 3.66 & 4.70 & 5.28 & 5.73 & 6.26 & 6.76 & 6.64 & 6.01 & 5.05 & 3.64 & 2.80 & 1809.2 & 5.0 \\
\hline 40.0 & 2.99 & 3.73 & 4.71 & 5.19 & 5.57 & 6.05 & 6.57 & 6.54 & 6.04 & 5.17 & 3.78 & 2.92 & 1805.2 & .4 .9 \\
\hline 45.0 & 3.07 & 3.78 & 4.70 & 5.09 & 5.38 & 5.81 & 6.34 & 6.41 & 6.04 & 5.26 & 3.90 & 3.03 & 1790.9 & 4.9 \\
\hline 50.0 & 3.14 & 3.80 & 4.66 & 4.95 & 5.18 & 5.56 & 6.09 & 6.25 & 6.00 & 5.32 & 4.00 & 3.11 & 1768.0 & 4.8 \\
\hline 60.0 & 3.21 & 3.78 & 4.50 & 4.61 & 4.69 & 4.97 & 5.50 & 5.81 & 5.80 & 5.34 & 4.11 & 3.22 & 1691.2 & 4.6 \\
\hline 70.0 & 3.20 & 3.67 & 4.23 & 4.17 & 4.12 & 4.29 & 4.79 & 5.24 & 5.47 & 5.21 & 4.11 & 3.24 & 1575.2 & 4.3 \\
\hline 80.0 & 3.11 & 3.48 & 3.87 & 3.65 & 3.47 & 3.57 & 3.99 & 4.56 & 5.00 & 4.95 & 4.01 & 3.18 & 1425.0 & 3.9 \\
\hline 90.0 & 2.93 & 3.20 & 3.42 & 3.06 & 2.82 & 2.82 & 3.19 & 3.78 & 4.41 & 4.57 & 3.80 & 3.04 & 1248.5 & 3.4 \\
\hline
\end{tabular}


AVERAGE DAILY TOTAL TERRESTRIAL INSOLATION ESTIMATES (KLH/SQ. M)

SITE: S.JOLX FALLS

LATITUDE: 43 TIEGREES 34 MINUTES

ALERAGE DAJLY AMOUNTS BY MONTH

\begin{tabular}{|c|c|c|c|c|c|c|c|c|c|c|c|c|c|c|}
\hline $\begin{array}{l}\text { ARRAY } \\
\text { TILT }\end{array}$ & J & $F$ & $M$ & A & $M$ & 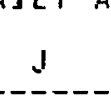 & $J$ & A & 5 & 0 & $N$ & D & $\begin{array}{l}\text { ANNUAL } \\
\text { AMIOUNT }\end{array}$ & $\begin{array}{c}\text { AVERAGE } \\
\text { DAY }\end{array}$ \\
\hline 0.0 & 1.68 & 2.53 & 3.63 & 4.86 & 5.97 & 6.62 & 6.77 & 5.82 & 4.44 & 3.17 & 1.52 & 1.39 & 1487.2 & 4.1 \\
\hline 10.0 & 2.06 & 2.89 & 3.95 & 5.07 & 6.07 & 6.66 & 6.89 & 6.10 & 4.89 & 3.71 & 2.39 & 1.76 & 1597.4 & 4.4 \\
\hline 15.0 & 2.23 & 3.05 & 4.07 & 5.13 & 6.07 & 6.62 & 6.90 & 6.19 & 5.07 & 3.95 & 2.60 & 1.93 & 1639.2 & 4.5 \\
\hline 20.0 & 2.39 & 3.19 & 4.18 & 5.16 & 6.03 & 6.58 & 6.86 & 6.24 & 5.22 & 4.17 & 2.8 .0 & 2.09 & 1671.8 & 4.6 \\
\hline 25.0 & 2.53 & 3.31 & 4.26 & 5.16 & 5.96 & 6.45 & 6.78 & 6.26 & 5.33 & 4.36 & 2.98 & 2.24 & 1694.6 & 4.6 \\
\hline 30.0 & 2.66 & 3.42 & 4.31 & 5.14 & 5.86 & 6.31 & 6.67 & 6.24 & 5.42 & 4.52 & 3.14 & 2.37 & 1707.6 & 4.7 \\
\hline 35.0 & 2.77 & 3.50 & 4.35 & 5.09 & 5.73 & 6.14 & 6.51 & 6.18 & 5.47 & 4.68 & 3.28 & 2.49 & 1710.7 & 4.7 \\
\hline 40.0 & 2.87 & 3.55 & 4.35 & 5.00 & 5.57 & 5.93 & 6.32 & 6.09 & 5.50 & 4.76 & $3 .<1$ & 2.59 & 1703.8 & 4.7 \\
\hline 45.0 & 2.94 & 3.60 & 4.34 & 4.90 & 5.38 & 5.70 & 6.10 & 5.96 & 5.48 & 4.84 & 3.51 & 2.67 & 1687.6 & 4.6 \\
\hline 50.0 & 3.00 & 3.62 & 4.29 & 4.77 & 5.17 & 5.45 & 5.86 & 5.80 & 5.44 & 4.89 & 3.58 & 2.74 & 1663.2 & 4.6 \\
\hline 60.0 & 3.06 & 3.60 & 4.13 & 4.43 & 4.68 & 9.87 & 5.29 & 5.39 & 5.25 & 4.88 & 3.67 & 2.83 & 1585.9 & 4.3 \\
\hline 70.0 & 3.05 & 3.49 & 3.88 & 4.01 & 4.10 & 4.20 & 4.60 & 4.86 & 4.93 & 4.76 & 3.66 & 2.84 & 1472.5 & 4.0 \\
\hline 80.0 & 2.95 & 3.29 & 3.55 & 3.50 & 3.45 & 3.43 & 3.84 & 4.23 & 4.50 & 4.51 & 3.56 & 2.78 & 1328.3 & 3.6 \\
\hline 90.0 & 2.79 & 3.02 & 3.13 & 2.94 & 2.79 & 2.75 & 3.07 & 3.51 & 3.97 & 4.15 & 3.36 & 2.65 & 1160.4 & 3.2 \\
\hline
\end{tabular}


AVERAGE DAILY TOTAL TERRESTRIAL INSOLATION ESTIMATES (KWH, SQ. M)

SITE: CHATTANOOGA
LATITUDE: 35 DEGREES 2 MINUTES

AVERAGE DAILY AMOUNTS BY MONTH

\begin{tabular}{|c|c|c|c|c|c|c|c|c|c|c|c|c|c|c|}
\hline ARRAY & $J$ & $F$ & $M$ & A & $M$ & $J$ & J & $A$ & $s$ & 0 & $H$ & D & $\begin{array}{l}\text { ANNUAL } \\
\text { AMOUNT }\end{array}$ & $\begin{array}{c}\text { AVERAGE } \\
\text { DAY }\end{array}$ \\
\hline 0.0 & 1.98 & 2.71 & 3.70 & 4.89 & 5.45 & 5.78 & 5.46 & 5.14 & 4.21 & 3.50 & 2.43 & 1.83 & 1433.7 & 3.9 \\
\hline 10.0 & 2.25 & 2.97 & 3.91 & 5.00 & 5.45 & 5.73 & 5.46 & 5.26 & 4.47 & 3.90 & 2.82 & 2.14 & 1503.5 & 4.1 \\
\hline 15.0 & 2.37 & 3.07 & 3.99 & 5.02 & 5.40 & 5.66 & $5.4 ?$ & 5.28 & $4.5^{\prime} 6$ & 4.07 & 3.00 & 2.28 & 1526.3 & 4.2 \\
\hline 20.0 & 2.48 & 3.16 & 4.04 & 5.01 & 5.33 & 5.56 & 5.34 & 5.27 & 4.63 & 4.21 & 3.15 & 2.41 & 1540.7 & 4.2 \\
\hline 25.0 & 2.57 & 3.23 & 4.07 & 4.97 & 5.23 & 5.43 & 5.24 & 5.23 & 4.67 & 4.33 & 3.29 & 2.52 & 1546.6 & 4.2 \\
\hline 30.0 & 2.65 & 3.28 & 4.08 & 4.90 & 5.10 & 5.27 & 5.11 & 5.17 & 4.69 & 4.43 & 3.41 & 2.62 & 1544.1 & 4.2 \\
\hline 35.0 & 2.72 & 3.32 & 4.07 & 4.81 & 4.94 & 5.08 & 4.95 & 5.07 & 4.68 & 4.50 & 3.51 & 2.70 & 1533.0 & 4.2 \\
\hline 40.0 & 2.76 & 3.34 & 4.04 & 4.70 & 4.77 & 4.88 & 4.78 & 4.95 & 4.64 & 4.54 & 3.59 & 2.77 & 1515.1 & 4.2 \\
\hline 45.0 & 2.80 & 3.33 & 3.98 & 4.56 & 4.58 & 4.66 & 4.59 & 4.81 & 4.58 & 4.55 & 3.64 & 2.82 & 1488.8 & 4.1 \\
\hline 50.0 & 2.81 & 3.31 & 3.90 & 4.40 & 4.36 & 4.42 & 4.37 & 4.54 & 4.49 & 4.54 & 3.68 & 2.85 & 1454.5 & 4.0 \\
\hline 60.0 & 2.80 & 3.22 & 3.69 & 4.02 & 3.87 & 3.87 & 3.87 & 4.22 & 4.24 & 4.44 & 3.68 & 2.87 & 1362.9 & 3.7 \\
\hline 70.0 & 2.72 & 3.05 & 3.39 & 3.55 & 3.31 & 3.28 & 3.31 & 3.73 & 3.90 & 4.23 & 3.59 & 2.82 & 1243.6 & 3.4 \\
\hline 80.0 & 2.57 & 2.82 & 3.03 & 3.02 & 2.74 & 2.67 & 2.73 & 3.17 & $3.48^{\circ}$ & 3.92 & 3.41 & 2.70 & 1103.4 & 3.0 \\
\hline 90.0 & 2.37 & 2.54 & 2.62 & 2.46 & 2.15 & 2.08 & 2.14 & 2.57 & 2.99 & 3.53 & 3.15 & 2.51 & 945.9 & 2.6 \\
\hline
\end{tabular}


AVERAGE DAILY TOTAL TEPRESTRIAL INSOLATION ESTIMATES (KWH/SQ. M)

SITE: KNOXVILLE

LATITUDE: 35 DEGF:EES 49 MINUTES

AVERAGE DAILY AMCUNTS BY MONTH

\begin{tabular}{|c|c|c|c|c|c|c|c|c|c|c|c|c|c|c|}
\hline ARRAY & $J$ & $F$ & $M$ & A & $M$ & \rfloor & J & A & $s$ & 0 & $N$ & $D$ & $\begin{array}{l}\text { ANNUAL } \\
\text { AMOUNT }\end{array}$ & $\begin{array}{c}\text { AVERAGE } \\
\text { DAY }\end{array}$ \\
\hline 0.0 & 1.95 & 2.73 & 3.75 & 5.05 & 5.68 & 5.99 & 5.68 & 5.25 & 4.36 & 3.53 & 2.39 & 1.80 & 1466.8 & 4.0 \\
\hline 10.0 & 2.23 & 3.00 & 3.98 & 5.17 & 5.68 & 5.95 & 5.69 & 5.39 & 4.64. & 3.96 & 2.79 & 2.12 & 1541.3 & 4.2 \\
\hline 15.0 & 2.36 & 3.11 & 4.06 & 5.20 & 5.64 & 5.88 & 5.64 & 5.42 & 4.75 & 4.14 & 2.97 & 2.26 & 1566.0 & 4.3 \\
\hline 20.0 & 2.47 & 3.20 & 4.12 & 5.19 & 5.57 & 5.78 & 5.57 & 5.41 & 4.83 & 4.30 & 3.13 & 2.39 & 1582.1 & 4.3 \\
\hline 25.0 & 2.55 & 3.28 & 4.16 & 5.15 & 5.47 & 5.65 & $5.47^{\circ}$ & 5.38 & 4.88 & 4.43 & 3.27 & 2.50 & 1589.4 & 4.4 \\
\hline 30.0 & 2.65 & 3.34 & 4.17 & 5.99 & 5.33 & 5.48 & 5.33 & 5.31 & 4.90 & 4.53 & 3.40 & 2.61 & 1587.9 & 4.4 \\
\hline 35.0 & 2.71 & 3.38 & 4.16 & 5.30 & 5.17 & 5.29 & 5.17 & 5.22 & 4.90 & 4.61 & 3.50 & 2.69 & 1577.5 & 4.3 \\
\hline 40.0 & 2.77 & 3.40 & 4.13 & 4.39 & 5.00 & 5.09 & 4.99 & 5.10 & 4.87 & 4.66 & 3.58 & 2.76 & 1559.8 & 4.3 \\
\hline 45.0 & 2.80 & 3.40 & 4.08 & 4.75 & 4.80 & 4.36 & 4.79 & 4.95 & 4.81 & 4.68 & 3.64 & 2.82 & 1533.7 & 4.2 \\
\hline 50.0 & 2.82 & 3.39 & 4.00 & 4.58 & 4.57 & 4.51 & 4.57 & 4.78 & 4.72 & 4.68 & 3.68 & 2.86 & 1499.2 & 4.1 \\
\hline 60.0 & 2.81 & 3.30 & 3.79 & c..$\because 3$ & 4.06 & 4.34 & 4.05 & 4.36 & 4.47 & 4.58 & 3.69 & 2.88 & 1406.2 & 3.9 \\
\hline 70.0 & 2.74 & 3.13 & 3.49 & $3 .: 1$ & 3.47 & $3 . \dot{4} 2$ & 3.45 & 3.86 & 4.12 & 4.37 & 3.61 & 2.83 & 1284.1 & 3.5 \\
\hline 80.0 & 2.60 & 2.90 & 3.13 & 3.16 & 2.88 & 2.78 & 2.86 & 3.28 & 3.68 & 4.06 & 3.43 & 2.72 & 1140.2 & 3.1 \\
\hline 90.0 & 2.40 & 2.61 & 2.70 & $2.5 ?$ & 2.25 & $2 .-6$ & 2.24 & 2.66 & 3.17 & 3.66 & 3.18 & 2.54 & 977.5 & 2.7 \\
\hline
\end{tabular}


AVERAGE DAILY TOTAL TERRESTRIAL INSOLATION ESTIMATES ( $K W H / S Q$. M)

SITE: MEMPHIS

SITE:
LATITUDE : 35 DEGREES 3 MINUTES

AVERAGE DAILY AMOUNTS BY MONTH

\begin{tabular}{|c|c|c|c|c|c|c|c|c|c|c|c|c|c|c|}
\hline ARRAY & $J$ & $F$ & $M$ & A & $M$ & $\mathrm{~J}$ & $\mathrm{~J}$ & A & 5 & 0 & $N$ & D & $\begin{array}{l}\text { ANNUAL } \\
\text { APOOUNT }\end{array}$ & $\begin{array}{l}\text { AVERAGE } \\
\text { DAY }\end{array}$ \\
\hline 0.0 & 2.15 & 2.98 & 4.02 & 5.17 & 5.94 & 6.44 & 6.21 & 5.75 & 4.63 & 3.80 & 2.57 & 1.98 & 1573.1 & 4.3 \\
\hline 10.0 & 2.47 & 3.28 & 4.27 & 5.29 & 5.94 & 6.39 & 6.21 & 5.91 & 4.94 & 4.26 & 3.00 & 2.34 & 1653.9 & 4.5 \\
\hline 15.0 & 2.61 & 3.41 & 4.35 & 5.31 & 5.89 & 6.30 & 6.16 & 5.94 & 5.05 & 4.46 & 3.19 & 2.50 & 1680.5 & 4.6 \\
\hline 20.0 & 2.73 & 3.52 & 4.42 & 5.30 & 5.81 & 6.19 & 6.07 & 5.93 & 5.14 & 4.63 & 3.37 & 2.65 & 1697.7 & 4.7 \\
\hline 25.0 & 2.84 & 3.60 & 4.46 & 5.26 & 5.70 & 6.04 & 5.95 & 5.89 & 5.19 & 4.77 & 3.52 & 2.78 & 1705.4 & 4.7 \\
\hline 30.0 & 2.94 & 3.67 & 4.47 & 5.19 & 5.55 & 5.85 & 5.80 & 5.81 & 5.22 & 4.89 & 3.65 & 2.90 & 1703.4 & 4.7 \\
\hline 35.0 & 3.01 & 3.71 & 4.46 & 5.10 & 5.38 & 5.64 & 5.62 & 5.71 & 5.21 & 4.97 & 3.76 & 2.99 & 1691.7 & 4.6 \\
\hline 40.0 & 3.07 & $\Xi .74$ & 4.43 & $4 . .98$ & 5.19 & 5.41 & 5.42 & 5.57 & 5.18 & 5.02 & 3.84 & 3.07 & 1672.3 & 4.6 \\
\hline 45.0 & 3.11 & 3.74 & 4.37 & 4.83 & 4.98 & 5.16 & 5.19 & 5.41 & 5.11 & 5.05 & 3.91 & 3.13 & 1643.5 & 4.5 \\
\hline 50.0 & 3.13 & $\geq .72$ & 4.29 & 4.66 & 4.74 & 4.88 & 4.93 & 5.21 & 5.01 & 5.04 & 3.95 & 3.18 & 1605.5 & 4.4 \\
\hline 60.0 & 3.12 & 3.63 & 4.05 & 4.25 & 4.19 & 4.25 & 4.35 & 4.74 & 4.74 & 4.93 & 3.95 & 3.20 & 1503.3 & 4.1 \\
\hline 70.0 & 3.04 & ?. 44 & 3.73 & 3.75 & 3.56 & 3.56 & 3.68 & 4.17 & 4.35 & 4.71 & 3.86 & 3.15 & 1369.6 & 3.8 \\
\hline 80.0 & 2.88 & 3.19 & 3.33 & 3.19 & 2.93 & 2.87 & 3.01 & 3.52 & 3.88 & 4.37 & 3.67 & 3.02 & 1212.3 & 3.3 \\
\hline 90.0 & 2.66 & 2.86 & 2.87 & 2.59 & 2.27 & 2.19 & 2.32 & 2.82 & 3.33 & 3.93 & 3.39 & 2.82 & 1035.3 & 2.8 \\
\hline
\end{tabular}


AVERAGE DAILY TOTAL TERRESTREAL INSOLATION ESTIMATES (KWH/SQ. M)

SITE:
LATITUDE:
36 DEGRELES 7 MINUTES

ALERAGE EAJLY AMOUNTS BY MONTH

\begin{tabular}{|c|c|c|c|c|c|c|c|c|c|c|c|c|c|c|}
\hline ARRAY & $J$ & $F$ & $M$ & $A$ & $M$ & $J$ & $J$ & A & 5 & 0 & $N$ & $D$ & $\begin{array}{l}\text { ANNUAL } \\
\text { ANOUNT }\end{array}$ & $\begin{array}{c}\text { AVER AGE } \\
\text { DAY }\end{array}$ \\
\hline 0.0 & 1.82 & 2.60 & 3.56 & 4.87 & 5.74 & G.IE. & 5.96 & 5.48 & 4.40 & 3.52 & 2.24 & 1.64 & 1463.1 & 4.0 \\
\hline 10.0 & 2.07 & 2.85 & 3.77 & 5.00 & 5.76 & 6.14 & E. .98 & 5.63 & 4.70 & 3.95 & 2.61 & 1.92 & 1534.4 & 4.2 \\
\hline 15.0 & 2.18 & 2.96 & 3.34 & 5.02 & 5.71 & 6.07 & 5.93 & 5.66 & 4.81 & 4.13 & 2.77 & 2.04 & 1557.5 & 4.3 \\
\hline 20.0 & .2 .28 & 3.04 & 3.70 & 5.01 & 5.64 & 5.97 & 5.86 & 5.66 & 4.89 & 4.29 & 2.92 & 2.15 & 1572.1 & 4.3 \\
\hline 25.0 & 2.37 & 3.11 & 3.93 & 4.98 & 5.54 & .5 .83 & 5.75 & 5.63 & 4.95 & 4.42 & 3.05 & 2.25 . & 1577.9 & 4.3 \\
\hline 30.0 & 2.44 & 3.17 & 3.94 & 4.92 & 5.41 & 5.6 .6 & 5.61 & 5.56 & 4.97 & 4.53 & 3.16 & 2.34 & 1574.9 & 4.3 \\
\hline 35.0 & 2.50 & 3.21 & 3.94 & 4.83 & 5.25 & 5.46 & 5.44 & 5.47 & 4.97 & 4.61 & 3.26 & 2.41 & 1563.2 & 4.3 \\
\hline 40.0 & 2.54 & 3.22 & 3.91 & 4.72 & 5.07 & 5.25 & 5.25 & 5.34 & 4.94 & 4.66 & 3.33 & 2.47 & 1544.2 & 4.2 \\
\hline 45.0 & 2.57 & 3.23 & 3.85 & 4.59 & 4.87 & 5.02 & 5.04 & 5.19 & 4.88 & 4.68 & 3.39 & 2.52 & 1517.0 & 4.2 \\
\hline 50.0 & 2.59 & 3.21 & 3.78 & 4.44 & 4.64 & 4.75 & 4.80 & 5.01 & 4.80 & 4.68 & 3.42 & 2.55 & 1481.5 & 4.1 \\
\hline 60.0 & 2.58 & 3.12 & 3.58 & 4.06 & 4.12 & 4.16 & 4.25 & 4.57 & 4.54 & 4.58 & 3.42 & 2.56 & 1386.8 & 3.8 \\
\hline 70.0 & 2.50 & 2.97 & 3.50 & 3.60 & 3.52 & 3.52 & 3.62 & 4.04 & 4.19 & 4.38 & 3.35 & 2.52 & 1263.5 & 3.5 \\
\hline 80.0 & 2.38 & 2.75 & 2.96 & 3.07 & 2.92 & 2.85 & 2.99 & 3.43 & 3.75 & 4.07 & 3.17 & 2.41 & 1119.0 & 3.1 \\
\hline 90.0 & 2.20 & 2.48 & 2.5 .6 & 2.51 & $? .28$ & 2.21 & 2.32 & 2.78 & 3.23 & 3.67 & 2.95 & 2.25 & 956.3 & 2.6 \\
\hline
\end{tabular}


AUERAGE DAIL TOTAL TERRESTRIAL INSOLATION ESTIMATES (KWH/SQ. M)

SITE: ABILENE

LATITUDE: 32 DEGREES 26 MINUTES

A!ERAGE DAILY AMOUNTS BY MONTH

\begin{tabular}{|c|c|c|c|c|c|c|c|c|c|c|c|c|c|c|c|}
\hline ARRAY & & $\mathrm{J}$ & $=$ & $M$ & $A$ & $M$ & $\jmath$ & J & A & 5 & 0 & $N$ & D & $\begin{array}{l}\text { ANNUAL } \\
\text { AMOUNT }\end{array}$ & $\begin{array}{c}\text { AUERAGE } \\
\text { DAY }\end{array}$ \\
\hline 0.0 & . & 2.91 & 3.74 & 4.96 & 5.81 & 6.42 & 6.96 & 6.74 & 6.17 & 5.03 & 4.15 & 3.17 & 2.72 & 1790.0 & 4.9 \\
\hline 10.0 & & 3.35 & 4.12 & 5.27 & 5.93 & 6.59 & 6.87 & 6.71 & 6.31 & 5.34 & 4.62 & 3.70 & 3.24 & 1883.2 & 5.2 \\
\hline 15.0 & & 3.55 & 4.28 & 5.38 & 5.94 & 6.33 & 6.76 & 6.63 & 6.32 & 5.45 & 4.82 & 3.93 & 3.47 & 1913.8 & 5.2 \\
\hline 20.0 & & 3.73 & 4.41 & 5.46 & 5.92 & 6.22 & 6.61 & 6.52 & 6.30 & 5.53 & 4.99 & 4.14 & 3.68 & 1933.3 & 5.3 \\
\hline 25.0 & & 3.88 & 4.52 & 5.50 & 5.86 & 6.08 & 6.43 & 6.37 & 6.24 & 5.57 & 5.13 & 4.32 & 3.87 & 1941.6 & 5.3 \\
\hline 30.0 & & 4.01 & 4.60 & 5.52 & 5.77 & 5.91 & 6.21 & 6.19 & 6.15 & 5.59 & 5.24 & 4.48 & 4.03 & 1938.6 & 5.3 \\
\hline $35.0^{\circ}$ & & 4.12 & 4.66 & 5.50 & 5.66 & 5.71 & 5.97 & 5.98 & 6.02 & 5.57 & 5.31 & 4.51 & 4.17 & 1925.3 & 5.3 \\
\hline 40.0 & . & 4.20 & 4.69 & 5.45 & 5.51 & 5.49 & 5.70 & 5.74 & 5.86 & 5.52 & 5.36 & 4.71 & 4.28 & 1901.8 & 5.2 \\
\hline 45.0 & & 4.25 & 4.69 & 5.37 & 5.33 & 5.24 & 5.40 & 5.47 & 5.67 & 5.43 & 5.37 & 4.78 & 4.37 & 1867.3 & 5.1 \\
\hline 50.0 & & 4.28 & 4.66 & 5.26 & 5.12 & 4.96 & 5.08 & 5.18 & 5.44 & 5.32 & 5.35 & 4.82 & 4.43 & 1822.2 & 5.0 \\
\hline 60.0 & & 4.26 & 4.52 & 4.94 & 4.63 & 4.33 & 4.36 & 4.50 & 4.91 & 5.00 & 5.20 & 4.81 & 4.46 & 1701.2 & 4.7 \\
\hline 70.0 & - & 4.14 & 4.28. & 4.52 & 4.04 & 3.64 & 3.60 & 3.77 & 4.27 & 4.56 & 4.94 & 4.68 & 4.37 & 1545.1 & 4.2 \\
\hline 80.0 & & 3.91 & 3.94 & 4.00 & 3.37 & 2.93 & 2.82 & 3.01 & 3.55 & 4.03 & 4.55 & 4.43 & 4.18 & 1359.8 & 3.7 \\
\hline 90.0 & & 3.59 & 3.51 & 3.39 & 2.68 & 2.22 & 2.10 & 2.26 & 2.80 & 3.41 & 4.06 & 4.07 & 3.89 & 1154.8 & 3.2 \\
\hline
\end{tabular}


AVERAGE EAILY TOTAL TERRESTRIAL INSOLATION ESTIMATES (KWH/SQ. M)

SITE: AMARILLO TX

AVERAGE DAILY AMOUNTS BY MONTH

\begin{tabular}{|c|c|c|c|c|c|c|c|c|c|c|c|c|c|c|}
\hline ARRAY & $\mathrm{J}$ & $F$ & $M$ & A & $M$ & $J$ & $J$ & A & 5 & 0 & $N$ & $D$ & $\begin{array}{l}\text { ANNUAL } \\
\text { AMOUNT }\end{array}$ & $\begin{array}{c}\text { AVERAGE } \\
\text { DAY }\end{array}$ \\
\hline 0.0 & 3.02 & 3.92 & 5.14 & 6.37 & 6.97 & 7.54 & 7.19 & 6.63 & 5.55 & 4.42 & 3.25 & 2.75 & 1910.7 & 5.2 \\
\hline 10.0 & 3.59 & 4.41 & 5.51 & 6.56 & 6.98 & 7.47 & 7.19 & 6.83 & 5.98 & 5.03 & 3.90 & 3.38 & 2035.3 & 5.6 \\
\hline 15.0 & 3.85 & 4.62 & 5.65 & 6.60 & 6.92 & 7.33 & 7.13 & 6.88 & 6.14 & 5.29 & 4.20 & 3.67 & 2079.7 & 5.7 \\
\hline 20.0 & 4.08 & 4.80 & 5.76 & 6.59 & 6.83 & 7.24 & 7.03 & 6.88 & 6.26 & 5.52 & 4.46 & 3.93 & 2111.7 & 5.8 \\
\hline 25.0 & 4.29 & 4.95 & 5.84 & 6.55 & 6.69 & 7.05 & 6.89 & 6.83 & 6.34 & 5.71 & 4.70 & 4.16 & 2131.0 & 5.8 \\
\hline 30.0 & 4.47 & 5.07 & 5.87 & 6.47 & 6.52 & 6.83 & 6.71 & 6.75 & 6.39 & 5.87 & 4.91 & 4.38 & 2137.5 & 5.9 \\
\hline 35.0 & 4.62 & 5.15 & 5.88 & 6.36 & 6.31 & 6.57 & 6.49 & 6.63 & 6.40 & 5.99 & 5.09 & 4.56 & 2131.2 & 5.8 \\
\hline 40.0 & 4.74 & 5.21 & 5.85 & 6.21 & 6.08 & 6.29 & 6.25 & 6.48 & 6.37 & 6.07 & 5.23 & 4.71 & 2114.3 & 5.8 \\
\hline 45.0 & 4.83 & 5.24 & 5.78 & 6.03 & 5.82 & 5.9 .8 & 5.98 & 6.28 & 6.30 & 6.12 & 5.34 & 4.83 & 2084.8 & 5.7 \\
\hline 50.0 & 4.89 & 5.23 & 5.68 & 5.81 & 5.52 & 5.64 & 5.67 & 6.05 & 6.19 & 6.12 & 5.41 & 4.93 & 2042.8 & 5.6 \\
\hline 60.0 & 4.92 & $5 . .12$ & 5.38 & 5.28 & 4.85 & 4.87 & 4.97 & 5.50 & 5.86 & 6.02 & 5.46 & 5.01 & 1923.0 & 5.3 \\
\hline 70.0 & 4.82 & 4.89 & $4 . \$ 5$ & 4.63 & 4.08 & 4.03 & 4.17 & 4.81 & 5.39 & 5.76 & 5.36 & 4.96 & 1759.4 & 4.8 \\
\hline 80.0 & 4.60 & 4.53 & 4.42 & 3.89 & 3.31 & 3.18 & 3.36 & 4.03 & 4.80 & 5.35 & 5.12 & 4.79 & 1562.0 & 4.3 \\
\hline 90.0 & 4.26 & 4.08 & 3.79 & 3.10 & 2.50 & 2.35 & 2.52 & 3.20 & 4.09 & 4.81 & 4.75 & 4.48 & 1335.5 & 3.7 \\
\hline
\end{tabular}


AVERAGE DAILY TOTAL TERRESTRIAL INSOLATION ESTIMATES ( $K W H / S Q$. M)

SITE: AUSTIN
TUX

AVERAGE DAILY AMOUNTS BY MONTH

\begin{tabular}{|c|c|c|c|c|c|c|c|c|c|c|c|c|c|c|}
\hline ARRAY & $\jmath$ & $F$ & $M$ & A & M & $J$ & 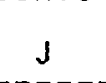 & A & $s$ & 0 & $N$ & $D$ & $\begin{array}{l}\text { ANNUAL } \\
\text { AMOUNT }\end{array}$ & $\begin{array}{c}\text { AVERAGE } \\
\text { DAY }\end{array}$ \\
\hline 0.0 & 2.72 & 3.55 & 4.50 & 5.06 & 5.78 & 6.53 & 6.64 & 6.09 & 5.06 & 4.21 & $3 . \because 1$ & 2.60 & 1700.7 & 4.7 \\
\hline 10.0 & 3.08 & 3.87 & 4.73 & 5.14 & 5.73 & 6.42 & 6.58 & 6.19 & 5.34 & 4.64 & 3.56 & 3.01 & 1774.8 & 4.9 \\
\hline 15.0 & 3.23 & 4.00 & 4.81 & 5.13 & 5.66 & 6.30 & 6.49 & 6.19 & 5.43 & 4.82 & 3.75 & 3.20 & 1797.0 & 4.9 \\
\hline 20.0 & 3.37 & 4.10 & 4.86 & 5.10 & 5.56 & 6.16 & 6.37 & 6.16 & 5.49 & 4.97 & 3.32 & 3.36 & 1809.0 & 5.0 \\
\hline 25.0 & 3.48 & 4.19 & 4.88 & 5.04 & 5.43 & 5.98 & 6.21 & 6.09 & 5.52 & 5.09 & 4.07 & 3.51 & 1810.8 & 5.0 \\
\hline 30.0 & 3.58 & 4.25 & 4.87 & 4.95 & 5.27 & 5.76 & 6.02 & 5.98 & 5.52 & 5.18 & 4.20 & 3.63 & 1802.4 & 4.9 \\
\hline 35.0 & 3.66 & 4.28 & 4.84 & 4.84 & 5.09 & 5.53 & 5.80 & 5.84 & 5.49 & 5.24 & 4.30 & 3.74 & 1785.2 & 4.9 \\
\hline 40.0 & 3.71 & 4.29 & 4.78 & 4.70 & 4.88 & 5.27 & 5.56 & 5.68 & 5.43 & 5.27 & 4.37 & 3.82 & 1758.1 & 4.8 \\
\hline 45.0 & 3.74 & 4.28 & 4.70 & 4.54 & 4.66 & 4.99 & 5.28 & 5.48 & 5.33 & 5.27 & 4.72 & 3.88 & 1721.2 & 4.7 \\
\hline 50.0 & 3.75 & 4.24 & 4.59 & 4.36 & 4.40 & 4.68 & 4.98 & 5.25 & 5.20 & 5.23 & $4 . .+4$ & 3.91 & 1674.6 & 4.6 \\
\hline 60.0 & 3.71 & 4.09 & 4.29 & 3.93 & 3.84 & 4.00 & 4.30 & 4.70 & 4.86 & 5.06 & 4.70 & 3.90 & 1554.4 & 4.3 \\
\hline 76.0 & 3.57 & 3.85 & 3.90 & 3.43 & 3.23 & 3.30 & 3.58 & 4.06 & 4.42 & 4.78 & 4.25 & 3.80 & 1404.5 & 3.8 \\
\hline 80.0 & 3.36 & 3.52 & 3.44 & 2.86 & 2.61 & 2.57 & 2.83 & 3.35 & 3.88 & 4.38 & 4.00 & 3.61 & 1228.4 & 3.4 \\
\hline 90.0 & 3.06 & 3.12 & 2.91 & 2.29 & $\therefore .00$ & 1.93 & 2.11 & 2.62 & 3.26 & 3.88 & 3.55 & 3.33 & 1038.4 & 2.8 \\
\hline
\end{tabular}


AVERAGE DAILY TOTAL TERRESTREAL INSOLATION ESTIMATES (KWH/SQ. M)

SITE: BFOWNSUILLE TX

LATITUDE: 25 DEGREES 54 MINUTES

AVERAGE DAILY AMOLNTS BY MONTH

\begin{tabular}{|c|c|c|c|c|c|c|c|c|c|c|c|c|c|c|}
\hline ARRAY & $j$ & $F$ & $M$ & A & $M$ & $\mathbf{J}$ & J & A & 5 & 0 & $N$ & $D$ & $\begin{array}{l}\text { AMNUAL } \\
\text { AMOUNT }\end{array}$ & $\begin{array}{c}\text { AVERAGE } \\
\text { DAY }\end{array}$ \\
\hline 0.0 & 2.88 & 3.59 & 4.59 & 5.48 & 6.08 & 6.67 & 6.98 & 6.39 & 5.34 & 4.55 & 3.32 & 2.72 & 1784.1 & 4.9 \\
\hline 10.0 & 3.19 & 3.85 & 4.77 & 5.52 & 5.97 & 6.49 & 6.85 & 6.44 & 5.57 & 4.95 & 3.72 & 3.07 & 1839.5 & 5.0 \\
\hline 15.0 & 3.32 & 3.95 & 4.83 & 5.49 & 5.87 & 6.35 & 6.73 & 6.41 & 5.64 & 5.10 & 3.89 & 3.22 & 1851.8 & 5.1 \\
\hline 20.0 & 3.43 & 4.03 & $\mp .85$ & 5.43 & $5: 74$ & 6.17 & 6.57 & 6.35 & 5.68 & 5.23 & 4.04 & 3.35 & 1853.6 & 5.1 \\
\hline 25.0 & 3.53 & 4.09 & 4.85 & 5.35 & 5.58 & 5.96 & 6.37 & 6.24 & 5.69 & 5.33 & 4.15 & 3.46 & 1845.0 & 5.1 \\
\hline 30.0 & 3.60 & 4.12 & 4.83 & 5.23 & 5.39 & 5.72 & 6.14 & 6.11 & 5.66 & 5.40 & 4.25 & 3.56 & 1827.1 & 5.0 \\
\hline 35.0 & 3.65 & 4.13 & 4.77 & 5.09 & 5.18 & 5.46 & 5.89 & 5.94 & 5.60 & 5.43 & 4.33 & 3.63 & 1799.3 & 4.9 \\
\hline 40.0 & 3.69 & 4.12 & 4.69 & 4.93 & 4.94 & 5.17 & 5.60 & 5.73 & 5.51 & 5.43 & 4.33 & 3.68 & 1761.3 & 4.8 \\
\hline 45.0 & 3.70 & 4.08 & 6.59 & 4.73 & 4.67 & 4.35 & 5.28 & 5.50 & 5.38 & 5.40 & 4.40 & 3.71 & 1713.7 & 4.7 \\
\hline 50.0 & 3.69 & 4.03 & 4.46 & 4.52 & 4.39 & 4.51 & 4.93 & 5.23 & 5.23 & 5.34 & 4.40 & 3.72 & 1656.6 & 4.5 \\
\hline 60.0 & 3.61 & 3.85 & 4.13 & 4.01 & 3.75 & 3.79 & 4.17 & 4.62 & 4.83 & 5.12 & 4.31 & 3.67 & 1516.9 & 4.2 \\
\hline 70.0 & 3.44 & 3.58 & 3.71 & 3.44 & 3.10 & 3.35 & 3.39 & 3.92 & 4.33 & 4.78 & 4.13 & 3.53 & 1350.6 & 3.7 \\
\hline 80.0 & 3.20 & 3.24 & 3.23 & 2.82 & 2.42 & 2.32 & 2.57 & 3.16 & 3.74 & 4.33 & 3.84 & 3.32 & 1161.2 & 3.2 \\
\hline 90.0 & 2.89 & 2.84 & 2.69 & 2.18 & 1.81 & 1.73 & 1.86 & 2.38 & 3.08 & 3.78 & 3.47 & 3.02 & 965.0 & 2.6 \\
\hline
\end{tabular}


AUERAGE DAILY TOTAL TERRESTRIAL INSOLATION ESTIMATES (KLH/SQ. M)

SITE: CORPUS CHRISTI TX

LATITUDE: 27 DEGREES 46 MINUTES

AUERAGE DAILY AMOUNTS BY MONTH

\begin{tabular}{|c|c|c|c|c|c|c|c|c|c|c|c|c|c|c|}
\hline ARRAY & J & $F$ & $M$ & A & $M$ & J & $J$ & A & $\mathrm{s}$ & 0 & $N$ & D & $\begin{array}{l}\text { ANNUAL } \\
\text { AMOUNT }\end{array}$ & $\begin{array}{c}\text { AVERAGE } \\
\text { DAY }\end{array}$ \\
\hline 0.0 & 2.83 & 3.62 & 4.50 & 5.18 & 5.88 & 6.60 & 6.89 & 6.28 & 5.31 & 4.47 & 3.28 & 2.66 & 1751.5 & 4.8 \\
\hline 10.0 & 3.16 & 3.91 & 4.70 & 5.23 & 5.81 & 6.45 & 6.80 & 6.35 & 5.57 & 4.90 & 3.72 & 3.03 & 1815.8 & 5.0 \\
\hline 15.0 & 3.30 & 4.02 & 4.76 & 5.22 & 5.72 & 6.32 & 6.69 & 6.34 & 5.65 & 5.07 & 3.90 & 3.19 & 1832.7 & 5.0 \\
\hline 20.0 & 3.43 & 4.12 & 4.79 & 5.17 & 5.60 & 6.15 & 6.54 & 6.28 & 5.71 & 5.21 & 4.06 & 3.34 & 1839.3 & 5.0 \\
\hline 25.0 & 3.53 & 4.19 & 4.80 & 5.10 & 5.46 & 5.95 & 6.36 & 6.20 & 5.72 & 5.33 & 4.20 & 3.46 & 1835.5 & 5.0 \\
\hline 30.0 & 3.61 & 4.23 & 4.78 & 5.00 & 5.28 & 5.73 & 6.14 & 6.07 & 5.71 & 5.41 & 4.31 & 3.57 & 1821.9 & 5.0 \\
\hline 35.0 & 3.68 & 4.25 & 4.74 & 4.88 & 5.09 & 5.48 & 5.90 & 5.92 & 5.66 & 5.45 & 4.40 & 3.65 & 1798.9 & 4.9 \\
\hline 40.0 & 3.72 & 4.25 & 4.67 & 4.73 & 4.87 & 5.21 & 5.63 & 5.73 & 5.58 & 5.47 & 4.46 & 3.71 & 1765.9 & 4.8 \\
\hline 45.0 & 3.74 & 4.22 & 4.57 & 4.55 & 4.62 & 4.91 & 5.33 & 5.51 & 5.46 & 5.45 & 4.50 & 3.75 & 1723.0 & 4.7 \\
\hline 50.0 & 3.74 & 4.17 & 4.45 & 4.36 & 4.36 & 4.58 & 5.00 & 5.26 & 5.32 & 5.40 & 4.50 & 3.77 & 1670.5 & 4.6 \\
\hline 60.0 & 3.67 & 4.00 & 4.14 & 3.90 & 3.76 & 3.87 & 4.26 & 4.67 & 4.94 & 5.20 & 4.44 & 3.74 & 1539.2 & 4.2 \\
\hline 70.0 & 3.52 & 3.75 & 3.74 & 3.37 & 3.14 & 3.16 & 3.50 & 3.99 & 4.45 & 4.87 & 4.26 & 3.62 & 1379.9 & 3.8 \\
\hline 80.0 & 3.29 & 3.41 & 3.27 & 2.79 & 2.48 & 2.43 & 2.70 & 3.25 & 3.87 & 4.44 & 3.99 & 3.41 & 1195.6 & 3.3 \\
\hline 90.0 & 2,98 & 3.00 & 2.74 & 2.20 & 1.88 & 1.81 & 1.96 & 2.49 & 3.21 & 3.90 & 3.62 & 3.12 & 1000.8 & 2.7 \\
\hline
\end{tabular}


AVERAGE DFILY TOTAL TERRESTREAL INSOLATION ESTIMATES (KWH/SQ. M)

SITE: DALLAS

TX

LATITUDE: 32 DEGREES 51 MINUTES

AVERAGE GAILY AMOUNTS BY MONTH

\begin{tabular}{|c|c|c|c|c|c|c|c|c|c|c|c|c|c|c|}
\hline ARRAY & $\mathrm{J}$ & $F$ & $M$ & A & M & $J$ & $J$ & A & $s$ & 0 & $N$ & D & $\begin{array}{l}\text { ANNUAL } \\
\text { AMOUNT }\end{array}$ & $\begin{array}{l}\text { AVERAGE } \\
\text { DAY }\end{array}$ \\
\hline 0.0 & 2.59 & 3.38 & 4.47 & 5.13 & 5.95 & 6.73 & 6.68 & 6.15 & 5.00 & 4.03 & 2.95 & 2.46 & 1690.9 & 4.6 \\
\hline 10.0 & 2.97 & 3.71 & 4.73 & 5.23 & 5.93 & 6.64 & $\epsilon .66$ & 6.29 & 5.31 & 4.49 & 3.42 & 2.90 & 1775.2 & 4.9 \\
\hline 15.0 & 3.14 & 3.85 & 4.82 & 5.24 & 5.87 & 6.54 & 6.59 & 6.31 & 5.42 & 4.68 & 3.63 & 3.10 & 1802.5 & 4.9 \\
\hline 20.0 & 3.28 & 3.96 & 4.89 & 5.22 & 5.77 & 6.40 & 6.48 & 6.29 & 5.50 & 4.85 & 3.82 & 3.28 & 1819.5 & 5.0 \\
\hline 25.0 & 3.41 & 4.06 & 4.93 & 5.17 & 5.65 & 6.23 & 6.34 & 6.23 & 5.55 & 4.98 & 3.99 & 3.44 & 1826.0 & 5.0 \\
\hline 30.0 & 3.52 & 4.13 & 4.73 & 5.09 & 5.49 & 6.02 & 6.15 & 6.14 & 5.57 & 5.09 & 4.13 & 3.58 & 1822.2 & 5.0 \\
\hline 35.0 & 3.61 & 4.17 & 4.92 & 4.99 & 5.32 & 5.79 & 5.95 & 6.02 & 5.55 & 5.16 & 4.24 & 3.70 & 1808.7 & 5.0 \\
\hline 40.0 & 3.68 & 4.19 & 4.87 & 4.855 & 5.12 & 5.54 & 5.72 & 5.86 & 5.50 & 5.21 & 4.33 & 3.79 & 1785.9 & 4.9 \\
\hline 45.0 & 3.72 & 4.19 & 4.80 & 4.71 & 4.89 & 5.26 & 5.45 & 5.67 & 5.42 & 5.22 & 4.40 & 3.87 & 1752.9 & 4.8 \\
\hline 50.0 & 3.74 & 4.17 & 4.70 & $4.5: 3$ & 4.64 & 4.95 & 5.16 & 5.45 & 5.31 & 5.20 & 4.43 & 3.91 & 1710.1 & 4.7 \\
\hline $6 c .0$ & 3.72 & 4.04 & 4.42 & 4.11 & 4.07 & 4.26 & 4.50 & 4.92 & 4.99 & 5.06 & 4.42 & 3.94 & 1596.2 & 4.4 \\
\hline 70.0 & 3.61 & 3.82 & 4.04 & 3.61 & 3.44 & 3.54 & 3.77 & 4.29 & 4.56 & 4.81 & 4.30 & 3.86 & 144.9 .9 & 4.0 \\
\hline 80.0 & 3.41 & 3.52 & 3.59 & 3.04 & $\therefore 80$ & 2.79 & 3.02 & 3.57 & 4.04 & 4.44 & 4.07 & 3.69 & 1277.0 & 3.5 \\
\hline 90.0 & 3.13 & 3.15 & 3.06 & 2.45 & 2.16 & 2.09 & 2.28 & 2.82 & 3.43 & 3.96 & 3.75 & 3.43 & 1085.7 & 3.0 \\
\hline
\end{tabular}


AVERAGE DAILY TOTAL TERRESTRIAL INSOLATION ESTIMATES (KWH,SQ. M)

STTE: DEL RIO
ITUDE: 29 DEGREES 22 MINUTES

AVERAGE DAILY AMOUNTS BY MONTH

\begin{tabular}{|c|c|c|c|c|c|c|c|c|c|c|c|c|c|c|}
\hline ARRAY & $J$ & $F$ & $M$ & $A$ & $M$ & $\begin{array}{ll}0 \\
0\end{array}$ & 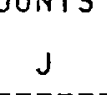 & 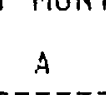 & $\mathrm{s}$ & 0 & $r$ & $D$ & $\begin{array}{l}\text { ANNUAL } \\
\text { AMOUNT }\end{array}$ & $\begin{array}{c}\text { AVERAGE } \\
\text { DAY }\end{array}$ \\
\hline 0.0 & 3.02 & 3.81 & 4.97 & 5.36 & 5.76 & 6.38 & 6.48 & 6.11 & 4.99 & 4.29 & 3.33 & 2.84 & 1746.4 & 4.8 \\
\hline 10.0 & 3.42 & 4.15 & 5.23 & 5.43 & 5.71 & 6.25 & 6.41 & 6.20 & 5.25 & 4.72 & 3.82 & 3.30 & 1823.7 & 5.0 \\
\hline 15.0 & 3.60 & 4.29 & 5.32 & 5.42 & 5.63 & 6.14 & 6.32 & 6.19 & 5.34 & 4.90 & 4.02 & 3.50 & 1847.0 & 5.1 \\
\hline 20.0 & 3.75 & 4.40 & 5.37 & 5.39 & 5.52 & 5.99 & 6.20 & 6.15 & 5.39 & 5.04 & 4.21 & 3.68 & 1859.9 & 5.1 \\
\hline 25.0 & 3.88 & 4.49 & 5.40 & 5.32 & 5.39 & 5.81 & 6.04 & 6.07 & 5.41 & 5.16 & 4.37 & 3.84 & 1862.2 & 5.1 \\
\hline 30.0 & 3.99 & 4.55 & 5.39 & 5.22 & 5.22 & 5.60 & 5.85 & 5.96 & 5.41 & 5.25 & 4.50 & 3.98 & 1854.1 & 5.1 \\
\hline 35.0 & 4.07 & 4.59 & 5.35 & 5.10 & 5.04 & 5.37 & 5.64 & 5.82 & 5.37 & 5.30 & 4.61 & 4.09 & 1836.7 & 5.0 \\
\hline 40.0 & 4.13 & 4.60 & .5 .29 & 4.95 & 4.84 & 5.12 & 5.39 & 5.65 & 5.30 & 5.32 & 4.69 & 4.17 & 1809.0 & 5.0 \\
\hline 45.0 & 4.17 & 4.58 & 5.19 & 4.78 & 4.60 & 4.84 & 5.12 & 5.44 & 5.20 & 5.31 & 4.74 & 4.23 & 1771.1 & 4.9 \\
\hline 50.0 & 4.18 & 4.54 & 5.06 & 4.58 & 4.35 & 4.53 & 4.82 & 5.21 & 5.07 & 5.27 & 4.76 & 4.27 & 1723.3 & 4.7 \\
\hline 60.0 & 4.13 & 4.37 & 4.73 & 4.11 & 3.78 & 3.86 & 4.16 & 4.65 & 4.73 & 5.10 & 4.71 & 4.26 & 1599.6 & 4.4 \\
\hline 70.0 & 3.97 & 4.11 & 4.29 & 3.56 & 3.18 & 3.19 & 3.45 & 4.01 & 4.28 & 4.80 & 4.54 & 4.14 & 1445.5 & 4.0 \\
\hline 80.0 & 3.73 & 3.75 & 3.76 & 2.96 & 2.55 & 2.48 & 2.72 & 3.29 & 3.75 & 4.39 & 4.27 & 3.93 & 1263.8 & . 3.5 \\
\hline 90.0 & 3.40 & 3.32 & 3.15 & 2.34 & 1.95 & 1.87 & 2.03 & 2.56 & 3.14 & 3.88 & 3.89 & 3.62 & 1068.2 & 2.9 \\
\hline
\end{tabular}


AUERAGE DAILY TOTAL TERRESTRIAL INSOLATION ESTIMATES (KWH/SQ. M)

$\begin{aligned} \text { SITE: } & \text { EL PASC } \\ \text { LATHTUDE: } & \text { \I DEGREES } 48 \text { MINUTES }\end{aligned}$

AVERAGE DAILY AMOUNTS BY MONTH

\begin{tabular}{|c|c|c|c|c|c|c|c|c|c|c|c|c|c|c|}
\hline ARRAY & $J$ & $F$ & $M$ & $A$ & $M$ & Strat & - & riom & $s$ & 0 & $N$ & $D$ & $\begin{array}{l}\text { ANNUAL } \\
\text { AMOUNT }\end{array}$ & $\begin{array}{c}\text { AVERAGE } \\
\text { DAY }\end{array}$ \\
\hline 0.0 & 3.54 & 4.67 & 6.02 & 7.45 & 8.20 & 8.46 & 7.73 & 7.20 & 6.26 & 5.17 & 3.92 & 3.25 & 2187.8 & 6.0 \\
\hline 10.0 & 4.15 & 5.22 & 6.43 & 7.63 & 8.15 & 8.32 & 7.68 & 7.37 & 6.70 & 5.84 & 4.55 & 3.92 & 2315.1 & 6.3 \\
\hline 15.0 & 4.42 & 5.45 & 6.58 & 7.65 & 8.05 & 8.17 & 7.59 & 7.39 & 6.86 & 6.12 & 4.97 & 4.22 & 2357.8 & 6.5 \\
\hline 20.0 & $4.60^{\circ}$ & 5.65 & 6.69 & 7.62 & 7.91 & 7.98 & 7.45 & 7.37 & 6.97 & 6.36 & 5.26 & 4.49 & 2386.0 & 6.5 \\
\hline 25.0 & 4.87 & 5.81 & 6.76 & 7.55 & 7.71 & 7.73 & 7.27 & 7.30 & 7.04 & 6.57 & 5.52 & 4.74 & 2399.6 & 6.6 \\
\hline 30.0 & 5.05 & 5.93 & 6.79 & 7.43 & 7.47 & 7.44 & 7.05 & 7.18 & 7.07 & 6.73 & 5.74 & 4.96 & 2398.3 & 6.6 \\
\hline 35.0 & 5.20 & 6.02 & 6.77 & 7.27 & 7.20 & 7.12 & 6.79 & 7.03 & 7.06 & 6.84 & 5.92 & 5.14 & 2383.9 & 6.5 \\
\hline 40.0 & 5.32 & 6.07 & 6.72 & 7.07 & 6.89 & 6.77 & 6.51 & 6.83 & 7.00 & 6.92 & 6.37 & 5.29 & 2355.8 & 6.5 \\
\hline 45.0 & 5.40 & 6.09 & 6.62 & 6.83 & 6.54 & 6.38 & 6.18 & 6.60 & 6.89 & 6.94 & 6.17 & 5.40 & 2313.2 & 6.3 \\
\hline 50.0 & 5.45 & 6.06 & 6.48 & 6.54 & 6.15 & 5.96 & 5.82 & 6.32 & 6.75 & 6.93 & 5.24 & 5.48 & 2256.5 & 6.2 \\
\hline 60.0 & 5.44 & 5.90 & 6.09 & 5.86 & 5.28 & 5.01 & 5.02 & 5.67 & 6.33 & 6.76 & 5.24 & 5.54 & 2102.5 & 5.8 \\
\hline 70.0 & 5.29 & 5.59 & 5.55 & 5.05 & 4.33 & 4.04 & 4.13 & 4.89 & 5.77 & 6.42 & 5.39 & 5.44 & 1902.7 & 5.2 \\
\hline 80.0 & 5.00 & 5.15 & 4.89 & 4.13 & 3.35 & 3.03 & 3.23 & 4.01 & 5.07 & 5.92 & 5.77 & 5.21 & 1663.6 & 4.6 \\
\hline 90.0 & 4.59 & 4.58 & 4.11 & 3.18 & 2.40 & 2.11 & 2.34 & 3.09 & 4.25 & 5.27 & 5.30 & 4.84 & 1399.4 & 3.8 \\
\hline
\end{tabular}


AVERAGE DAILY TOTAL TERRESTRIAL INSOLATION ESTIMATES (KWH/JQ. M)

SITE: FORT WORTH
LATITUDE: 32 DEGREES 50 MINUTES

AYERAGE DAILY AMOUNTS BY MONTH

\begin{tabular}{|c|c|c|c|c|c|c|c|c|c|c|c|c|c|c|}
\hline ARRAY & $J$ & $F$ & $M$ & A & $M$ & 3 & 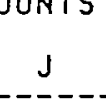 & 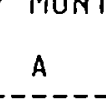 & 5 & 0 & $N$ & D & $\begin{array}{l}\text { ANNUAL } \\
\text { ANIOUNT }\end{array}$ & $\begin{array}{c}\text { AVERAGE } \\
\text { DAY }\end{array}$ \\
\hline 0.0 & 2.53 & 3.37 & 4.44 & 5.10 & 5.96 & 6.78 & 6.80 & 6.25 & 5.11 & 4.08 & 2.95 & 2.41 & 1699.5 & 4.7 \\
\hline 10.0 & 2.90 & 3.70 & 4.69 & 5.20 & 5.94 & 6.70 & 6.77 & 6.40 & 5.43 & 4.55 & 3.43 & 2.85 & 1783.6 & 4.9 \\
\hline 15.0 & 3.06 & 3.84 & 4.78 & 5.21 & 5.88 & 6.59 & 6.70 & 6.42 & 5.55 & 4.75 & 3.64 & 3.04 & 1810.6 & 5.0 \\
\hline 20.0 & 3.20 & 3.96 & 4.85 & 5.19 & 5.78 & 6.46 & 6.59 & 6.40 & 5.63 & 4.92 & 3.83 & 3.21 & 1827.3 & 5.0 \\
\hline 25.0 & 3.33 & 4.05 & 4.88 & 5.14 & 5.66 & 6.28 & 6.44 & 6.34 & 5.68 & 5.05 & 4.00 & 3.37 & 1833.5 & 5.0 \\
\hline 30.0 & 3.43 & 4.12 & 4.89 & 5.06 & 5.50 & 6.07 & 6.26 & 6.25 & 5.70 & 5.16 & 4.14 & 3.50 & 1829.3 & 5.0 \\
\hline 35.0 & 3.52 & 4.16 & 4.87 & 4.96 & 5.32 & 5.84 & 6.04 & 6.12 & 5.68 & 5.24 & 4.26 & 3.62 & 1815.4 & 5.0 \\
\hline$\dot{40.0}$ & 3.58 & 4.18 & 4.83 & 4.83 & 5.13 & 5.58 & 5.81 & 5.96 & 5.63 & 5.28 & 4.35 & 3.71 & 1792.1 & 4.9 \\
\hline 45.0 & 3.62 & 4.18 & 4.75 & 4.68 & 4.90 & 5.30 & 5.54 & 5.77 & 5.55 & 5.30 & 4.91 & 3.78 & 1758.6 & 4.8 \\
\hline $5 r .0$ & 3.64 & 4.16 & 4.65 & 4.50 & 4.65 & 4.99 & 5.24 & 5.54 & 5.44 & 5.28 & 4.94 & 3.82 & 1715.2 & 4.7 \\
\hline 60.0 & 3.62 & 4.03 & 4.38 & 4.08 & 4.08 & 4.29 & 4.57 & 5.00 & 5.11 & 5.14 & 4.43 & 3.84 & 1600.0 & 4.4 \\
\hline$=0.0$ & 3.51 & 3.81 & 4.01 & 3.58 & 3.45 & 3.56 & 3.82 & 4.36 & 4.67 & 4.88 & 4.31 & 3.77 & 1452.3 & 4.0 \\
\hline 80.0 & 3.32 & 3.51 & 3.56 & 3.02 & 2.81 & 2.80 & 3.06 & 3.62 & 4.13 & 4.51 & 4.88 & 3.60 & 1278.0 & 3.5 \\
\hline $30: 0$ & 3.05 & 3.14 & 3.03 & 2.44 & 2.16 & 2.10 & 2.30 & 2.86 & 3.50 & 4.03 & 3.76 & 3.34 & 1085.4 & 3.0 \\
\hline
\end{tabular}


AVERAG: DAILY TOTAL TEREESTRIAL INSOLATION ESTIMATES (KWH/SQ. M)

\begin{aligned} SITE: & FOLSTON \\ LATITUDE: & $\angle 9$ DEGREES \\ \hline 9 & MINUTES \end{aligned}

AVERAGE LAILY AMOUNTS BY MONTH

\begin{tabular}{|c|c|c|c|c|c|c|c|c|c|c|c|c|c|c|}
\hline ARRAY & $\begin{array}{l}\mathrm{J} \\
--1\end{array}$ & $F$ & $M$ & A & $M$ & $y^{-2}$ & J & trom & 5 & 0 & $N$ & D & $\begin{array}{l}\text { ANNUAL } \\
\text { APIOUNT }\end{array}$ & $\begin{array}{c}\text { AVERAGE } \\
\text { DAY }\end{array}$ \\
\hline 0.0 & 2.43 & 3.26 & 4.08 & 4.80 & 5.60 & E. 58 & 5.77 & 5.32 & 4.63 & 4.03 & 2.91 & 2.29 & 1556.4 & 4.3 \\
\hline 10.0 & 2.72 & 3.54 & 4.27 & 4.87 & 5.55 & 5.88 & 5.71 & 5.40 & 4.87 & 4.42 & 3.30 & 2.63 & 1618.4 & 4.4 \\
\hline 15.0 & 2.84 & 3.64 & 4.34 & 4.86 & 5.48 & 5.78 & 5.64 & 5.39 & 4.94 & 4.59 & 3.47 & 2.77 & 1636.2 & 4.5 \\
\hline 20.0 & 2.95 & 3.73 & 4.37 & 4.83 & 5.38 & E. 65 & 5.54 & 5.36 & 4.99 & 4.72 & 3.62 & 2.90 & 1645.1 & 4.5 \\
\hline 25.0 & 3.04 & 3.80 & 4.39 & 4.77 & 5.25 & 5.48 & 5.40 & 5.29 & 5.01 & 4.83 & 3.75 & 3.02 & 1644.9 & 4.5 \\
\hline 30.0 & 3.12 & 3.85 & 4.38 & 4.68 & 5.10 & 5.29 & c. .24 & 5.20 & 5.01 & 4.91 & 3.86 & 3.11 & 1635.7 & 4.5 \\
\hline 35.0 & 3.18 & 3.87 & 4.35 & 4.58 & 4.92 & 5.09 & 5.06 & 5.08 & 4.97 & 4.96 & 3.95 & 3.19 & 1618.7 & 4.4 \\
\hline 40.0 & 3.22 & 3.88 & 4.29 & 4.45 & 4.73 & 4.8 .6 & 4.85 & 4.94 & 4.91 & 4.98 & 4.01 & 3.25 & 1593.0 & 4.4 \\
\hline 45.0 & 3.24 & 3.86 & 4.21 & 4.30 & 4.51 & 4.60 & 4.62 & 4.76 & 4.82 & 4.97 & 4.04 & 3.29 & 1558.6 & 4.3 \\
\hline 50.0 & 3.24 & 3.82 & 4.11 & 4.12 & 4.26 & 4.32 & 4.37 & 4.57 & 4.70 & 4.93 & 4.06 & 3.31 & 1515.8 & 4.2 \\
\hline 60.0 & 3.19 & 3.68 & 3.94 & 3.72 & 3.72 & 3.71 & 3.81 & 4.11 & 4.39 & 4.77 & 4.01 & 3.29 & 1406.3 & 3.9 \\
\hline 70.0 & 3.06 & 3.46 & 3.49 & 3.24 & 3.14 & 3.09 & $\Xi .20$ & 3.57 & 3.99 & 4.49 & 3.87 & 3.20 & 1271.2 & 3.5 \\
\hline 80.0 & 2.87 & 3.16 & 3.08 & 2.72 & 2.53 & 2.4 .4 & 2.57 & 2.97 & 3.50 & 4.11 & 3.63 & 3.03 & 1113.3 & 3.1 \\
\hline 90.0 & 2.62 & 2.80 & 2.61 & 2.18 & 1.95 & 1.86 & ]. .97 & 2.35 & 2.95 & 3.65 & 3.32 & 2.79 & 943.6 & 2.6 \\
\hline
\end{tabular}


AVERAGE DAILY TOTAL TERRESTRIAL INSOLATION ESTIMATES (KUH/SQ. M)

$\begin{aligned} \text { SITE: } & \text { KINGSUILLE } \\ \text { LATITUDE: } & 27 \text { DEGREES } 31 \text { MIHUTES }\end{aligned}$

A:IERAGE DAILY AMOUNTS BY MONTH

\begin{tabular}{|c|c|c|c|c|c|c|c|c|c|c|c|c|c|c|}
\hline ARRAY & J & $F$ & $M$ & A & $M$ & $J$ & $J$ & $A$ & $s$ & 0 & $N$ & $D$ & $\begin{array}{l}\text { ANNUAL } \\
\text { AMOUNT }\end{array}$ & $\begin{array}{c}\text { AVERAGE } \\
\text { DAY }\end{array}$ \\
\hline 0.0 & 2.88 & 3.67 & 4.52 & 5.24 & 5.88 & 6.42 & 6.65 & 6.06 & 5.11 & 4.39 & 3.26 & 2.68 & 1728.6 & 4.7 \\
\hline 10.0 & 3.22 & 3.96 & 4.71 & 5.29 & 5.80 & 6.28 & 6.56 & 6.13 & 5.36 & 4.80 & 3.68 & 3.05 & 1791.2 & 4.9 \\
\hline 15.0 & 3.36 & 4.08 & 4.77 & 5.27 & 5.72 & 6.15 & 6.45 & 6.11 & 5.43 & 4.96 & 3.86 & 3.21 & 1807.6 & 5.0 \\
\hline 20.0 & 3.49 & 4.17 & 4.81 & 5.22 & 5.60 & 5.99 & 6.31 & 6.06 & 5.48 & 5.10 & 4.02 & 3.35 & 1813.8 & 5.0 \\
\hline 25.0 & 3.59 & 4.24 & 4.82 & 5.15 & 5.45 & 5.79 & 6.14 & 5.97 & 5.49 & 5.20 & 4.15 & 3.48 & 1809.9 & 5.0 \\
\hline 30.0 & 3.68 & 4.28 & 4.80 & 5.05 & 5.27 & 5.58 & 5.93 & 5.85 & 5.47 & 5.27 & 4.26 & 3.58 & 1796.4 & 4.9 \\
\hline 35.0 & 3.74 & 4.30 & 4.75 & 4.92 & 5.08 & 5.34 & 5.70 & 5.70 & 5.42 & 5.32 & 4.34 & 3.66 & 1773.7 & 4.9 \\
\hline 40.0 & 3.78 & 4.30 & 4.68 & 4.77 & 4.86 & 5.07 & 5.44 & 5.52 & 5.34 & 5.33 & 4.40 & 3.72 & 1741.0 & 4.8 \\
\hline 45.0 & 3.80 & 4.27 & 4.58 & 4.59 & 4.61 & 4.78 & 5.15 & 5.30 & 5.23 & 5.31 & 4.43 & 3.76 & 1698.7 & 4.7 \\
\hline 50.0 & 3.80 & 4.22 & 4.46 & 4.39 & 4.34 & 4.46 & 4.83 & 5.06 & 5.09 & 5.25 & 4.43 & 3.78 & 1647.1 & 4.5 \\
\hline 60.0 & 3.73 & 4.05 & 4.15 & 3.93 & 3.75 & 3.78 & 4.13 & 4.50 & 4.73 & 5.05 & 4.36 & 3.75 & 1517.9 & 4.2 \\
\hline 70.0 & 3.58 & 3.79 & 3.75 & 3.39 & 3.12 & 3.08 & 3.40 & 3.85 & 4.26 & 4.74 & 4.19 & 3.62 & 1361.3 & 3.7 \\
\hline 80.0 & 3.34 & 3.44 & 3.27 & 2.80 & 2.47 & 2.38 & 2.63 & 3.14 & 3.70 & 4.31 & 3.92 & 3.41 & 1180.3 & 3.2 \\
\hline 90.0 & 3.03 & 3.03 & 2.74 & 2.20 & 1.87 & 1.78 & 1.93 & 2.42 & 3.08 & 3.79 & 3.55 & 3.13 & 989.0 & 2.7 \\
\hline
\end{tabular}


AVERAGE DAILY TOTAL TEFRESTRIAL INSOLATION ESTIMATES (KWH/SQ. M)

SITE: LFREDO TXX

AVERAGE DAILY AMOUNTS BY MONTH

\begin{tabular}{|c|c|c|c|c|c|c|c|c|c|c|c|c|c|c|}
\hline $\begin{array}{l}\text { ARRAY } \\
\text { TILT }\end{array}$ & 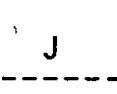 & $F$ & $M$ & A & $M$ & $J$ & J & A & 5 & 0 & $\mathrm{~N}$ & $D$ & $\begin{array}{l}\text { ANNUAL } \\
\text { ANIOUNT }\end{array}$ & $\begin{array}{c}\text { AVERAGE } \\
\text { DAY }\end{array}$ \\
\hline 0.0 & 3.02 & 3.78 & 4.77 & 5.44 & 6.15 & 6.54 & 6.72 & 6.33 & 5.37 & 4.44 & 3.28 & 2.80 & 1786.0 & 4.9 \\
\hline 10.0 & 3.39 & 4.09 & 4.99 & 5.49 & 6.07 & 6.39 & 6.62 & 6.41 & 5.63 & 4.86 & 3.70 & 3.20 & 1852.5 & 5.1 \\
\hline 15.0 & 3.54 & 4.21 & 5.06 & 5.48 & 5.97 & 6.26 & 6.52 & 6.39 & 5.71 & 5.03 & 3.83 & 3.37 & 1870.1 & 5.1 \\
\hline 20.0 & 3.68 & 4.30 & 5.09 & 5.43 & 5.85 & 6.09 & 6.38 & 6.34 & 5.76 & 5.17 & 4.04 & 3.53 & 1877.2 & 5.1 \\
\hline 25.0 & 3.80 & 4.38 & 3.10 & 5.35 & 5.69 & 5.89 & 6.20 & 6.24 & 5.78 & 5.27 & 4.13 & 3.66 & 1873.7 & 5.1 \\
\hline 30.0 & 3.89 & 4.43 & 5.09 & 5.24 & 5.51 & 5.67 & 5.99 & 6.12 & 5.76 & 5.35 & 4.29 & 3.78 & 1860.2 & 5.1 \\
\hline 35.0 & 3.96 & 4.45 & 3.04 & 5.11 & 5.30 & 5.43 & 5.76 & 5.96 & 5.71 & 5.39 & 4.37 & 3.87 & 1836.9 & 5.0 \\
\hline 40.0 & 4.01 & 4.45 & 4.96 & 4.95 & 5.07 & 5.15 & 5.49 & 5.77 & 5.63 & 5.41 & 4.43 & 3.93 & 1803.3 & 4.9 \\
\hline 45.0 & 4.03 & 4.42 & 4.8 .6 & 4.77 & 4.80 & 4.85 & 5.20 & 5.54 & 5.51 & 5.39 & 4.45 & 3.98 & 1759.6 & 4.8 \\
\hline 50.0 & 4.03 & 4.37 & 4.73 & 4.56 & 4.52 & 4.53 & 4.88 & 5.29 & 5.37 & 5.33 & 4.47 & 4.00 & 1706.2 & 4.7 \\
\hline 60.0 & 3.96 & 4.19 & 4.40 & 4.07 & 3.89 & 3.53 & 4.15 & 4.70 & 4.98 & 5.13 & 4.40 & 3.95 & 1572.1 & 4.3 \\
\hline 70.0 & 3.80 & 3.92 & 3.57 & 3.51 & 3.23 & 3.12 & 3.42 & 4.01 & 4.49 & $4.8 I$ & 4.22 & 3.84 & 1409.3 & 3.9 \\
\hline 80.0 & 3.55 & 3.57 & 3.47 & 2.89 & 2.53 & $2 .+0$ & 2.65 & 3.26 & 3.90 & 4.38 & 3.95 & 3.62 & 1220.8 & 3.3 \\
\hline 90.0 & 3.22 & 3.14 & 2.90 & 2.26 & 1.90 & 1.79 & 1.94 & 2.49 & 3.23 & 3.85 & 3.58 & 3.31 & 1021.3 & 2.8 \\
\hline
\end{tabular}


AVERAGE [AILY TOTAL TERRESTRIAL INSOLATION ESTIMATES (KWH/SQ. M)

SITE: LUBBOCK

LATITUDE: 33 DEGREES 39 MINUTES

AVERAGE DAILY AMOUNTS BY MONTH

\begin{tabular}{|c|c|c|c|c|c|c|c|c|c|c|c|c|c|c|}
\hline ARRAY & J & $F$ & M & A & $M$ & J & J & A & 5 & 0 & $N$ & $D$ & $\begin{array}{l}\text { ANNUAL } \\
\text { AMOUNT }\end{array}$ & $\begin{array}{l}\text { AVERAGE } \\
\text { DAY }\end{array}$ \\
\hline 0.0 & 3.25 & 4.20 & 5.55 & 6.83 & 7.56 & 8.02 & 7.60 & 6.97 & 5.74 & 4.63 & 3.52 & 2.95 & 2034.1 & 5.6 \\
\hline 10.0 & 3.83 & 4.70 & 5.95 & 7.02 & 7.55 & 7.92 & 7.59 & 7.16 & 6.16 & 5.24 & 4.19 & 3.58 & 2157.9 & 5.9 \\
\hline 15.0 & 4.09 & 4.71 & 6.10 & 7.05 & 7.47 & 7.80 & 7.51 & 7.19 & 6.31 & 5.49 & 4.49 & 3.87 & 2200.6 & 6.0 \\
\hline 20.0 & 4.33 & 5.109 & 6.21 & 7.04 & 7.35 & 7.63 & 7.39 & 7.18 & 6.42 & 5.72 & 4.77 & 4.13 & 2230.0 & 6.1 \\
\hline 25.0 & 4.53 & 5.25 & 6.28 & 6.98 & 7.19 & 7.42 & 7.23 & 7.13 & 6.50 & 5.90 & 5.01 & 4.37 & 2246.0 & 6.2 \\
\hline 30.0 & 4.71 & 5.36 & 6.31 & 6.39 & 6.99 & 7.17 & 7.02 & 7.03 & 6.54 & 6.05 & 5.22 & 4.58 & 2248.2 & 6.2 \\
\hline 35.0 & 4.86 & 5.45 & 6.31 & 6.75 & 6.75 & 6.88 & 6.78 & 6.89 & 6.53 & 6.17 & 5.40 & 4.76 & 2237.5 & 6.1 \\
\hline 40.0 & 4.98 & 5.50 & 6.27 & 6.58 & 6.49 & 6.57 & 6.51 & 6.72 & 6.49 & 6.24 & 5.54 & 4.91 & 2215.0 & 6.1 \\
\hline 45.0 & 5.07 & 5.52 & 6.19 & 6.37 & 6.19 & 6.22 & 6.21 & 6.50 & 6.41 & 6.27 & 5.65 & 5.03 & 2179.1 & 6.0 \\
\hline 50.0 & 5.12 & 5.51 & 6.07 & $6 .: 3$ & 5.85 & 5.84 & 5.87 & 6.25 & 6.28 & 6.27 & 5.71 & 5.11 & 2130.1 & 5.8 \\
\hline 60.0 & 5.13 & 5.38 & 5.73 & 5.54 & 5.09 & 4.99 & 5.10 & 5.64 & 5.93 & 6.14 & 5.74 & 5.18 & 1994.2 & 5.5 \\
\hline 70.0 & 5.01 & 5.11 & 5.26 & 4.8 .2 & 4.23 & 4.08 & 4.23 & 4.91 & 5.43 & 5.85 & 5.62 & 5.11 & 1814.0 & 5.0 \\
\hline 80.0 & 4.76 & 4.73 & 4.66 & $4 . C 1$ & 3.37 & 3.15 & 3.36 & 4.07 & 4.80 & 5.41 & 5.35 & 4.91 & 1598.1 & 4.4 \\
\hline 90.0 & 4.39 & 4.23 & 3.96 & 3.15 & 2.49 & 2.28 & 2.47 & 3.18 & 4.07 & 4.85 & 4.94 & 4.58 & 1355.1 & 3.7 \\
\hline
\end{tabular}


AVERAGE DAILY TOTAL TERRESTRIAL INSOLATION ESTIMATES (KWH/SQ. M)

SITE: LUFKIN
LATITUDE:
31 DEGREES IS MINUTES

AVERAGE DAILY AMOUNTS BY MONTH

\begin{tabular}{|c|c|c|c|c|c|c|c|c|c|c|c|c|c|c|}
\hline ARRAY & 3 & $F$ & $M$ & A & $M$ & $\mathrm{~J}$ & $\mathrm{~J}$ & A & s & 0 & $N$ & D & $\begin{array}{l}\text { ANNUAL } \\
\text { AMOUNT }\end{array}$ & $\begin{array}{c}\text { AVERAGE } \\
\text { DAY }\end{array}$ \\
\hline 0.0 & 2.50 & 3.38 & 4.33 & 5.12 & 5.89 & 6.48 & 6.33 & 5.87 & 4.82 & 4.25 & 3.03 & 2.42 & 1657.3 & 4.5 \\
\hline 10.0 & 2.82 & 3.69 & 4.56 & 5.20 & 5.85 & 6.38 & 6.28 & 5.98 & 5.09 & 4.72 & 3.49 & 2.81 & 1731.7 & 4.7 \\
\hline 15.0 & 2.97 & 3.81 & $4.64^{\circ}$ & 5.20 & 5.78 & 6.27 & 6.21 & 5.99 & 5.18 & 4.91 & 3.69 & 2.98 & 1754.5 & 4.8 \\
\hline 20.0 & 3.09 & 3.91 & 4.69 & 5.18 & 5.63 & 6.13 & 5.10 & 5.96 & 5.25 & 5.07 & 3.86 & 3.14 & 1767.4 & 4.8 \\
\hline 25.0 & 3.20 & 4.00 & 4.71 & 5.12 & 5.55 & 5.96 & 5.95 & 5.89 & 5.28 & 5.21 & 4.02 & 3.28 & 1770.3 & 4.9 \\
\hline 30.0 & 3.28 & 4.05 & 4.71 & 5.03 & 5.37 & 5.75 & 5.78 & 5.80 & 5.28 & 5.31 & 4.15 & 3.40 & 1763.2 & 4.8 \\
\hline 35.0 & 3.35 & 4.09 & 4.68 & 4.93 & 5.21 & 5.53 & 5.58 & 5.67 & 5.25 & 5.38 & 4.25 & 3.49 & 1747.4 & 4.8 \\
\hline 40.0 & 3.40 & 4.10 & 4.63 & 4.79 & 5.00 & 5.28 & 5.35 & 5.51 & 5.19 & 5.41 & 4.33 & 3.57 & 1722.2 & 4.7 \\
\hline 45.0 & 3.44 & 4.05 & 4.55 & 4.63 & 4.78 & 5.00 & 5.10 & 5.33 & 5.11 & 5.42 & 4.38 & 3.63 & 1687.3 & 4.6 \\
\hline 50.0 & 3.45 & $4.0 E$ & 4.45 & 4.45 & 4.52 & 4.70 & 4.82 & 5.11 & 4.99 & 5.39 & 4.41 & 3.66 & 1643.1 & 4.5 \\
\hline 60.0 & 3.41 & 3.92 & 4.17 & 4.02 & 3.95 & 4.03 & 4.19 & 4.60 & 4.67 & 5.23 & 4.38 & 3.66 & 1528.1 & 4.2 \\
\hline 70.0 & 3.29 & 3.70 & 3.80 & 3.51 & 3.33 & 3.34 & 3.51 & 3.99 & 4.26 & 4.95 & 4.24 & 3.57 & 1383.4 & 3.8 \\
\hline 80.0 & 3.09 & 3.39 & 3.36 & 2.94 & 2.69 & 2.62 & 2.80 & 3.31 & 3.75 & 4.55 & 4.00 & 3.40 & 1213.3 & 3.3 \\
\hline 90.0 & 2.83 & 3.01 & 2.85 & 2.36 & 2.06 & 1.97 & 2.12 & 2.61 & 3.17 & 4.04 & 3.66 & 3.14 & 1028.3 & 2.8 \\
\hline
\end{tabular}


AVERAGE DAILY TOTAL TERRESTRIAL INSOLATION ESTIMATES (KWH/SQ. M)

SITE: MIDLAND-ODESSA TX
LATITUDE: 31 DEGREES 56 MINUTES

AVERAGE DAILY AMOUNTS BY MONTH

\begin{tabular}{|c|c|c|c|c|c|c|c|c|c|c|c|c|c|c|}
\hline ARRAY & $J$ & $F$ & $M$ & $A$ & $M$ & 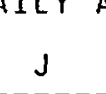 & 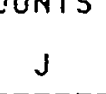 & 180 & $s$ & 0 & $N$ & $D$ & $\begin{array}{l}\text { ANNUAL } \\
\text { ANMUNT }\end{array}$ & $\begin{array}{c}\text { AVERAGE } \\
\text { DAY }\end{array}$ \\
\hline 0.0 & 3.40 & 4.36 & 5.79 & 6.91 & 7.67 & 8.08 & 7.53 & 6.97 & 5.81 & 4.80 & 3.70 & 3.15 & 2075.9 & 5.7 \\
\hline 10.0 & 3.97 & 4.86 & 6.18 & 7.07 & 7.63 & 7.95 & 7.49 & 7.13 & 6.20 & 5.40 & 4.37 & 3.79 & 2193.0 & 6.0 \\
\hline 15.0 & 4.22 & 5.06 & 6.33 & 7.09 & 7.54 & 7.81 & 7.40 & 7.15 & 6.34 & 5.64 & 4.66 & 4.08 & 2232.0 & 6.1 \\
\hline 20.0 & 4.45 & 5.23 & 6.43 & 7.06 & 7.40 & 7.63 & 7.26 & 7.13 & 6.44 & 5.86 & 4.93 & 4.34 & 2257.5 & 6.2 \\
\hline 25.0 & 4.65 & 5.38 & 6.49 & 6.99 & 7.22 & 7.40 & 7.09 & 7.06 & 6.50 & 6.04 & 5.16 & 4.58 & 2269.4 & 6.2 \\
\hline 30.0 & 4.82 & 5.49 & 6.52 & 6.8 .8 & 7.00 & 7.13 & 6.87 & 6.95 & 6.52 & 6.18 & 5.37 & 4.78 & 2267.5 & 6.2 \\
\hline 35.0 & 4.96 & 5.56 & 6.50 & 6.74 & 6.75 & 6.84 & 6.63 & 6.80 & 6.51 & 6.28 & 5.53 & 4.96 & 2253.3 & 6.2 \\
\hline 40.0 & 5.07 & 5.61 & 6.45 & 6.55 & 6.47 & 6.51 & 6.35 & 6.61 & 6.45 & 6.34 & 5.66 & 5.10 & 2226.3 & 6.1 \\
\hline 45.0 & 5.14 & 5.61 & 6.35 & 6.33 & 6.16 & 6.14 & 6.04 & 6.39 & 6.35 & 6.36 & 5.76 & 5.21 & 2186.0 & 6.0 \\
\hline 50.0 & 5.19 & 5.59 & 6.22 & 6.07 & .5 .80 & 5.75 & 5.69 & 6.13 & 6.22 & 6.34 & 5.82 & 5.29 & 2132.5 & 5.8 \\
\hline 60.0 & 5.17 & 5.43 & 5.85 & 5.46 & 5.01 & 4.86 & 4.91 & 5.50 & 5.84 & 6.18 & 5.82 & 5.34 & 1988.0 & 5.4 \\
\hline 70.0 & 5.03 & 5.15 & 5.33 & 4.72 & 4.13 & 3.95 & 4.06 & 4.75 & 5.32 & 5.87 & 5.67 & 5.24 & 1800.7 & 4.9 \\
\hline 80.0 & 4.76 & 4.74 & 4.70 & 3.88 & 3.24 & 3.00 & 3.19 & 3.90 & 4.68 & 5.41 & 5.37 & 5.02 & 1577.2 & 4.3 \\
\hline 90.0 & 4.37 & 4.21 & 3.96 & 3.02 & 2.36 & 2.14 & 2.33 & 3.03 & 3.94 & 4.81 & 4.93 & 4.66 & 1330.2 & 3.6 \\
\hline
\end{tabular}


AVERAGE DAILY TOTAL TERRESTRIAL INSOLATION ESTIMATES (KWH/SQ. M)

SITE: PORT ARTHUR

LATITUDE: 29 DEGREES 57 MINUTES

AVERAGE DAILY AMOUNTS BY MONTH

\section{ARRAY \\ TILT}

0.0

10.0

15.0

20.0

25.0

30.0

35.0

40.0

45.0

50.0

60.0

70.0

80.0

90.0

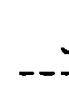

J

2.52

2.83

2.96

3.0

3.17

3.25

3.32

3.36

3.38

3.39

3.3

3.2

3.01

2.74

$F$

$F \quad M$. A

$M$

$\mathrm{J}$

$\begin{array}{lll}3.38 & 4.26 \quad 5.08\end{array}$

3.67

4.47

5.15

5.90

6.34

J $A$

A

$\begin{array}{lll}3.79 & 4.53 & 5.14 \\ 3.88 & 4.57 & 5.11\end{array}$

5.85

6.23

$5.82 \quad 5.48 \quad 4.81$

4.17

N D

$\begin{array}{llll}4.57 & 5.11 & 5.67\end{array}$

6.12

5.77

5.58

5.06

4.59

3.00

$5.695 .55 \quad 5.14$

$\begin{array}{lll}4.77 & 3.60 & 2.88\end{array}$

3.42

2.37

5.97

5.59

5.52

5.19

$4.913 .75 \quad 3.02$

$5.80 \quad 5.45$

5.45

5.22

5.03

5.595 .29

$5.36 \quad 5.21$

5.03

3.89

$\begin{array}{lll}5.59 & 5.29 & 5.36 \\ 5.37 & 5.11 & 5.23\end{array}$

5.18

$\begin{array}{lll}5.11 & 4.00 & 3.24 \\ 5.17 & 4.09 & 3.32\end{array}$

5.12

$4.90 \quad 5.08 \quad 5.11$

$\begin{array}{lll}5.17 & 4.09 & 3.32\end{array}$

$\begin{array}{lll}5.19 & 4.16 \quad 3.39\end{array}$

4.84

$4.66 \quad 4.90 \quad 5.02$

5.19

$4.41 \quad 4.70 \quad 4.90$

5.15

4.54

4.41

$.70 \quad 4.90$
.22

$\begin{array}{lll}5.15 & 4.21 & 3.46 \\ 4.98 & 4.17 & 3.44\end{array}$

$\begin{array}{lllllll}3.88 & 3.83 & 4.22 & 4.57 & 4.98 & 4.17 & 3.44 \\ 3.21 & 3.22 & 3.66 & 4.15 & 4.69 & 4.02 & 3.34 \\ 2.51 & 2.58 & 3.04 & 3.64 & 4.30 & 3.78 & 3.17\end{array}$

$3.30 \quad 3.22$

$2.92 \quad 2.72$

2.85

2.62

2.28

$2.00 \quad 1.89 \quad 1.97$

$2.40 \quad 3.06 \quad 3.81$

$3.45 \quad 2.92$

ANNUAL

AMOUNT

1618.0

1683.8

1702.8

1712.4

1712.5

1703.0

1685.5

1658.7

1622.7

1577.9

1463.2

1321.5

1155.6

977.6 
AVERAGE DAILY TOTAL TERRESTRIAL INSOLATION ESTIMATES (KMH/SQ. M)

$\begin{aligned} \text { SITE: } & \text { SAN ANGELO } \\ \text { LATITUDE: } & 31 \text { TE DEREES } 22 \text { MINUTES }\end{aligned}$

AVERAGE DAILY AMOUNTS BY MONTH

\begin{tabular}{|c|c|c|c|c|c|c|c|c|c|c|c|c|c|c|}
\hline ARRAY & $\mathrm{J}$ & $F$ & $M$ & A & $M$ & $J$ & $J$ & A & $\mathrm{s}$ & 0 & $N$ & $D$ & $\begin{array}{l}\text { ANNUAL } \\
\text { ANIOUNT }\end{array}$ & $\begin{array}{c}\text { AVERAGE } \\
\text { DAY }\end{array}$ \\
\hline 0.0 & 3.02 & 3.81 & 5.05 & 5.83 & 6.41 & 6.89 & 6.69 & 6.19 & 5.06 & 4.22 & 3.29 & 2.82 & 1805.2 & 4.9 \\
\hline 10.0 & 3.48 & 4.19 & 5.35 & 5.94 & 6.37 & 6.78 & 6.64 & 6.32 & 5.35 & 4.68 & 3.81 & 3.33 & 1895.1 & 5.2 \\
\hline 15.0 & 3.67 & 4.34 & 5.46 & 5.95 & 6.29 & 6.66 & 6.56 & 6.32 & 5.46 & 4.87 & 4.04 & 3.56 & 1923.9 & 5.3 \\
\hline 20.0 & 3.85 & 4.47 & 5.53 & 5.92 & 6.18 & 6.51 & 6.45 & 6.29 & 5.53 & 5.04 & 4.25 & 3.77 & 1941.6 & 5.3 \\
\hline 25.0 & 4.00 & 4.5 .8 & 5.57 & 5.85 & 6.04 & 6.32 & 6.29 & 6.23 & 5.57 & 5.17 & 4.43 & 3.95 & 1948.0 & 5.3 \\
\hline 30.0 & 4.13 & 4.65 & 5.58 & 5.76 & 5.86 & 6.10 & 6.10 & 6.13 & 5.57 & 5.27 & 4.59 & 4.11 & 1943.2 & 5.3 \\
\hline 35.0 & 4.23 & 4.70 & 5.56 & 5.63 & 5.66 & 5.86 & 5.89 & 5.99 & 5.55 & 5.34 & 4.71 & 4.24 & 1928.4 & 5.3 \\
\hline 40.0 & 4.31 & 4.73 & 5.50 & 5.48 & 5.43 & 5.59 & 5.65 & 5.83 & 5.49 & 5.38 & 4.81 & 4.35 & 1903.0 & 5.2 \\
\hline 45.0 & 4.36 & 4.72 & 5.41 & 5.30 & 5.18 & 5.29 & 5.38 & 5.63 & 5.40 & 5.38 & 4.87 & 4.43 & 1866.7 & 5.1 \\
\hline 50.0 & 4.39 & 4.69 & 5.29 & 5.08 & 4.89 & 4.97 & 5.08 & 5.40 & 5.28 & 5.35 & 4.91 & 4.48 & 1819.6 & 5.0 \\
\hline 60.0 & 4.36 & 4.54 & 4.96 & 4.58 & 4.26 & 4.24 & 4.40 & 4.85 & 4.94 & 5.20 & 4.89 & 4.50 & 1695.1 & 4.6 \\
\hline 70.0 & 4.22 & 4.29 & 4.52 & 3.98 & 3.56 & 3.50 & 3.67 & 4.21 & 4.50. & 4.92 & $4.74^{\circ}$ & 4.41 & 1536.5 & 4.2 \\
\hline 80.0 & 3.98 & 3.94 & 3.99 & 3.31 & 2.85 & 2.72 & 2.92 & 3.48 & 3.96 & $4.52^{\circ}$ & 4.48 & 4.20 & 1348.3 & 3.7 \\
\hline 90.0 & 3.64 & 3.50 & 3.37 & 2.62 & 2.15 & 2.02 & 2.18 & 2.73 & 3.34 & 4.02 & 4.10 & 3.89 & 1142.3 & 3.1 \\
\hline
\end{tabular}


AVERAGE DAILY TOTAL TERRESTRIAL INSOLATION ESTIMATES (KWH/SQ. M)

SITE: SAN ANTONIO TX
LATITUDE: 29 DECREES 32 MINUTES

AVERAGE DAILY AMOUNTS BY MONTH

\begin{tabular}{|c|c|c|c|c|c|c|c|c|c|c|c|c|c|c|}
\hline ARRAY & $\mathrm{J}$ & $F$ & $M$ & $A$ & $M$ & $J$ & $J$ & A & $\mathrm{s}$ & 0 & $N$ & $\mathrm{D}$ & $\begin{array}{l}\text { ANNUAL } \\
\text { AMOUNT }\end{array}$ & $\begin{array}{c}\text { AVERAGE } \\
\text { DAY }\end{array}$ \\
\hline 0.0 & 2.82 & 3.64 & 4.56 & 5.09 & 5.98 & 6.52 & 6.69 & 6.13 & 5.16 & 4.26 & 3.18 & 2.67 & 1726.8 & 4.7 \\
\hline 10.0 & 3.18 & 3.97 & 4.79 & 5.15 & 5.92 & 6.39 & 6.62 & 6.23 & 5.44 & 4.69 & 3.62 & 3.08 & 1799.2 & 4.9 \\
\hline 15.0 & 3.34 & 4.09 & 4.86 & 5.14 & 5.84 & 6.28 & 6.53 & 6.23 & 5.53 & 4.87 & 3.82 & 3.26 & 1820.3 & 5.0 \\
\hline 20.0 & 3.48 & 4.20 & 4.91 & 5.11 & 5.73 & 6.13 & 6.40 & 6.18 & 5.59 & 5.02 & 3.99 & 3.43 & 1831.2 & 5.0 \\
\hline 25.0 & 3.60 & 4.28 & 4.93 & 5.04 & 5.59 & 5.94 & 6.23 & 6.11 & 5.62 & 5.13 & 4.14 & 3.57 & 1831.6 & 5.0 \\
\hline 30.0 & 3.69 & 4.34 & 4.92 & 4.95 & 5.42 & 5.72 & 6.03 & 6.00 & 5.61 & 5.22 & 4.26 & 3.69 & 1821.9 & 5.0 \\
\hline 35.0 & 3.77 & 4.37 & 9.88 & 4.84 & 5.23 & 5.49 & 5.81 & 5.86 & 5.57 & 5.27 & 4.36 & 3.79 & 1803.2 & 4.9 \\
\hline 40.0 & 3.82 & 4.38 & 4.82 & 4.70 & 5.01 & 5.23 & 5.56 & 5.68 & 5.50 & 5.30 & 4.43 & 3.87 & 1774.3 & 4.9 \\
\hline 45.0 & 3.85 & 4.36 & 4.73 & 4.53 & $: 7.77$ & 4.94 & 5.28 & 5.48 & 5.40 & 5.29 & 4.47 & 3.93 & 1735.5 & 4.8 \\
\hline 50.0 & 3.86 & 4.32 & 4.61 & 4.35 & 4.50 & 4.63 & 4.97 & 5.24 & 5.27 & 5.25 & 4.49 & 3.96 & 1687.1 & 4.6 \\
\hline 60.0 & 3.81 & 4.16 & $\dot{4} .31$ & 3.91 & 3.91 & 3.94 & 4.28 & 4.69 & 4.92 & 5.07 & 4.44 & 3.94 & 1562.9 & 4.3 \\
\hline 70.0 & 3.66 & 3.91 & 3.91 & 3.40 & 3.27 & 3.25 & 3.55 & 4.04 & 4.45 & 4.78 & 4.28 & 3.84 & 1409.4 & 3.9 \\
\hline 80.0 & 3.44 & 3.57 & 3.44 & 2.83 & 2.61 & 2.52 & 2.78 & 3.31 & 3.90 & 4.37 & 4.02 & 3.63 & 1229.5 & 3.4 \\
\hline 90.0 & 3.13 & 3.16 & 2.90 & 2.26 & 1.99 & 1.89 & 2.06 & 2.58 & 3.26 & 3.87 & 3.67 & 3.35 & 1036.7 & 2.8 \\
\hline
\end{tabular}


AVERAGE DAILY TOTAL TERRESTRIAL INSOLATION ESTIMATES (KRH/SQ. M)

$\begin{aligned} \text { SITE: } & \text { SHERMAN } \\ \text { LATITUDE: } & 33 \text { DEGREES } 43 \text { MINUTES }\end{aligned}$

AVERAGE DAILY AMOUNTS BY MONTH

\begin{tabular}{|c|c|c|c|c|c|c|c|c|c|c|c|c|c|c|}
\hline ARRAY & $\mathrm{J}$ & $F$ & $M$ & A & $M$ & $\mathrm{~J}$ & J & $A$ & $s$ & 0 & $N$ & $D$ & $\begin{array}{l}\text { ANNUAL } \\
\text { AMOUNT }\end{array}$ & $\begin{array}{c}\text { AVERAGE } \\
\text { DAY }\end{array}$ \\
\hline 0.0 & 2.50 & $3 . \hat{2} 7$ & 4.30 & 5.08 & 5.84 & 6.67 & 6.55 & 6.09 & 4.98 & 4.00 & 2.89 & 2.34 & 1660.3 & 4.5 \\
\hline 10.0 & 2.88 & 3.60 & 4.55 & $5 .: 9$ & 5.82 & 6.59 & 6.54 & 6.24 & 5.30 & 4.48 & 3.38 & 2.78 & 1746.7 & 4.8 \\
\hline 15.0 & 3.04 & 3.74 & 4.64 & 5.20 & 5.77 & 6.50 & 6.47 & 6.26 & 5.42 & 4.68 & 3.60 & 2.97 & 1775.2 & 4.9 \\
\hline 20.0 & 3.19 & 3.8 .5 & 4.71 & 5.19 & 5.68 & 6.37 & 6.37 & 6.25 & 5.51 & 4.85 & 3.79 & 3.15 & 1793.6 & 4.9 \\
\hline 25.0 & 3.32 & 3.95 & 4.75 & 5.14 & 5.57 & 6.21 & 6.24 & 6.20 & 5.56 & 4.99 & 3.96 & 3.31 & 1801.9 & 4.9 \\
\hline 30.0 & 3.43 & 4.02 & 4.76 & 5.07 & 5.42 & 6.01 & 6.07 & 6.11 & 5.58 & 5.10 & 4.11 & 3.44 & 1799.8 & 4.9 \\
\hline 35.0 & 3.52 & 4.06 & 4.74 & 4.97 & 5.25 & 5.78 & 5.87 & 5.99 & 5.57 & 5.19 & 4.23 & 3.56 & 1787.9 & 4.9 \\
\hline 40.0 & 3.59 & 4.09 & 4.70 & 4.8 .5 & 5.06 & 5.53 & 5.64 & 5.84 & 5.53 & 5.24 & 4.33 & 3.66 & 1767.1 & 4.8 \\
\hline 45.0 & 3.64 & 4.09 & 4.63 & 4.70 & 4.84 & 5.26 & 5.39 & 5.66 & 5.45 & 5.25 & 4.39 & 3.73 & 1736.3 & 4.8 \\
\hline 50.0 & 3.67 & 4.07 & 4.54 & 4.53 & 4.60 & 4.96 & 5.11 & 5.45 & 5.35 & 5.24 & 4.44 & 3.78 & 1695.7 & 4.6 \\
\hline 60.0 & 3.65 & 3.95 & 4.28 & 4.11 & 4.05 & 4.29 & 4.48 & 4.93 & 5.04 & 5.12 & 4.43 & 3.81 & 1586.5 & 4.3 \\
\hline 70.0 & 3.55 & 3.75 & 3.93 & 3.62 & 3.44 & 3.58 & 3.76 & 4.31 & 4.62 & 4.87 & 4.32 & 3.74 & 1444.3 & 4.0 \\
\hline 80.0 & 3.36 & 3.46 & 3.49 & 3.06 & 2.82 & 2.84 & 3.04 & 3.60 & 4.09 & 4.50 & 4.10 & 3.58 & 1276.1 & 3.5 \\
\hline $90: 0$ & 3.09 & 3.10 & 2.99 & 2.48 & 2.18 & 2.14 & 2.31 & 2.86 & 3.49 & 4.03 & 3.78 & 3.33 & 1088.1 & 3.01 \\
\hline
\end{tabular}


AVERAGE DAILY TOTAL TERRESTRIAL INSOLATION ESTIMATES (KWH/SQ. M)

SITE: L'ACO

LATITUDE: 31 DEGREES 37 MINUTES

AVERAG = CAILY AMOUNTS BY MONTH

\begin{tabular}{|c|c|c|c|c|c|c|c|c|c|c|c|c|c|c|}
\hline ARRAY & J & $F$ & $M$ & $A$ & $M$ & $\mathrm{~J}$ & $j$ & A & $\mathrm{s}$ & 0 & $N$ & D & $\begin{array}{l}\text { ANNUAL } \\
\text { AMOUNT }\end{array}$ & $\begin{array}{c}\text { AVERAGE } \\
\text { DAY }\end{array}$ \\
\hline 0.0 & 2.62 & 3.46 & 4.50 & 5.08 & 5.53 & E. 66 & 6.71 & 6.18 & 5.05 & 4.10 & 3.01 & 2.53 & 1690.2 & 4.6 \\
\hline 10.0 & 2.99 & 3.78 & 9.74 & 5.17 & 5.55 & E. 56 & 6.67 & 6.30 & 5.34 & 4.55 & 3.47 & 2.96 & 1769.3 & 4.8 \\
\hline 15.0 & 3.14 & 3.92 & 4.83 & 5.17 & 5.53 & 6.45 & 6.59 & 6.31 & 5.45 & 4.74 & 3.67 & 3.15 & 1794.0 & 4.9 \\
\hline 20.0 & 3.28 & 4.03 & 7.88 & 5.14 & 5.41 & $E .31$ & 6.48 & 6.28 & 5.52 & 4.89 & 3.85 & 3.32 & 1808.5 & 5.0 \\
\hline 25.0 & 3.40 & 4.12 & 4.91 & 5.09 & 5.25 & 6.13 & 6.32 & 6.22 & 5.56 & 5.02 & 4.01 & 3.48 & 1812.8 & 5.0 \\
\hline 30.0 & 3.50 & 4.18 & 4.92 & 5.01 & 5.17 & 5.92 & 6.13 & 6.12 & 5.57 & 5.12 & 4.14 & 3.61 & 1806.8 & 5.0 \\
\hline 35.0 & 3.58 & 4.22 & 4.89 & $4.9 C$ & 4.97 & 5.69 & 5.92 & 5.99 & 5.54 & 5.19 & 4.25 & 3.72 & 1791.6 & 4.9 \\
\hline 40.0 & 3.64 & 4.24 & 4.84 & 4.77 & 4.73 & 5.43 & 5.63 & 5.82 & 5.49 & 5.22 & 4.33 & 3.81 & 1766.8 & 4.8 \\
\hline 45.0 & 3.68 & 4.23 & 4.76 & 4.61 & 4.57 & 5.15 & 5.41 & 5.63 & 5.40 & 5.22 & 4.38 & 3.87 & 1732.1 & 4.7 \\
\hline 50.0 & 3.70 & 4.20 & 4.65 & 4.43 & 4.33 & 4.84 & 5.11 & 5.40 & 5.28 & 5.20 & 4.41 & 3.92 & 1687.6 & 4.6 \\
\hline 60.0 & 3.66 & 4.06 & 4.36 & 4.01 & $3.8 \mathrm{C}$ & 4.14 & 4.43 & 4.86 & 4.95 & 5.05 & 4.38 & 3.92 & 1571.1 & 4.3 \\
\hline 70.0 & 3.54 & 3.83 & 3.98 & 3.51 & $3.2 z$ & 3.43 & 3.70 & 4.21 & 4.51 & 4.78 & 4.25 & 3.84 & 1423.7 & 3.9 \\
\hline 80.0 & 3.33 & 3.52 & 3.52 & 2.94 & 2.62 & 2.68 & 2.94 & 3.49 & 3.97 & 4.40 & 4.01 & 3.65 & 1249.8 & 3.4 \\
\hline 90.0 & 3.05 & 3.13 & 2.99 & 2.36 & 2.03 & 2.01 & 2.20 & 2.74 & 3.36 & 3.91 & 3.68 & 3.38 & 1059.7 & 2.9 \\
\hline
\end{tabular}


AVERAGE DAILY TOTAL TERRESTRIAL INSOLATION ESTIMATES (KLH/SQ. M)

SITE: WICHITA FALLS MX
LATITUDE: 33 DEGREES 58 MINUTES

AVERAGE DAILY AMOUNTS BY MONTH.

\begin{tabular}{|c|c|c|c|c|c|c|c|c|c|c|c|c|c|c|}
\hline ARRA“ & $J$ & $F$ & $M$ & A & $M$ & $J$ & $\mathrm{~J}$ & A & $\mathrm{s}$ & 0 & $N$ & D & $\begin{array}{l}\text { ANNUAL } \\
\text { AMOUHT }\end{array}$ & $\begin{array}{c}\text { AVERAGE } \\
\text { DAY }\end{array}$ \\
\hline 0.0 & 2.71 & 3.55 & 4.64 & 5.56 & 6.36 & 7.00 & 6.82 & $6: 20$ & 5.04 & 4.07 & 3.01 & 2.52 & 1750.6 & 4.8 \\
\hline 10.0 & 3.15 & 3.93 & 4.93 & 5.69 & 6.35 & 6.92 & 6.81 & 6.37 & 5.38 & 4.57 & 3.54 & 3.02 & 1847.0 & 5.1 \\
\hline 15.0 & 3.34 & 4.08 & 5.04 & 5.70 & 6.29 & 6.82 & 6.75 & 6.39 & 5.50 & 4.78 & 3.78 & 3.24 & 1879.4 & 5.1 \\
\hline 20.0 & 3.51 & 4.22 & 5.12 & 5.69 & 6.19 & 6.69 & 6.64 & 6.38 & 5.59 & 4.96 & 3.99 & 3.45 & 1901.0 & 5.2 \\
\hline 25.0 & 3.67 & 4.33 & 5.17 & $5 . \dot{3} 4$ & 6.07 & 6.51 & 6.50 & 6.33 & 5.65 & 5.11 & 4.18 & 3.63 & 1911.5 & 5.2 \\
\hline 30.0 & 3.80 & 4.41 & 5.19 & 5.57 & 5.90 & 6.30 & 6,32 & 6.24 & 5.68 & 5.23 & 4.34 & 3.79 & 1911.0 & 5.2 \\
\hline 35.0 & 3.90 & 4.47 & 5.18 & 5.76 & 5.71 & 6.06 & 6.11 & 6.12 & 5.67 & 5.32 & 4.48 & 3.93 & 1899.8 & 5.2 \\
\hline 40.0 & 3.99 & 4.51 & 5.14 & 5.32 & 5.50 & 5.80 & 5.88 & 5.97 & 5.63 & 5.37 & 4.59 & 4.05 & 1879.1 & 5.1 \\
\hline 45.0 & 4.05 & 4.51 & 5.07 & 5.16 & 5.26 & 5.51 & 5.62 & 5.79 & 5.55 & 5.39 & 4.66 & 4.13 & 1847.5 & 5.1 \\
\hline 50.0 & 4.08 & 4.49 & 4.97 & 4.97 & 5.00 & 5.20 & 5.33 & 5.57 & 5.44 & 5.38 & 4.71 & 4.19 & 1805.4 & 4.9 \\
\hline 60.0 & 4.07 & 4.37 & 4.69 & 4.51 & 4.39 & 4.48 & 4.66 & 5.04 & 5.13 & 5.26 & 4.72 & 4.24 & 1690.6 & 4.6 \\
\hline 70.0 & 3.97 & 4.15 & 4.30 & 3.96 & 3.70 & 3.73 & 3.91 & 4.41 & 4.71 & 5.01 & 4.61 & 4.17 & 1539.9 & 4.2 \\
\hline 80.0 & 3.76 & 3.84 & 3.83 & 3.34 & 3.01 & 2.94 & 3.15 & 3.69 & 4.18 & 4.64 & 4.38 & 4.00 & 1360.9 & 3.7 \\
\hline 90.0 & 3.47 & 3.44 & 3.27 & 2.68 & 2.30 & 2.21 & 2.37 & 2.93 & 3.56 & 4.15 & 4.04 & 3.73 & 1160.0 & 3.2 \\
\hline
\end{tabular}


AVERAGE DAILY TOTAL TERRESTRIAL INSOLATION ESTIMATES (KWH/SQ. M)

$\begin{aligned} \text { SITE: } & \text { BRYCE CANYON U UT } \\ \text { LATITLDE: } & 37 \text { DEGREES } 42 \text { MINUTES }\end{aligned}$

AVERACE DAILY AMOUNTS BY MONTH

\begin{tabular}{|c|c|c|c|c|c|c|c|c|c|c|c|c|c|c|}
\hline ARRAY & $J$ & $F$ & $M$ & $A$ & $M$ & $\mathrm{~J}$ & $J$ & A & $s$ & 0 & N & D. & $\begin{array}{l}\text { ANNUAL } \\
\text { AMOUNT }\end{array}$ & $\begin{array}{c}\text { AVERAGE } \\
\text { DAY }\end{array}$ \\
\hline 0.0 & 2.88 & 3.89 & 5.31 & 6.72 & 7.73 & 8.37 & 7.65 & 6.80 & 6.05 & 4.62 & 3.20 & 2.58 & 2004.2 & 5.5 \\
\hline 10.0 & 3.49 & $4.4 c$ & 5.76 & 6.97 & 7.79 & 8.34 & 7.70 & 7.06 & 6.62 & 5.36 & 3.95 & 3.26 & 2154.0 & 5.9 \\
\hline 15.0 & 3.77 & 4.67 & 5.94 & 7.04 & 7.75 & 8.25 & 7.66 & 7.13 & 6.84 & 5.69 & 4.29 & 3.56 & 2209.6 & 6.1 \\
\hline 20.0 & 4.03 & 4.88 & 6.08 & 7.06 & 7.66 & 8.11 & 7.57 & 7.15 & 7.02 & 5.98 & 4.60 & 3.85 & 2251.7 & 6.2 \\
\hline 25.0 & 4.25 & 5.06 & 6.18 & 7.03 & 7.53 & 7.93 & 7.44 & 7.13 & 7.15 & 6.23 & 4.88 & 4.11 & 2279.9 & 6.2 \\
\hline 30.0 & 4.46 & 5.20 & 6.25 & 6.97 & 7.35 & 7.69 & 7.26 & 7.06 & 7.24 & 6.43 & 5.13 & 4.34 & 2294.0 & 6.3 \\
\hline 35.0 & 4.63 & 5.31 & 6.28 & 6.86 & 7.13 & 7.41 & 7.04 & 6.96 & 7.28 & 6.60 & 5.34 & 4.55 & 2294.0 & 6.3 \\
\hline 40.0 & 4.77 & 5.39 & 6.26 & 6.72 & 6.87 & 7.10 & 6.79 & 6.81 & 7.28 & 6.73 & 5.52 & 4.72 & 2281.1 & 6.2 \\
\hline 45.0 & 4.89 & 5.44 & 6.22 & 6.54 & 6.57 & 5.76 & 6.51 & 6.63 & 7.22 & 6.81 & 5.66 & 4.86 & 2255.7 & 6.2 \\
\hline 50.0 & 4.97 & 5.45 & 6.13 & 6.32 & 6.23 & 5.39 & 6.19 & 6.41 & 7.13 & 6.84 & 5.77 & 4.98 & 2216.3 & 6.1 \\
\hline 60.0 & 5.03 & 5.37 & 5.84 & 5.78 & 5.54 & 5.54 & 5.46 & 5.85 & 6.80 & 6.78 & 5.86 & 5.10 & 2097.4 & 5.7 \\
\hline 70.0 & 4.96 & 5.16 & 5.42 & 5.11 & 4.63 & 4.58 & 4.61 & 5.17 & 6.30 & 6.53 & 5.80 & 5.09 & 1928.3 & 5.3 \\
\hline 80.0 & 4.77 & 4.82 & 4.87 & 4.33 & 3.79 & 3.63 & 3.74 & 4.37 & 5.65 & 6.12 & 5.58 & 4.94 & 1720.4 & 4.7 \\
\hline 90.0 & 4.45 & 4.37 & 4.20 & 3.47 & 2.85 & 2.66 & 2.82 & 3.49 & 4.86 & 5.55 & 5.21 & 4.66 & 1476.9 & 4.0 \\
\hline
\end{tabular}


AVERAGE DAILY TOTAL TERRESTRIAL INSOLATION ESTIMATES (KWH/SQ. M)

SITE: CEDAR CITY

LATITUDE: 37 DEGREES 42 MINUTES

AVERAGE DAILY AMOUNTS BY MONTH

\begin{tabular}{|c|c|c|c|c|c|c|c|c|c|c|c|c|c|c|}
\hline ARRAY & $\mathbf{J}$ & $F$ & $M$ & A & $M$ & $J$ & $J$ & A & 5 & 0 & $N$ & $D$ & $\begin{array}{l}\text { ANNUAL } \\
\text { AMIOUNT }\end{array}$ & $\begin{array}{c}\text { AVERAGE } \\
\text { DAY }\end{array}$ \\
\hline 0.0 & 2.78 & 3.72 & 5.16 & 6.60 & 7.78 & 8.53 & 7.89 & 7.07 & 6.21 & 4.60 & 3.13 & 2.48 & 2008.5 & 5.5 \\
\hline 10.0 & 3.36 & 4.22 & 5.59 & 6.84 & 7.84 & 8.51 & 7.95 & 7.34 & 6.80 & 5.34 & 3.85 & 3.11 & 2154.3 & 5.9 \\
\hline 15.0 & 3.63 & 4.44 & 5.75 & 6.90 & 7.80 & 8.41 & 7.91 & 7.41 & 7.03 & 5.66 & 4.17 & 3.39 & 2207.7 & 6.0 \\
\hline 20.0 & 3.86 & 4.53 & 5.89 & 6.92 & 7.71 & 8.27 & 7.82 & 7.44 & 7.21 & 5.95 & 4.47 & 3.66 & 2247.7 & 6.2 \\
\hline 25.0 & 4.08 & 4.79 & 5.98 & 6.90 & 7.57 & 8.08 & 7.68 & 7.42 & 7.36 & 6.19 & 4.74 & 3.90 & 2273.9 & 6.2 \\
\hline $30 . c$ & 4.27 & 4.92 & 6.04 & 6.84 & 7.39 & 7.84 & 7.49 & 7.35 & 7.45 & 6.40 & 4.98 & 4.11 & 2286.0 & 6.3 \\
\hline 35.0 & 4.43 & 5.03 & 6.07 & 6.73 & 7.17 & 7.55 & 7.27 & 7.24 & 7.50 & 6.57 & 5.19 & 4.30 & 2284.0 & 6.3 \\
\hline 40.0 & 4.57 & 5.10 & 6.06 & 6.59 & 6.91 & 7.23 & 7.00 & 7.09 & 7.49 & 6.69 & 5.36 & 4.46 & 2269.2 & 6.2 \\
\hline 45.0 & 4.67 & 5.34 & 6.01 & 6.42 & 6.63 & 6.89 & 6.72 & 6.90 & 7.44 & 6.77 & 5.49 & 4.60 & 2242.0 & 6.1 \\
\hline 50.0 & 4.75 & 5.15 & 5.92 & 6.20 & 6.31 & 6.50 & 6.39 & 6.67 & 7.35 & 6.80 & 5.59 & 4.70 & 2201.0 & 6.0 \\
\hline 60.0 & 4.80 & 5.07 & 5.64 & 5.67 & 5.57 & 5.63 & 5.62 & 6.09 & 7.01 & 6.74 & 5.68 & 4.81 & 2079.0 & 5.7 \\
\hline 70.0 & 4.74 & 4.86 & 5.23 & 5.01 & 4.70 & 4.65 & 4.74 & 5.37 & 6.50 & 6.50 & 5.62 & 4.79 & 1907.5 & 5.2 \\
\hline 80.0 & 4.55 & 4.54 & 4.70 & 4.25 & 3.81 & 3.66 & 3.83 & 4.53 & 5.82 & 6.08 & 5.40 & 4.65 & 1697.9 & 4.7 \\
\hline 90.0 & 4.24 & 4.11 & 4.06 & 3.41 & 2.87 & 2.68 & 2.87 & 3.62 & $5=00$ & 5.52 & 5.05 & 4.38 & 1453.4 & 4.0 \\
\hline
\end{tabular}


AVERAGE DAILY TOTAL TERRESTRIAL INSOLATION ESTIMATES (KWH/SQ. M)

SITE: SALT LAKE CITY UT

LATITUEE: 40 DEGREES 46 MINUTES

AVERAG D DAILY AMOUNTS BY MONTH

\begin{tabular}{|c|c|c|c|c|c|c|c|c|c|c|c|c|c|c|}
\hline ARRAY & $J$ & $F$ & $M$ & A & $M$ & $J$ & $\mathrm{~J}$ & A & $S$ & 0 & N & $D$ & $\begin{array}{l}\text { ANNUAL } \\
\text { AMOUNT }\end{array}$ & $\begin{array}{c}\text { AVERAGE } \\
\text { DAY }\end{array}$ \\
\hline 0.0 & 2.02 & 3.12 & 4.58 & 5.97 & 7.45 & 8.07 & 8.17 & 7.10 & 5.81 & 4.07 & 2.49 & 1.80 & 1848.4 & 5.1 \\
\hline 10.0 & 2.43 & 3.55 & 4.99 & 6.22 & 7.55 & 8.09 & 8.29 & 7.44 & 6.42 & 4.78 & 3.09 & 2.25 & 1984.3 & 5.4 \\
\hline 15.0 & 2.62 & 3.74 & 5.16 & 6.29 & 7.54 & 8.04 & 8.27 & 7.55 & 6.67 & 5.09 & 3.36 & 2.46 & 2034.8 & 5.6 \\
\hline 20.0 & 2.79 & 3.91 & 5.29 & 6.32 & 7.43 & 7.93 & 8.21 & 7.60 & 6.88 & 5.37 & 3.61 & 2.65 & 2072.9 & 5.7 \\
\hline 25.0 & 2.95 & 4.06 & 5.39 & 6.32 & 7.37 & 7.78 & 8.09 & 7.61 & 7.04 & 5.62 & 3.84 & 2.83 & 2098.5 & 5.7 \\
\hline 36.0 & 3.08 & 4.18 & 5.46 & 6.28 & 7.23 & 7.58 & 7.92 & 7.57 & 7.16 & 5.83 & 4.04 & 2.99 & 2111.2 & 5.8 \\
\hline 35.0 & 3.20 & 4.27 & 5.50 & 6.20 & 7.04 & 7.33 & 7.71 & 7.49 & 7.23 & 6.00 & 7.22 & 3.13 & 2111.1 & 5.8 \\
\hline 40.0 & 3.30 & 4.34 & 5.50 & 6.09 & i. 81 & 7.05 & 7.45 & 7.35 & 7.25 & 6.13 & 4.37 & 3.25 & 2098.0 & 5.7 \\
\hline 45.0 & 3.38 & 4.38 & 5.47 & 5.95 & 6.55 & 6.75 & 7.17 & 7.18 & 7.23 & 6.23 & 4.49 & 3.35 & 2074.6 & 5.7 \\
\hline 50.0 & 3.44 & 4.40 & 5.41 & 5.78 & 6.27 & 6.41 & 6.85 & 6.97 & 7.16 & 6.23 & 4.59 & 3.42 & 2039.0 & 5.6 \\
\hline 60.0 & 3.49 & 4.35 & 5.19 & 5.33 & 5.6 .0 & 5.64 & 6.09 & 6.42 & 6.89 & 6.26 & 4.68 & 3.51 & 1931.2 & 5.3 \\
\hline 70.0 & 3.45 & 4.20 & 4.85 & 4.77 & 4.80 & 4.74 & 5.19 & 5.72 & 6.44 & 6.07 & 4.65 & 3.51 & 1777.4 & 4.9 \\
\hline 80.0 & 3.33 & 3.95 & 4.40 & 4.10 & 3.95 & 3.84 & 4.23 & 4.89 & 5.83 & 5.73 & 4.50 & 3.42 & 1587.0 & 4.3 \\
\hline 90.0 & 3.12 & 3.60 & 3.85 & 3.36 & 3.07 & 2.89 & 3.24 & 3.96 & 5.07 & 5.24 & 4.23 & 3.24 & 1364.8 & 3.7 \\
\hline
\end{tabular}


AVERAGE DAILY TOTAL TERRESTRIAL INSOLATION ESTIMATES (KLH/SQ. M)

SITE: NORFOLK

LATITUDE: 36 DEGREES 54 MINUTES

AVERAGE DAILY AMOUNTS BY MONTH

\begin{tabular}{|c|c|c|c|c|c|c|c|c|c|c|c|c|c|c|}
\hline ARRAY & $\mathrm{J}$ & $F$ & $M$ & $A$ & $M$ & $\mathrm{~J}$ & $\jmath$ & $A$ & $\mathrm{~s}$ & 0 & $\mathbf{N}$ & $\mathrm{D}$ & $\begin{array}{l}\text { ANNUAL } \\
\text { AMOUNT. }\end{array}$ & $\begin{array}{c}\text { AVERAGE } \\
\text { DAY }\end{array}$ \\
\hline 0.0 & 2.13 & 2.94 & 4.03 & 5.29 & 5.95 & 6.31 & 5.84 & 5.30 & 4.39 & 3.42 & 2.55 & 1.97 & 1526.0 & 4.2 \\
\hline 10.0 & 2.49 & 3.26 & $4: 30$ & 5.4 .4 & 5.97 & 6.27 & 5.86 & 5.45 & 4.70 & 3.84 & 3.03 & 2.37 & 1613.5 & 4.4 \\
\hline 15.0 & 2.65 & 3.40 & 4.40 & 5.47 & 5.93 & 6.21 & 5.82 & 5.48 & 4.82 & 4.02 & 3.25 & 2.55 & 1644.0 & 4.5 \\
\hline 20.0 & 2.79 & 3.52 & 4.48 & 5.47 & 5.86 & 6.10 & 5.75 & 5.48 & 4.91 & $4: 18$ & 3.45 & 2.72 & 1665.2 & 4.6 \\
\hline 25.0 & 2.91 & 3.61 & 4.53 & 5.44 & 5.76 & 5.97 & 5.65 & 5.45 & 4.97 & 4.31 & 3.62 & 2.87 & 1677.0 & 4.6 \\
\hline 30.0 & 3.02 & 3.69 & 4.56 & 5.38 & 5.62 & 5.80 & 5.51 & 5.39 & 5.00 & 4.42 & 3.77 & 3.00 & 1679.3 & 4.6 \\
\hline 35.0 & 3.11 & 3.75 & 4.56 & 5.29 & 5.46 & 5.60 & 5.35 & 5.30 & 5.00 & 4.50 & 3.90 & 3.12 & 1672.2 & 4.6 \\
\hline 40.0 & 3.19 & 3.78 & 4.53 & 5.17 & 5.27 & 5.38 & .5 .17 & 5.19 & 4.97 & 4.56 & 4.01 & 3.21 & 1656.7 & 4.5 \\
\hline 45.0 & 3.24 & 3.79 & 4.48 & 5.03 & 5.07 & 5.14 & 4.97 & 5.05 & 4.92 & 4.58 & 4.09 & 3.29 & 1632.5 & 4.5 \\
\hline 50.0 & 3.27 & 3.78 & 4.40 & 4.86 & 4.83 & 4.88 & 4.74 & 4.88 & 4.84 & 4.58 & 4.14 & 3.34 & 1599.2 & 4.4 \\
\hline 60.0 & 3.28 & 3.70 & 4.18 & 4.45 & 4.29 & 4.28 & 4.21 & 4.46 & 4.59 & 4.50 & 4.17 & 3.39 & 1506.3 & 4.1 \\
\hline 70.0 & 3.21 & 3.53 & 3.86 & 3.95 & 3.68 & 3.62 & 3.61 & 3.95 & 4.24 & 4.31 & 4.10 & 3.36 & 1381.1 & 3.8 \\
\hline 80.0 & 3.06 & 3.2 .3 & 3.47 & 3.37 & 3.04 & 2.94 & 2.99 & 3.37 & 3.80 & 4.01 & 3.92 & 3.23 & 1231.4 & 3.4 \\
\hline 90.0 & 2.84 & 2.95 & 3.00 & 2.74 & 2.37 & 2.27 & 2.33 & 2.74 & 3.28 & 3.62 & 3.64 & 3.03 & 1059.7 & 2.9 \\
\hline
\end{tabular}


AVERAGE DAILY TOTAL TERRESTRIAL INSOLATION ESTIMATES (KWH/SQ. M)

SITE: RICHMOND

LATITUDE: 37 DEGREES 30 MINUTES

AVERAGE DAILY AMOUNTS BY MONTH

\begin{tabular}{|c|c|c|c|c|c|c|c|c|c|c|c|c|c|c|}
\hline ARRAY & $J$ & $F$ & $M$ & A & $M$ & ل & $J$ & A & $S$ & 0 & $N$ & D & $\begin{array}{l}\text { ANNUAL } \\
\text { AMOUNT }\end{array}$ & $\begin{array}{c}\text { AVERAGE } \\
\text { DAY }\end{array}$ \\
\hline 0.0 & 1.99 & 2.77 & 3.81 & 4.94 & 5.55 & 5.90 & 5.59 & 5.05 & 4.24 & 3.26 & 2.31 & 1.79 & 1437.2 & 3.9 \\
\hline 10.0 & 2.32 & 3.07 & 4.06 & 5.08 & 5.57 & 5.88 & 5.61 & 5.19 & 4.54 & 3.66 & 2.73 & 2.15 & 1518.4 & 4.2 \\
\hline 15.0 & 2.46 & 3.20 & 4.15 & 5.11 & 5.54 & 5.82 & 5.58 & 5.22 & 4.65 & 3.83 & 2.92 & 2.31 & 1546.7 & 4.2 \\
\hline 20.0 & 2.59 & 3.30 & 4.23 & 5.11 & 5.48 & 5.73 & 5.51 & 5.23 & 4.74 & 3.98 & 3.09 & 2.46 & 1566.4 & 4.3 \\
\hline 25.0 & 2.71 & 3.40 & 4.27 & 5.05 & 5.39 & $=.61$ & 5.42 & 5.20 & 4.80 & 4.11 & 3.24 & 2.59 & 1577.5 & 4.3 \\
\hline 30.0 & 2.81 & 3.47 & 4.30 & $5.0 \geq$ & 5.26 & 5.45 & 5.30 & 5.14 & 4.83 & 4.21 & 3.38 & 2.71 & 1579.7 & 4.3 \\
\hline 35.0 & 2.89 & 3.52 & 4.30 & $4.9 E$ & 5.12 & 5.27 & 5.15 & 5.06 & 4.83 & 4.29 & 3.49 & 2.81 & 1573.2 & 4.3 \\
\hline 40.0 & 2.96 & 3.55 & 4.28 & $4.8 c$ & 4.95 & 5.07 & 4.97 & $4.95^{\circ}$ & 4.81 & 4.34 & 3.58 & 2.90 & 1558.7 & 4.3 \\
\hline 45.0 & 3.01 & 3.56 & 4.23 & 4.71 & 4.76 & 4.86 & 4.73 & 4.82 & 4.76 & 4.37 & 3.65 & 2.97 & 1536.4 & 4.2 \\
\hline 50.0 & 3.04 & 3.56 & 4.16 & 4.56 & 4.55 & 4.62 & 4.57 & 4.66 & 4.68 & 4.37 & 3.70 & 3.01 & 1505.6 & 4.1 \\
\hline 60.0 & 3.05 & 3.48 & 3.95 & 4.18 & 4.06 & 4.07 & 4.08 & 4.27 & 4.45 & 4.29 & 3.72 & 3.06 & 1419.5 & 3.9 \\
\hline 70.0 & 2.98 & 3.32 & 3.66 & 3.72 & 3.49 & 3.46 & 3.51 & 3.80 & 4.12 & 4.11 & 3.66 & 3.02 & 1303.6 & 3.6 \\
\hline 80.0 & 2.85 & 3.09 & 3.29 & 3.19 & 2.91 & 2.85 & 2.92 & 3.26 & 3.69 & 3.83 & 3.50 & 2.91 & 1164.6 & 3.2 \\
\hline 90.0 & 2.64 & 2.79 & 2.86 & 2.62 & 2.30 & 2.23 & 2.30 & 2.66 & 3.20 & 3.47 & 3.25 & 2.74 & 1005.2 & 2.8 \\
\hline
\end{tabular}


AVERAGE DAILY TOTAL TERRESTRIAL INSOLATION ESTIMATES (KLH/SQ. M)

SITE: ROANOKE
LATITUDE:
37 DEGREES 19 MINUTES

AVERAGE DAILY AMOUNTS BY MONTH

\begin{tabular}{|c|c|c|c|c|c|c|c|c|c|c|c|c|c|c|}
\hline ARRAY & $\mathrm{J}$ & $F$ & $M$ & $A$ & $M$ & $\mathrm{~J}$ & $J$ & A & $s$ & 0 & $\mathrm{~N}$ & D & $\begin{array}{l}\text { ANNUAL } \\
\text { ANIOUNT }\end{array}$ & $\begin{array}{c}\text { AVERAGE } \\
\text { DAY }\end{array}$ \\
\hline 0.0 & 2.08 & 2.84 & 3.89 & 4.99 & 5.56 & 5.94 & 5.66 & 5.11 & 4.28 & 3.41 & 2.41 & 1.87 & 1462.7 & 4.0 \\
\hline 10.0 & 2.43 & 3.15 & 4.15 & 5.13 & 5.58 & 5.91 & 5.68 & 5.26 & 4.58 & 3.84 & 2.86 & 2.25 & 1547.5 & 4.2 \\
\hline 15.0 & 2.58 & 3.28 & 4.25 & 5.16 & 5.55 & 5.85 & 5.64 & 5.29 & 4.69 & 4.03 & 3.06 & 2.42 & 1577.2 & 4.3 \\
\hline 20.0 & 2.72 & 3.39 & 4.32 & 5.16 & 5.49 & 5.76 & 5.58 & 5.29 & 4.78 & 4.19 & 3.24 & 2.58 & 1598.2 & 4.4 \\
\hline 25.0 & 2.85 & 3.49 & 4.37 & 5.13 & 5.39 & 5.63 & 5.48 & $5.27^{\circ}$ & 4.84 & 4.33 & 3.40 & 2.72 & 1610.3 & 4.4 \\
\hline 30.0 & 2.96 & 3.56 & 4.40 & 5.07 & 5.27 & 5.48 & 5.36 & 5.21 & 4.87 & 4.44 & 3.54 & 2.84 & 1613.3 & 4.4 \\
\hline 35.0 & 3.05 & 3.62 & 4.40 & 4.99 & 5.12 & 5.30 & 5.20 & 5.12 & 4.87 & 4.52 & 3.66 & 2.95 & 1607.3 & 4.4 \\
\hline 40.0 & 3.12 & 3.65 & 4.38 & 4.88 & 4.95 & 5.10 & 5.03 & 5.01 & 4.85 & 4.58 & 3.76 & 3.04 & 1593.2 & 4.4 \\
\hline 45.0 & 3.17 & 3.66 & 4.33 & 4.75 & 4.76 & 4.88 & 4.83 & 4.88 & 4.79 & 4.61 & 3.83 & 3.11 & 1571.0 & 4.3 \\
\hline 50.0 & 3.21 & 3.66 & 4.25 & 4.6 .0 & 4.55 & 4.63 & 4.62 & 4.72 & 4.72 & 4.61 & 3.88 & 3.17 & 1540.1 & 4.2 \\
\hline 60.0 & 3.22 & 3.53 & 4.04 & 4.21 & 4.06 & 4.08 & 4.11 & 4.32 & 4.48 & 4.53 & 3.91 & 3.21 & 1453.0 & 4.0 \\
\hline 70.0 & 3.15 & 3.41 & 3.74 & 3.75 & 3.49 & 3.47 & 3.53 & 3.84 & 4.14 & 4.34 & 3.84 & 3.18 & 1335.2 & 3.7 \\
\hline 80.0 & 3.01 & 3.1 .3 & 3.36 & 3.21 & 2.91 & 2.85 & 2.94 & 3.29 & 3.72 & 4.05 & 3.68 & 3.07 & 1193.5 & 3.3 \\
\hline 90.0 & 2.79 & 2.87 & 2.92 & 2.63 & 2.29 & 2.23 & 2.31. & 2.68 & 3.21 & 3.67 & 3.42 & 2.88 & 1030.7 & 2.8 \\
\hline
\end{tabular}


AVERAGE DAILY TOTEL TERRESTRIAL INSOLATION ESTIMATES (KLH/SQ. M)

SITE: BURLINGTON

LATITUQE: 44 DECREES 28 MINUTES

AVERAGE DEILY AMOUNTS BY MONTH

\begin{tabular}{|c|c|c|c|c|c|c|c|c|c|c|c|c|c|c|}
\hline ARRAY & $J$ & $F$ & $M$ & A & $M$ & 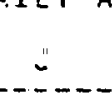 & $\mathrm{J}$ & $A$ & 5 & 0 & $N$ & $\mathrm{D}$ & $\begin{array}{l}\text { ANNUAL } \\
\text { AMOUNT }\end{array}$ & $\begin{array}{c}\text { AVERAGE } \\
\text { DAY }\end{array}$ \\
\hline 0.0 & 1.22 & 1.91 & 2.96 & 4.09 & 4.96 & $=.45$ & 5.43 & 4.65 & 3.53 & 2.34 & 1.18 & 0.89 & 1177.3 & 3.2 \\
\hline 10.0 & 1.44 & 2.14 & 3.19 & 4.25 & 5.08 & 5.49 & 5.52 & 4.85 & 3.85 & 2.68 & 1.39 & 1.14 & 1248.9 & 3.4 \\
\hline 15.0 & 1.54 & 2.24 & 3.28 & 4.29 & 5.04 & 5.46 & 5.52 & 4.91 & 3.97 & 2.83 & 1.49 & 1.26 & 1274.9 & 3.5 \\
\hline 20.0 & 1.63 & 2.33 & 3.36 & 4.32 & $5.0 \mathrm{I}$ & 5.41 & 5.49 & 4.95 & 4.08 & 2.96 & 1.57 & 1.36 & 1294.2 & 3.5 \\
\hline 25.0 & 1.71 & 2.40 & 3.42 & 4.32 & $4.9 E$ & 5.33 & 5.43 & 4.95 & 4.16 & 3.08 & 1.65 & 1.46 & 1306.5 & 3.6 \\
\hline 30.0 & 1.79 & 2.47 & 3.45 & 4.30 & 4.83 & 5.22 & 5.34 & 4.93 & 4.21 & 3.18 & 1.72 & 1.55 & 1311.6 & 3.6 \\
\hline 35.0 & 1.85 & 2.51 & 3.47 & 4.25 & 4.77 & 5.09 & 5.23 & 4.89 & 4.25 & 3.26 & $\mathbb{1} .78$ & 1.63 & 1309.5 & 3.6 \\
\hline 40.0 & 1.90 & 2.55 & 3.47 & 4.19 & 4.64 & 4.92 & 5.08 & 4.81 & 4.26 & 3.32 & 1.83 & 1.70 & 1300.3 & 3.6 \\
\hline 45.0 & 1.94 & 2.57 & 3.46 & 4.10 & 4.47 & 4.74 & 4.91 & 4.71 & 4.24 & 3.36 & J. 87 & 1.76 & 1284.2 & 3.5 \\
\hline 50.0 & 1.97 & 2.57 & 3.42 & 3.99 & 4.35 & 4.55 & 4.73 & 4.59 & 4.20 & 3.39 & J. 89 & 1.81 & 1262.5 & 3.5 \\
\hline 60.0 & 2.00 & 2.54 & 3.29 & 3.72 & 3.94 & 4.10 & 4.30 & 4.28 & 4.05 & 3.37 & 1.92 & 1.87 & 1199.1 & 3.3 \\
\hline 70.0 & 1.98 & 2.46 & 3.09 & 3.38 & 3.43 & 3.57 & 3.78 & 3.88 & 3.81 & 3.27 & 1.89 & 1.88 & 1110.3 & 3.0 \\
\hline 80.0 & 1.91 , & 2.32 & 2.83 & 2.97 & 2.95 & 3.01 & 3.20 & 3.40 & 3.48 & 3.10 & i. 83 & 1.85 & 1000.0 & 2.7 \\
\hline 90.0 & 1.80 & 2.12 & 2.51 & 2.51 & 2.47 & 2.44 & 2.62 & 2.86 & 3.08 & 2.85 & i. 72 & 1.76 & 874.0 & 2.4 \\
\hline
\end{tabular}


AVERAGE DAILY TOTAL TERRESTRIAL INSOLATION ESTIMATES (KLH/SQ. M)

$\begin{aligned} \text { SITE: } & \text { OLYMPIA } \\ \text { LATITUDE: } & 46 \text { DEGREES } 58 \text { MINUTES }\end{aligned}$

AVERAGE DAILY AMOUNTS BY MONTH

\begin{tabular}{|c|c|c|c|c|c|c|c|c|c|c|c|c|c|c|}
\hline ARRAY & $\mathrm{J}$ & $F$ & $M$ & $A$ & $M$ & $\mathrm{~J}$ & $J$ & A & 5 & 0 & $N$ & $\mathrm{D}$ & $\begin{array}{l}\text { ANNUAL } \\
\text { AMOUNT }\end{array}$ & $\begin{array}{c}\text { AVERAGE } \\
\text { DAY }\end{array}$ \\
\hline 0.0 & 0.85 & 1.58 & 2.66 & 3.96 & 5.14 & 5.34 & 6.04 & 4.88 & 3.65 & 2.01 & 1.07 & 0.70 & 1155.2 & 3.2 \\
\hline 10.0 & 1.05 & 1.77 & 2.87 & 4.13 & 5.25 & 5.39 & 6.17 & 5.13 & 4.02 & 2.31 & 1.29 & 0.90 & 1228.8 & 3.4 \\
\hline 15.0 & 1.14 & 1.85 & 2.96 & 4.19 & 5.26 & 5.37 & 6.19 & 5.22 & 4.17 & 2.45 & 1.39 & 1.00 & 1255.9 & 3.4 \\
\hline 20.0. & 1.22 & 1.92 & 3.03 & 4.22 & 5.24 & 5.33 & 6.17 & 5.27 & 4.30 & 2.57 & 1.49 & 1.09 & 1276.4 & 3.5 \\
\hline 25.0 & 1.30 & 1.98 & 3.09 & 4.23 & 5.20 & 5.27 & 6.12 & 5.29 & 4.41 & 2.68 & 1.57 & 1.17 & 1290.0 & 3.5 \\
\hline 30.0 & 1.37 & 2.03 & 3.13 & $4.2 ?$ & 5.12 & 5.17 & 6.04 & 5.29 & 4.49 & 2.77 & 1.65 & 1.25 . & 1296.5 & 3.6 \\
\hline 35.0 & 1.43 & $2.0 ?$ & 3.15 & 4.13 & 5.03 & 5.05 & 5.92 & 5.25 & 4.54 & 2.85 & 1.71 & 1.32 & 1295.7 & 3.5 \\
\hline 40.0 & 1.48 & 2.09 & 3.16 & 4.13 & 4.90 & 4.90 & 5.77 & 5.19 & 4.57 & 2.91 & 1.77 & 1.38 & 1287.8 & 3.5 \\
\hline 45.0 & 1.53 & 2.11 & 3.15 & 4.05 & 4.75 & 4.73 & 5.59 & 5.10 & 4.57 & 2.95 & 1.81 & 1.43 & 1272.8 & 3.5 \\
\hline 50.0 & 1.56 & 2.12 & 3.12 & 3.95 & 4.58 & 4.54 & 5.39 & 4.98 & 4.54 & 2.98 & 1.85 & 1.47 & 1251.9 & 3.4 \\
\hline 60.0 & 1.60 & 2.09 & 3.01 & $3.7 !$ & 4.19 & 4.12 & 4.92 & 4.66 & 4.41 & 2.98 & 1.89 & 1.52 & 1191.3 & 3.3 \\
\hline 70.0 & 1.60 & 2.02 & 2.84 & 3.38 & 3.72 & 3.62 & 4.34 & 4.25 & 4.17 & 2.90 & 1.88 & 1.54 & 1105.2 & 3.0 \\
\hline 80.0 & 1.56 & 1.91 & 2.61 & 2.93 & 3.19 & 3.07 & 3.69 & 3.75 & 3.84 & 2.76 & 1.82 & 1.52 & 996.6 & 2.7 \\
\hline 90.0 & 1.48 & 1.76 & 2.34 & 2.55 & 2.64 & 2.52 & 3.02 & 3.18 & 3.42 & 2.56 & 1.73 & 1.46 & 872.4 & 2.4 \\
\hline
\end{tabular}


AVERAGE DAILY TOTAL TERRESTRIAL INSOLATION ESTIMATES (KWH:SQ. M)

$\begin{aligned} \text { SITE: } & \text { SEATTLE-TACOMA WA } \\ \text { LATITUDE : } & 47 \text { DEG }\end{aligned}$

AVERAGE DAILY AMJUNTS BY MONTH

\begin{tabular}{|c|c|c|c|c|c|c|c|c|c|c|c|c|c|c|}
\hline $\begin{array}{l}\text { ARRAY } \\
\text { TILT }\end{array}$ & $\begin{array}{c}J \\
---\end{array}$ & $F$ & $M$ & A & $M$ & 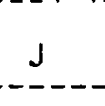 & --- & $A$ & 5 & 0 & $H$ & $\mathrm{D}$ & $\begin{array}{l}\text { ANNUAL } \\
\text { AMOUNT }\end{array}$ & $\begin{array}{c}\text { AVERAGE } \\
\text { DAY }\end{array}$ \\
\hline 0.0 & 0.83 & 1.56 & 2.68 & 4.07 & 5.41 & 5.68 & 7.08 & 5.10 & 3.61 & 2.07 & 1.06 & 0.67 & 1215.0 & 3.3 \\
\hline 10.0 & 1.02 & 1.74 & 2.90 & 4.26 & 5.52 & 5.74 & 7.27 & 5.38 & 3.99 & 2.40 & 1.29 & 0.86 & 1293.6 & 3.5 \\
\hline 15.0 & 1.11 & 1.82 & 3.00 & 4.32 & 5.53 & 5.72 & 7.30 & 5.47 & 4.15 & 2.55 & 1.40 & 0.96 & 1322.5 & 3.6 \\
\hline 20.0 & 1.20 & 1.89 & 3.07 & 4.36 & 5.52 & 5.68 & 7.38 & 5.54 & 4.28 & 2.69 & 1.49 & 1.04 & 1344.4 & 3.7 \\
\hline 25.0 & 1.28 & 1.95 & 3.13 & 4.37 & $5: 48$ & 5.61 & 7.24 & 5.57 & 4.39 & 2.81 & 1.58 & 1.13 & 1358.9 & 3.7 \\
\hline 30.0 & 1.35 & 2.01 & 3.17 & 4.37 & 5.40 & 5.52 & 7.15 & 5.57 & 4.47 & 2.91 & 1.66 & 1.20 & 1365.8 & 3.7 \\
\hline 35.0 & 1.41 & 2.05 & 3.20 & 4.33 & 5.30 & 5.39 & 7.02 & 5.54 & 4.52 & 2.99 & 1.73 & 1.27 & 1364.9 & 3.7 \\
\hline 40.0 & 1.46 & 2.07 & 3.21 & 4.28 & 5.17 & 5.23 & 6.84 & 5.47 & 4.55 & 3.06 & 1.79 & 1.32 & 1356.4 & 3.7 \\
\hline 45.0 & 1.51 & 2.09 & 3.20 & 4.20 & 5.01 & 5.05 & 6.63 & 5.38 & 4.55 & 3.11 & 1.84 & 1.37 & 1340.2 & 3.7 \\
\hline 50.0 & 1.54 & 2.10 & 3.17 & 4.10 & 4.84 & 4.85 & 6.38 & 5.26 & 4.53 & 3.14 & $1 . .88$ & 1.42 & 1317.6 & 3.6 \\
\hline 60.0 & 1.58 & 2.08 & 3.07 & 3.85 & 4.43 & 4.40 & 5.82 & 4.93 & 4.40 & 3.15 & 1.92 & 1.47 & 1252.9 & 3.4 \\
\hline 70.0 & 1.58 & 2.01 & 2.90 & 3.51 & 3.93 & 3.86 & 5.12 & 4.50 & 4.17 & 3.08 & 1.91 & 1.49 & 1160.8 & 3.2 \\
\hline 80.0 & 1.54 & 1.90 & 2.67 & 3.11 & 3.37 & $\$ .27$ & 4.32 & 3.97 & 3.84 & 2.93 & 1.86 & 1.47 & 1044.6 & 2.9 \\
\hline 90.0 & 1.47 & 1.76 & 2.39 & 2.66 & 2.78 & $\tilde{c} .68$ & 3.50 & 3.36 & 3.43 & 2.72 & 1.76 & 1.41 & 911.9 & 2.5 \\
\hline
\end{tabular}


AVERAGE DAILY TOTAL TERRESTRIAL INSOLATION ESTIMATES (KLH/SQ. M)

SITE:
LATITUDE: 47 DARANE

$\begin{array}{ll}\text { SITE: } & \text { SPOKANE } \\ \text { LATITUDE: } & 47 \text { DEGREES } 38 \text { MINUTES }\end{array}$

AVERAGE DAILY AMOUNTS BY MONTH
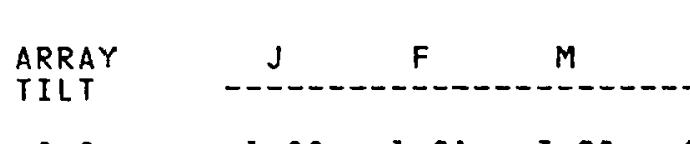

0.0

$\begin{array}{llll}1.00 & 1.91 \quad 3.28 \quad 4.71\end{array}$

$M$

$--$

10.0

$1.19 \quad 2.19 \quad 3.61$

4.96

6.05

6.57

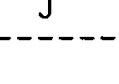

A

$S$

15.0

$\begin{array}{lll}1.28 & 2.32 \quad 3.75\end{array}$

5.04

6.19

6.65

7.64

6.12

6.21

6.64

7.64
7.68

6.51

6.51

4.52

5.09

5.09

6.20

6.597 .68

\title{
25.0
}

$1.44 \quad 2.53 \quad 3.95$

5.126 .16

6.52

5

5.11

6.16

$6.52 \quad 7.63 \quad 6.80$

5.32

5.53

$1.512 .62 \quad 4.02$

5.08

6.08

$\begin{array}{llllllll}6.59 & 7.68 & 6.75 & 5.53 & 3.60 & 1.86 & 1.19 & 1590 \\ 6.52 & 7.63 & 6.80 & 5.70 & 3.79 & 1.98 & 1.27 & 1612 \\ 6.40 & 7.53 & 6.82 & 5.83 & 3.96 & 2.10 & 1.34 & 1625 \\ 6.25 & 7.39 & 6.79 & 5.93 & 4.10 & 2.20 & 1.41 & 162 \\ 6.07 & 7.21 & 6.72 & 5.99 & 4.21 & 2.29 & 1.47 & 162 \\ 5.85 & 6.98 & 6.61 & 6.01 & 4.30 & 2.36 & 1.52 & 1507 \\ 5.61 & 6.72 & 6.47 & 6.00 & 4.36 & 2.42 & 1.56 & 158 \\ 5.08 & 6.13 & 6.08 & 5.86 & 4.39 & 2.49 & 1.61 & 1511 \\ 4.44 & 5.39 & 5.54 & 5.57 & 4.32 & 2.50 & 1.62 & 1404 \\ 3.73 & 4.54 & 4.88 & 5.15 & 4.13 & 2.44 & 1.59 & 1266 \\ 3.02 & 3.67 & 4.11 & 4.60 & 3.85 & 2.32 & 1.52 & 1106 \\ & & & & & & & \end{array}$

$\begin{array}{llllllll}6.59 & 7.68 & 6.75 & 5.53 & 3.60 & 1.86 & 1.19 & 1590 . \\ 6.52 & 7.63 & 6.80 & 5.70 & 3.79 & 1.98 & 1.27 & 161 \\ 6.40 & 7.53 & 6.82 & 5.83 & 3.96 & 2.10 & 1.34 & 1625 . \\ 6.25 & 7.39 & 6.79 & 5.93 & 4.10 & 2.20 & 1.41 & 1629 . \\ 6.07 & 7.21 & 6.72 & 5.99 & 4.21 & 2.29 & 1.47 & 162 \\ 5.85 & 6.98 & 6.61 & 6.01 & 4.30 & 2.36 & 1.52 & 160 \\ 5.61 & 6.72 & 6.47 & 6.00 & 4.36 & 2.42 & 1.56 & 158 \\ 5.08 & 6.13 & 6.08 & 5.86 & 4.39 & 2.49 & 1.61 & 151 \\ 4.44 & 5.39 & 5.54 & 5.57 & 4.32 & 2.50 & 1.62 & 140 \\ 3.73 & 4.54 & 4.88 & 5.15 & 4.13 & 2.44 & 1.59 & 1266 . \\ 3.02 & 3.67 & 4.11 & 4.60 & 3.85 & 2.32 & 1.52 & 1106 .\end{array}$

5.70

3.79

$\begin{array}{llllllll}6.59 & 7.68 & 6.75 & 5.53 & 3.60 & 1.86 & 1.19 & 1590 . \\ 6.52 & 7.63 & 6.80 & 5.70 & 3.79 & 1.98 & 1.27 & 161 \\ 6.40 & 7.53 & 6.82 & 5.83 & 3.96 & 2.10 & 1.34 & 1625 . \\ 6.25 & 7.39 & 6.79 & 5.93 & 4.10 & 2.20 & 1.41 & 1629 . \\ 6.07 & 7.21 & 6.72 & 5.99 & 4.21 & 2.29 & 1.47 & 162 \\ 5.85 & 6.98 & 6.61 & 6.01 & 4.30 & 2.36 & 1.52 & 160 \\ 5.61 & 6.72 & 6.47 & 6.00 & 4.36 & 2.42 & 1.56 & 158 \\ 5.08 & 6.13 & 6.08 & 5.86 & 4.39 & 2.49 & 1.61 & 151 \\ 4.44 & 5.39 & 5.54 & 5.57 & 4.32 & 2.50 & 1.62 & 140 \\ 3.73 & 4.54 & 4.88 & 5.15 & 4.13 & 2.44 & 1.59 & 1266 . \\ 3.02 & 3.67 & 4.11 & 4.60 & 3.85 & 2.32 & 1.52 & 1106 .\end{array}$

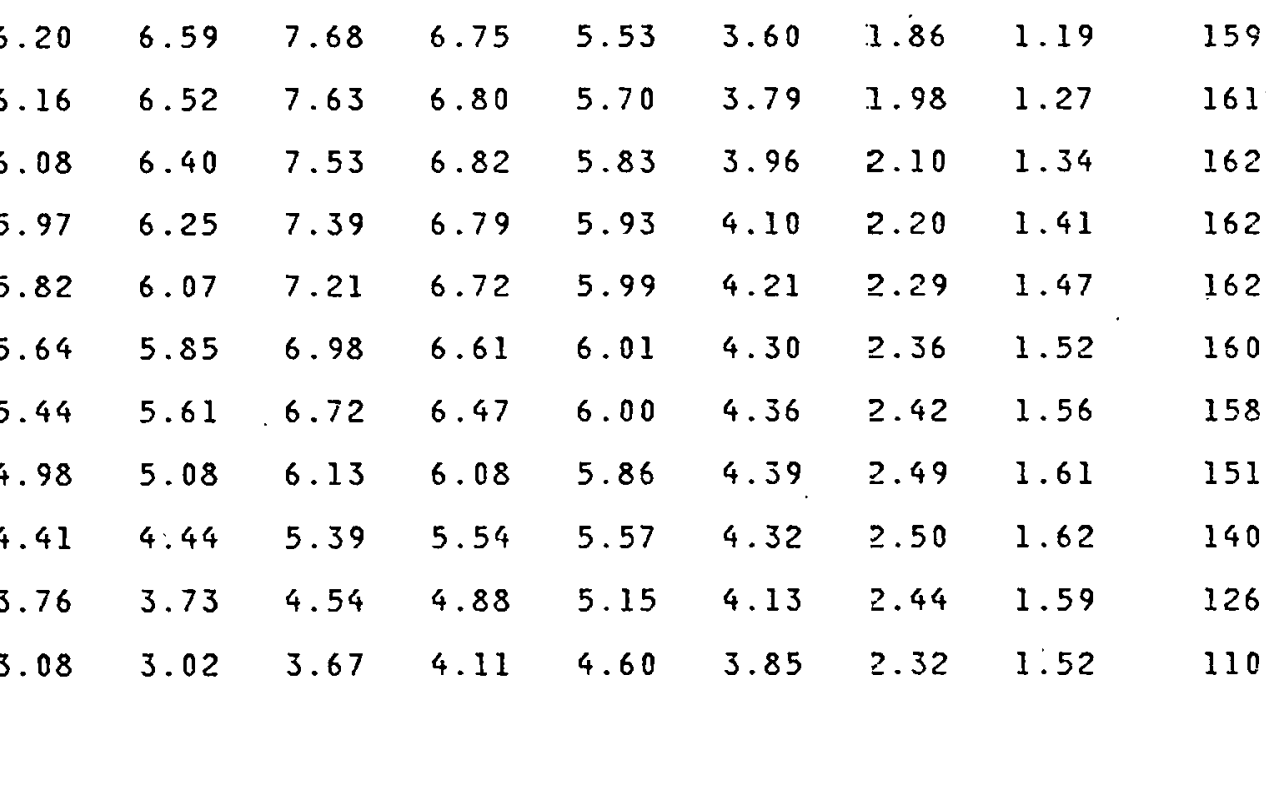

$\begin{array}{llllllll}6.59 & 7.68 & 6.75 & 5.53 & 3.60 & 1.86 & 1.19 & 1590 . \\ 6.52 & 7.63 & 6.80 & 5.70 & 3.79 & 1.98 & 1.27 & 161 \\ 6.40 & 7.53 & 6.82 & 5.83 & 3.96 & 2.10 & 1.34 & 1625 . \\ 6.25 & 7.39 & 6.79 & 5.93 & 4.10 & 2.20 & 1.41 & 1629 . \\ 6.07 & 7.21 & 6.72 & 5.99 & 4.21 & 2.29 & 1.47 & 162 \\ 5.85 & 6.98 & 6.61 & 6.01 & 4.30 & 2.36 & 1.52 & 160 \\ 5.61 & 6.72 & 6.47 & 6.00 & 4.36 & 2.42 & 1.56 & 158 \\ 5.08 & 6.13 & 6.08 & 5.86 & 4.39 & 2.49 & 1.61 & 151 \\ 4.44 & 5.39 & 5.54 & 5.57 & 4.32 & 2.50 & 1.62 & 140 \\ 3.73 & 4.54 & 4.88 & 5.15 & 4.13 & 2.44 & 1.59 & 1266 . \\ 3.02 & 3.67 & 4.11 & 4.60 & 3.85 & 2.32 & 1.52 & 1106 .\end{array}$

5.02

$\begin{array}{lll}1.62 & 2.74 & 4.10\end{array}$

$1.66 \quad 2.78 \quad 4.10$

4.93

5.82

$\begin{array}{llllllll}6.59 & 7.68 & 6.75 & 5.53 & 3.60 & 1.86 & 1.19 & 1590 \\ 6.52 & 7.63 & 6.80 & 5.70 & 3.79 & 1.98 & 1.27 & 1612 \\ 6.40 & 7.53 & 6.82 & 5.83 & 3.96 & 2.10 & 1.34 & 1625 \\ 6.25 & 7.39 & 6.79 & 5.93 & 4.10 & 2.20 & 1.41 & 1629 \\ 6.07 & 7.21 & 6.72 & 5.99 & 4.21 & 2.29 & 1.47 & 1623 \\ 5.85 & 6.98 & 6.61 & 6.01 & 4.30 & 2.36 & 1.52 & 1507 \\ 5.61 & 6.72 & 6.47 & 6.00 & 4.36 & 2.42 & 1.56 & 158 \\ 5.08 & 6.13 & 6.08 & 5.86 & 4.39 & 2.49 & 1.61 & 1511 \\ 4.44 & 5.39 & 5.54 & 5.57 & 4.32 & 2.50 & 1.62 & 1404 \\ 3.73 & 4.54 & 4.88 & 5.15 & 4.13 & 2.44 & 1.59 & 1266.52 \\ 3.02 & 3.67 & 4.11 & 4.60 & 3.85 & 2.32 & 1.52 & 1106 .\end{array}$

5.64

\begin{tabular}{|c|c|c|c|c|c|c|}
\hline 6.59 & 7.68 & 6.75 & 5.53 & 3.60 & 7.86 & 1.19 \\
\hline 6.52 & 7.63 & 6.80 & 5.70 & 3.79 & 1.98 & 1.27 \\
\hline 6.40 & 7.53 & 6.82 & 5.83 & 3.96 & 2.10 & 1.34 \\
\hline 6.25 & 7.39 & 6.79 & 5.93 & 4.10 & 2.20 & 1.41 \\
\hline 6.07 & 7.21 & 6.72 & 5.99 & 4.21 & 2.29 & 1.47 \\
\hline 5.85 & 6.98 & 6.61 & 6.01 & 4.30 & 2.36 & 1.52 \\
\hline 5.61 & 6.72 & 6.47 & 6.00 & 4.36 & 2.42 & 1.56 \\
\hline 5.08 & 6.13 & 6.08 & 5.86 & 4.39 & 2.49 & 1.61 \\
\hline $4: 44$ & 5.39 & 5.54 & 5.57 & 4.32 & 2.50 & 1.62 \\
\hline 3.73 & 4.54 & 4.88 & 5.15 & 4.13 & 2.44 & 1.59 \\
\hline 3.02 & 3.67 & 4.11 & 4.60 & 3.85 & 2.32 & 1.52 \\
\hline
\end{tabular}

$\begin{array}{llllllll}6.59 & 7.68 & 6.75 & 5.53 & 3.60 & 1.86 & 1.19 & 1590 . \\ 6.52 & 7.63 & 6.80 & 5.70 & 3.79 & 1.98 & 1.27 & 161 \\ 6.40 & 7.53 & 6.82 & 5.83 & 3.96 & 2.10 & 1.34 & 1625 . \\ 6.25 & 7.39 & 6.79 & 5.93 & 4.10 & 2.20 & 1.41 & 1629 . \\ 6.07 & 7.21 & 6.72 & 5.99 & 4.21 & 2.29 & 1.47 & 162 \\ 5.85 & 6.98 & 6.61 & 6.01 & 4.30 & 2.36 & 1.52 & 160 \\ 5.61 & 6.72 & 6.47 & 6.00 & 4.36 & 2.42 & 1.56 & 158 \\ 5.08 & 6.13 & 6.08 & 5.86 & 4.39 & 2.49 & 1.61 & 151 \\ 4.44 & 5.39 & 5.54 & 5.57 & 4.32 & 2.50 & 1.62 & 140 \\ 3.73 & 4.54 & 4.88 & 5.15 & 4.13 & 2.44 & 1.59 & 1266 . \\ 3.02 & 3.67 & 4.11 & 4.60 & 3.85 & 2.32 & 1.52 & 1106 .\end{array}$

$\begin{array}{llllllll}6.59 & 7.68 & 6.75 & 5.53 & 3.60 & 1.86 & 1.19 & 1590 . \\ 6.52 & 7.63 & 6.80 & 5.70 & 3.79 & 1.98 & 1.27 & 161 \\ 6.40 & 7.53 & 6.82 & 5.83 & 3.96 & 2.10 & 1.34 & 1625 . \\ 6.25 & 7.39 & 6.79 & 5.93 & 4.10 & 2.20 & 1.41 & 1629 . \\ 6.07 & 7.21 & 6.72 & 5.99 & 4.21 & 2.29 & 1.47 & 162 \\ 5.85 & 6.98 & 6.61 & 6.01 & 4.30 & 2.36 & 1.52 & 160 \\ 5.61 & 6.72 & 6.47 & 6.00 & 4.36 & 2.42 & 1.56 & 158 \\ 5.08 & 6.13 & 6.08 & 5.86 & 4.39 & 2.49 & 1.61 & 151 \\ 4.44 & 5.39 & 5.54 & 5.57 & 4.32 & 2.50 & 1.62 & 140 \\ 3.73 & 4.54 & 4.88 & 5.15 & 4.13 & 2.44 & 1.59 & 1266 . \\ 3.02 & 3.67 & 4.11 & 4.60 & 3.85 & 2.32 & 1.52 & 1106 .\end{array}$

$\begin{array}{llllllll}6.59 & 7.68 & 6.75 & 5.53 & 3.60 & 1.86 & 1.19 & 1590 . \\ 6.52 & 7.63 & 6.80 & 5.70 & 3.79 & 1.98 & 1.27 & 161 \\ 6.40 & 7.53 & 6.82 & 5.83 & 3.96 & 2.10 & 1.34 & 1625 . \\ 6.25 & 7.39 & 6.79 & 5.93 & 4.10 & 2.20 & 1.41 & 1629 . \\ 6.07 & 7.21 & 6.72 & 5.99 & 4.21 & 2.29 & 1.47 & 162 \\ 5.85 & 6.98 & 6.61 & 6.01 & 4.30 & 2.36 & 1.52 & 160 \\ 5.61 & 6.72 & 6.47 & 6.00 & 4.36 & 2.42 & 1.56 & 158 \\ 5.08 & 6.13 & 6.08 & 5.86 & 4.39 & 2.49 & 1.61 & 151 \\ 4.44 & 5.39 & 5.54 & 5.57 & 4.32 & 2.50 & 1.62 & 140 \\ 3.73 & 4.54 & 4.88 & 5.15 & 4.13 & 2.44 & 1.59 & 1266 . \\ 3.02 & 3.67 & 4.11 & 4.60 & 3.85 & 2.32 & 1.52 & 1106 .\end{array}$

$\begin{array}{llllllll}6.59 & 7.68 & 6.75 & 5.53 & 3.60 & 1.86 & 1.19 & 1590 . \\ 6.52 & 7.63 & 6.80 & 5.70 & 3.79 & 1.98 & 1.27 & 161 \\ 6.40 & 7.53 & 6.82 & 5.83 & 3.96 & 2.10 & 1.34 & 1625 . \\ 6.25 & 7.39 & 6.79 & 5.93 & 4.10 & 2.20 & 1.41 & 1629 . \\ 6.07 & 7.21 & 6.72 & 5.99 & 4.21 & 2.29 & 1.47 & 162 \\ 5.85 & 6.98 & 6.61 & 6.01 & 4.30 & 2.36 & 1.52 & 160 \\ 5.61 & 6.72 & 6.47 & 6.00 & 4.36 & 2.42 & 1.56 & 158 \\ 5.08 & 6.13 & 6.08 & 5.86 & 4.39 & 2.49 & 1.61 & 151 \\ 4.44 & 5.39 & 5.54 & 5.57 & 4.32 & 2.50 & 1.62 & 140 \\ 3.73 & 4.54 & 4.88 & 5.15 & 4.13 & 2.44 & 1.59 & 1266 . \\ 3.02 & 3.67 & 4.11 & 4.60 & 3.85 & 2.32 & 1.52 & 1106 .\end{array}$

$\begin{array}{llllllll}6.59 & 7.68 & 6.75 & 5.53 & 3.60 & 1.86 & 1.19 & 1590 . \\ 6.52 & 7.63 & 6.80 & 5.70 & 3.79 & 1.98 & 1.27 & 161 \\ 6.40 & 7.53 & 6.82 & 5.83 & 3.96 & 2.10 & 1.34 & 1625 . \\ 6.25 & 7.39 & 6.79 & 5.93 & 4.10 & 2.20 & 1.41 & 1629 . \\ 6.07 & 7.21 & 6.72 & 5.99 & 4.21 & 2.29 & 1.47 & 162 \\ 5.85 & 6.98 & 6.61 & 6.01 & 4.30 & 2.36 & 1.52 & 160 \\ 5.61 & 6.72 & 6.47 & 6.00 & 4.36 & 2.42 & 1.56 & 158 \\ 5.08 & 6.13 & 6.08 & 5.86 & 4.39 & 2.49 & 1.61 & 151 \\ 4.44 & 5.39 & 5.54 & 5.57 & 4.32 & 2.50 & 1.62 & 140 \\ 3.73 & 4.54 & 4.88 & 5.15 & 4.13 & 2.44 & 1.59 & 1266 . \\ 3.02 & 3.67 & 4.11 & 4.60 & 3.85 & 2.32 & 1.52 & 1106 .\end{array}$

6.01

$\begin{array}{lllllllll}6.20 & 6.59 & 7.68 & 6.75 & 5.53 & 3.60 & 1.86 & 1.19 & 1590 \\ 6.16 & 6.52 & 7.63 & 6.80 & 5.70 & 3.79 & 1.98 & 1.27 & 1612 \\ 6.08 & 6.40 & 7.53 & 6.82 & 5.83 & 3.96 & 2.10 & 1.34 & 1625 \\ 5.97 & 6.25 & 7.39 & 6.79 & 5.93 & 4.10 & 2.20 & 1.41 & 1629 \\ 5.82 & 6.07 & 7.21 & 6.72 & 5.99 & 4.21 & 2.29 & 1.47 & 1623 \\ 5.64 & 5.85 & 6.98 & 6.61 & 6.01 & 4.30 & 2.36 & 1.52 & 1607 \\ 5.44 & 5.61 & 6.72 & 6.47 & 6.00 & 4.36 & 2.42 & 1.56 & 158 \\ 4.98 & 5.08 & 6.13 & 6.08 & 5.86 & 4.39 & 2.49 & 1.61 & 1511 \\ 4.41 & 4.44 & 5.39 & 5.54 & 5.57 & 4.32 & 2.50 & 1.62 & 14041.59 \\ 3.76 & 3.73 & 4.54 & 4.88 & 5.15 & 4.13 & 2.44 & 1.59 & 1266 \\ 3.08 & 3.02 & 3.67 & 4.11 & 4.60 & 3.85 & 2.32 & 1.52 & 1106 .\end{array}$

$\begin{array}{llllllll}6.59 & 7.68 & 6.75 & 5.53 & 3.60 & 1.86 & 1.19 & 1590 . \\ 6.52 & 7.63 & 6.80 & 5.70 & 3.79 & 1.98 & 1.27 & 161 \\ 6.40 & 7.53 & 6.82 & 5.83 & 3.96 & 2.10 & 1.34 & 1625 . \\ 6.25 & 7.39 & 6.79 & 5.93 & 4.10 & 2.20 & 1.41 & 1629 . \\ 6.07 & 7.21 & 6.72 & 5.99 & 4.21 & 2.29 & 1.47 & 162 \\ 5.85 & 6.98 & 6.61 & 6.01 & 4.30 & 2.36 & 1.52 & 160 \\ 5.61 & 6.72 & 6.47 & 6.00 & 4.36 & 2.42 & 1.56 & 158 \\ 5.08 & 6.13 & 6.08 & 5.86 & 4.39 & 2.49 & 1.61 & 151 \\ 4.44 & 5.39 & 5.54 & 5.57 & 4.32 & 2.50 & 1.62 & 140 \\ 3.73 & 4.54 & 4.88 & 5.15 & 4.13 & 2.44 & 1.59 & 1266 . \\ 3.02 & 3.67 & 4.11 & 4.60 & 3.85 & 2.32 & 1.52 & 1106 .\end{array}$

$\begin{array}{llllllll}6.59 & 7.68 & 6.75 & 5.53 & 3.60 & 1.86 & 1.19 & 1590 . \\ 6.52 & 7.63 & 6.80 & 5.70 & 3.79 & 1.98 & 1.27 & 161 \\ 6.40 & 7.53 & 6.82 & 5.83 & 3.96 & 2.10 & 1.34 & 1625 . \\ 6.25 & 7.39 & 6.79 & 5.93 & 4.10 & 2.20 & 1.41 & 1629 . \\ 6.07 & 7.21 & 6.72 & 5.99 & 4.21 & 2.29 & 1.47 & 162 \\ 5.85 & 6.98 & 6.61 & 6.01 & 4.30 & 2.36 & 1.52 & 160 \\ 5.61 & 6.72 & 6.47 & 6.00 & 4.36 & 2.42 & 1.56 & 158 \\ 5.08 & 6.13 & 6.08 & 5.86 & 4.39 & 2.49 & 1.61 & 151 \\ 4.44 & 5.39 & 5.54 & 5.57 & 4.32 & 2.50 & 1.62 & 140 \\ 3.73 & 4.54 & 4.88 & 5.15 & 4.13 & 2.44 & 1.59 & 1266 . \\ 3.02 & 3.67 & 4.11 & 4.60 & 3.85 & 2.32 & 1.52 & 1106 .\end{array}$

\begin{tabular}{|c|c|c|c|c|c|c|c|}
\hline 6.20 & 6.59 & 7.68 & 6.75 & 5.53 & 3.60 & 1.86 & 1.19 \\
\hline 6.16 & 6.52 & 7.63 & 6.80 & 5.70 & 3.79 & 1.98 & 1.27 \\
\hline 6.08 & 6.40 & 7.53 & 6.82 & 5.83 & 3.96 & 2.10 & 1.34 \\
\hline 5.97 & 6.25 & 7.39 & 6.79 & 5.93 & 4.10 & 2.20 & 1.41 \\
\hline 5.82 & 6.07 & 7.21 & 6.72 & 5.99 & 4.21 & 2.29 & 1.47 \\
\hline 5.64 & 5.85 & 6.98 & 6.61 & 6.01 & 4.30 & 2.36 & 1.52 \\
\hline 5.44 & 5.61 & 6.72 & 6.47 & 6.00 & 4.36 & 2.42 & 1.56 \\
\hline 4.98 & 5.08 & 6.13 & 6.08 & 5.86 & 4.39 & 2.49 & 1.61 \\
\hline 4.41 & $4: 44$ & 5.39 & 5.54 & 5.57 & 4.32 & 2.50 & 1.62 \\
\hline 3.76 & 3.73 & 4.54 & 4.88 & 5.15 & 4.13 & 2.44 & 1.59 \\
\hline 3.08 & 3.02 & 3.67 & 4.11 & 4.60 & 3.85 & 2.32 & 1.52 \\
\hline
\end{tabular}

$\begin{array}{llllllll}6.59 & 7.68 & 6.75 & 5.53 & 3.60 & 1.86 & 1.19 & 1590 . \\ 6.52 & 7.63 & 6.80 & 5.70 & 3.79 & 1.98 & 1.27 & 161 \\ 6.40 & 7.53 & 6.82 & 5.83 & 3.96 & 2.10 & 1.34 & 1625 . \\ 6.25 & 7.39 & 6.79 & 5.93 & 4.10 & 2.20 & 1.41 & 1629 . \\ 6.07 & 7.21 & 6.72 & 5.99 & 4.21 & 2.29 & 1.47 & 162 \\ 5.85 & 6.98 & 6.61 & 6.01 & 4.30 & 2.36 & 1.52 & 160 \\ 5.61 & 6.72 & 6.47 & 6.00 & 4.36 & 2.42 & 1.56 & 158 \\ 5.08 & 6.13 & 6.08 & 5.86 & 4.39 & 2.49 & 1.61 & 151 \\ 4.44 & 5.39 & 5.54 & 5.57 & 4.32 & 2.50 & 1.62 & 140 \\ 3.73 & 4.54 & 4.88 & 5.15 & 4.13 & 2.44 & 1.59 & 1266 . \\ 3.02 & 3.67 & 4.11 & 4.60 & 3.85 & 2.32 & 1.52 & 1106 .\end{array}$

$\begin{array}{llllllll}6.59 & 7.68 & 6.75 & 5.53 & 3.60 & 1.86 & 1.19 & 1590 . \\ 6.52 & 7.63 & 6.80 & 5.70 & 3.79 & 1.98 & 1.27 & 161 \\ 6.40 & 7.53 & 6.82 & 5.83 & 3.96 & 2.10 & 1.34 & 1625 . \\ 6.25 & 7.39 & 6.79 & 5.93 & 4.10 & 2.20 & 1.41 & 1629 . \\ 6.07 & 7.21 & 6.72 & 5.99 & 4.21 & 2.29 & 1.47 & 162 \\ 5.85 & 6.98 & 6.61 & 6.01 & 4.30 & 2.36 & 1.52 & 160 \\ 5.61 & 6.72 & 6.47 & 6.00 & 4.36 & 2.42 & 1.56 & 158 \\ 5.08 & 6.13 & 6.08 & 5.86 & 4.39 & 2.49 & 1.61 & 151 \\ 4.44 & 5.39 & 5.54 & 5.57 & 4.32 & 2.50 & 1.62 & 140 \\ 3.73 & 4.54 & 4.88 & 5.15 & 4.13 & 2.44 & 1.59 & 1266 . \\ 3.02 & 3.67 & 4.11 & 4.60 & 3.85 & 2.32 & 1.52 & 1106 .\end{array}$

$\begin{array}{llllllll}6.59 & 7.68 & 6.75 & 5.53 & 3.60 & 1.86 & 1.19 & 1590 . \\ 6.52 & 7.63 & 6.80 & 5.70 & 3.79 & 1.98 & 1.27 & 161 \\ 6.40 & 7.53 & 6.82 & 5.83 & 3.96 & 2.10 & 1.34 & 1625 . \\ 6.25 & 7.39 & 6.79 & 5.93 & 4.10 & 2.20 & 1.41 & 1629 . \\ 6.07 & 7.21 & 6.72 & 5.99 & 4.21 & 2.29 & 1.47 & 162 \\ 5.85 & 6.98 & 6.61 & 6.01 & 4.30 & 2.36 & 1.52 & 160 \\ 5.61 & 6.72 & 6.47 & 6.00 & 4.36 & 2.42 & 1.56 & 158 \\ 5.08 & 6.13 & 6.08 & 5.86 & 4.39 & 2.49 & 1.61 & 151 \\ 4.44 & 5.39 & 5.54 & 5.57 & 4.32 & 2.50 & 1.62 & 140 \\ 3.73 & 4.54 & 4.88 & 5.15 & 4.13 & 2.44 & 1.59 & 1266 . \\ 3.02 & 3.67 & 4.11 & 4.60 & 3.85 & 2.32 & 1.52 & 1106 .\end{array}$

$\begin{array}{llllllll}6.59 & 7.68 & 6.75 & 5.53 & 3.60 & 1.86 & 1.19 & 1590 . \\ 6.52 & 7.63 & 6.80 & 5.70 & 3.79 & 1.98 & 1.27 & 161 \\ 6.40 & 7.53 & 6.82 & 5.83 & 3.96 & 2.10 & 1.34 & 1625 . \\ 6.25 & 7.39 & 6.79 & 5.93 & 4.10 & 2.20 & 1.41 & 1629 . \\ 6.07 & 7.21 & 6.72 & 5.99 & 4.21 & 2.29 & 1.47 & 162 \\ 5.85 & 6.98 & 6.61 & 6.01 & 4.30 & 2.36 & 1.52 & 160 \\ 5.61 & 6.72 & 6.47 & 6.00 & 4.36 & 2.42 & 1.56 & 158 \\ 5.08 & 6.13 & 6.08 & 5.86 & 4.39 & 2.49 & 1.61 & 151 \\ 4.44 & 5.39 & 5.54 & 5.57 & 4.32 & 2.50 & 1.62 & 140 \\ 3.73 & 4.54 & 4.88 & 5.15 & 4.13 & 2.44 & 1.59 & 1266 . \\ 3.02 & 3.67 & 4.11 & 4.60 & 3.85 & 2.32 & 1.52 & 1106 .\end{array}$

\begin{tabular}{|c|c|c|c|c|c|c|c|}
\hline 6.20 & 6.59 & 7.68 & 6.75 & 5.53 & 3.60 & 1.86 & 1.19 \\
\hline 6.16 & 6.52 & 7.63 & 6.80 & 5.70 & 3.79 & 1.98 & 1.27 \\
\hline 6.08 & 6.40 & 7.53 & 6.82 & 5.83 & 3.96 & 2.10 & 1.34 \\
\hline 5.97 & 6.25 & 7.39 & 6.79 & 5.93 & 4.10 & 2.20 & 1.41 \\
\hline 5.82 & 6.07 & 7.21 & 6.72 & 5.99 & 4.21 & 2.29 & 1.47 \\
\hline 5.64 & 5.85 & 6.98 & 6.61 & 6.01 & 4.30 & 2.36 & 1.52 \\
\hline 5.44 & 5.61 & 6.72 & 6.47 & 6.00 & 4.36 & 2.42 & 1.56 \\
\hline 4.98 & 5.08 & 6.13 & 6.08 & 5.86 & 4.39 & 2.49 & 1.61 \\
\hline 4.41 & $4: 44$ & 5.39 & 5.54 & 5.57 & 4.32 & 2.50 & 1.62 \\
\hline 3.76 & 3.73 & 4.54 & 4.88 & 5.15 & 4.13 & 2.44 & 1.59 \\
\hline 3.08 & 3.02 & 3.67 & 4.11 & 4.60 & 3.85 & 2.32 & 1.52 \\
\hline
\end{tabular}

70.0

$1.72 \quad 2.73 \quad 3.75$

80.0

$1.67 \quad 2.60 \quad 3.46$

4.13

$3.65^{\circ}$

\begin{tabular}{|c|c|c|c|c|c|c|c|}
\hline 6.20 & 6.59 & 7.68 & 6.75 & 5.53 & 3.60 & 1.86 & 1.19 \\
\hline 6.16 & 6.52 & 7.63 & 6.80 & 5.70 & 3.79 & 1.98 & 1.27 \\
\hline 6.08 & 6.40 & 7.53 & 6.82 & 5.83 & 3.96 & 2.10 & 1.34 \\
\hline 5.97 & 6.25 & 7.39 & 6.79 & 5.93 & 4.10 & 2.20 & 1.41 \\
\hline 5.82 & 6.07 & 7.21 & 6.72 & 5.99 & 4.21 & 2.29 & 1.47 \\
\hline 5.64 & 5.85 & 6.98 & 6.61 & 6.01 & 4.30 & 2.36 & 1.52 \\
\hline 5.44 & 5.61 & 6.72 & 6.47 & 6.00 & 4.36 & 2.42 & 1.56 \\
\hline 4.98 & 5.08 & 6.13 & 6.08 & 5.86 & 4.39 & 2.49 & 1.61 \\
\hline 4.41 & 4.44 & 5.39 & 5.54 & 5.57 & 4.32 & 2.50 & 1.62 \\
\hline 3.76 & 3.73 & 4.54 & 4.88 & 5.15 & 4.13 & 2.44 & 1.59 \\
\hline 3.08 & 3.02 & 3.67 & 4.11 & 4.60 & 3.85 & 2.32 & 1.52 \\
\hline
\end{tabular}

5.54

4.88

5.154 .13

$\begin{array}{llll}3.08 & 3.02 & 3.67 & 4.11\end{array}$

$4.60 \quad 3.85$

$\mathrm{N}$
$\mathrm{N}$

\begin{abstract}
$\begin{array}{lll}1.58 & 2.41 & 3.10\end{array}$
\end{abstract}
3.113 .0

$\begin{array}{lc}\text { ANNUAL } & \begin{array}{c}\text { AVERAGE } \\ \text { ANOUNT }\end{array} \\ 1412.4 & 3.9 \\ 1518.3 & 4.2 \\ 1558.6 & 4.3 \\ 1590.2 & 4.4 \\ 1612.7 & 4.4 \\ 1625.8 & 4.5 \\ 1629.3 & 4.5 \\ 1623.3 & 4.4 \\ 1607.7 & 4.4 \\ 1583.9 & 4.3 \\ 1511.6 & 4.1 \\ 1404.7 & 3.8 \\ 1266.8 & 3.5 \\ 1106.8 & 3.0 \\ 1106 & \end{array}$


AVERAGE DAILY TOTAL. TERRESTRIAL INSOLATION ESTIMATES (KINH/SQ. M)

SITE: WHIDBEY ISLAND WA

LATITUEE: 48 DEGREES 21 MINUTES

AVERAGE DAILY AMOUNTS BY MONTH

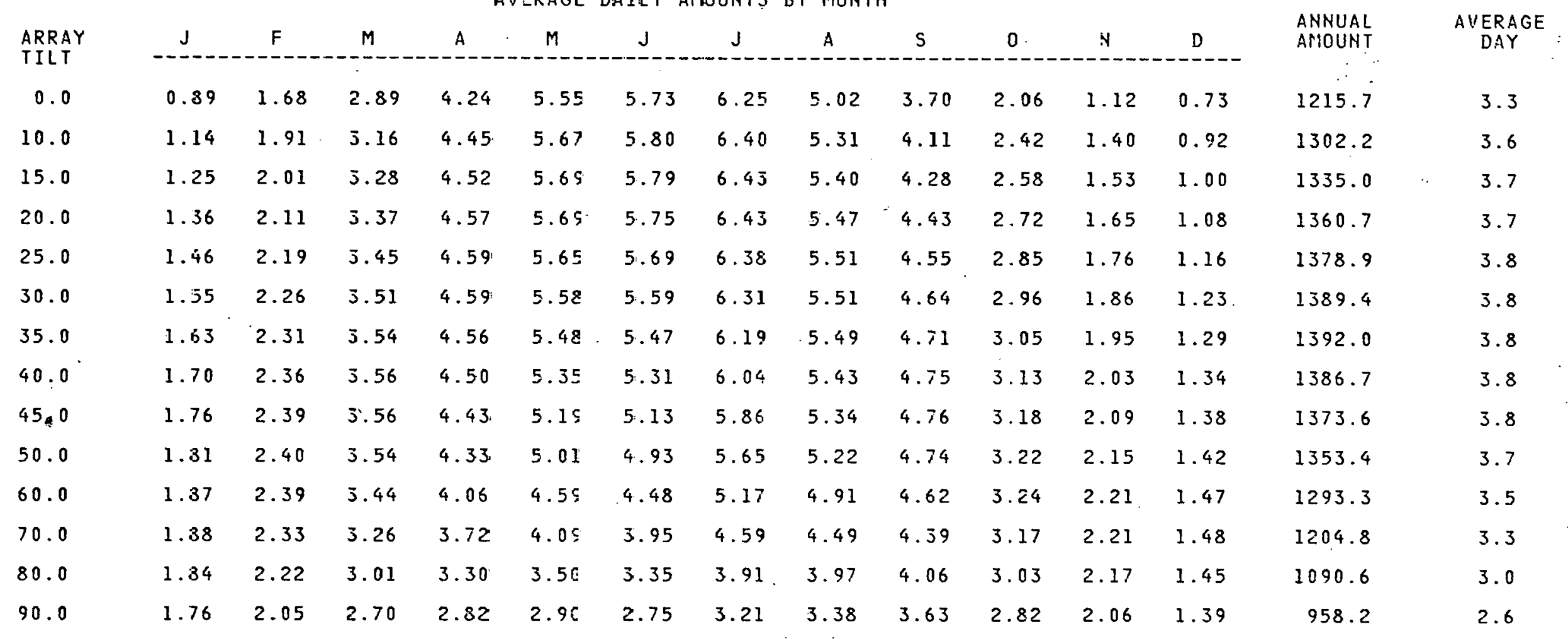


AVERAGE DAILY TOTAL TERRESTRIAL INSOLATION ESTIMATES (KNH/SQ. M)

$\begin{aligned} \text { SITE: } & \text { YAKIMA } \\ \text { LATITUDE : } & 45 \text { DEGREES } 34 \text { MINUTES }\end{aligned}$

AVERAGE DAILY AMOUNTS BY MONTH

\begin{tabular}{|c|c|c|c|c|c|c|c|c|c|c|c|c|c|c|}
\hline ARRAY & $\mathbf{J}$ & $F$ & $M$ & $A$ & $M$ & $\begin{array}{ll}2 \\
\end{array}$ & $J$ & trom & $S$ & 0 & $N$ & D & $\begin{array}{l}\text { ANNUAL } \\
\text { AMOUNT }\end{array}$ & $\begin{array}{c}\text { AVERAGE } \\
\text { DAY }\end{array}$ \\
\hline 0.0 & 1.15 & 2.10 & 3.54 & 5.74 & 6.33 & 6.83 & 7.43 & 6.23 & 4.68 & 2.81 & 1.40 & 0.93 & 1478.0 & 4.0 \\
\hline 10.0 & 1.40 & 2.42 & 3.89 & 5.30 & 6.47 & 6.91 & 7.62 & 6.61 & 5.24 & 3.34 & 1.75 & 1.17 & 1588.9 & 4.4 \\
\hline 15.0 & 1.51 & 2.56 & 4.03 & 5.38 & 6.49 & 6.89 & 7.65 & 6.74 & 5.48 & 3.58 & 1.92 & 1.28 & 1631.0 & 4.5 \\
\hline 20.0 & 1.61 & 2.68 & 4.16 & 5.44 & 6.48 & 6.84 & 7.64 & 6.83 & 5.68 & 3.79 & 2.07 & 1.38 & 1664.0 & 4.6 \\
\hline 25.8 & 1.70 & 2.79 & 4.25 & 5.46 & $i .43$ & 6.75 & 7.58 & 6.87 & 5.85 & 3.98 & 2.20 & 1.48 & 1687.3 & 4.6 \\
\hline 30.0 & 1.78 & 2.88 & 4.33 & 5.45 & 6.34 & 6.63 & 7.48 & 6.88 & 5.98 & 4.15 & 2.33 & 1.56 & 1700.6 & 4.7 \\
\hline 35.0 & 1.86 & 2.96 & 4.38 & 5.42 & 6.21 & 6.46 & 7.33 & 6.85 & $6: 07$ & 4.29 & 2.44 & 1.64 & 1703.9 & 4.7 \\
\hline 40.0 & 1.92 & 3.02 & 4.40 & 5.35 & 6.05 & 6.26 & 7.14 & 6.77 & 6.13 & 4.41 & 2.54 & 1.71 & 1697.1 & 4.6 \\
\hline 45.0 & 1.97 & 3.06 & 4.40 & 5.25 & 5.86 & 6.03 & 6.90 & 6.65 & 6.14 & 4.49 & 2.62 & 1.77 & 1680.3 & 4.6 \\
\hline 50.0 & 2.01 & 3.09 & 4.37 & 5.13 & 5.65 & 5.78 & 6.65 & 6.50 & 6.12 & 4.55 & 2.68 & 1.81 & 1655.4 & 4.5 \\
\hline 60.0 & 2.05 & 3.08 & .4 .24 & 4.80 & 5.15 & 5.21 & 6.04 & 6.09 & 5.96 & 4.58 & 2.75 & 1.87 & 1578.4 & 4.3 \\
\hline 70.0 & 2.04 & 3.00 & 4.01 & 4.37 & 4.54 & 4.53 & 5.29 & 5.53 & 5.66 & 4.49 & 2.76 & 1.88 & 1465.0 & 4.0 \\
\hline 80.0 & 1.98 & 2.85 & 3.69 & 3.85 & 3.84 & 3.79 & 4.44 & 4.85 & 5.21 & 4.29 & 2.69 & 1.84 & 1319.2 & 3.6 \\
\hline 90.0 & 1.88 & 2.63 & 3.29 & 3.26 & $3: 13$ & 3.04 & 3.57 & 4.07 & 4.64 & 3.98 & 2.56 & 1.76 & 1150.6 & 3.2 \\
\hline
\end{tabular}


AVERAGE DAILY TOTAL TERRESTRIAL INSOLATION ESTIMATES (KWH/SQ. M)

SITE:
LATITUDE : WLAIRE
44 DESREES 52 MINUTES

AVERAG: DAILY A MOUNTS BY MONTH

\begin{tabular}{|c|c|c|c|c|c|c|c|c|c|c|c|c|c|c|}
\hline ARRAY & $J$ & $F$ & $M$ & A & $M$ & N12 & 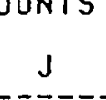 & $A$ & $s$ & 0 & $N$ & D & $\begin{array}{l}\text { ANNUAL } \\
\text { AMOUNT }\end{array}$ & $\begin{array}{c}\text { AVERAGE } \\
\text { DAY }\end{array}$ \\
\hline 0.0 & 1.43 & 2.35 & 3.43 & 4.49 & $5.3 L$ & 5.90 & 5.95 & 5.11 & 3.77 & 2.60 & 1.42 & 1.07 & 1305.3 & 3.6 \\
\hline 10.0 & 1.74 & $2.70 !$ & 3.74 & 4.69 & 5.37 & 5.94 & 6.06 & 5.35 & 4.12 & 3.02 & 1.74 & 1.34 & 1396.1 & 3.8 \\
\hline 15.0 & 1.88 & 2.85 & 3.86 & 4.75 & 5.43 & 5.91 & 6.07 & 5.43 & 4.26 & 3.20 & 1.88 & 1.46 & 1430.3 & 3.9 \\
\hline 20.0 & 2.02 & 2.99 & 3.97 & 4.78 & 5.37 & 5.86 & 5.04 & 5.48 & 4.38 & 3.37 & 2.01 & 1.57 & 1456.8 & 4.0 \\
\hline 25.0 & 2.14 & 3.11 & 4.05 & 4.79 & 5.32 & 5.78 & 5.98 & 5.49 & 4.48 & 3.52 & 2.13 & 1.67 & 1475.1 & 4.0 \\
\hline 30.0 & 2.24 & 3.21 & 4.11 & 4.77 & 5.23 & 5.66 & 5.88 & 5.48 & 4.54 & 3.64 & 2.24 & 1.77 & 1485.0 & 4.1 \\
\hline 35.0 & 2.34 & 3.29 & 4.14 & 4.72 & 5.12 & 5.52 & 5.76 & 5.43 & 4.59 & 3.74 & 2.33 & 1.85 & 1486.6 & 4.1 \\
\hline 40.0 & 2.42 & 3.35 & 4.15 & 4.65 & 4.93 & 5.34 & 5.60 & 5.35 & 4.60 & 3.82 & 2.42 & 1.92 & 1479.8 & 4.1 \\
\hline 45.0 & 2.48 & 3.39 & 4.14 & 4.56 & 4.82 & 5.14 & 5.41 & 5.24 & 4.59 & 3.88 & 2.48 & 1.98 & 1464.7 & 4.0 \\
\hline 50.0 & 2.53 & 3.42 & 4.10 & 4.44 & 4.64 & 4.93 & 5.21 & 5.11 & 4.55 & 3.91 & 2.53 & 2.03 & 1443.3 & 4.0 \\
\hline 60.0 & 2.59 & 3.40 & 3.96 & 4.14 & 4.23 & 4.44 & 6.73 & 4.77 & 4.40 & 3.91 & 2.58 & 2.09 & 1376.3 & 3.8 \\
\hline 70.0 & 2.57 & 3.30 & 3.73 & 3.76 & 3.73 & 3.86 & 4.15 & 4.32 & 4.14 & 3.81 & 2.57 & 2.10 & 1279.3 & 3.5 \\
\hline 80.0 & 2.50 & 3.13 & 3.42 & 3.30 & 3.17 & 3.25 & 3.50 & 3.78 & 3.79 & 3.61 & 2.50 & 2.05 & 1155.8 & 3.2 \\
\hline 90.0 & 2.36 & 2.88 & 3.03 & 2.79 & 2.63 & 2.62 & 2.85 & 3.18 & 3.36 & 3.33 & 2.36 & 1.95 & 1013.1 & 2.8 \\
\hline
\end{tabular}


AVERAGE DAILY TOTAL TERRESTRIAL INSOLATION ESTIMATES (KLH/SQ. M)

SITE: GREEN BAY

LATITUDE: 44 DEGREES 29 MINUTES

AVERAGE DAILY AMOUNTS BY MONTH

\begin{tabular}{|c|c|c|c|c|c|c|c|c|c|c|c|c|c|c|}
\hline ARRAir & $\mathrm{J}$ & $F$ & $M$ & A & $\mathrm{M}$ & $J$ & J & A & $s$ & 0 & $N$ & $D$ & $\begin{array}{l}\text { ANNUAL } \\
\text { APIOUNT }\end{array}$ & $\begin{array}{c}\text { AVERAGE } \\
\text { DAY }\end{array}$ \\
\hline 0.0 & 1.42 & 2.29 & 3.48 & 4.54 & 5.42 & 6.02 & 5.96 & 5.12 & 3.84 & 2.58 & 1.47 & 1.10 & 1317.3 & 3.6 \\
\hline 10.0 & 1.73 & 2.61 & 3.79 & 4.73 & 5.51 & 6.06 & 6.06 & 5.35 & 4.20 & 2.99 & 1.79 & 1.37 & 1406.9 & 3.9 \\
\hline 15.0 & 1.86 & 2.75 & 3.91 & 4.79 & 5.51 & 6.03 & 6.06 & 5.43 & 4.34 & 3.17 & 1.94 & 1.49 & 1440.4 & 3.9 \\
\hline 20.0 & 1.99 & 2.88 & 4.01 & 4.82 & 5.48 & 5.97 & 6.03 & 5.47 & 4.46. & 3.33 & 2.08 & 1.61 & 1466.1 & 4.0 \\
\hline 25.0 & 2.11 & 2.98 & 4.09 & 4.82 & 5.43 & 5.88 & 5.97 & 5.49 & 4.55 & 3.47 & 2.20 & 1.71 & 1483.5 & 4.1 \\
\hline 30.0 & 2.21 & 3.08 & 4.15 & 4.80 & 5.34 & 5.76 & 5.87 & 5.47 & 4.62 & 3.59 & 2.31 & 1.81 & 1492.6 & 4.1 \\
\hline 35.0 & 2.30 & 3.15 & 4.18 & 4.75 & 5.22 & 5.61 & 5.75 & 5.42 & 4.66 & 3.68 & 2.41 & 1.89 & 1493.2 & 4.1 \\
\hline 40.0 & 2.37 & 3.21 & 4.19 & 4.63 & 5.08 & 5.43 & 5.59 & 5.34 & 4.68 & 3.76 & 2.49 & 1.96 & 1485.4 & 4.1 \\
\hline 45.0 & 2.44 & 3.24 & 4.18 & 4.57 & 4.91 & 5.23 & 5.40 & 5.23 & 4.67 & 3.81 & 2.56 & 2.02 & 1469.5 & 4.0 \\
\hline 50.0 & 2.48 & 3.26 & 4.14 & 4.47 & 4.73 & 5.01 & 5.20 & 5.10 & 4.62 & 3.84 & 2.61 & 2.07 & 1447.0 & 4.0 \\
\hline 60.0 & 2.53 & 3.24 & 3.99 & 4.16 & 4.30 & 4.50 & 4.71 & 4.75 & 4.46 & 3.83 & 2.66 & 2.13 & 1378.1 & 3.8 \\
\hline 70.0 & 2.51 & 3.14 & 3.76 & 3.78 & 3.79 & 3.91 & 4.13 & 4.30 & 4.20 & 3.73 & 2.65 & 2.13 & 1279.0 & 3.5 \\
\hline 80.0 & 2.44 & 2.97 & 3.44 & 3.31 & 3.21 & 3.28 & 3.48 & 3.76 & 3.84 & 3.53 & 2.57 & 2.08 & 1153.8 & 3.2 \\
\hline 90.0 & 2.30 & 2.73 & 3.05 & 2.79 & 2.63 & 2.63 & 2.83 & 3.15 & 3.40 & 3.26 & 2.43 & 1.98 & 1009.5 & 2.8 \\
\hline
\end{tabular}


AVERAGE DAI:Y TOTAL TEZZESTRIAL INSOLATION ESTIMATES (KWH.SQ. M)

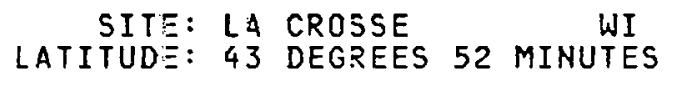

AVERAGE DAILY AMOUNTS BY MONTH

\begin{tabular}{|c|c|c|c|c|c|c|c|c|c|c|c|c|c|c|}
\hline ARRAY & $J$ & $\mathrm{~F}$ & M & A & $M$ & $J$ & $\mathrm{~J}$ & A & $S$ & 0 & iv & D & $\begin{array}{l}\text { ANNUAL } \\
\text { ANIOUNT }\end{array}$ & $\begin{array}{l}\text { AVERAGE } \\
\text { DAY }\end{array}$ \\
\hline 0.0 & 1.52 & 2.41 & 3.47 & 4.50 & 5.41 & 6.01 & 6.00 & 5.26 & 3.91 & 2.72 & 1.56 & 1.16 & 1338.2 & 3.7 \\
\hline 10.0 & 1.84 & 2.75 & 3.76 & 4.68 & 5.49 & 6.04 & 6.10 & 5.50 & 4.27 & 3.14 & 1.90 & 1.44 & 1429.5 & 3.9 \\
\hline 15.0 & 1.99 & 2.90 & 3.88 & 4.73 & 5.49 & 6.01 & 6.10 & 5.57 & 4.42 & 3.33 & 2.06 & 1.57 & 1463.6 & 4.0 \\
\hline 20.0 & 2.12 & 3.03 & 3.98 & 4.76 & 3.46 & 5.95 & 6.06 & 5.52 & 4.54 & 3.49 & 2.20 & 1.69 & 1489.7 & 4.1 \\
\hline 25.0 & 2.25 & 3.15 & 4.06 & 4.77 & 5.39 & 5.86 & 6.00 & 5.63 & 4.63 & 3.64 & 2.33 & 1.80 & 1507.4 & 4.1 \\
\hline 30.0 & 2.36 & 3.24 & 4.11 & 4.74 & 5.31 & 5.74 & 5.90 & 5.61 & 4.70 & 3.76 & 2.45 & 1.90 & 1516.6 & 4.2 \\
\hline 35.0 & 2.45 & 3.32 & 4.14 & 4.69 & 5.19 & 5.58 & 5.76 & 5.55 & 4.74 & 3.87 & 2.55 & 1.98 & 1517.2 & 4.2 \\
\hline 40.0 & 2.53 & 3.38 & 4.15 & 4.62 & 5.04 & 5.40 & 5.60 & 5.47 & 4.75 & 3.94 & 2.64 & 2.06 & 1509.2 & 4.1 \\
\hline 45.0 & 2.30 & 3.42 & 4.13 & 4.52 & 4.87 & 5.19 & 5.41 & 5.35 & 4.73 & 4.00 & 2.71 & 2.12 & 1493.2 & 4.1 \\
\hline 5.0 .0 & 2.55 & 3.44 & 4.09 & 4.40 & $4.6 \%$ & 4.97 & 5.20 & 5.21 & 4.69 & 4.03 & 2.76 & 2.17 & 1470.2 & 4.0 \\
\hline 60.0 & 2.59 & 3.41 & 3.94 & 4.10 & 4.26 & 4.46 & 4.71 & 4.85 & 4.52 & 4.01 & 2.82 & 2.23 & 1399.9 & 3.8 \\
\hline 70.0 & 2.68 & 3.31 & 3.70 & 3.71 & 3.76 & 3.87 & 4.12 & 4.38 & 4.25 & 3.90 & 2.80 & 2.23 & 1298.8 & 3.6 \\
\hline 80.0 & 2.59 & 3.12 & 3.38 & 3.25 & 3.17 & 3.24 & 3.47 & 3.82 & 3.88 & 3.70 & 2.72 & 2.18 & 1171.6 & 3.2 \\
\hline 90.0 & 2.45 & 2.87 & 2.99 & 2.73 & 2.59 & 2.59 & 2.81 & 3.19 & 3.43 & 3.40 & 2.56 & 2.07 & 1024.3 & 2.8 \\
\hline
\end{tabular}


AVERAGE [AILY TOTAL TERRESTRIAL INSOLATION ESTIMATES (KWH/SQ. M)

SITE: MADISON
LATITUDE: 43 DEGREES 8 MINUTES

AVERAGE DAILY AMOUNTS BY MONTH

\begin{tabular}{|c|c|c|c|c|c|c|c|c|c|c|c|c|c|c|}
\hline ARR AY & $\mathrm{J}$ & $F$ & $M$ & $A$ & $M$ & $J$ & $J$ & $A$ & $S$ & 0 & $N$ & D & $\begin{array}{l}\text { ANNUAL } \\
\text { AMOUNT }\end{array}$ & $\begin{array}{c}\text { AVERAGE } \\
\text { DAY }\end{array}$ \\
\hline 0.3 & 1.63 & 2.54 & 3.58 & 4.41 & 5.49 & 6.15 & 6.10 & 5.38 & 4.09 & 2.87 & 1.59 & 1.23 & 1372.8 & 3.8 \\
\hline 10.3 & 1.97 & 2.89 & 3.88 & 4.58 & 5.57 & 6.18 & 6.19 & 5.62 & 4.47 & 3.32 & 1.93 & 1.51 & 1465.7 & 4.0 \\
\hline 15.0 & 2.13 & 3.05 & 4.00 & 4.63 & 5.56 & 6.14 & 6.19 & 5.70 & 4.62 & 3.51 & 2.08 & 1.64 & 1500.2 & 4.1 \\
\hline 20.01 & 2.27 & 3.18 & 4.10 & 4.65 & 5.53 & 6.08 & 6.15 & 5.74 & 4.74 & 3.69 & 2.22 & 1.76 & 1526.4 & 4.2 \\
\hline 25.0 & 2.40 & 3.30 & 4.17 & 4.65 & 5.46 & 5.98 & 6.08 & 5.75 & 4.84 & 3.84 & 2.35 & 1.87 & 1544.0 & 4.2 \\
\hline 30.0 & 2.52 & 3.40 & 4.23 & 4.63 & 5.37 & 5.85 & 5.97 & 5.72 & 4.91 & 3.97 & 2.46 & 1.97 & 1552.9 & 4.3 \\
\hline 35.0 & 2.62 & 3.48 & 4.25 & 4.57 & 5.25 & 5.69 & 5.83 & 5.66 & 4.95 & 4.08 & 2.56 & 2.06 & 1553.0 & 4.3 \\
\hline 40.0 & 2.71 & 3.54 & 4.26 & 4.50 & 5.10 & 5.50 & 5.66 & 5.57 & 4.96 & 4.16 & 2.64 & 2.14 & 1544.2 & 4.2 \\
\hline 45.0 & 2.77 & 3.58 & 4.24 & 4.40 & 4.92 & 5.28 & 5.46 & 5.45 & 4.94 & 4.21 & 2.71 & 2.20 & 1527.4 & 4.2 \\
\hline 50.0 & 2.83 & 3.60 & 4.19 & 4.28 & 4.74 & 5.05 & 5.25 & 5.31 & 4.89 & 4.24 & 2.76 & 2.25 & 1503.2 & 4.1 \\
\hline 60.0 & 2.88 & 3.57 & 4.03 & 3.98 & 4.29 & 4.52 & 4.74 & 4.93 & 4.71 & 4.22 & 2.81 & 2.31 & 1429.8 & 3.9 \\
\hline 70.0 & 2.35 & 3.45 & 3.78 & 3.60 & 3.76 & 3.91 & 4.14 & 4.44 & 4.42 & 4.10 & 2.79 & 2.31 & 1324.9 & 3.6 \\
\hline 80.0 & 2.76 & 3.26 & 3.45 & 3.15 & 3.18 & 3.26 & 3.47 & 3.87 & 4.03 & 3.88 & 2.70 & 2.25 & 1193.6 & 3.3 \\
\hline 90.0 & 2.60 & 2.99 & 3.05 & 2.64 & 2.59 & 2.59 & 2.80 & 3.21 & 3.55 & 3.56 & 2.54 & 2.13 & 1041.5 & 2.9 \\
\hline
\end{tabular}


AVERAGE DAILY TOTAL TERRESTRIAL INSOLATION ESTIMATES (KWH/SQ. M)

SITE: MILWAUKEE
LATITUDE:
42 DEGREES 57 MINUTES

AVERAGE DAILY AMOUNTS BY MONTH

\begin{tabular}{|c|c|c|c|c|c|c|c|c|c|c|c|c|c|c|}
\hline ARRAY & $\mathrm{J}$ & $F$ & $M$ & A & $M$ & 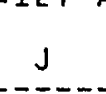 & $j$ & A & 5 & 0 & $N$ & $D$ & $\begin{array}{l}\text { ANNUAL } \\
\text { AMOUNT }\end{array}$ & $\begin{array}{c}\text { A VERAGE } \\
\text { DAY }\end{array}$ \\
\hline 0.0 & 1.51 & 2.32 & 3.43 & 4.55 & 5.57 & 6.23 & 5.19 & 5.42 & 4.13 & 2.86 & 1.66 & 1.20 & 1373.4 & 3.8 \\
\hline 10.0 & 1.81 & 2.62 & 3.71 & 4.72 & 5.65 & 6.26 & 6.28 & 5.66 & 4.51 & 3.30 & 2.01 & 1.46 & 1462.7 & 4.0 \\
\hline 15.0 & 1.95 & 2.75 & 3.82 & 4.77 & 5.64 & 6.22 & 5.28 & 5.73 & 4.66 & 3.49 & 2.17 & 1.59 & 1495.5 & 4.1 \\
\hline 20.0 & 2.07 & 2.87 & 3.91 & 4.80 & 5.61 & 6.16 & 6.24 & 5.77 & 4.78 & 3.67 & 2.32 & 1.70 & 1520.0 & 4.2 \\
\hline 25.0 & 2.18 & 2.97 & 3.98 & 4.80 & 5.54 & 6.06 & 6.16 & 5.78 & 4.88 & 3.82 & 2.45 & 1.80 & 1535.9 & 4.2 \\
\hline 30.0 & 2.28 & 3.05 & 4.03 & 4.77 & 5.45 & 5.92 & 6.05 & 5.75 & 4.95 & 3.94 & 2.57 & 1.90 & 1543.2 & 4.2 \\
\hline 35.0 & 2.37 & 3.11 & 4.05 & 4.72 & 5.52 & 5.76 & 5.91 & 5.70 & 4.99 & 4.05 & 2.68 & 1.98 & 1541.7 & 4.2 \\
\hline 40.0 & 2.44 & 3.15 & 4.05 & 4.64 & 5.27 & 5.56 & 5.74 & 5.60 & 5.00 & 4.13 & 2.76 & 2.05 & 1531.5 & 4.2 \\
\hline 45.0 & 2.50 & 3.13 & 4.03 & 4.54 & 4.59 & 5.34 & 5.54 & 5.48 & 4.98 & 4.18 & 2.84 & 2.10 & 1513.4 & 4.1 \\
\hline 50.3 & 2.54 & 3.23 & 3.98 & 4.41 & 4.80 & 5.11 & 5.32 & 5.34 & 4.93 & 4.21 & 2.89 & 2.15 & 1488.0 & 4.1 \\
\hline 60.0 & 2.58 & 3.15 & 3.83 & 4.10 & 4.34 & 4.57 & 4.30 & 4.95 & 4.74 & 4.19 & 2.94 & 2.20 & 1412.5 & 3.9 \\
\hline 70.0 & 2.55 & 3.05 & 3.59 & 3.70 & 3.81 & 3.94 & 4.18 & 4.46 & 4.45 & 4.06 & 2.92 & 2.20 & 1306.1 & 3.6 \\
\hline 80.0 & 2.46 & 2.8 .2 & 3.27 & 3.23 & 3.21 & 3.28 & 3.50 & 3.88 & 4.05 & 3.84 & 2.82 & 2.14 & 1173.9 & 3.2 \\
\hline 90.0 & 2.32 & 2.67 & 2.89 & 2.71 & $2 . E 1$ & 2.60 & 2.32 & 3.22 & 3.57 & 3.52 & 2.66 & 2.03 & 1021.6 & 2.8 \\
\hline
\end{tabular}


AVERAGE DAILY TOTAL TERRESTRIAL INSOLATION ESTIMATES (KAH/SQ. M)

$\begin{aligned} \text { SITE: } & \text { CHARLESTON } \\ \text { LATITUDE: } & 38 \text { DEGREES } 22 \text { MINUTES }\end{aligned}$

AVERAGE DAILY AMOUNTS BY MONTH

\begin{tabular}{|c|c|c|c|c|c|c|c|c|c|c|c|c|c|c|}
\hline ARRAY & $\mathrm{J}$ & $F$ & $M$ & $A$ & $M$ & $J$ & $\mathbf{J}$ & $A$ & $S$ & 0 & $N$ & D & $\begin{array}{l}\text { ANNUAL } \\
\text { ANOOUNT }\end{array}$ & $\begin{array}{c}\text { AVERAGE } \\
\text { DAY }\end{array}$ \\
\hline 0.0 & 1.57 & 2.23 & 3.18 & 4.28 & 5.16 & 5.60 & 5.30 & 4.78 & 4.00 & 3.07 & 1.93 & 1.39 & 1294.8 & 3.5 \\
\hline 10.0 & 1.79 & 2.44 & 3.37 & 4.39 & 5.19 & 5.59 & 5.33 & 4.92 & 4.29 & 3.45 & 2.27 & 1.63 & 1350.5 & 3.7 \\
\hline 15.0 & 1.89 & 2.52 & 3.44 & $4 . \div 2$ & 5.16 & 5.54 & 5.30 & 4.95 & 4.39 & 3.61 & 2.42 & 1.73 & 1382.5 & 3.8 \\
\hline 20.0 & 1.97 & 2.60 & 3.49 & $4 . \div 2$ & 5.11 & 5.45 & 5.25 & 4.96 & 4.48 & 3.76 & 2.55 & 1.83 & 1397.1 & 3.8 \\
\hline 25.0 & 2.05 & 2.66 & 3.53 & 4.80 & 5.03 & 5.34 & 5.16 & 4.94 & 4.53 & 3.88 & 2.67 & 1.92 & 1404.2 & 3.8 \\
\hline 30.0 & 2.12 & 2.71 & 3.54 & 4.35 & 4.92 & 5.21 & 5.05 & 4.89 & 4.57 & 3.97 & 2.77 & 1.99 & 1403.6 & 3.8 \\
\hline 35.0 & 2.17 & 2.74 & 3.54 & 4.28 & 4.79 & 5.04 & 4.91 & 4.81 & 4.57 & 4.05 & 2.86 & 2.06 & 1395.5 & 3.8 \\
\hline $40.0^{\prime}$ & 2.21 & 2.75 & 3.52 & $4 . \approx 9$ & 4.63 & 4.85 & 4.75 & 4.71 & 4.55 & 4.10 & 2.93 & 2.11 & 1380.2 & 3.8 \\
\hline 45.0 & 2.24 & 2.76 & 3.48 & 4.18 & 4.46 & 4.65 & 4.58 & 4.59 & 4.51 & 4.13 & 2.99 & 2.15 & 1358.6 & 3.7 \\
\hline 50.0 & 2.26 & 2.74 & 3.42 & 3.55 & 4.27 & 4.43 & 4.38 & 4.44 & 4.44 & 4.13 & 3.02 & 2.18 & 1329.6 & 3.6 \\
\hline 60.0 & 2.25 & 2.68 & 3.25 & 3.64 & 3.83 & 3.93 & 3.92 & 4.08 & 4.22 & 4.06 & 3.0 .4 & 2.20 & 1250.9 & 3.4 \\
\hline 70.0 & 2.19 & 2.55 & 3.01 & 3.25 & 3.32 & 3.35 & 3.39 & 3.64 & 3.91 & 3.90 & 2.98 & 2.17 & 1145.5 & 3.1 \\
\hline 80.0 & 2.09 & 2.37 & 2.71 & 2.81 & 2.78 & 2.78 & 2.83 & 3.13 & 3.52 & 3.64 & 2.85 & 2.09 & 1023.0 & 2.8 \\
\hline 90.0 & 1.94 & 2.15 & 2.37 & 2.53 & 2.22 & 2.19 & 2.26 & 2.58 & 3.06 & 3.30 & 2.66 & 1.95 & 882.3 & 2.4 \\
\hline
\end{tabular}


AVERAGE DAILY TOTAL TERRESTRIAL INSOLATION ESTIMATES (KWH/SQ. M)

SITE: HUNTIVGTON
LATITUDE: 38 DESREES 22 MINUTES

AYERAGE DAILY AMOJNTS BY MONTH

\begin{tabular}{|c|c|c|c|c|c|c|c|c|c|c|c|c|c|c|}
\hline ARRAY & $\mathrm{J}$ & $F$ & $M$ & A & $M$ & $41 L A$ & $\mathrm{~J}$ & roun & $\mathrm{s}$ & 0 & $N$ & $D$ & $\begin{array}{l}\text { ANNUAL } \\
\text { AMOUNT }\end{array}$ & $\begin{array}{c}\text { AVERAGE } \\
\text { DAY }\end{array}$ \\
\hline 0.0 & 1.65 & 2.39 & 3.36 & 4.57 & 5.37 & 5.82 & 5.58 & 4.98 & 4.11. & 3.17 & 2.01 & 1.47 & 1355.8 & 3.7 \\
\hline 10.0 & 1.90 & 2.63 & 3.58 & 4.70 & 5.42 & 5.80 & 5.61 & 5.14 & 4.41 & 3.57 & 2.36 & 1.74 & .1427 .4 & 3.9 \\
\hline 15.0 & 2.01 & 2.73 & 3.65 & 4.73 & 5.37 & 5.74 & 5.58 & 5.17 & 4.52 & 3.74 & 2.52 & 1.86 & 1451.7 & 4.0 \\
\hline 20.0 & 2.11 & 2.82 & 3.71 & 4.73 & 5.33 & 5.66 & 5.52 & 5.18 & 4.61 & 3.89 & 2.67 & 1.97 & 1468.2 & 4.0 \\
\hline 25.0 & 2.19 & 2.89 & 3.75 & 4.71 & 5.25 & 5.54 & 5.43 & 5.15 & 4.67 & 4.02 & 2.80 & 2.07 & 1476.5 & 4.0 \\
\hline 30.0 & 2.27 & 2.95 & 3.77 & 4.66 & 5.14 & 5.40 & 5.31 & 5.10 & 4.70 & 4.13 & 2.91 & 2.16 & 1476.8 & 4.0 \\
\hline 35.0 & 2.33 & 2.99 & 3.77 & 4.59 & 5.00 & 5.23 & 5.16 & 5.03 & 4.71. & 4.21 & 3.00 & 2.24 & 1469.0 & 4.0 \\
\hline 40.0 & 2.38 & 3.01 & 3.75 & 4.510 & 4.83 & 5.03 & 4.99 & 4.92 & 4.69 & 4.26 & 3.08 & 2.30 & 1453.7 & 4.0 \\
\hline 45.0 & 2.41 & 3.02 & 3.71 & 4.38 & 4.65 & 4.82 & 4.81 & 4.79 & $4.64 \%$ & 4.29 & 3.14 & 2.35 & 1431.4 & 3.9 \\
\hline 50.0 & 2.43 & 3.01 & 3.65 & 4.24 & 4.45 & 4.59 & 4.60 & 4.64 & $4.57^{7}$ & 4.29 & 3.18 & 2.38 & 1401.4 & 3.8 \\
\hline 60.0 & 2.43 & 2.94 & 3.47 & 3.90 & 3.99 & 4.06 & 4.11 & 4.26 & 4.35 & 4.23 & 3.20 & 2.41 & 1319.1 & 3.6 \\
\hline 70.0 & 2.37 & 2.80 & $3: 22$ & 3.48 & 3.45 & 3.46 & 3.55 & 3.80 & 4.03 & 4.06 & 3.14 & 2.39 & 1209.4 & 3.3 \\
\hline 80.0 & 2.26 & 2.61 & 2.90 & 3.00 & 2.88 & 2.86 & 2.96 & 3.27 & 3.63 & 3.79 & 3.01 & 2.29 & 1079.0 & 3.0 \\
\hline 90.0 & 2.10 & 2.36 & 2.53 & 2.48 & 2.30 & 2.25 & 2.35 & 2.68 & 3.15 & 3.44 & 2.80 & 2.15 & 930.1 & 2.5 \\
\hline
\end{tabular}


AVERAGE DAILY TOTAL TERRESTRIAL INSOLATION ESTIMATES (KLIH/SQ. M)

SITE:
LATITUDE:
42 DESPER
LATEES 55 MINUTES

AVERAGE DAILY AMOUNTS BY MONTH

\begin{tabular}{|c|c|c|c|c|c|c|c|c|c|c|c|c|c|c|}
\hline ARRAY & $\mathrm{J}$ & $F$ & $M$ & A & $M$ & $\mathrm{~J}$ & $J$ & A & $s$ & 0 & $N$ & & $\begin{array}{l}\text { ANNUAL } \\
\text { AHOUNT }\end{array}$ & $\begin{array}{c}\text { AUERAGE } \\
\text { DAY }\end{array}$ \\
\hline 0.0 & 2.16 & 3.19 & 4.54 & 5.83 & 6.94 & 7.88 & 7.99 & 7.02 & 5.52 & $3.84^{\circ}$ & 2.42 & 1.87 & 1803.8 & 4.9 \\
\hline 10.0 & 2.70 & 3.71 & 4.99 & 6.10 & 7.06 & 7.93 & 8.15 & 7.40 & 6.14 & 4.56 & 3.08 & 2.45 & 1958.2 & 5.4 \\
\hline 15.0 & 2.95 & 3.94 & 5.17 & 6.18 & 7.06 & 7.89 & 8.15 & 7.52 & 6.39 & $4: 88$ & 3.39 & 2.72 & 2018.2 & 5.5 \\
\hline 20.0 & 3.18 & 4.15 & 5.32 & 6.23 & 7.02 & 7.80 & 8.11 & 7.60 & 6.61 & 5.17 & 3.67 & 2.97 & 2066.0 & 5.7 \\
\hline 25.0 & 3.39 & 4.33 & 5.44 & 6.24 & 6.94 & 7.67 & 8.01 & 7.63 & 6.78 & 5.43 & 3.93 & 3.20 & 2101.2 & 5.8 \\
\hline 30.0 & 3.58 & 4.48 & 5.53 & 6.21 & 6.82 & 7.50 & 7.87 & 7.61 & 6.91 & 5.65 & 4.16 & 3.42 & 2123.6 & 5.8 \\
\hline 35.0 & 3.74 & 4.60 & 5.58 & 6.1 .5 & 6.66 & 7.28 & 7.68 & 7.54 & 7.00 & 5.84 & 4.37 & 3.60 & 2132.8 & 5.8 \\
\hline 40.0 & 3.89 & 4.70 & 5.60 & 6.05 & 6.46 & 7.01 & 7.44 & 7.43 & 7.04 & 5.98 & 4.55 & 3.77 & 2128.9 & 5.8 \\
\hline 45.0 & 4.00 & 4.77 & 5.59 & 5.93 & 6.23 & 6.72 & 7.17 & 7.28 & 7.03 & 6.09 & 4.69 & 3.91 & 2113.2 & 5.8 \\
\hline 50.0 & 4.09 & 4.80 & 5.54 & 5.77 & 5.98 & 6.41 & 6.87 & 7.08 & 6.98 & 6.16 & 4.81 & 4.02 & 2086.1 & 5.7 \\
\hline 60.0 & 4.19 & 4.79 & 5.34 & 5.35 & 5.38 & 5.69 & 6.16 & 6.57 & 6.75 & $6: 17$ & 4.95 & 4.17 & 1994.0 & 5.5 \\
\hline 70.0 & 4.19 & 4.65 & 5.02 & 4.82 & $4.68^{\circ}$ & 4.84 & 5.31 & 5.89 & 6.35 & 6.02 & 4.95 & 4.21 & 1853.7 & 5.1 \\
\hline 80.0 & 4.07 & 4.40 & 4.58 & 4.13 & 3.89 & 3.96 & 4.37 & 5.08 & 5.79 & 5.72 & 4.82 & 4.13 & 1672.3 & 4.6 \\
\hline 90.0 & 3.84 & 4.04 & 4.03 & 3.46 & 3.09 & 3.04 & 3.42 & 4.16 & 5.08 & 5.26 & 4.56 & 3.94 & 1457.7 & 4.0 \\
\hline & & & ; & & & & $c-2$ & & & & & & & . \\
\hline
\end{tabular}


AVERAGE DAILY TOTAL TERRESTRIAL INSOLATION ESTIMATES (KWH/SQ. M)

SITE: CHEYENNE

LATITUIIE: 41 DEGREES 9 MINUTES

AVERAGE DAILY AMOUNTS BY MONTH

\begin{tabular}{|c|c|c|c|c|c|c|c|c|c|c|c|c|c|c|}
\hline ARRAY & $\therefore$ & $F$ & $M$ & A & $M$ & $J$ & $J$ & A & $\mathrm{S}$ & 0 & $N$ & D & $\begin{array}{l}\text { ANNUAL } \\
\text { AMOUNT }\end{array}$ & $\begin{array}{c}\text { AVERAGE } \\
\text { DAY }\end{array}$ \\
\hline 0.0 & 2.42 & 3.36 & 4.52 & 5.59 & 5.29 & 7.12 & 7.02 & 6.20 & 5.26 & 3.91 & 2.60 & 2.12 & 1717.6 & 4.7 \\
\hline 10.0 & 3.00 & 3.87 & 4.93 & 5.81 & 3.36 & 7.13 & 7.12 & 6.48 & 5.78 & 4.59 & 3.26 & 2.74 & 1859.7 & 5.1 \\
\hline 15.0 & 3.26 & 4.10 & 5.09 & 5.88 & 6.35 & 7.08 & 7.11 & 6.56 & 5.99 & 4.89 & 3.56 & 3.02 & 1914.8 & 5.2 \\
\hline 20.0 & 3.50 & 4.30 & 5.22 & 5.91 & 6.30 & 7.00 & 7.05 & 6.60 & 6.17 & 5.16 & 3.84 & 3.29 & 1958.4 & 5.4 \\
\hline 25.0 & 3.73 & 4.47 & 5.32 & 5.90 & 6.22 & 6.87 & 6.95 & 6.60 & 6.30 & 5.39 & 4.10 & 3.53 & 1990.3 & 5.5 \\
\hline 30.0 & 3.92 & 4.61 & 5.39 & 5.87 & 6.10 & 6.70 & 6.82 & 6.56 & 6.40 & 5.59 & 4.32 & 3.76 & 2010.3 & 5.5 \\
\hline 35.0 & 4.10 & 4.73 & 5.43 & 5.80 & 5.94 & 6.49 & 6.64 & 6.49 & 6.46 & 5.76 & 4.52 & 3.95 & 2018.2 & 5.5 \\
\hline 40.0 & 4.24 & 4.82 & 5.44 & 5.39 & 5.75 & 6.25 & 6.43 & 6.37 & 6.48 & 5.89 & 4.69 & 4.13 & 2013.9 & 5.5 \\
\hline 45.0 & 4.36 & 4.88 & 5.41 & 5.56 & 5.55 & 5.99 & 6.19 & 6.23 & 6.45 & 5.97 & 4.83 & 4.27 & 1999.3 & 5.5 \\
\hline 50.0 & 4.45 & 4.90 & 5.35 & 5.40 & 5.32 & 5.71 & 5.93 & 6.05 & 6.39 & 6.02 & 4.94 & 4.39 & 1973.2 & 5.4 \\
\hline 60.0 & 4.54 & 4.87 & 5.14 & 4.99 & 4.78 & 5.05 & 5.30 & 5.58 & 6.14 & 6.01 & 5.06 & 4.53 & $1885: 9$ & 5.2 \\
\hline 70.0 & 4.52 & 4.71 & 4.81 & 4.47 & 4.14 & 4.29 & 4.57 & 4.99 & 5.74 & 5.83 & 5.04 & 4.55 & 1753.9 & 4.8 \\
\hline 80.0 & 4.37 & 4.43 & 4.36 & 3.86 & 3.45 & 3.52 & 3.77 & 4.30 & 5.21 & 5.51 & 4.88 & 4.45 & 1585.1 & 4.3 \\
\hline 90.0 & 4.12 & 4.05 & 3.82 & 3.18 & 2.74 & 2.71 & 2.96 & 3.51 & 4.55 & 5.04 & 4.60 & 4.23 & 1383.8 & 3.8 \\
\hline
\end{tabular}


AVERAGE DAILY TOTAL TERRESTTRIAL INSOLATION ESTIMATES (HWH/SQ. M)

SITE: ROCK SPRINGS

LATITUDE: 41 DEGREES 36 MINUTES

AVERAGE DAILY AMOUNTS BY MONTH

\begin{tabular}{|c|c|c|c|c|c|c|c|c|c|c|c|c|c|c|}
\hline ARRAY & $\mathrm{J}$ & $F$ & M & A & $M$ & $\mathbf{J}$ & $J$ & A & 5 & 0 & $N$ & D & $\begin{array}{l}\text { ANNUAL } \\
\text { AMOUNT }\end{array}$ & $\begin{array}{c}\text { AVERAGE } \\
\text { DAY }\end{array}$ \\
\hline 0.0 & 2.32 & 3.43 & 4.82 & 6.13 & 7.38 & 8.11 & 8.03 & 7.06 & 5.78 & 4.11 & 2.61 & 2.05 & 1884.3 & 5.2 \\
\hline 10.0 & 2.88 & 3.97 & 5.29 & 6.40 & 7.50 & 8.14 & 8.16 & 7.42 & 6.41 & 4.87 & 3.30 & 2.66 & 2041.1 & 5.6 \\
\hline 15.0 & 3.13 & 4.21 & 5.48 & 6.48 & 7.49 & 8.09 & 8.16 & 7.53 & 6.67 & 5.20 & 3.61 & 2.94 & 2101.4 & 5.8 \\
\hline 20.0 & 3.37 & 4.42 & 5.63 & 6.53 & 7.44 & 7.99 & 8.10 & 7.59 & 6.89 & 5.50 & 3.90 & 3.20 & 2149.0 & 5.9 \\
\hline 25.0 & 3.58 & 4.61 & 5.75 & $6 . .53$ & 7.34 & 7.85 & 7.99 & 7.61 & 7.06 & 5.77 & 4.17 & 3.44 & 2183.3 & 6.0 \\
\hline 30.0 & 3.77 & 4.77 & 5.84 & 6.50 & 7.20 & 7.65 & 7.84 & 7.58 & 7.19 & 5.99 & 4.41 & 3.66 & 2204.1 & 6.0 \\
\hline 35.0 & 3.94 & 4.89 & 5.89 & 6.53 & 7.02 & 7.42 & 7.63 & 7.50 & 7.27 & 6.18 & 4.62 & 3.85 & 2211.3 & 6.1 \\
\hline 40.0 & 4.08 & 4.99 & 5.90 & 6.32 & 6.80 & 7.13 & 7.39 & 7.38 & 7.30 & 6.33 & 4.80 & 4.02 & 2204.8 & 6.0 \\
\hline 45.0 & 4.19 & 5.05 & 5.88 & $6 . i 7$ & 6.55 & 6.83 & 7.11 & 7.21 & 7.28 & 6.43 & 4.95 & 4.17 & 2186.8 & 6.0 \\
\hline 50.0 & 4.28 & 5.09 & 5.83 & 6.00 & 6.27 & 6.50 & 6.80 & 7.01 & 7.22 & 6.50 & 5.06 & 4.28 & 2156.1 & 5.9 \\
\hline 60.0 & 4.37 & 5.06 & 5.61 & 5.5 .5 & 5.61 & 5.73 & 6.07 & 6.47 & 6.96 & 6.50 & 5.19 & 4.43 & 2055.4 & 5.6 \\
\hline 70.0 & 4.35 & 4.90 & 5.25 & 4.97 & 4.84 & 4.84 & 5.20 & 5.78 & 6.53 & 6.32 & 5.18 & 4.45 & 1904.8 & 5.2 \\
\hline 80.0 & 4.22 & 4.62 & 4.77 & 4.29 & 3.99 & 3.93 & 4.26 & 4.96 & 5.92 & 5.98 & 5.03 & 4.36 & 1713.0 & 4.7 \\
\hline 90.0 & 3.97 & 4.23 & 4.18 & 3.52 & 3.12 & 2.97 & 3.29 & 4.03 & 5.17 & 5.49 & 4.74 & 4.14 & 1486.0 & 4.1 \\
\hline
\end{tabular}


AVERAGE DAILY TOTAL TERRESTRIAL INSOLATION ESTIMATES (KWH/SQ. M)

SITE: SHERIDAN WY W

LATITUDE: 44 DEGREES 46 MINUTES

AVERAG D DAILY AMOUNTS BY MONTH

\begin{tabular}{|c|c|c|c|c|c|c|c|c|c|c|c|c|c|c|}
\hline ARRAY & 3 & $F$ & $M$ & $A$ & $M$ & $J$ & $J$ & A & $S$ & 0 & $N$ & D & $\begin{array}{l}\text { ANNUAL } \\
\text { AMOUNT }\end{array}$ & $\begin{array}{c}\text { A YERAGE } \\
\text { DAY }\end{array}$ \\
\hline 0.0 & 1.63 & 2.49 & 3.79 & 4.85 & 5.93 & 6.80 & 7.34 & 6.33 & 4.73 & 3.17 & 1.86 & 1.39 & 1534.0 & 4.2 \\
\hline 10.0 & 2.03 & 2.86 & 4.15 & 5.07 & 6.04 & 6.86 & 7.50 & 6.68 & 5.26 & 3.75 & 2.35 & 1.80 & 1656.6 & 4.5 \\
\hline 15.0 & 2.21 & 3.03 & 4.30 & 5.13 & 6.05 & 6.83 & 7.51 & 6.80 & 5.48 & 4.01 & 2.58 & 1.99 & 1704.0 & 4.7 \\
\hline $2 i .0$ & 2.37 & 3.18 & 4.42 & 5.17 & 6.02 & 6.77 & 7.49 & 6.87 & 5.66 & 4.24 & 2.79 & 2.17 & 1741.6 & 4.8 \\
\hline 25.0 & 2.52 & 3.31 & 4.52 & 5.13 & 5.96 & 6.67 & 7.41 & 6.91 . & 5.81 & 4.45 & 2.98 & 2.33 & 1769.0 & 4.8 \\
\hline 30.0 & 2.66 & 3.42 & 4.59 & 5.15 & .3 .87 & 6.53 & 7.30 & 6.90 & 5.92 & 4.63 & 3.16 & 2.48 & $/ 1785.9$ & 4.9 \\
\hline 35.0 & 2.78 & 3.51 & 4.63 & 5.12 & 5.77 & 6.36 & 7.14 & 6.85 & 6.00 & 4.78 & 3.31 & 2.61 & 1792.3 & 4.9 \\
\hline 40.0 & 2.88 & 3.58 & 4.65 & 5.04 & 5.5 .3 & 6.15 & 6.94 & 6.76 & 6.04 & 4.90 & 3.44 & 2.73 & 1788.0 & 4.9 \\
\hline 45.0 & 2.97 & 3.63 & 4.64 & 4.94 & 5.40 & 5.91 & 6.69 & 6.63 & 6.04 & 4.99 & 3.55 & 2.83 & 1773.2 & 4.9 \\
\hline 50.0 & 3.03 & 3.65 & 4.60 & 4.811 & 5.20 & 5.66 & 6.44 & 6.46 & 6.00 & 5.05 & 3.64 & 2.91 & 1750.3 & 4.8 \\
\hline 60.0 & 3.11 & 3.64 & 4.45 & 4.49 & 4.72 & 5.08 & 5.82 & 6.03 & 5.82 & 5.06 & 3.74 & $3 / .02$ & 1673.7 & 4.6 \\
\hline 70.0 & 3.10 & 3.54 & 4.19 & 4.0 .7 & 4.15 & 4.39 & 5.07 & 5.45 & 5.49 & 4.95 & 3.75 & 3.04 & 1558.3 & 4.3 \\
\hline 80.0 & 3.02 & 3.35 & 3.84 & 3.57 & $3.5=0$ & 3.65 & 4.22 & 4.74 & 5.03 & 4.71 & 3.65 & 2.99 & 1408.7 & 3.9 \\
\hline 90.0 & 2.86 & 3.09 & 3.41 & 3.00 & 2.85 & 2.90 & 3.37 & 3.94 & 4.45 & 4.35 & 3.46 & 2.86 & 1233.7 & 3.4 \\
\hline
\end{tabular}

